\title{
FACE RECOGNITION VENDOR TEST 2002
}

\section{Overview and Summary \\ March 2003}

\section{P. Jonathon Phillips ${ }^{1,2}$, Patrick Grother ${ }^{2}$, Ross J. Micheals ${ }^{2}$, Duane M. Blackburn ${ }^{3}$, Elham Tabassi ${ }^{2}$, Mike Bone ${ }^{4}$}

${ }^{1}$ DARPA

3701 North Fairfax Dr.

Arlington, VA 22203

${ }^{2}$ National Institute of Standards and Technology

100 Bureau Drive, Stop 8940

Gaithersburg, MD 20899

${ }^{3}$ DoD Counterdrug Technology Development Program Office

17320 Dahlgren Rd, Code B07

Dahlgren, VA 22448

${ }^{4}$ NAVSEA Crane Division

300 Highway 361, Code 4041

Crane, IN 47522

\section{Sponsors and Supporters:}

Defense Advanced Research Projects Agency Department of State

Federal Bureau of Investigation

National Institute of Justice

National Institute of Standards and Technology

Transportation Security Administration

ONDCP Counterdrug Technology Assessment Center

United States Customs Service
Department of Energy

Drug Enforcement Administration

Immigration and Naturalization Service

United States Secret Service

Technical Support Working Group

Australian Customs

Canadian Passport Office

United Kingdom Biometric Working Group 


\section{This page intentionally blank.}


Face Recognition Vendor Test 2002:

Overview and Summary

\title{
P. Jonathon Phillips ${ }^{1,2}$, Patrick Grother ${ }^{2}$, Ross Micheals ${ }^{2}$, Duane M. Blackburn ${ }^{3}$, Elham Tabassi ${ }^{2}$, J. Mike Bone ${ }^{4}$
}

\author{
${ }^{1}$ DARPA \\ 3701 North Fairfax Drive \\ Arlington, VA 22203 \\ ${ }^{2}$ National Institute of Standards and Technology \\ 100 Bureau Drive; Stop 8940 \\ Gaithersburg, MD 20899 \\ ${ }^{3}$ DoD Counterdrug Technology Development Program Office \\ 17320 Dahlgren Road, Code B07 \\ Dahlgren, VA 22448 \\ ${ }^{4}$ NAVSEA Crane Division \\ 300 Highway 361, Code 4041 \\ Crane, IN 47522
}

\begin{abstract}
The Face Recognition Vendor Test (FRVT) 2002 is an independently administered technology evaluation of mature face recognition systems. FRVT 2002 provides performance measures for assessing the capability of face recognition systems to meet requirements for large-scale real world applications. Ten commercial firms participated in FRVT 2002. FRVT 2002 computed performance statistics on an extremely large dataset-121,589 operational facial images of 37,437 individuals. FRVT 2002 1) characterized identification and watch list performance as a function of database size, 2) estimated the variability in performance for different groups of people, 3) characterized performance as a function of elapsed time between enrolled and new images of a person and 4) investigated the effect of demographics on performance. FRVT 2002 shows that recognition from indoor images has made substantial progress since FRVT 2000. Demographic results show that males are easier to recognize than females and that older people are easier to recognize than younger people. FRVT 2002 also assessed the impact of two techniques for improving face recognition: three-dimensional morphable models, and face recognition from video sequences. Results show that three-dimensional morphable models increases performance, and that face recognition from video sequences offers only a limited increase in performance over still images. For FRVT 2002, a new XML-based evaluation protocol was developed. This protocol is flexible and supports evaluations of biometrics in general.
\end{abstract}

\footnotetext{
${ }^{1}$ Please direct correspondence to Jonathon Phillips at jphillips@darpa.mil or jonathon@nist.gov.
} 


\section{Introduction \& Executive Summary}

The Face Recognition Vendor Test (FRVT) 2002 was a large-scale evaluation of automatic face recognition technology. The primary objective of FRVT 2002 was to provide performance measures for assessing the ability of automatic face recognition systems to meet real-world requirements. FRVT 2002 measures performance of the core capabilities of face recognition technology. It provides an assessment of the potential for face recognition technology to meet the requirements for operational applications. However, it does not address many application specific issues and, therefore, is not a "buyer's guide" to face recognition.

FRVT 2002 was an independently administered technology evaluation. Ten participants were evaluated under the direct supervision of the FRVT 2002 organizers at a U.S. Government facility in Dahlgren, Virginia in July and August 2002. Participants were tested using data that they had not previously seen.

The heart of the FRVT 2002 was the high computational intensity test (HCInt). The HCInt consisted of 121,589 operational images of 37,437 people. The images were provided from the U.S. Department of State's Mexican non-immigrant Visa archive. From this data, real-world performance figures on a very large data set were computed. Performance statistics were computed for verification, identification, and watch list tasks ${ }^{2}$.

The most likely application of face recognition technology would use images taken indoors. FRVT 2002 results show that normal changes in indoor lighting do not significantly affect performance of the top systems. Approximately the same performance results were obtained using two indoor data sets, with different lighting, in FRVT 2002. In both experiments, the best performer had a $90 \%$ verification rate at a false accept rate of $1 \%$.

- For the best face recognition systems, the recognition rate for faces captured outdoors, at a false accept rate of $1 \%$, was only $50 \%$. Thus, face recognition from outdoor imagery remains a research challenge area.

- The FRVT 2002 database also consisted of images of the same person taken on different days. The performance results in this case, using indoor imagery, shows improvement in the capabilities of the face recognition systems over the last two years. Compared with similar experiments conducted two years earlier in FRVT 2000, the results of FRVT 2002 indicate there has been a $50 \%$ reduction in error rates ${ }^{3}$.

A very important question for real-world applications is the rate of decrease in performance as time increases between the acquisition of the database of image and new images presented to a system. FRVT 2002 found that for the top systems, performance degraded at approximately $5 \%$ points per year.

One open question in face recognition is: How does database and watch list size effect performance? Because of the large number of people and images in the FRVT 2002 data set, we were able to report the first large-scale results on this question. For the best system, the top-rank identification rate was $85 \%$ on a database of 800 people, $83 \%$ on a database of 1,600 , and $73 \%$ on a database of 37,437 . For every doubling of database size, performance decreases by two to three overall percentage points. In mathematical terms, identification performance decreases linearly with respect to the logarithm of the database size.

\footnotetext{
${ }^{2}$ See Section 4 for an overview of the verification, identification and watch list tasks.

${ }^{3}$ D. M. Blackburn, J. M. Bone, and P. J. Phillips (2001), FRVT 2000 Report, Technical Report, http://www.frvt.org.
} 
A similar effect was observed for the watch list task. As the watch list size increases, performance decreases. For the best system, the identification and detection rate was $77 \%$ at a false alarm rate of $1 \%$ for a watch list of 25 people. For a watch list of 300 people, the identification and detection rate was $69 \%$ at a false alarm rate of $1 \%$. In general, a watch list with 25 to 50 people will perform better than a larger size watch list.

Previous evaluations have reported face recognition performance as a function of imaging properties. For example, previous reports compared the differences in performance when using indoor versus outdoor images, or frontal versus non-frontal images. FRVT 2002, for the first time, examined the effects of demographics on performance. Two major effects were found. First, recognition rates for males were higher than females. For the top systems, identification rates for males were $6 \%$ to $9 \%$ points higher than that of females. For the best system, identification performance on males was $78 \%$ and for females was $79 \%$. Second, recognition rates for older people were higher than younger people. For 18 to 22 year olds the average identification rate for the top systems was $62 \%$, and for 38 to 42 year olds was $74 \%$. For every ten years increase in age, on average performance increases approximately $5 \%$ through age 63 . All identification rates were computed from a database of 37,437 individuals.

Since FRVT 2000, new techniques and approaches to assist face recognition have emerged. FRVT 2002 looked at two of these new techniques. The first was the three-dimensional morphable models technique of Blanz and Vetter. Morphable models are a technique for improving recognition of non-frontal images. We found that Blanz and Vetter's technique significantly increased recognition performance. The second technique is recognition from video sequences. We found that, using FRVT 2002 data sets, recognition performance using video sequences was the same as the performance using still images.

In summary, the key lessons learned in FRVT 2002 were:

- Given reasonable controlled indoor lighting, the current state of the art in face recognition is $90 \%$ verification at a $1 \%$ false accept rate.

- The use of morphable models can significantly improve non-frontal face recognition.

- Watch list performance decreases as a function of size - performance using smaller watch lists is better than performance using larger watch lists.

- In face recognition applications, accommodations should be made for demographic information since characteristics such as age and sex can significantly affect performance.

These findings are discussed in detail in the other FRVT 2002 documents. The complete FRVT 2002 report has three volumes: Summary and Overview, Evaluation Report, and Technical Appendices (all three are available at http://www.frvt.org). This document, the Summary and Overview, briefly presents the key results from the FRVT 2002. The Evaluation Report is a detailed description of FRVT 2002 procedures, experiments, and results. The Technical Appendices provide supplementary material, detailed documentation of the FRVT 2002 evaluation protocol, participant system and software descriptions, and participant responses to the FRVT 2002 (pre-release versions of the Evaluation Report and Technical Appendices).

\section{FRVT 2002 Design}

FRVT 2002 was designed to allow participation by as many face recognition research groups and companies as possible. FRVT 2002 consisted of two sub-tests - the high computational intensity (HCInt) test and medium computational intensity (MCInt) test. Each sub-test was designed to encourage broad participation in the evaluation. The HCInt was designed to evaluate the performance of state-of-the-art systems on extremely challenging real-world problems. The MCInt was designed to provide an understanding of a participant's capability to perform face recognition tasks with several different formats of imagery (still and video) under varying conditions. The MCInt was also designed to help identify promising new face recognition technologies not identified in the HCInt. The HCInt had to be performed on the equivalent of three high-end workstations, the MCInt on a single workstation. Technical specifications for each participant's HCInt and MCInt systems are provided in the Face Recognition Vendor Test 2002 Technical Appendices. Participants were given 11 days to complete each test. 
FRVT 2002 was announced on 25 April 2002 and was open to all developers and providers of core face recognition technology. This included academia, research laboratories, and commercial companies.

Participants could take the HCInt, MCInt, or both. The participants and the tests they took are provided in Table $1^{4}$. FRVT 2002 was administered at the U.S. Naval base at Dahlgren, Virginia between 10 July and 9 August 2002.

Table 1. FRVT 2002 participants and tests completed.

\begin{tabular}{lcc}
\hline \multicolumn{1}{c}{ Participant } & MCInt & HCInt \\
\hline AcSys Biometrics Corp & $\mathrm{X}$ & \\
Cognitec Systems GmbH & $\mathrm{X}$ & $\mathrm{X}$ \\
C-VIS Computer Vision und Automation GmbH & $\mathrm{X}$ & $\mathrm{X}$ \\
Dream Mirh Co., Ltd & $\mathrm{X}$ & $\mathrm{X}$ \\
Eyematic Interfaces Inc. & $\mathrm{X}$ & $\mathrm{X}$ \\
Iconquest & $\mathrm{X}$ & \\
Identix & $\mathrm{X}$ & $\mathrm{X}$ \\
Imagis Technologies Inc. & $\mathrm{X}$ & $\mathrm{X}$ \\
Viisage Technology & $\mathrm{X}$ & $\mathrm{X}$ \\
VisionSphere Technologies Inc. & $\mathrm{X}$ & $\mathrm{X}$ \\
\hline
\end{tabular}

All images and video sequences in FRVT 2002 were sequestered prior to the test and had not been seen by any participant. Testing on sequestered data has a number of advantages. First it provides a level playing field. Second, it ensures that systems are evaluated on the general face recognition task, not the ability to tune a system to a particular data set. FRVT 2002 was administered under strict U.S. Government supervision.

\section{Image Data Sets}

This section describes the data sets used in FRVT 2002. A common aspect of all images used in FRVT 2002 is that they contained the face of exactly one individual. The HCInt data set is a subset of a much larger collection provided by the Visa Services Directorate, Bureau of Consular Affairs of the U.S. Department of State. The HCInt data set consisted of 121,589 images of 37,437 individuals with at least three images of each person. The images are of good quality and were gathered in a consistent manner. The background is universally uniform. The names of the individuals were encoded to protect the privacy of the subjects. Due to privacy considerations, representative, not actual, images of the data set are shown in Figure 1.

\footnotetext{
${ }^{4}$ The identification of any commercial product or trade name does not imply endorsement or recommendation by the National Institute of Standards and Technology or any other FRVT 2002 Sponsor or Supporter.
} 

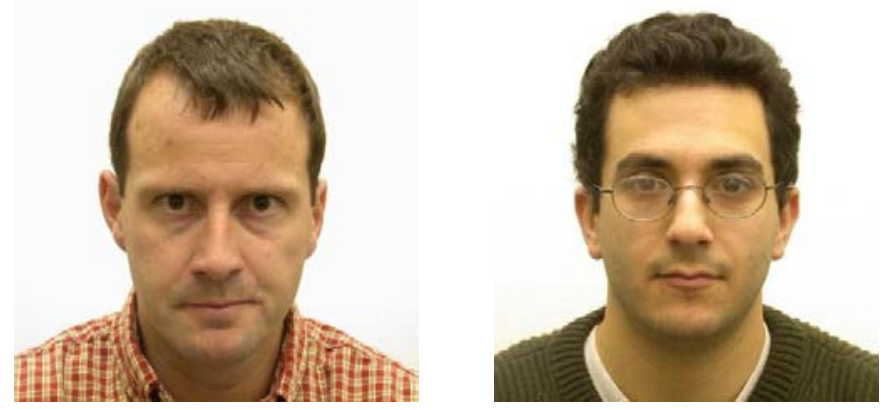

Figure 1. Images included here are reasonable representations of those used in the FRVT 2002 High Computational Intensity test.

The MCInt data set is composed of a heterogeneous set of still images and video sequences of subjects in a variety of poses, activities and illumination conditions. The images originate from two sources. The first is the still facial image data set collected at the National Institute of Standards and Technology (NIST), Naval Surface Warfare Center (NSWC, Dahlgren), and the University of South Florida (USF) between 1999 and 2002. The second set is from The University of Texas at Dallas and consists of video sequences and still images taken in 2001. The NIST-NSWC-USF data set is comprised of images taken indoors and outdoors. The images were taken over more than three years at three sites. The images in Figure 2 are from NIST, the NSWC, and the USF dataset. The outdoor stills are characterized by changing background and directional sunlight illumination. Figure 3 shows selected frames from 150-frame UT Dallas "facial speech" videos. The two rows show the same subject gathered on different occasions.

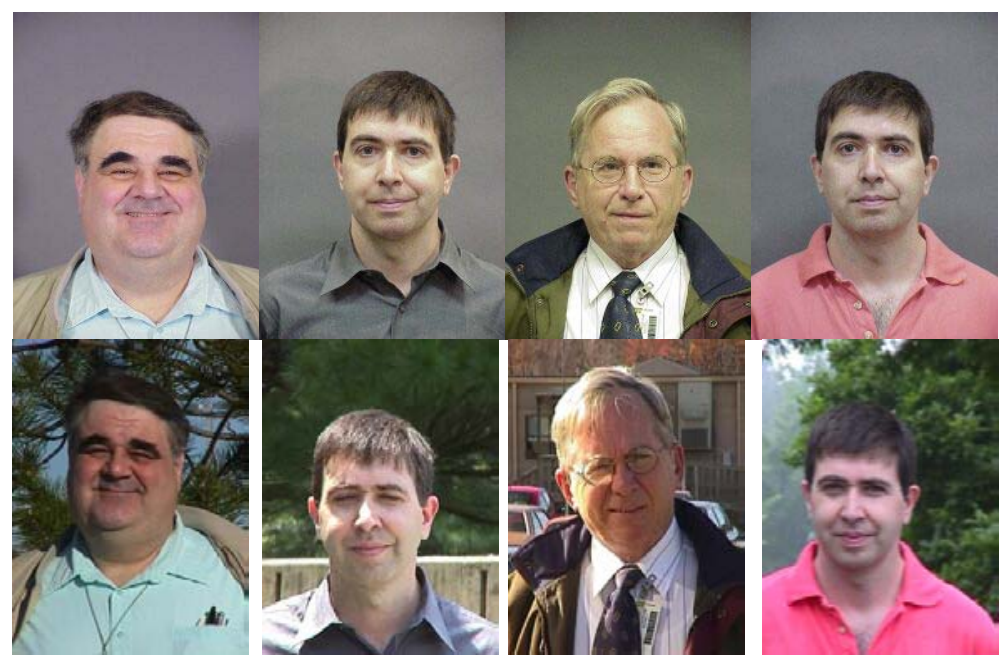

Figure 2. Indoor and outdoor images from the NIST-NSWC-USF data set. The top row contains images taken indoors and the bottom contains outdoor images taken on the same day as the indoor images. 


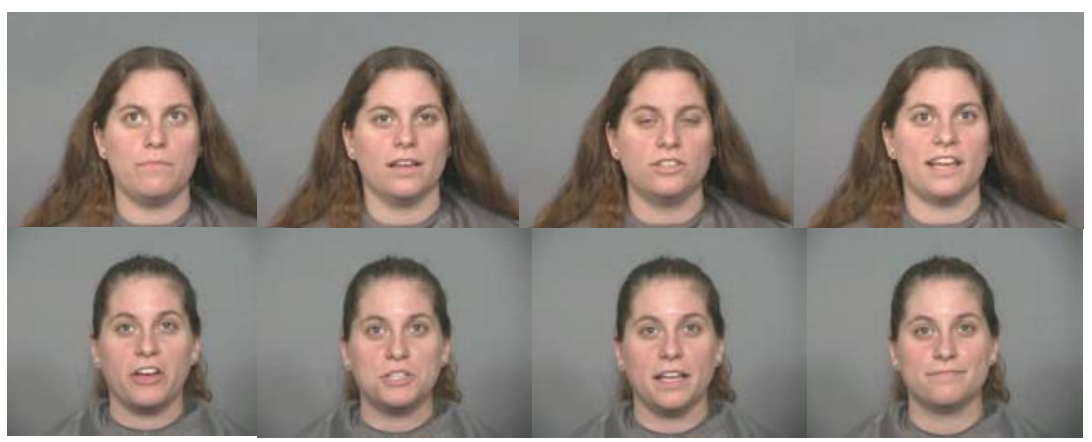

Figure 3. Selected frames sampled from two University of Texas at Dallas video sequences.

\section{Overview of Results}

FRVT 2002 addressed several important face recognition topics. Performance on frontal faces was examined under different environmental conditions for three tasks - verification, identification and watch lists. Newly developed technologies were examined to understand recent progress in the field and to identify promising avenues of future research. Finally, demographics of the subject population were examined. The three primary face recognition tasks are listed below:

- Verification: "Am who I say I am?"

- Identification: "Who am I?"

- Watch list: "Are you looking for me?"

In a verification task, a person presents their biometric and an identity claim to a face recognition system. The system then compares the presented biometric with a stored biometric of the claimed identity. Based on the results of comparing the new and stored biometric, the system either accepts or rejects the claim. From an evaluation point of view, there are two types of system users. The first is a legitimate user. The second is a person who attempts to impersonate a legitimate user. Verification performance is characterized by two performance statistics. The two statistics characterize the success rate of the two types of users. The first is the rate at which legitimate users are granted access. This is the verification rate. The second is the rate at which imposters are granted access. This is the false accept rate. The ideal system would have a verification rate of $100 \%$ and a false accept rate of $0 \%$. Unfortunately, such a system does not exist. In real-world systems, there is a trade-off between verification and false accept rates.

It is critically important to consider the false accept rates and verification rates together in order to understand the performance capabilities of a face recognition system. It is easy to build a system that always grants access to a subject. This system will have a $100 \%$ verification rate since access will always be granted in response to a legitimate user's request. Conversely, this system will also have a $100 \%$ false accept rate because it also grants access to imposters. The best system is one that balances the verification rate with a false accept rate in a manner consistent with operational needs.

Examples of this trade-off can be viewed at Figure 4. Verification performance results are shown for the top three systems. For each system, verification performance is reported for three false accept rates: $0.1 \%$, $1 \%$ and $10 \%$. Cognitec and Identix have verification rates of $82 \%$ with a false accept rate of $0.1 \%$. With a false accept rate of $1 \%$, their verification rates are both $90 \%$. With a false accept rate of $10 \%$, their verification rates are $96 \%$. This illustrates the trade-off between the verification rate and false alarm rate. 


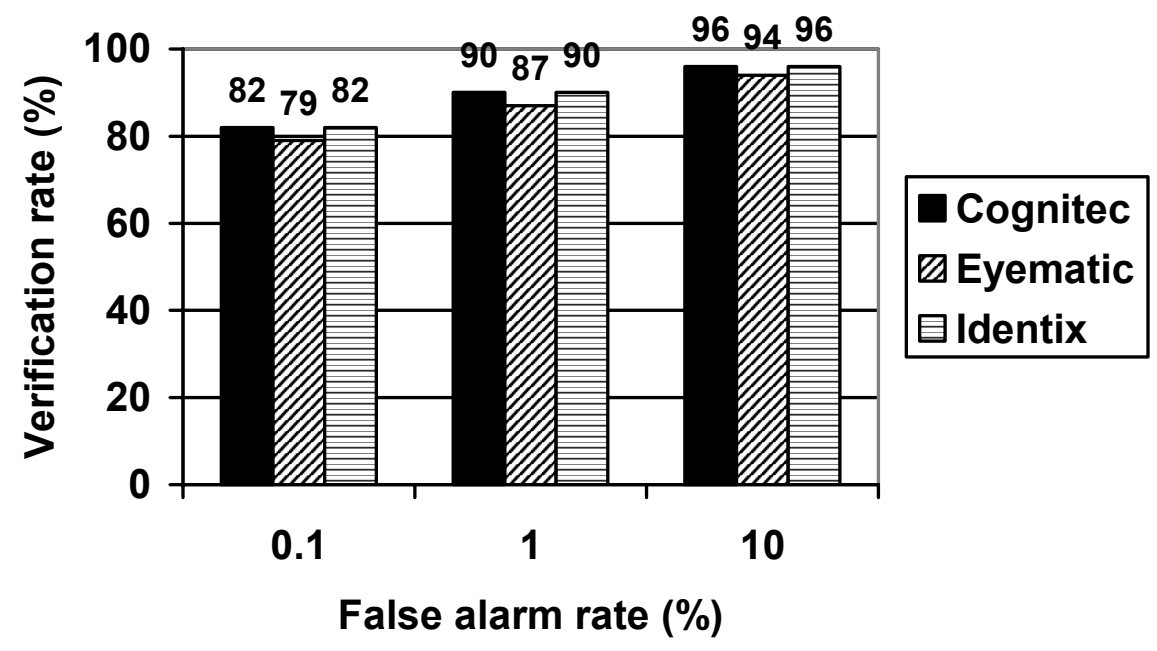

Figure 4. Verification performance is shown for the top three systems on HCInt visa images. Verification rates are reported for false accept rates of $0.1 \%, 1 \%$, and $10 \%$.

The results in Figure 4 were generated using the HCInt visa dataset (37,437 individuals). The database images consisted of multiple images of the same individuals taken on different days. There is up to three years difference between some pictures in the database. The verification performance from the HCint visa images provides a reliable estimate of performance because of the large number of images in the data set. Figure 5 depicts all FRVT 2002 participants' verification rates (at a 1\% false accept rate) using the HCInt visa dataset.

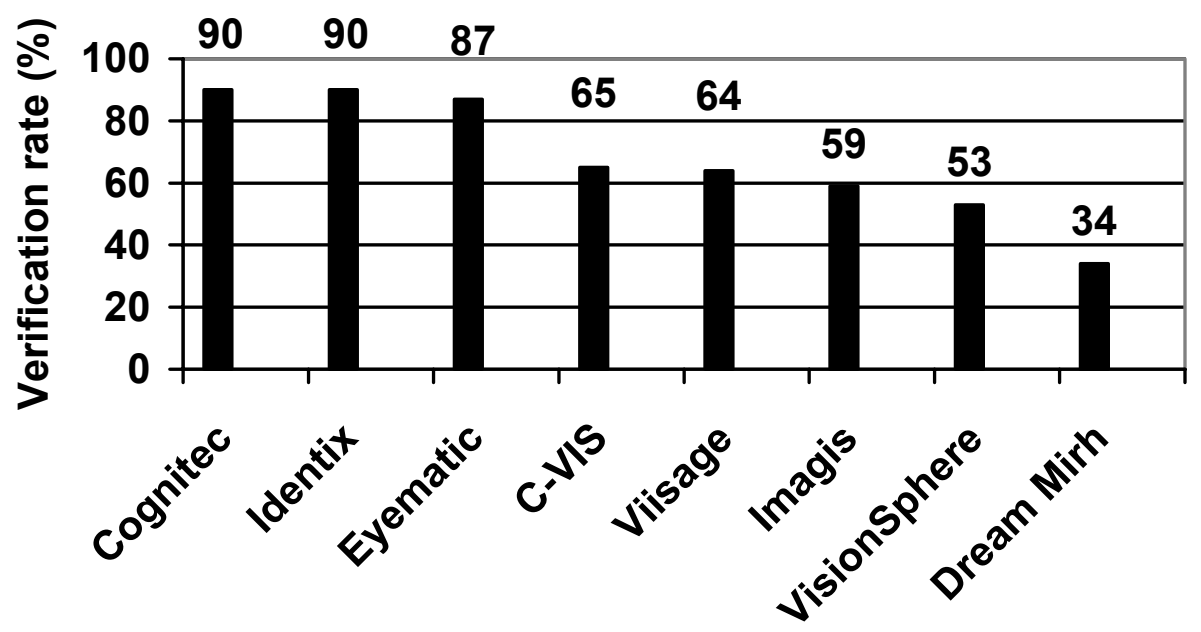

Participant

Figure 5. Verification performance is reported for all participants on the HCInt visa dataset. Verification performance is reported at a false accept rate of $1 \%$.

Recognition from frontal images is an important capability of face recognition systems. FRVT 2002 examined recognition performance using frontal images taken under varied conditions. The analysis included the results using the HCInt and MCInt data sets. In all cases, the database consists of images taken indoors under good lighting conditions. Results are reported for five different conditions (Figure 6): 
- Matching images of a person taken indoors on the same day with the same illumination.

- Matching images of a person taken indoors on the same day with different illumination.

- Matching images of a person taken indoors on different days with the same illumination.

- HCInt visa images (matching images of a person taken on different days).

- Matching of an image of a person taken indoors with an image taken outdoors. Both images were taken on the same day.

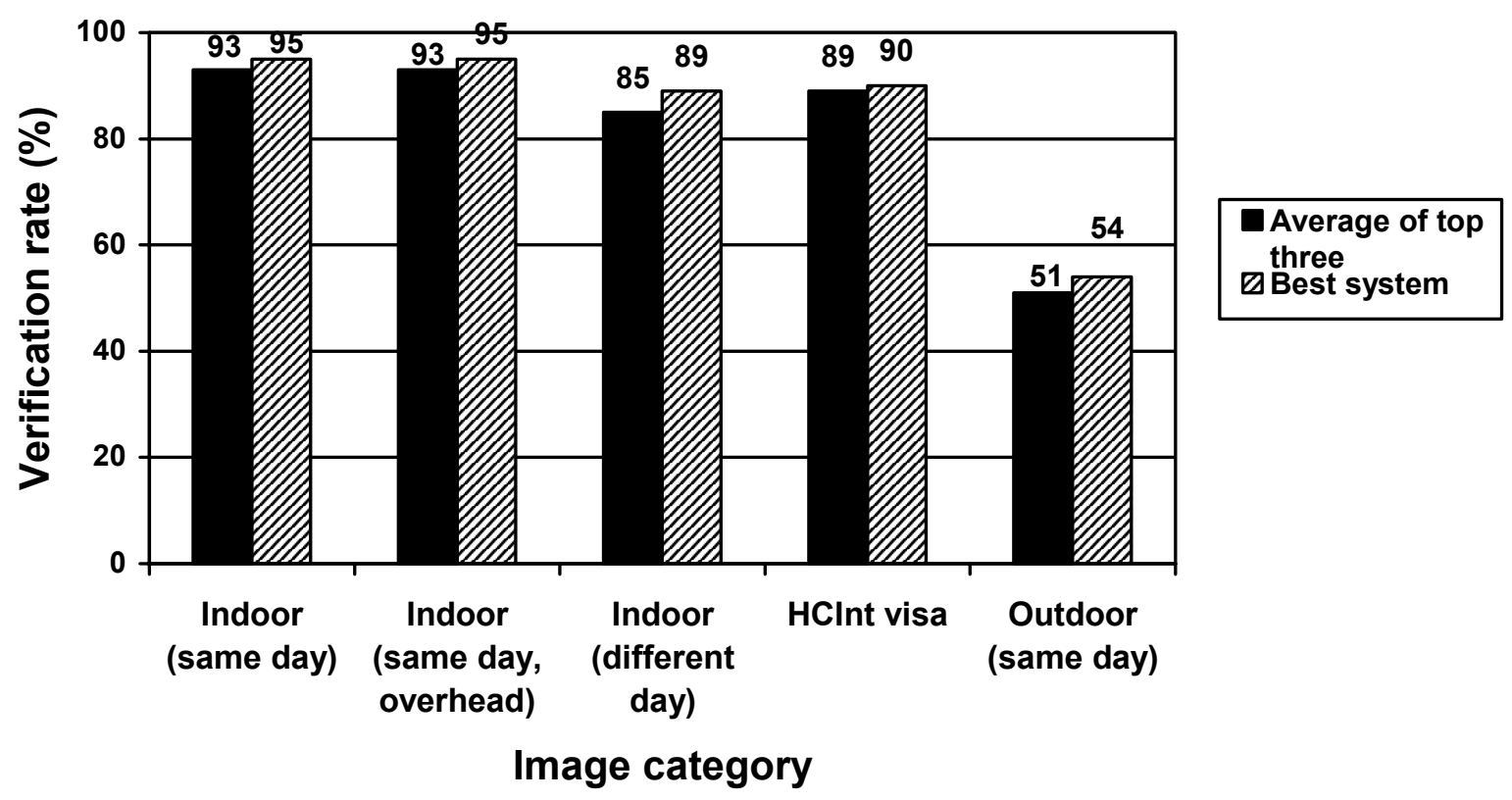

Figure 6. Verification performance is reported for five categories of frontal facial images.

Performance is reported for the best system and average of the top three systems in each category. The verification rate is reported at a false accept rate $1 \%$.

Figure 6 shows performance of the best system in each category and average performance for the three best systems in each category. Our analysis shows that reasonable changes in indoor lighting do not affect performance (the two indoor same categories) for top systems. These results are consistent across multiple databases for these systems (indoor different day and HCInt visa categories). Invariance to normal changes in indoor illumination is an issue for some of the other systems that participated in the FRVT 2002. This suggests that recognition from indoor images is reasonably stable. Performance using images of subjects that were taken outdoors, even on the same day, was drastically reduced. This is a trend that was also apparent in FRVT 2000, indicating that the variation and structure of outdoor lighting has a drastic affect on performance. For example, for the best systems, verification performance drops from $95 \%$ to $54 \%$ - a $40 \%$ drop - going from indoors to outdoors.

In the identification task, an image of an unknown person is provided to a system. (In the identification task, we assume that through some other method we know the person is in the database.) The system then compares the unknown image to the database of known people. The results of this comparison are then presented by the system to an operator in a ranked listing of the top $n$ 'candidates' (typically anywhere from one to 50). If the correct image is somewhere in the top $n$, then the system is considered to have performed the identification task correctly.

Figure 7 depicts the top three participants' identification rate performance at three different 'ranks'. In this test, the database consisted of 37,437 different images. A rank of ' 1 ' is the rate at which the system's 'best' or 'most likely' candidate was correct; the rank 10 results depicts the rate at which the correct identity was 
within the top ten candidates. Notice that the performance goes up for higher ranks. Our analysis has also shown that a system's identification performance capability is dependent upon the size of the database. For the best system, the top-rank identification rate was $85 \%$ on a database of 800 people, $83 \%$ on a database of 1,600 , and $73 \%$ on a database of 37,437 . For every doubling of database size, performance decreases by $2 \%$ to $3 \%$ points. In mathematical terms, identification performance decreases linearly with respect to the logarithm of the database size.

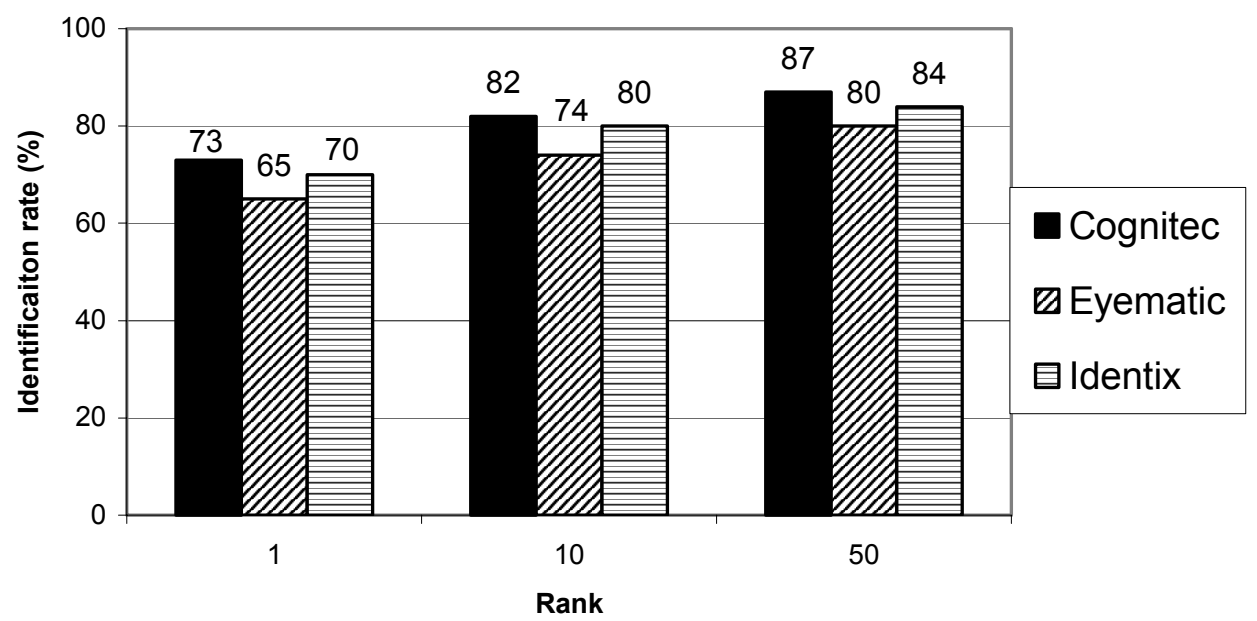

Figure 7. Identification performance for the three best system on the HCInt visa dataset. The database consisted of 37,437 individuals. Identification rates are reported for ranks 1,10 , and 50 .

FRVT 2002 also evaluated face recognition technology with respect to a watch list task. In the watch list task, a face recognition system must first detect if an individual is, or is not, on the watch list. If the individual is on the watch list, the system must then correctly identify the individual. The statistic for correctly detecting and identifying an individual on the watch list is called the detection and identification rate. In some instances, the system may incorrectly alarm on an individual that is not on the watch list. The rate at which an individual that is not on the watch list is incorrectly alarmed is called the false alarm rate.

Typically, the watch list task is more difficult than the identification or verification tasks alone. Figure 8 shows detection and identification rates for varying watch list sizes at a false alarm rate of $1 \%$. For the best system using a watch list of 25 people, the detection and identification rate is $77 \%$. Increasing the size watch list to 3,000 people, decreases the detection and identification rate to $56 \%$. Figure 8 also indicates that the systems achieve better performance for a smaller watch list. If the impetus of the watch list application is to detect and identify the "most wanted" individuals, the watch list should be kept as small as possible. Increasing the size of the watch list reduces the probability that an individual on the watch list is correctly detected and identified when presented to the system. 


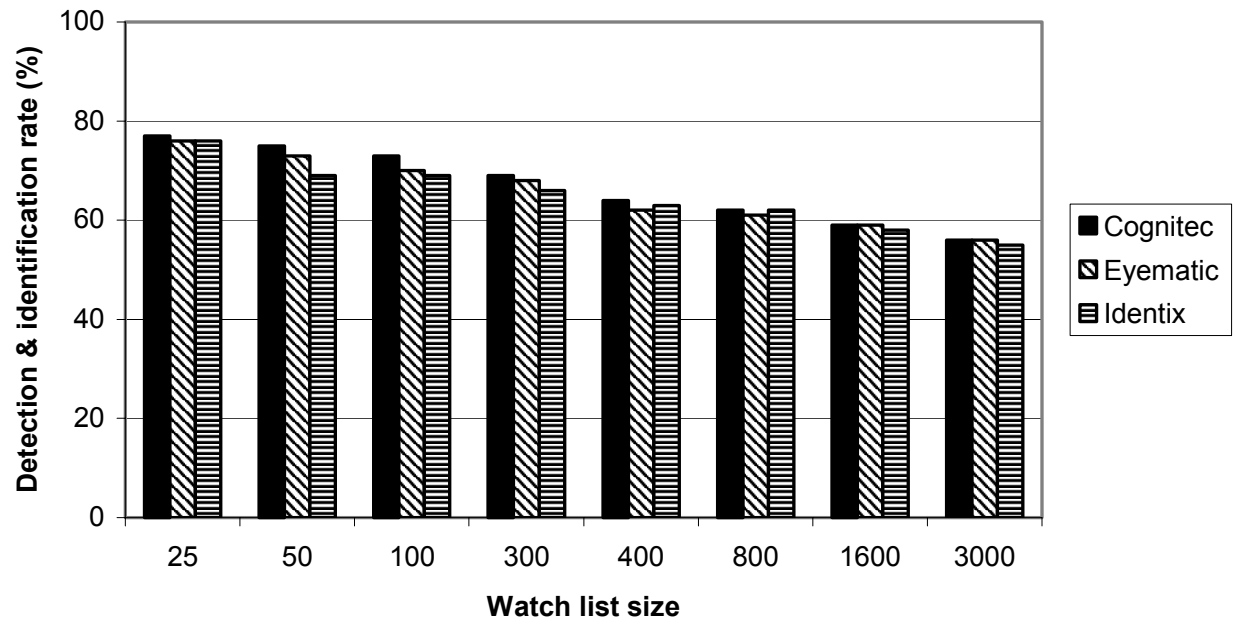

Figure 8. Watch list performance is shown for the three best systems on the HCInt visa dataset. Detection and identification rates are reported at a false alarm rate of $1 \%$ for eight watch list sizes.

So far we have concentrated in recognition performance from frontal images. The general face recognition problem requires recognition from non-frontal images. FRVT 2000 and FRVT 2002 show that one of the more difficult tasks for modern face recognition systems is recognizing faces in non-frontal imagery. Most face recognition systems perform well when all of the images are frontal. But, as a subject becomes more and more off angle (both horizontally and vertically), performance decreases.

One potential solution to this problem is the use of 'morphable models.' Although the details of how a morphable model works are quite complex, their use with respect to any face recognition system is straightforward. A morphable model takes a facial image taken from any angle as input, and outputs what that subject might look like if they were facing forward (the model leaves images already facing forward mostly unchanged). If the morphable model does this prediction well, then a face recognition system could use the output from the morphable model as a substitute for the original, off-angle image.

The efficiency of a morphable model was evaluated in FRVT 2002. Baseline performance was measured on a set of non-frontal images. The set contained people looking left, right, up and down. Performance was also measured on the same set of imagery after they had been processed by the morphable model. The transformed non-frontal faces in the FRVT 2002 were generated by the techniques of Blanz and Vetter ${ }^{5}$. As shown in Figure 9, there was a dramatic improvement in performance using the imagery provided from the morphable models. For example, on non-frontal images rotated either to left or right, Identix's performance increases from $26 \%$ on the original non-frontal images to $84 \%$ on the morphed images. FRVT 2002 participants were not informed prior to testing that the provided input would include morphable model imagery. Therefore, it is likely that with better vendor integration, morphable models might be able to boost performance even more than the preliminary, exploratory data presented here.

\footnotetext{
${ }^{5}$ V. Blanz, S. Romdhami, and T. Vetter (2002), "Face identification across different poses and illuminations with a 3D morphable model," Proceedings of International Conference on Automatic Face and Gesture Recognition, pp. 202-207.

V. Blanz and T. Vetter (1999), "A morphable model for the synthesis of 3D faces," Computer Graphics Proceeding SIGGRAPH' '99, pp. 187-194.
} 


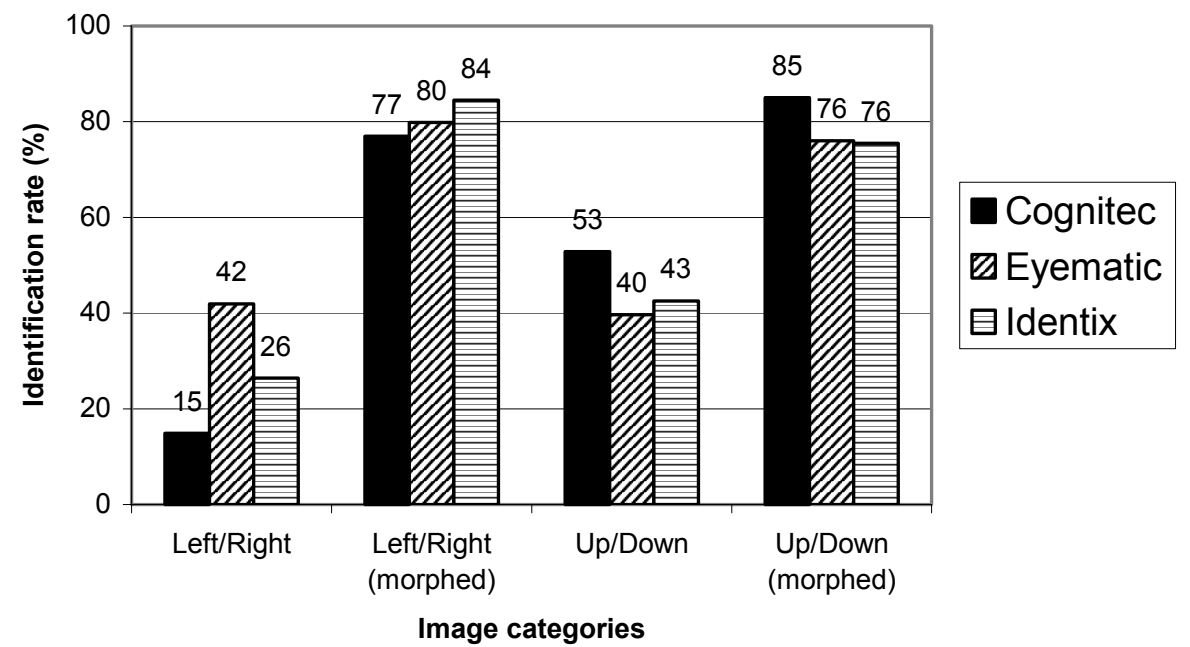

Figure 9. Identification performance is shown on non-frontal and morphed non-frontal images. The left/right and up/down categories are top identification rates for the original non-frontal images. The left/right (morphed) and up/down (morphed) categories are top identification rates for the morphed non-frontal images. Performance is on a database of 87 individuals.

Another important component of FRVT 2002 was the use of still versus video imagery. Our analysis has determined that there is little recognition benefit gained by using video images in lieu of still images. For the top three performers, performance drops $3 \%$ points when using video images instead of still images.

The results indicate that for pure recognition, video does not appear to make any difference in performance. FRVT 2002 did not address the issue of detecting faces in video. Using video to detect faces could greatly assist a face recognition system.

FRVT 2002 investigated several demographic aspects of face recognition. Specifically, we examined how sex, age, and time-delay affect recognition. Males are easier to recognize than females. For the top systems, identification rates for males were $6 \%$ to $9 \%$ points higher than that of females. For the best system, identification performance on males was $78 \%$ and for females was $79 \%$. Older people are easier to identify than younger people. Figure 10 depicts the average identification rate for the top three performers, as a function of age. In Figure 10, each bar is the identification performance for a five-year range of performance. For example, the first two bins are 18 to 22 year olds and 23 to 27 year olds. For 18 to 22 year olds the average identification rate for the top systems was $62 \%$, and for 38 to 42 year olds was $74 \%$. For every ten years increase in age, on average performance increases approximately $5 \%$ points. Identification results are computed from a database of 37,437 . 


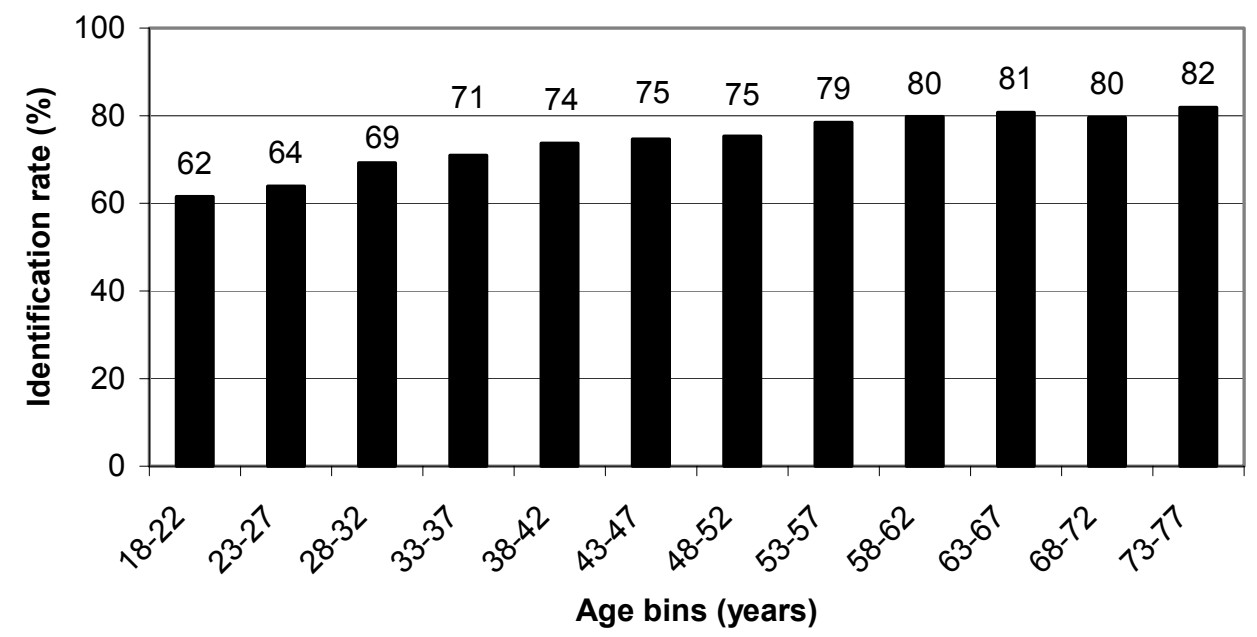

Figure 10. Identification performance is shown broken out by age of an individual. Each bin is labeled by the age range it contains (five year intervals). Identification rate is the average for the top three systems. Performance is on a database of 37,437.

Although it is less of a 'demographic' than sex or age, the elapsed time between database and new images, also affects performance. Figure 11 shows this difference with respect to 60 day 'bins.' As the elapsed time between the database and new images increases, performance decreases. For the better systems, identification performance decreases at $5 \%$ points per year. This makes sense intuitively, since we expect that as the face changes over time, face recognition algorithms cannot as easily model these facial variations.

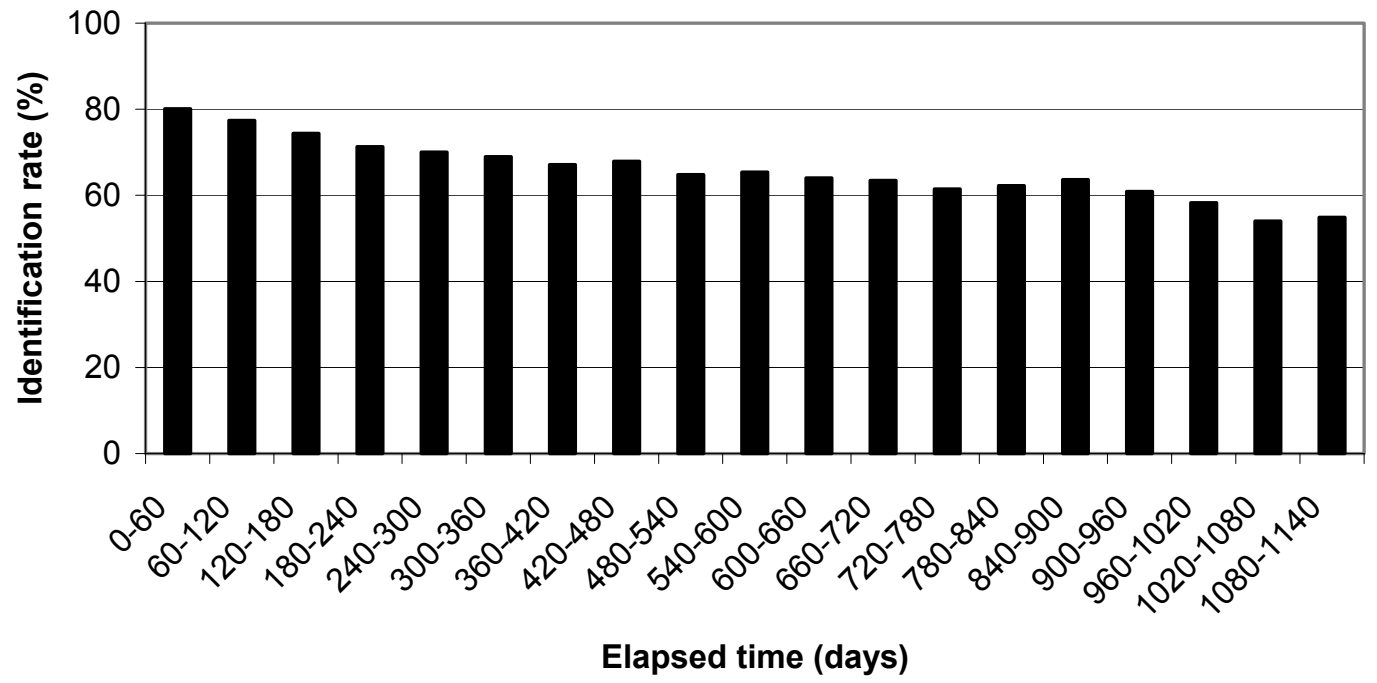

Figure 11. Identification performance is reported broken out by elapsed time between database and new image. Performance is reported in 60 day intervals. The average rank one identification rate for the top three systems is reported on a database of 37,437 individuals. 


\section{Conclusion}

At a simple level, FRVT 2002 was an evaluation and comparison of ten face recognition systems. Upon closer examination, FRVT 2002 will have a much broader impact. From an operational perspective, FRVT 2002 results will impact policy, the engineering design of large-scale biometric systems, and how future technology, scenario, and operation evaluations will be designed. From a scientific point of view, FRVT 2002 will have an impact on future directions of research in the computer vision and pattern recognition, psychology, and statistics fields. FRVT 2002 results raise many more questions than they answer.

Before summarizing the findings of FRVT 2002, two potentially important issues need to be addressed:

1) Does face recognition work?

2) Which system is best for my application?

The answers to both of these questions are closely related to one another. Face recognition performance, like other biometric types, is application-dependent. Just as there is no best biometric type for all operational applications, there is no best face recognition system for all operational applications. FRVT 2002 was not designed to be a "buyer's guide for face recognition" - where one looks at graphs or scores and selects a system for installation. Rather, it is a technology evaluation that should assist decisionmakers in determining (1) if face recognition technology could potentially meet the performance requirements for an operational application, and (2) which systems should be selected for applicationspecific scenario evaluations.

In order to determine if face recognition works and which system(s) should be deployed, one first needs to properly define the operational application of interest and operational performance requirements. These requirements need to be as specific as possible because even a small change in operational requirements can sometimes significantly alter anticipated performance. Questions to ask when defining an application include:

- Identification, verification or watch list mode of operation?

- The size of the database for identification or watch list?

- Demographics of the anticipated users (age, sex, etc.)?

- Lighting conditions - indoor/outdoor? Supplemental lighting?

- Is the system to be installed overtly or covertly?

- What is the anticipated user behavior?

- How long has it been since the images in the database were taken?

- What is the required throughput rate?

- How many "exception handling" cases can you handle for a given period of time?

- For each mode of operation, which parameter (identification: rank or identification rate; verification: false alarm or probability of verification; watch list: false alarm or correct alarm) is most vital?

- What are the minimum accuracy requirements?

FRVT 2002 can provide input to several, but not all of these questions. Questions associated with anticipated user behavior, exception handling, human computer interaction, and how a system is integrated into the business model are not addressed in a technology evaluation such as FRVT 2002. Providing answers to these types of questions are in the province of scenario and operational evaluations. Answers to some of these questions will identify which experiments in FRVT 2002 are relevant to a given application. Results from the relevant experiments will 1) show if face recognition could potentially meet the performance requirements for the application, 2) identify which systems should be selected for follow-up scenario evaluations, and 3) provide a starting point for designing and conducting scenario and operational evaluations for a specific application. Without specifying requirements, implementation constraints, and process models for an application, one cannot accurately determine if face recognition will work or which system should be selected.

FRVT 2002 is the most thorough and comprehensive evaluation of automatic face recognition technology to date. The evaluation has examined many long-standing questions and raised several new questions for 
further study. These are discussed in detail in Section 9. The conclusions from FRVT 2002 are summarized below:

- Indoor face recognition performance has substantially improved since FRVT 2000.

- Face recognition performance decreases approximately linearly with elapsed time database and new images.

- Better face recognition systems do not appear to be sensitive to normal indoor lighting changes.

- Three-dimensional morphable models substantially improve the ability to recognize non-frontal faces.

- On FRVT 2002 imagery, recognition from video sequences was not better than from still images.

- Males are easier to recognize than females.

- Younger people are harder to recognize than older people.

- Outdoor face recognition performance needs improvement.

- For identification and watch list tasks, performance decreases linearly in the logarithm of the database or watch list size.

Other major FRVT 2002 accomplishments include the evaluation protocol developed for this test and the associated scoring suite. The evaluation protocol and scoring suite are XML-based. They were designed to be applicable to general biometric evaluations, not just restricted for use in face recognition evaluations.

Face recognition and processing are important research problems spanning numerous fields and disciplines. This is because face recognition, in addition to having numerous practical applications, is a fundamental human behavior that is essential for effective communications and interactions among people. Researchers are interested in how people process faces, and scientists and engineers are working on techniques to replicate human face processing functions. Research advances along two intertwined paths. One path has an application orientation and the other a scientific orientation. Advances on both paths reinforce each other, with FRVT 2002 providing research directions for both paths. In the 1990's, the FERET evaluations stimulated research in face recognition technology and in doing so helped to advance automatic face recognition during its infancy. With the numerous questions it raises, FRVT 2002 is poised to play a similar role in stimulating future face recognition and processing research.

\section{Acknowledgements}

The organizers of the FRVT 2002 gratefully acknowledge the Defense Advanced Research Projects Agency, Department of State, Federal Bureau of Investigation, National Institute of Justice, National Institute of Standards and Technology, and Transportation Security Administration as evaluation sponsors and the ONDCP Counterdrug Technology Assessment Center, United States Customs Service, Department of Energy, Drug Enforcement Administration, Immigration and Naturalization Service, U.S. Secret Service, Technical Support Working Group, Australian Customs, Canadian Passport Office, and United Kingdom Biometric Working Group as evaluation supporters.

The authors extend their thanks to:

The Department of State, specifically Travis Farris for allowing NIST to use the Mexican nonimmigrant visa images for FRVT 2002; John Atkins and Rasool Azad for their invaluable assistance with the images, meta data, and background information on the images' origins and properties.

Volker Blanz and Thomas Vetter at the University of Freiburg for supplying us with the three-dimensional morphable images. Volker Blanz expeditiously provided us with frontal reconstructions obtained from their 3D Morphable Model implementation. Alice O'Toole at The University of Texas at Dallas, for supplying us with the video sequence database. Tom Gandy and Cathy Schott for their general assistance and their assistance in editing and proofreading the FRVT 2002 reports. Charlie Wilson at NIST for his general assistance and insightful comments. Kevin Bowyer, Travis Farris, and Russ Neuman for their comments on preliminary drafts of the report. 


\title{
FACE RECOGNITION VENDOR TEST 2002
}

\section{Evaluation Report \\ March 2003}

\section{P. Jonathon Phillips ${ }^{1,2}$, Patrick Grother ${ }^{2}$, Ross J. Micheals ${ }^{2}$, Duane M. Blackburn ${ }^{3}$, Elham Tabassi ${ }^{2}$, Mike Bone ${ }^{4}$}

\author{
${ }^{1}$ DARPA \\ 3701 North Fairfax Dr. \\ Arlington, VA 22203 \\ ${ }^{2}$ National Institute of Standards and Technology \\ 100 Bureau Drive, Stop 8940 \\ Gaithersburg, MD 20899 \\ ${ }^{3}$ DoD Counterdrug Technology Development Program Office \\ 17320 Dahlgren Rd, Code B07 \\ Dahlgren, VA 22448 \\ ${ }^{4}$ NAVSEA Crane Division \\ 300 Highway 361, Code 4041 \\ Crane, IN 47522
}

\section{Sponsors and Supporters:}

Defense Advanced Research Projects Agency Department of State

Federal Bureau of Investigation

National Institute of Justice

National Institute of Standards and Technology

Transportation Security Administration

ONDCP Counterdrug Technology Assessment Center

United States Customs Service
Department of Energy

Drug Enforcement Administration

Immigration and Naturalization Service

United States Secret Service

Technical Support Working Group

Australian Customs

Canadian Passport Office

United Kingdom Biometric Working Group 


\section{This page intentionally blank.}




\title{
Face Recognition Vendor Test 2002: Evaluation Report
}

\section{P. Jonathon Phillips ${ }^{1,2}$, Patrick Grother ${ }^{2}$, Ross J. Micheals ${ }^{2}$, Duane M. Blackburn ${ }^{3}$, Elham Tabassi ${ }^{2}$, Mike Bone ${ }^{4}$}

\author{
${ }^{1}$ DARPA \\ 3701 North Fairfax Dr. \\ Arlington, VA 22203 \\ ${ }^{2}$ National Institute of Standards and Technology \\ 100 Bureau Drive, Stop 8940 \\ Gaithersburg, MD 20899 \\ ${ }^{3}$ DoD Counterdrug Technology Development Program Office \\ 17320 Dahlgren Rd, Code B07 \\ Dahlgren, VA 22448 \\ ${ }^{4}$ NAVSEA Crane Division \\ 300 Highway 361, Code 4041 \\ Crane, IN 47522
}

\begin{abstract}
The Face Recognition Vendor Test (FRVT) 2002 is an independently administered technology evaluation of mature face recognition systems. FRVT 2002 provides performance measures for assessing the capability of face recognition systems to meet requirements for large-scale, real-world applications. Ten commercial firms participated in FRVT 2002. FRVT 2002 computed performance statistics on an extremely large data set-121,589 operational facial images of 37,437 individuals. FRVT 2002 1) characterized identification and watch list performance as a function of database size, 2) estimated the variability in performance for different groups of people, 3) characterized performance as a function of elapsed time between enrolled and new images of a person, and 4) investigated the effect of demographics on performance. FRVT 2002 shows that recognition from indoor images has made substantial progress since FRVT 2000. Demographic results show that males are easier to recognize than females and that older people are easier to recognize than younger people. FRVT 2002 also assessed the impact of three new techniques for improving face recognition: three-dimensional morphable models, normalization of similarity scores, and face recognition from video sequences. Results show that three-dimensional morphable models and normalization increase performance and that face recognition from video sequences offers only a limited increase in performance over still images. A new XML-based evaluation protocol was developed for FRVT 2002. This protocol is flexible and supports evaluations of biometrics in general.
\end{abstract}

1.Please direct correspondence to Jonathon Phillips at jphillips@darpa.mil or jonathon@nist.gov 


\section{Table of Contents}

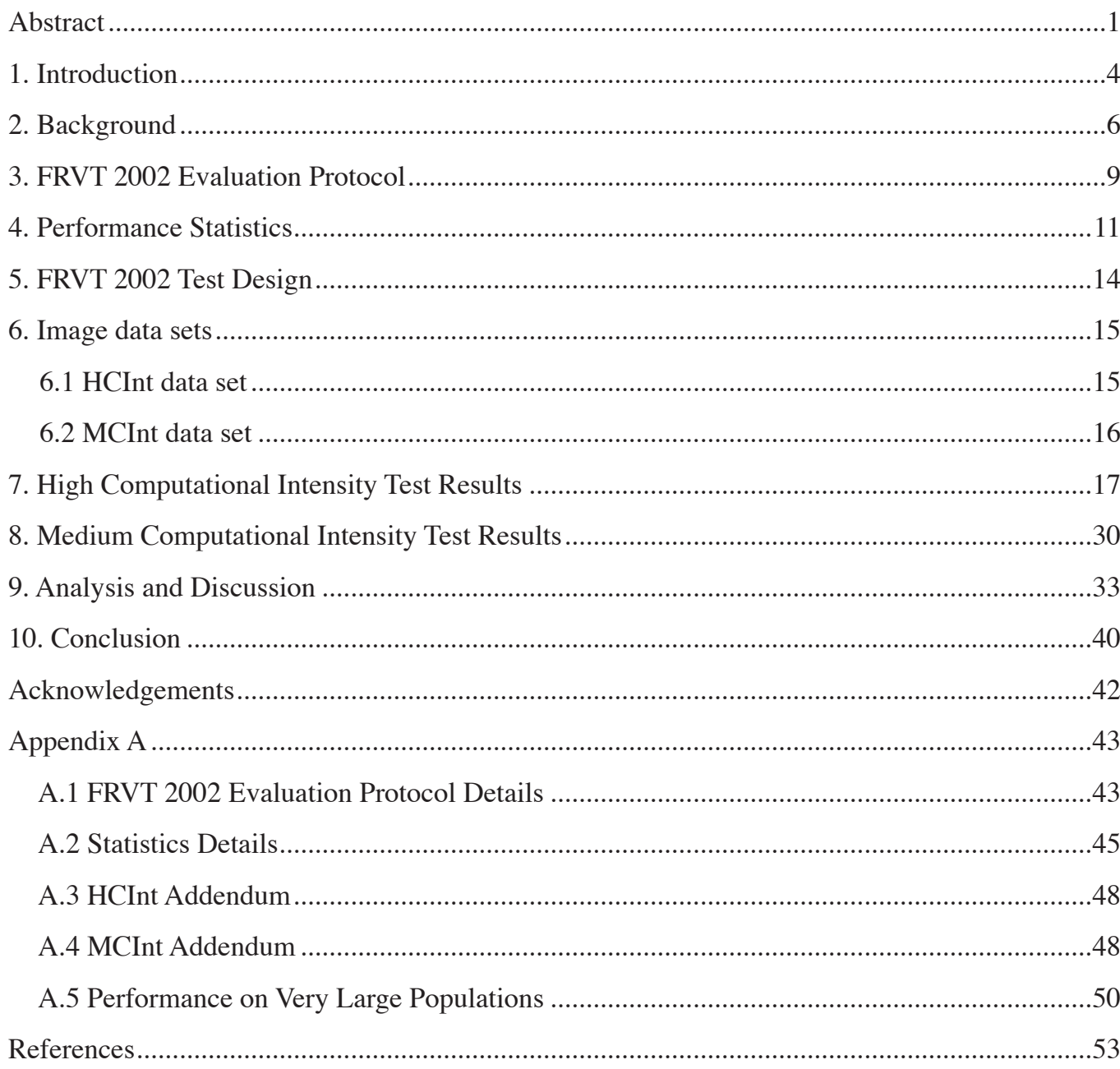




\section{TABLES AND Figures}

Table 1. Measurements of the size of the FERET Sep96 and FRVT evaluations ..........................6

Table 2. Summary of experiments performed in the FERET and FRVT evaluations ......................7

Table 3. List of FRVT 2002 participants and tests completed ......................................................14

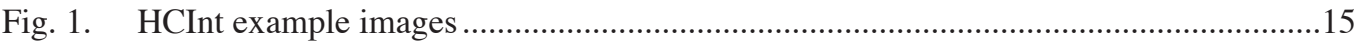

Fig. 2. Indoor and outdoor images from the NIST-NSWC-USF data set...................................16

Fig. 3. Example of morphed images ...................................................................................17

Fig. 4. Example of UT Dallas videos ..............................................................................17

Fig. 5. Histogram of elapsed time between gallery and probe images of a person ....................18

Fig. 6. Histogram of age distribution of people in the HCInt large gallery ...............................18

Fig. 7. ROC for HCInt large gallery and probe set..................................................................19

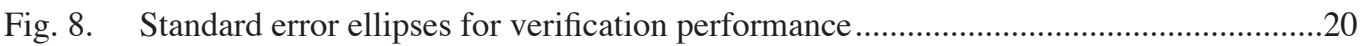

Fig. 9. Plots showing the difference between normalized and non-normalized verification performance

Fig. 10. Identification performance on HCInt large gallery and probe set ..................................22

Fig. 11. Rank 1 identification performance as a function of gallery size ....................................22

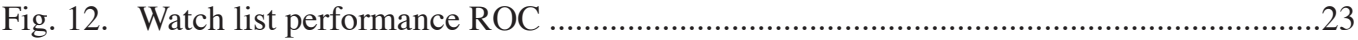

Fig. 13. Watch list performance for Cognitec as a function of rank for eight false alarm rates..24

Fig. 14. Watch list performance as a function of gallery size for a false alarm rate of $0.01 \ldots \ldots .25$

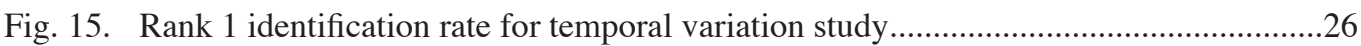

Fig. 16. Verification performance for temporal variation study ..............................................26

Fig. 17. CMC showing the difference in performance between male and female probes...........27

Fig. 18. ROC showing the difference in performance between male and female probe sets ......28

Fig. 19. Difference in verification performance for male and female probe sets ........................28

Fig. 20. Rank 1 identification performance broken out by age .................................................29

Fig. 21. Interaction between age and sex for rank 1 identification on HCInt large gallery.........29

Fig. 22. Comparison of system performance on different categories of frontalprobes .................31

Fig. 23. The effect of still versus three-dimensional morphable models.......................................32

Fig. 24. Plot showing still versus video recognition for frontal imagery .....................................33

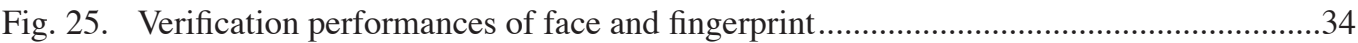

Fig. 26. Rank 1 identification rate as a function of gallery size for face and fingerprint .............35

Fig. 27. Interaction between age and sex identification on HCInt large gallery .........................48

Fig. 28. ID performance of different categories of frontal probes................................................48

Fig. 29. ID performance of still versus three-dimensional morphable models ...........................49

Fig. 30. ID performance of plot showing still versus video recognition for frontal imagery......49

Fig. 31. Study of identification performance as a function of gallery size ....................................52 


\section{INTRODUCTION}

The primary objective of the Face Recognition Vendor Test (FRVT) 2002 is to provide performance measures for assessing the capability of automatic face recognition systems to meet real world applications. Achieving this objective required an evaluation that was much larger and broader scale than any previous biometric evaluations. The increase in scale included the number of individuals in the evaluation as well as the detail and depth of analysis performed. This required designing a new biometric evaluation protocol and establishing a new standard for evaluations. This applies not only to face recognition, but biometric evaluations in general.

FRVT 2002 was an independently administered technology evaluation. Ten participants were evaluated under direct supervision of the FRVT 2002 organizers at the U.S. Government facility in Dahlgren, Virginia, in July and August 2002. The participants were tested on sequestered data sets. Sequestering the data sets guaranteed that all the participants were tested on a level field and the systems were tested on their recognition ability on new facial imagery. Digital data sets ensured that all the systems were tested on exactly the same data and performance among the systems could be directly compared.

The heart of FRVT 2002 was the high computational intensity test (HCInt) that measured system performance on 121,589 operational images of 37,437 people. The images were provided from the U.S. Department of State's Mexican non-immigrant visa archive. From this data, real-world performance figures on a very large data set were computed. Performance statistics were computed for verification, identification, and watch list tasks. The performance results on the HCInt show an improvement in the capabilities of the face recognition systems over the last two years. On comparable experiments in FRVT 2000, there has been a 50 percent reduction in error rates (Blackburn et al. 2001; Phillips et al. 2003).

In previous evaluations, performance was broken out by coarse imaging properties: facial images taken on the same day, images taken on different days, effects of pose changes, and effects of lighting changes. As face recognition technology matured from infancy to the first generation of commercial systems, this level of detail spurred research, identified the most promising approaches, and identified interesting research directions. Now, as face recognition technology is being considered for large-scale implementations and new research avenues are being developed, more detailed evaluations are required. FRVT 2002 is the first generation of large-scale face recognition evaluations. The broad scale of FRVT 2002 consists of an experimental design that, for the first time, investigates key imaging and demographic factors affecting performance.

One possible face recognition application is to identify an unknown person from a large database. Effectiveness of face recognition technology for this application depends upon how database size affects performance. Previously, the largest performance evaluation was conducted on a data set of 1,196 people (Phillips, Moon, et al. 2000). The FRVT 2002 measures recognition performance on databases of up to 37,437 people. This provides the first characterization of how database size affects performance.

In real-world applications, systems are required to recognize faces from images taken on different days. From earlier evaluations, we know that as the elapsed time between the enrolled image and a new image of person increases, recognition rates decrease. However, the rate at which performance declines had not been thoroughly investigated. In FRVT 2002, this temporal effect is reported in intervals of 60 days. This provides the first characterization of how elapsed time between image acquisitions affects performance.

As face recognition technology advances, new techniques are being proposed to improve performance. Evaluations provide an opportunity to assess if new techniques improve performance. FRVT 2002 assesses the impact of three new techniques: three-dimensional morphable models, normalization of similarity scores, and face recognition from video sequences. Three-dimensional morphable models correct for pose variations by fitting a three-dimensional model to a non-frontal facial image. The model then transforms the non-frontal facial image to a frontal facial image. The transformed frontal image 
is then matched with other frontal images. Normalization is a post-matching, statistical technique for correcting differences in templates among people. Compensating for the statistical variation among people in a database potentially reduces the effect of differences among faces. The majority of face recognition systems perform recognition from still imagery. New techniques are being developed that explicitly incorporate temporal information in video sequences into face recognition algorithms. The theory is that the temporal information in video sequences contains more information than a still image and exploiting this information will improve performance. FRVT 2002 performed experiments to see if these three techniques improved performance. Experiments showed that three-dimensional morphable models and normalization could significantly improve performance. Experiments showed that for the video sequences in FRVT 2002, there was negligible change in performance.

An operational face recognition system will process and recognize different groups of people at different locations. Performance will vary for the different groups of people. To measure the potential variations among groups of people, FRVT 2002 calculates performance for different subsets of the 37,437 people in the Mexican Visa database. This provides an estimate of the variability of performance over different groups of people using a face recognition system.

One application for face recognition technology is to detect people on a watch list. The watch list task has elements of both the verification and identification tasks. In the watch list task, a photo of an unknown person is provided to a system and the system must determine if the unknown person is on a watch list. If a system determines that a person is on the watch list, the system then provides the identity of the unknown person. FRVT 2002 provides the first in-depth examination and analysis of the watch list task for multiple face recognition systems. Experiments measure the effect of watch list size on performance and performance as a function of the number of matches displayed. These results can provide a basis for establishing conditions under which watch lists can be effective in real-world applications.

Face recognition system performance is a function of the imaging conditions and the demographics of the population that will use the system. To date, evaluations of face recognition systems has not considered the effects of demographics on performance. FRVT 2002 examines the effects of sex and age of a person on performance. Experiments show that males are easier to recognize than females and older people are easier to recognize than younger people. Results also show that the difference in performance between males and females decreases with age. Thus the demographics of a data set do affect reported performance. Demographic information needs to be considered when setting specifications for operational systems.

As face recognition technology has matured and is being considered for more applications, the demand for evaluations is increasing. At the same time, the complexity and sophistication of the evaluations is increasing. This results in rising evaluation costs and increases the chance that mistakes will occur. To overcome these challenges, the FRVT 2002 introduced an evaluation protocol that is XML-based and contains a package of standard scoring routines. The XML specifications include formats for test data sets, raw output formats from systems, and performance scores. The scoring routines are designed to work with XML file formats. The FRVT 2002 evaluation protocol, XML specification, and scoring package are general and can be used to evaluate other types of biometrics. The FRVT 2002 evaluation protocol is a solid basis for establishing a standard evaluation protocol.

The complete FRVT 2002 report comes in the volumes: Evaluation Report, Summary and Overview, and Technical Appendices (all three are available at http://www.frvt.org). The Evaluation Report is the document you are reading. It contains a detailed description of the FRVT 2002 procedures, experiments, and results. The Summary and Overview briefly presents the key results from the FRVT 2002. The Technical Appendices provide supplementary material, detailed documentation of the FRVT 2002 evaluation protocol, and participant description of their systems and response to the FRVT 2002. 


\section{BACKGROUND}

FRVT 2002 follows four previous face recognition technology evaluations - three FERET evaluations (1994, 1995,1996) and FRVT 2000 (Phillips et al. 1996, 1998; Phillips, Moon, et al. 2000; Blackburn et al. 2001; Phillips et al. 2003). The FERET program introduced evaluations to the face recognition community and helped to advance face recognition from its infancy to the prototype system stage. By 2000, face recognition technology had matured from prototype systems to commercial systems. The Face Recognition Vendor Test 2000 (FRVT 2000) measured the capabilities of these systems and their technical progress since the last FERET evaluation. Public interest in face recognition technology had risen significantly by 2002. FRVT 2002 was designed to measure technical progress since 2000, to evaluate performance on real-life, large-scale databases, and to introduce new experiments to better understand face recognition performance.

In the literature, results are reported on three tasks: identification, verification, and watch list. Not every paper or report presents results for all three tasks. Computing performance requires two board sets. The first is the gallery which contains the database on individuals known to the system. The second is the probe set that contains the images (biometric signature) of an unknown individuals presented to the system for recognition. A probe is one signature in a probe set.

Each successive evaluation increased in size, difficulty and complexity, reflecting the maturing of face recognition technology as well as evaluation theory. Table 1 shows the increase in difficulty of the evaluations. Table 1 lists the number of signatures in each evaluation, the number of comparisons between signatures required to complete the evaluation, the evaluation time limit, and the minimum number of comparisons per second required to complete the evaluations with in the time limit. The FERET Aug94 and Mar95 evaluations are not included in the table because their designed was based on a different evaluation protocol. Table 2 summarizes the experiments conducted in the FERET and FRVT evaluations. The experiments are broken into different categories. The first category lists the different types of experiments conducted broken out by task and the maximum number of individuals in an experiment. The basic experiment category lists standard experiments included in face recognition evaluations. For the basic experiments, the gallery consisted of frontal facial images taken indoors. The indoor same day-expression change experiment consisted of probes taken on the same day as the gallery image, but with a change in expression. The indoor same day - illumination change experiments investigated the effects of changes of illumination indoors. In the indoor different day experiments, the probe image was taken on a different day than the gallery image. For the indoor different day - greater than 18 months, the probe image was taken at least 18 months after the gallery image of a person. In the outdoor same day experiments, the probe was taken outdoors on the same day as the gallery image. For the outdoor different day, the probe was taken outdoors on a different than the gallery image. In the pose experiments the probes were non-frontal facial images. In the left or right experiments the face is looking either to the left or right at varying angles. In the up or down experiments, the face is looking either up or down. The detailed analysis experiments look at results in greater depth. The resolution experiments examine the effects of changing the size of the face. The size of the face is changed by digital manipulation. The compression experiments examined the effect on performance of compressing the probe image. The media experiments examined the effect on performance of switching between

Table 1. Measurements of the size of the FERET Sep96 and FRVT evaluations

\begin{tabular}{ccccc}
\hline Evaluation & $\begin{array}{c}\text { No. of } \\
\text { Signatures }\end{array}$ & $\begin{array}{c}\text { No. of } \\
\text { Comparisons }\end{array}$ & $\begin{array}{c}\text { Evaluation Time } \\
\text { Limt }\end{array}$ & $\begin{array}{c}\text { Minimum No. of } \\
\text { Comparisons per } \\
\text { Second }\end{array}$ \\
\hline FERET Sep96 & 3,813 & $\sim 14.5$ million & 72 hours & 56 \\
FRVT 2000 & 13,872 & $\sim 192$ million & 72 hours & 742 \\
FRVT 2002 - MCINT & 9,612 & $\sim 63$ million & 264 hours & 66 \\
FRVT 2002 - HCINT & 12,1589 & $\sim 15$ billion & 264 hours & 15,555 \\
\hline
\end{tabular}

${ }^{\dagger}$ Note: The MCINT portion of FRVT 2002 is the only test in this chart that included "video" signatures. Signatures in all other tests were a single still image. 
Table 2. Summary of experiments performed in the FERET and FRVT evaluations

\begin{tabular}{|c|c|c|c|c|c|}
\hline Measurable & $\begin{array}{l}\text { FERET } \\
\text { Aug94 }\end{array}$ & $\begin{array}{l}\text { FERET } \\
\text { Mar95 }\end{array}$ & $\begin{array}{l}\text { FERET } \\
\text { Sep96 }\end{array}$ & $\begin{array}{l}\text { FRVT } \\
2000\end{array}$ & $\begin{array}{l}\text { FRVT } \\
2002\end{array}$ \\
\hline \multicolumn{6}{|l|}{ Largest number of individuals in: } \\
\hline A verification experiment & & & 1,196 & 1,196 & 37,437 \\
\hline An identification experiment & 498 & 831 & 1,196 & 1,196 & 37,437 \\
\hline A watch list experiment & 25 & & & & 3,000 \\
\hline \multicolumn{6}{|l|}{ Basic experiment categories } \\
\hline Indoor same day -expression change & $*$ & $*$ & $*$ & * & $*$ \\
\hline Indoor same day - illumination change & $*$ & & $*$ & $*$ & $*$ \\
\hline Indoor different day & $*$ & $*$ & $*$ & * & $*$ \\
\hline Indoor different day - greater than 18 months & & & $*$ & $*$ & $*$ \\
\hline Outdoor same day & & & & $*$ & $*$ \\
\hline Outdoor different day & & & & & $*$ \\
\hline Pose-left or right & $*$ & & & $*$ & * \\
\hline Pose - up or down & & & & & $*$ \\
\hline \multicolumn{6}{|l|}{ Detailed analysis } \\
\hline Resolution of face & $*$ & & & * & \\
\hline Image compression & & & & * & \\
\hline Media & & & & $*$ & \\
\hline Distance of face from camera & & & & $*$ & \\
\hline Standard error ellipses & & & & & $*$ \\
\hline Id. Performance as a function of gallery size & & & & & $*$ \\
\hline $\begin{array}{l}\text { Watch list performance as a function of } \\
\text { gallery size }\end{array}$ & & & & & $*$ \\
\hline Watch list performance as a function of rank & & & & & $*$ \\
\hline \multicolumn{6}{|l|}{ Technologies evaluated } \\
\hline 3D morphable models & & & & & $*$ \\
\hline Normalization & & & & & $*$ \\
\hline Video & & & & & $*$ \\
\hline \multicolumn{6}{|l|}{ Demographic factors } \\
\hline Sex & & & & & $*$ \\
\hline Age & & & & & $*$ \\
\hline Interaction between sex and age & & & & & $*$ \\
\hline
\end{tabular}

film and digital facial images. The distance experiment looked at the effects of distance from the camera on performance. Unlike the resolution experiment, these images were not digital manipulated. The error ellipse experiments looked the effects of different galleries on performance. The gallery size experiments looked the effects of increasing the gallery size on identification and watch list performance. The watch list rank experiment looked at the effect of increasing the number matches examined on performance. The technology experiments investigated three new techniques: three-dimensional morphable models, normalization, and video-based recognition algorithms. The demographic experiments examined the effect of sex and age on performance.

Biometrics testing is an active area. Unfortunately, most results are not publicly released. Reasons for not releasing results include sensitivity of performance figures for operational systems and proprietary rights associated with the performance data. Aside from security concerns, it is advantageous to the biometrics community if results are released. Releasing the results allows for peer assessment of the evaluation procedure and results, makes the biometrics community aware of the latest results, and informs the biometric community of the latest advances in testing 
methodology. If a biometric evaluation is designed correctly, the results should be reproducible. This means that the results reported in one evaluation should be observed in evaluations conducted by different groups. For example, in FRVT 2002, we found that males are easier to identify than females. If this was a general property of face recognition algorithms, then other face recognition evaluations should also observe this phenomenon. If other groups do not observe this phenomenon, there could a problem in one of the groups' test methodologies or the phenomenon could just be a property of the data set in the evaluation or systems tested. If subsequent evaluations replicate the phenomenon, this provides further evidence that the phenomenon is a general property -in this example, that male faces are easier to identify than female faces. Public release of results helps the community to differentiate between general properties of a biometric and properties of a particular evaluation. As the testing community matures, this will evolve into a set of standard evaluation procedures.

Other publicly available evaluations have also significantly contributed to our understanding of biometric system performance. The NIST speaker recognition evaluations are annual technology evaluations that measure verification performance (Martin and Przybocki 2000). The overall goals of the evaluations have always been to drive the technology forward, to measure the state-of-the-art, and to find the most promising algorithmic approaches. As in the FERET and FRVT programs, the NIST speaker recognition evaluations have increased in size and scope since the first evaluation in 1996.

The Fingerprint Verification Competition (FVC) 2000 and 2002 are technology evaluations that measure verification performance of single-finger fingerprint devices (Maio et al. 2002a, 2002b). These evaluations were the first common benchmarks for fingerprint developers, thus allowing them to unambiguously compare their algorithms.

The UK Biometrics Working Group's Biometric Test Programme Report is a scenario evaluation report that compared six different biometrics (Mansfield et al. 2001). Representative face, fingerprint, hand geometry, iris, vein and voice recognition systems were tested for verification performance in a normal office environment with cooperative, non-habituated users. The objectives of the test program were to show the level of performance attainable by a selection of biometric systems, to determine the feasibility of demonstrating satisfactory performance through testing, to encourage more testing to be sponsored, and to promote methodologies contributing to the improvement of biometric testing. The report is groundbreaking in that it is the first opensource evaluation that directly compares performance of different biometrics for the same application. "Best practices in testing and reporting performance of biometric devices" was developed in part based on the lessons learned from performing the Biometrics Working Group's biometric test program (Mansfield and Wayman 2002).

San Jose State University has been evaluating biometric systems since 1994, and was the Biometric Consortium's National Biometric Test Center from 1997-2000. The Test Center explored a number of essential questions relating to the science underpinning biometric technologies. The Collected Works document from these endeavors is publicly available and contains evaluation results from the INSPASS Hand Geometry System, the Philippine AFIS System and numerous small-scale evaluations (Wayman 2000).

Bone and Blackburn (2002) performed a scenario on the watch list task and the effect of gallery size on verification performance. This study is the first publicly available evaluation to report in-depth on the watch list task. 


\section{FRVt 2002 Evaluation Protocol}

A design principle and testing protocol describes how evaluations are designed and conducted. Design principles outline the core philosophy and guiding beliefs in designing an evaluation; the evaluation protocol provides the implementation details.

The FRVT 2002 evaluation protocol is based on the September 1996 evaluation protocol (Phillips, Moon, et al. 2000). The protocol added general biometric signatures, normalization of similarity scores, and an XML-based specification. The XML-based specification is extensible to other biometrics and is being used for fingerprint recognition evaluation.

The design of FRVT 2002, along with the FERET evaluations and FRVT 2000, followed the precepts for biometrics evaluations articulated in Phillips, Martin, et al. (2000). Succinctly stated, the precepts are:

1. Evaluations are designed and administered by groups that are independent of algorithm developers and vendors being tested.

2. Test data is sequestered and not seen by the participants prior to an evaluation.

3. The evaluation test design, protocol, and methodology are published.

4. Performance results are spread in a manner that allows for meaningful differences among the participants.

Points 1 and 2 ensure fairness in an evaluation. Point 1 provides assurance that the test is not designed to favor one participant over another. Independent evaluations help enforce points 2 and 4. In addition, point 2 ensures that systems are evaluated on their ability to generalize performance to new sets of faces, not the ability of the system to be tuned to a particular set of faces. When judging and interpreting results, it is necessary to understand the conditions under which algorithms and systems are tested. These conditions are described in the evaluation test design, protocol and methodology. Tests are administered using an evaluation protocol that identifies the mechanics of the tests and the manner in which the tests will be scored. In face recognition, the protocol states the number of images of each person in the test, how the output from the algorithm is recorded, and how the performance results are reported. Publishing the evaluation protocol, as recommended in point 3 , lets the readers of published results understand how the results were computed.

Point 4 addresses the 'three bears' problem. Phillips, Moon, et al. (2000) first articulated the 'three bears' problem in designing face recognition evaluations. The 'three bears' problem sets guiding principles for designing an evaluation of the right level of difficulty. If all the scores for all algorithms are too high and within the same error margin, then one cannot distinguish among the algorithms tested. In addition, if the scores are too high in an evaluation, then that is an indication that the evaluation was in reality an exercise in 'tuning' algorithm parameters. If the scores are too low, then it is not possible to determine what problems have been solved. The goal in designing an evaluation is to have variation among the scores. There are two sorts of variation. The first type is variation among the experiments in an evaluation. Most evaluations consist of a set of experiments, where each experiment reports performance on different problems in face recognition. For example, experiments might look at changes in lighting or subject pose of a face. The second type of variation is among algorithms for each experiment. The variation in performance among the experiments lets one know which problems are currently sufficiently solved for consideration in field testing, which problems are research problems, and which problems are beyond the capabilities of the field. The variation among algorithm performance lets one know which techniques are best for a particular experiment. If all the scores for all algorithms across all experiments are virtually the same, then one cannot distinguish among the algorithms.

The key elements that ease adoption of points three and four can be incorporated into the evaluation protocol. For FRVT 2002, this was the FRVT 2002 evaluation protocol. This evaluation 
protocol was designed to assess the state of the art, advance the state of the art, and point to future directions of research. The ability to accomplish these three goals simultaneously was through a protocol whose framework allows for the computation of performance statistics for multiple galleries and probe sets. This allows for the FRVT 2002 evaluation protocol to solve the 'three bears' problem by including galleries and probe sets of different difficulties into the evaluation. This produces a comprehensive set of performance statistics that assess the state of the art, progress in face recognition, and point to future directions of research. The use of an XML-based specification allows for this evaluation protocol to become a formal standard for biometric evaluation.

The solution to the 'three bears' problem lies in the selection of images used in the evaluation. The characteristics and quality of the images are major factors in determining the difficulty of the problem being evaluated. For example, the location of the face in an image can affect problem difficulty. The problem is much easier if a face must be in the center of image compared to the case where a face can be located anywhere within the image. In FRVT 2002 data sets, variability was introduced by the size of the database, inclusion of images taken at different dates and both outdoor and indoor locations. This resulted in changes in lighting, scale, and background.

The testing protocol is based upon a set of design principles. The design principles directly relate the evaluation to the face recognition problem being evaluated. In particular, for FERET and FRVT 2000, the driving applications were searching large databases and access control. Stating the design principles allows one to assess how appropriate the FERET tests and FRVT 2000 are for a particular face recognition algorithm. Also, design principles assist in determining if an evaluation methodology for testing algorithm(s) for a particular application is appropriate.

The FRVT 2002 evaluation protocol consists of two parts. The first is the rules for conducting an evaluation, and the second is the format of the results that allow for scoring. The specific file format specifications are XML-based. Formal details can be found in "Face Recognition Vendor Test 2002: The Technical Appendices."

The input to an algorithm or system being evaluated is two sets of signatures, the target and query sets. Galleries and probe sets are constructed from the target and query sets respectively. The output from an algorithm is a similarity measure between all pairs of images from the target and query sets. A similarity measure is a numerical measure of how similar two faces are. Performance statistics are computed from the similarity measures. A complete set of similarity scores between all pairs of signatures from the target and query set is referred to as a similarity matrix. The first rule in the FRVT 2002 evaluation protocol is that a complete similarity matrix must be computed. This rule guarantees that performance statistics can be computed for all algorithms.

To be able to compute performance for multiple galleries and probe sets requires that multiple signatures of a person are placed in both the target and query sets. This leads to the second rule: Each signature in the target and query sets is considered to contain a unique face. In practice, this rule is enforced by giving every signature in the target and query sets a unique random identifier.

The third rule is that training is completed prior to the start of an evaluation. This forces each algorithm to have a general representation for faces, not a representation tuned to a specific gallery. Also, if training were specific to a gallery, it would not be possible to construct multiple galleries and probe sets from a single run. An algorithm would have to be retrained and the evaluation rerun for each gallery.

Using target and query sets allows us to compute performance for different categories of images. Possible probe categories include (1) gallery and probe images taken on the same day, (2) duplicates taken within a week of the gallery image, and (3) duplicates where the time between the images is at least one year. This is illustrated in the following example. A target and query set consists of the same set of facial images. Eight images of each face are taken. Each face is taken 
both indoors and outdoors, with two different facial expressions on two different days. From these target and query sets, one can measure the effects of indoor versus outdoor illumination by constructing a gallery of indoor images and a probe set of outdoor images, both consisting of neutral expressions taken on the first day. Construction of similar galleries and probe sets would allow one to test the effects of temporal or expression changes. The effect of covariates such as age and sex of a person can also be measured. It is the ability to construct galleries from the target set and probe sets from the query set that allows the FRVT 2002 protocol to perform a detailed analysis.

The FRVT 2002 evaluation protocol allows for the computation of performance statistics for identification, verification, and watch list tasks. The protocol is sufficiently flexible that one can estimate performance using sub-sampling and re-sampling techniques. For example, galleries of varying sizes are created to measure the effects of gallery size on performance. To estimate the variability of performance, multiple galleries are created.

\section{Performance Statistics}

In FRVT 2002, performance results are reported for identification, verification, and watch list tasks. In addition to computing classical performance statistics, new statistical methods were developed to estimate variation in performance over multiple galleries and to explore the effect of covariates on performance. This section presents an overview of the performance statistics that describe the FRVT 2002 experiments. Mathematical details are provided in Appendix A.2.

The identification task allows one to ask how good an algorithm is at identifying a probe image. The question is not always "is the top match correct?," but rather "is the correct answer in the top $k$ matches?" The identification task is modeled after real-world law enforcement applications in which a large electronic mug-book is searched to identify suspects. In identification scoring, the similarity scores between the probe and all the gallery images are sorted. A higher similarity score means there is a greater resemblance between two faces. In identification, every probe has a match with one of the gallery images - this is known as the correct match (identification is a closed universe evaluation). A probe is correctly identified if the correct match has the highest similarity score. More generally, a probe has rank $k$ if the correct match is the $k^{\text {th }}$ largest similarity score. For example, a probe has rank 5 if the correct match is the fifth largest similarity score. A probe is correctly identified if it has rank 1 . Rank can vary between 1 and the size of the gallery. The identification rate at rank $k$ is the fraction of probes that have rank $k$ or higher. Identification performance is plotted on a cumulative match characteristic (CMC). Figure 10 from Section 7 shows a CMC. The horizontal axis of the graph is rank on a logarithmic scale. A logarithmic scale was chosen to emphasize the lower ranks. Most applications are based on performance at lower ranks. The vertical axis is the identification rate. Identification is also known as 'one to many' matching because a probe is compared to an entire gallery.

In FRVT 2002, we assume the following operational model for biometric verification or authentication systems. In a typical verification task using, a subject presents his biometric signature to the system and claims to be a person in the system's gallery. The presented biometric signature is a probe. The system then compares the probe with the stored signature of the subject in the gallery. The comparison produces a similarity score. The system accepts the identity claim if the similarity score is greater than the system's operating threshold. The operational threshold is determined by the applications, and different applications will have different operational thresholds. Otherwise, the system rejects the claim. In analyzing verification performance, two cases need to be considered. The first case concerns when a person makes a legitimate identity claim - the person is who he claims to be. The performance measure for this case is the correct verification rate. The second case is when the claimed identity is incorrect-the person is not who he claims to be. The performance measure for the second case is the false accept rate. The ideal system would have a verification rate of 1.0 and a false accept rate of 0.0 . All legitimate claims are 
accepted and all false claims are rejected. However, there is a trade-off between the verification and false accept rates in real-world systems. By changing the operating threshold, the verification and false accept rate change. A higher operating threshold results in lowering the false accept rate and the verification rate.

Verification and false accept rates characterize verification performance. Both these performance rates cannot be maximized simultaneously; there is a trade-off between them. Verification performance is reported by showing the trade-off between the verification and false accept rates on a receiver operator characteristic (ROC). An example of a ROC is shown at figure 7, Section 7. The horizontal axis is the false accept rate (scaled logarithmically). A logarithmic scale is used because operating points of interest have low false accept rates and a logarithmic scale emphasizes low false accept rates. The vertical axis is the verification rate. Verification is also known as 'one to one' matching because a decision is made by comparing one probe signature with one gallery signature. Because probes presented to a system are not necessarily in the gallery, verification is considered an open universe task.

In FRVT 2002, we examine how verification performance varies under two conditions. The first is how performance varies with different galleries. This models the performance of a system that might be installed at different locations. The second is how performance varies for different classes of probes. For example, what is the difference in performance for male and female probes? Each combination of the gallery and probe sets generates a different ROC. To study the variation, it is necessary to combine results over a set of ROCs. One method of combining results is to measure the variation of the verification rate for each false alarm rate. This models the situation where one can readjust the operating threshold for each gallery or probe set. For many applications, this is not feasible or desirable. However, this is an appropriate technique for combining ROCs from multiple systems because it is not possible to set uniform operating thresholds across different systems. For the same system, it is possible to set one operating threshold across all galleries and probe sets. Using this 'base-operating threshold,' one computes the verification and false accept rate for each gallery and probe set. The resulting verification and false alarm rates will vary across the different galleries and probe sets. This method for computing variance in performance models the situation in which the operating threshold is set once for an application. Setting the base-operating threshold can be based upon an overall desired performance level for the population that will use the system. In FRVT 2002, the base-operating threshold is set based upon the system performance on an aggregate population. The base-operating threshold corresponds to a specific false accept rate on the aggregate population - this is referred to as the nominal false accept rate.

The last task examined was the watch list. The watch list task is a generalization of verification and identification. Like verification, watch list is an open universe task. In fact, verification and identification are special cases of the watch list task. In the watch list task, a system determines if a probe corresponds to a person on the watch list, and then identifies the person in the probe. When a probe is provided to the system, it is compared to the entire gallery. The gallery is also known as the watch list. As in identification, the similarity score between the probe and the gallery are sorted. If the top match is above an operating threshold, an alarm is declared and the estimated identity is reported. This is the verification portion of the watch list task. As in verification, there are two performance statistics: detection and identification rate, and false alarm rate. We will first look at the case where the identity of a probe is someone in the gallery (in the watch list). A probe is detected and identified if the probe is correctly identified and the correct match score is above the operating threshold. The detection and identification rate is the fraction of probes of people in the gallery who are detected and identified. In the second case, a probe is not of someone in the gallery. This type of probe is also referred to as an imposter. A false alarm occurs when the top match score for an imposter is above the operating threshold. Watch list performance is reported on a ROC, where the $x$-axis is the false alarm rate and the $y$-axis is the detection and identification rate (see figure 12 in Section 7). A watch list ROC plots the trade-off between the detection and identification rate, and false alarm rate. 
In the general case, the system examines the top $k$ matches with the gallery. A probe of a person in the gallery is detected and identified at rank $k$ if the probe is of rank $k$ or less and the correct match is above the operating threshold. For imposter probes, a false alarm at rank $k$ occurs if any of the top $k$ matches are above the operating threshold. The full watch list performance is plotted along three axes: detection and identification rate, false alarm rate, and rank. The performance of a system is represented as a surface in the three-dimensional parameter space. When the size of the gallery is one, the watch list reduces to verification. Identification is the special case when the false alarm rate is 1.0. Formally, the identification $\mathrm{CMC}$ is the curve that results from intersecting the watch list performance surface with the false alarm rate equals 1.0 plane.

FRVT 2002 allowed participants to submit normalization procedures. Normalization is a post processing function that adjusts similarity scores based on a specific gallery. FRVT 2002 allowed a different normalization function for each task: identification, verification, and watch list. The input to a normalization routine is the set of all similarity scores between a probe and a gallery. The output is a new set of normalized similarity scores between a probe and a gallery. The normalization function attempts to adjust for variations among probes and to emphasize differences among the gallery signatures. If the gallery changes, then similarity scores need to be normalized again. This has implications for scoring techniques that require performance on multiple galleries. Traditionally, verification has been referred to as 'one to one' matching. This is because, in verification, one probe is matched with one gallery signature. Normalization requires that a probe be compared with a gallery. When normalization is applied, is verification still 'one to one' matching?

All the results in this report are performance statistics estimated from participant report similarity scores. As with all estimation procedures there is an uncertainty associated with the measurement. In face recognition and biometrics, it is an active area of research to develop techniques to measure the uncertainty of an estimator (Bolle et al. 2000; Beveridge et al. 2001; Micheals and Boult 2001; Moon and Phillips 2001). Unfortunately, there are no accepted methods for computing confidence intervals in the biometric community. Therefore, we do not place confidence intervals on performance statistics reported. Another measure of uncertainty is the variance of an estimator. For verification performance on the HCInt we report the observed variance of the verification performance estimators. In this case we were able to report the observed variance because of the large size of the HCInt data set.

Mansfield and Wayman (2002) describe four performance statistics that are not reported in FRVT 2002. These statistics provide an additional level of detail in performance and are mainly designed for scenario and fingerprint evaluations. FRVT 2002 views the systems being tested as black boxes and reports performance for the complete system. Two of the statistics are failure to enroll and failure to acquire. The analogy of failure to enroll in FRVT 2002 is that a template of sufficient quality could not be extracted for a gallery signature. In FRVT 2002, if a system could not extract a template for a gallery signature, then most likely, all matches with that signature would produce low similarity scores. This would be reflected in the performance statistics. The FRVT 2002 design is transparent to these types of internal errors that may be handled differently by each participant system. The analogy for failure to acquire is that a template of sufficient quality could not be extracted from a probe signature. This is handled in the same manner as a failure to enroll. Binning error rate and penetration rate are system-level statistics of the success of limiting the expense of one to many searches by suitably partitioning the gallery. FRVT 2002 is transparent to partitioning; any binning undertaken by a system is internal and not explicitly reported. 


\section{FRVT 2002 Test Design}

FRVT 2002 was designed to allow participation by as many face recognition research groups and companies as possible. FRVT 2002 consisted of two sub-tests. These were the high computational intensity (HCInt) and medium computational intensity (MCInt) tests. The two sub-tests were designed to encourage broad participation in the evaluation. The HCInt was designed to evaluate the performance of state-of-the-art systems on extremely challenging real-world problems. The MCInt was designed to provide an understanding of a participant's capability to perform face recognition tasks with several different formats of imagery (still and video) under varying conditions. The MCInt was also designed to help identify promising new face recognition technologies not identified in the HCInt.

FRVT 2002 was announced on 25 April 2002 in Federal Business Opportunities, the Biometric Consortium list server (listserv), and in e-mails to members of the face recognition and biometrics communities. FRVT 2002 was open to all developers and providers of core face recognition technology. This included academia, research laboratories, and commercial companies. There were neither fees nor compensation for participating. Participants were required to provide all hardware, software, and personnel necessary to complete the test. Participants could take the HCInt, MCInt, or both. The participants and the tests they took are provided in table $3^{2}$. FRVT 2002 was administered at the U.S. Naval base at Dahlgren, Virginia between 10 July and 9 August 2002.

Table 3. List of FRVT 2002 participants and tests completed

\begin{tabular}{lcc}
\hline \multicolumn{1}{c}{ Participant } & MClnt & HClnt \\
\hline AcSys Biometrics Corp & $\mathrm{X}$ & \\
Cognitec Systems GmbH & $\mathrm{X}$ & $\mathrm{X}$ \\
C-VIS Computer Vision und Automation GmbH & $\mathrm{X}$ & $\mathrm{X}$ \\
Dream Mirh Co., Ltd & $\mathrm{X}$ & $\mathrm{X}$ \\
Eyematic Interfaces Inc. & $\mathrm{X}$ & $\mathrm{X}$ \\
Iconquest & $\mathrm{X}$ & $\mathrm{X}$ \\
Identix & $\mathrm{X}$ & $\mathrm{X}$ \\
Imagis Technologies Inc. & $\mathrm{X}$ & $\mathrm{X}$ \\
Viisage Technology & $\mathrm{X}$ & $\mathrm{X}$ \\
VisionSphere Technologies Inc. & $\mathrm{X}$ & \\
\hline
\end{tabular}

All images and video sequences in FRVT 2002 were sequestered prior to the test and had not been seen by any participant. Testing on sequestered data has a number of advantages. First it provides a level playing field. Second, it ensures that systems are evaluated on the general face recognition task, not the ability to tune a system to a particular data set. FRVT 2002 was administered under strict U.S. Government supervision. All tuning and adjustment to participants' systems had to be complete prior to arrival at the test site.

The HCInt contained one target and query set. The target and query set were the same and contained 121,589 full frontal facial images (see Section 6.1 for details). The HCInt was fully automatic. In a fully automatic test, the input to the system is the facial image or video sequence. The system must automatically locate the face in the image and find any facial features that are required by the system. The HCInt had to be performed on the equivalent of three high-end workstations. Technical specifications for each participant's HCInt and MCInt systems are provided in the Face Recognition Vendor Test 2002: Technical Appendices. Participants were given 264 hours to complete the test and output the complete set of similarity files. The complete set of similarity files consisted of approximately 15 billion similarity scores.

The MCInt consisted of two sub-tests: still and video. The still and video sub-tests each had a target and query set. In both sub-tests the target and query set were the same. Both sub-tests were fully automatic. Participants had to complete both sub-tests in 264 hours on one high-end

2. The identification of any commercial product or trade name does not imply endorsement or recommendation by the National Institute of Standards and Technology, or any other FRVT 2002 sponsor or supporter. 
workstation. The output from the MCInt consisted of approximately 56 million similarity scores. The still target and query set consisted of 7,722 digital facial images. The types of facial images varied-details can be found in Section 6.2. The video target and query set consisted of 1,890 signatures. The signatures consisted of 882 still facial images and 1008 facial video sequences.

\section{IMAGE DATA SETS}

This section describes the data sets used in FRVT 2002. Some common aspects of all images used in FRVT 2002 are that they contained the face of exactly one individual, that the face (and neck and part of the chest) was the only foreground object, and that the image was acquired in the visible spectrum in color. All images provided to FRVT 2002 participants were in standard JPEG format.

\subsection{HCINT DATA SET}

The HCInt data set is a subset of a much larger collection provided by the Visa Services Directorate, Bureau of Consular Affairs of the U.S. Department of State. That collection consists of approximately 6.8 million images from about 6.1 million visa applicants collected from 1996 to 2002. The HCInt data set consisted of 121,589 images from the database. HCInt contained at least three images of each of the 37,437 subjects in the data set.

The images are of good quality and were gathered in a consistent manner. They were collected at U.S. consular offices using standard issue digital imaging apparatus whose specification remained fixed over the collection period. The result is a set of well-posed (i.e., frontal to within 10 degrees) images of cooperative subjects usually with a mouth-closed neutral facial expression. The subject usually occupies a quarter of the image area. The top of the subject's shoulders is almost always visible. The background is universally uniform, with a white background (in most cases). The names of the individuals were encoded to protect the privacy of the subjects. Year of birth was provided. Due to privacy considerations, representative images of the actual data set are shown in figure 1.

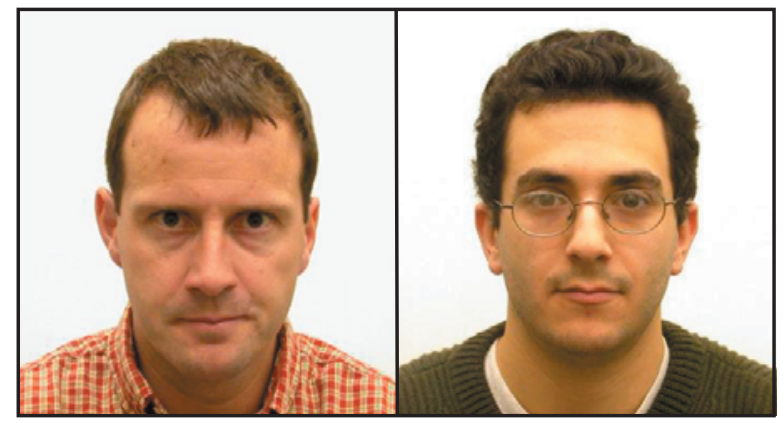

Fig. 1. Images included here are reasonable representations of those used in the FRVT 2002 High Computational Intensity test.

One concern with operational databases is the integrity of the identities associated with the images. There are two errors that can occur. First, two different persons can have the same identify. Since the identifier is based on a hash of the person's name and other identifying information, it is very unlikely that two people will have the same identifier. Second, a person has two identifiers. This usually occurs because of clerical errors. Since each individual in the data set has at least three images, the same clerical error would have had to occur three times. Thus, the inclusion of three images of each person in the data set makes it very unlikely that a person in the HCInt data set will have two identifiers. 


\subsection{MCINT DATA SET}

In contrast to the HCInt image set, the MCInt data set is composed of a heterogeneous set of still images and video sequences of subjects in a variety of poses, activities and illumination conditions. The images originate from two sources. The first is the still facial image data set collected the National Institute of Standards and Technology (NIST), Naval Surface Warfare Center (NSWC, Dahlgren), and the University of South Florida (USF) between 1999 and 2002. The second, from The University of Texas at Dallas, consists of video and stills taken in 2001.

The NIST-NSWC-USF data set is comprised of images taken indoors and outdoors. Although the images were taken over more than three years at three sites, they were acquired in almost identical circumstances such that their geometric and photometric characteristics are approximately the same. While the imagery itself has consistent properties, the subject populations of the three sites differ. The USF segment consists of subject population of diverse ethnicity that is distinct from the older, predominantly Caucasian mix at NIST and NSWC. The images in figure 2 are examples of the gallery and probe set images used for testing changes in expression, overhead lighting and outdoor lighting, and elapsed time. The outdoor stills are characterized by changing background and directional sunlight illumination.

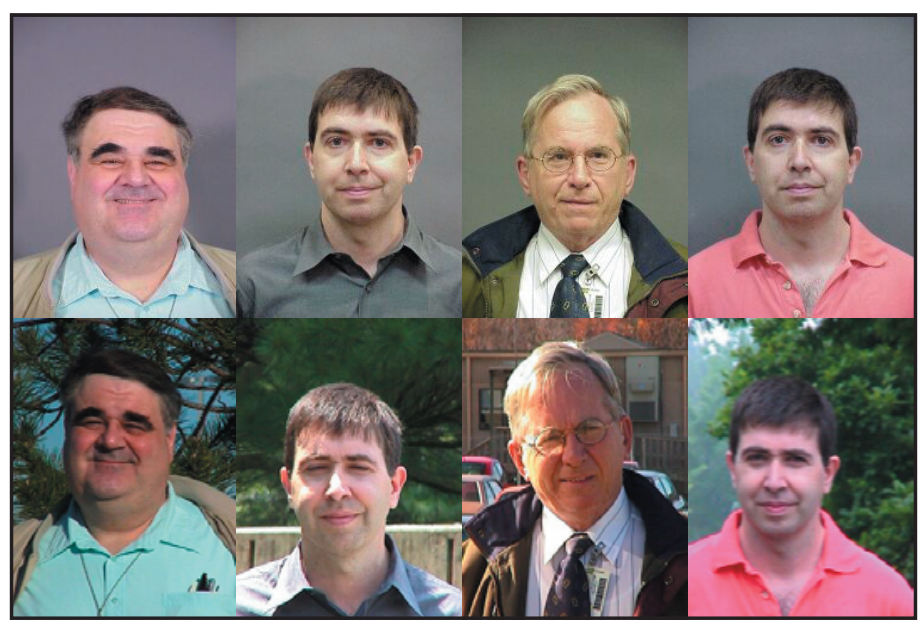

Fig. 2. Indoor and outdoor images from the NIST-NSWC-USF data set. The top row contains images taken indoors and the bottom contains outdoor images taken on the same day as the indoor images.

The MCInt image set included partitions to test the efficacy of using 3D morphable models as a preprocessing step in the recognition of non-frontal images (Blanz and Vetter 1999; Blanz et al. 2002). The concept is that a non-frontal image is fitted to a $3 \mathrm{D}$ model of human heads, and then the morphable model generates a frontal view based on the non-frontal image. In the MCInt, morphed images were generated by the algorithm of Blanz et al. (2002)(See Technical Appendice O).

The University of Texas at Dallas data set was created for use in human memory experiments. Close-range video clips and static images were taken of more than a hundred individuals on at least two different occasions to make duplicate sets separated by one week to six months. Included in the MCInt was a 63-person subset of this data set of subjects that appeared on two occasions.

Still Images. Nine high quality still images of each individual were taken from equally spaced viewpoints spanning angles from left profile to right profile.

Exploration Video Sequences. A video was taken of each individual's face moving through the nine facial poses used for the still images.

Facial Speech Videos. Two video clips were taken of individuals speaking, first in a neutral way, then in an animated way. Figure 4 shows two examples. 


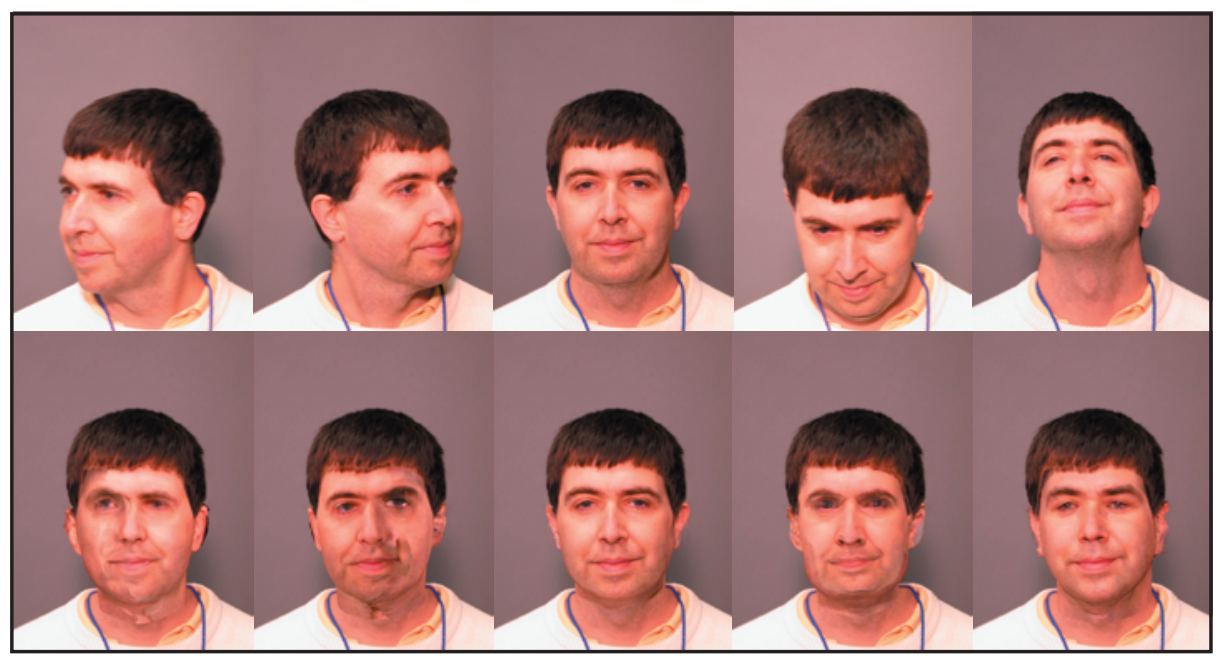

Fig. 3. The top row shows original images. The subject, illuminated by one light source, is looking left and right at about 45 degrees, straight at the camera, and up and down at about 30 degrees. The second row shows the corresponding frontal reconstructions from the 3D morphable model. The center column shows the effect of fitting the morphable model to a frontal image.

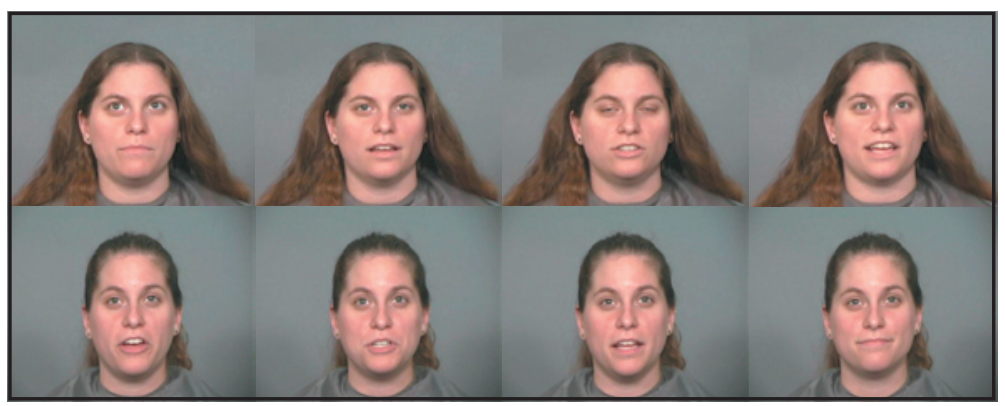

Fig. 4. The rows show selected frames from examples of the UT Dallas "facial speech" videos lasting 150 frames. The two rows show the subject gathered on different occasions.

\section{High COMPUTATIONAL INTENSITY TEST RESULTS}

The HCInt target set contains 121,589 images from 37,437 individuals, with at least three images of each person. The query and target set are identical. The design of the HCInt experiments is based on the HCInt large gallery and HCInt large probe set. The large gallery consists of the earliest dated image for the 37,437 individuals in the target set. The large probe set consists of two images of each person in the gallery. The first image is the most recent image. This ensures that performance can be computed for the greatest possible elapsed time for each person in the HCInt data set. The second image of each person placed in the probe set is that with a median time between the gallery and all images of a person. For people with three images in the query set, the oldest image is placed in the gallery and the two most recent images are placed in the probe set. A histogram of the distribution of elapsed time between probe and gallery images is given in figure 5. The number of probes in each bin is broken out by sex.

The HCInt large gallery contains 18,468 males and 18,878 females (there were 91 individuals where the sex could not be determined). The numbers in figure 5 and figure 6 do not always add up. In figure 5 the histogram is terminated at 1,140 days. Some of the probes show the elapsed 


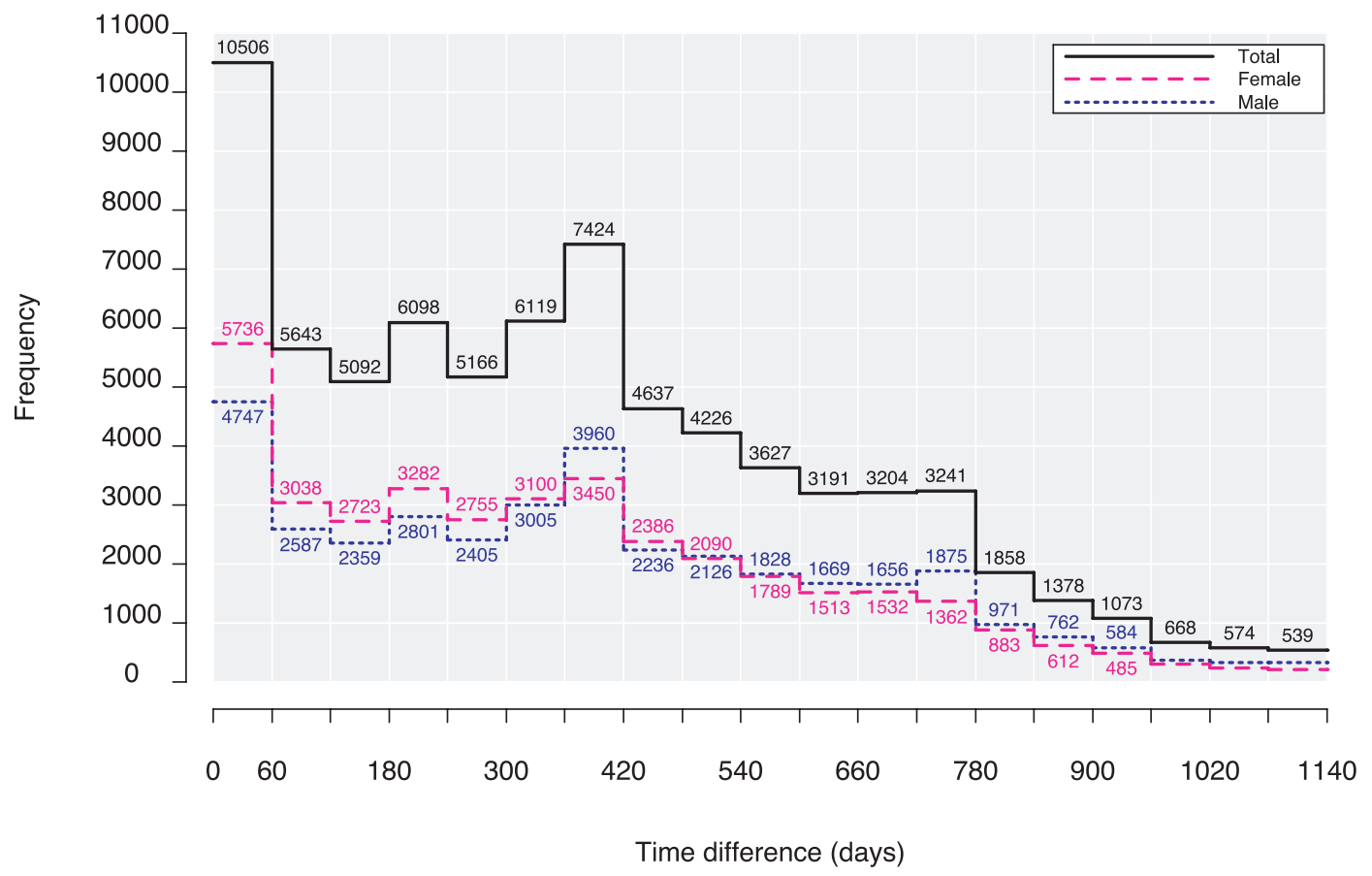

Fig. 5. Histogram of elapsed time between gallery and probe images of a person. The time intervals are divided into 60-day bins. The number of probes in each bin is broken out by sex.

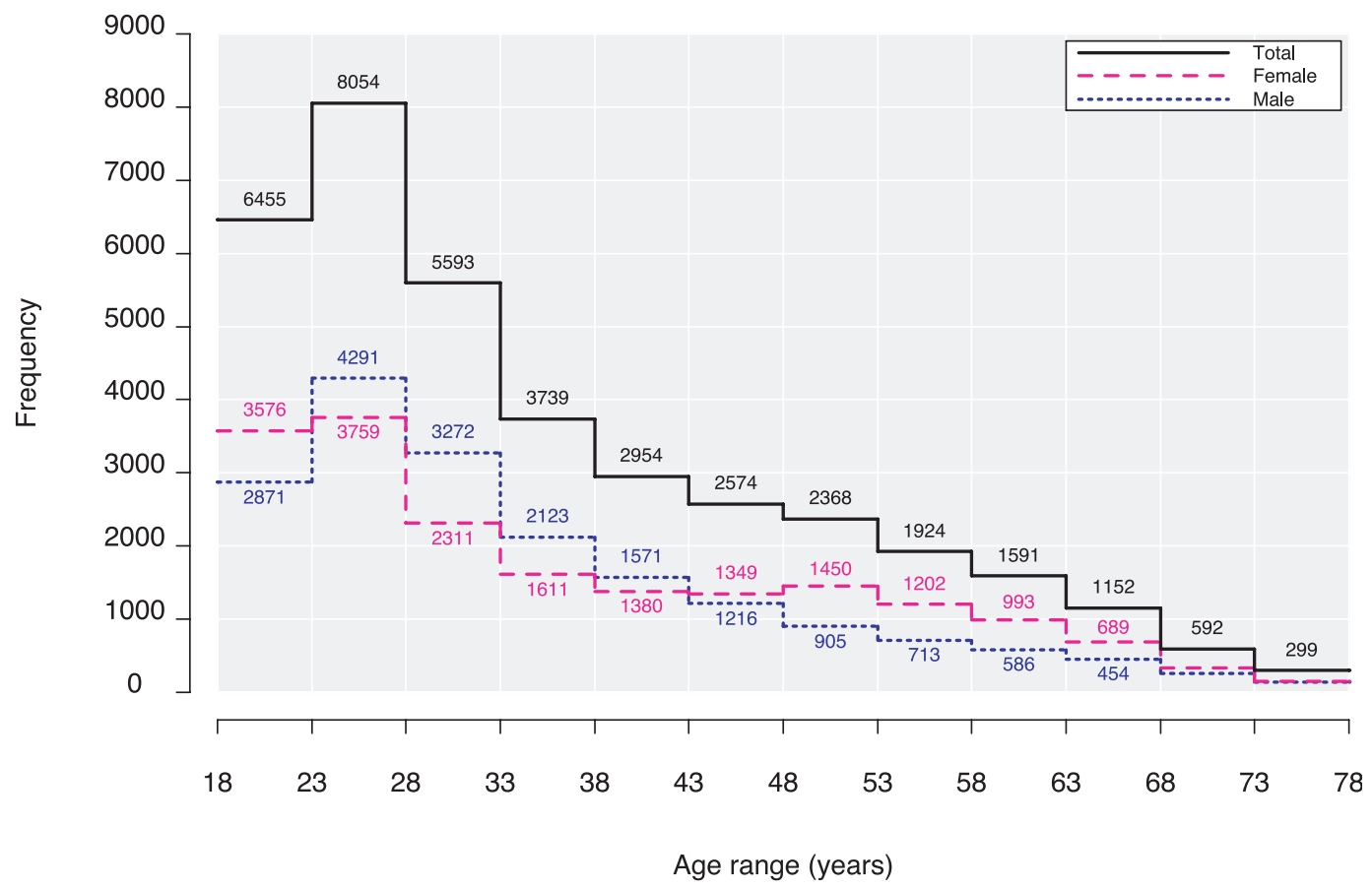

Fig. 6. Histogram of age distribution of people in the HCInt large gallery. The number of people in each bin is broken out by sex. 
time is greater than 1,140 days, however, the number is sufficiently small so performance was not calculated. The people in the HCInt data set were divided into 12 age categories. Each age category is a five-year bin, with the first bin containing 18 to 22 -year olds. The age of a person is set at the age when the gallery image was taken. A histogram documenting the distribution of elapsed time between probe and gallery images is shown in figure 6 . The number of people in each bin is broken out by sex. The numbers do not add up to 37,437 . People older than 78 were not included in the histogram. This is because there are too few of them to justify experimentation.

Additional galleries and probe sets are derived from the large gallery and probe set. The exact composition depends on the experiment run. Unless otherwise stated, if the image of a person is placed in the gallery, then the two images of that person in the large probe set are placed in the new probe set.

One of the new features introduced in FRVT 2002 is measuring the variance in the statistics computed to determine how performance changes if the people in the gallery are different. Computing the variance requires computing performance statistics on multiple galleries and probe sets. To perform the variance experiments, a set of twelve HCInt small galleries were generated from the large HCInt gallery. Each gallery consists of 3,000 individuals. The twelve galleries are disjoint. There are twelve corresponding small probe sets. A small probe sets consists of the two probe images in the large probe set for each person in the gallery. Thus, each small probe set consists of 6,000 images from 3,000 individuals; each probe is an image of a person in the gallery.

The first experiment is the HCInt large gallery verification experiment. Figure 7 shows verification performance on a ROC for the eight participants. The false accept rate axis is on a logarithmic scale. The verification performance was computed by the round-robin technique. The false alarm rate was computed from probes in the large probe set; i.e., the match and non-match distributions were both generated from the large probe set.

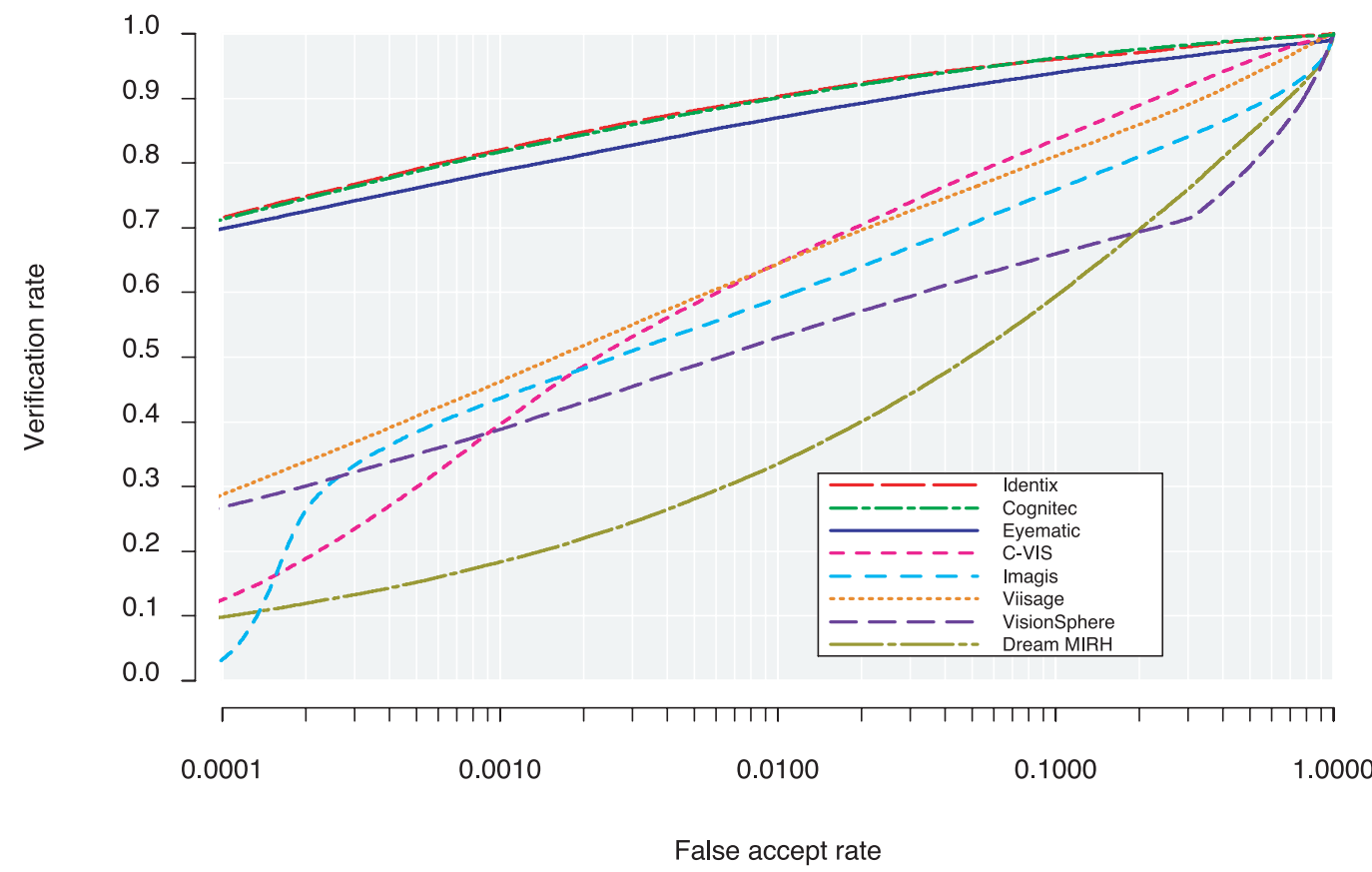

Fig. 7. Verification performance ROC for eight participants on the HCInt large gallery and probe set. The gallery consisted of 37,437 individuals with one image per person, and the probe set consisted of $\mathbf{7 4 , 8 7 4}$ probes with two images per person. 
The results in figure 7 report verification performance for a single large gallery. The results do not address the important question of how performance varies if the people in the gallery are different. To measure this variation, verification performance was computed for the twelve HCInt small galleries. The false alarm rate was computed from 6,000 true imposters (two images of 3,000 individuals). The results for this experiment are presented in figure 8 for Cognitec, Eyematic, and Identix. The centerline is the aggregate performance for the twelve galleries. For selected operating points, performance was computed for the twelve small galleries and probe sets. For each of the twelve galleries, verification rates and false accept rates were computed. The false accept rate was computed with true imposters. Thus, at each operating threshold, there are twelve pairs of verification and false accept rates. A standard error ellipse was computed for each set of verification and false accept rates.

Error ellipses in figure 8 are two times the standard deviation of the verification and false accept rates along the appropriate axes. An ellipse gives an estimate of the range in performance that could result if the people in the gallery are changed. If the large gallery were larger, it would be possible to compute performance for more small galleries of size 3,000. The greater number of small galleries would increase the accuracy of the error ellipse. However, the size of the ellipses would not decrease as the number of small galleries increased. This is because the error ellipses are a function of the multiple small galleries, and composition of the small galleries reflects the natural variation in the population. The natural variation will always be present-more small galleries increase the accuracy of the estimated variation in the performance due to the natural composition of the population. In the HCInt the ellipses are estimated from disjoint galleries and probe sets. This avoids many of the issues associated with re-sampling techniques. Re-sampling techniques require making assumptions about the distributional properties of the similarity scores. Typical assumptions are that similarity scores are independent and identification distributed (iid). In interpreting the meaning of error ellipses, a number of subtle facts need to be noted. The error ellipses are not error bounds on the ROC. Rather, error ellipses are a measure of the variance in

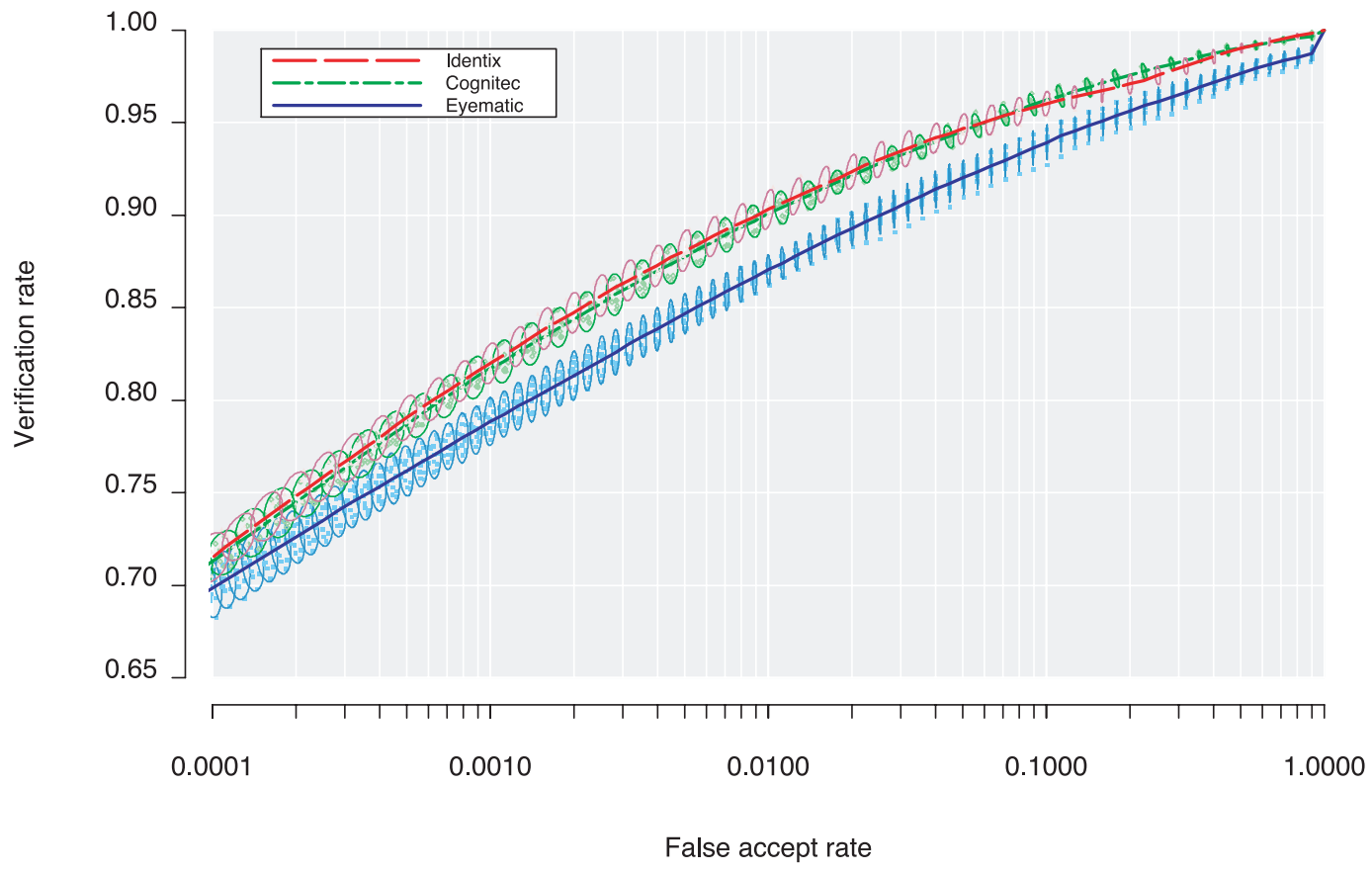

Fig. 8. Standard error ellipses for verification performance for Cognitec, Eyematic, and Identix. The standard error was computed from twelve HCInt small galleries of size 3,000. The center line is the ROC performance for the aggregate of all twelve galleries. The ellipses are two times the standard deviation at selected performance points, and the points clustered around the ellipses represent the performance of one of the twelve galleries at the selected performance point. 
performance that occurs by changing the gallery. The standard error is an empirical estimate of the variation. They are not confidence intervals. Confidence intervals decrease in size as the number of samples increase. To estimate confidence intervals requires that one knows or can estimate the underlying distribution.

One of the recent innovations in face recognition has been the adoption of normalization. C-VIS, Eyematic, Identix, and Viisage submitted normalization routines in FRVT 2002. In two experiments, we investigate the contribution of normalization to verification and identification performance.

To measure the effect of normalization on verification, performance was computed for normalized and non-normalized versions for the twelve small galleries and probe sets. The difference in the verification rate was computed for four false accept rates: 0.0001, 0.001, 0.01, and 0.1 . Figure 9 plots the normalized score minus the non-normalized score. Figure 9 contains four panels, one for each false alarm rate. In each panel, the mean change in verification rate over the twelve small galleries is plotted. For example, at a false accept rate of 0.001 , the mean change in performance for Eyematic is 0.089. This means that normalization improved Eyematic's verification rate by 0.089 . In this experiment, the improvement in verification rate over the twelve galleries was very stable. The variance in the change is sufficiently small that it would not show up in figure 9 if plotted. The results in figure 9 show that normalization improves performance. All verification performance results in FRVT 2002 are computed with normalized scores for those who submitted normalization functions.
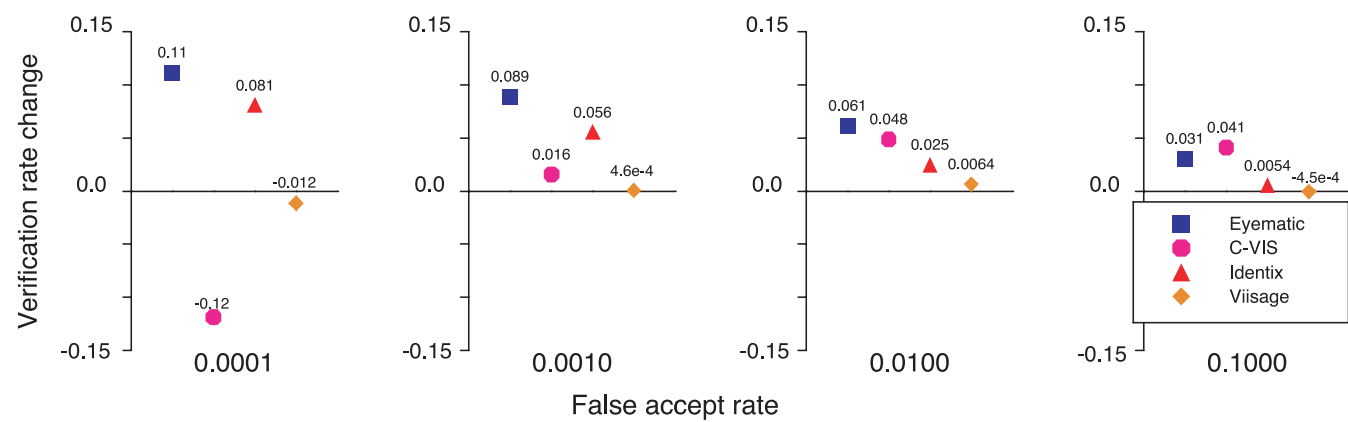

False accept rate

Fig. 9. Plots showing the difference between normalized and non-normalized verification performance. Performance was computed for the twelve galleries in the experiment. Relative performance of the verification rate is shown for false accept rates of $0.0001,0.001,0.01$, and 0.1 .

The same experiment was repeated with identification. The results showed that normalization did not improve performance for identification. Because normalization did not improve identification performance, all identification scores in FRVT 2002 are computed without normalization. For the watch list task, the improvement from normalized scores was comparable to verification, and therefore, watch list performance is computed using normalized scores.

The results of the HCInt large gallery identification experiment are shown in figure 10. Identification results are reported on a CMC with rank scaled logarithmically.

While the HCInt large gallery experiment measured performance on a large gallery of 37,437 individuals, the HCInt gallery size experiment examines how rank 1 identification performance decreases as gallery size increases. Figure 11 shows performance as a function of gallery size. The horizontal axis is gallery size on a logarithmic scale and the vertical axis is rank 1 identification performance. The performance was computed for thirteen difference gallery sizes: 25, 50, 100, $200,400,800,1,600,3,000,3,200,6,400,12,800,25,600$, and 37,437. The spread of the galleries sizes is approximately logarithmic in gallery size. 


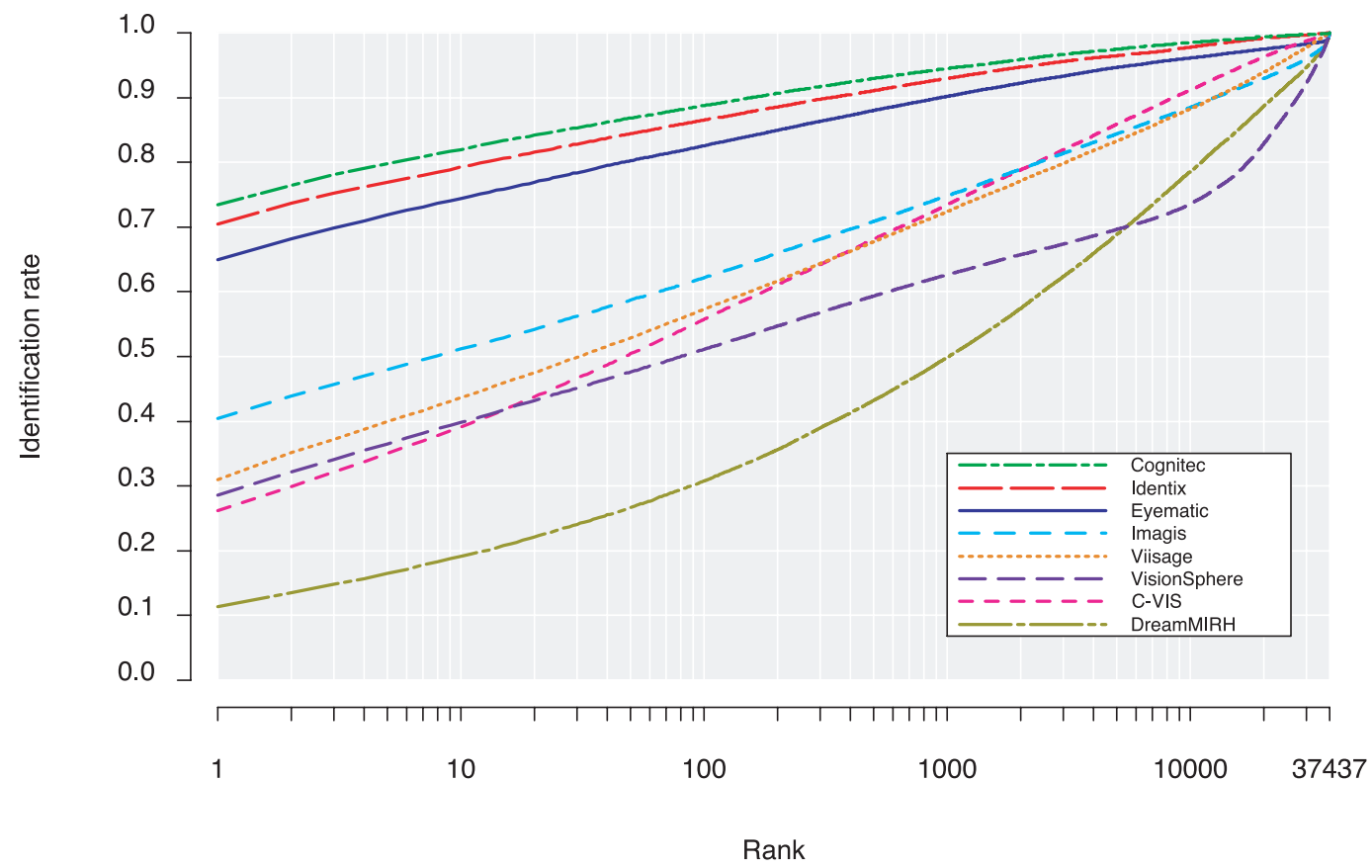

Fig. 10. Identification performance reported on a $\mathrm{CMC}$ for the large gallery and probe set. The large gallery contained images of 37,437 individuals. The horizontal axis is on a logarithmic scale

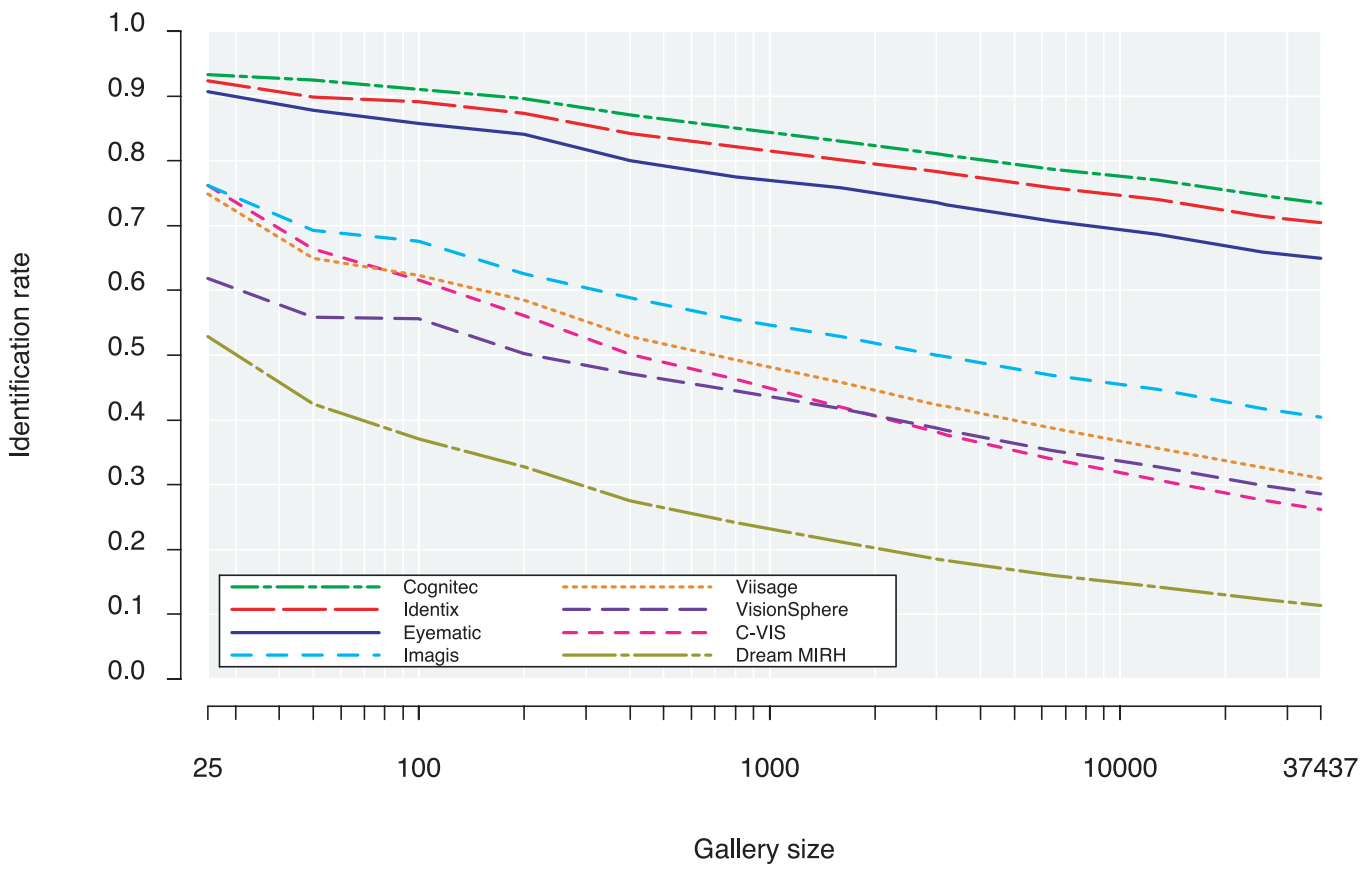

Fig. 11. Rank 1 identification performance as a function of gallery size.

The identification rates plotted in figure 11 are an average identification rate over a set of galleries. For each gallery size, disjoint galleries were generated and the mean identification was computed. Where possible, performance was computed from twelve galleries. However, the number of disjoint galleries generated was limited by the size of the HCInt data set. For example, it was not possible to generate more than one gallery of size 25,600 and 37,437. Where possible, performance is the average over multiple galleries. The averaging was done to provide a reliable estimate of performance. 
The following experiments examined watch list performance: the watch list ROC experiment, the watch list rank experiment, and the watch list gallery size experiment. Performance of a system on a watch list task is characterized by four values: detect and identify rate, false alarm rate, gallery size, and rank. To understand the effect of each of the values on performance, we performed three watch list experiments. In each of the experiments, one or two of the values are held constant so that the effect of changing the remaining values can be examined.

The watch list ROC experiment computes a watch list ROC for all eight participants. In this experiment, performance was computed for twelve galleries. To provide a reliable estimate, average performance over the twelve galleries was plotted. All galleries consisted of 3,000 individuals. The probe set consisted of 6,000 probes of people in the gallery and 6,000 imposters (there were two images per person in the probe set). Rank 1 identification performance is computed. Specifically, is the top match with a given probe a person on the watch list? Figure 12 shows the watch list performance for all eight participants.

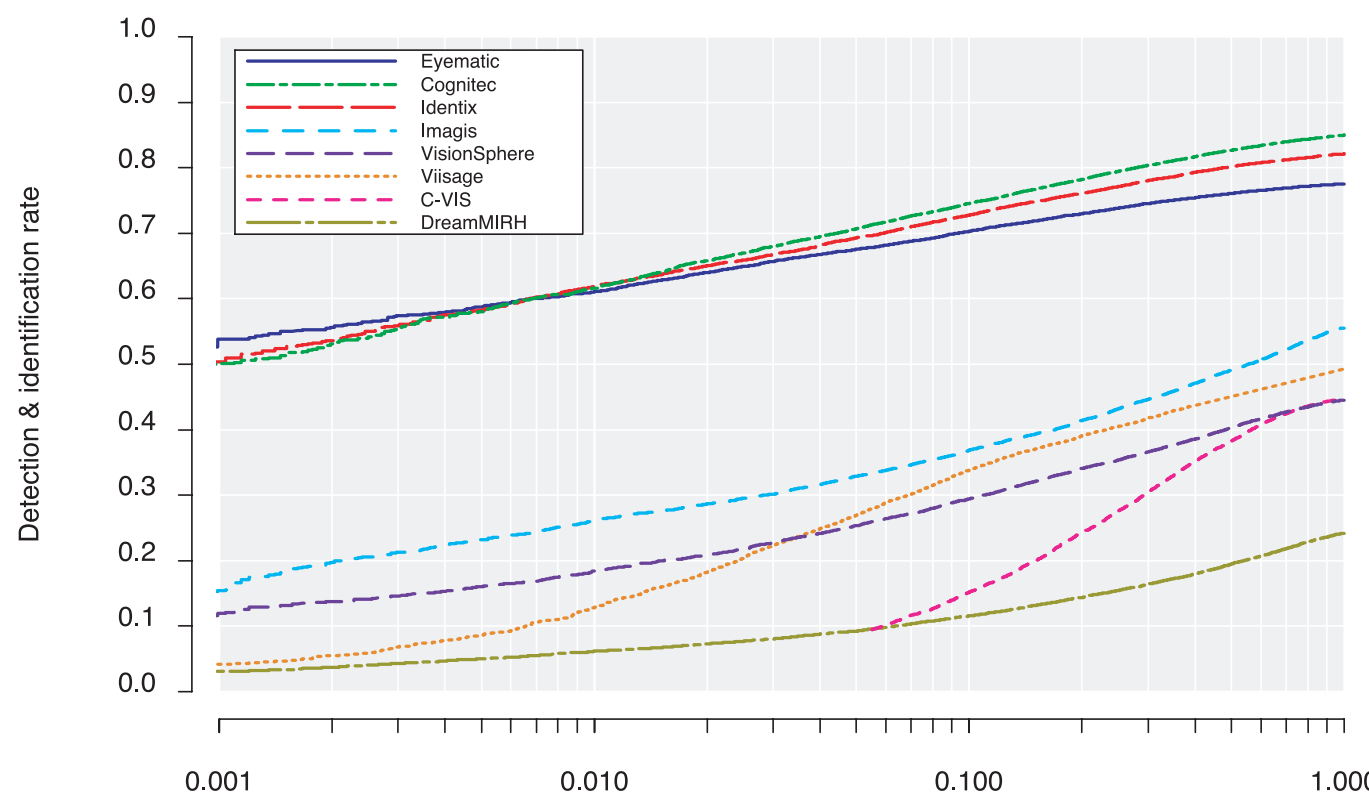

False alarm rate

Fig. 12. Watch list performance ROC. Gallery size is 3,000. Rank 1 performance is reported. The C-VIS system does not operate for the full range of false alarm rates plotted in this experiment.

In a normal watch list task, the rank 1 match between a probe and a gallery is found. If the rank 1 match score is above a threshold, an alarm is issued. The general case finds the top $k$ matches between a probe and a gallery, and reports those matches above an operating threshold. The watch list rank experiment investigates the effect of examining the top $k$ matches on performance. In this experiment, results are only reported for Cognitec in order to keep the graph readable-the overall trend applies to all participants. In the watch list rank experiment, the gallery consists of 800 individuals. The probe sets consist of 1,600 probes of people in the gallery (two per person in the gallery) and 1,600 imposters of 800 individuals. Results for this experiment are shown in figure 13. Full CMCs are computed for eight false alarm rates. The $x$-axis is rank and the $y$-axis is the detection and identification rate. Each curve in the CMC is calculated for a given false alarm rate. The false alarm rate associated with each curve is adjacent to the curve on the right-hand side. For a false alarm rate of 0.01 , performance is the same for all ranks. Even at a false alarm rate of 0.1, performance increases very slightly for $k=2$. This suggests that in watch list applications, one only needs to examine the top match. It is worth noting that in figure 13 the curve with a false alarm rate of 1.0 is the $\mathrm{CMC}$ for identification against the gallery of 800 individuals. 


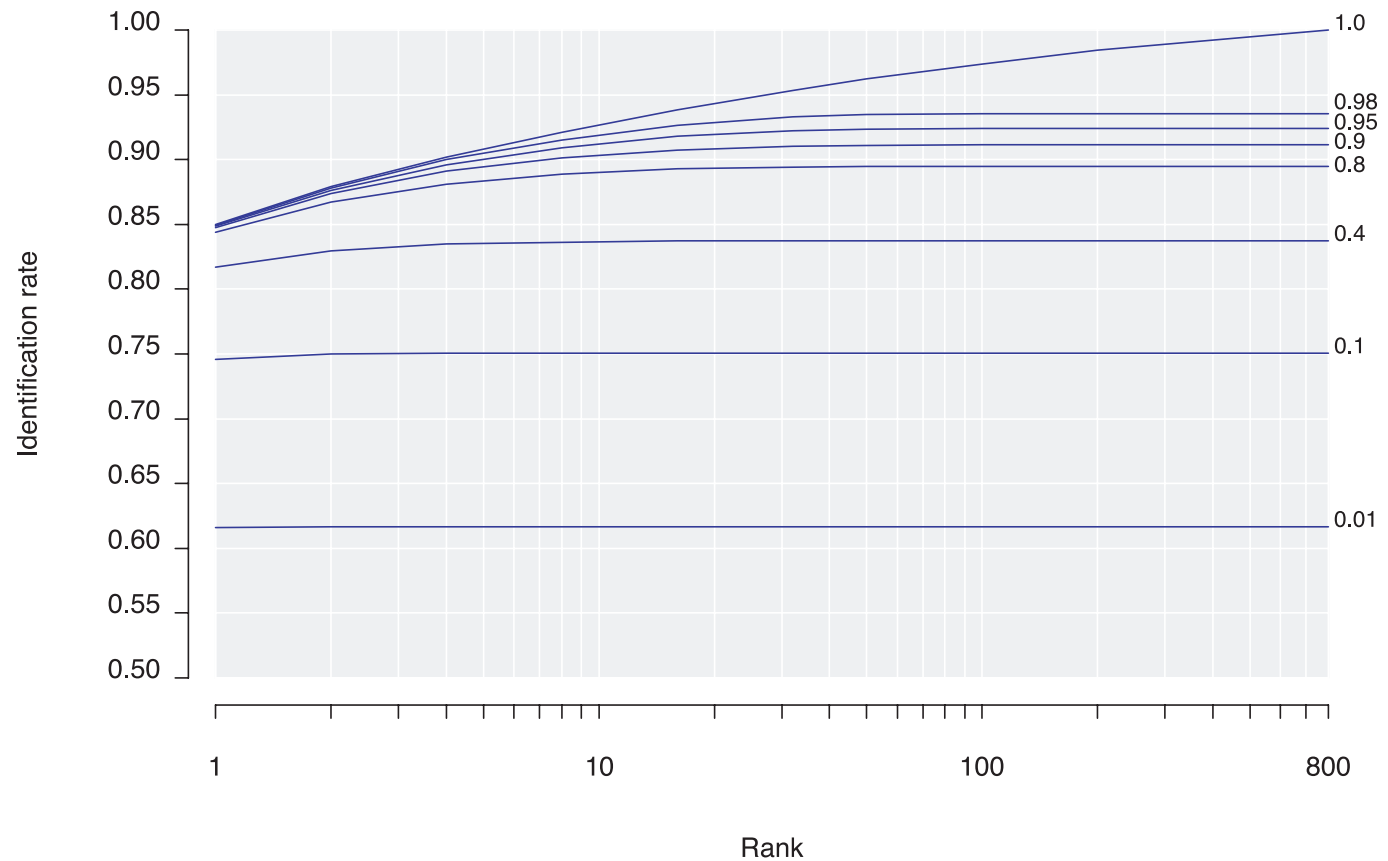

Fig. 13. Watch list performance for Cognitec as a function of rank for eight false alarm rates. The false alarm rates for each curve are on the right side of the graph. The gallery size is 800 . The top curve (false alarm rate of 1.0) is the CMC for the gallery in this experiment.

The watch list gallery size experiment examines how watch list size (gallery size) affects performance. Performance was computed for eight galleries sizes: 25, 50, 100, 200, 400, 800, 1,600 , and 3,000. The spacing of the gallery sizes is approximately logarithmic in size of the gallery. Each probe set contained two images of each person in the gallery and an equal number of imposters. To provide a reliable estimate of performance for each gallery size, watch list performance was computed on twelve disjoint galleries. The average performance is plotted in figure 14 . The curves correspond to a false alarm rate of 0.01 . The decrease in the detection and identification rate mirrors that of the decrease in identification. The decrease is approximately linear in the logarithm of the gallery size.

The performance analysis on the HCInt data set has, so far, concentrated on tasks: verification, identification, and watch list. The next step is to look at the effect of three covariates on performance. The covariates are: elapsed time between acquisitions of gallery and probe images of a person, sex, and age of an individual. Since there are multiple images of an individual in the data set, we fixed age by the age of an individual when the gallery image was taken.

Previous evaluations have examined the effect of temporal variations at a very coarse level. In past evaluations, the categories were: Images Collected on the Same Day, Images Collected on Different Days, and Images Collection over a Year Apart. FERET and FRVT 2000 showed that there was a major difference in performance between same-day images and images collected on different days. However, the rate at which performance declined as a function of elapsed time between gallery and probe could not be accurately estimated. This was due to the small size of the probe set.

In the HCInt, the effects of temporal variation are studied by computing identification and verification performance on a large gallery and probe set. The probe set is partitioned into 19 bins. Each bin corresponds to a 60-day interval. The first bin corresponds to 1 to 60 days. A probe is in the 1 to 60-day bin if the time between the acquisition of the gallery and probe image is between 1 and 60 days. The second bin corresponds to 61 to 120 . The remaining 17 bins are constructed in a similar manner in 60 -day intervals. Figure 5 shows the number of probes in each 60 -day interval. 


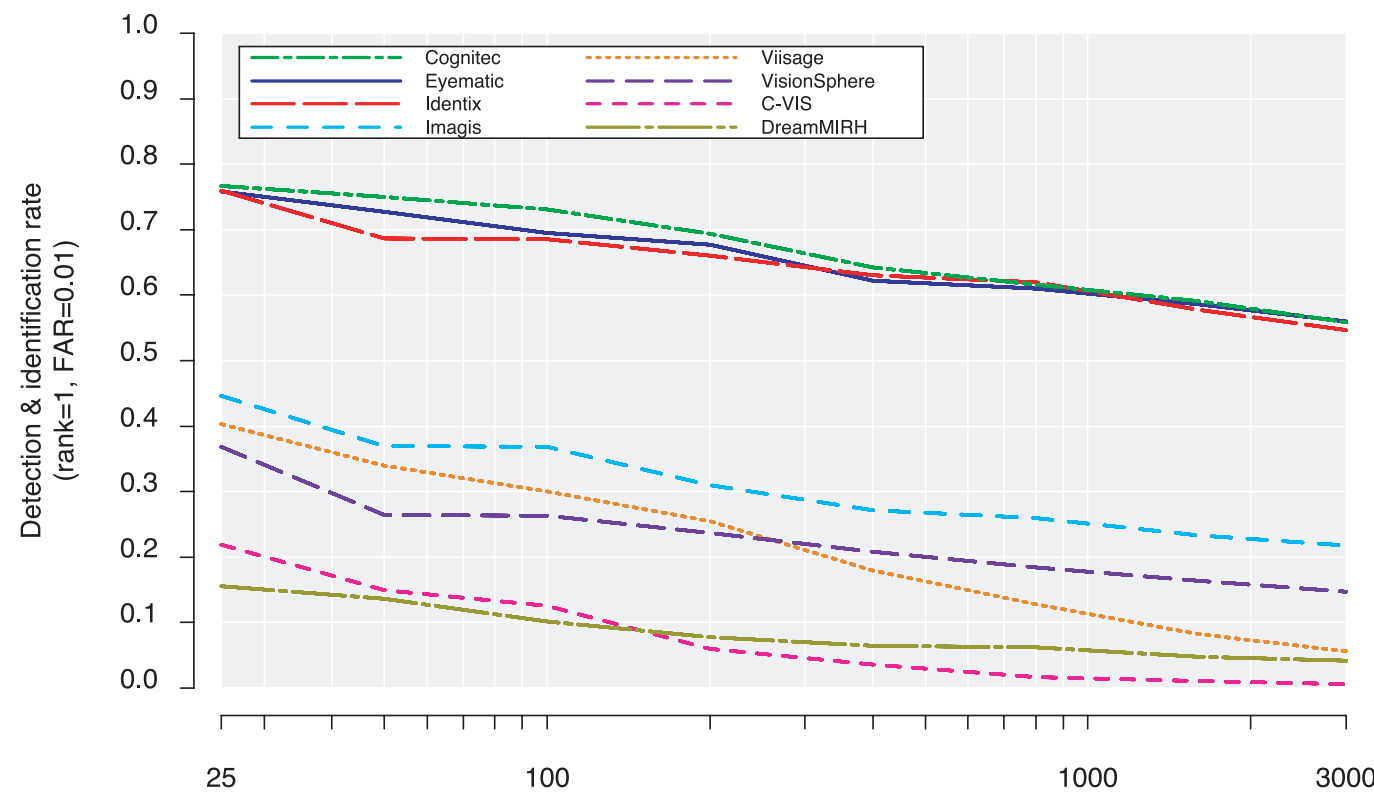

Gallery size

Fig. 14. Watch list performance as a function of gallery size for a false alarm rate of 0.01 . Rank 1 performance is measured.

Both identification and verification performance were computed. For all 19 bins, performance was computed against the large gallery, and the size of the probe set varied according to the bin. Figure 15 shows the rank one identification rate. Figure 16 plots the verification rate at a nominal false accept rate of 0.01 . The false accept rate is nominal because there is a change in the false accept rate for each bin. Details on how the verification and false alarm rate are computed for each bin are explained in more detail in Section 4. Briefly, from the large gallery verification experiment, the threshold that corresponds to a false accept rate of 0.01 is selected. Using this threshold, verification and false accept rates are computed for the different probe sets. The different probe sets correspond to the elapsed time bins. This results in different verification and false accept rates for each probe set. This method of computing effects for both verification and false accept rates is the basis for analysis in the HCInt covariate experiments.

Imaging conditions and elapsed time between images are one set of conditions that affect performance. These effects have been extensively studied in this and previous evaluations. In addition, the population in a database can significantly affect performance. Two examples are the sex and the age of people being recognized. Experiments on the HCInt show that the sex and age of a person affects performance.

To measure the effect of sex on performance, the HCInt large probe set was divided into male and female probe sets. Identification performance was computed for the male and female probe sets against the large gallery. Note: the large gallery was not divided into male and female galleries. For all systems, the identification rate for males was higher than the identification rate for females. Figure 17 shows the difference in identification performance for the male and female probe sets. For each rank, male minus female performance is plotted.

The calculation of the difference in performance for identification is straightforward; subtract the male and female identification rates. For verification, the procedure is a bit more complicated. Two statistics characterize the performance difference between males and females, the difference in the verification rate and the difference in the false accept rate. Figure 18 shows the difference in performance between the male and female probe sets in a ROC. The difference is shown by a 


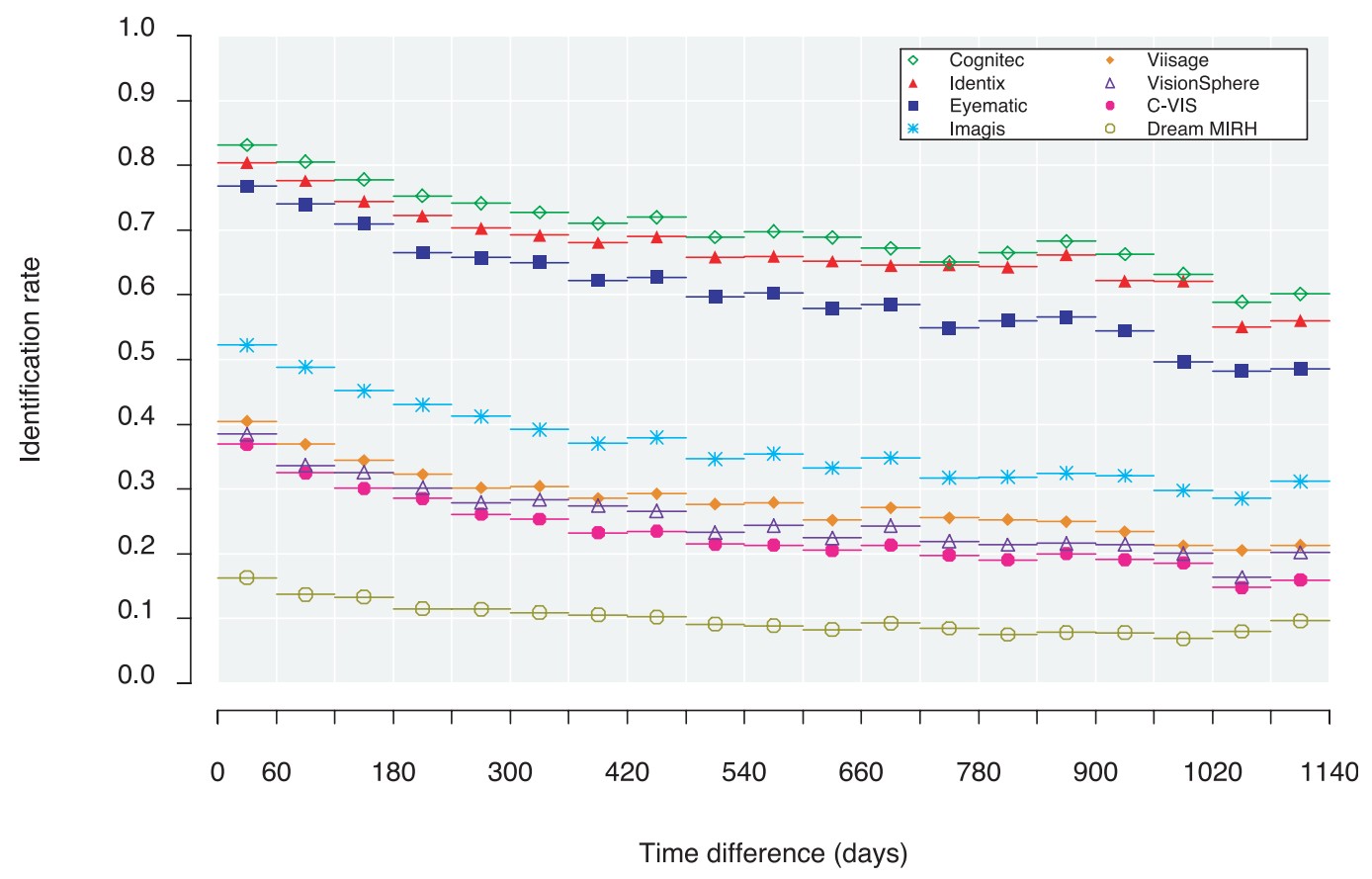

Fig. 15. Rank 1 identification rate for temporal variation study. The identification performance is broken out by 60-day intervals. The identification rate is for probes against the HCInt large gallery.

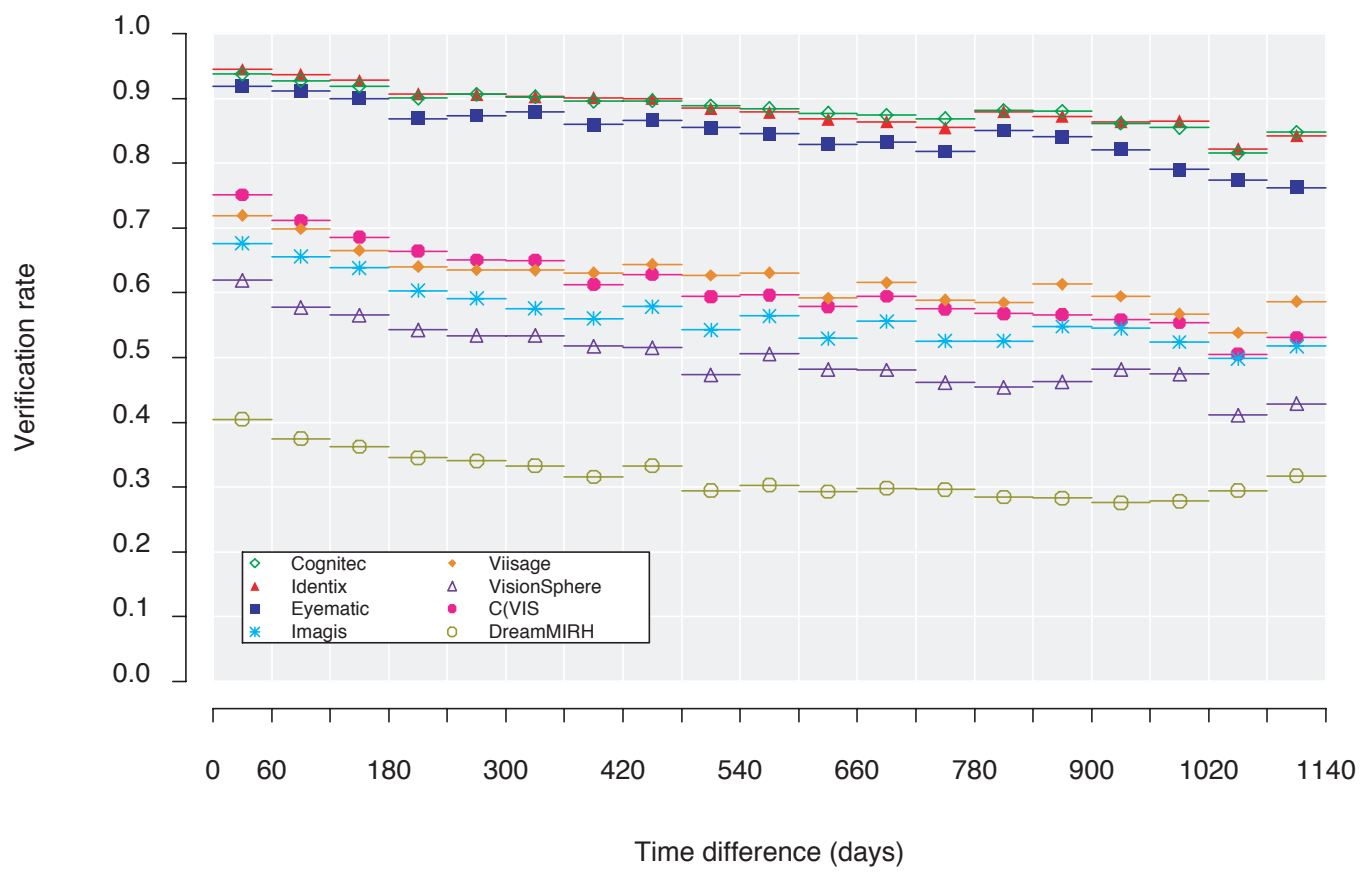

Fig. 16. Verification performance for temporal variation study. The verification rate is given at a false accept rate of 0.01 .

series of line segments for each system. The dot at approximately the center of each line segment is the aggregate performance for both male and female probe sets. This point is found on the large gallery ROC in figure 7. The bottom end of each line segment is the corresponding performance point for the female probe set. The top end of each line segment is the corresponding performance point for the male probe set. For all systems, the verification rate for males is better than for females. However, the same is not true for the false accept rates. For Cognitec, Eyematic, Viisage, 
VisionSphere, the false accept rate for males is better than for females (line segments go from the top left to bottom right). For these four systems, performance on males is better than females. For C-Vis, DreamMIRH, Identix, and Imagis, the false accept rate for females is better than males (line segments go from the top right to bottom left). For these systems, neither female nor male performance is better than the other. Rather, the decision depends on the scenario.

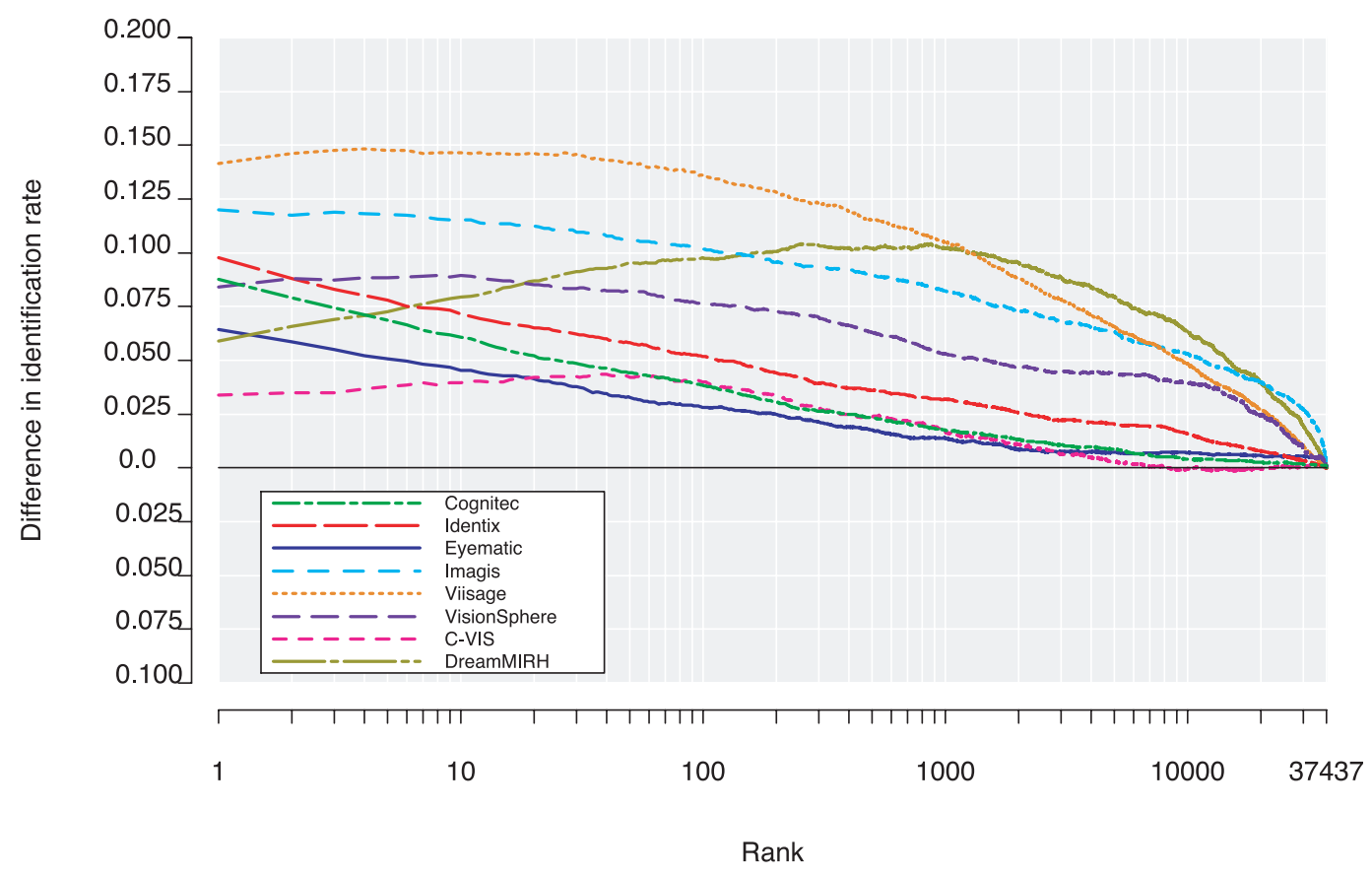

Fig. 17. CMC showing the difference in performance between male and female probes. For each rank, male minus female performance is plotted. The CMC shows that the identification rate of males is better than females.

Figure 17 and figure 18 break out performance by sex for the large gallery and large probe set, and show noticeable differences in performance between the sexes. This generates a follow-up question: Is the difference in performance consistent, or is it a property of the large gallery and large probe set? To address this question, verification and identification performance was broken out by sex and was computed on the twelve small gallery and probe sets. Verification and false accept rates were computed for both male and female probe sets. The performance figures were computed at a nominal false accept rate of 0.01 . For each of the twelve galleries the male minus female performance figures were calculated for both verification and false accept rates. Figure 19 plots these differences for all twelve small galleries and participants. Each symbol is the performance of one of the participants in one of the twelve galleries. The $x$-axis is the difference in the false accept rate and the $y$-axis is the difference in the verification rate. For each participant, the results for all twelve galleries cluster. With the exception of three runs, the verification rate for males was better than females, and for the three exceptions, the rate is very close to zero. There is an order of magnitude difference between the two axes. This shows that there is a much larger change in the verification rate than in the false accept rate. The same computations were performed for identification, and the results are consistent with the results in figure 17. For all galleries and all participants, the identification rate for males was better than females. This shows that the bias towards males in the large gallery is consistent with the results of the twelve small galleries and provides strong evidence that the observed sex bias is a property of the HCInt data set.

The effect of age on performance has not been previously examined. To examine the effect of age on performance the HCInt large probe set was divided into 12 age categories. Each age category is a five-year bin, with the first bin containing 18 to 22 year olds. All probes of a person 


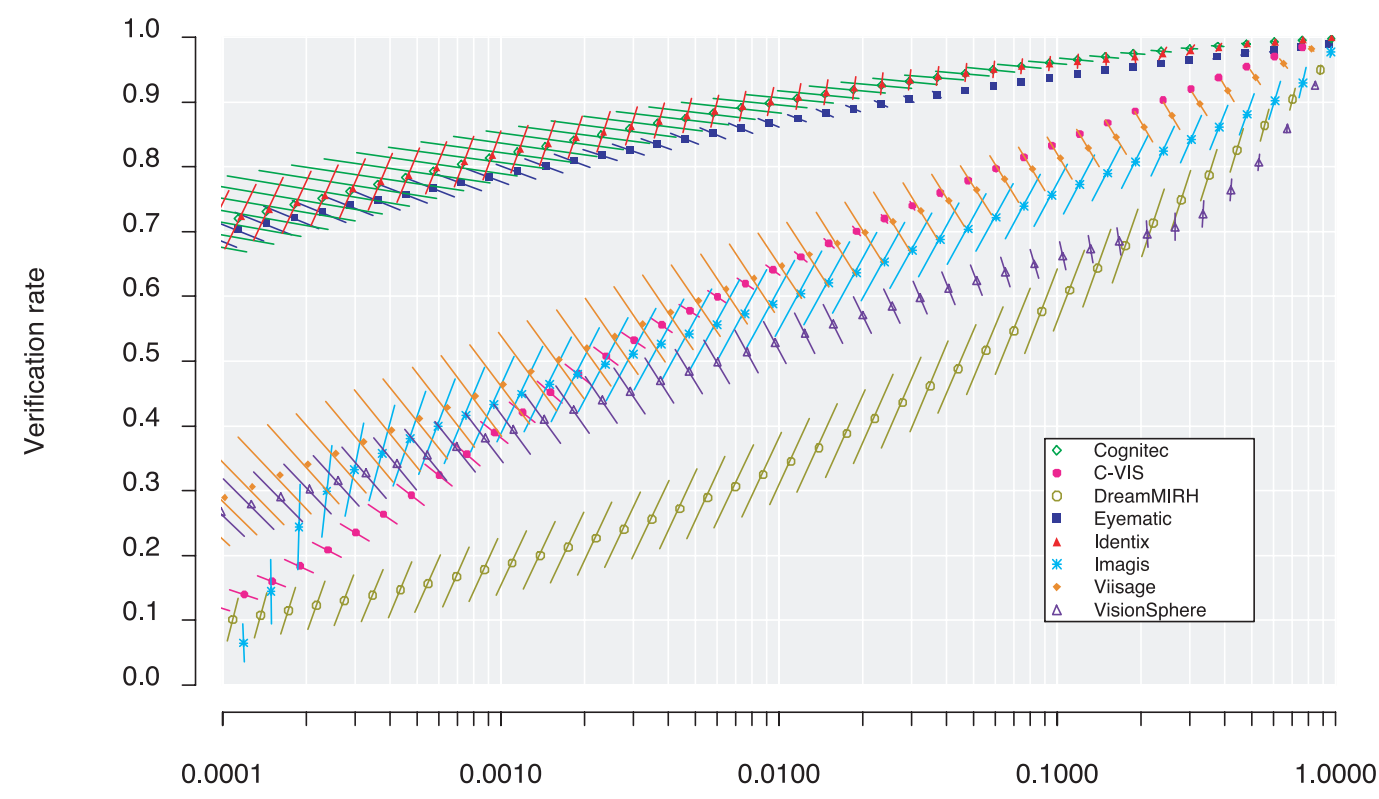

False accept rate

Fig. 18. ROC showing the difference in performance between male and female probe sets. For each line segment, the center point is the aggregate performance for male and female probes. The end of the line segment below the center is the performance on the females, and the end above the center point is performance for the males. For each $\mathrm{ROC}$, the difference in performance at multiple operating points is shown.

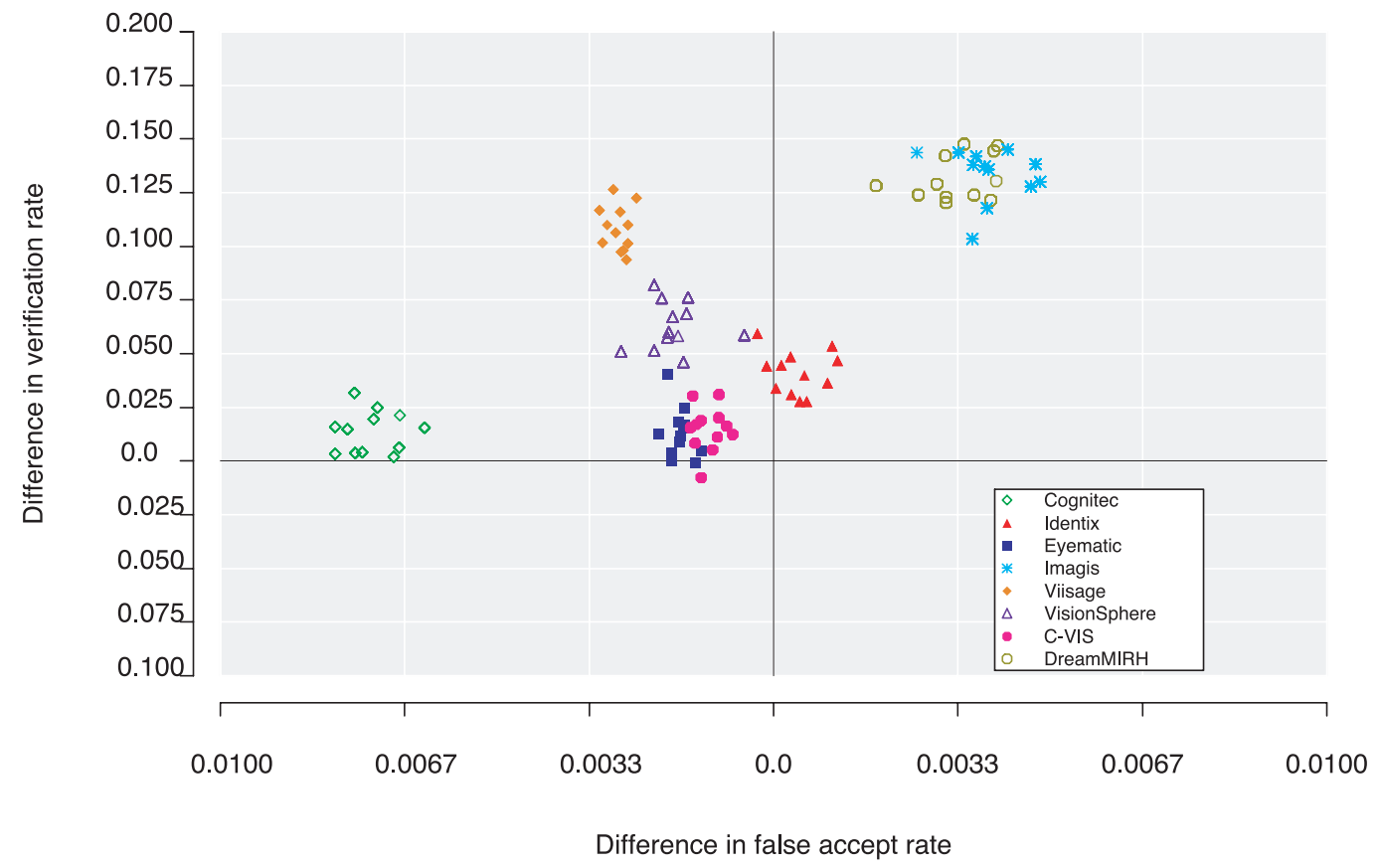

Fig. 19. Difference in verification performance for male and female probe sets for the twelve small galleries and probe sets. The $x$-axis plots the change in the false accept rate and the $y$-axis plots the change in the verification rate. Note that there is an order of magnitude difference in the scales of the two axes. 
are placed in the same bin, and the bin is determined by the age of a person when the gallery image was taken. For example, if the gallery was taken when a person was 21 year old, and the probes where taken when the person was 24 and 25, then both probes were placed in the 18 to 22 year bin. The performance for all age bins was measured against the HCInt large gallery. The top rank identification performance was broken out by age and is plotted in figure 20.

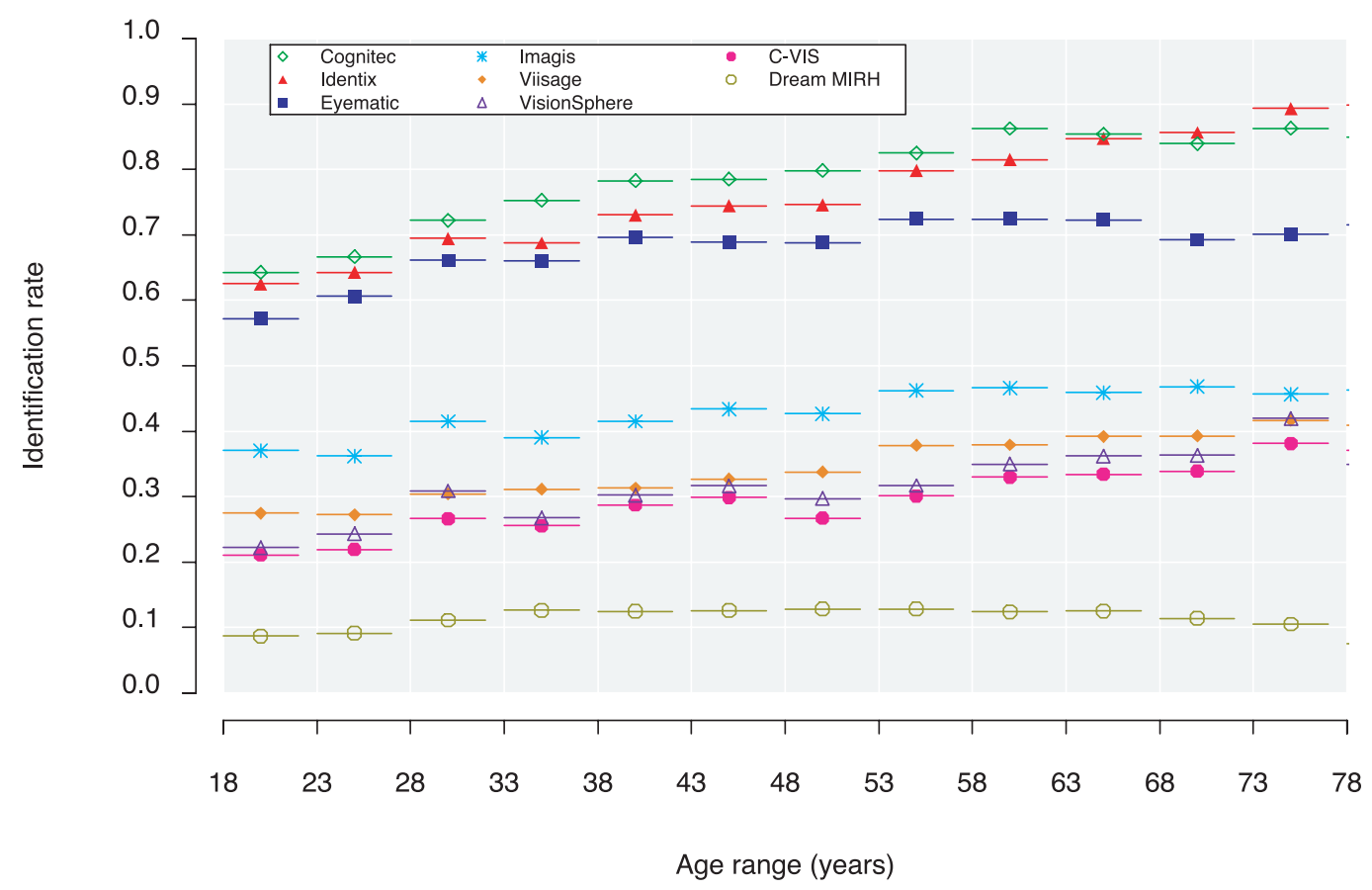

Fig. 20. Rank 1 identification performance on the HCInt large gallery broken out by age.

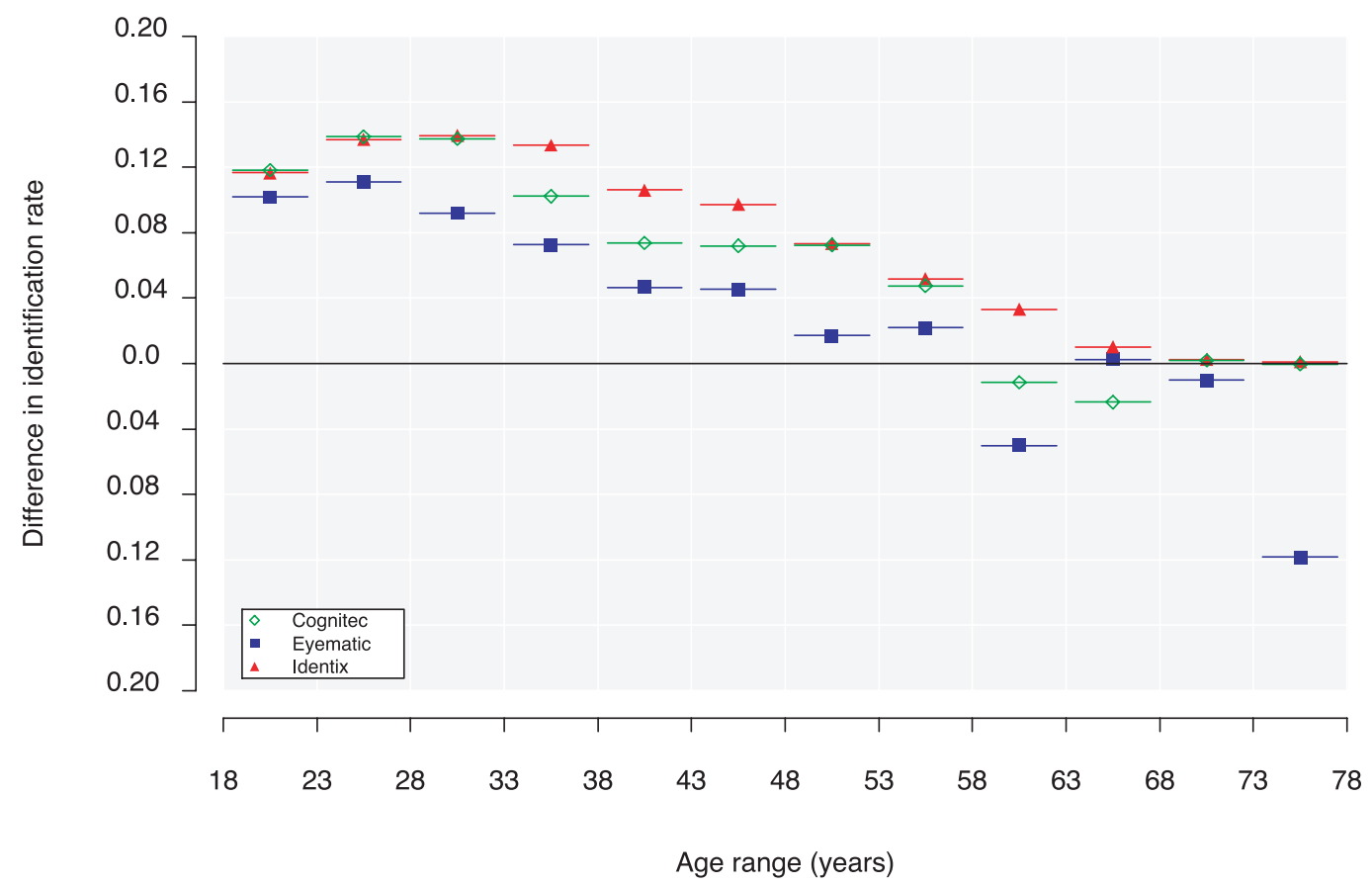

Fig. 21. Interaction between age and sex for rank 1 identification on HCInt large gallery (for Cognitec, Eyematic, and Identix). Rank 1 performance for males minus females is plotted for each age bin. 
Figures 17-20 show that both the sex and age of a person affect performance. However, it is possible that the age and sex effects are the same. This could happen if the males are much older than the females. Thus, we need to check the interaction of the two factors. This is accomplished by dividing each of the 12 age probe sets into male and female probe sets. For each of the 12 age bins, we compute the difference in the male and female rank 1 identification rates (male minus female identification rate). The results for Cognitec, Eyematic, and Identix are shown in figure 21. Figure 21 shows that the difference in performance for the sexes decreases as age increases, and shows an interaction between sex and age. Figure 27 shows rank 1 identification performance for all participants.

The HCInt experiments have provided answers to a number of long-standing questions and raised new questions. The long-standing questions have been the effect of gallery size on performance, variation in performance for different galleries, effect of elapsed time on performance, and characterization of watch list performance. New questions emerge from studying the effect of covariates on performance. The new questions arise from the determination that sex and age, and their interaction, do affect performance. This will lead to new research and insights into how face recognition systems work as well as issues associated with deploying automatic face recognition systems.

\section{Medium Computational Intensity Test Results}

The MCInt was designed to measure performance under different imaging conditions. MCInt reports three sets of the experiments and their results. The first set investigates recognition from frontal images under different imaging conditions. The second set looks at how pose variations affect performance and the impact of using three-dimensional morphable models as a preprocessing stage. The third set compares recognition performance using still and video sequences. The experiments also provide another assessment - the effect of pose variations on performance.

The goal of the MCInt is to provide a broad assessment of progress and the state-of-the-art. To provide a clear and concise assessment, performance is characterized in the Evaluation Report by two statistics: rank 1 identification rate and verification rate at a false accept rate of 0.01 . Full identification CMCs and verification ROCs for each experiment and participant can be found in the Technical Appendices. In figure 22 through figure 24, the verification rate is plotted for a false accept rate of 0.01. In figure 28 through figure 30 in Section A.4, the experiment performance is reported for rank 1 identification rate. Verification rate was selected for the main body of the report because verification performance is not a function of the gallery size. This allows for a degree of comparison among the results from the three sets of experiments. A strict comparison among the experiments is not possible because performance was computed from different galleries.

The first experiment is the MCInt frontal face experiment, which investigates how changes in lighting conditions affect face recognition from frontal mugshot style images. Performance is computed for five probe sets against the same gallery. The gallery consisted of 787 people taken under incandescent studio lighting. The subjects had a neutral expression. To provide an empirical upper bound on system performance, the first probe set consisted of images taken within five minutes of the gallery image under the same imaging conditions. This is referred to as the indoor-same day probe set. The indoor-same day probe set had a different expression than the gallery image and contained 786 images from 781 individuals. The gallery images the expression was usually neutral, and in the probe the expression was usually a smile.

The indoor-overhead lighting probe set consisted of images taken with five minutes of the gallery images but with overhead fluorescent illumination. This probe set contained 786 images from 786 individuals. The indoor-overhead lighting probe set tested the effects of indoor lighting changes on performance. 
The indoor-different day probe set contained probes taken under the indoor studio setup on a different day than the gallery image of a person. This probe set contained 320 images from 320 individuals and measured the effects of temporal variation on indoor probes. The median elapsed time between gallery and probe image was 147 days and the maximum was 730 days.

The outdoor-same day probe set contained images taken outdoors on the same day as the gallery image of a person. This probe set contained 444 images from 435 individuals and measured the effects of outdoor illumination.

The outdoor-different day probe set contained images taken outdoors on a different day than the gallery image of a person. This probe set contained 145 images from 103 individuals. The outdoor-different day probe set measured the effects of temporal variation and outdoor illumination. The median elapsed time between gallery and probe image was 152 days and the maximum was 505 days.

The results from the MCInt frontal face experiment are presented in figure 22 and figure 28. Figure 22 shows verification performance at a false accept rate of 0.01 for each participant and each probe set. The $y$-axis is verification performance. The $x$-axis is categorical, with one category for each of the five probe sets. The marks on the vertical line above Indoor (same day) are verification performance for the indoor-same day probe set. The performance results for the other four probe sets are reported in the same manner. Lines are drawn between the results for each participant on different probe sets. This is done to make it easier to examine how the properties of different probe sets affect performance. Figure 28 plots identification performance in the same manner.

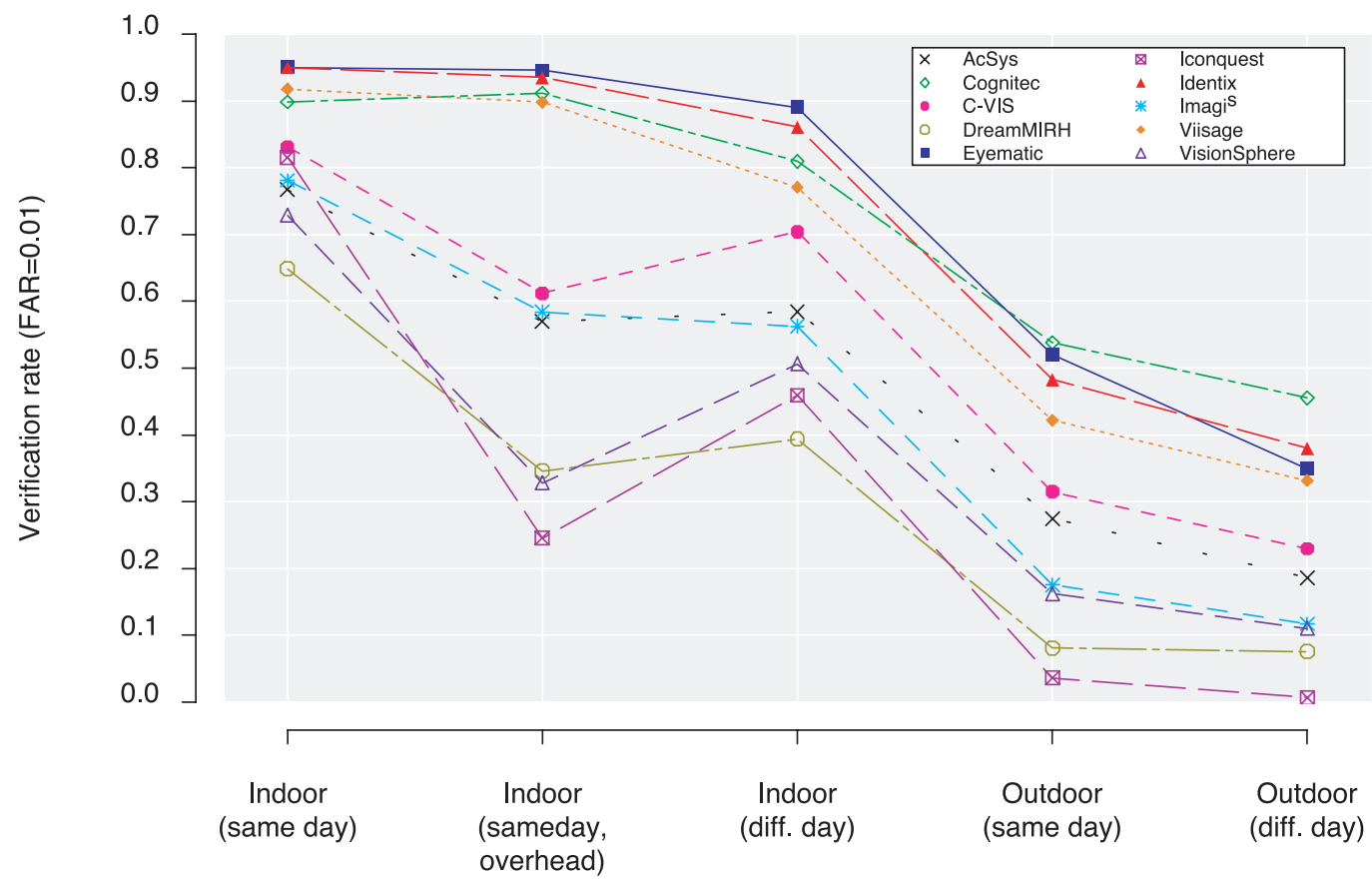

Fig. 22. Comparison of system performance on different categories of probes. The verification rate at a false accept rate of 0.01 is plotted.

The MCInt morphable model experiment examined the effects of pose variation and morphable models on performance. This experiment consisted of one gallery and nine probe sets. The gallery consisted of full frontal images of 87 individuals taken indoors under studio conditions with a single incandescent photo floodlight. Each probe set consisted of 87 images of 87 people. All probe images were taken within five minutes of the gallery image under the same conditions. The only difference is the pose. 
Figure 3 shows examples of the nine probe sets. The 45 left and 45 right probe sets contained facial images facing 45 degrees to the left and right of center respectively. The $45 \mathrm{~L}$ and $45 \mathrm{R}$ columns in figure 23 and figure 29 report verification and identification results for the 45 left and 45 right probe sets. Line segments are drawn between original and corresponding morphed probe sets to emphasize the effect of morphing. The $30 \mathrm{up}$ and 30 down probe sets contain facial images facing 30 degrees up and down respectively. The performance results for these two probe sets are reported in the $30 \mathrm{U}$ and $30 \mathrm{~L}$ columns. In the remaining five probe sets, a three-dimensional morphable model has been applied to the probes. See figure 3 for examples of applying a threedimensional morphable model to a probe.

The frontal morph probe set provides a baseline for how morphing affects a system. In the frontal morph probe set, the morphable model is applied to the gallery images. Thus, the difference between a gallery and a frontal morph image is that the morphable model has transformed the probe. The results for the frontal morph probe set are in column frontal (morph). If a system were insensitive to the artifacts introduced by the morphable model, then the verification and identification rates would be 1.0. In Figure 23, sensitivity to morphable models range from 0.98 down to 0.45 .

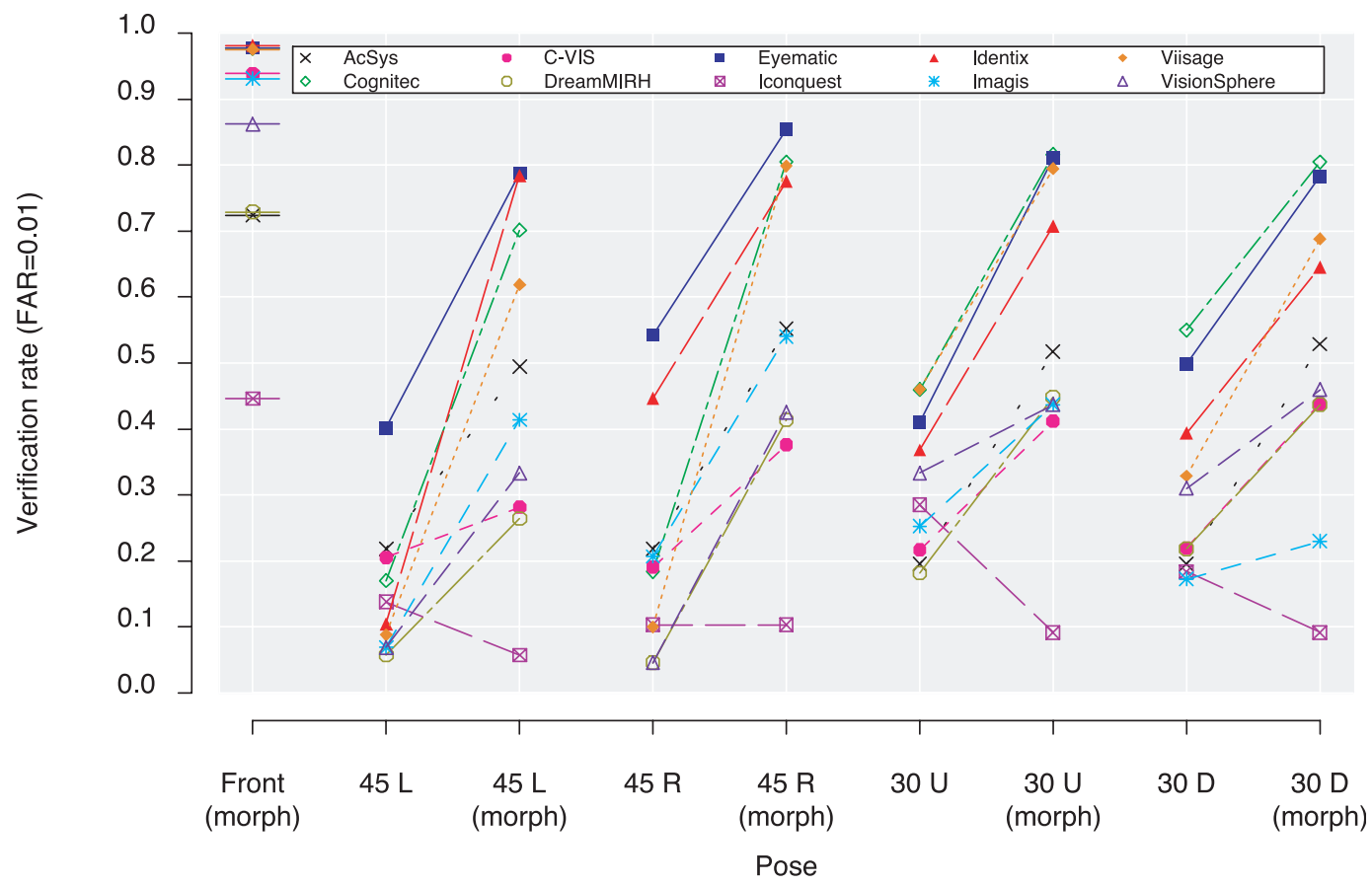

Fig. 23. The effect of still versus three-dimensional morphable models. The verification rate at a false accept rate of 0.01 is plotted.

To investigate the effects of morphable models, performance was computed for four probe sets: 45 left morphed, 45 right morphed, 30 up morphed, and 30 down morphed. These probe sets were produced by applying the morphable model to the 45 left, 45 right, 30 up, and 30 down probe sets respectively. The results for the morphed probe sets are in columns $45 \mathrm{~L}$ (morph), $45 \mathrm{R}$ (morph), $30 \mathrm{U}$ (morph), and $30 \mathrm{D}$ (morph). The results show that with the exception of Iconquest, morphable models significantly improved performance.

The MCInt still-video experiment compares performance of still and video-style probe sets. This experiment consisted of one gallery of still full frontal digital images of 63 individuals taken indoors under studio conditions. In the still-video experiment, there are two probe sets: still and video. Both probe sets contained 63 signatures from 63 individuals. The probes in both probe 
sets were taken indoors under studio conditions, consist of the same individuals, and are taken on different days than the gallery images. The still and video signature probes of a person were taken on the same day. The still probe set consisted of a single still full frontal image. A video probe was extracted from a 5 second video sequence of a person speaking (see figure 4). A video probe consisted of a sequence of 100 images (3.33 seconds), where each image was a frame in the original digital video sequence. We called this probe set video-style because the probe set consists of a sequence of frames.

The results of the MCInt still-video experiment are shown in figure 24 and figure 30. (Human inspection of the Viisage similarity files confirmed their performance on the still probe set.) With the exception of DreamMIRH and VisionSphere, performance was better on the still probe set than the video probe set. This was true for both verification and identification.

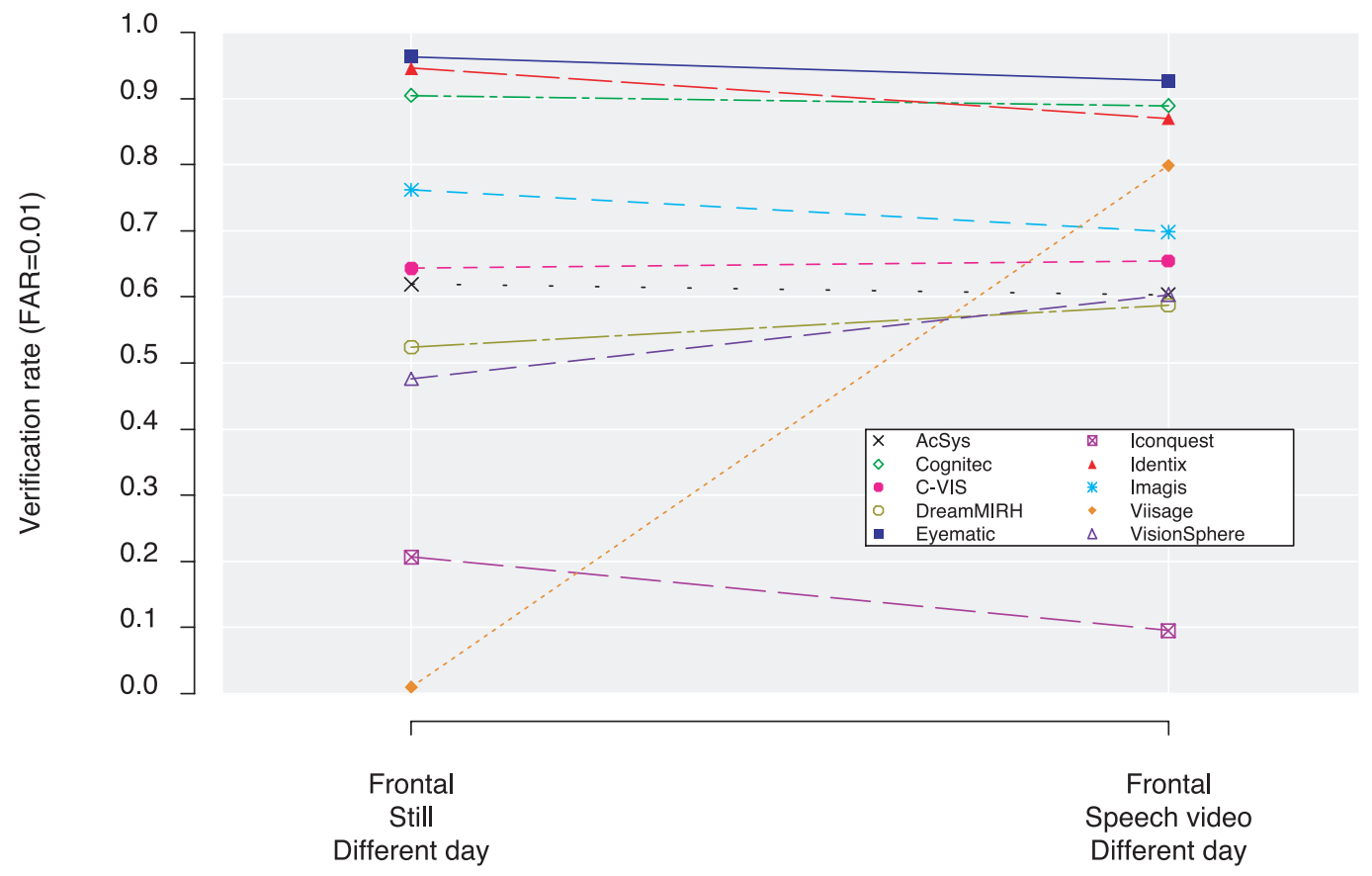

Fig. 24. Plot showing still versus video recognition for frontal imagery. The verification rate at a false accept rate of 0.01 is plotted.

\section{Analysis and Discussion}

The large number of images and people in the HCInt allow for a much more detailed analysis than in previous face recognition and biometric evaluations. The HCInt measures performance on indoor images taken on different days. Performance for this category was also reported in FRVT 2000 (Blackburn et al. 2001). Because the gallery sizes in FRVT 2000 and FRVT 2002 vary, comparing the results between the two must be restricted to verification. On comparable experiments, the error rate has dropped by approximately 50 percent between FRVT 2000 and 2002. This shows substantial improvement in performance since FRVT 2000.

A companion NIST study on fingerprints highlights progress in face recognition and points to directions of research to improve identification rates for large galleries (NIST 2002). Performance was computed for the verification test bed (VTB), an in-house NIST algorithm based on an automated fingerprint identification system (AFIS). The matching technology in the VTB is comparable to commercial systems in 1998. The recognition rates were computed from fingerprints provided to NIST by the U.S. Immigration and Naturalization Service's IDENT system. 
Fingerprint verification performance was computed for twelve independent galleries and probe sets. The gallery consisted of the fingerprint of one index finger for 6,000 individuals. The probe set consisted of a single fingerprint for the 6,000 people in the gallery.

Figure 25 shows verification performance with error ellipses for the VTB, Cognitec, Eyematic, and Identix. At false accept rates around 0.01 , verification performance is comparable. In fact, this is the cross-over point between face recognition and fingerprint performance. At false accept rates below 0.01, fingerprint performance is better. At rates above 0.01 , the best face recognition systems perform better. The good news is for false accepts around 0.01 , that face recognition performance is now comparable to large-scale fingerprint systems available in 1998. This suggests that a dual face recognition and fingerprint system is viable.

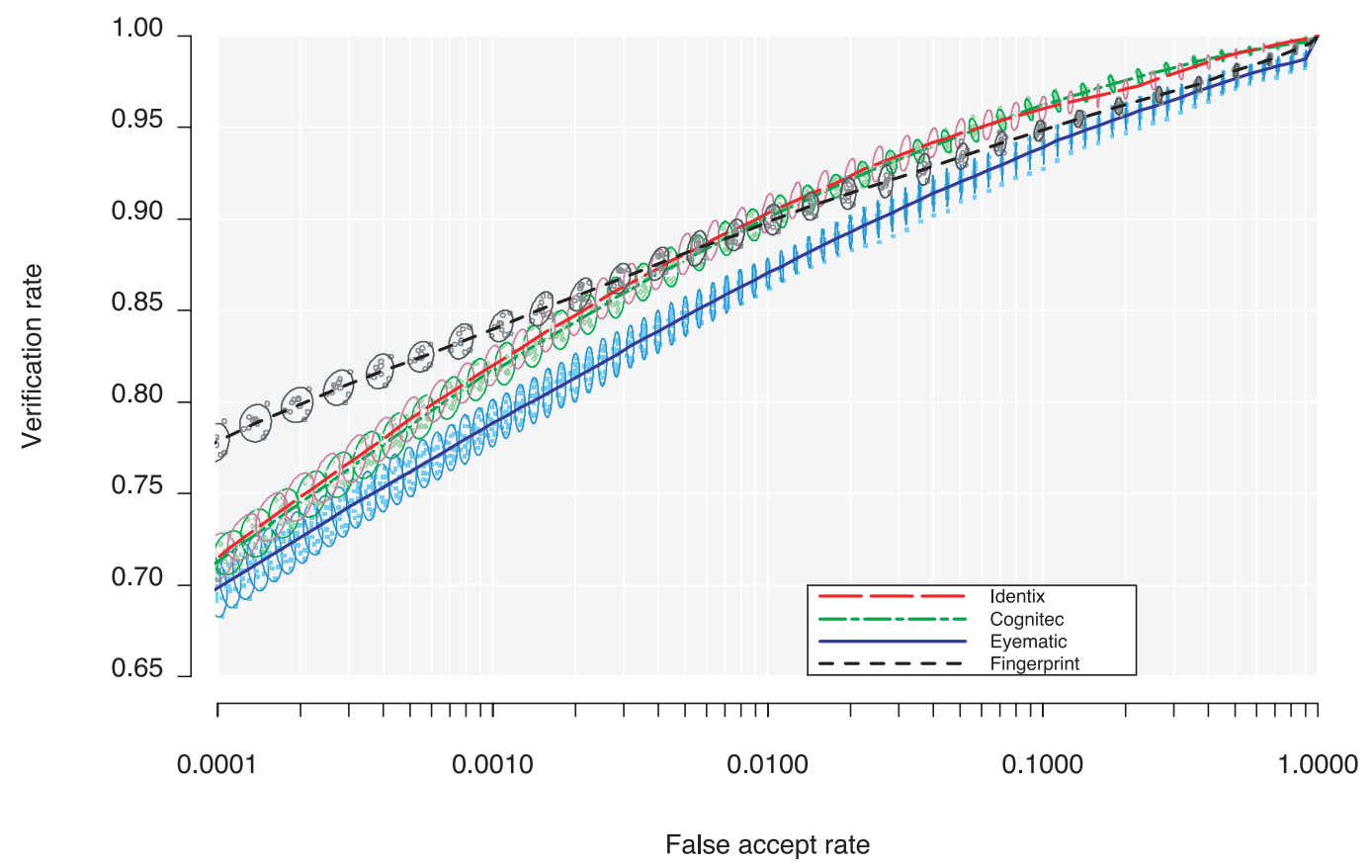

Fig. 25. Verification performances of Cognitec, Identix, and Eyematic on Visa images and NIST VTB single fingerprint matcher on IDENT data.

We now turn our attention to identification rates for large galleries. Figure 26 shows rank 1 identification rate as a function of gallery size for Cognitec, Identix, Eyematic, and NIST VTB (single index finger). The face and fingerprint curves do not completely overlap because identification rates were computed for different sized galleries for face and finger. The results from the VTB clearly show that fingerprint performance on identification is superior to face. In Appendix A.5, we present a moment model for predicting performance as a function of gallery size (see later in this section for a more detailed discussion). The moment model relates verification and identification performance. The model predicts that the verification rate for false accept rates at 0.001 and 0.0001 have the most significant impact on identification performance for large galleries. This could explain why face and finger performance are comparable at a false accept rate of 0.01 , but do not have comparable identification performance. Verification performance at false accept rates of 0.001 and 0.0001 has a greater impact on identification rate.

The fingerprint verification ROC is flatter than the face ROC (figure 25). A ROC that is flat means that a system either recognizes a person or completely fails. This suggests that a fingerprint recognition algorithm is much more likely to recognize a majority with high confidence and the remainder with low confidence. Face recognition, on the other hand, is more "egalitarian;" it recognizes most people at about the same level. 


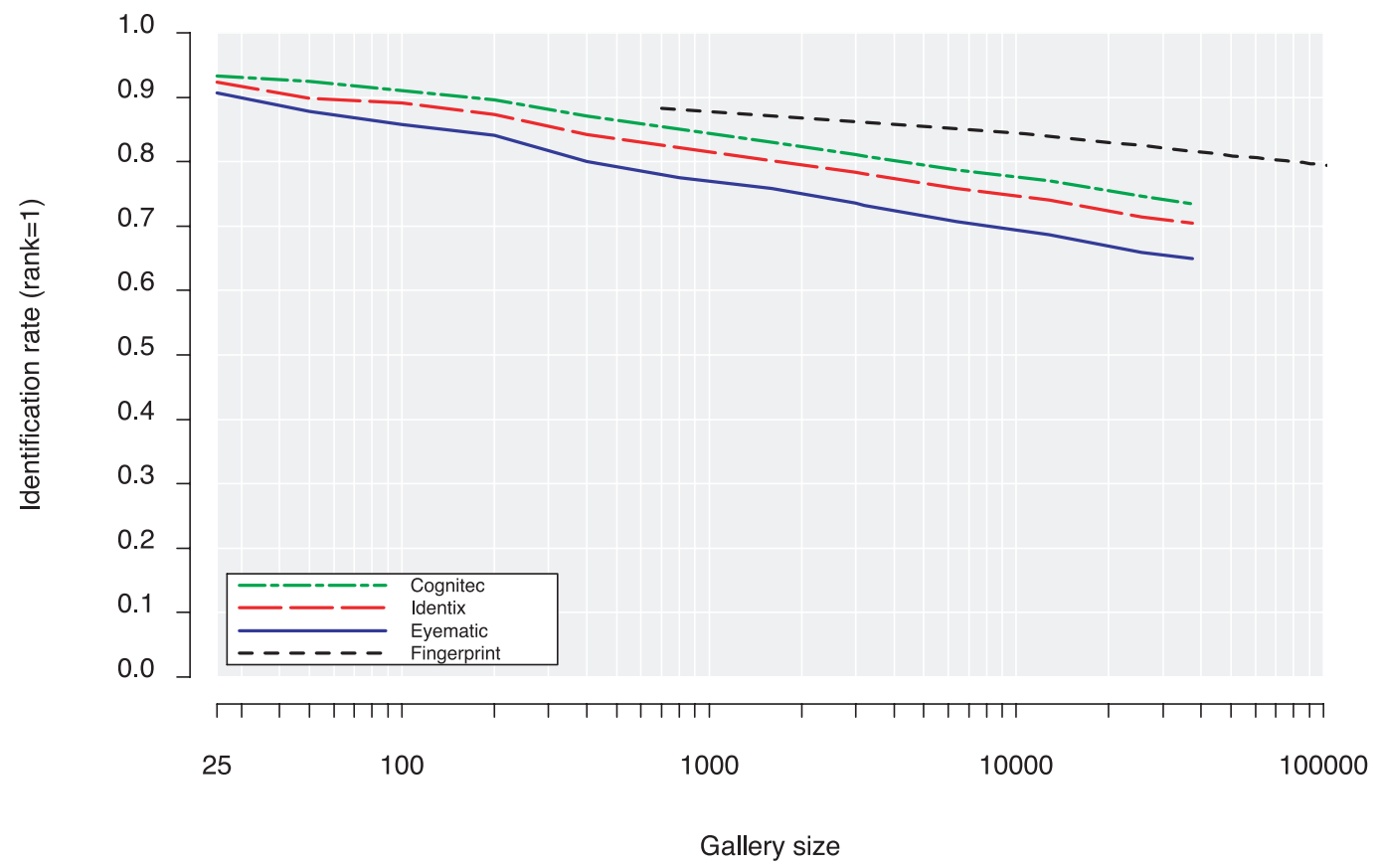

Fig. 26. Rank 1 identification rate as a function of gallery size for Cognitec, Eyematic, Identix, and NIST VTB single fingerprint matcher on IDENT data.

The results from FRVT 2002, the VTB, and the moment model indicate that to improve identification performance, effort should be made to increase verification performance for low false alarm rates. One possible approach is to concentrate on the vast majority of faces that are relatively easy to recognize and to not concentrate on the hardest faces to recognize. This would flatten out the resulting ROC, but at the same time, this could have a significant effect on identification performance for large galleries. To be able to increase the verification rate for very low false accept rates may require new features that are tailored to an individual or group of individuals. This differs from current approaches that select features that distinguish among a large population of people. Developing and evaluating performance for improvements at very low false accept rates will require experiments to be performed on large databases.

The large number of images and people in the HCInt data set made it possible to estimate the variance in performance statistics. For verification performance, error ellipses were computed. This provided an estimate of the change in verification when different people are in the gallery. Knowledge of the amount of variation is necessary for understanding the predictive value of an evaluation. If the error ellipses are large, the actual performance of a deployed system could be substantially different than performance predicted in an evaluation. If this is the case, the predictive value of the evaluation is small. Also, for applications in which a system will be deployed at multiple locations, the ellipses can provide an estimate of the range of performances that will be observed over the multiple locations. From a scientific point of view, error ellipses are one method of investigating the natural variation of faces in the population. This is because part of the variation that contributes to the error ellipses is generated by the natural variation in the population.

One of the primary purposes of the evaluation was to assess the state-of-the-art in face recognition. The HCInt provided a robust assessment of performance on digital visa images. The visa images were taken indoors under controlled lighting conditions. While the HCInt provides an assessment of performance for one category of images, the MCInt provides an assessment over a wider range of imaging conditions. 
The results from the HCInt provide a baseline to calibrate the difficulty of image categories in the MCInt. The primary performance measure for comparing results from different experiments is the verification rate at a false accept rate of 0.01 . Verification performance was selected because it is independent of gallery size. A specific false accept rate was selected because it makes the comparison manageable and is representative of a real-world operational setting.

To confirm that it is possible to compare results from the HCInt with the MCInt, a set of 1,024 visa images of 512 individuals was included in the MCInt. Verification results from the visa images in the MCInt and HCInt are comparable and consistent. Performance on the MCInt visa image experiment is within the error ellipses on the HCInt data.

We first examined the probe categories taken indoors. The frontal facial image experiment examined three categories of probes that were taken indoors: same-day different expression, sameday different illumination, and different day. Verification performance on the MCInt different-day probe set is comparable to the performance on the HCInt visa images. The top three performers do not have the same verification performance on the HCInt and MCInt indoor different day probe sets. However, the top three verification scores, without consideration of which participant produced the score, are comparable.

For the better systems, performance on the same-day expression and overhead lighting probe sets is comparable. The difference between these probe sets is primarily lighting. The indoor-different day and visa probe sets are comparable. Here again, the primary difference is lighting. This suggests that the best face recognition systems are not sensitive to normal indoor lighting changes.

Next we examined the effect of faces taken outdoors on performance. Compared to the indoor probe set, there was a large decrease in performance on the outdoor probe categories - same day and different day. For the better systems, the change in performance going from indoor to outdoor probes was much greater than the change going from same day to different-day probes. We also ran the experiment with a gallery consisting of outdoor images. We then measured performance on same-day and different-day outdoor probes. Performance was comparable to the results reported for the indoor gallery and different-day outdoor probe set (figure 22). This suggests that the main cause of the drop in performance from indoor to outdoor imagery is that the images were taken outdoors. MCInt recognition results on outdoor probe sets are consistent with the results of FRVT 2000. This indicates that recognition of faces in outdoor images needs to be a focus of research.

Figure 15 and figure 16 show the results for the HCInt time-lapse experiment. Identification performance dropped off roughly linearly in the time elapsed between acquisition of the gallery and probe images. Identification performance drops are approximately 0.05 points per year. For verification, performance dropped off more slowly than for identification. The next step was to make sure that the drop-off in performance was not confounded with the sex effect. To address this, performance was broken out by sex for each elapsed time bin. The results were consistent with previous results (figure 15 and figure 16). This shows that the time difference results and sex of the subject are not confounded.

FRVT 2002 looked at three new face recognition techniques: three dimensional morphable models, normalization of similarity scores, and face recognition from video sequences. The results of preprocessing non-frontal images with three-dimensional morphable models are shown in figure 23 and figure 29. The effects of pose changes in looking right and left, and up and down were examined. For all but one system, Iconquest, preprocessing with three-dimensional morphable models produced a substantial increase in performance.

Participants were not aware that the MCInt would include non-frontal images that had been preprocessed by three-dimensional morphable models. Therefore, the systems were not tuned to artifacts that the morphing may have introduced into the images, and the morphing technique was 
not tuned to any of the systems. These results provide a baseline for improvements in recognition of non-frontal images after preprocessing by a morphable model.

Normalization is a post-processing procedure that adjusts for variations in the composition of galleries. Four of the evaluation participants submitted normalization routines. FRVT 2002 results show that for identification, normalization did not improve performance. For verification, three of the normalization routines increased performance; but the amount of increase depended on the false accept rate. Similar results were found for verification in the watch list experiments. Our results are consistent with those found by Mansfield et al. (2001) for fingerprints.

The third technique investigated was face recognition from video sequences. There are two theories supporting the hypothesis that face recognition from video is better than stills. The first is that there are more images of person acquired. The second is that video techniques can explicitly incorporate temporal information in a sequence the representation of a face. Video sequences can potentially improve the performance of a system at two stages. One is at the detection and location stage. A test of this stage would involve video where the face size and angle would vary through the video sequence. The other is at the recognition stage. FRVT 2002 examined performance at the recognition stage. To test recognition at this stage, the probe video sequences contained a face of a person talking - sometimes expressively. Each frame in the video sequence was the quality of a mugshot image. The difference between a mugshot image and the frames in the video was that the collection of frames contained a greater range of expressions and motions. By testing systems on high quality facial imagery, FRVT 2002 tested the recognition ability from video. If the sequences had contained people walking towards the camera where the face varied in size and orientation, the results would have confounded detection and recognition.

FRVT 2002 results show that with the exception of DreamMirh and VisionSphere, recognition performance did not improve using video. While DreamMirh and VisionSphere performance was better on video, their performance from video sequences was significantly worse than the recognition rate on stills for the best systems. Our results show that for the FRVT 2002 video sequences, and the system tested, pure recognition from video does not improve performance.

Previous evaluations have broken out performance by imaging conditions, but have not previously examined the effects of demographics on performance. The HCInt evaluation showed that demographics can have a significant effect on performance. The HCInt results showed that the sex and age of a person affect performance. The effect of race on performance was not examined because the overwhelming majority of faces in the HCInt data set were Mexican. It is known that the race of a person does affect algorithm performance (Furl et al. 2002). The second largest racial group in the HCInt data consisted of 629 people born in China. Because of the limited size of this population, we were not able to perform a detailed study. However, results based on the limited amount of data suggest that people of Chinese origin are easier to recognize than people born in Mexico. Thus, race is another demographic factor that needs to be considered when fielding a system.

It is important to differentiate between two types of effects of covariates on performance. The first examines the effects on a specific population or set of users of a biometric system. In the HCInt data set, FRVT 2002 measured the performance of a population that was roughly equally divided between male and female, contained significantly more young people than older people, and consisted mostly of Mexicans. The performance would have been different if the data set had consisted of primarily males in their twenties. Because of the observed effect of covariates on performance, it is recommended that prior to fielding a system, the performance for that system is estimated for the demographic make-up of the user population.

The second type of effect of covariates consists of fundamental properties of the systems or images themselves. The most prominent covariate result in the HCInt evaluations is that males are easier to recognize than females. Is the difference in performance a fundamental property of male 
and female faces, or is it a function of ratio of male to females in the HCInt data set? To provide insight into this question, we ran a number of additional experiments on balanced data sets. A balanced data set consists of an equal number of gallery images from each covariate. By balancing on the covariate, we control for the effect of the covariate in the data set.

Two experiments were run to measure the effects of the sex of a person on performance. The first examined sex alone. The other looked at the interaction of sex and age. For the first experiment, two galleries were created of the same size: one all female and one all male. Identification performance for the male-only gallery was higher than the female-only gallery.

In the second experiment, one gallery was created that was balanced for both sex and age. Thus, there were the same number of male and female individuals in each age bin, and all age bins were the same size. The results are consistent with the unbalanced experiment. Both unbalanced and balanced experiments showed a sex effect, an age effect, and an interaction between the two.

One of the lessons of the covariate experiments is that covariates cannot be examined in isolation. The interactions among the covariates need to be examined. If the interactions are not examined, it is possible the observed effects of two covariates are from the same source. For example, it may have been the case the younger age bins had contained significantly more females and the older age bins may have contained significantly more males. If this were the case, the observed age and sex effects would have been caused by the same underling phenomena. But, as was observed in the HCInt data set, the performance gap between males and females declines with age. Without examining the interaction, this effect would not have been found.

There are a number of results in the literature that support the observation that base recognition rates are higher for males and females. Givens et al. (2002) found a small sex effect, and also found a statistically significant age effect. The effects are reported for a principal component-base face recognition algorithm using images from the FERET database. In the Givens et al. study, there were only two age groups: young and old. Our findings differ with those of Gross et al. (2001). Gross et al. found females easier to recognize than males from the AR database (Martinez and Benavente 1998). However, their findings were on a small data set of 130 people.

A related problem in face processing is automatically determining the sex of an unknown face. While there is an extensive literature on this subject, very few papers report classification rates for males and females. Moghaddam and Yang (2002) report male and female classification errors for eight algorithms. Their experiments were conducted on images from the FERET database. For all eight algorithms, the error rates for males were lower than females. Shaknarovich et al. (2002) find similar results on a database of images collected on the World Wide Web.

The results from FRVT 2002 and in the literature provide evidence that automatic recognition tasks are easier for males than for females. The underlying reason that males are easier to recognize is not known. Additional experiments are required to provide an explanation. Possible explanations range from facial hair on men to the general observation that women are more likely to have greater day-to-day variation in their appearance than men. However, follow-up experiments are required to determine the explanation for the bias.

It is known that the training set for a face recognition algorithm does affect its performance on different demographic groups. Systems were trained and tuned prior to starting the FRVT 2002. The FRVT 2002 evaluation protocol did not specify a training set or restrict the training set that a participant could use in training and tuning their system. Thus, the FRVT 2002 did not control for the composition of the training set, and this could have contributed to the covariate effects. However, the covariate effects existed for all eight systems. This suggests that the composition of 
the training set is not the only possible source of the covariates effects. Additional experiments are required to determine the contribution of the composition of the training set to covariate effects.

The identification results in figure 11 show that identification performance decreases as gallery size increases. The rate of decrease in gallery size appears to be linear in the logarithm of gallery size. Performance decreases by approximately 0.06 for every order magnitude increase in gallery size (base 10). The one exception is DreamMirh. The log-linear performance in figure 11 is observed up to a gallery of 37,437. Does the log-linear performance continue indefinitely or change at some point? The same trend was observed for performance as a function of gallery in the watch list task (see figure 14). An empirical answer to this question requires experimental runs on even larger galleries. The other challenge is to develop a mathematical model to explain this phenomenon. One current model regards identification from a gallery of size $N$ as $N$ attempts at verification. Wayman (1999) and Daugman (2003) formalized this as a binomial model, where the binomial coefficient is constant for all gallery sizes. This model does not explain the observed data because the model predicts an exponential decrease in performance as gallery size increases. Appendix A.5 presents a more sophisticated model that is based on moments of the match distribution. This model predicts the log-linear behavior, but underestimates the identification rate. The probable cause of the underestimation is that the model assumes that the similarity scores are independent and identically distributed (iid). The iid assumption fails because there are complex interactions among the similarity scores.

The face recognition community, and biometrics in general, has developed a range of evaluations in terms of number of people and images. To provide a rough guide to evaluation size, we introduce the following nomenclature:

- Small: $~ 1,000$ signatures and $\sim 330$ individuals

- Medium: 10,000 signatures and 3,300 individuals

- Large: $~ 100,000$ signatures and $\sim 33,000$ individuals

- Very large: 1,000,000 signatures and $\sim 330,000$ individuals

- Extremely large: $\sim 10,000,000$ signatures and $\sim 3,300,000$ individuals

Each size has its own role and place. A larger evaluation is not inherently better, especially when cost is considered. Most previous evaluations have been small, but they have had a positive impact on the development and assessment of biometrics.

The MCInt is a small to medium evaluation, and was able to differentiate between large and small effects on performance. For example, the MCInt results showed a large difference in performance between recognition of non-frontal images and non-frontal images that have been morphed. Thus, the MCInt results showed that morphable models improved performance for non-frontal images. In another example, the MCInt results showed a small change in performance for video versus still signatures, and we concluded that video signatures do not improve performance. An evaluation such as MCInt, is good for making an assessment on 1) a specified set of experiments, and 2) where one is looking to distinguish between large and small effects.

The HCInt allowed for a detailed analysis and was able to estimate the variance of performance statistics and measure the effects of covariates on performance. This analysis required not only a large numbers of images and people, but also an appropriate number of errors. If there had only been ten or hundred errors, we would not have been able to perform detailed covariate analysis. In designing very large and extremely large evaluations one needs to state the object of the evaluation and have an idea of the overall accuracy of the biometric being tested. For example, if a biometric has an identification rate of 0.9999 (error rate of one in 10,000), then an evaluation on a data set of 100,000 images would on average produce ten errors. To be able to perform a detailed analysis of performance, such as in HCInt, would require a test set several orders of magnitude larger. 
Evaluations of all sizes are needed and have their role in assessing performance of biometrics. Factors effecting the size and design of an evaluation include the evaluations goals and the overall accuracy of a biometric. The greater the accuracy of biometric, the larger the required size of an evaluation. The more detailed analysis needed, the larger the required size of an evaluation. At the other end of the scale, an evaluation with very specific and defined purposes maybe able to meets its goals with a small evaluation.

\section{Conclusion}

At a simple level, FRVT 2002 was an evaluation and comparison of ten face recognition systems. Upon closer examination, FRVT 2002 will have a much broader impact. From an operational perspective, FRVT 2002 results will impact policy, the engineering design of largescale biometric systems, and how future technology, scenario, and operation evaluations will be designed. From a scientific point of view, FRVT 2002 will have an impact on future directions of research in the computer vision and pattern recognition, psychology, and statistics fields. FRVT 2002 results raise many more questions than they answer.

Before summarizing the findings of FRVT 2002, two potentially important issues need to be addressed:

1) Does face recognition work?

2) Which system is best for my application?

The answers to both of these questions are closely related to one another. Face recognition performance, like other biometric types, is application-dependent. Just as there is no best biometric type for all operational applications, there is no best face recognition system for all operational applications. FRVT 2002 was not designed to be a "buyer's guide for face recognition" -where one looks at graphs or scores and selects the best system for installation. Rather, it is a technology evaluation that should assist decision-makers in determining (1) if face recognition technology could potentially meet the performance requirements for an operational application, and (2) which systems should be selected for application-specific scenario evaluations.

In order to determine if face recognition works and which system(s) should be deployed, one first needs to properly define the operational application of interest and operational performance requirements. These requirements need to be as specific as possible because even a small change in operational requirements can sometimes significantly alter anticipated performance. Questions to ask when defining an application include:

- Identification, verification or watch list mode of operation?

- The size of the database for identification or watch list?

- Demographics of the anticipated users (age, sex, etc.)?

- Lighting conditions - indoor/outdoor? Supplemental lighting?

- Is the system to be installed overtly or covertly?

- What is the anticipated user behavior?

- How long has it been since the images in the database were taken?

- What is the required throughput rate?

- How many "exception handling" cases can you handle for a given period of time?

- For each mode of operation, which parameter (identification: rank or identification rate; verification: false alarm or probability of verification; watch list: false alarm or correct alarm) is most vital?

- What are the minimum accuracy requirements?

FRVT 2002 can only provide input to several, but not all of these questions. Questions associated with anticipated user behavior, exception handling, human computer interaction, and how a system is integrated into the business model are not addressed in a technology evaluation such as FRVT 2002. Providing answers to these types of questions are the province of scenario 
and operational evaluations. Answers to some of these questions will identify which experiments in FRVT 2002 are relevant to a given application. Results from the relevant experiments will 1) show if face recognition could potentially meet the performance requirements for the application, 2) identify which systems should be selected for follow-up scenario evaluations, and 3) provide a starting point for designing and conducting scenario and operational evaluations for a specific application. Without specifying requirements, implementation constraints, and process models for an application, one cannot accurately determine if face recognition will work or which system should be selected.

FRVT 2002 is the most thorough and comprehensive evaluation of automatic face recognition technology to date. The evaluation has examined many long-standing questions and raised several new questions for further study. These are discussed in detail in Section 9. The conclusions from FRVT 2002 are summarized below:

- Indoor face recognition performance has substantially improved since FRVT 2000.

- Face recognition performance decreases approximately linearly with elapsed time gallery and probe images.

- Better face recognition systems do not appear to be sensitive to normal indoor lighting changes.

- Three-dimensional morphable models substantially improve the ability to recognize nonfrontal faces.

- Normalization improves verification and watch list performance.

- On FRVT 2002 imagery, recognition from video sequences was not better than from still images.

- Males are easier to recognize than females.

- Younger people are harder to recognize than older people.

- Outdoor face recognition performance needs improvement.

- For identification and watch list tasks, performance decreases linearly in the logarithm of the gallery size.

One of the goals of a technology evaluation is to identify future directions of research. Among the research directions identified by FRVT 2002 are:

- Recognition from outdoor facial images.

- Recognition from non-frontal facial images.

- Recognition at low false accept/alarm rates.

- Understanding why males are easier to recognize than females.

- Greater understanding of the effects of demographic factors on performance.

- Development of better statistical methods for understanding performance.

- Develop improved models for predicting identification performance on very large galleries.

- Effect of algorithm and system training on covariate performance.

- Integration of morphable models into face recognition performance.

- Understanding the video sequences in FRVT 2002 did not improve performance.

Other major FRVT 2002 accomplishments include the evaluation protocol developed for this test and the associated scoring suite. The evaluation protocol and scoring suite are XML-based. They were designed to be applicable to general biometric evaluations, not just restricted for use in face recognition evaluations.

Face recognition and processing are important research problems spanning numerous fields and disciplines. This is because face recognition, in addition to having numerous practical applications, is a fundamental human behavior that is essential for effective communications and interactions among people. Researchers are interested in how people process faces, and scientists and engineers are working on techniques to replicate the human face processing functions. Research advances along two intertwined paths. One path has an application orientation and the other, a scientific orientation. Advances on both paths reinforce each other, with FRVT 2002 providing 
research directions for both paths. In the 1990's, the FERET evaluations stimulated research in face recognition technology and in doing so helped advancing automatic face recognition during its infancy. With the numerous questions it raises, FRVT 2002 is poised to play a similar role in stimulating future face recognition and processing research.

\section{ACKNOWLeDgements}

The organizers of the FRVT 2002 gratefully acknowledge the Defense Advanced Research Projects Agency, Department of State, Federal Bureau of Investigation, National Institute of Justice, National Institute of Standards and Technology, and Transportation Security Administration as evaluation sponsors and the ONDCP Counterdrug Technology Assessment Center, United States Customs Service, Department of Energy, Drug Enforcement Administration, Immigration and Naturalization Service, U.S. Secret Service, Technical Support Working Group, Australian Customs, Canadian Passport Office, and United Kingdom Biometric Working Group as evaluation supporters.

The authors extend their thanks to:

The Department of State, specifically Travis Farris for allowing NIST to use the Mexican nonimmigrant visa images for FRVT 2002; John Atkins and Rasool Azad for their invaluable assistance with the images, meta-data, and background information on the images' origins and properties.

Volker Blanz and Thomas Vetter at the University of Freiburg for supplying us with the threedimensional morphable images. Volker Blanz expeditiously provided us with frontal reconstructions obtained from their 3D Morphable Model implementation.

Alice O'Toole at The University of Texas at Dallas, for supplying us with the video sequence database.

Karen Marshall at NIST for manually classifying the sex of the individuals in the HCInt subset. Also, for the flawless preparation of the media used for conveying the images and similarity files to and from the vendors during the testing phase.

Sam Trahan at NIST for the maintenance of the alternative scoring code branch, and for authoring the sex classification tool.

Tom Gandy and Cathy Schott for their general assistance and their assistance in editing and proofreading the FRVT 2002 reports.

Michael Garris at NIST for his careful review and comments on the XML specification.

Charlie Wilson at NIST for his general assistance and insightful comments.

Kevin Bowyer, Travis Farris, and Russ Neuman for their comments on preliminary drafts of the report. 


\section{Appendix A}

\section{A.1 FRVT 2002 EVALUATION PROTOCOL DETAILS}

The FRVT 2002 evaluation protocol is a general framework for conducting technology biometric evaluations. The details presented are for FRVT 2002, but the framework is applicable to biometrics in general. The protocol encodes the philosophy and design of the evaluation, specifies the properties of the input to and output from a system, and dictates how performance statistics and results should be computed. The properties of the protocol are:

- All participants are tested in the same manner.

- Training is completed prior to the start of the evaluation.

- Biometric signatures are completely general.

- The input to a system is a target and query set.

- The output is a complete similarity matrix.

- All similarity scores $s(q, t)$ are a function of a query/target pair $(q, t)$.

- A gallery can be any arbitrary subset of the target set with at most one biometric signature per person in the gallery.

- Normalization is a post-processing function.

- Normalization is a function of a gallery and a probe.

- All performance scores are computed from sub-matrices of the complete similarity matrix.

In the FRVT 2002 evaluation protocol, a system is given two sets of biometric signatures: a target and a query set. A target set $T$ contains the set of signatures that are known to a system. A query set $Q$ contains signatures of unknown identity that are to be recognized. The term 'recognition' covers identification, verification, and watch list tasks. In FRVT 2002, biometric signatures are either digital still images or digital video sequences. The FRVT 2002 evaluation protocol is general enough to be extensible to general biometrics signatures.

For each pair of query and target signatures $(q, t) \in Q \times T$, a system reports a similarity score $s(q, t)$. A similarity score is a measure of how similar two biometric signatures are. The FRVT 2002 evaluation protocol was designed with the assumption that each system has its own similarity measure. Systems that report distances can be incorporated into the scoring framework by negating their similarity scores.

The complete set of similarity scores over $Q \times T$ is a similarity matrix. The key property of the FRVT 2002 evaluation protocol, which allows for great flexibility in scoring, is that for any target-query pair, $(q, t)$ we have $s(q, t)$. From a full similarity matrix, "virtual" experiments are performed from subsets of $Q$ and $T$. The basic components of such a 'virtual' experiment are a gallery and a probe set. In traditional experiments, such as those in the FERET evaluations and FRVT 2000, an experiment consisted of a single gallery $G$ and probe set $P$. A gallery $G$ is a subset of a target set $T$ and a probe set is a subset of a query set $Q$. For a virtual experiment, performance scores are computed from the similarity scores in $P \times G$. The scores in $P \times G$ are extracted from $Q \times T$.

Operationally, it was expected (but not required) that a FRVT 2002 participant generated the similarity matrix as follows. Given a query element $q$, the system compared $q$ with all targets $t \in T$ to generate a vector, or single column of the similarity matrix. We denote this vector as $s(q, T)$. This process is repeated for all query signatures to generate the full similarity matrix. We denote this matrix as $s(Q, T)$. 
In summary, we have,

- $s(q, t)$, a single scalar value, representing the similarity between query element $q$ and target element $t$,

- $s(q, T)$, a vector of $|T|$ elements, representing the similarity between query element $q$ and each target element $t \in T$, and

- $s(Q, T)$, a matrix of $|Q||T|$ elements, representing the similarity between every pair of target and query signatures, $(q, t) \in Q \times T$.

In constructing galleries from a target set, a gallery must contain only one biometric signature for each person. This rule does not present a limitation for scoring, but may require additional planning in designing target and query sets. The most common objection to this rule is creating a gallery that contains multiple images of an individual. One method to put multiple images of a person in the gallery is to place $n$ images of a person in the gallery as $n$ separate biometric signatures. When a probe is presented to a system, this will produce $n$ similarity scores between the probe and the images of this person in the gallery. During scoring, the question is how to make a decision based on the $n$ similarity scores? Does the scoring algorithm take the smallest, largest, or maybe the mean? The trouble with this approach is that the scoring code resolves the issues, not the system being evaluated. The approach taken by the FRVT 2002 protocol is to create a single biometric signature $t$ that contains the $n$ images. Thus, for a probe, a single similarity score is returned and the system decides how to integrate the information contained in the multiple images. In the FRVT 2002 protocol, a biometric signature is a list of data in a specified format. The data in a biometric signature can be heterogeneous. For example, it could consist of a facial image and a fingerprint.

Using gallery and probes allows us to compute performance measures for different categories of images. For example, FRVT 2002 included: (1) probe and galleries that varied according to the subject's age, (2) measurement of the empirical variation of the verification rate and false alarm rate across disjoint probe and gallery sets, (3) the effect of gallery size within a watch list scenario, and so on. Naturally, the possibilities for different experiments are limitless.

At the request of an FRVT 2002 participant, all participants had the opportunity to provide a custom normalization function as a component of their system. Operationally, a normalization function is applied to an appropriate subset of the score matrix, just before the scoring algorithms are applied. In its most general form, normalization is any post-processing transform performed on a subset of the similarity matrix. For an algorithm that uses normalization, the final performance scores are computed over the transformed values.

In FRVT 2002, we distinguish between two families of normalization functions. One possible normalization function, say $f_{1}$, takes each $s(p, G)$ as input, and gives as output, a new set of similarity, or "normalized" scores, $s_{1}(p, G ; G)=f_{1}(s(p, G))$. Here, we use a semi-colon to emphasize that $s_{1}(p, G ; G)$ is a vector of similarity scores that is dependent or parameterized by a particular gallery. Recall that $s(p, G)$ and $s_{1}(p, G ; G)$ are all the similarity scores for a given probe. This means that normalization occurs on a probe-by-probe basis, and is not a function of the entire similarity matrix $s(P, G)$. If the results for each probe are joined together, we have a new similarity score matrix $s_{1}(P, G ; G)$, where each column of the matrix has been normalized with respect to the gallery.

The second type of normalization function $f_{2}$, which also operates on a probe-by-probe basis, incorporates the similarity scores between all pairs of gallery images ${ }^{3}$ as well as the vector of similarity scores $s(p, G)$. That is, $s_{2}(p, G ; G)=f_{2}(s(p, G), s(G, G))$. Note that if $|G|$ is large, it may not be practical to use such an algorithm, since the matrix $s(G, G)$ may exceed memory limits.

3. This implies that in order to use such a normalization routine, the target and query sets must share a set of (gallery) images. 


\section{A.2 STATISTICS DETAILS}

In FRVT 2002, we evaluate an algorithm on three related tasks: identification, verification, and watch list. Each of these tasks can be mapped to sets of operations on the similarity scores.

For the identification task, the operational model for FRVT 2002 is a closed universe. That is, for each probe $p \in P$ there exists one (and only one) gallery signature $g^{*} \in G$ such that signatures $p$ and $g^{*}$ are of the same subject. In the remainder of this Appendix, we will use $g *$ to represent the mate of probe $p$.

During identification, for a probe $p \in P$, a system reports the gallery elements that have the highest similarities to the probe image. If the subject appears in the top $\mathrm{n}$ candidate gallery images, then the subject is correctly identified. For each probe $p \in P$, we sort $s(p, G)$. A probe $p$ is said to be of rank $k$ if $s\left(p, g^{*}\right)$ is the $k^{\text {th }}$ largest similarity score in $s(p, G)$. The definition of correct identification can therefore be parameterized with respect to the rank $k$. Over all probes, let $R_{k}$ denote the number of probes in the top $k$. Then, $R_{k} /|P|$ is the fraction of probes in the top $k$.

So far, our scoring algorithm assumed that there are no ties among the similarity scores. To handle cases where $s\left(p, g^{*}\right)$ is tied with other values, the FRVT 2002 scoring software reports the mean value of two different ranks - the optimistic and pessimistic rank. The optimistic rank is (one plus) the number of similarity scores in $s(p, G)$ that are strictly greater than $s\left(p, g^{*}\right)$. The pessimistic rank is the number of similarity scores in $s(p, G)$ that are greater to or equal to $s\left(p, g^{*}\right)$. For example, suppose that for a particular probe, an algorithm reported identical similarity scores for each element in the gallery. Then, the optimistic rank would be one, since there would be zero scores strictly greater than $s\left(p, g^{*}\right)$. The pessimistic rank would be the same size as the gallery, $|G|$ , since in this case, all $|G|$ scores would be equal to or greater than $s\left(p, g^{*}\right)$. The final reported rank would be the average of the two ranks, or $(1+|G|) / 2$.

For the verification task, the operational model for FRVT 2002 is as follows. A system performs a verification when a probe $p$ is presented to a system along with a claim of identity. The system computes the similarity $s\left(p, g^{*}\right)$ where $g^{*}$ is the stored signature corresponding to the claimed identity. Naturally, for evaluation purposes, it is assumed that the $g^{*}$ exists -i.e., a subject does not claim to be someone not in the gallery. The claim of identity is accepted if $s\left(p, g^{*}\right) \geq \tau$, where $\tau$ is some a priori operating threshold. ${ }^{4}$

Performance statistics for the verification tasks are computed from collections of match and non-match scores. A match score is any similarity score generated by comparing a probe and gallery element from the same subject. Similarly, a non-match score is a similarity measure between signatures of different subjects.

A verification experiment requires three sets to be specified. The gallery $G$, a set of probes $P_{G}$ that contain probes of people who are in the gallery. The set $P_{G}$ determines the set of match scores-i.e., if $P_{G}$ represents the set of probes with facial imagery in the gallery, then all the match scores in the similarity matrix $s\left(G, P_{G}\right)$ may be used to calculate the verification rate. Let $P_{N}$ represent the set of probes that are used to generate the non-match scores. The false accept rate is computed from all the non-match scores in the similarity matrix $s\left(G, P_{N}\right)$. Unlike $P_{G}$, which, by definition of the verification problem must have corresponding gallery images, there is no such requirement for $P_{N}$. The number of non-match scores varies according on the overlap among the subjects in the gallery and $P_{N}$. If $P_{G}$ and $P_{N}$ are the same set, then the number of non-matches is $|G| \cdot\left|P_{G}\right|-\left|P_{G}\right|$. This was the case in the 'round-robin' method of computing verification performance in Phillips et al (2000). When $P_{N}$ does not contain any people in the gallery, then the number of non-matches is $|G| \cdot\left|P_{N}\right|$.

In the traditional 'round-robin' evaluations, $P_{G}$ and $P_{N}$ are often the same set. From an operational standpoint, this models the case where a subject, already with legitimate access to

4. This inclusive (as opposed to exclusive) method of accepting or rejecting a claim is compatible with a Neyman-Pearson observer. A Neyman-Pearson model maximizes the verification rate for a fixed false accept rate (Bickel and Doksum 1977; Egan 1975). 
the system (they are in $P_{G}$ ), attempts to gain access to the very same system, under a different identity. There may be some specialized scenarios where this is a valid model. However, we prefer to model the situation in which a person who does not already have access to the system makes a false verification attempt. In this model, the people (not just the signatures) in the probe set $P_{N}$ are different from the people in the gallery. The people in $P_{N}$ are sometimes referred to as true imposters. The rationale for having the non-match scores generated by true imposters is that non-match distributions from $s\left(G, P_{G}\right)$ may be different than those from $s\left(G, P_{N}\right)$.

From the match and non-match scores, the verification and false accept rates are respectively computed. The verification rate is calculated by dividing the number of match scores above the threshold $\tau, M_{\tau}$ by the total number of match scores $M$. For the threshold $\tau$, the verification rate (VR) is $M_{\tau} / M$. The false accept rate is computed in a similar manner. If the total number of nonmatch scores is $N$ and the number of non-match scores greater than or equal to $\tau$, then $N_{\tau} / N$ is the false accept rate (FAR).

We first present a simple algorithm for computing an ROC, and then streamline it into a faster version. In our implementation, we assume that $M \ll N$, and that $N=O\left(M^{2}\right)$. Strictly speaking, an ROC is not a 'curve' but a collection of operating points where the verification or false alarm rate changes.

The first step in the ROC algorithm is to sort all $M+N$ match and non-match scores. In computing the corresponding ROC, one only needs to compute performance at thresholds equal to unique values of these $M+N$ scores. Let $\tau_{i}$ be the ith largest similarity score-this can be either a match or nonmatch score. The verification and false accept rates are computed at each threshold $\tau_{i}$, by starting with $\tau_{1}$ (the largest score) and proceeding down the sorted list of similarity scores in ascending order.

The algorithm has some (simple) initial conditions. We add the artificial threshold $\tau_{0}=\infty$, and initialize an array of match and non-match counters $M_{\tau_{i}}$ and $N_{\tau_{i}}$ where we keep a match and non-match counter for each threshold. This initial condition corresponds to: 1) rejecting all identity claims, and 2) the operating point of both the verification and false accept rate are zero. The algorithm loops over each threshold, $\tau_{i}$, in order. For each threshold, the number of match and non-match scores with a similarity score in the range $\left[\tau_{i}, \tau_{i+1}\right)$ is recorded. If $m_{i}\left(n_{i}\right)$ is the number of match (non-match) scores with values in this range, then $M_{\tau_{i}}\left(N_{\tau_{i}}\right)$ are simply $M_{\tau_{i}}=M_{\tau_{(i-1)}}+m_{\tau_{i}}\left(N_{\tau_{i}}=N_{\tau_{(i-1)}}+n_{\tau_{i}}\right)$.

As stated, this algorithm computes the verification and false accept rate at more points than necessary. To show this, let $\tau_{i}$ and $\tau_{(i-1)}$ be two adjacent thresholds that each have one non-match score and zero match scores. For both thresholds, the verification rate will be the same, but the false accept rate (FAR) will be lower at $\tau_{(i-1)}$ than at $\tau_{i}$. Therefore, operationally, one would run the system at the operating point associated with $\tau_{i-1.1}$. To generalize this, performance only needs to be computed at the operating points $\tau_{i}$ corresponding to match scores. A scoring algorithm adjusting for this observation proceeds by sorting the unique match scores and using these for the set of thresholds that define the match and non-match score counts.

This algorithm computes an ROC for a single algorithm on a single set of data. In FRVT 2002 , we wish to examine how a system performance varies with different gallery or probe sets. To study this variation, it is necessary to combine results over a set of ROCs. One method to accomplish this is to measure the variation on the verification rate for each false alarm rate. This is appropriate for combining ROCs from different systems, because it is not possible (nor is it operationally feasible) to set a uniform threshold across different systems (they would be tuned individually). However, for the same system, it is possible to set one threshold across all galleries and probe sets. For each threshold, it is possible to compute the variation in the verification and false alarm rates. 
To be able to compute variation in verification and false accept rates across multiple galleries and probe sets, we need to again modify our ROC algorithm. Our goal is to combine the ROCs from $R$ experiments, where each experiment consists of a gallery, probe set for matches, and probe set for nonmatches. The first step in computing the combined ROC is to combine and sort all $R$ sets of match scores to use for our thresholds. Here, contiguous thresholds may correspond to match scores from different experiments. This is not a problem, however, since we are using these thresholds to 'oversample' each of the individual ROCs.

In our variance analysis, we select 'evenly spaced' thresholds. Each threshold generates $R$ operating points - there is a (VR,FAR) pair for each of the $R$ experiments. In the FRVT 2002 analysis, these individual points are plotted, along with an error ellipse that traces two standard errors in the TAR and FAR dimensions. From a linear algebra standpoint, the principal axes of the error ellipse are the eigenvectors of the covariance matrix of the $R$ operating points.

The operational model of the watch list scenario is as follows. In some sense, the watch scenario is a generalization of both identification and verification. In the watch list scenario, a probe $p$ is presented to a system. The system then compares the probe to a gallery, which plays the role of the watch list. The system then: a) determines if the person is on the watch list and b) produces an estimate of the identity of the person. Alternately, the system can report the top $n$ matches if there are sufficient matches above a threshold.

More formally, suppose a watch list system is presented with a probe $p$ of a subject that is on the watch list. For a correct result, a probe must pass a verification and an identification requirement. A similarity threshold parameterizes the verification requirement $\tau$ and a rank $k$ parameterizes the identification requirement. The identification requirement is fulfilled if the rank of probe $p$ is $k$ or better (lower). Similarly, the detection requirement is met if $s\left(p, g^{*}\right) \geq \tau$. Both requirements must be met for a correct watch list detection and identification. If the probe is not on the watch list, then the probe gives a false alarm if there is any gallery (a.k.a. watch list) element $g \in G$ such that $s\left(p, g^{*}\right) \geq \tau$. This is akin to someone not on the watch list being "similar" enough to someone on the watch list to warrant a 'false alarm.' For a watch list, the detection and identification rate is the fraction of probes (also on the watch list) that are detected and identified correctly. The false alarm rate is the fraction of probes (without corresponding gallery elements) that have a similarity to any gallery element that is greater than the threshold. 


\section{A.3 HCINT ADDENDUM}

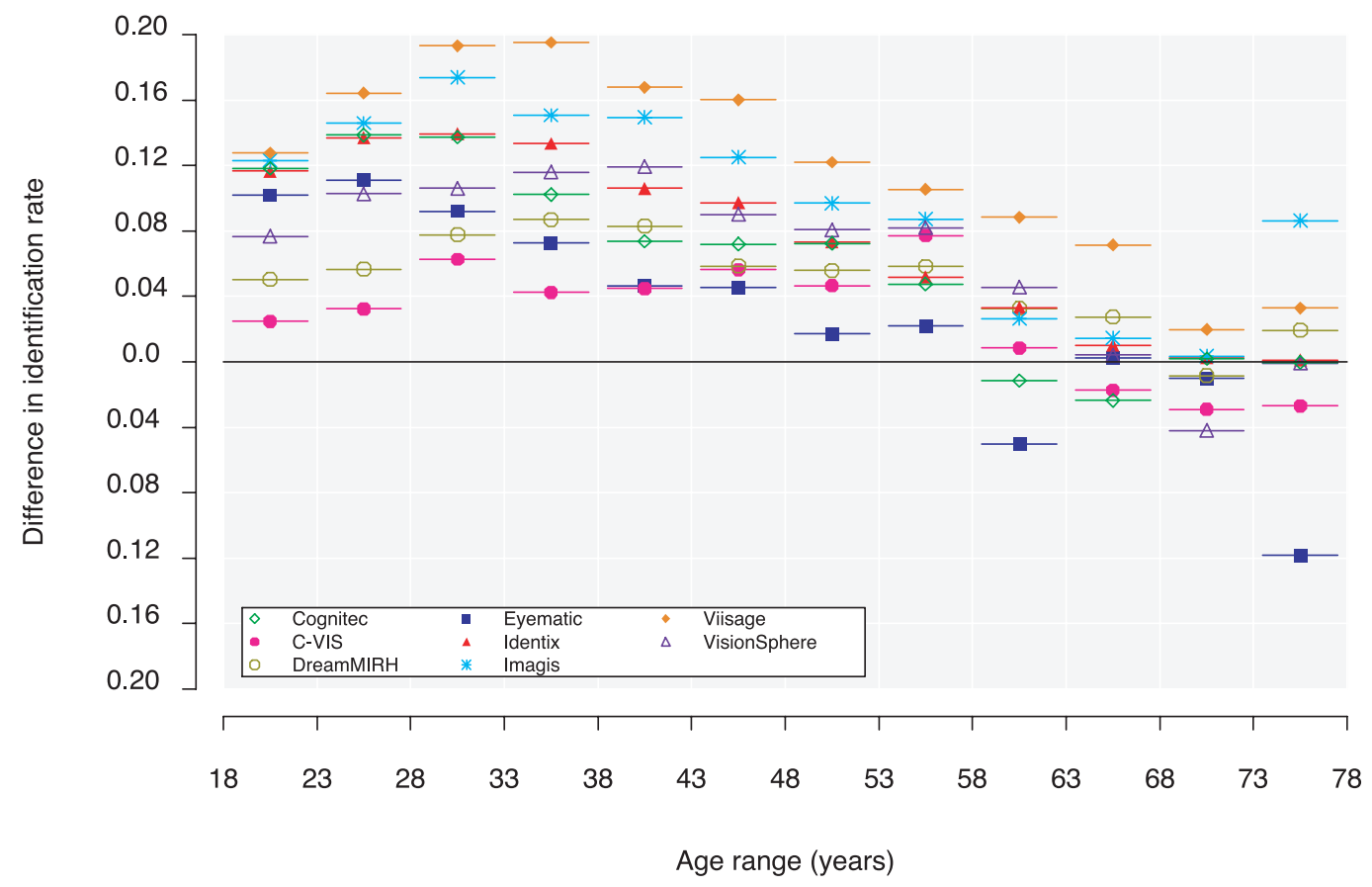

Fig. 27. Interaction between age and sex for rank 1 identification on HCInt large gallery (for all eight participants). Rank 1 performance for males minus females is plotted for each age bin.

\section{A.4 MCINT ADDENDUM}

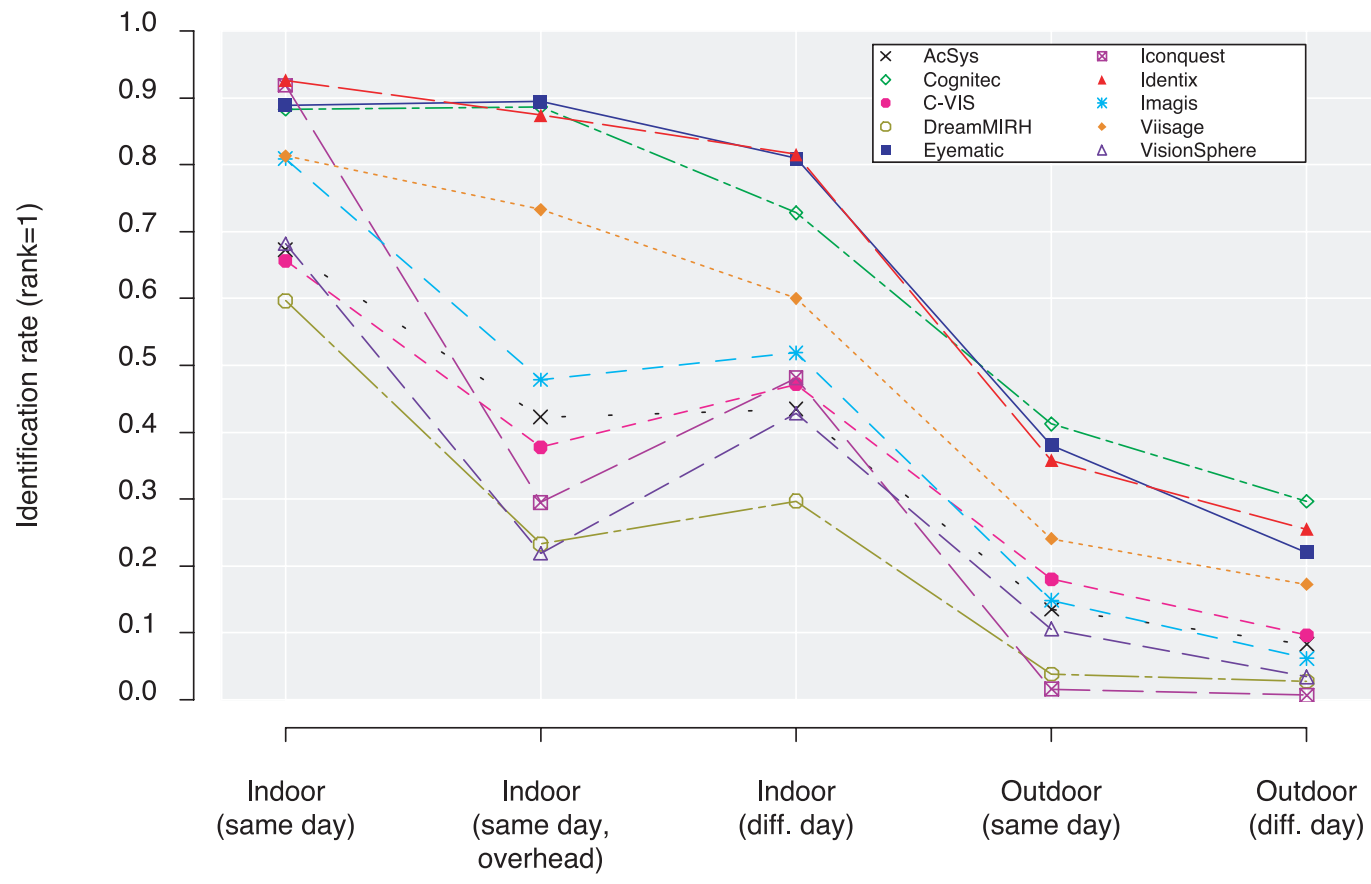

Fig. 28. Comparison of system performance of different categories of probes. The rank 1 identification rate is plotted. 


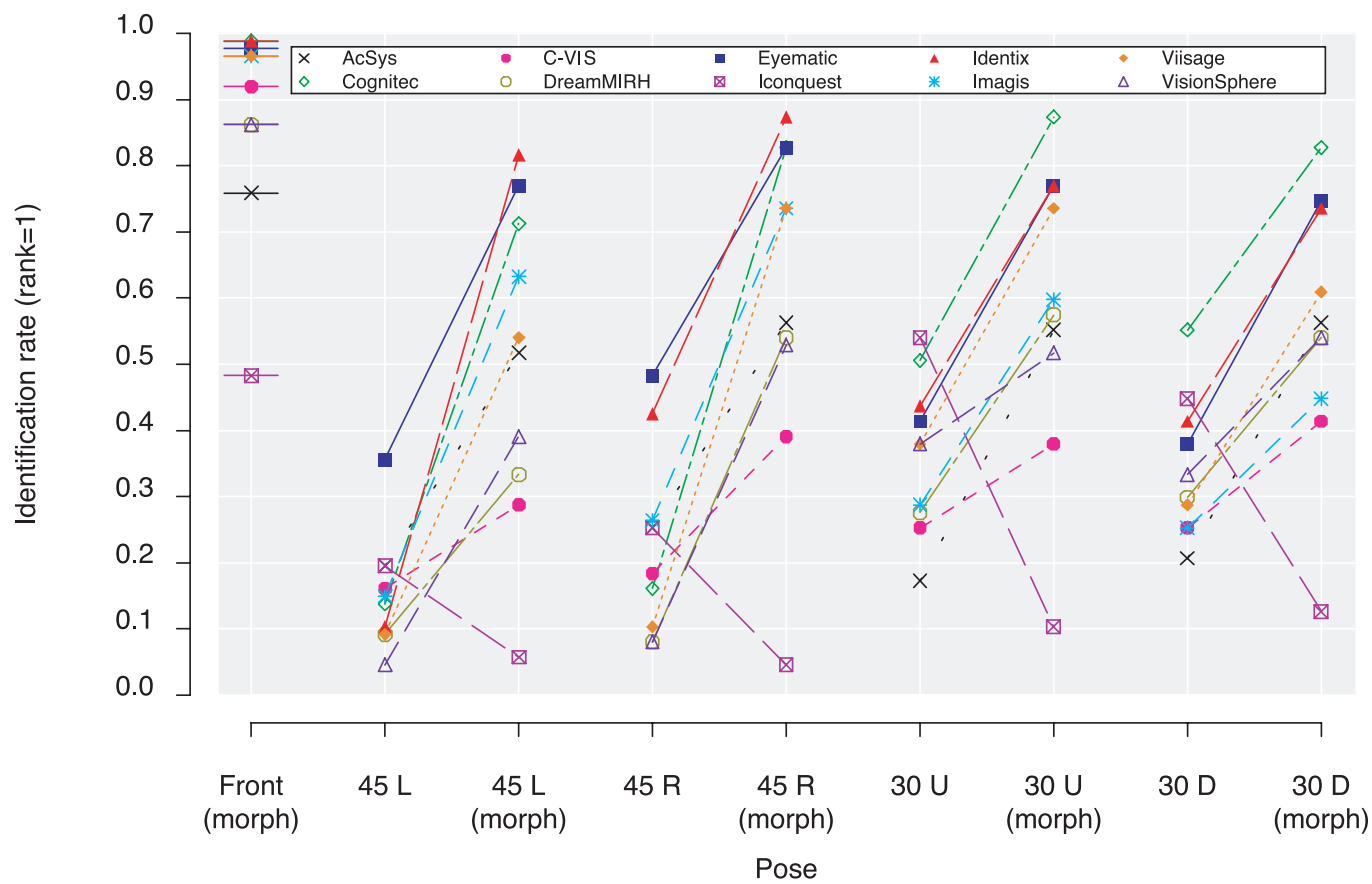

Fig. 29. The effect of still versus three-dimensional morphable models. The rank 1 identification rate is plotted.

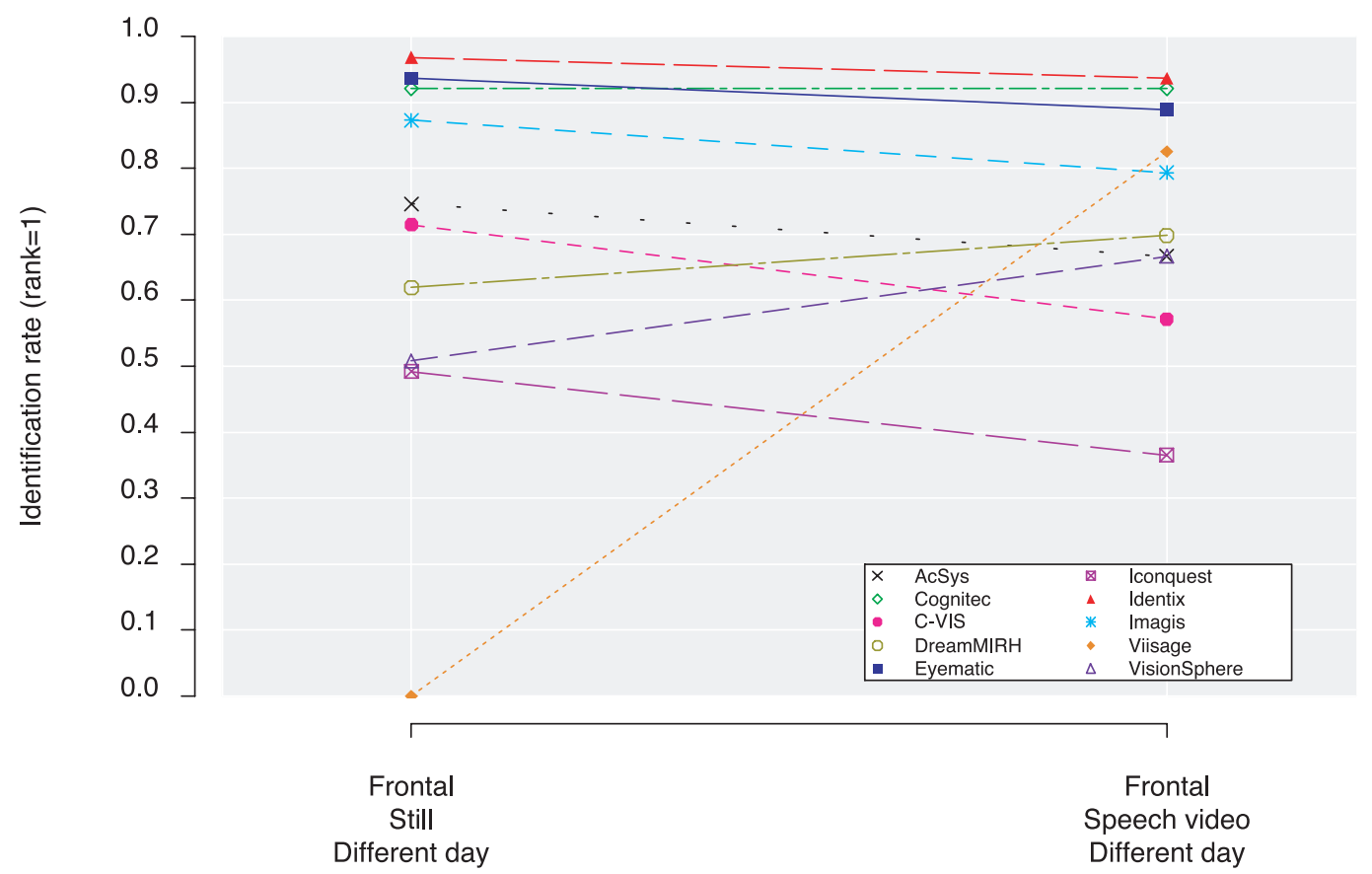

Fig. 30. Plot showing still versus video recognition for frontal imagery. The rank 1 identification rate is plotted. 


\section{A.5 PERFORMANCE ON VERY LARGE POPULATIONS}

An important question is how recognition performance decreases as the gallery size increases. In HCInt, it was empirically observed rank-one identification rate drops linearly with the logarithm of the gallery size (Figure 11). Our goal was to develop a model that explains the log-linear behavior from the match and non-match distributions. We formalize the model of the log-linear behavior by $P_{I}(G)=1-\alpha \log G$.

Gallery size is denoted by $G, P_{I}(G)$ is rank 1 performance for gallery size $G$, and $\alpha$ is a system performance parameter. The difference among the systems tested is the parameter $\alpha$. Three different models are discussed in this section, and their prediction for the performance for Eyematic is shown in Figure 31. The dashed red line in Figure 31 is Eyematic's actual performance as a function of gallery size.

A lower bound is $P_{I}(G)=1 / G$, which is the performance of randomly guessing the identity of a probe. Wayman (1999) and Daugman (2002) present models that model identification as $N$ verification attempts. Both present a binomial model ${ }^{5}{ }^{6} P_{I}(G)=\left(1-P_{F}(\tau)\right)^{G-1}$.

There are four problems with this model. First, $P_{F}(\tau)$ is function of $\tau$, and they do not specify how $\tau$ is selected. Figure 31 plots the binomial model for three values of $P_{F}: 0.1,0.01$, and 0.001 . The binomial model underestimates actual performance dramatically for anything other than small $G$ or very small $P_{F}$. To obtain the observed performance for the Eyematic system on the HCInt large gallery, i.e., $P_{I}(37,437)=0.65$, the needed value is $P_{F}=1.15 \times 10^{-5}$. In addition, the shape of the curve is not representative. Second, the model does not include the match distribution. The prediction is the same for all match distributions. Third, the model does not fit the observed data. Fourth, asymptotically the random algorithm is a better predictor. The expected performance of randomly guessing the identity of a probe is $P_{I}(G)=1 / G$. For a given $P_{F}(\tau)$, there is sufficiently large such for

$$
1 / G>\left(1-P_{F}(\tau)\right)^{G-1}
$$

In this appendix, we present a moment model for predicting identification performance as a function of gallery size. The model is a function of both the match and non-match distribution. The moment model is more accurate than proceeding models. The model predicts the log-linear behavior, however, as a rule, it under estimates performance. Before proceeding, we introduce some notation. The probability density function (pdf) of the match distribution is $m(\tau)$ and the cumulative density function (cdf) of the non-match distribution is $N(\tau)$. The model is presented for similarity scores; therefore, we assume that the match distribution is to the right of the nonmatch distribution. For an operating threshold $\tau, N(\tau)$ is the associated false alarm rate.

We will proceed by discussing the case where the size of the gallery is two. For a probe $p$, there is one match score and one non-match score. In the moment model, the match score is sampled from $m(\tau)$ and the non-match score is sampled from $N(\tau)$. We assume that the sampling from the match and non-match distributions is independent. For an operating threshold $\tau$, the probability that the match score will be greater than the non-match score is $N(\tau)$. Since the match score is randomly sampled from $m(\tau)$, the probability that the match score will be greater than the non-match score is

$$
P_{I}(2)=\int_{-\infty}^{\infty} N(\tau) m(\tau) d \tau
$$

However, Eq (1.1) is the area under the ROC (AUC) for the verification ROC (see Egan (1975) page 45). In signal detection theory, there is an experiment design called two interval forced choice (2IFC). In this experimental design, an observer is presented with two signals. One signal contains the true signal and the other, noise. The observer must decide which signal contains the true signal and which contains noise. The probability of making the correct decision is the area under the ROC. There is a direct relationship between 2IFC and closed universe identification.

5. See discussion of eq. 13 in Daugman (2003).

6. See discussion around eq. 31 in Wayman (1999). Our penetration rate is 1 and the number of templates is 1. 
In identification, the true signal is the correct match, and the noise is the incorrect match. The observer is presented with two matches, and the observer must decide which match is the correct match.

We now proceed to the general case for a gallery of size $G$. For a gallery of size $G$ and an operating threshold $\tau$, the probability that the match score will be greater than all $G-1$ non-match scores is $N(\tau)^{G-1}$. Since the match score is randomly sampled from $m(\tau)$, the probability that all match scores will be greater than the non-match score is

$$
P_{I}(G)=\int_{-\infty}^{\infty} N(\tau)^{G-1} m(\tau) d \tau
$$

We refer to this as a moment model because for a gallery size $G$, the expected identification rate is a function of the $G-1^{\text {th }}$ moment of $m(\tau)$ about $N(\tau)$.

The 2IFC experimental design can be generalized to $n$ IFC. In the generalization, an observer is presented with $n-1$ noise signals and one true signal. The observer must than decide which of the $n$ signals is the true signal. Identification from a gallery of size $G$ is equivalent to a $n$ IFC experimental design.

We ran two simulations to see how well the moment model predicted empirical performance. The probability $P_{I}(G)$ is computed from empirical match and non-match distributions. In our simulations, the match distribution was estimated from 6,000 match scores and the non-match distribution was estimated from 18 million non-match scores. Simulations were run on both non-normalized and normalized scores from Eyematic. The results are in Figure 31. The non-normalized simulation is labeled non-normalized moment and the normalized is labeled normalized moment. The non-normalized simulation underestimates the empirical slope. Similar results were found for the other participants. However, the normalized simulation fits quiet well with the empirical results. For the other participants that submitted non-normalization functions, the normalized scores were a better fit to the observed data. However, they did not fit the data.

One of the assumptions made in the moment model is that all similarity scores are sampled independently. This is the reason that our model underestimates the identification performance. Interesting, however, the fit is much better for normalized scores. This suggests that one of the features of normalization is that it increases independence among the match and non-match scores.

Next we generalize the moment one more time, to predict performance at rank $k$. Putting the appropriate Bernoulli coefficients into the model, the probability that a probe is at rank $k$ is

$$
\int_{-\infty}^{\infty}\left(\begin{array}{c}
G-1 \\
k-1
\end{array}\right)(1-N(\tau))^{k-1} N(\tau)^{G-1} m(\tau) d \tau,
$$

and the performance for gallery size $G$ for rank $k$ is

$$
P(G, k)=\sum_{i=1}^{k} \int_{-\infty}^{\infty}\left(\begin{array}{c}
G-1 \\
i-1
\end{array}\right)(1-N(\tau))^{i-1} N(\tau)^{G-i} m(\tau) d \tau .
$$

The availability of a large database allowed a more thorough investigation into the effect of gallery size on performance. To predict the performance, we introduced the moment model and showed its connection with classical signal detection theory. In turn, this connection established a link between verification and identification performance. In addition, the model provides insight into how normalization works. Further research is needed to correct the underestimation in the moment model. 


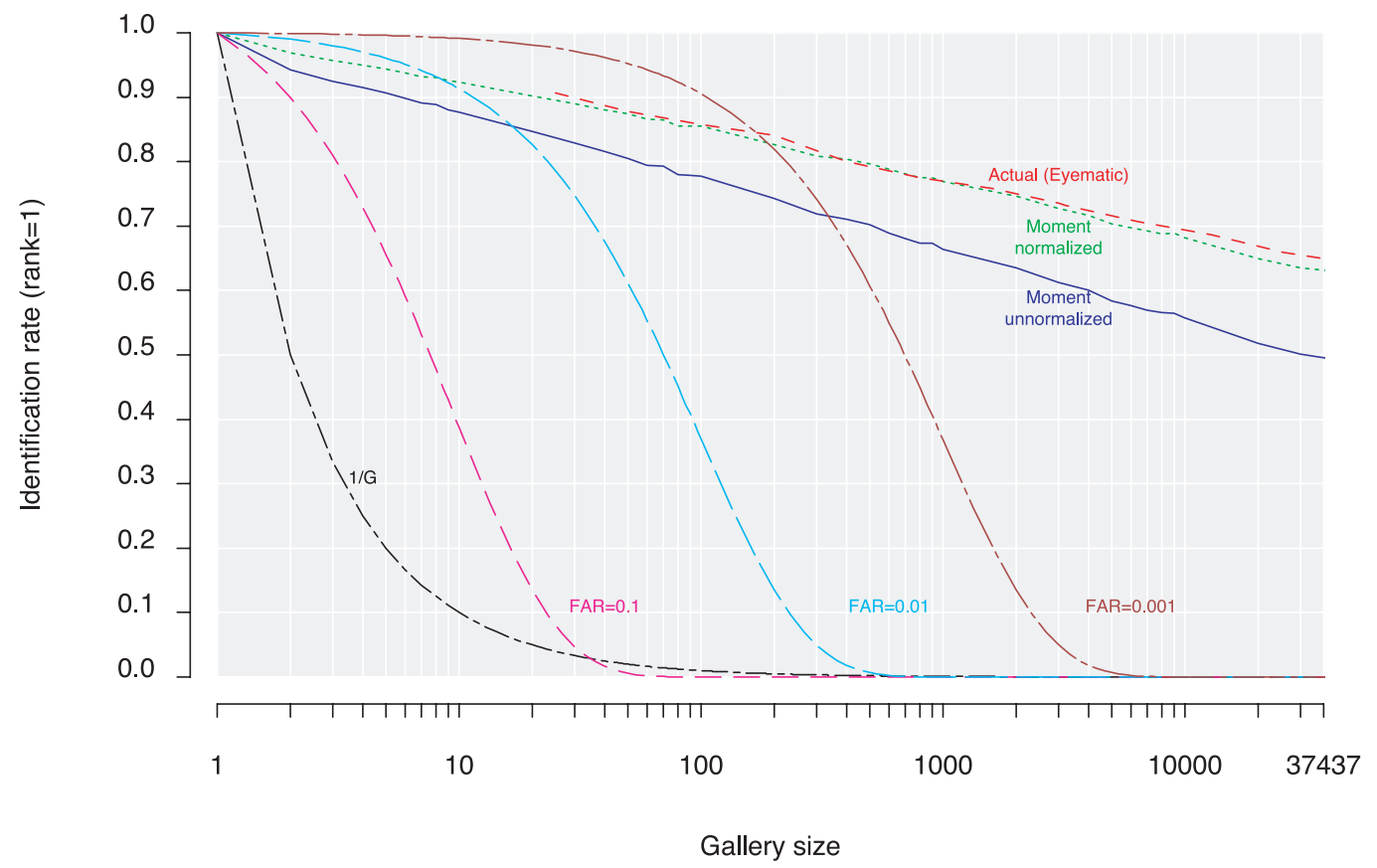

Fig. 31. Study of identification performance as a function of gallery size. 


\section{REFERENCES}

Beveridge, J. R., K. She, B. A. Draper, and G. H. Givens. 2001. "A nonparametric statistical comparison of principal component and linear discriminant subspaces for face recognition," Proceedings of IEEE Conference on Computer Vision and Pattern Recognition, Vol. 1, pp 535542.

Blackburn, D. M., J. M. Bone, and P. J. Phillips. 2001. FRVT 2000 Report, Technical Report, http://www.frvt.org.

Blanz, V., and T. Vetter. 1999. "A morphable model for the synthesis of 3D faces," Computer Graphics Proceeding SIGGRAPH '99, pp. 187-194.

Blanz,V., S. Romdhami, and T. Vetter. 2002. "Face identification across different poses and illuminations with a 3D morphable model," Proceedings of International Conference on Automatic Face and Gesture Recognition, pp. 202-207.

Bickel, P. J., and K. A. Doksum. 1977. Mathematical Statistics, Oakland, CA: Holden-Day.

Bone, J. M., and D.M. Blackburn. 2002. "Face Recognition at a Chokepoint - Scenario Evaluation Results," Technical Report, http://www.dodcounterdrug.com/facialrecognition.

Bolle, R. M., N. K. Ratha, and S. Pankanti. 2000. "Evaluating authentication systems using bootstrap confidence intervals," In Proceedings of $15^{\text {th }}$ International conference on pattern recognition, pp. 835-841.

Daugman, J. 2003. "The importance of being random: statistical principals of iris recognition," Pattern Recognition, Vol. 36, No. 2, pp 279-291.

Egan, J. P. 1975. Signal detection theory and ROC analysis, New York: Academic Press.

Furl, N., P. J. Phillips, and A. J. O'Toole. 2002. "Face recognition algorithms and the other-race effect: computational mechanisms for a developmental contact hypothesis," Cognitive Science Vol. 26, pp. 797-815.

Givens, G., J. R. Beveridge, B. A. Draper, and D. Bolme. 2002. "A statistical assessment of subject factors in PCA recognition of human faces," NIPS Workshop on Statistics for Computational Experiments.

Gross, R., J. Shi, and J. F. Cohn. 2001. "Quo vadis face recognition," Proceedings of Third workshop on empirical evaluation methods in face recognition.

MacMillian, N. A., and C. D. Creelman. 1991. Detection theory: A user's guide, Cambridge: Cambridge University Press.

Maio, D., D. Maltoni, R. Cappelli, J. L. Wayman, and A. K. Jain. 2002a. "FVC 2000: fingerprint verification competition," IEEE Transactions on Pattern Analysis and Machine Intelligence, Vol. 24, No. 3, pp. 402-412.

Maio, D., D. Maltoni, R. Cappelli, J. L. Wayman, and A. K. Jain. 2002b. "FVC 2002: second fingerprint verification competition," Proceedings $16^{\text {th }}$ International Conference on Pattern Recognition, Vol. 3, pp. 811-814. http://bias.csr.unibo.it/fvc2002/

Mansfield, T., G. Kelly, D. Chandler, and J. Kane. 2001. "Biometric Product Testing Final Report," Technical Report, CESG, http://www.cesg.gov.uk/technology/biometrics/index.htm.

Mansfield, T., and J. Wayman. 2002. "Best practices in testing and reporting performance of biometric devices Version 2.01," Technical Report, National Physical Laboratory, UK, http://www.cesg.gov.uk/technology/biometrics/index.htm.

Martin, A., and M. Przybocki. 2000. "The NIST 1999 speaker recognition evaluation-An overview," Digital Signal Processing Vol. 10, pp. 1-18.

Martinez, A. R., and R. Benavente. 1998. The AR face database, Technical Report 24, Computer Vision Center, Barcelona, Spain.

Micheals, R. J., and T. Boult. 2001. "Efficient evaluation of classification and recognition systems," Proceedings of IEEE Conference on Computer Vision and Pattern Recognition, Vol. 1, pp 50-57.

Moghaddam, B., and M.-H. Yang. 2002. "Learning gender with support faces," IEEE Trans. Pattern Analysis and Machine Intelligence, Vol. 24, No. 5, pp. 707-711.

Moon, H., and P.J. Phillips. 2001. "Computational and performance aspects of PCA-based face recognition algorithms," Perception, 30, pp. 301-321. 
NIST. 2002. Use of Technology Standards and Interoperable Databases with Machine-Readable, Tamper-Resistant Travel Documents. http://www.itl.nist.gov/iad/894.03/fing/fing.html

Phillips, P. J., P. J. Rauss, and S. Der. 1996. "FERET (face recognition technology) recognition algorithm development and test results," Army Research Laboratory technical report, ARL-TR995. http://www.frvt.org.

Phillips, P. J., H. Wechsler, J. Huang, and P. Rauss. 1998. "The FERET database and evaluation procedure for face-recognition algorithms," Image and Vision Computing, Vol. 16, No. 5, pp. 295-306.

Phillips, P. J., A. Martin, C. L. Wilson, and M. Przybocki. 2000. "An introduction to evaluating biometric systems," Computer, Vol. 33, pp. 56-63.

Phillips, P. J., H. Moon, S. Rizvi, and P. Rauss. 2000. "The FERET Evaluation methodology for face-recognition algorithms," IEEE Trans. Pattern Analysis and Machine Intelligence, Vol. 22, No. 10.

Phillips, P. J., D. Blackburn, P. Grother, E. Newton, and J. M. Bone. 2003. "Methods for assessing progress in face recognition," In Biometric Systems: Technology, Design and Performance Evaluation (eds.) J.L. Wayman, A.K. Jain, D. Maltoni and D. Maio, Surrey, UK: Springer-Verlag London.

Shakhnarovich, G., P Viola, and B. Moghaddam. 2002. "A unified learning framework for real time face detection and classification," Proceedings Fifth IEEE International Conference on Automatic Face and Gesture Recognition, pp. 14-21.

Wayman, J. L. 1999. "Error-rate equations for the general biometric system", IEEE Robotics \& Automation Magazine, Vol. 6, No. 1, pp. 35-48.

Wayman, J. L. ed. 2000. National biometric test center collected works 1997-2000, Version 1.3, Technical Report, San Jose State University, http://www.engr.sjsu.edu/biometrics. 


\section{FACE RECOGNITION VENDOR TEST 2002}

\section{Technical Appendices \\ March 2003}

\section{P. Jonathon Phillips ${ }^{1,2}$, Patrick Grother ${ }^{2}$, Ross J. Micheals ${ }^{2}$, Duane M. Blackburn ${ }^{3}$, Elham Tabassi ${ }^{2}$, Mike Bone ${ }^{4}$}

${ }^{1}$ DARPA

3701 North Fairfax Dr.

Arlington, VA 22203

${ }^{2}$ National Institute of Standards and Technology

100 Bureau Drive, Stop 8940

Gaithersburg, MD 20899

${ }^{3}$ DoD Counterdrug Technology Development Program Office

17320 Dahlgren Rd, Code B07

Dahlgren, VA 22448

${ }^{4}$ NAVSEA Crane Division

300 Highway 361, Code 4041

Crane, IN 47522

\section{Sponsors and Supporters:}

Defense Advanced Research Projects Agency Department of State

Federal Bureau of Investigation

National Institute of Justice

National Institute of Standards and Technology

Transportation Security Administration

ONDCP Counterdrug Technology Assessment Center

United States Customs Service
Department of Energy

Drug Enforcement Administration

Immigration and Naturalization Service

United States Secret Service

Technical Support Working Group

Australian Customs

Canadian Passport Office

United Kingdom Biometric Working Group 


\section{This page intentionally blank.}


Face Recognition Vendor Test 2002:

Technical Appendices

\title{
P. Jonathon Phillips ${ }^{1,2}$, Patrick Grother ${ }^{2}$, Ross Micheals ${ }^{2}$, Duane M. Blackburn ${ }^{3}$, Elham Tabassi ${ }^{2}$ J. Mike Bone ${ }^{4}$
}

\author{
${ }^{1}$ DARPA \\ 3701 North Fairfax Drive \\ Arlington, VA 22203 \\ ${ }^{2}$ National Institute of Standards and Technology \\ 100 Bureau Drive; Stop 8940 \\ Gaithersburg, MD 20899 \\ ${ }^{3}$ DoD Counterdrug Technology Development Program Office \\ 17320 Dahlgren Rd, Code B07 \\ Dahlgren, VA 22448 \\ ${ }^{4}$ NAVSEA Crane Division \\ 300 Highway 361, Code 4041 \\ Crane, IN 47522
}

\begin{abstract}
The Face Recognition Vendor Test 2002: Technical Appendices is a companion document to the Face Recognition Vendor Test 2002: Evaluation Report. This document provides supplemental material that is not included in the Evaluation Report. The Technical Appendices present all material provided to the Face Recognition Vendor Test (FRVT) 2002 participants, detailed descriptions of the evaluation protocols and testing procedures, additional performance results, and description of participant systems and comments on the results ${ }^{2}$. Appendix E contains submissions to the frequently asked questions section of the website and the answers to the questions.
\end{abstract}

\footnotetext{
${ }^{1}$ Please direct correspondence to Jonathon Phillips at jphillips@darpa.mil or Jonathon@ nist.gov. The Face Recognition Vendor Test 2002: Evaluation Report is available at http://www.frvt.org.

${ }^{2}$ Participant documents (system descriptions and comments on results) are the opinions of the Participants and are provided in this text for reference. Inclusion of these documents does NOT imply that FRVT 2002 Authors/Sponsors/Supporters agree with any statements or derivative results within those documents. The identification of any commercial product or trade name does not imply endorsement or recommendation by the National Institute of Standards and Technology or any other FRVT 2002 Author/Sponsor/Supporter.
} 
The Face Recognition Vendor Test 2002: Technical Appendices is a companion document to the Face Recogntion Vendor Test 2002: Evaluation Report. The Technical Appendices provide supplemental information about the procedures used in FRVT 2002, documentation provided to participants prior to testing, additional Face Recognition Vendor Test (FRVT) 2002 results and participant-provided documentation describing their systems and their comments to the Evaluation Report. The appendices provide a comprehensive description of the evaluation protocol and testing procedure, and provide the necessery background for perparing and administering future technology evaluations. Table $\mathrm{i}$ list the title of the appendices in the Technical Appendices. A short description of each appendix follows.

Table i - List and Title of Appendices.

\begin{tabular}{cl}
\hline Appendix & \multicolumn{1}{c}{ Content } \\
\hline B & FRVT 2002 Announcement \\
C & FRVT 2002 Website \\
D & Forms to Participate in FRVT 2002 \\
E & Frequently Asked Questions \\
F & FRVT 2002 Methods and Procedures \\
G & FRVT 2002 Technical Data Set \\
H & FRVT 2002 Test Plan \\
I & Image Collection Process and Image Description \\
J & FRVT 2002 Normalization API \\
K & Development Image Set \\
L & Detailed Results \\
M & Participant Product Description \\
N & Participant Comments on FRVT 2002 Evaluation Report \\
O & Additional Information on the 3D Morphable Model Used in \\
& FRVT 2002 \\
\hline
\end{tabular}

Appendix B contains the documents that announced the FRVT 2002.

Appendix $C$ contains a copy of the FRVT 2002 Website that was posted during the evaluation. This includes the publicly available portion of the website as well as the restricted-access participant area. The participant area contained information that was only available to participants and tentative participants during the evaluation process.

Appendix D contains copies of the two forms that FRVT 2002 participants were required to complete and sign to participate in FRVT 2002. One form explained the ground rules associate with participating in FRVT 2002. The second form explained restrictions on the use of FRVT 2002 imagery.

Appendix $\boldsymbol{E}$ contains a copy of submitted frequently asked questions and their answers. The questions and answers provide additional clarification on the FRVT 2002.

Appendix $\boldsymbol{F}$ is a description of the methods, procedures and timelines used to manage FRVT 2002.

Appendix $\boldsymbol{G}$ contains the documentation that was included in the FRVT 2002 technical datasets. The technical dataset documented the interfaces for reading target and query sets and writing the similarity files.

Appendix $\boldsymbol{H}$ contains the documentation provided to FRVT 2002 participants that describes the on-site procedures followed during the administration of the test. 
Appendix I describes the imagery in the HCInt and MCInt datasets.

Appendix $\boldsymbol{J}$ describes procedures and API documentation for the normalization component of FRVT 2002.

Appendix $\boldsymbol{K}$ shows the sample images given to FRVT 2002 participants prior to testing. The sample image was designed to give participants a basic "feel" for the types of images that were included in the FRVT 2002 data sets. The sample image set was not designed for either developing or tuning of face recognition systems.

Appendix L. In order to clearly present an assessment of the state-of-the-art in face recognition, it was necessary in the Evaluation Report to report summary statistics of performance. Appendix L presents full cumulative match charateristics (CMC) and receiver operating characteristics (ROC) for all MCInt experiments (Section 8 and Appendix A.4 in the Evaluation Report). Also, this appendix presents HCInt verification error ellipses ROCs (Figure 8) for all participants in Figure 8.

Appendix $M$ provides a description and cost of each system that was tested. The descriptions were given to the FRVT 2002 organizers by the participants prior to commencement of the FRVT 2002 evaluation.

Appendix $N$ contains the position papers written by FRVT 2002 Participants after viewing the evaluation results.

Appendix $\boldsymbol{O}$ contains additional information on the 3D Morphable Model used in FRVT 2002. 


\section{This page intentionally blank.}


Appendix B

FRVT 2002 Announcements 


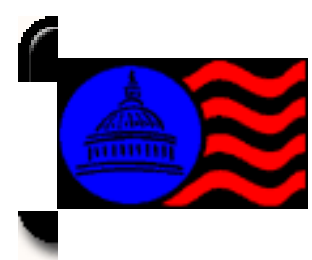

FBO DAILY ISSUE OF APRIL 27, 2002 FBO \#0146

SOURCES SOUGHT

\section{6 -- SOURCES SOUGHT FOR FACIAL RECOGNITION SYSTEMS}

Notice Date

$4 / 25 / 2002$

Notice Type

Sources Sought

\section{Contracting Office}

Department of Commerce, National Institute of Standards and Technology (NIST), Acquisition and Logistics Division, 100 Bureau Drive, Building 301, Room B129, Mail Stop 3571, Gaithersburg, MD, 20899-3571

ZIP Code

20899-3571

Solicitation Number

SOURCESSOUGHTFACIALRECOG

Response Due

$5 / 10 / 2002$

\section{Archive Date}

$5 / 25 / 2002$

\section{Point of Contact}

Tamara Grinspon, Contract Specialist, Phone (301) 975-4390, Fax (301) 975-8884, - Tamara Grinspon, Contract Specialist, Phone (301) 975-4390, Fax (301) 975-8884,

\section{E-Mail Address}

tamara.grinspon@nist.gov, tamara.grinspon@nist.gov

\section{Description}

The National Institute of Standards and Technology (NIST) wishes to identify potential sources to provide commercial, or soon-to-be commercial, face (facial) recognition systems. This is a market research survey being conducted for planning purposes only and is not to be construed as a commitment by the Government. THIS IS NOT A REQUEST FOR PROPOSALS. Available systems may be selected by the Government to participate in the Facial Recognition Vendor Test 2002, a government-sponsored technology evaluation of face recognition technologies. Potential sources of face recognition technology will not be funded, nor charged, to participate in FRVT 
SRCSGT | 66 | SOURCES SOUGHT FOR FACIAL RECOGNITION SYSTEMS | 27-A... Page 2 of 2

2002. A special format for gathering this information has been established in the FRVT 2002 "How to Participate" page at http://www.frvt.org . A deadline for submission is shown on the "Calendar" page of http://www.frvt.org .--

\section{Place of Performance}

Address: National Institute of Standards and Technology, Shipping and Receiving, Building 301, 100 Bureau Drive, Gaithersburg, MD 20899-0001

\section{Record}

SN00066141-W 20020427/020425213206 (fbodaily.com)

\section{Source}

FedBizOpps.gov Link to This Notice

(may not be valid after Archive Date)

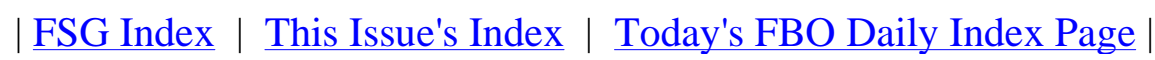




\section{Blackburn Duane M DLVA}

From:

Duane Blackburn [BlackburnDM@nswc.navy.mil]

Sent:

Thursday, April 25, 2002 7:56 PM

To:

Subject:

BIOMETRICS@PEACH.EASE.LSOFT.COM

FRVT 2002 Announcement

In the USA Patriot Act, the National Institute of Standards (NIST) is mandated to measure the accuracy of biometric technologies. In accordance

with this legislation, NIST, in cooperation with 14 other Government agencies, will conduct the Facial Recognition Vendor Test 2002. The evaluation will be held in the summer of 2002 with published results expected in the fall. The same individuals that managed the FRVT 2000 are

also managing the FRVT 2002 evaluation.

Information about FRVT 2002 has been made available on the Internet at http://www.frvt.org including:

Overview of FRVT 2002

Sponsoring agencies

Qualification requirements for participants

Calendar of events

The FRVT 2002 is a government-sponsored technology evaluation of face recognition technology. Participation is open to anyone selling a commercial or mature prototype face recognition system, subject to the requirements and procedures outlined on the FRVT 2002 website.

The sponsors and supporters of FRVT 2002 thank you for the opportunity of

providing this service for the government, public and biometrics industry .

The preceding was forwarded by the Biometric Consortium's Electronic Discussion Group. Any opinions expressed here do not necessarily reflect those of the Biometric Consortium. Further distribution is prohibited.

Problems and questions regarding this list should be sent to BIOMETRICS-request@PEACH.EASE. LSOFT . COM.

To remove your name from this list please send the command "SIGNOFF BIOMETRICS" to <LISTSERV@PEACH.EASE.LSOFT.COM>. Please do not send the "SIGNOFF BIOMETRICS" command to the BIOMETRICS list.

To update membership information (new e-mail address etc.), please send a message to <baileyabiometrics.org> providing the updated information. 


\begin{tabular}{|l|l|}
\hline From: "FRVT2002" <frvt2002@brtrc.com> & 葍 \\
Subject: FRVT 2002 Announcement & 兽 \\
Date: Thu, 25 Apr 2002 19:57:22-0400 & \\
\hline To: frvt2002@brtrc.com & \\
\hline
\end{tabular}

In the USA Patriot Act, the National Institute of Standards (NIST) is mandated to measure the accuracy of biometric technologies. In accordance with this legislation, NIST, in cooperation with 14 other Government agencies, will conduct the Facial Recognition Vendor Test 2002. The evaluation will be held in the summer of 2002 with published results expected in the fall. The same individuals that managed the FRVT 2000 are also managing the FRVT 2002 evaluation. 


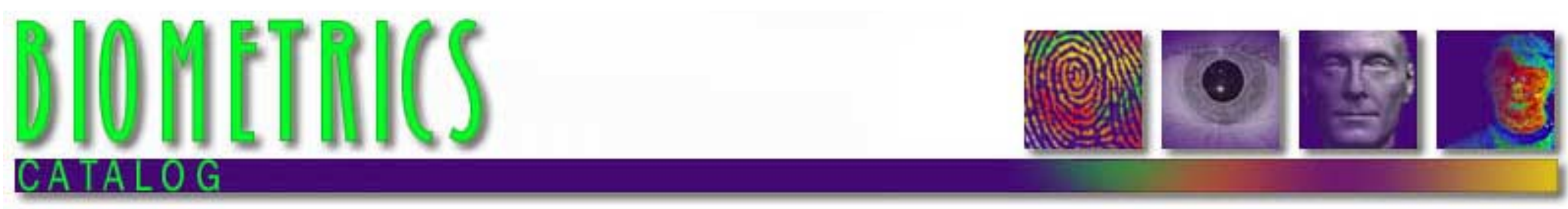

Results List Modify Catalog Entry

Modified: 4/25/02 8:05:00 PM

Category: Government Tests and Deployments

Biometric Type: Facial Recognition

Vendor: $n / a$

Title: Facial Recognition Vendor Test 2002

Description: In the USA Patriot Act, the National Institute of Standards (NIST) is mandated to measure the accuracy of biometric technologies. In accordance with this legislation, NIST, in cooperation with 14 other Government agencies, will conduct the Facial Recognition Vendor Test 2002. The evaluation will be held in the summer of 2002 with published results expected in the fall. The same individuals that managed the FRVT 2000 are also managing the FRVT 2002 evaluation.

Information about FRVT 2002 has been made available on the Internet at http://www.frvt.org including:

Overview of FRVT 2002

Sponsoring agencies

Qualification requirements for participants

Calendar of events

The FRVT 2002 is a government-sponsored technology evaluation of face recognition technology. Participation is open to anyone selling a commercial or mature prototype face recognition system, subject to the requirements and procedures outlined on the FRVT 2002 website.

The sponsors and supporters of FRVT 2002 thank you for the opportunity of providing this service for the government, public and biometrics industry.

\section{Additional References}

Related sites: FRVT Homepage 
Appendix C

FRVT 2002 Website 
Status Update 26 November 2002

How to Participate

\section{Participants}

\section{Sponsors}

\section{FAQ}

\section{Calendar}

\section{Participant Area}

Facial Recognition

Vendor Test 2000

\section{FERET}

\section{Evaluation}

Methodology

\section{Home}

\section{FACE RECOGNITION VENDOR TEST 2002}

\section{Introduction}

In the USA Patriot Act, the National Institute of Standards (NIST) is mandated to measure the accuracy of biometric technologies. In accordance with this legislation, NIST, in cooperation with other Government agencies, is conducting the Face Recognition Vendor Test 2002. The evaluation is being held in the summer of 2002 with results expected to be published in the fall 2002. FRVT 2002 Sponsors and Supporters are:

- Defense Advanced Research Projects Agency

- Department of State

- Federal Bureau of Investigation

- National Institute of Justice

- National Institute of Standards and Technology

- Transportation Security Administration

- ONDCP Counterdrug Technology Assessment Center

- United States Customs Service

- Department of Energy

- Drug Enforcement Administration

- Immigration and Naturalization Service

- Secret Service

- Technical Support Working Group

- Australian Customs

- Canadian Passport Office

- United Kingdom Biometric Working Group

The following individuals supervise and manage FRVT 2002:

- Dr. P. Jonathon Phillips, Test Director

- Duane Blackburn

- Mike Bone

- Patrick Grother

- Dr. Ross Michaels

One objective of the Patriot Act legislation is to "... develop and certify a technology standard, including appropriate biometric identifier standards, that can be used to verify the identity of persons applying for a US visa or such persons seeking to enter the US pursuant to a visa for the purposes of 
conducting background checks, confirming identity, and ensuring that a person has not received a visa under a different name...". FRVT 2002 satisfies the US Patriot Act face recognition evaluation mandate. Results provide as input for future decisions to be made in accordance with the Patriot Act and subsequent legislation.

\section{Participation}

To assess the state-of-the-art in face recognition technology, the FRVT 2002 is conducting a technology evaluation of both mature prototype and commercially available systems face recognition systems. Core face recognition technology developers were invited to participate. The deadline for signing up for the FRVT 2002 was 10 May 2002. A list of FRVT 2002 Participants can be found on the Participants Page.

\section{FRVT 2002 Status Update - 17 August 2002}

Administering the FRVT 2002 to the participants has been completed, and the FRVT 2002 organizers are in the processing of computing the performance measures and writing the report. The report is due to be released in November 2002, and will be available from this website.

To find out more about the FRVT 2002, attend the two FRVT 2002 talks at the Biometric Consortium Conference 2002 23-25 September in Crystal City, VA. The talks are "Overview of FRVT 2002" to be presented Monday morning 23 September, and "New Evaluation Statistics for Measuring the Performance of Biometric Technologies", Wednesday morning.

FRVT 2002 was administered to fourteen participants at the Navy facility in Dahlgren, Virginia, from 10 July through 9 August 2002.

In the original proposed design, the FRVT 2002 consisted of four tests. However, because of higher than expected response, the organizers redesigned the FRVT 2002 to consist of two tests. These are the High Computational Intensity ( $\mathrm{HClnt}$ ) Test and the Medium Computational Intensity (MCInt) Test. Both test required the systems to be full automatic, and manual intervention was not allowed. Participants could sign up to take either or both tests.

The High Computational Intensity ( $\mathrm{HClnt}$ ) Test was designed to test state-of-the-art systems on extremely challenging real-world images. These were full-face still frontal images. This test compares still database images against still images of an unknown person. The $\mathrm{HClnt}$ required participants to process a set of approximately 121,000 images, and match all 
possible pairs of images from the 121,000 image set. This required performing 15 billion matches. The results from the $\mathrm{HCl}$ t will measure performance of face recognitions systems on large databases, examine the effect of database size on performance and estimate variability in system performance.

The Medium Computational Intensity ( MCInt) Test consists of two separate parts: still and video. The Medium Computational Intensity Subtest was designed to provide an understanding of a participant's capability to perform face recognition tasks with several different formats of imagery (still and video) under varying conditions. This subtest will also help identify promising face recognition technologies that did not take the High Computational Intensity test. The still portion of the $\mathrm{MCI}$ t is similar to the FERET and FRVT 2000 evaluations. It compares a database of still images against still images of unknown people. The still portion of the MCInt is designed to measure performance on different categories of images. Examples of different effects that will be measured are time between images, changes in illumination, and variation in pose. The video portion is designed to provide an initial assessment of whether or not video helps increase face recognition performance. This portion uses video style imagery that is extracted from digital video sequences. The video portion compares both a database of still images against video sequences of an unknown person and a database of video sequences against video sequences of an unknown person.

\section{FRVT 2002 Status Update - 26 November 2002}

The Face Recognition Vendor Test (FRVT) 2002 conducted experiments with approximately 121,000 images in its database. This resulted in approximately 15 billion comparisons and 60 gigabytes of raw data for each participant. This represents a major advancement in face recognition evaluations.

The FRVT 2002 Evaluation Team has completed scoring on the initial set of experiments planned for FRVT 2002. In the course of scoring the initial experiments, the FRVT 2002 Evaluation Team discovered a number of new and interesting results. These results led to the creation of additional experiments that will describe strengths and weaknesses of face recognition systems that have not previously been studied. The FRVT 2002 Evaluation Team is currently in the process of scoring participants for these additional experiments.

The decision was made to include these additional experiments in the FRVT 2002 Evaluation Report, 
thus causing a slight delay in its release. The FRVT 2002 Evaluation Team apologizes for this delay, but feels it is justified as the results of these new experiments have the potential to directly impact how and where face recognition systems should be fielded.

The experiments, analysis, and report generation will be completed in December 2002. Once the report is completed it will be submitted for public release approval. Once this approval is received, the FRVT 2002 Evaluation Report will be posted on the FRVT 2002 website.

\section{Communications}

To maintain fairness, the Government is adhering to the following procedures for FRVT 2002. Information provided to any single participant will also be provided to all participants. The primary and preferred source of communications between Participants and FRVT 2002 government personnel is the frequently asked question (FAQ) page. A government employee has been named as the FRVT Liaison. The FRVT Liaison will be the primary point of contact and will manage communications with Participants. Questions that are outside the purview of the FAQ can be submitted to the FRVT Liaison via email at frvt2002@nist.gov. All e-mails sent to the FRVT Liaison and germane to the FRVT 2002 will be posted on the FAQ page. This policy ensures all Participants are guaranteed equal access to information concerning FRVT 2002. Contact with the FRVT Liaison other than via the FAQ areas, or contact with someone other than the non-FRVT Liaison is only allowed for extenuating circumstances. Media inquiries should be sent to Philip Bulman of the NIST Public Affairs Office at philip.bulman@nist.gov.

The FRVT 2002 Evaluation Report will be placed on the FRVT website at http:// www. frvt.org when it has been completed and approved for public release. We will announce the release on the Biometrics Consortium listserv. 
Status Update

26 November 2002

How to Participate

\section{Participants}

\section{Sponsors}

\section{$F A Q$}

Calendar

\section{Participant Area}

\section{Facial Recognition}

Vendor Test 2000

Face Recognition Vendor Test 2002

\section{FERET}

Evaluation

Methodology

\section{Home}

\section{FACE RECOGNITION VENDOR TEST 2002 \\ How to Participate}

In order to request participation in the FRVT 2002, potential participants must electronically fill out two forms:

Application to Participate in FRVT 2002

Application for Access to the FRVT 2002 Technical

Datasets

Both forms are to be filled out on-line, printed, signed, and sent to the locations designated on each form. Upon receipt of both original signed forms by the Government, applicants will be classified as a Tentative Participant. The Government must receive both forms by the due date described in the FRVT 2002 Calendar Page.

The Application to Participate in the FRVT 2002 explains the rules, process, and procedures governing the administration of FRVT 2002. Additional technical details will be made available at a later date in the FRVT2002 Test Plan.

The Application for Access to the FRVT 2002 Technical Datasets explains rules governing access and use of the:

- FRVT 2002 evaluation data sets.

- FRVT 2002 training set.

- Software provided with the FRVT 2002 training set.

Conducting a technology evaluation requires that the sets of imagery provided to the Participants and results of the evaluation are in specified formats. The FRVT 2002 training set and associated software provides an example of the formats that will be used in FRVT 2002. This will provide Participants with the necessary imagery and tools to check that their system can read FRVT 2002 test image sets and that their systems output the results in the correct format. The imagery data sets and results format are in XML. The specifications for the XML format are included in the software provided with the FRVT 2002 training set. Very important: The baseline PCA algorithm included in the FRVT 2002 Technical Datasets requires normalized (eye-coordinates given) images. I mages provided during FRVT 2002 will NOT be normalized. Systems evaluated will need to automatically process facial imagery without eye coordinates being provided.

The FRVT 2002 evaluation protocol is based on the evaluation protocol used in the September 1996 FERET and Face

Recognition Vendor Test 2000 (FRVT 2000) evaluations. FRVT 2002 will evaluate systems that can automatically process facial imagery without eye coordinates being provided. For a subtest, 
a system is given two lists of signatures; list $\mathbf{A}$ and list $\mathbf{B}$. In the FRVT 2002, a signature consists one still image or a set of still images for the video subset. The system then has to match every signature in list $\mathbf{A}$ with every signature in list B. Each match yields a similarity score. The complete set of similarity scores between list $A$ and list $B$ is a similarity matrix. The format for the similarity matrix is contained in the FRVT 2002 Technical Datasets distribution. From the similarity matrix, performance scores and statistics are computed.

The desired method of communications between participants and the FRVT 2002 is the Participant FAQ page in the Participant Area. The Participant Area FAQ is not limited to simple questions, but will serve as the method of communications between the FRVT 2002 Liaison and Participants. All pertinent e-mail sent to the FRVT 2002 Liaison will be posted on the Participant FAQ page. This policy is to ensure that all participants are guaranteed equal access to information concerning FRVT 2002. Exceptions will only be allowed for extenuating circumstances. Two examples of the exceptions are delivery of login information for Participant Area or resolving major issues with similarity files. 
Status Update 26 November 2002

How to Participate

\section{Participants}

\section{Sponsors}

\section{FAQ}

\section{Calendar}

\section{Participant Area}

\section{Facial Recognition}

Vendor Test 2000

Face Recognition

Vendor Test 2002

\section{FERET}

Evaluation

Metbodology

\section{Home}

\section{FACE RECOGNITION VENDOR TEST 2002 \\ Participants}

Company: AcSys Biometrics Corp

Web Site: http://www.acsysbiometricscorp.com

Company: C-VIS Computer Vision und Automation GmbH Web Site: http://www.c-vis.com

Company: Cognitec Systems GmbH

Web Site: http://www.cognitec-systems.com

Company: Dream Mirh Co., Ltd

Web Site: http://www.dreammirh.com

Company: Electronic System Products Inc.

Web Site: http://www. espipd.com

Company: Eyematic Interfaces Inc.

Web Site: http://www. eyematic.com

Company: I conquest

Company: Identix

Web Site: http://www. identix.com

Company: Imagis Technologies Inc.

Web Site: http://www. imagistechnologies.com

Company: IRID Inc.

Web Site: http://iridinc.com

Company: Phoenix Systems Corporation

Web Site: http://www. itsphoenix.com

Company: VicarVision

Web Site: http://www. vicarvision.nl

Company: Viiasge Technology

Web Site: http://www. viisage.com

Company: VisionSphere Technologies Inc.

Web Site: http://www. visionspheretech.com 
Status Update

26 November 2002

How to Participate

\section{Participants}

\section{Sponsors}

\section{$F A Q$}

Calendar

\section{Participant Area}

\section{Facial Recognition Vendor Test 2000}

Face Recognition Vendor Test 2002

\section{FERET}

Evaluation

Methodology

\section{Home}

\section{FACE RECOGNITION VENDOR TEST 2002 \\ Sponsors}

Multiple federal agencies are sponsoring and supporting the FRVT 2002. Sponsoring agencies are those providing financial, physical, and manpower support for FRVT 2002. Supporting agencies are not providing direct sponsorship, but have given their support for FRVT 2002.

\section{Sponsors}

\section{Defense Advanced Research Projects Agency. DARPA is the central} research and development organization for the Department of Defense (DoD). It manages and directs selected basic and applied research and development projects for DoD, and pursues research and technology where risk and payoff are both very high and where success may provide dramatic advances for traditional military roles and missions and dual-use applications.

\section{U.S. Department of State. As the lead U.S. foreign affairs agency, the} Department of State helps to shape a freer, more secure, and more prosperous world through formulating, representing, and implementing the President's foreign policy.

Federal Bureau of Investigation. The Mission of the FBI is to uphold the law through the investigation of violations of federal criminal law; to protect the United States from foreign intelligence and terrorist activities; to provide leadership and law enforcement assistance to federal, state, local, and international agencies; and to perform these responsibilities in a manner that is responsive to the needs of the public and is faithful to the Constitution of the United States.

National Institute of Justice. NIJ is the research agency of the U.S.

Department of Justice. Created by the Omnibus Crime Control and Safe Streets Act of 1968, as amended, NIJ is authorized to support research, evaluation, and demonstration programs, development of technology, and both national and international information dissemination.

National Institute of Standards and Technology. NIST was established by Congress "to assist industry in the development of technology ... needed to improve product quality, to modernize manufacturing processes, to ensure product reliability ... and to facilitate rapid commercialization ... of products based on new scientific discoveries." An agency of the U.S. Department of Commerce's Technology Administration, NIST strengthens the U.S. economy and improves the quality of life by working with industry to develop and apply technology, measurements, and standards.

Transportation Security Administration. On November 19, 2001, the President signed into law the Aviation and Transportation Security Act, which among other things established a new Transportation Security Administration (TSA) within the Department of Transportation. TSA's 
mission is to protect the Nation's transportation systems to ensure freedom of movement for people and commerce.

\section{Supporters}

\section{Counterdrug Technology Assessment Center. The Counterdrug}

Technology Assessment Center (CTAC) was established within the Office of National Drug Control Policy as the central counterdrug technology research and development organization of the U.S. Government. In 1998, CTAC's role was expanded to support a program to transfer successfully developed counterdrug technologies directly to state and local law enforcement agencies. The Technology Transfer Program concentrates on providing state-of-the-art, affordable, easily integrated and maintainable tools to enhance the capabilities of state and local law enforcement agencies for counterdrug missions.

\section{Customs Service. The United States Customs Service is the primary} enforcement agency protecting the Nation's borders. It is the only border agency with an extensive air, land, and marine interdiction force and with an investigative component supported by its own intelligence branch.

\section{Department of Energy. The Department of Energy develops and} implements energy policy and manages a vast array of technical programs. The DOE complex includes unique capabilities in science and engineering that are applied to meet the Department's goals in Energy Resources, Nuclear National Security, Environmental Quality, and Science.

\section{Drug Enforcement Administration. The mission of the Drug Enforcement}

Administration (DEA) is to enforce the controlled substances laws and regulations of the United States and bring to the criminal and civil justice system of the United States, or any other competent jurisdiction, those organizations and principal members of organizations, involved in the growing, manufacture, or distribution of controlled substances appearing in or destined for illicit traffic in the United States; and to recommend and support non-enforcement programs aimed at reducing the availability of illicit controlled substances on the domestic and international markets.

Immigration and Naturalization Service. The INS is a Federal agency within the US Department of Justice (DOJ) that administers the nation's immigration laws. The INS conducts immigration inspections of travelers entering (or seeking entry) to the United States as they arrive at officially designated ports of entry, regulates permanent and temporary immigration to the United States, maintains control of U.S. borders, and identifies and removes people who have no lawful immigration status in the United States.

\section{Secret Service. The mission of the Secret Service is to protect the}

President, the Vice President, and their families, heads of state, and other designated individuals; the investigation of threats against these protectees; the protection of the White House, Vice President's Residence, Foreign Missions, and other buildings within Washington, D.C.; and security design, planning, and implementation at designated National Special Security Events. The Secret Service is also responsible for the enforcement of laws relating to counterfeiting of obligations and securities of the United States, investigation of financial crimes including, but not limited to access device fraud, financial institution fraud, identity theft, 
computer fraud, telecommunications fraud, and computer based attacks on our nation's financial, banking, and telecommunications infrastructure.

Technical Support Working Group. The Technical Support Working Group (TSWG) is the U.S. national forum that identifies, prioritizes, and coordinates interagency and international research and development (R\&D) requirements for combating terrorism. The TSWG rapidly develops technologies and equipment to meet the high-priority needs of the combating terrorism community, and addresses joint international operational requirements through cooperative $R \& D$ with major allies.

\section{Australian Customs Service. The Australian Customs Service is}

responsible for managing the integrity and security of Australia's borders. It facilitates legitimate movements of people and goods into and out of Australia, while detecting and deterring unlawful movements. Australian Customs works closely with other government and international agencies. It has a fleet of ocean-going patrol vessels and contracts two major aerial surveillance providers for civil maritime surveillance and response. Interception of illegal drugs is a high priority and sophisticated techniques are used to target high risk aircraft, vessels, cargo, postal items and travellers. This includes intelligence analysis, computer-based analysis, detector dogs and various other technologies. Customs vision is to be a world leader in Customs administration delivering high quality service to the community, industry and commerce.

Canadian Passport Office. The Passport Office of Canada, an Agency of the Department of Foreign Affairs and International Trade, is charged with the issuing, revoking, withholding, recovery and use of Canadian passports. It provides guidance to missions issuing passports abroad and supervises all matters relating to Canadian travel documents. The fundamental purpose of the Passport Office is to facilitate the travel of Canadians throughout the world by providing internationally respected travel documents and services. The Passport Office works in alliance with other government agencies to provide secure identification documents and services.

\section{United Kingdom Biometric Working Group. The UK Biometrics Working} Group (BWG) co-ordinates the Office of the e-Envoy (OeE) Biometrics Programme, the goal of which is to enable the use of biometric authentication technology to support the OeE e-government aims and to facilitate the adoption of biometrics in support of wider government business. The BWG's mission includes providing general advice on biometric technology and procurement, addressing privacy and Human Rights issues, directing technical areas such as evaluation and testing, and offering biometric policy advice and security assurance. 
Status Update

26 November 2002

How to Participate

\section{Participants}

\section{Sponsors}

\section{FAQ}

\section{Calendar}

\section{Participant Area}

\section{Facial Recognition}

Vendor Test 2000

Face Recognition

Vendor Test 2002

\section{FERET}

Evaluation

Methodology

\section{Home}

\section{FACE RECOGNITION VENDOR TEST 2002 \\ Calendar}

April 25 Announcement to Public

May 10 Deadline to Request Participation

July 10 - August 9 FRVT 2002 Testing

November FRVT 2002 Evaluation Report Released 


\section{Status Update \\ 26 November 2002}

How to Participate

\section{Participants}

\begin{tabular}{l} 
Sponsors \\
\hline$A A$
\end{tabular}

\section{Calendar}

\section{Participant Area}

\section{Facial Recognition}

Vendor Test 2000

Face Recognition

Vendor Test 2002

\section{FERET}

\section{Evaluation}

Methodology

\section{Home}

\section{FACE RECOGNITION VENDOR TEST 2002 \\ Participant Area}

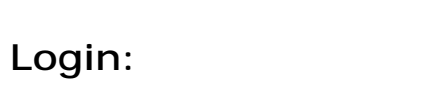

Password:

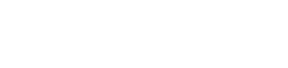

Change Password 


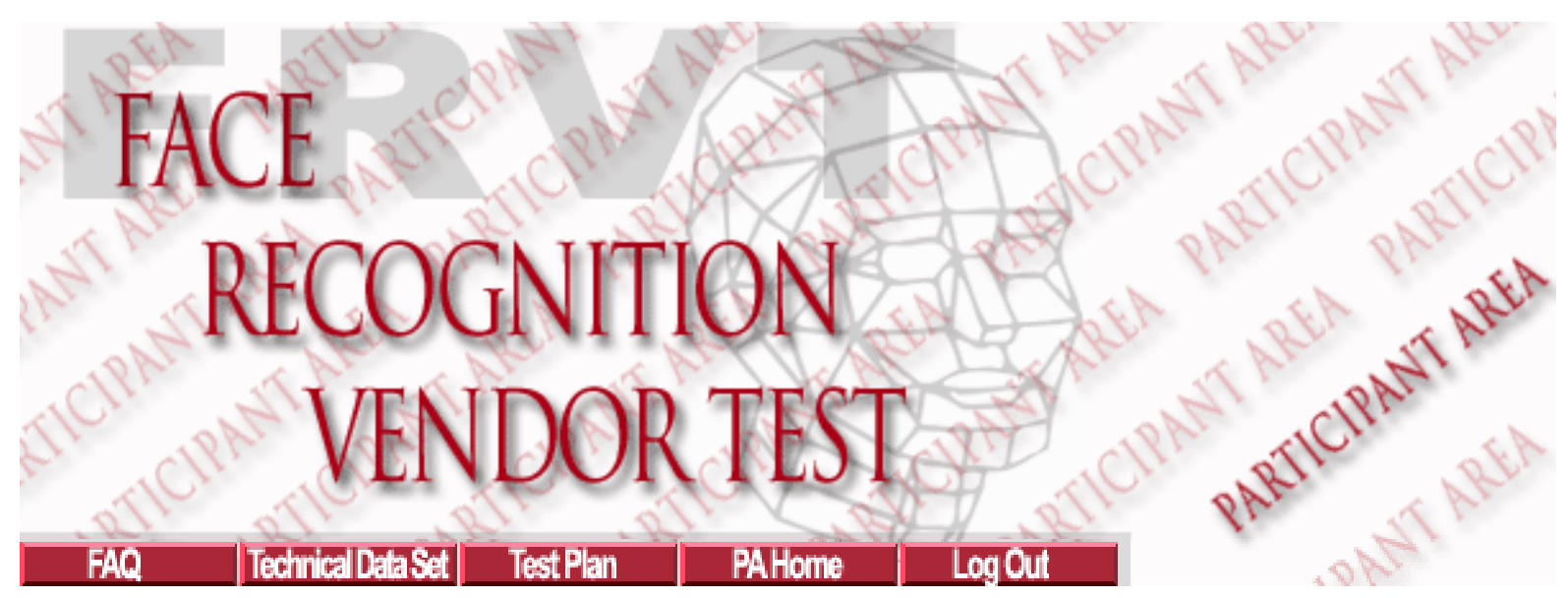

Welcome to the Participant Area of the Face Recognition Vendor Test 2002 (FRVT 2002). This area is for participants only and contains information that is not to be divulged to anyone (even others within participant's companies) besides those directly involved with the FRVT 2002.

\section{Technical Data Set Overview}

The Technical Data Set has been made available on the participant area of the FRVT 2002 webpage. Three documents are included in this set:

1. FRVT 2002 Implementer's Guide. Explains the format you will be receiving image data and the requirements for your output similarity data. This is the document that needs to be read to be able to take the FRVT 2002.

2. Computational Requirements for FRVT 2002 High Computational Intensity Test. An example of how to compute the time required for participant systems to complete the subtests. Participants taking the high computation intensity test need to read this and complete a short white paper described in this document. 3. HEF Guide. Sample image files, FR algorithm, and scoring code. This is a reference guide.

\section{Schedule}

\begin{tabular}{|l|l|}
\hline June 3 & Technical Data Set available on Participant Area \\
\hline June 14 & $\begin{array}{l}\text { Deadline for High Computational Intensity Test } \\
\text { Participants to submit their computational } \\
\text { requirement white paper. Details for this white can } \\
\text { be found in Computational Requirements for FRVT } \\
2002 \text { High Computational Intensity Test. }\end{array}$ \\
\hline June 14 & $\begin{array}{l}\text { Last day for Participants to withdraw from FRVT 2002 } \\
\text { without the withdrawal being mentioned in the FRVT } \\
\text { 2002 Evaluation Report }\end{array}$ \\
\hline June 14 & $\begin{array}{l}\text { Last day to reach agreement with FRVT Liaison for } \\
\text { non-standard hardware requests }\end{array}$ \\
\hline June 14 & $\begin{array}{l}\text { Names of participants announced on FRVT 2002 } \\
\text { website }\end{array}$ \\
\hline
\end{tabular}




\begin{tabular}{|c|c|}
\hline June 19 & $\begin{array}{l}\text { FRVT } 2002 \text { Organizers release test schedule to } \\
\text { participants }\end{array}$ \\
\hline J une 28 & $\begin{array}{l}4 \text { pm, EDT. Deadline for participants to provide FRVT } \\
2002 \text { Organizers sample similarity files based on the } \\
\text { Technical Data Set \& } 11 \text { June Supplemental. Note } \\
\text { that this is the final deadline. Participants are } \\
\text { encouraged to submit the sample similarity files as } \\
\text { early as possible. Providing these files early will } \\
\text { enable participants to fix any mistakes in their files. } \\
\text { Resubmissions of similarity files are encouraged prior } \\
\text { to the } 28 \text { June deadline, but may not be accepted } \\
\text { after this date. }\end{array}$ \\
\hline July 9 & FRVT 2002 testing begins at NSWC Dahlgren \\
\hline
\end{tabular}




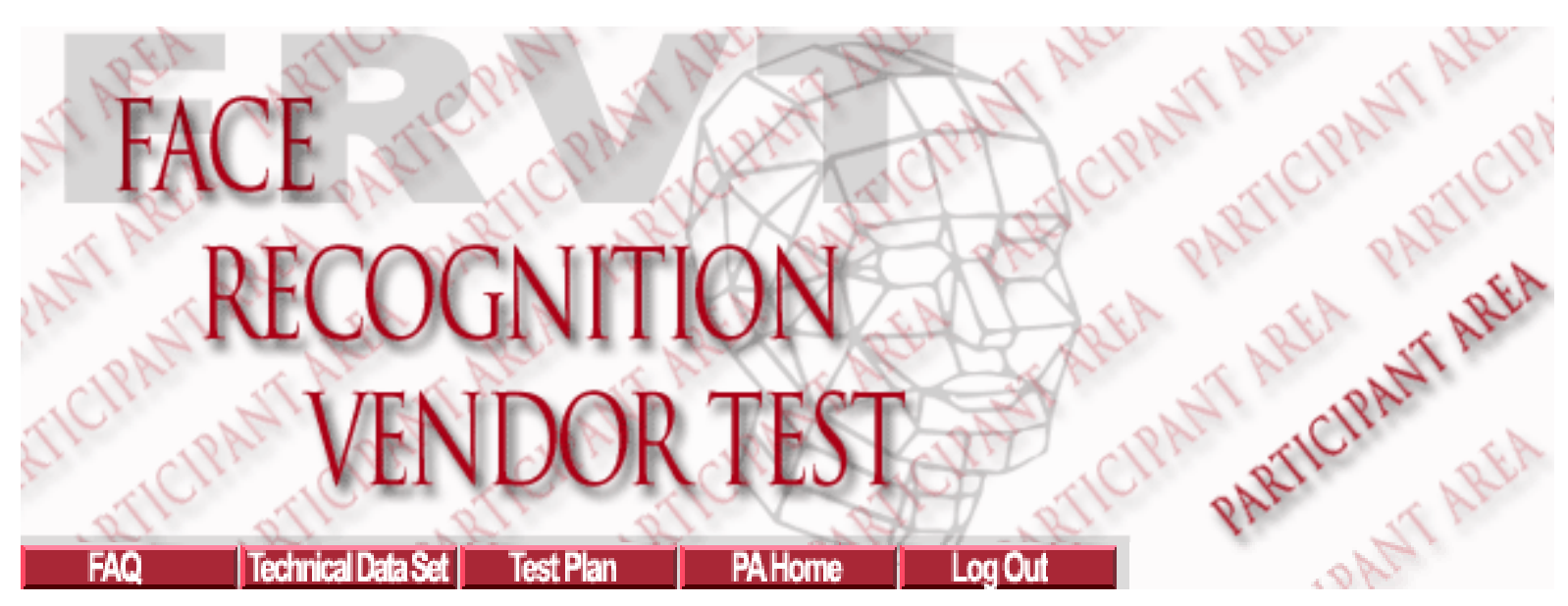

\section{Original Technical Data Set Release}

HEF-0.99.0.zip (Windows)

HEF-0.99.0.tar.gz (Unix)

Two files above are formatted for different operating systems but contain the same information.

\section{J une Supplemental}

In response to some participants concerns that the example Technical Datasets was misleading, we have assembled a limited set of files that are in exactly the same form as participants can expect to see during the tests.

The supplemental .zip (alternatively .tar) archives contain:

1. the $\mathrm{XML}$ files for the target and query sets.

2. the "images" directory containing FERET images in JPEG format. 3. the empty "output" directory where participants output files will be written.

Participants should be able to take SOLELY the contents of this .zip archive and produce valid binary similarity files. The image quality itself may or may not be representative of that included in the tests. This supplemental data, like the HEF Technical Datasets before it, is included ONLY to allow participants to see the input-output relationships.

Notes:

1. This supplemental data does not include video or still-set signature data. Participants should read the FRVT 2002 Implementer's Guide and code accordingly.

2. The code distributed with the Technical Datasets does not include a face detector; instead it uses prior manually located eye coordinates from a text file. This kind of information will NOT be made available to FRVT 2002 participants. The supplemental .zip archive is a real distribution that vendors should be able to 
process as is. It does not include face or eye localization information and therefore the PCA implementation that accompanies the original Technical Datasets will not run on it.

HEF-supplement.zip (Windows)

HEF-supplement.tar (Unix)

In summary, this zip/tar file contains:

1. 512 FERET images in JPG format ( 256 targ +256 query) in a directory "images"

2. An empty directory "output"

3. FERET_target. $x \mathrm{ml}$

4. FERET_query.xml

5. A short README.txt file

It does NOT contain:

1. Any nrm files

2. Any eye coordinates

3. Any code

\section{FRVT 2002 Scoring Suite for Participant Review}

FRVT2002-SS-PR1.tar.gz (Unix) 


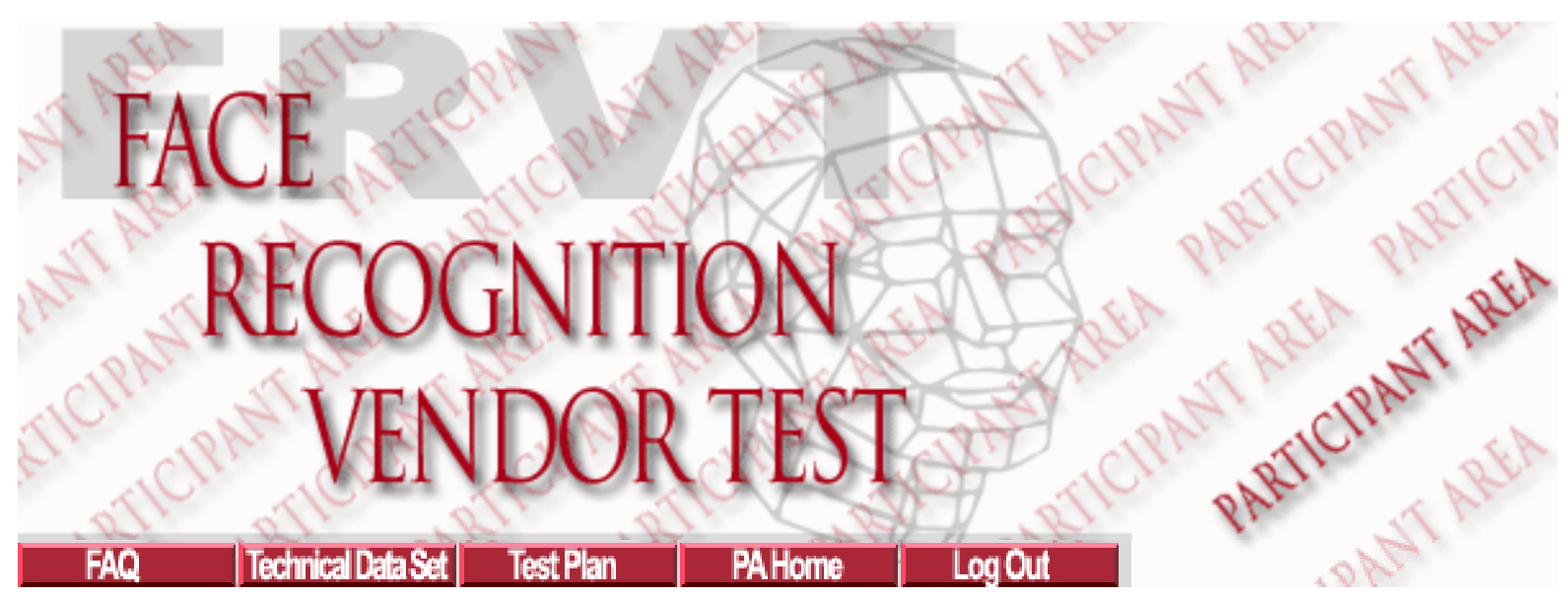

FRVT 2002 Test Plan

\section{FRVT 2002 Normalization Specifications}

\section{FRVT 2002 Visitor I nformation}

The FRVT 2002 Visitor Information document contains information regarding your upcoming visit to NSWC Dahlgren Division for the purpose of participating in the FRVT 2002. Notice that some personal information must be gathered for access to the base. This information will only be used for gaining access to the facilities. Deadlines for sending this information are very soon, so please review this attachment as soon as possible. 
Appendix D

Forms to Participate in FRVT 2002 
Status Update

26 November 2002

How to Participate

\section{Participants}

\section{Sponsors}

\section{$F A Q$}

\section{Calendar}

\section{Participant Area}

Facial Recognition

Vendor Test 2000

Face Recognition

Vendor Test 2002

\section{FERET}

Evaluation

\section{Metbodology}

\section{Home}

\section{FACIAL RECOGNITION VENDOR TEST 2002 \\ Formts}

\section{Application for Participating in Face Recognition Vendor Test 2002}

FRVT 2002 consists of four independent subtests. Each subtest will be performed separately and will have a maximum amount of time for completion. For each subtest, Participants will provide computer(s) and a face recognition system. Participants are required to complete each subtest in the allotted time. Time cannot be saved from one subtest for use in another subtest. Results will be provided on a subtest by subtest basis (scores for participants will only be provided for subtests they fully complete). The four FRVT 2002 subtests are:

Low Computational Intensity. This subtest consists of a relatively small number of images (approximately $1,000)$. It is designed to test slower systems and to determine if additional allotted time yields superior performance.

Medium Computational Intensity. This subtest is designed to allow for the measurement of performance under a variety of conditions. For example, images will be included with changes in illumination and subject pose. This subtest will require the processing of approximately 10,000 images.

High Computational Intensity. This subtest evaluates a systems ability to perform face recognition tasks using very large data sets. This subtest will require the processing of approximately 120,000 images.

Video. This subtest is designed to evaluate face recognition performance on short video sequences of faces.

Participants will be able to select their own computer equipment following these guidelines:

Low Computational Intensity. One desktop computer Medium Computational Intensity. One desktop computer High Computational Intensity. One stand-alone computer network consisting of up to three desktop computers. Video. One desktop computer

For commercially available systems, desktop computers and networked computers must only contain hardware and software that is commercially available. For mature prototypes, participants must provide a list of system components and identify those that are and are not commercially available. Component and cost information 
about the computer(s) each participant is using must be included in their system description document.

To maintain fairness, the Government will adhere to the following procedures for FRVT 2002. Information provided to any single Participant will also be provided to all Participants. The primary and preferred source of communications between Participants and FRVT 2002 government personnel is the frequently asked question (FAQ) page. A government employee has been named as the FRVT Liaison. The FRVT Liaison will be the primary point of contact and will manage communications with Participants. Questions that are outside the purview of the FAQ can be submitted to the FRVT Liaison via email at frvt2002@nist.gov. All e-mails sent to the FRVT Liaison and germane to the FRVT 2002 will be posted on the FAQ page. This policy ensures all Participants are guaranteed equal access to information concerning FRVT 2002. Contact with the FRVT Liaison other than via the FAQ areas, or contact with someone other than the non-FRVT Liaison will only be allowed for extenuating circumstances (for example, delivery of login information for Participant Area or major issues with similarity files).

\section{Evaluation Procedure}

\subsection{The FRVT 2002 technology evaluation commenced 25 April 2002.}

1.2 In order to request participation in the FRVT 2002, potential participants must electronically fill out this form (Application to Participate in FRVT 2002) and the image data set form (Application for Access to the FRVT 2002 Technical Datasets), sign the forms and send to the locations designated on each form. Upon receipt of both original signed forms by the Government, you will be classified as a "Tentative Participant". The Government must receive both forms by the due date described in the FRVT 2002 Calendar Area.

1.3 The Government will determine which Tentative Participants will be selected as FRVT 2002 Participants. It is the Government's desire to select all Tentative Participants as Participants. However, if demand for participation exceeds the Government's ability to properly evaluate the technology, the Government will be required to select Participants from the Tentative Participant list. If this occurs, the Government will instruct all Tentative Participants to provide the Government a white paper that will be used as the basis for selection as a FRVT 2002 Participant. The Government will provide Tentative Participants with the format and requirements for this white paper, if the need exists. A list of Tentative Participants, and later, Participants will be made available on the FRVT 2002 web site.

1.4 FRVT 2002 Technical Data Sets will be made available in the Participant Area of the FRVT 2002 web site (Application for Access to the FRVT 2002 Technical Datasets). Participants will be required to perform trials using this test set, at their own facilities, and provide similarity files to the Government. The Government will check these similarity files for compliance. A deadline for delivery of these similarity files will be provided on the web site. Participants who miss this deadline may not be evaluated. Should the similarity files not 
comply with standards, the Government will notify Participants and may allow re-submission of new similarity files to ensure compliance. Very important: The baseline PCA algorithm included in the FRVT 2002 Technical Datasets requires normalized (eye-coordinates given) images. I mages provided during FRVT 2002 will NOT be normalized. Systems evaluated will need to automatically process facial imagery without eye coordinates being provided.

1.5 Evaluation dates and locations will be provided at a later date. The Government will attempt to accommodate each Participant on the determination of dates to the maximum extent possible.

1.6 FRVT 2002 Participants will be given a large database of images on the day they arrive for actual testing. The images will be provided on a USB Hard Drive (IDE hard drive, formatted NTFS with one partition, in an external enclosure connected to host computer via USB port). Participants will be required to partition their hard drives into two areas, one for the OS and face recognition algorithms, and the other for working space. Participants will be allowed to copy these images onto their working space hard drive partition only. The participant perform a low-level format on the working space partition of their hard drive. The Government will assure that none of the images, or data derived from the images, are still resident on the test computer. Participants will allow the Government to inspect all disks on the system to verify compliance. This inspection will involve, at a minimum, the government deleting files generated during testing and wiping free space on all disk drives. At the completion of the test the Participants will transfer all the required output files from their system onto the original hard drive via USB. The drive will then be returned to the Government.

1.7 Participants will be required to submit a five-page (maximum) system description document, in electronic form, on the first day of testing. These documents will be included in the final FRVT 2002 report that will be released to the public. Failure to provide this document, in its proper form, may result in not being evaluated in FRVT 2002. This document must adequately address the following topics:

- Overview of the evaluated system.

- Component list for the evaluated system.

- Detailed cost breakdown of the submitted system (commercial vendors only).

- Detail any modifications required to take FRVT 2002.

1.8 Precise details of the FRVT 2002 Test Plan will be made available on the FRVT 2002 web site at a later date.

1.9 FRVT 2002 Participants will not be allowed to comment on their participation in FRVT 2002 until the FRVT 2002 Evaluation Report is released.

1.10 Testing activities will be recorded using video cameras for documentation of the evaluations. Footage from this documentation will not be made available to the public without review and comment from any participant that is named in the video. 


\section{Release of Evaluation Results}

Upon the completion of the evaluations, the Government will combine all results into a final report. The final report will contain, at a minimum, descriptive information concerning FRVT 2002, descriptions of each experiment, evaluation results, and each Participant's five-page system description document. A pre-release version of this report will be made available to Participants. Participants will be invited to provide comments which will be included as an appendix to the FRVT 2002 Evaluation Report. More specific guidance concerning the report and Participant comments will be provided at a later date. Participants are reminded that they will not comment publicly or privately on the pre-release version of the FRVT 2002 Report until it has been released to the public.

\section{Additional Information}

\subsection{Any data obtained during these evaluations, as well as any} documentation required by the Government from the participants, becomes the property of the Government. Participants will not possess a proprietary interest in the data and/or submitted documentation.

3.2 By signing and submitting the FRVT 2002 forms, Tentative Participants and Participants attest that they will not file any claim against FRVT 2002 Sponsors, Supporters, or other agency of the U.S. Government, or otherwise seek compensation for any equipment, materials, supplies, information, travel, labor and/or other participant provided services.

\subsection{The Government is not bound or obligated to follow any} recommendations of the Participant. The United States Government, or any individual agency, is not bound, nor is it obligated, in any way to give any special consideration to FRVT 2002 Participants on future contracts.

3.4 If a Participant decides to use results of these evaluations in any way for their own purpose, it must be accompanied by the following phrase: "Results shown from the Face Recognition Vendor Test 2002 do not constitute endorsement of any particular system by the Government." It must also be accompanied by an internet hyperlink to the final report on the FRVT 2002 web site.

3.5 With the signing of this form, Tentative Participants and Participants are authorizing their company/organization to participate in the Face Recognition Vendor Test 2002 according to the provisions in this form and later documents supplied by the Government.

3.6 With the signing of this form, Tentative Participants and Participants realize that they must send signed original copies of this form as well as "Application for Access to the FRVT 2002 Technical Datasets" to the locations documented on each form.

3.7 With the signing of this form, Tentative Participants and Participants realize that any test details and/or modifications that are provided in the Participant Area of the FRVT 2002 web site 
supersede the information on this form.

3.8 With the signing of this form, Tentative Participants and Participants realize that they can withdraw from the FRVT 2002 evaluations at any time up to the day prior to the start of their testing. Tentative Participant and Participant withdrawals will be documented in the FRVT 2002 Evaluation Report.

\section{Request to Participate}

4.1 Participant I nformation

\section{Company Name:}

Responsible Party:
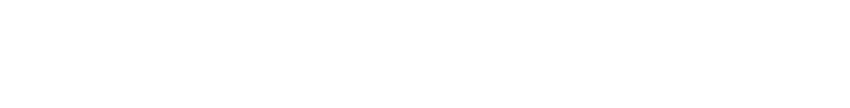

(Prefix/First Name -- Last Name/Suffix)

\section{Responsible Party Title: \\ Alternate/ Witness:}
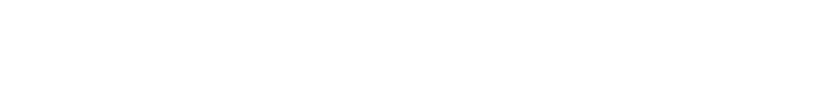

(Prefix/First Name -- Last Name/Suffix)

Mailing Address:
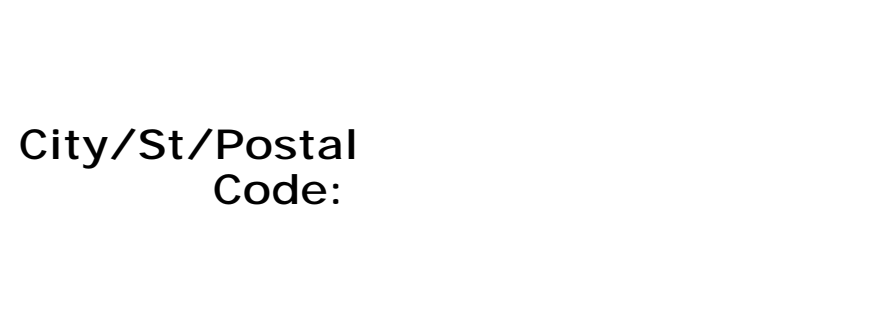

Phone Number:

Fax Number:
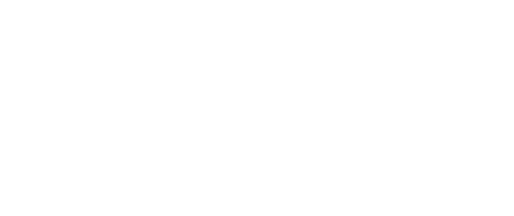

Email Address:

Web Site Address:

FRVT 2002 POC:
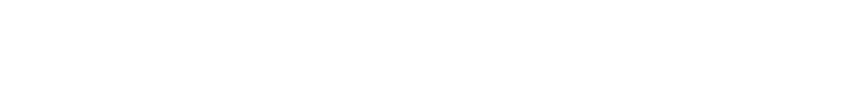

(Prefix/First Name -- Last Name/Suffix)

POC Phone:

POC Email:

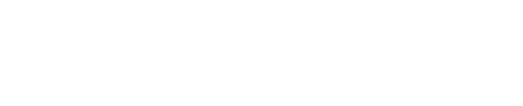




\section{Evaluation Groups \& Operating System}

Please check the Evaluton Group(s) you wish to participate in. Indicate your Operating System for each Evaluaton Group you select.

$\square$ Low Computational Intensity

Operating System: Select... Version: $\square$ Other OS:

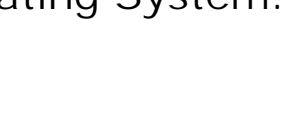

$\square$ Medium Computational Intensity

Operating System:

$$
\text { Select... }
$$

Version:

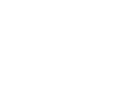

Other

OS:

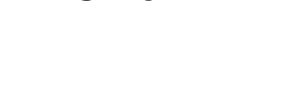

High Computational Intensity

Operating System:

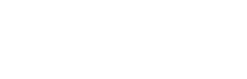

Version:

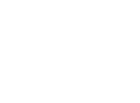

Other

OS:

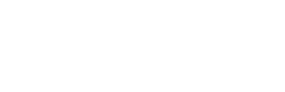

$\square$ Video

Operating System:

Select...

Version:

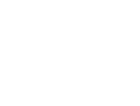

Other

OS:

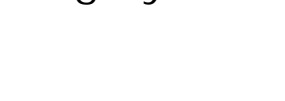

\section{Note: The application deadline of 10 May 2002 has past and we are no longer accepting applications for FRVT 2002.}

\subsection{Signatures}

With my signature, I hereby request consideration as a Tentative Participant and Participant in the Face Recognition Vendor Test 2002 according to the rules and limitations listed on this form and the Participant Area of the FRVT 2002 web site.

4.3 Application Fulfillment

To Complete the application process:

1. Print this page(s) from your browser

2. Obtain the appropriate signatures

3. Mail the completed form to:

FRVT 2002 Liaison

Code B06

1470 Room 1101

17320 Dahlgren Road

Dahlgren, VA 22448 


\section{USA}

You will receive a confirmation note that has your ID and password for access to the participant area of the web site upon receipt of both required forms. 
Status Update 26 November 2002

How to Participate

\section{Participants}

Sponsors

$F A Q$

Calendar

Participant Area

Facial Recognition

Vendor Test 2000

Face Recognition Vendor Test 2002

\section{FERET}

\section{Evaluation} Metbodology

Home

\section{FACIAL RECOGNITION VENDOR TEST 2002 \\ Formis}

\section{Application for Access to the FRVT 2002 Technical Datasets}

The FRVT 2002 Technical Data Sets consist of

- FRVT 2002 evaluation data sets.

- FRVT 2002 training set.

- Software provided with the FRVT 2002 training set.

FRVT 2002 evaluation data sets are used in the evaluation and will be made available at the time a system is evaluated. FRVT 2002 Training Data and Supporting Software are supplied to Participants to assist in preparing for the FRVT 2002. These two components will be made available to Participants in the FRVT 2002 Participant Area. To receive access to the FRVT 2002 Technical Data Sets, participants must sign the agreement at the bottom of this page. Failure to observe the restrictions in this document will result in access being denied for future releases of the image database and removal from FRVT 2002. Offenders may also be subject to civil damages.

\section{Restrictions for the FRVT 2002 Training Data and Supporting Software}

The FRVT 2002 training data and supporting software includes:

- A subset of normalized images from the FERET database

- A baseline PCA face recognition algorithm

- Scoring code to generate receiver operator characteristics (ROC) and cumulative match characteristics (CMC)

- Instructions on how to use the software tools

- Documentation on similarity file output requirements for your algorithms

- Instructions for submission of sample similarity files for Government review

Very important: The baseline PCA algorithm included in the FRVT 2002 Technical Datasets requires normalized (eye-coordinates given) images. I mages provided during FRVT 2002 NOT be normalized. Systems evaluated will need to automatically process facial imagery without eye coordinates being provided.

The Participant agrees to the following restrictions on the FRVT 2002 Training data and supporting software:

1. The FRVT 2002 Technical Datasets will not be further distributed, published, copied, or further disseminated in any way or form whatsoever, whether for profit or not. This includes further distributing, copying or disseminating to a different facility or organizational unit in the requesting university, organization, or company.

2. All requests for copies of the FRVT 2002 Technical Datasets will be forwarded to the Agent at the National Institute of Standards and Technology (NIST). 
3. All publication or other release of any part of the Technical Datasets, or data derived from using the Technical Datasets, in reports, papers or other documents must first be approved in writing. Approval may be sought by writing from the NIST technical agent.

\section{Restrictions for the FRVT 2002 Evaluation Data Sets}

The Participant agrees to the following restrictions on the FRVT 2002 Technology Evaluation Test Set:

1. The database will not be used for any purpose except for participation in FRVT 2002 Technology Evaluations.

2. The database will only be copied to the Participant's system in the working space partition of their hard drive. Participants will perform a low-level format of this partition at completion of their testing.

3. If required, a separate laptop may be used to facilitate transfer to/from USB hard drive and face recognition system.

4. All data generated from this database during FRVT 2002 will deleted from all Participant systems by the Government at the conclusion of the evaluation.

\section{Request for Access to Databases}

3.1 Participant Information

\section{Company Name:}

Responsible Party:
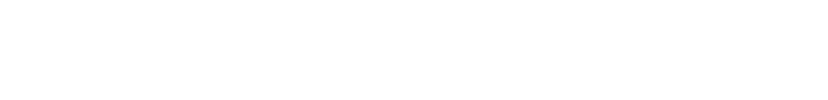

(Prefix/First Name -- Last Name/Suffix)

Responsible Party Title:

Alternate/ Witness:

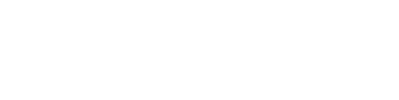

(Prefix/First Name -- Last Name/Suffix)

Mailing Address:

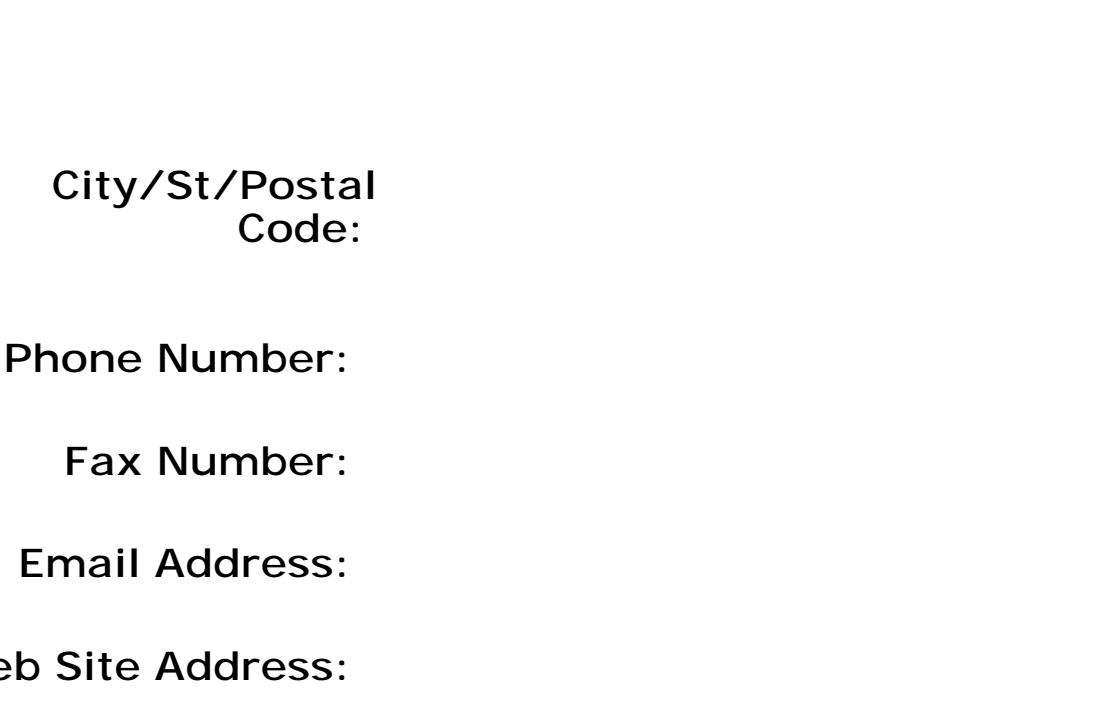




\section{$* * * *$ Note: The application deadline of 10 May 2002 has past and we are no longer accepting applications for FRVT 2002.}

\subsection{Signatures}

With my signature, I authorize my company/organization to use the FRVT 2002 Technical Datasets in association with the Facial Recognition Vendor Test 2002, and promise to do so according to the rules and limitations listed on this form.

3.3 Application Fulfillment

To Complete the application process:

1. Print this page(s) from your browser

2. Obtain the appropriate signatures

3. Mail the completed form to:

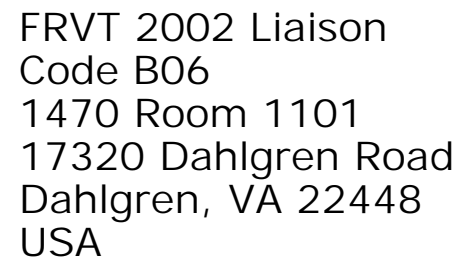

You will receive a confirmation note that has your ID and password for access to the Participant Area of the web site upon receipt of both required forms. 


\section{This page intentionally blank.}


Appendix E

Frequently Asked Questions 
Status Update

26 November 2002

How to Participate

\section{Participants}

\section{Sponsors}

\section{$F A Q$}

Calendar

\section{Participant Area}

\section{Facial Recognition} Vendor Test 2000

\section{Face Recognition} Vendor Test 2002

\section{FERET}

\section{Evaluation}

Methodology

\section{Home}

\section{FACE RECOGNITION VENDOR TEST \\ $F A Q$}

\section{Submit an unanswered question}

1. On the FRVT 2002 Application to Participate Form, what is the difference between 'Responsible Party', 'Alternate/Witness', and 'FRVT 2002 POC'?

2. For the low/medium/high computational intensity tests, can we use a desktop computer with multiple-processors and count that as one desktop computer?

3. For the Video Subtest, what is the video data format (framerate, file format)?

4. Is there a fee to participate in FRVT 2002?

5. Is there a penalty or fine for withdrawing from FRVT 2002?

6. For the first three subtests (low, medium and high computational subtests), can participants use the exact same code that was used for the Recognition Performance Test of FRVT2000?

7. The calendar lists June 21st as the date testing begins. Does that mean systems and software must be ready for all four subtests by that date? Or, will each vendor perform the first subtest in turn, then the second, and so forth?

8. For the video subtest, how many computers can be used? Can co-processing devices be used in addition to the $\mathrm{PC}(\mathrm{s})$ ?

9. For the video subtest, do the video images have to be analyzed in real-time, or can we take as long as we like to analyze each frame?

10. For the video subtest, how many seconds of video are available in each video sequence?

11. For the video subtest, what is the total number of images?

12. For the video subtest, can the video watch list be hand-aligned (human processed)?

13. For the low, medium, and high computational subtests, are the numbers provided ( $1 \mathrm{~K}, 10 \mathrm{~K}$ and $120 \mathrm{~K})$ the number of images that have to be processed? Or are these numbers the total number of comparisons to be made?

14. For each of the subtests, how many images per person does the gallery include?

15. What metrics will be used to define performance for the 
static and video subtests?

16. Will infrared systems be evaluated in FRVT 2002?

17. What is the purpose and requirements for the white paper?

18. Can non-US companies participate in FRVT 2002?

19. What are the time periods allowed for each test?

20. Why don't you 'level the playing field' by making all Participants perform on the same hardware configuration?

21. Is it possible to submit more than one facial recognition system for each sub test?

22. Will the actual time a participant uses for a subtest appear in the evaluation report?

23. Is it possible for the public to witness any of the face recognition testing that is taking place?

24. Could a detailed definition of the face recognition tests, possibly what is contained in the Test Plan, be sent to me?

25. How will the public be notified that the test results from FRVT 2002 are available?

26. Do you know the exact date the FRVT 2002 results will be published online?

27. Will the data released publicly from FRVT 2002 be selective due to homeland security concerns?

28. Would it be possible to obtain, purchase, or rent the FRVT 2002 dataset(s)?

1. On the FRVT 2002 Application to Participate Form, what is the difference between 'Responsible Party', 'Alternate/ Witness', and 'FRVT 2002 POC'?

Responsible Party: An individual at a potential participant's company that has the authority to request participation in FRVT 2002 on behalf of that company.

Alternate/Witness: An individual who is witnessing the company application and signature of the responsible party.

FRVT 2002 POC (Point of Contact): This individual will be the lead person on-site during the evaluations and will be the company's designated individual for contact with the FRVT Evaluation Team for all aspects of the evaluation.

2. For the low/ medium/ high computational intensity tests, can we use a desktop computer with multiple-processors and count that as one desktop 


\section{computer?}

For FRVT 2002, a "single desktop computer" is defined to be a computer with case dimensions and power requirements similar to commercially-available computers for an individual's use. Multiple processors are allowed inside that case and will be counted as a single desktop computer. Other configurations approximating a single desktop may be allowed on a case-by-case basis, but must be coordinated with the FRVT 2002 Liaison prior to participation. A detailed component list, complete with pricing information, must be included in the participant's write-up that explains the system the participant used for FRVT 2002.

\section{For the Video Subtest, what is the video data format (framerate, file format)?}

The video will be supplied as a sequence of separate standard JPEG files. The frame rate of the camera was 29.97 frames per second. The frames were obtained by transcoding from the original DV format.

\section{Is there a fee to participate in FRVT 2002?}

There is no participation fee for FRVT 2002.

Participants will be required to provide hardware and software necessary for evaluating their system, as well as on-site representation during the evaluation.

\section{Is there a penalty or fine for withdrawing from FRVT 2002?}

Tentative Participants and Participants can withdraw from the FRVT 2002 evaluations at any time up to the day prior to the start of their testing. Tentative Participant and Participant withdrawals will be documented in the FRVT 2002 Evaluation Report. There are no other penalties or fines.

\section{For the first three subtests (low, medium and high computational subtests), can participants use the exact same code that was used for the Recognition Performance Test of FRVT2000?}

No. The required input and output files have different formats than those used in FRVT 2000. In the FRVT 2002, the input and output files will be in specified XML formats. The formats will be documented when the "Technical Datasets" are released to participating vendors. That distribution will include working code that demonstrates the use of XML. 
7. The calendar lists J une 21 st as the date testing begins. Does that mean systems and software must be ready for all four subtests by that date? Or, will each vendor perform the first subtest in turn, then the second, and so forth?

A test schedule will be developed and published at the conclusion of the application process. It is our intention to have participants perform different subtests simultaneously on different machines. It may be possible, or desirable for some vendors to perform some subtests sequentially. For example, low and medium tests on the same machine.

8. For the video subtest, how many computers can be used? Can co-processing devices be used in addition to the PC(s)?

A single desktop computer is allowed for the video subtest. For FRVT 2002, a "single desktop computer" is defined to be a computer with case dimensions and power requirements similar to commercially-available computers for an individual's use. Multiple processors are allowed inside that case and will be counted as a single desktop computer. Other configurations approximating a single desktop may be allowed on a case-by-case basis, but must be coordinated with the FRVT 2002 Liaison prior to participation. A detailed component list, complete with pricing information, must be included in the participant's write-up that explains the system the participant used for FRVT 2002.

9. For the video subtest, do the video images have to be analyzed in real-time, or can we take as long as we like to analyze each frame?

Individual video sequences may be analyzed over an extended period of time. Real-time processing is not required as long as the video subtest is completed in the allocated time. This subtest is intended to determine if superior performance can be obtained from video sequences rather than still images.

10. For the video subtest, how many seconds of video are available in each video sequence?

The video sequences have a mean duration of approximately 3 seconds. They were obtained from cameras operating at approximately 30 frames per second. 
The precise numbers have not yet been determined. There will likely be fewer than two thousand sequences each being comprised of approximately 90 frames. Note that because subjects' faces are visible in all frames a vendor may use just one frame from a sequence in order to attempt recognition.

\section{For the video subtest, can the video watch list be hand-aligned (human processed)?}

No. All systems for all sub-tests must be fully automated. All image enrollments and subsequent recognition computations for all the FRVT 2002 tests must be performed automatically, without human inspection or intervention.

13. For the low, medium, and high computational subtests, are the numbers provided (1K, 10K and 120K) the number of images that have to be processed? Or are these numbers the total number of comparisons to be made?

There will be approximately $1 \mathrm{~K}, 10 \mathrm{~K}$, and $120 \mathrm{~K}$ images that need to be processed. This implies that there could be approximately $1 \mathrm{M}, 100 \mathrm{M}$ and 14.4 Billion required comparisons between images. However, a subtest may be designed so that fewer comparisons are required. A complete discussion of the FRVT 2002 computational requirements will be included with the release of the Technical Datasets.

\section{For each of the subtests, how many images per person does the gallery include? \\ The image sets contain between 1 and 20 images per person. The test is structured such that the identities of the subjects are not given to participants.}

\section{What metrics will be used to define performance for the static and video subtests?}

The FRVT 2002 report will give a richer set of performance numbers than those quoted in FRVT 2000. The FRVT 2002 will report figures appropriate to assessing performance on tasks that include identification, verification, and watch-list operations. In addition, the effect of gallery size on performance will be included. Other analyses may be performed and reported. These include measures of statistical significance and estimates of variance of performance statistics.

\section{Will infrared systems be evaluated in FRVT 2002?}

Infrared imagery will not be included in the FRVT 
17. What is the purpose and requirements for the white paper?

If demand for participation in FRVT 2002 exceeds the ability of the Government to properly test all participant face recognition systems, the Government may ask potential participants to submit a technical paper describing their system and its capabilities. The Government will review all submitted 'white papers' and use them as a basis for selecting final participants in FRVT 2002. Format and requirements for white papers will be provided should the need arise.

\section{Can non-US companies participate in FRVT 2002?} Yes.

\section{What are the time periods allowed for each test?}

We have established the following tentative time limits:
Low computational: 1 Week
Medium Computational: 1 Week
High Computational: 2 Weeks
Video: 1 Week

\section{Why don't you 'level the playing field' by making all Participants perform on the same hardware configuration?}

FRVT 2002's objective is to "...provide independent government evaluations of commercially available and mature prototype face recognition systems." Vendor/Participants are generally free to have their systems (software and hardware) evaluated as they are sold commercially. This may include non-desktop based systems on a case-by-case basis as some vendors sell systems with specific hardware/software integrations. FRVT 2002 will publish information concerning each Participant's system in its final report to support FRVT 2002 Sponsor requirements. This policy is in the best interests of both participants and FRVT 2002 Sponsors.

\section{Is it possible to submit more than one facial recognition system for each sub test?}

Multiple face recognition systems from one participant may be allowed on a case-by-case basis, but must be coordinated/approved with the FRVT 2002 Liaison. The deadline for approval is 20 May 2002. 
22. Will the actual time a participant uses for a subtest appear in the evaluation report?

No. The report will only document if the participant finished the subtest or not.

23. Is it possible for the public to witness any of the face recognition testing that is taking place?

No. To ensure fairness for all participants, and thus a fair evaluation, no public visits to the test facility are permitted.

24. Could a detailed definition of the face recognition tests, possibly what is contained in the Test Plan, be sent to me?

No. A thorough description of the test procedures will be provided in the FRVT 2002 Evaluation Report.

25. How will the public be notified that the test results from FRVT 2002 are available?

Results will be made available on

http://www.frvt.org. A notification message will also be sent to the Biometrics Consortium listserv.

26. Do you know the exact date the FRVT 2002 results will be published online?

It is not possible to forecast a specific date at this time.

27. Will the data released publicly from FRVT 2002 be selective due to homeland security concerns?

FRVT 2002 is a technology evaluation, so it is not application-specific. All results will be published in the FRVT 2002 Evaluation Report

28. Would it be possible to obtain, purchase, or rent the FRVT 2002 dataset(s)?

The images contained in the FRVT 2002 datasets are for use in the FRVT 2002 evaluation and are not currently available for other purposes. At a later date, some of the images in the MCInt may be released. Images in the $\mathrm{HCl}$ nt will not be released. If you desire face image data for your own development or baseline evaluation efforts, the entire FERET database is currently available. Please see 
http://www. nist.gov/humanid/feret. 


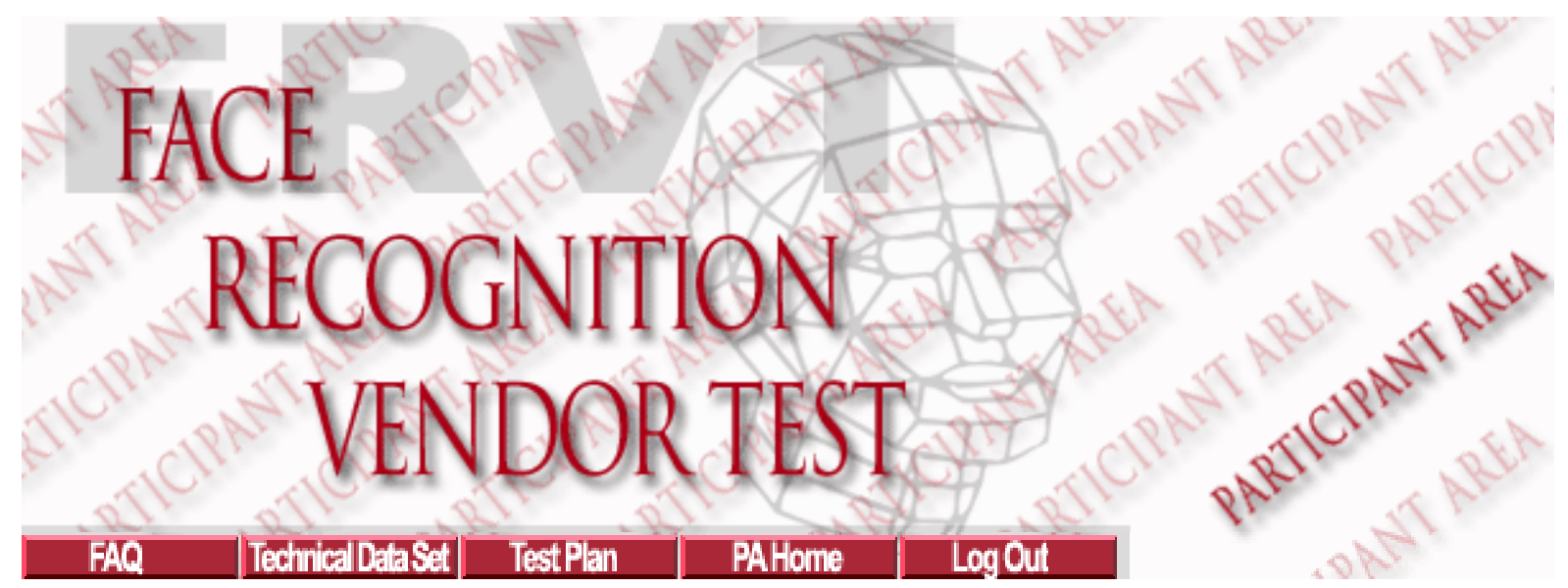

\section{Submit an unanswered question}

1. At what location will the tests be held?

2. What is the reason for not using a video file format for the video subtest?

3. What will be the range of inter-eye distances, in terms of pixels, for each subtest?

4. Will there be experiments within subtests? If so, what will they be?

5. Do any of the tests involve scenes that are cluttered, busy, or contain moving background objects?

6. Is the score format similar to FRVT2000? How about the Large DB Test for 120,000 images?

7. Will the USB protocol (used to transfer data to and from vendor machines) be 1 or 2?

8. IS JPEG only image format used for FRVT 2002? What is the percentage of color and grayscale images for each test?

9. What kind of USB drive will be used? Let us know exact specification so that we can buy one and test it.

10. Will all participants be required to arrive on July 9 th, or will some participants be scheduled to arrive later to stagger the testing?

11. Are we required to send a white paper based on "Computational Requirements for FRVT 2002 High Computational Intensity Test" from the technical dataset if we are not participating in the High Computational Intensity Test?

12. The FRVT test begins with the organizers providing a dataset to the participants. Will there be any other forms that need to be signed to receive this information?

13. Where do Participants send the sample similarity files based on the sample images in the Technical Dataset?

14. Is there a typo on page 5 of "The FRVT I mplementer's Guide"? We read "4N + 24 bytes". Shouldn't this be "4N +28 bytes"?

15. Are the variations (lighting, facial expression, etc) in the Technical Data Set representative of the type of variations that the vendor software will be tested on?

16. For the Medium Computational Intensity Subtests, will the query signature_set contain still_set or video type images?

17. Your e-mail message dated J une 6 announces the test reorganization and the fact that the new Medium test will contain 7500 
signatures. Where are we supposed to get this new data/subset for the Medium test?

18. What can we expect for backgrounds in the images in FRVT 2002? (For example, complex outdoor B/D, Etc.)

19. Regarding "The FRVT 2002 I mplementer's Guide": (1) It is not clear which $\mathrm{xml}$ is the target and which one is the query. (2) Also, the actual content of the $\mathrm{xml}$ files is confusing. The <file / > entries (ex.: <file name="00003fa010_940307.nrm"/>) point to inexistant files of unknown format (p. 3 of "The FRVT 2002 Implementer's Guide" states that all pictures in the FRVT2002 are JPEG).

20. What is the exact number of images for each subtest? (We need exact number in order to calculate processing time for white paper.)

21. The Document "The FRVT2002 I mplementer's Guide" lists 3 kinds of signatures to be used in the test (Fig 2.1). How does each signature relate to each subtest?

22. Signature \#2 ("The FRVT2002 I mplementer's Guide" figure 2.1) suggests a stereo camera system. Will stereo data be used in any subtest?

23. Why are your HEF_TD library just suitable for debug version?

24. Do we need to enroll total of $\mathrm{N}$ images for query and target set in each test?

25. Is it possible for a participant to start the test run and then leave the testing? FRVT Organizers would need to copy results to disk and then ship the computer back.

26. Will FRVT Organizers be providing UPS for participants use during testing?

27. We downloaded everything for generating the sample similarity files. In one of your documents it says that we need to produce some XML documents from the results. However, from the stuff we downloaded it seems as if the result files are just binary files. Did we misunderstand something? Do we have to deal with XML files at all?

28. Is the query and target set be the exactly same or different?

29. Why have you provided the technical dataset in a format and structure different from the procedural requirements of the test? Why not provide a technical dataset that is identical to what we will receive? Am I really supposed to convert all of the PGM format images to JPG and modify the target and query XML files accordingly? This seems to be required if I am to create a similarity file based on the target and query XML documents provided (FERET-256-1.sigs.xml, FERET-256-2.sigs.xml)

30. Am I really supposed to convert all of the PGM format images to JPG and modify the target and query XML files accordingly? This seems to be required if I am to create a similarity file based on the target and query XML documents provided (FERET256-1.sigs. xml, FERET-256-2.sigs. xml)

31. Can FRVT 2002 Organizers provide monitors for participants to use during the evaluations.

32. What is the minimum and the maximum relative size of a face in the image? Can the image be of factor 1000 wider than the eye distance, for example?

33. Do all images show one face only, or do you have images with several faces, e.g. in the video test?

34. What will be the total size of images (in GB) for 121,000 images?

35. How does a participant withdraw from participating in the FRVT 2002?

36. Will we need to modify the target.xml and query.xml files to get the schema resources from a local drive during FRVT 2002 tests?

37. Please elaborate on how the FRVT 2002 will evaluate 3D, infrared, or other novel face recognition systems.

38. Is the test site available 24 hours a day during the test period including weekends?

39. I am still having trouble understanding the use of XML in FRVT 2002. Can you help me?

40. How many target set and query set will be given for each subtest? 
41. Is it necessary that a participant's POC be on site, or could it be a different employee of the participant or some other person engaged by the participant?

42. Each output file consists of similarity data. Must this data be sorted in a specific format?

43. Can you supply some sample data in color, or indicate where a database of color face image data which is representative of test data may be obtained?

44. What is the minimum and maximum for the width and height (in pixels) of the imagery in each test?

45. What is the scaling for similarity and distance values $(0-10,0-100 \ldots)$ ?

46. Medium Computational Intensity Test will be administered with two targets and query sets - one for still images, one for video images. Are you going to provide us with a disk containing tree structure (Chapter 3 of I mplementers guide) for each set?

47. Will the subtests be run sequentially or in parallel? Can we use the same machine for both subtests?

48. You have said that the query \& target set of HCInT are identical. Will FRVT 2002 organizers provide two XML files or only one XML file?

49. Are we allowed to bring other people, such as a key technician or software engineer, in addition to the Technical Point of Contact during the 9 to 5 period when we have access to the system?

50. FAQ \# 3 states that the number of pixels between the eyes will be $30-500$ pixels. How does this relate to FAQ \# 44 ?

51. The technical dataset contains 3 video signatures named "video 00-02". We do not find any video sigs in the FRVT supplemental directory, so we assume that we do not have to process any video sigs for the sample similarity files we will submit for the FRVT evaluation procedure. Is this correct?

52. If the test program crashes, may our representative or representatives restart the program?

53. Can we place phone calls from the test facility to our offices? Are cell phones allowed? Are laptop PC's allowed?

54. Will the test area in Dahlgren have 20-amp AC outlets?

55. Do we need to get clearance from NSWC Dahlgren in advance for anyone that we bring, both the Technical Point of Contact and others? Are there special issues, especially long delays, for clearance for non-US citizens?

56. Can you provide an example of the 5-page document we are required to submit?

57. Are face locations nearly center in the image or could the face be located on a side?

58. What is the approximate breakdown of signatures in the Medium Computational Intensity Test?

59. How many still images are in a still set signature?

60. Is any remote access to the system such as TELNET or FTP available? Can we monitor the test remotely, e.g. over phone lines or the Internet? If the test program crashes, may we restart another debugged version of the test program?

61. Participants have been given 264 hours for testing. A participant finishes early, will they be permitted to leave early?

62. In the event that the voice-only telephone is not available and cell phone calls cannot be placed from within the building due to the metal covering mentioned in the FRVT 2002 Test Plan or other reasons, may participants leave the building to place cell phone calls from outside for technical support or other reasons?

63. May participants monitor the system at any time during the hour, or is it a specified time such as the start of the hour (9:00, 10:00, 11:00,...)? How much time do we have for the hourly monitoring, such as a maximum of five minutes?

64. May participants examine the contents of files generated by the test program such as similarity files or other intermediate status files written by the application?

65. May participants view a console output from the program, messages such as "processing probe $64,123 \ldots .$. ?

66. What is the extension for the similarity file? .SIM? .BIN?

67. Will you supply the power and data cables for the IDE drives in USB2.0 enclosures containing your image database? 
68. We will bring only Linux based systems for the test. How shall we do the virus scan?

69. How often does power go out at NSWC Dahlgren?

70. What are the deadlines for submitting a normalization function?

71. Would a windows based library be acceptable as well for the normalization function?

72. What is the number of signatures and images for the tests in FRVT 2002?

73. Are each signatures type and modality name in XML file? What about directory structure?

74. After completing the medium computational intensity test, may we add that computer to the system that is running the high computational intensity test?

75. We are planning to bring more than two people for FRVT2002 because of our functional role and contractual schedule. If you are very strict about the number of people (only two people will be allowed at the test site) for the testing, we want to be able to switch people during the test still maintain two people at the test site (including POC).

76. What is the directory structure and naming format for files we generate in the $\mathrm{MCI}$ nt?

77. In the Outline and Overview for Use of Normalization Functions in FRVT 2002 we are told that a possible normalization can be based, in part, on similarity scores for all pairs of gallery images in G. Does this imply that G consists of a subset of the target set which is also represented as probe sets in the query set Q? Will there be a way to identify these subsets other than by brute force searches of the XML target and probe set definitions?

78. For the High Computational Intensity test, are we allowed to copy the FRVT data (pictures, $\mathrm{xml}, \ldots$ ) on the three computers? At the end, are we allowed to copy back to the USB drive from each of the three computers or do we need to centralize all similarity files on one computer before copying back?

Participant Area FAQ closed during evaluation period from 2 July through 13 August.

79. When is the normalization function due?

80. There seem to be time limits imposed on the normalization functions. And if a F2 normalization is taking too long, the testers will revert to using the F1 normalization function. How much time is allotted per gallery siz?. Meaning, for a gallery size of $X$ how long should F2 or the F1 function take before it is deemed too long?

81. There are several math functions that our normalization function uses such as sqrt and exp. Is it okay to expect that whatever application is linking in our .o file will also link in the standard c/c++ code for these functions (either libm.a or libstdc++. so seems to do it fine on our machines here)?

82. Will the normalization functions be used on the high computational intensity test as well as the medium computational intensity test? If so, will there be a way for the functions being called to know in advance whether the scores being passed in are coming from the high versus the medium computational intensity test?

83. What is the minimum size of the gallery being passed to the normalization functions?

84. In FRVT 2002 Normalization Specifications: What is the meaning of "identification, verification, and watch list tasks"?

85. Is it planned to send the participants a pre-release of the FRVT 2002 Evaluation Report, allowing them to comment on it (as was done for the FRVT 2000 Evaluation Report)? 


\section{At what location will the tests be held?}

FRVT 2002 will be administered at NSWC Dahlgren in Dahlgren, Va. Please see http://www.nswc.navy.mil/visitor info.html for general visitor information. Specific visitor procedures and requirements will be given at a later date.

\section{What is the reason for not using a video file format for the video subtest?}

There are a myriad of different methods/file formats to share video data. Video data, however, is a sequence of still images. Since the rest of the subtests use JPG, the evaluation team decided to also use a sequence of JPG images for the video test. Also, this evaluation addresses face recognition, not video signal handling.

\section{What will be the range of inter-eye distances, in terms of pixels, for each subtest?}

The distance between the eyes in all tests will be at least 30 pixels and not more than 500 .

\section{Will there be experiments within subtests? If so, what will they be?}

As in FRVT 2000 and the FERET evaluations, virtual galleries and probe sets will be constructed from the target and query sets. See "The FERET Evaluation Methodology for Face-recognition Algorithms" for a detailed description of these sets. The virtual gallery and probe sets will be designed to analyze the effects of non-frontal images, time delay between photographs, illumination changes, outdoor lighting and other factors.

5. Do any of the tests involve scenes that are cluttered, busy, or contain moving background objects?

No.

6. Is the score format similar to FRVT2000? How about the Large DB Test for 120,000 images?

This issue is addressed in the Technical Datasets.

7. Will the USB protocol (used to transfer data to and from vendor machines) be 1 or 2 ? 
The USB model supports both USB 1 and USB 2. We recommend using USB 2 as it is much faster.

8. Is J PEG only image format used for FRVT 2002? What is the percentage of color and grayscale images for each test?

JPEG is the only image format used for FRVT 2002. The vast majority of images will be color.

9. What kind of USB drive will be used? Let us know exact specification so that we can buy one and test it.

It's a standard IDE drive placed inside a USB-2.0 enabled enclosure. An example of such a product is http://www.adstech.com/products/USB 2 Drivekit/intro/USB2DriveKit.asp?pid=USBX-804. This may or may not be the actual device used. This commercial equipment was identified to adequately describe the subject matter above. In no case does such identification imply recommendation or endorsement by the National Institute of Standards and Technology, nor does it imply that the equipment is necessarily the best available for the purpose.

10. Will all participants be required to arrive on J uly 9th, or will some participants be scheduled to arrive later to stagger the testing?

Test times will be staggered.

11. Are we required to send a white paper based on "Computational Requirements for FRVT 2002 High Computational I ntensity Test" from the technical dataset if we are not participating in the High Computational I ntensity Test?

No. We recommend that you still perform the assessment for the Medium Computational Intensity Subtest to ensure that you will be able to complete the test in the allotted time.

12. The FRVT test begins with the organizers providing a dataset to the participants. Will there be any other forms that need to be signed to receive this information?

No, this was taken care of by submitting the "Application for Access to the FRVT 2002 Technical Datasets" that we have already received.

13. Where do Participants send the sample similarity files based on the sample images in the Technical Dataset? 
Zip them and attach it in an email to frvt2002@nist.gov

14. Is there a typo on page 5 of "The FRVT I mplementer's Guide"? We read " $4 \mathrm{~N}+24$ bytes". Shouldn't this be "4N + 28 bytes"?

Yes. The correct phrase should be " $4 \mathrm{~N}+28$ "

15. Are the variations (lighting, facial expression, etc) in the Technical Data Set representative of the type of variations that the vendor software will be tested on?

The FERET images in the Technical Datasets are not necessarily representative of the imagery to be used in FRVT 2002.

These images were included to allow replication of the required functionality.

16. For the Medium Computational I ntensity Subtests, will the query signature_set contain still_set or video type images?

In the Medium Computational Intensity Subtests, both target and query sets may contain both still and video imagery.

17. Your e-mail message dated J une 6 announces the test reorganization and the fact that the new Medium test will contain 7500 signatures. Where are we supposed to get this new data/ subset for the Medium test?

The Medium computational intensity test will contain approximately 7,500 signatures. The format for the signatures is contained in the documentation distributed in the Technical Data Sets.

18. What can we expect for backgrounds in the images in FRVT 2002? (For example, complex outdoor B/ D, Etc.)

The key feature of the imagery is that each image consists of a single face that is the largest object in the image. Background varies from a relatively uniform background to images taken outdoors. The distance between the eyes in all tests will be at least 30 pixels and not more than 500 .

19. Regarding "The FRVT 2002 I mplementer's Guide": (1) It is not clear which xml is the target and which one is the query. (2) Also, the actual content of the $x \mathrm{ml}$ files is confusing. The < file / > entries (ex.: < file 
name="00003fa010_940307.nrm" / >) point to inexistant files of unknown format (p. 3 of "The FRVT 2002 I mplementer's Guide" states that all pictures in the FRVT2002 are J PEG).

A1 - The two signature-set files in the distribution, FERET-256-1.sigs.xml and FERET-256-2.sigs.xml, can interchangeably serve as target or query sets.

A2 - The files specified in this format do not exist in the distribution as they're created as an intermediate step of our implementation. Once they are created (by csu-normalizer) the files can be used. The .nrm format is an internal binary format for our geometrically and photometrically normalized images; they're most likely not usable with any third party viewers.

FRVT 2002 participants should be able to read JPEG files specified in the general form: . The previous line should not be construed to indicate that the directory depth is 2: it may be shallower or deeper as needed.

Participants should also be able to write the required binary files in the form. They can assume that the required paths will already exist, so that any "mkdir"-like operation will not be necessary. The Technical Datasets signature-set files do not include paths, the result being that the output files would be written into the working directory.

\section{What is the exact number of images for each subtest? (We need exact number in order to calculate processing} time for white paper.)

The number of images in the High Computational Intensity Subtest is exactly 121590 . The number of images in the Medium Computational Intensity Subtest has not been finalized.

21. The Document "The FRVT2002 I mplementer's Guide" lists 3 kinds of signatures to be used in the test (Fig 2.1 ). How does each signature relate to each subtest?

For the High Computational Intensity Test only still signatures (and thus single still images) will be used. For the Medium Computational Intensity Test mixed still, still-set and video signatures will be used.

\section{Signature \#2 ("The FRVT2002 I mplementer's Guide" figure 2.1) suggests a stereo camera system. Will stereo} data be used in any subtest?

The still-set signature given as an example in figure 2.1 of the implementers guide lists two images. Their names should NOT be construed to indicate that they were taken simultaneously or under the same lighting conditions or even on the same day; there are just two images of one person. No stereoscopic imagery will be used in FRVT 2002. 


\section{Why are your HEF_TD library just suitable for debug version?}

To aid development, we recommend using the "debugging" version of Xerces and the HEF libraries. Help with the "release" version of the libraries will not be supported. Note that this refers primarily to the Windows version of the libraries.

\section{Do we need to enroll total of $\mathbf{N}$ images for query and target set in each test?}

The FRVT 2002 Implementer's Guide describes the three kinds of signature that may occur in the target and query sets: a "still" signature contains only one image, but the "still-set" signature and the "video" signature will contain one or more images.

In the High Computational Intensity test every signature is a "still" signature. Therefore the number of images is identical to the number of signatures. The Medium Computational Intensity test, however, contains all types of signature, and therefore there are more images than signatures. For the $\mathrm{MCInt}$, therefore, it is not strictly necessary to enroll or process all the images, although the expectation is that better performance would be obtained by doing so. It is only necessary to report for all signatures.

The FRVT 2002 Organizers do not make any recommendations on which images should be considered, or on how they should be processed.

\section{Is it possible for a participant to start the test run and then leave the testing? FRVT Organizers would need to copy results to disk and then ship the computer back.}

Participants must be on site for the setup and check out procedures. The check out includes (but not limited to) transferring the similarity files from the participant system to the FRVT 2002 test agent and the inspection of the participant's hardware. Participants may leave while the test is being conducted. Participants are, however, responsible for insuring their systems complete all tests. The FRVT 2002 organizers are not responsible for informing participants that their system has crashed or ceased to work for any reason. This includes (but is not limited to) power outages.

\section{Will FRVT Organizers be providing UPS for participants use during testing?}

No. Participants are encouraged to bring their own UPS. 
27. We downloaded everything for generating the sample similarity files. In one of your documents it says that we need to produce some XML documents from the results. However, from the stuff we downloaded it seems as if the result files are just binary files. Did we misunderstand something? Do we have to deal with XML files at all?

FRVT 2002 participants will need to be able to read signature set XML documents and output binary result files.

\section{Is the query and target set be the exactly same or different?}

For the High Test the target and query sets are identical.

For the Medium Computational Intensity test participants SHOULD assume the sets are DIFFERENT.

29. Why have you provided the technical dataset in a format and structure different from the procedural requirements of the test? Why not provide a technical dataset that is identical to what we will receive? Am I really supposed to convert all of the PGM format images to J PG and modify the target and query XML files accordingly? This seems to be required if $I$ am to create a similarity file based on the target and query XML documents provided (FERET-2561.sigs.xml, FERET-256-2.sigs.xml)

The purpose of the technical dataset is to provide an example system that can read XML signature sets and output binary similarity files. It is not the goal of the technical dataset to provide an example standalone face recognition system that is FRVT 2002 "ready." Note that successful compilation and execution of the Technical Dataset is not a requirement for participation in FRVT 2002, but was provided to enable participants to gain an end-to-end feel for how the evaluations will be run.

Other than the names, quantity, and type of files, the XML that vendors will receive during the FRVT 2002 are identical in structure to what is provided in the technical dataset (i.e., they will validate with respect to one of the provided signature set schemas).

A Technical Dataset Supplemental is now available that contains data in the same format as you will be expected to utilize for the FRVT 2002 Evaluation.

30. Am I really supposed to convert all of the PGM format images to JPG and modify the target and query XML files accordingly? This seems to be required if $I$ am to create a similarity file based on the target and query XML documents provided ( FERET-256-1.sigs.xml, FERET-256-2.sigs.xml)

During FRVT 2002, the images provided will be in "JPEG" format. If, for internal testing purposes, you wish to use the 
provided imagery and XML files for your own system, then converting the images to a format understood by your recognition system may be required.

\section{Can FRVT 2002 Organizers provide monitors for participants to use during the evaluations.}

We will not be able to accommodate this request.

32. What is the minimum and the maximum relative size of a face in the image? Can the image be of factor 1000 wider than the eye distance, for example?

The key feature of the imagery is that each image consists of a single face that is the largest object in the image.

33. Do all images show one face only, or do you have images with several faces, e.g. in the video test?

All images contain only one face.

34. What will be the total size of images (in GB) for 121,000 images?

The total amount is less than 5GB.

35. How does a participant withdraw from participating in the FRVT 2002?

The responsible party (as designated on the applicant's "Request to Participate in FRVT 2002" form must send an e-mail that explicitly states their desire to withdraw. This e-mail must be sent to frvt2002@nist.gov.

36. Please elaborate on how the FRVT 2002 will evaluate 3D, infrared, or other novel face recognition systems.

The FRVT 2002 is designed to evaluate the performance of face recognition from still and video still imagery in the visible part of the spectrum. The FRVT 2002 will not evaluate performance of face recognition signatures gathered by other means. This includes three-dimensional signatures and infrared imagery. Since other types of facial signatures will not be provided, participants that require other types of signatures are encouraged to withdraw from the FRVT 2002 by 14 J une 2002. To withdraw, the responsible party (as designated on the applicant's "Request to Participate in FRVT 
2002" form) must send an e-mail that explicitly states their desire to withdraw. This e-mail must be sent to frvt2002@nist.gov.

\section{Will we need to modify the target. $x \mathrm{ml}$ and query.xml files to get the schema resources from a local drive during} FRVT 2002 tests?

Vendors will not have Internet access during FRVT 2002. If validating the given signature sets is desired, it should be done from the provided schema files.

\section{Is the test site available 24 hours a day during the test period including weekends?}

The test site will be available from 0900-1700, seven days a week.

\section{I am still having trouble understanding the use of XML in FRVT 2002. Can you help me?}

There have been a number of questions regarding the use of XML in FRVT 2002. Please click here to review an important document where we attempt to clarify these issues. First is a recommendation to participants. After, is a detailed discussion of how validation works with respect to the technical dataset.

\section{How many target set and query set will be given for each subtest?}

The High Computational Intensity Subtest has one target set and one query set. The Medium Computational Intensity Subtest has two target and query sets. See the two tables below for more information.

\begin{tabular}{||c|c|}
\hline \multicolumn{2}{|c|}{ High computational I ntensity Test } \\
\hline \hline Target & \multicolumn{1}{|c|}{ Query } \\
\hline \hline Still Signature & Still Signature \\
\hline
\end{tabular}

\begin{tabular}{|c|c|c|}
\hline \multicolumn{3}{|c|}{ Medium Computational I ntensity Test } \\
\hline & Target & Query \\
\hline
\end{tabular}




\begin{tabular}{|l||l||l||} 
Still Target \& Query Set & $\begin{array}{l}\text { Still signature } \\
\text { Still-set signature }\end{array}$ & $\begin{array}{l}\text { Still signature } \\
\text { Still-set signature }\end{array}$ \\
\hline \hline $\begin{array}{l}\text { Video Target \& Query } \\
\text { Set }\end{array}$ & $\begin{array}{l}\text { Still signature } \\
\text { Video signature }\end{array}$ & Video signature \\
\hline \hline
\end{tabular}

41. Is it necessary that a participant's POC be on site, or could it be a different employee of the participant or some other person engaged by the participant?

A Participant's POC serves a very important role during the on-site portion of the evaluation as they will serve as that participant's official representative. To change the individual designated as a participant's POC for the on-site portion of the evaluation, the responsible party (as designated in the "Request to Participate in FRVT 2002" form you submitted), must send an e-mail to frvt2002@nist.gov stating the new POC name and e-mail address. FRVT Organizers must receive this change notification two weeks prior to the participant's scheduled arrive date in order to process visit requests to the test facility.

\section{Each output file consists of similarity data. Must this data be sorted in a specific format?}

It is very important that data in the similarity files are sorted in a specific way. Any reordering or missing values in the similarity files will make scoring impossible. Please see page 5 of the Implementer's Guide for a complete specification of the format.

\section{Can you supply some sample data in color, or indicate where a database of color face image data which is representative of test data may be obtained?}

Data being used for FRVT 2002 was collected using the DARPA HumanID data collection protocols. You can look at Appendices G, K and L of the FRVT 2000 Evaluation Report for an understanding of the specific collection setup and resultant images used for FRVT 2000. While somewhat similar, there will be some deviation in the collection setup and images used for FRVT 2002.

44. What is the minimum and maximum for the width and height (in pixels) of the imagery in each test?

\begin{tabular}{|l||c||c|c|c||}
\hline & Min Width & Min Height & Max Width & Max Height \\
\hline \hline
\end{tabular}




\begin{tabular}{|c|c|c|c|c|}
\hline $\mathrm{HClnt}$ & ||252 & $\mid 300$ & |252 & $\mid 300$ \\
\hline $\mathrm{MClnt}$ & 200 & 240 & 300 & 360 \\
\hline
\end{tabular}

45. What is the scaling for similarity and distance values $(0-10,0-100 \ldots)$ ?

The output values can be between -inf to +inf. Participants are however encouraged to use a limited scale (such as 0 - 1 ,

$0-10$, etc). If possible, try to avoid duplicate/tied scores.

46. Medium Computational I ntensity Test will be administered with two targets and query sets - one for still images, one for video images. Are you going to provide us with a disk containing tree structure (Chapter 3 of I mplementers guide) for each set?

The precise directory tree structure of the $\mathrm{MCl}$ nt test, like the $\mathrm{HClnt}$ test, should not be needed by a participant prior to testing. The input and output paths and filenames are embedded in the XML signature-set files.

To summarize the implementers guide:

Input: The JPEG images are named in the "name" attribute of the "file" element. This includes the path relative to the location of the XML file it is in. For example:

<file name="images/12345/zz98765.jpg/>

Output: The output similarity files MUST be written to the file named in the "name" attribute of the query signature, again with path relative to the current XML file. For example:

<signature name="output/abcde/54321.sim">

Participants are reminded that they can also send email to frvt2002@nist.gov with technical XML-specific questions.

\section{Will the subtests be run sequentially or in parallel? Can we use the same machine for both subtests?}

Participants have been given a 12-day window (approximately noon on day one until noon on day twelve) for testing their face recognition systems. Also, the total time for each subtest is 264 hours and there are different computer limitations for each subtest. Therefore, most participants will be performing both sub-tests in parallel on two computer 
systems. (Note: The two sets within the Medium Computational Intensity Test will be performed sequentially on the same computer.) The only way the two subtests can be performed sequentially is if a participant can complete both subtests, using the more rigid medium computational intensity test computer limitations, within there given 12-day testing window.

48. You have said that the query \& target set of $\mathrm{HCInT}$ are identical. Will FRVT 2002 organizers provide two XML files or only one XML file?

FRVT 2002 Organizers will provide two XML files. These files (target and query set) are identical.

$$
\begin{aligned}
& \text { frvt2002_high_intensity_target. xml } \\
& \text { frvt2002_high_intensity_query.xml }
\end{aligned}
$$

49. Are we allowed to bring other people, such as a key technician or software engineer, in addition to the Technical Point of Contact during the 9 to 5 period when we have access to the system?

Each participant will be allowed to bring two respresentatives: Their FRVT 2002 POC and one other individual.

50. FAQ \# 3 states that the number of pixels between the eyes will be $30-500$ pixels. How does this relate to FAQ \# 44?

FAQ \#3 was answered much earlier - before FRVT 2002 Organizers had finalized the imagery. The answer to FAQ \#3 was based on possible imagery. The answer to FAQ \#44 (a more recent FAQ) is based on actual imagery used. Answers to both questions are technically correct.

51. The technical dataset contains 3 video signatures named "video 00-02". We do not find any video sigs in the FRVT supplemental directory, so we assume that we do not have to process any video sigs for the sample similarity files we will submit for the FRVT evaluation procedure. Is this correct?

Correct. No sample submission from video files is required.

52. If the test program crashes, may our representative or representatives restart the program? 
Yes.

\section{Can we place phone calls from the test facility to our offices? Are cell phones allowed? Are laptop PC's allowed?}

Cell phones and laptops are allowed. We are working to get a dedicated land-line phone installed for participant's use but cannot guarantee its availability at this time. We will provide a number where participants can be reached for emergencies.

\section{Will the test area in Dahlgren have 20-amp AC outlets?}

Yes.

55. Do we need to get clearance from NSWC Dahlgren in advance for anyone that we bring, both the Technical Point of Contact and others? Are there special issues, especially long delays, for clearance for non-US citizens?

There are no long delays of getting individuals onto the base. The FRVT 2002 Liaison will be contacting all participants soon to gather personal information for access to the base.

56. Can you provide an example of the 5-page document we are required to submit?

See Appendix J from the FRVT 2000 Evaluation Report for examples.

57. Are face locations nearly center in the image or could the face be located on a side?

The key feature of the imagery is that each image consists of a single face that is the largest object in the image. The position of the face could be anywhere in the image.

\section{What is the approximate breakdown of signatures in the Medium Computational I ntensity Test?}

The following numbers are high-bound estimates and could change somewhat: 
Video approximately 2500

Still approximately 5000

Still-set approximately 500

\section{How many still images are in a still set signature?}

Between 2 and 10.

60. Is any remote access to the system such as TELNET or FTP available? Can we monitor the test remotely, e.g. over phone lines or the Internet? If the test program crashes, may we restart another debugged version of the test program?

The goal of FRVT 2002 is to test fully functioning systems. Each participant has signed up to have one system evaluated for each test. The option to have more than one system evaluated could have been negotiated up to the sign-up deadline. Participants are restricted to only changing system configuration components of their system (e.g. the USB drive was given a different drive position than you anticipated). See test plan for more details. Changing the code or otherwise modifying a system once it arrives on site is not allowed. This includes debugging, changing the code, downloading patches, or changing face recognition system parameters. External monitoring/control of the system during testing (via TELNET, FTP, PC Anywhere, dialup, Internet, wireless, etc.) is not permitted.

61. Participants have been given 264 hours for testing. A participant finishes early, will they be permitted to leave early?

Yes. Once you have completed the test(s) and successfully checked out, you will be permitted to leave.

62. In the event that the voice-only telephone is not available and cell phone calls cannot be placed from within the building due to the metal covering mentioned in the FRVT 2002 Test Plan or other reasons, may participants leave the building to place cell phone calls from outside for technical support or other reasons?

Yes. Foreign nationals will have to be escorted.

63. May participants monitor the system at any time during the hour, or is it a specified time such as the start of the hour $(9: 00,10: 00,11: 00, \ldots)$ ? How much time do we have for the hourly monitoring, such as a maximum of five 
minutes?

Participants will be taken into the testing area at the top of the hour for approximately 5-10 minutes. The timing and amount of time may change according to various circumstances (power failure, computer crash, etc.)

64. May participants examine the contents of files generated by the test program such as similarity files or other intermediate status files written by the application?

Yes.

65. May participants view a console output from the program, messages such as "processing probe 64,123...."?

Yes.

66. What is the extension for the similarity file? .SIM? .BI N?

The output name is EXACTLY that already specified in the name attribute of the query's signature. It MAY include an extension, or it may not. It may also include a path. In any case participants must NOT add to, subtract from, or change the name in any way. This is discussed more in section 2.2.1 in the Implementer's Guide.

67. Will you supply the power and data cables for the IDE drives in USB2.0 enclosures containing your image database?

Yes.

68. We will bring only Linux based systems for the test. How shall we do the virus scan?

Participants using Linux will not be required to install anti-virus programs.

69. How often does power go out at NSWC Dahlgren? 
Power is fairly stable at NSWC Dahlgren. Power occasionally goes out during large thunderstorms, usually for only a few minutes.

\section{What are the deadlines for submitting a normalization function?}

You must let the FRVT 2002 Liaison know by 5:00 PM EDT 3 July 2002 if you plan to submit a normalization function. A reasonable amount of time will be given to participants to submit their normalization functions. These may result in some participants submitting their normalization functions after they have completed taking the FRVT 2002.

\section{Would a windows based library be acceptable as well for the normalization function?}

In a separate document, we will release the specification for the normalization functions. The scoring package for the FRVT 2002 will run on a Linux. Hence, the normalization function will need to run on a Linux. The proposed method of implementation is: the function will be a gcc or g++ routine supplied to the FRVT 2002 organizers as a compiled object binary (.o file). Then we can link the object binary when we compile the code.

\section{What is the number of signatures and images for the tests in FRVT 2002?}

Approximate (non-statistically rounded) numbers are given below. Please see Notes below the chart for additional caveats for this data.

\begin{tabular}{|c|c|c|c|c|c|c|c|}
\hline \multicolumn{2}{|c|}{ Test } & \multicolumn{3}{|c|}{ Input } & \multicolumn{2}{|c|}{ Output } & \multirow{2}{*}{\begin{tabular}{|c|} 
Total \\
\#Megabytes
\end{tabular}} \\
\hline & & \#Signatures & \#I mages & \#Megabytes & \#Files & \#Megabytes & \\
\hline High & & 121589 & 121589 & 1500 & 121589 & 57000 & 58500 \\
\hline Med & Video & 1900 & 40000 & 1000 & 2000 & 20 & 1020 \\
\hline \multirow[t]{2}{*}{ Med } & Stills & 4500 & 4500 & 1200 & 4500 & 100 & 1300 \\
\hline & & & & & & & 61000 \\
\hline
\end{tabular}

\section{Notes:}

1. The numbers for the $\mathrm{MCl}$ nt will change somewhat before testing begins.

2. The MCInt Video segment includes signatures of two types: video and still; it does not contain still-set signatures. 
3. The MClnt Stills segment includes signatures of two types: still and still-set; it does not contain video signatures.

\section{Are each signatures type and modality name in XML file? What about directory structure?}

Please see the example XML file here. Participants should note that the tree structure of the tests is implied by the paths given in the $\mathrm{xml}$ lines:

$$
\begin{aligned}
& <\text { file name="apath/bpath/cimage.jpg"/> } \\
& \text { <signature name="output/dpath/"> }
\end{aligned}
$$

Users will not have to create any of the directories in the output tree, as they'll be present with the supplied materials. No tree structure will be supplied beforehand.

\section{After completing the medium computational intensity test, may we add that computer to the system that is} running the high computational intensity test?

Yes, under the following conditions:

1) For the $\mathrm{HCl}$ nt, time begins when the first computer starts. You will still be on the clock when you are adding the additional computer to the $\mathrm{HClnt}$ network.

2) At no time may the number of computers working on the problem exceed the limit of three workstations. For example, when the $\mathrm{MCl}$ t test is being taken, you will have one computer run the $\mathrm{MCI}$ t and two computers run the $\mathrm{HClnt}$. When the $\mathrm{MClnt}$ is finished, you will have three computers running the $\mathrm{HClnt}$.

75. We are planning to bring more than two people for FRVT2002 because of our functional role and contractual schedule. If you are very strict about the number of people (only two people will be allowed at the test site) for the testing, we want to be able to switch people during the test still maintain two people at the test site (including Poc).

Only two individuals will be permitted at the test site at any given time. The individuals at the test site can change, but only two will be allowed at one time. According to the test plan, participants are also allowed to switch POCs after notifying test agents and receiving their permission.

76. What is the directory structure and naming format for files we generate in the $\mathrm{MCl}$ nt? 
The output name is EXACTLY that already specified in the name attribute of the query's signature. It MAY include an extension, or it may not. It may also include a path. In any case participants must NOT add to, subtract from, or change the path and name in any way. All the subdirectories in the output tree will already be present; users will not have to call "mkdir" or an equivalent. They should be able to just open the output file for writing.

77. In the Outline and Overview for Use of Normalization Functions in FRVT 2002 we are told that a possible normalization can be based, in part, on similarity scores for all pairs of gallery images in G. Does this imply that $G$ consists of a subset of the target set which is also represented as probe sets in the query set $Q$ ? Will there be a way to identify these subsets other than by brute force searches of the XML target and probe set definitions?

Normalization is a post processing procedure that occurs when the FRVT 2002 organizers score the results. As such, the participants do not need to know what are the galleries at the time the test is administered. At the time scoring takes place, the FRVT 2002 organizers will give the appropriate gallery similarity scores to the normalization routine.

78. For the High Computational I ntensity test, are we allowed to copy the FRVT data (pictures, $x \mathrm{ml}, .$. ) on the three computers? At the end, are we allowed to copy back to the USB drive from each of the three computers or do we need to centralize all similarity files on one computer before copying back?

You may copy all data to the three machines as needed. For the output files you may transfer them among machines as you see fit. All the output files must ultimately be placed on the USB drive.

Participant Area FAQ closed during evaluation period from 2 July through 13 August.

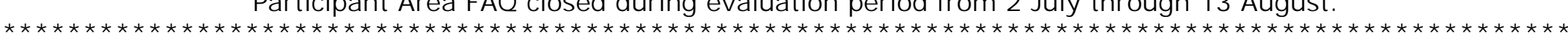

79. When is the normalization function due?

1700 EDT on 20 August

80. There seem to be time limits imposed on the normalization functions. And if a F2 normalization is taking too long, the testers will revert to using the F1 normalization function. How much time is allotted per gallery siz?. Meaning, for a gallery size of $X$ how long should $F 2$ or the $F 1$ function take before it is deemed too long? 
Participants that are concerned about the speed of their normalization functions should submit, to frvt2002@nist.gov, the following information:

- an approximation of how long the normalization routines will take on

- a $256 \times 256$ score matrix

- the same estimate for a $512 \times 512$ score matrix.

- the approximate machine specifications of the machine used for the timing test

- an order of speed in "big-O" notation

81. There are several math functions that our normalization function uses such as sqrt and exp. I s it okay to expect that whatever application is linking in our .o file will also link in the standard c/ c++ code for these functions (either libm.a or libstdc ++.so seems to do it fine on our machines here)?

It is safe to assume that the normalization code will be linked against the standard math library (libm). This includes, but it not limited to, functions such as abs( $), \exp (), \log (), \log 10(), \operatorname{pow}(), \operatorname{sqrt}(), \sin (), \cos (), \tan ()$, and so on.

82. Will the normalization functions be used on the high computational intensity test as well as the medium computational intensity test? If so, will there be a way for the functions being called to know in advance whether the scores being passed in are coming from the high versus the medium computational intensity test?

The normalization function could be used for either or both the $\mathrm{MCl}$ t and the $\mathrm{HClnt}$. Distinguishing between the two tests within the normalization function is not permitted nor necessary. Section 3 of the FRVT 2002 Normalization

Specifications clearly states the number and terms of normalization functions that can be submitted.

\section{What is the minimum size of the gallery being passed to the normalization functions?}

The FRVT 2002 Organizers will design experiments to adequately characterize the impact of normalization on performance. No further information regarding the experiments within FRVT 2002 will be discussed prior to release of the FRVT 2002 Evaluation Report.

84. In FRVT 2002 Normalization Specifications: What is the meaning of "identification, verification, and watch list tasks"? 
Identification: Closed universe test. Ranks the gallery by similarity to a probe. A probe is correctly identified if the identity of the top rank is the same as the probe.

Verification: Open universe test: Determines if the claimed identity of a face is correct.

Watch List: Open universe test: The gallery is the watch list. Consists of a two stage process. First - determine if the person in a probe is a person in the gallery (on the watch list). Next - if the probe is someone in the gallery, determine which person is it.

85. Is it planned to send the participants a pre-release of the FRVT 2002 Evaluation Report, allowing them to comment on it (as was done for the FRVT 2000 Evaluation Report)?

Yes 


\author{
Appendix F \\ Methods and Procedures
}

\title{
F.1 Introduction
}

To offer full public disclosure of the methods and procedures used during the Face Recognition Vendor Test 2002 (FRVT 2002) evaluation, this appendix provides a description of the administration of FRVT 2002. It is the authors' goal to fully convey to the reader the amount of work that is required to properly perform a technology evaluation of biometric systems. By stimulating discussion and analysis of the methods and procedures used for FRVT 2002, future evaluations may be improved.

The remainder of section F.1 provides a description of communication methods between the FRVT 2002 evaluation team and participants and a high-level timeline of important events in FRVT 2002. Section F.2 provides information regarding the announcement and invitation to participate in FRVT 2002, section F.3 discusses the requirements to participate and section F.4 discusses the participant selection process. Section F.5 discusses pre-test activities, and F.6 discusses post-test activities, that were required to successfully administer FRVT 2002.

\section{F.1.1 Communication Methods}

To maintain fairness, the FRVT 2002 evaluation team took special care to ensure that all participants received information about the evaluation at the same time. A government employee was named the FRVT 2002 Liaison. Except for extenuating circumstances, this individual was the only link from FRVT 2002 evaluation team to the outside world, including participants.

Two methods of communication were developed for discussions between the FRVT 2002 evaluation team and participants. First, the evaluation team established a frequently asked questions (FAQ) page on the participant area of the FRVT 2002 web site. Both tentative and committed participants could submit questions to the evaluation team through the web site, or through a dedicated e-mail address. The evaluation team then posted the questions on the FAQ page along with the answers. This primary and preferred method of communication ensured that all tentative participants and participants had equal access to information concerning FRVT 2002.

\section{F.1.2 Timeline}

FRVT 2002 was performed in a much more compressed timeframe than FRVT 2000. Whereas FRVT 2000 took thirteen months to prepare, six weeks to proctor, and eight months to develop a report, FRVT 2002 took six months to prepare, five weeks to administer, and five months to develop this report. Successful completion of FRVT 2002 in this timeline was only possible because the four principles from FRVT 2000 (Duane Blackburn, Mike Bone, Patrick Grother, and Jonathon Phillips) were able to perform similar duties in FRVT 2002, and Ross J. Micheals and Elham Tabassi were able to join the team at NIST with their own unique expertise. All told, approximately twenty individuals directly assisted in FRVT 2002 in some capacity.

Preliminary discussions regarding FRVT 2002 occurred at NIST and in a telephone discussion on 2 January 2002. Less than a week later, the FRVT 2002 evaluation team had agreed upon a rough outline. A brief review of available data sources that met the evaluation ideals was followed by an intense two-month period where the team gathered, analyzed and selected images for use in FRVT 2002. This activity culminated in the first formal meeting of the evaluation team in mid March. The purpose of this meeting was to finalize the makeup of FRVT 2002, review options for test location, define individual roles on the team for the remainder of the evaluation, and review participation forms and website content. 
Federal agencies were first told of the existence of FRVT 2002 on 24 January. A follow-up announcement to federal agencies was sent on 15 April to prepare them for the 25 April public announcement. Potential participants had from that date until 10 May to apply for participation. Participants were notified individually on 20 May and were provided their ID and Password for the Participant Area of the FRVT 2002 website. The Participant Area was an area of the FRVT 2002 website where additional information was provided to tentative participants and participants.

FRVT 2002 Evaluation team provided the FRVT 2002 Technical Dataset on 3 June. The technical data set contained documents that formally specified the input and output that would be used during the actual test, a sample face recognition system that operated under these protocols and an estimate of the computational power required to complete the evaluation. Participants were required to submit correct sample output files based on the dataset to FRVT 2002 Evaluation team by 28 June.

To ensure that applicants would be able to successfully complete the HCInt evaluation, the evaluation team mandated that applicants provide a resource assessment and to submit a white paper by 14 June that showed their anticipated time to complete the HCInt. The final list of FRVT 2002 participants was placed on the Participant page of the FRVT 2002 website on 18 June. Test dates were given to the participants on 13 June and the FRVT 2002 Test Plan was provided on 25 June. Testing occurred in two periods to help the government manage the evaluation process. The first period of testing went from 10 July through 21 July and the second from 24 July through 9 August.

\section{F.2 FRVT 2002 Announcement}

The FRVT 2002 Liaison announced the FRVT 2002 evaluation to the public on 25 April via five methods. First, an announcement was sent to the Biometrics Consortium listserv. ${ }^{1}$ This free and publicly available listserv currently has over 800 members from across the world and is generally regarded as one of the primary methods of sharing information throughout the biometrics community. Second, an announcement of the FRVT 2002 was placed on Federal Business Opportunities (formerly Commerce Business Daily or $\mathrm{CBD})^{2}$. Federal Business Opportunities is the single government point-of-entry for Federal government procurement opportunities over $\$ 25,000$. Through this one portal, commercial vendors seeking federal markets for their products and services can search, monitor and retrieve opportunities solicited by the entire federal community. Third, a posting on FRVT 2002 was placed in the free-to-use Biometrics Catalog ${ }^{3}$. Fourth, the FRVT 2002 Liaison sent an individual e-mail to 88 laboratories, universities and companies that had previously claimed to the U.S. government to have commercial or mature prototype face recognition products. Finally, e-mail was sent to various integrators, resellers, industry associations, conference organizers, and biometric reference publications/websites for them to further disseminate.

On 6 May, the FRVT 2002 Liaison sent a second series of e-mails announcing the FRVT 2002 to the Biometrics Consortium Listserv, the group of 88 agencies, universities and companies, and the group of other entities in the biometrics field.

\section{F.3 Qualifications to Participate}

To assess the state-of-the-art in face recognition technology, developers of the technology were invited to participate in FRVT 2002. The evaluation was limited to commercially available systems and mature prototypes. Additionally, only developers of core technology were permitted to participate, rather than developers licensing software from other core technology developers. In order to request participation, potential participants were required to electronically fill out the two forms shown in Appendix D, print and sign them, and send them back to the FRVT 2002 evaluation team. Those whose forms were received by 10 May were classified as tentative participants. Tentative participants were then reclassified as participants on 18 June after successful completion of several preliminary activities.

\footnotetext{
http://www.biometrics.org/html/listserv.html

http://fedbizopps.gov/

http://www.biometricscatalog.org/
} 


\section{F.4 Participation Overview and Test Schedule}

\section{F.4.1 Participation Overview}

Everyone that asked to participate in FRVT 2002, and successfully completed all pre-evaluation processes, was allowed to participate. Twenty-two tentative participants signed up to take FRVT 2002 and received the technical datasets. Of these, 14 later committed to participating and were listed on the FRVT 2002 website as "participants." Ten actually attempted the evaluation. All ten participants completed the evaluation.

\section{F.4.1 Developing the Test Schedule}

When creating the test schedule, the FRVT 2002 evaluation team planned on having no more than twelve participants on site at any time. Another constraint was to have no more than three participants start on the same day. The start dates were staggered to reduce the human traffic caused by multiple participants moving in equipment on the same day. Since there were more than twelve tentative participants at the time the schedule was originally created, it was decided to run the evaluation in two sessions with the second session starting after all participants in the first session had completed testing. Each participant was scheduled to have twelve days for testing, with actual test time of 264 continuous hours for each test.

On 13 June, there were 20 tentative participants. Each was randomly assigned one of the possible starting test dates and notified of this date via e-mail. Several tentative participants withdrew from participation after this date. Some participants also requested that their assigned dates be changed. The FRVT 2002 evaluation team granted these requests by reassigning them to empty slots in the schedule. Table 1 shows the start dates and latest allowed end dates used for FRVT 2002 testing.

\section{Table 1 - Scheduled start and end dates for each FRVT 2002 Participant. Note that not all participants required their full allotment of test dates.}

\begin{tabular}{lll}
\hline Participant & Start Date & End Date \\
\hline Eyematic Interfaces & 10 July & 21 July \\
Viisage & 24 July & 4 August \\
AcSys Biometrics & 25 July & 5 August \\
Cognitec Systems GmbH & 25 July & 5 August \\
Imagis Technologies & 25 July & 5 August \\
Dream Mirh & 26 July & 6 August \\
VisionSphere Technologies & 26 July & 6 August \\
C-Vis & 26 July & 6 August \\
Iconquest & 29 July & 9 August \\
Identix & 29 July & 9 August \\
\hline
\end{tabular}

\section{F.5 Pre-test Activities}

Before taking part in FRVT 2002, tentative participants and the FRVT 2002 evaluation team were required to complete several preliminary steps to prepare for the evaluation. These steps were designed to help guide them through some of the technical issues of the evaluation so that problems could be resolved before the actual testing began. The following subsections describe those activities.

\section{F.5.1 Technical Datasets}


A technical data set (Appendices $G$ ) was made available on the participant area of the web site on 3 June. It provided documentation on the format of the image data that would be distributed to participants during the evaluation and the required output format that participants would need to use for submitting results. It also contained a set of sample images, the source code for a sample face recognition algorithm, source code for software used to generate scores from matching results, and results based on the images and a sample face recognition system. Tentative participants were encouraged to use the source code as a guide for modifying their own software to run the evaluation. It also provided a means for them to test their own software and compare the resulting scores with those obtained using the sample face recognition algorithm. The technical data set also contained an example of the required HCInt Computational Intensity White Paper.

\section{F.5.2 HCInt Computational Intensity White Paper}

The FRVT 2002 evaluation team anticipated that many applicants might have underestimated the difficulty of the High Computational Intensity Test (HCInt). As part of the Technical Data Set, provided on 3 June, participants were given an example of how to derive their anticipated time and computational requirements to participate in that test. Applicants were mandated to perform a similar computation for their system and to submit a white paper by 14 June that shows their anticipated time to complete the HCInt. This allowed HCInt participants to prove that it was theoretically possible for their systems to completely process the large number of images included in the test. Failure to submit this paper resulted in their removal from participating in the HCInt. Applicants were also strongly encouraged to perform a similar assessment for the Medium Computational Intensity Test, but were not required to do so. All participants that started the HCInt and MCInt completed within the allotted times.

\section{F.5.3 Sample Similarity Files}

Without proper similarity files, the FRVT 2002 evaluation team could not produce the results shown in this report. The evaluation team and tentative participants worked together to ensure the accuracy of participant's similarity file development process prior to actual testing.

The technical data set contained a set of sample image files. Tentative participants were required to process these images using their software and produce similarity files in the proper format. They were required to submit these files to the FRVT 2002 evaluation team for analysis. If any problems were encountered with the submitted files, tentative participants were allowed to make corrections and resubmit as many times as necessary until the deadline of 28 June.

\section{F.5.4 Normalization Protocol}

The FRVT 2002 uses the Sep96 FERET evaluation protocol. The key innovation in this protocol is the usage of virtual galleries and probe sets. Virtual galleries and probe sets allow for the measurement of performance for different categories of images from one set of similarity files. This approach did not allow the use of normalization that some participants regularly use in their product. Similarity scores are classically computed by comparing two images and reporting a measure of similarity between the two faces in those images. Normalization adjusts these scores based on all the images in a gallery.

On 6 August, the FRVT 2002 Liaison announced an amendment to the test protocol to permit the usage of a participant's normalization routine if they desired. For FRVT 2002 purposes, normalization was a posttest administration procedure that was executed by NIST after similarity files had been submitted after testing. Participants who elected to utilize the FRVT 2002 normalization option were required to provide NIST, by 20 August, an object file that contains the result of the compilation of $\mathrm{C}$ code of specific functions. This file was then called at an appropriate time by the NIST scoring code during the scoring process. For intellectual property reasons participants were not allowed to submit source code or details of their normalization techniques to the FRVT 2002 evaluation team. The Normalization API is available in Appendix $\mathrm{J}$ of this document. 


\section{F.5.5 Dry Run}

The FRVT 2002 evaluation team performed a dry run at the test facilities on 9 July 2002. NIST provided a baseline face recognition system and acted as a participant as the FRVT 2002 test agents stepped through the entire test plan twice. Although no major issues were found, the dry run was very beneficial as the majority of the contracted test agents had not been previously involved in the planning and had several questions concerning what they were and were not allowed to do while proctoring the tests.

\section{F.6 Post-test Activities}

Participants were also required to perform several activities in support of the FRVT 2002 Evaluation Team after physical testing had been completed. These are discussed in the sections below.

\section{F.6.1 Scoring Code Inspection}

The scoring suite used for the Face Recognition Vendor Test 2002 was an updated version of those used for the FERET and FRVT 2000 evaluations. The FRVT 2002 Evaluation Team implemented a multi-step process to ensure that the newer version of the scoring suite is accurate. Several of these steps were performed by NIST to ensure that results using the FRVT 2002 scoring code would be identical to results using either the FRVT 2000 scoring code or the FERET scoring code.

The last step in this process was to provide the FRVT 2002 participants a copy of the scoring suite and the similarity files from a baseline system so that they could study the suite and report any implementation errors. The FRVT 2002 Liaison provided participants a set of similarity files, the scoring software and the results from that scoring software on 30 October 2002. Participants were asked to study the validity of the scoring code and provide feedback to the evaluation sponsors if they found any software implementation errors by 7 November 2002. No errors were reported.

\section{F.6.2 Participant Review of FRVT 2002 Evaluation Report}

The FRVT 2002 Evaluation Team provided a copy of this report to the participants for their review seven days prior to public release. Participants were given the option of preparing and submitting a position paper on the evaluation results within these seven days. These position papers are included in Appendix $\mathrm{N}$ of this report without modification. 


\section{This page intentionally blank.}


Appendix G

Technical Data Set 


\title{
The FRVT 2002 Implementer's Guide
}

\author{
Version 0.99.0 \\ Initial FRVT-2002 Technical Dataset Release
}

Patrick Grother Ross J. Micheals $\quad$ P. Jonathon Phillips

National Institute of Standards and Technology

Information Access Division, Image Group

Gaithersburg, MD 20899

May 31, 2002 


\section{Chapter 1}

\section{Introduction}

This paper details what Face Recognition Vendor Test 2002 (FRVT 2002) participants need to be able to do to complete the FRVT 2002 tests. The document is split into four chapters. This introduction gives an overview of the face recognition evaluation protocol in use. Chapter 2 describes the format of the files given to, and required from, FRVT 2002 participants. Chapter 3 is a procedural description of what participants should do during the test. Chapter 4 details a PCA based face recognition algorithm that is capable of reading and writing the required FRVT 2002 files.

Participants are also directed to the following related documents:

1. The FRVT 2002 Technical Datasets. This collection contains a face recognition system capable of reading and writing the files used in FRVT 2002. It also includes a small extract of the FERET database (256 images) used for demonstration purposes.

2. The short paper Computational Requirements of the FRVT 2002 is an analysis of the time required to complete the FRVT 2002. It is vital that participants read this document, consider its implications for their systems, complete the attached form, and return it to the FRVT 2002 organizers.

3. The logistical aspects of the FRVT 2002, for example times, dates, locations are formally described in the Face Recognition Vendor Test 2002 Test Plan.

4. FRVT 2002 is an implementation of The HumanID Evaluation Framework that defines a general mechanism for the evaluation of arbitrary multimodal biometric systems. The document is not required reading, but participants may refer to it for a more complete reference on the use of XML in FRVT 2002.

Readers of this document are directed to specific files in the Technical Datasets archive that accompany this document. Such pointers are intended to make concrete the concepts explained here.

\subsection{Overview}

Image recognition systems and biometric based identification systems usually operate by comparing new unknown images with a set of known images, often referred to as the gallery. The unknown, or probe images, are usually obtained some time after those in the enrolled gallery. For example, in a verification setting the new image is obtained from a person claiming to already be in the gallery. In an identifcation setting the new image may belong to a person who is, or is not, already present in the gallery. The performance of recognition systems on these various tasks can be estimated off-line in a so-called technology evaluation by requiring the system to report numerical comparisons between pairs of images which act respectively as probe and gallery images. The FERET protocol ${ }^{1}$ formalized this

\footnotetext{
${ }^{1}$ See Phillips et al. The FERET Evaluation Methodology For Face Recognition Algorithms, IEEE PAMI 22:1090-1104, 2000.
} 
procedure, and the FRVT 2002, like the FRVT $2000^{2}$ before it, uses the FERET methodology. One of the FERET protocol's features is that images of subjects taken under many different conditions are present in the testing sets, and consequently the performance of a system may be estimated for any image or subject condition represented by a subset of the image set. For example, if verification performance is required on a population of persons who have aged by six years and who have grown beards, then that number can be computed from just that subset of the reported recognition scores that correspond to those specific images. Many such recognition tasks can be embedded in the one test.

The FRVT 2002 uses an extented version of the FERET protocol known as the Human ID Evaluation Framwork $^{3}$ (HEF) that allows for the quantitative testing of recognition systems on heterogeneous sets of multimodal biometric signals. The multiple face-face comparisons of FERET are replaced by the abstract notion of comparing a signature with a signature. In this context a signature could be a single face image as before, or a set of faces, or a video of a person, or a set of ten fingerprint images, a speech recording, a composite fingerprint and face record, or, indeed, any other biometric information in any data format.

Thus we define the terms of FRVT 2002. Two tests are planned, referred to as the Medium Computational Intensity and High Computational Intensity tests. Each contain imagery representative of several imaging and/or subject circumstances. The imagery is specified by two signature sets called the target and query sets. The related terms gallery and probe refer, respectively, to subsets of target and query, and it is these subsets that specify the targeted application-specific experiments embedded in a test .

The test proceeds by a recognition system reporting real scalar similarity or distance values for all pairs of signatures in the target and query sets. The resulting similarity matrix forms the basis for many statistical analyses that result in various performance values.

\subsection{Contact Information}

The primary and preferred source of communications between participants and FRVT 2002 government personnel is the FRVT 2002 Frequently Asked Question (FAQ) page, which is located off of the FRVT 2002 website, http://www.frot.org. A government employee has been named as the FRVT Liason. The FRVT Liason will be the primary point of contact and will manage communications with participants. Questions that are outside the purview of the FAQ can be submitted to the FRVT Liason via email at frot2002@nist.gov. All e-mails sent to the FRVT Liason and germane to the FRVT 2002 will be posted on the FAQ page. This policy ensures all participants are guaranteed equal access to information concerning FRVT 2002. Contact with the FRVT 2002 Liason other than via the FAQ areas, or contact with someone other than the non-FRVT Liason will only be allowed under extenuating circumstances.

\footnotetext{
${ }^{2}$ See Blackburn et al. Facial Recognition Vendor Test 2000, February 2001.

${ }^{3}$ See Grother et al. The Human ID Evaluation Framework a copy of which accompanies the FRVT 2002 Technical Datasets.
} 


\section{Chapter 2}

\section{Input and Output File Specifications}

The primary requirement for FRVT 2002 is that for each test that participants undertake they must have the ability to read two files, one each for the target and query sets, compare the named signatures therein, and write binary files that contain measures of the likeness of the individual appearing in those signatures.

The formats of the input and output files are detailed in the following sections.

\subsection{Signatures}

The input signature set files are in an XML format ${ }^{1}$. XML is distinguished by its flexible and extensible usability in document mark up, and its machine readability. The ability of vendors to read XML is a fundamental requirement of FRVT 2002.

The target and query sets are signature sets containing many signatures . A signature is a named collection of arbitrary biometric information from one individual. FRVT 2002 is structured around the ability of a Face Recognition algorithm to compare any two signatures and produce a scalar real number, either a distance or a similarity value. Specifically, participants are required to compute and write a scalar for each signature pair occurring in the target and query sets. Further there are three kinds of signature in FRVT 2002, each of which contains only face imagery. Their definitions follow, and an example of each kind is given in figure 2.1.

1. A still is a single still image of one person's face,

2. A still-set is a set of still images of one subject, possibly taken at different times or under different conditions

3. A video is a sequence of frames of an individual taken at regular intervals over some short period of time.

Note the file elements at the deepest level of XML nesting are the most concrete. They give the physical location of the images, including a path relative to the location of the XML signature set file it is in. In FRVT 2002, all images specified in the name attribute are standard JPEG files, as indicated in the type="jpeg" attribute.

When a FRVT 2002 participant's algorithm encounters a pair of single-file still signatures it is clear that the system should do the conventional matching and report a real scalar number. For multi-file signatures (i.e. still set or video) the same is true, a real scalar must be reported. How participants effectively use all $n$ files of signature A and $k$ files of signature B is up to the particpant's application,

\footnotetext{
${ }^{1}$ See http://www.w3c.org/xml for example
} 


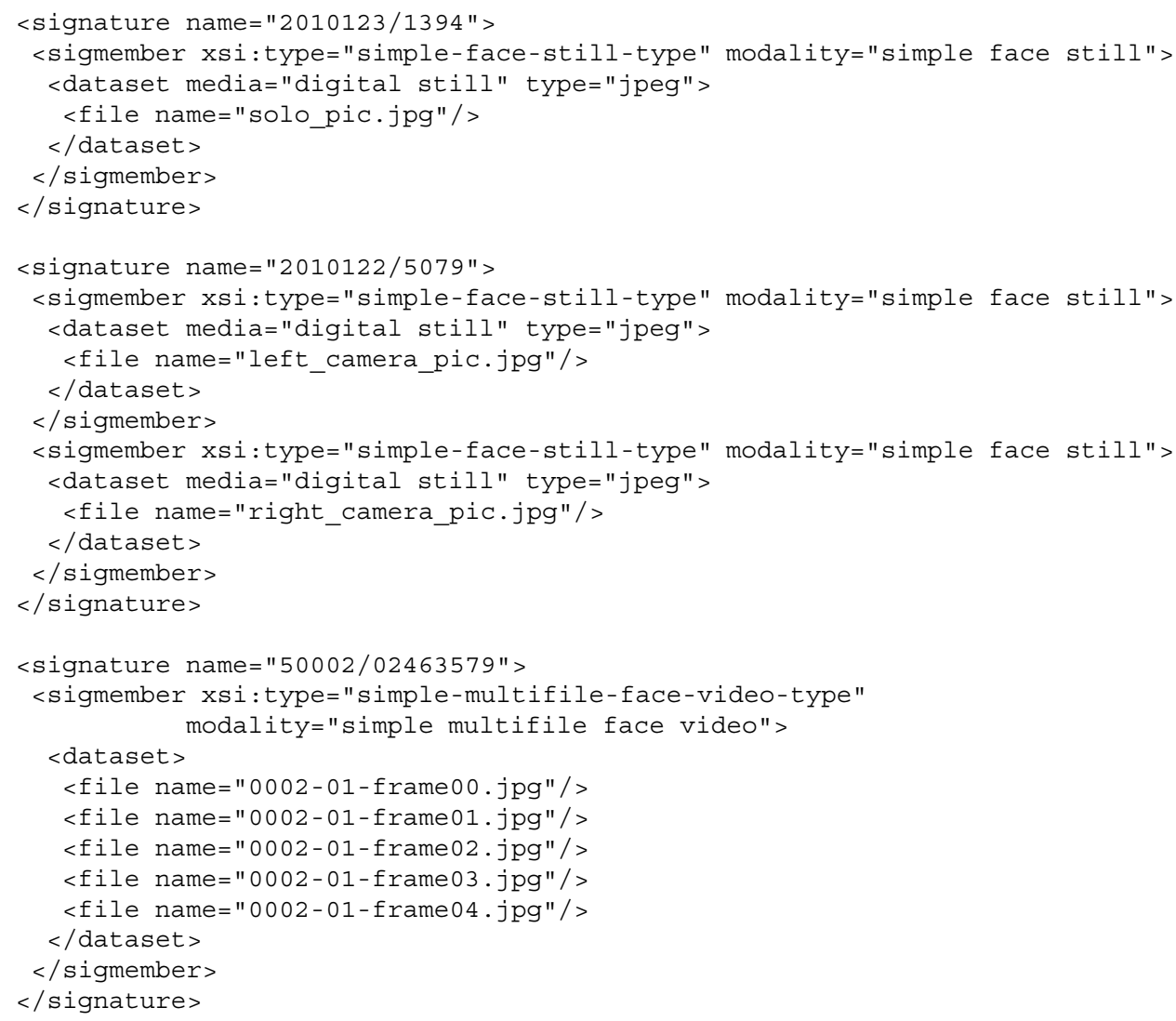

Figure 2.1: The three kinds of signatures that will be used present in FRVT 2002.

Examples of XML target and query sets are FERET/FERET-256-1.sigs.xml and FERET/FERET-256-2.sigs.xml in the Technical Datasets distribution. Code for reading such signature sets, and constructing a usable internal representation thereof, is contained in the file software/src/SigSet_XML.cpp as called from the application software/src/apps/HEF-Validator-app.cpp on the Technical Datasets distribution.

\subsection{Similarity Files}

While section 2.1 was concerned primarily with marking up standardized input for evaluations, this section defines the binary format that face recognition systems must output.

The overall requirement for a specific test is that if there are $N$ signatures in the target set and $M$ signatures in the query set, then a participant's code must produce $M$ output files each containing $N$ similarities or distances. The matrix of $N M$ values is referred to as a similarity matrix although is not usually stored or manipulated as such.

\subsubsection{File Names}

The output files take their names from the signature name attribute. This will generally include a path so that an application that has parsed the query signature set can write the similarity scores to a file whose name is exactly that in query signature. The application may assume that any directories present in the name's path will already be present in the provided output directory tree. 


\subsubsection{File Format}

For all tests these output files are in a binary, unformatted I/O, format. The XML format described in The HumanID Evaluation Framework document is disallowed in FRVT 2002 because, for large signature sets, the total storage requirement for such XML similarity files could be several hundred gigabytes.

The file consists of a 20 byte header, followed by as many similarity or distance values as there are signatures in the target set, and a final closing 8 byte footer. The header consists of four items: a "magic number" ${ }^{2}$, a value for testing the byte order (endian-ness) of the subsequent values, a count of the number of similarity or distance values, and a value for polarity (see section 2.2.4). The bulk of the data, $N$ binary floats, follows. It is imperative that the $i$-th element of the similarity data corresponds to the $i$-th target signature. Any reordering or missing values will make scoring impossible. The file is terminated with a repeat of the magic number.

The similarities of the $N$ target signatures to each query signature are stored in a single file whose name is taken from the name $="$ "' attribute of the query . For a target set of size $N$, the file must contain precisely $4 \mathrm{~N}+24$ bytes. The 4 arises because the required number of bytes used to represent one scalar real number is the size of a single precision float on most contemporary architectures. Participants must not output higher precision values, and they should ensure that their values are representable in the range of a float. Either Big or Little Endian byte ordering must be used consistently throughout. Sample binary files must be submitted to the FRVT 2002 organizers before the tests are commenced. Formally, the numbers must be reported in IEEE 754 format that is used on Intel based PCs, and many UNIX platforms. Participants with any concerns as to their floating point formats should collaborate with the FRVT 2002 organizers proactively.

In summary, the contents of each binary file is, in order,

\begin{tabular}{|l|l|l|l|l|}
\hline Description & Value & Data type & Number of items & Total Bytes \\
\hline Magic number & FRVT2002 & char & 8 & 8 \\
Endian Indicator & 0x12345678 & unsigned int & 1 & 4 \\
Number of Distances & $N$ & unsigned int & 1 & 4 \\
Polarity & 0 or 1 & unsigned int & 1 & 4 \\
\hline Similarity & 3.14159 & float & 1 & 4 \\
$\ldots$ & $\ldots$ & float & $N-2$ & $4(N-2)$ \\
Similarity & 8.31441 & float & 1 & 4 \\
\hline Magic number & FRVT2002 & char & 8 & 8 \\
\hline
\end{tabular}

Strict adherence to this format is mandatory because recovery from erroneously generated files may not be possible.

\subsubsection{Endian Indicator}

The endian indicator is used to ensure that when similarity files are written and read across architectures, different byte orderings ${ }^{3}$ can be corrected for. A mechanism for detecting the byte order of the machine that wrote the simliarity scores is a follows. First, take an integer of value, and store it in memory (line 34), and write it to a file one byte at a time (line 35). Later, when this file is read in the same fashion, the "natural" byte ordering imposed by the machine architecture is reflected in the input.

\subsubsection{Polarity}

For each signature in the query set, the FRVT 2002 participant must produce a similarity file. All such files may contain one of two categories of value, termed similarity or distance. A large value for a similarity implies sameness of identity of the subject appearing in the signature. A large value for distance, on the other hand, implies difference of identity. This concept is termed polarity and must be recorded in all similarity files as discussed above. The permissible values are 0 for similarity values and 1 for distances.

\footnotetext{
${ }^{2}$ A simple heuristic means for distinguishing this kind of file from all others.

${ }^{3}$ Byte order is also known as endianness or byte sex.
} 


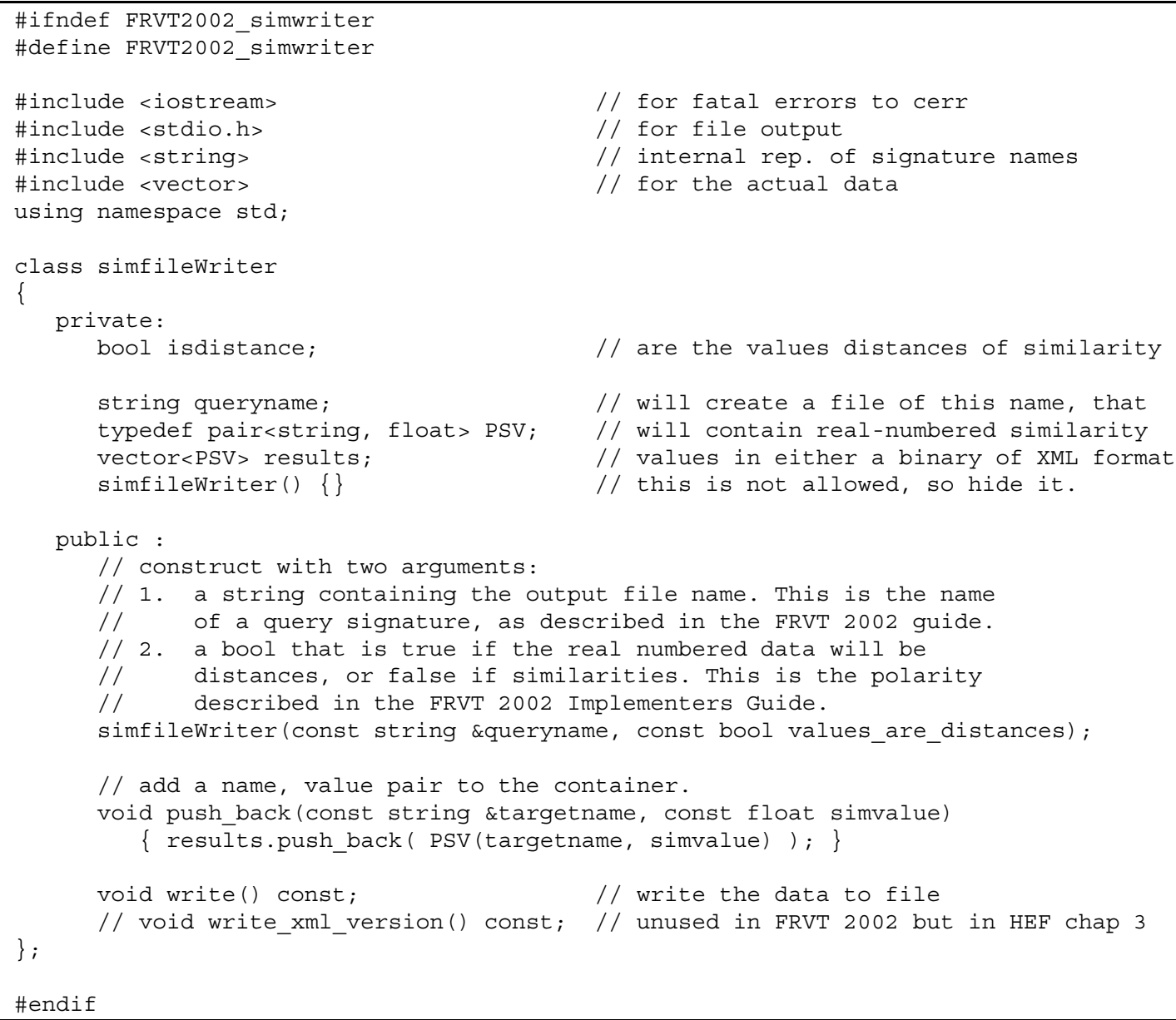

Figure 2.2: A C++ class specifying a container for holding similarity data during processing.

\subsubsection{Sample Code}

Figure 2.4 gives $\mathrm{C}++$ code for writing similarity files. It shows the output method of the simfileWriter container class shown in Figure 2.2. The pseudo code in figure 2.3 shows how the class could be used in a real FRVT 2002 system. Alternative working C++ writing code is included with the accompanying Technical Datasets distribution; specifically the file software/src/CSU/csuSubspaceTest.cpp contains a function called writeDistances() that outputs binary data to file. 


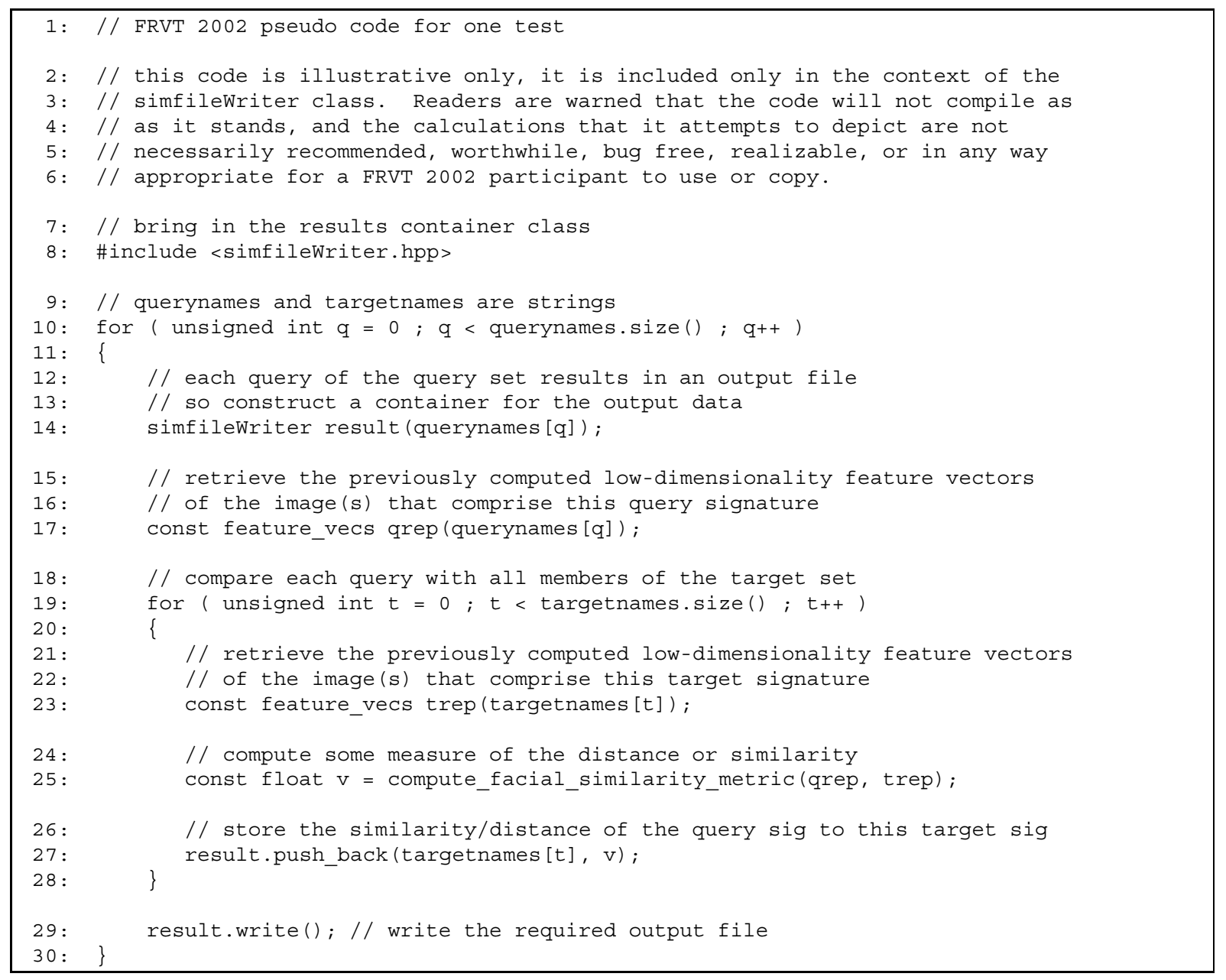

Figure 2.3: Pseudo code representing how the simfileWriter class could be used in a working FRVT 2002 implementation. 


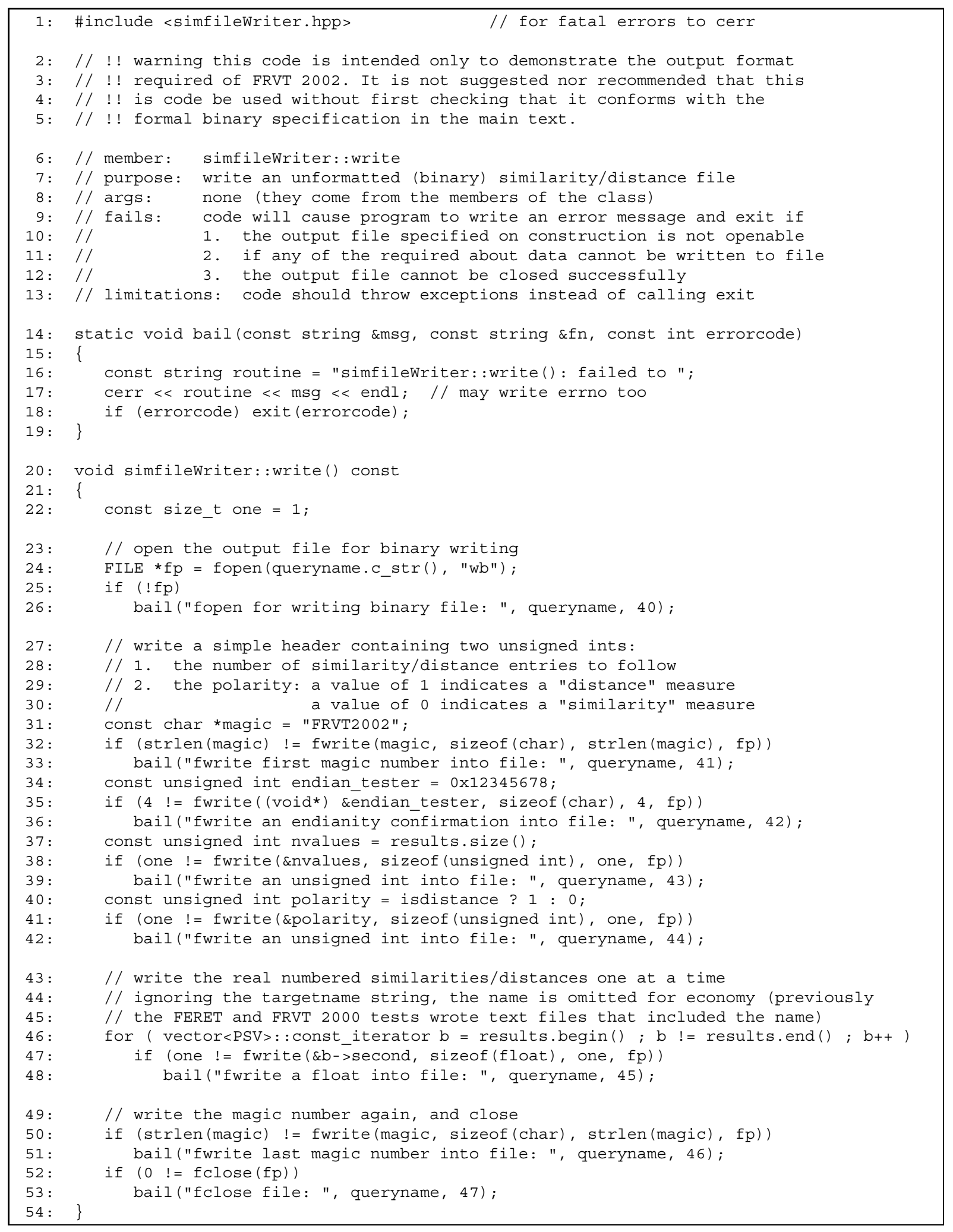

Figure 2.4: The write method code for outputing FRVT 2002 similarity files. The functionality of this routine must be emulated by participants. 


\section{Chapter 3}

\section{Procedural Requirements}

For each test, participants will be given a disk containing tree structures of the form in figure 3.1. The two XML files at the top level will be named after the test, one for each of the target and query sets as shown. Those files will contain file elements whose name attributes will include paths, having the general form images/somemajor/someminor/someimage.jpg. Participants are required to write output files specified by the name attribute of the query signatures, not the file's name, that also will generally include a pathname into the output directory tree.

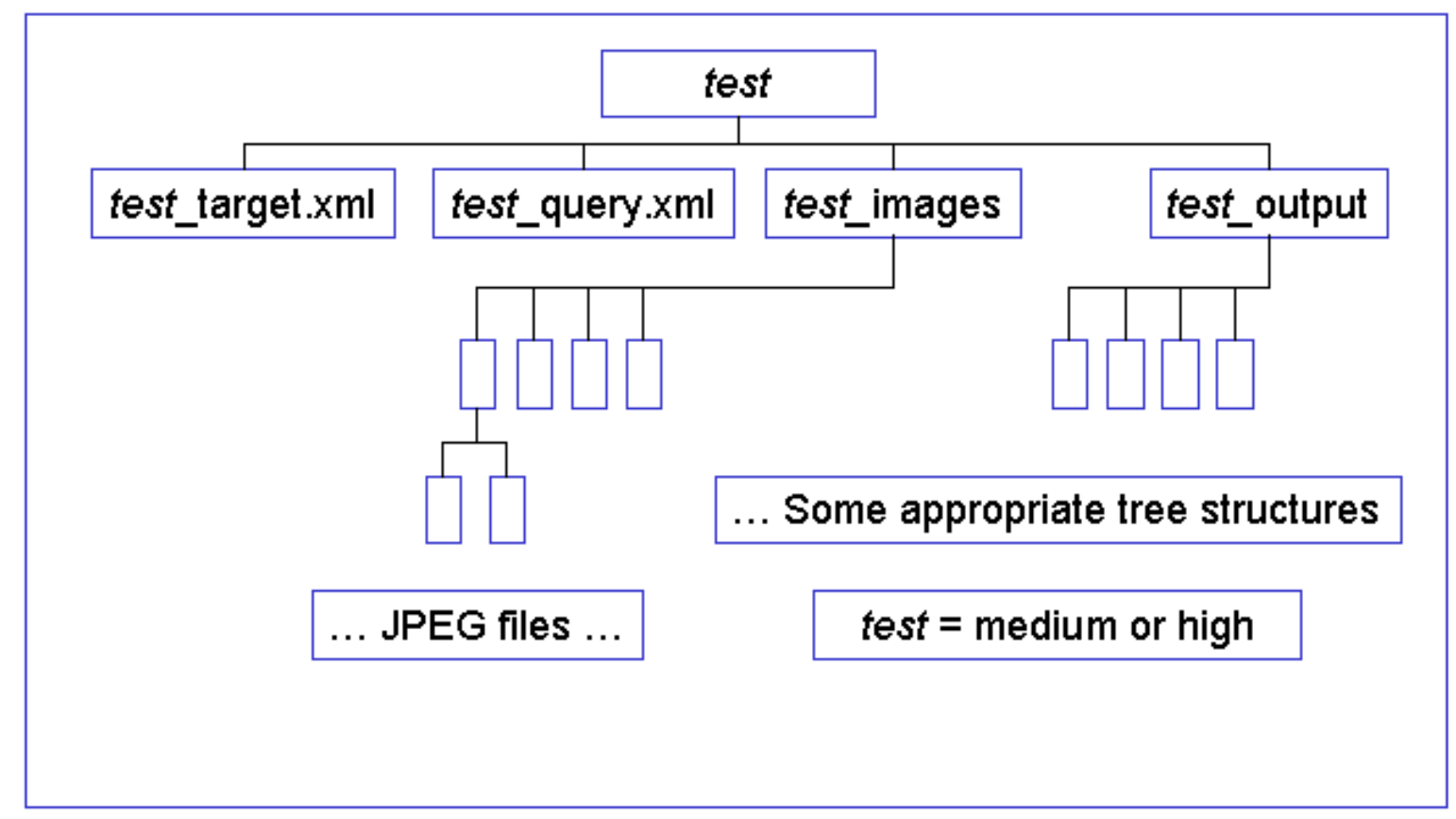

Figure 3.1: The tree structure given to participants for each test .

Thus a procedural description of what vendors must do is given below, but the document FRVT 2002 Test Plan should be consulted also.

1. Mount the drive supplied by the FRVT 2002 Organizers. This may simply consist of plugging in a USB drive. Alternative media such as CD or DVD may be available, by prior request.

2. Recursively copy the whole tree to a working partition. Note that the output directory tree should 
also be copied because it contains subdirectories that the application will populate as it proceeds.

3. Change directory into the top-level directory. This implies that the participants application has this as its working directory.

4. Execute the test. Participants must prepare to do this successfully before attending the formal FRVT 2002 test. In this respect they should see the document Computational Requirements of the FRVT 2002 that addresses this issue more extensively.

5. Ensure the correctness of the output files. This should be done by participants own software.

6. Recursively copy the output tree back to the supplied media (the USB drive), overwriting the (empty) one that was there.

7. The FRVT 2002 Test Agents will then delete all data on the working partition. There are strict requirements that all the material supplied to participants must be purged from the host machines. The FRVT 2002 proctors will further specify this process in the FRVT 2002 Test Plan. 


\section{Chapter 4}

\section{FRVT 2002 Technical Datasets Reference Implementation}

The HumanID Evaluation Framework (HEF), is a mechanism for the quantitative testing of recognition systems. The FRVT 2002 Technical Datasets Reference Implementation is a software package that implements a variety of functionality described by the HEF, with respect to the components of the HEF that will be used in FRVT 2002. Throughout this document, the term HEF-TD will be used to refer to this, the HumanID Evaluation Framework Technical Dataset software.

\subsection{Caveats}

Using this code in your own software for FRVT 2002 is neither required, nor necessarily recommended. It is provided only as an example implementation of a system that can read XML and output binary files of the same structure to be used during the actual tests. The provided code has been tested, but should not be considered to be "bug-free."

\subsection{Directory Structure}

The HEF-TD has the following directory structure. The top directory has three main subdirectories, documents/, FRVT-2002/ and software/. The contents of the documents/ and software/ directories are selfexplanatory. Files similar in structure and nature to those that will be used in FRVT-2002 are located in the FRVT-2002/ directory.

The software/ directory has the most complex structure. Unless noted otherwise, there is a single copy of each source code file (shared between both Unix and Windows) in software/src/ or software/include/. Under Unix-like operating systems, object files are stored on the objs/ directory. Under both Unix and Windows, library files and executable binaries and are stored in software/lib/ and software/bin/respectively. The files required to build HEF-TD under windows are in the Projects directory. Each supported compiler has its own directory within Projects/VC6/. At this time, because Microsoft Visual Studio 6 is the only supported Windows compiler, there is a single subdirectory, Projects/VC6/ in this folder. The software/import/ directory is reserved for external packages (such as Xerces). This directory is further subdivided by operating system i.e., software/import/unix/ and software/import/Win32/.

A full listing of the contents of the technical dataset are as follows: 
documents/

documents/implementers_guide/

documents/implementers_guide/FRVT2002-implementers_guide.ps

documents/implementers_guide/FRVT2002-implementers_guide.pdf documents/manual/

documents/manual/HEF-FRVT2002-TDS.ps

documents/manual/HEF-FRVT2002-TDS.pdf

documents/xml/0.99.0

documents/xml/0.99.0/sigset-schemas

documents/xml/0.99.0/sigset-schemas/multiple-simple-face-stills-set.xsd

documents/xml/0.99.0/sigset-schemas/sigset-general.xsd

documents/xml/0.99.0/sigset-schemas/sigset-unrestricted.xsd

documents/xml/0.99.0/sigset-schemas/simple-face-still-set.xsd

documents/xml/0.99.0/sigset-schemas/simple-face-still-type.xsd

documents/xml/0.99.0/sigset-schemas/simple-face-video-set.xsd

documents/xml/0.99.0/sigset-schemas/simple-face-video-type.xsd

documents/xml/0.99.0/sigset-schemas/simple-multifile-face-video-set.xsd

documents/xml/0.99.0/sigset-schemas/simple-multifile-face-video-type.xsd

documents/xml/0.99.0/sigset-schemas/single-sigmember.xsd

FRVT-2002/

FRVT-2002/FERET/

FRVT-2002/FERET/coords.512

FRVT-2002/FERET/FERET-256-1.sigs.xml

FRVT-2002/FERET/FERET-256-2.sigs.xml

FRVT-2002/FERET/m150x130.dat

FRVT-2002/FERET/raw/

FRVT-2002/FERET/raw/*.pgm

FRVT-2002/FERET/norms/

FRVT-2002/video/

FRVT-2002/video/raw/

FRVT-2002/video/raw/*.pgm

FRVT-2002/video/video-coords

FRVT-2002/video/video.nrm.ss.xml

\section{README}

softwarel

software/bin/

software/bin/demos/

software/bin/apps/

software/config.guess

software/config.sub

software/configure

software/configure.in

software/import/

software/import/unix/

software/import/Win32/

software/include/

software/include/CSU/

software/include/CSU/

software/include/CSU/csuDebuglevel.hpp

software/include/CSU/csuFileCommon.hpp

software/include/CSU/csuGlobal.hpp

software/include/CSU/csuMatrix.hpp

software/include/getopt.hpp

software/include/HEF/

software/include/HEF/config.hpp.in

software/include/HEF/dummy.h

software/include/HEF/Signature.hpp

software/include/HEF/SigSet.hpp

software/include/HEF/SigSet_XML.hpp

software/include/HEF/std_DefaultHandler.hpp

software/include/HEF/string-utils.hpp

software/install-sh

software/lib/

software/Makefile

software/Makefile.in
Documentation directory

Implementer's Guide directory

Implementer's Guide (Postscript version)

Implementer's Guide (PDF version)

HEF Manual directory

HEF Manual (Postscript version)

HEF Manual (PDF version)

Version 0.99.0 XML Schemas

Signature Set Schemas

HEF Multiple Simple Face Stills Set schema

General (abstract) signature set schemas

HEF Unrestricted Signature Set schema

HEF Simple Face Still Set schema

Simple Face Still schema type definition

HEF Simple Face Video Set Schema

Simple Face Video schema type definition

HEF Multifile Face Video Set schema

Simple Multifile Face Video type definition

HEF Single Sigmember schema

FRVT 2002 data and examples directory

FERET data directory

FERET imagery ground truth data

Example signature set

Example signature set

Image mask used during normalization

Directory containing raw image data

FERET imagery

Target directory for image norms

Video data directory

Directory containing raw video frames

Example (short) video sequences

Ground truth data for video clips (for normalizer)

Video data signature set

Main README file (points to this document)

HEF Technical Dataset software directory

General binaries and executables directory

Target directory for demonstration binaries

Target directory for application binaries

Required component of configure script

Required component of configure script

Unix configuration script

Autoconf script (generates configure)

General target directory for external (imported) software

Target directory for external (imported) Unix software

Target directory for external (imported) Win32 software

General include file directory

Directory for CSU include files

Directory for CSU include files

CSU debugging macros

CSU common function declarations

CSU globals

CSU matrix declarations

Helps provide getopt() capabilities under Win32

Directory for HEF include files

Used by configure script to generate config.hpp

Dummy header file for configure script

Signature class declarations

Abstract Signature Set class declaration

XML-based Signature Set class declaration

std_DefaultHandler class declaration

String utility function declarations

Binary installation script

Target library directory

Default Makefile

With configure, used to generate Makefile 


\begin{tabular}{|c|c|}
\hline File or Directory & Description \\
\hline$\vdots$ & $\vdots$ \\
\hline software/objs/ & Target directory for object files \\
\hline software/Projects/ & General IDE directory \\
\hline software/Projects/VC6/ & Directory for Visual C ++6 files \\
\hline software/Projects/VC6/HEF/ & Directory for HEF Visual C ++6 files (redundant) \\
\hline software/Projects/VC6/HEF/CSU Normalizer/ & VC6 directory for CSU Normalizer \\
\hline software/Projects/VC6/HEF/CSU Normalizer/CSU Normalizer.dsp & VC6 CSU Normalizer project \\
\hline software/Projects/VC6/HEF/CSU Subspace Engine/ & VC6 directory for CSU Subspace Engine \\
\hline software/Projects/VC6/HEF/CSU Subspace Engine/CSU Subspace Engine.dsp & VC6 CSU Subspace Engine project \\
\hline software/Projects/VC6/HEF/CSU Subspace Train/ & VC6 directory for CSU Subspace Engine \\
\hline software/Projects/VC6/HEF/CSU Subspace Train/CSU Subspace Train.dsp & VC6 CSU Subspace Train project \\
\hline software/Projects/VC6/HEF/HEF.dsw & VC6 HEF workspace \\
\hline software/Projects/VC6/HEF/LibHEF/ & VC6 LibHEF directory \\
\hline software/Projects/VC6/HEF/LibHEF/LibHEF.dsp & VC6 LibHEF project \\
\hline software/src/ & General source directory \\
\hline software/src/CSU/ & CSU source files directory \\
\hline software/src/CSU/csu1face2norm.cpp & CSU/NIST normalization code \\
\hline software/src/CSU/csuEigenTrain.cpp & CSU Eigenface training code \\
\hline software/src/CSU/csuFileCommon.cpp & Common file manipulation code \\
\hline software/src/CSU/csuFisherTrain.cpp & CSU Fisherface training code \\
\hline software/src/CSU/csuMatrix.cpp & CSU Matrix code \\
\hline software/src/CSU/csuSubspaceTest.cpp & CSU subspace training code \\
\hline software/src/CSU/csuSubspaceTrain.cpp & CSU subspace engine code \\
\hline software/src/CSU/cveigen.cpp & CSU eigenvector code \\
\hline software/src/CSU/getopt.cpp & Provides getopt() under Win32 \\
\hline software/src/demos/ & General demonstration code directory \\
\hline software/src/HEF-config.in & With configure generates HEF-config \\
\hline software/src/Signature.cpp & Signature class code \\
\hline software/src/SigSet.cpp & SigSet class code \\
\hline software/src/SigSet_XML.cpp & XML SigSet class code \\
\hline software/src/std_DefaultHandler.cpp & std_DefaultHandler class code \\
\hline software/src/string-utils.cpp & string utility functions code \\
\hline
\end{tabular}

\subsection{Required Software}

HEF-TD has been designed to be platform agnostic — currently, the supported platforms are Linux and Microsoft Windows. In order to build HEF-TD on either system, you will need the following software.

\subsubsection{HumanID Evaluation Framework FRVT-2002 Reference Implementation}

Naturally, to build the HEF-TD, you will need the latest version of the HEF-TD source code. If you do not have it already (i.e., you are reading a standalone version of this document) HEF-TD can be

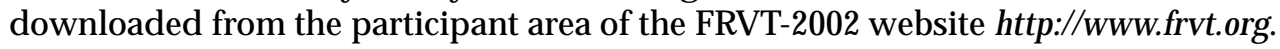

\subsubsection{C++ Compiler Suite}

HEF was designed to work with GCC (the GNU compiler collection) and Microsoft Visual C++. Although GCC is supported, HEF-TD currently cannot be compiled under the Cygwin environment, since Xerces-C++ does not yet support Cygwin.

To build HEF-TD under Linux, you'll need GCC 2.96 or better. Because HEF-TD makes heavy use of the C++ Standard Library (i.e., C++ strings, and templates), you'll need a version of GCC that has fairly mature template support. As of early 2002, most Linux distributions come with a sufficient compiler. If

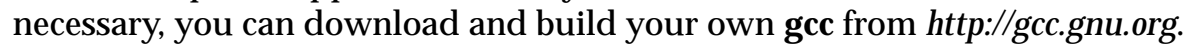

To build HEF-TD under Microsoft Windows, you'll need Microsoft Visual Studio 6 with the components necessary for compiling $\mathrm{C}++$ programs already installed. If you have not done so already, apply Microsoft Visual Studio 6.0 Service Pack 5. The code should also compile under Microsoft Visual Studio .NET, although .NET is not yet an "officially" supported platform. 


\subsection{Xerces-C++ 1.7.0}

The HEF-TD makes heavy use of the Apache Group's free XML parser, Xerces-C++. To build under Linux, you will need the Xerces-C++ 1.7.0 Stable Source Package for Unix's, or xerces-c 1 J 1 .tar.gz. This can be downloaded for free from the Apache Group at $h t t p: / / x m l . a p a c h e . o r g$. For Windows, you will want to download the Xerces-C++ 1.7.0 Stable Source Package for Windows, or Xerces- $c \_1 J \_$.zip. To minimize the potential for link-related problems, it is recommended that you use the same compiler for both HEF-TD and Xerces-C++.

\subsection{Suggested Software}

Although the following are not required to compile and run the HEF-TD, they are recommended if you want to see the original code used for the PCA implementation, and how it was adapted (a.k.a. "hacked") to accept XML input and output.

\subsubsection{CSU Face Recognition Algorithm Source Code}

Although HumanID Evaluation Framework does not include any face recognition algorithms, so that developers can see an example of a working system, in this reference implementation, we have included a version of the Colorado State University PCA implementation which has been modified to work with

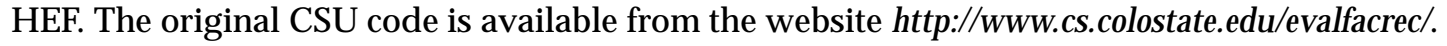

\subsubsection{The FERET Database}

To run the code with the same datasets used in developing and testing this package, you will want a copy of the FERET Database: March 2001 Release. For more information about obtaining your own

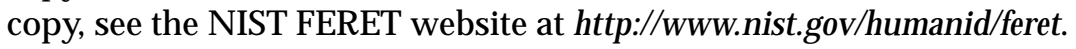

\subsection{Build Instructions}

If you are compiling the HEF-TD for Windows, you can skip directly to the next section (4.6.2). Otherwise, continue on to section (4.6.1). We recommend that you carefully read the installation instructions completely before you begin.

\subsubsection{Linux Build Instructions}

To build HEF-TD under Linux using the GCC compiler, follow these instructions:

1. On your development machine, untar the file HEF-0.99.0.tar.gz in a directory of your choosing. This can be accomplished with the command ${ }^{1}$

ㄴ. tar xvfz HEF-0.99.0.tar.gz

The package will untar into its own directory.

2. Next, you will need to compile Xerces. If you already have Xerces on your system, set the XERCESCROOT environment variable and continue to step 3.

\footnotetext{
${ }^{1}$ The leading "을 represents the system prompt (which on your system, will most likely look different than this) and should not be typed.
} 
(a) Copy the xerces-c-src1_7_0.tar.gz into the HEF-0.99.0/software/import/unix and untar it. (You will need to adjust these commands for your system - below it is assumed that the Xerces tarball is located in your current directory, which may not be the case for your system).

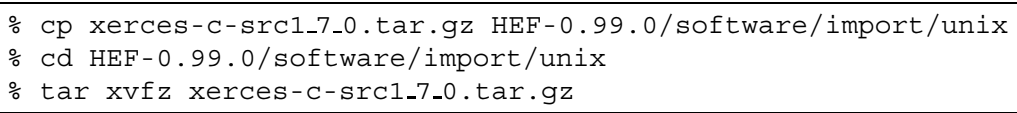

(b) Set your XERCESCROOT environment variable to the Xerces root directory. (Make sure you do this with export (bash) or setenv (csh), so that the configure scripts can see it).

(c) Out of the box, Xerces needs some modification. From the xerces-c-src1_7_0/ subdirectory, change into the $s r c / x e r c e s /$. And run:

\% chmod $755 . /$ runConfigure

\% chmod 755 ./configure

to make make runConfigure and configure executable.

(d) Next, run the runConfigure command with arguments appropriate to your system (run it without arguments to see the complete usage information). On a "typical" Linux system the command

$\div$./runConfigure $-\mathrm{p}$ linux $-\mathrm{c}$ gCC $-\mathrm{x}$ g++

should suffice.

(e) Finally, from the HEF-0.99.0/software/import/unix/xerces-c-src1 $7 \_/$src/xerces/ directory, compile Xerces with

\% gmake

or

make

Xerces suggests using the command "gmake" in case you have a version of make on your system other than GNU make. This will take some time. If Make returns with no errors, then Xerces was probably built correctly. Note that a "make install" is not required.

3. From the HEF-0.99.0/software/directory, run the configure command.

$\%$. / configure

The leading "./" is necessary if "." (the current directory) is not part of your PATH. ${ }^{2}$

4. Finally, to make the example PCA implementation, run

make csu-pca

This will make the libHEF.a library (saved in lib/) and variety of executables in the bin/ directory.

\subsubsection{Windows Build Instructions}

If you are compiling the HEF-TD under Linux, you can skip this section. Otherwise, to build HEF-TD under Windows using Microsoft Visual Studio, follow these instructions: ${ }^{3}$

1. On your development machine, unzip the file HEF-0.99.0.zip into a directory of your choosing. You will be accessing this directory many times, so it is not recommended that you nest it more than one or two levels deep from a drive's root.

\footnotetext{
${ }^{2}$ This Unix feature that is seemingly an annoyance is actually for security. If the current directory is in your path, you might unknowingly be running someone else's malicious executable if you happen to be in their directory.

${ }^{3}$ Note that we use the forward-slash (/) in these instructions, instead of the backslash, ( $\backslash$ ) the "correct," but not strictly enforced, directory delimiter in DOS/Windows.
} 
2. Unzip the file xerces-c_1_7_0.zip into the directory HEF-0.99.0/software/import/Win32/. Afterward, you should have a directory called HEF-0.99.0/software/import/Win32/xerces-c-src1 $7 \_$/ which contains the Xerces- $\mathrm{C}++1.7 .0$ source.

3. Start up Microsoft Visual Studio 6.0. Open the Xerces workspace (xerces-all.dsw), located in HEF0.99.0/software/import/Win32/xerces-c-src1 7 _0/Projects/Win32/VC6/xerces-all/.

4. Within this workspace, you will see a large number of projects. To the XercesLib project, within the workspace panel, right-click on XercesLib and select "Set as Active Project". Build the project by selecting Build xerces-c_1_7_0D.dll from the Build menu. Depending on your system, building the library will take some time. Upon successful compilation, you should see the message

xerces-c_1_7_0D.dll - 0 error(s), 0 warning(s)

under the Build tab of the output panel. This is a good time to build and play with other projects in the workspace. However, for HEF, you will only need to build XercesLib.

5. Close the xerces-all workspace.

6. From Visual Studio, open the HEF workspace (HEF.dsw), which is located in the directory HEF0.99.0/software/Projects/VC6/HEF/.

7. Build HEF-D.lib, the HEF static library. From within the workspace panel, right-click on the LibHEF project and select "Set as Active Project". Build the project by selecting Build LibHEF-D.lib from the Build menu.

8. Build the rest of the software. From the Build menu, select "Batch Build...". Unselect all of the "Release" versions of the projects - there is no real need to build these. Although it is not necessary, you may also want to unselect LibHEF, since it was built already. Make sure "Selection only" is marked (i.e., has a check in it), and select the "Build" button. As part of the build process, the static library file LibHEF-D.lib will be copied to the software/lib/directory, and a variety of executables will be copied into software/bin/.

9. Close the workspace and quit Microsoft Visual Studio 6.0.

\subsection{Run Instructions}

In this section, a full walk-through of a face recognition experiment is given. Before beginning, make sure the that your PATH environment variable includes the HEF-0.99.0/software/bin/directory. Additionally, under Unix, you'll want your LD_LIBRARY_PATH environment variable to include the location of the xerces-c_1_7_0D.so or xerces-c_1_7_0.so library (the latter, if you chose to compile the release version of the library). Under Windows, there is no separation of executable path, and dynamic library path. Your Windows PATH must include a path to the directory containing xerces-c_1_7_0D.dll (or xerces-c_1_7_0.dll). You might just want to copy into C:/Windows/System. In either case, make sure you point to the same library that you linked against during the compilation of HEF.

Here is a command-by-command example run of a "typical" experiment, as run on a Linux system, under the (GNU) bash shell. Under Windows, you can use the cmd shell (with slightly modified commands), or use the bash shell that comes packaged with the Cygwin environments (http://www.cygwin.com).

1. Convert the Imagery (if necessary). If you are working with the original FERET distribution (and not the PGMs included with HEF), then you will need to convert the TIF images to PGMs, so that the normalization code can operate on the images. This can be done with the "convert" command provided by the ImageMagick package. To convert the TIFF file.tiff to a pgm with the name file.pgm use the command:

$\%$ convert file.tiff pgm 
To convert all of the files in a directory, use the bash "for" loop construction:

\% for file in *tif; do convert \$file pgm; done

2. Normalize the Imagery. From this step on, we assume that our current directory is this package's FRVT-2002/FERET/directory, and that we have all of our images, as PGMs, in FRVT-2002/FERET/raw/. In this directory, you should have files FERET-256-1.sigs.xml, FERET-256-2.sigs.xml,coords.512, and m150x130.dat. These are included in the distribution.

To normalize the images, we use the CSU normalization code, which does not yet support XML. We run the command

\% csu-normalizer coords.512 raw norms -m m150x130.dat

where

- coords.512 is the ground truth file provided with FERET,

- raw is the directory containing the PGM imagery,

- norms is the directory to put the normalized images, and

- - m m150x130.dat indicates an image mask file, (also from the FERET distribution).

This generates normalized images, and puts them in the norms/directory. Notice that csu-normalizer tries to normalize every file listed in the ground truth file, and may complain if it does not find a corresponding PGM.

3. Make a copy or symbolic link to the schemas. When using a validating XML parser, the application will need access to the schema documents themselves. If you are running under Linux, then, from the FRVT-2002/FERET/directory, run the command

$\circ \ln -\mathrm{s} \ldots / \ldots /$ documents/xml/0.99.0.

which will create a symbolic link to the XML schema directories. Under Windows, you will need to copy the 0.99.0/directory tree, from documents $/ x \mathrm{ml} / 0.99 .0 /$ to FRVT-2002/FERET. This can be accomplished via the command line, or Windows explorer. If the schemas are not copied or symlinked correctly, then the example implementations will fail to run correctly, since the applications will not be able to properly locate the HEF schema files.

4. Train the algorithm. Next, we need to train the PCA algorithm (i.e., generate the feature space upon which we will project the normalized images). Do this with the command:

\% csu-subspace-train -imDir norms/ FERET-256-1.sigs.xml FERET-256-1.fs

where

- - imDir norms/is a directory (note the trailing forward-slash) where the normalized images can be found,

- FERET-256-1.sigs. $x m l$ is an XML document listing 32 normalized images. (This file actually contains more that 256 norms, but 224 of them have been commented out).

- FERET-256-1.fs is the name of the output file in which the generated feature space will be saved.

The feature space file is used in the next step, where we actually run the algorithm. Generating the feature space can take some time, especially with a large number of images.

5. Run the algorithm. Finally, we can run the PCA algorithm itself. First a "results" directory should be present to store the similarity data. If not:

$\%$ mkdir results 
Then, run PCA with the following command (shown on multiple lines for clarity)

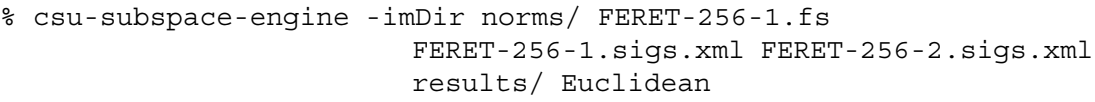

where

- -imDir norms/is the path to the normalized images

- FERET-256-1.fs is the feature space (a.k.a. the output from the training step)

- FERET-256-1.sigs.xml is the target file

- FERET-256-2.sigs.xml is the query file

- results/is a directory where the similarity scores will be written

- Euclidean is the distance metric to use (run csu-subspace-engine without arguments to see more metrics).

Note that in this run, we used the same file for the training, gallery, and query sets, but in a real experiment, this would likely not be the case. The outputs of the experiment are saved in the results/directory.

\subsubsection{About the Code}

There are two major components that were added for accepting XML input. First, is the class std_DefaultHandler (defined in src/std_DefaultHandler.cc and include/HEF/std_DefaultHandler.h). These files are wrappers for the Xerces-C++ DefaultHandler class, but replace signatures that use the Xerces structures (such as XMLCh and Attributes) with the C++ standard library classes string and map. The class SigSet_XML, which inherits from the class std_DefaultHandler and the purely virtual class SigSet, can be used to take an XML-based signature set file, and parse it into a collection of data structures. These data structures, defined in Signature.h, are based on the HEF XML specification.

If you are integrating HEF into your own code (or vice versa) here are a few helpful tips.

- Under both Linux and Windows, it's easy to get paths, binaries, and libraries inconsistent with each other. Most of the time, such errors manifest themselves in the form of either static or dynamic linking problems. Whenever you encounter such a problem, look carefully at your paths, libraries, and versions first.

- Under both Linux and Windows, the order in which files are linked is critical. When linking your executables, make sure that your own object files are first, with the libraries last.

\subsubsection{Known Bugs}

There is a bug in Xerces-C++ 1.7.0 which causes uniqueness constraints in schemas to not always be applied properly. To patch this bug "by hand," then you will need to apply the following diff to the file QName.cpp.

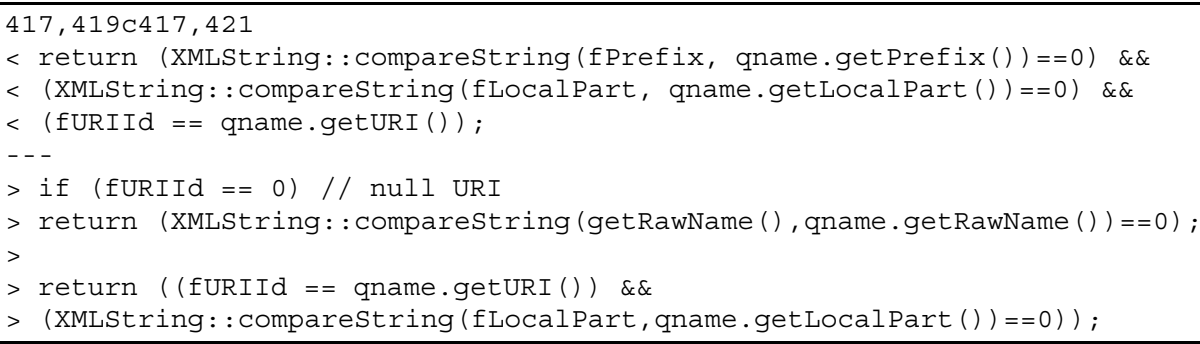


This is not necessary to ensure proper operation of the software, but is recommended if you want to check yourself for duplicate signature name and ids. 


\section{Computational Requirements for FRVT 2002}

\section{Introduction}

This document addresses the significant computational requirements of the FRVT 2002. It is imperative that participants understand the implications of this material as it pertains to their own ssystems. Participants are directed to the form included on the last page: It must be completed by participants and returned to the organizers at frot2002@nist.gov by 17:00 on June 142002.

This document's scope is limited. The correct references for implementers are:

1. The FRVT 2002 Technical Datasets. This collection contains a face recognition system capable of reading and writing the files used in FRVT 2002. It also includes a small extract of the FERET database (256 images) used for demonstration purposes.

2. The logistical aspects of the FRVT 2002, for example times, dates, locations are formally described in the Face Recognition Vendor Test 2002 Test Plan.

The FRVT 2002 tests are extensions of the FERET protocol [2] as used previously in FRVT 2000 [1]. Because the number of images used in the High Computational Intensity test, $\approx 121000$, is an order of magnitude larger than that used in FRVT 2000, the computational loads are significantly larger. Indeed, the amount of computation required grows superlinearly with the number of images, as discussed below. Also the storage requirements are large also.

\section{Timing Model}

The FERET evaluation requires that $N$ pieces of imagery in the target set $\mathcal{T}$ are compared with $M$ pieces in the query set $\mathcal{Q}$. These two sets are not necessarily disjoint i.e. $|\mathcal{T} \cap \mathcal{Q}| \geq 0$

The discussion that follows assumes the use of a modular recognition system comprised grossly of two parts: the first performs image analysis and dimensionality reduction on the two input sets; the second computes pairwise comparisons between those processed features. FRVT 2002 participants should augment this structure as necessary.

Thus the total processing time for any of the FRVT 2002 tests can be modelled as the sum of the times for input and image processing (enrollment), feature comparison, and output.

$$
T(N)=\frac{N}{R_{E}}+\frac{M}{R_{T}}+\frac{N M}{R_{C}}+\frac{N M}{R_{W}}
$$

where

\begin{tabular}{|l|l|r|}
\hline Symbol & Name of Operation & ${\text { NIST's Empirical Rate }\left(s^{-1}\right)}^{\prime}$ \\
\hline$R_{E}$ & target image analysis and enrollment & 1 \\
$R_{T}$ & query image analysis and enrollment & 1 \\
$R_{C}$ & Pairwise comparisons of $k$-dimensional feature vectors & $1.610^{8} / k$ \\
$R_{W}$ & Four byte binary floats output to file & $2.510^{6}$ \\
\hline
\end{tabular}

Note the dependence of the comparison rate on feature space dimensionality, $k$. Note the quadratic nature of equation 1. Given the very large rates for the quadratic terms it is clear that for small $N$ and $M$ the expensive enrollment process dominates the total time. But as $N$ increases the quadratic terms eventually dominate. The graph in 1 plots the linear enrollment times, the quadratic components, and 


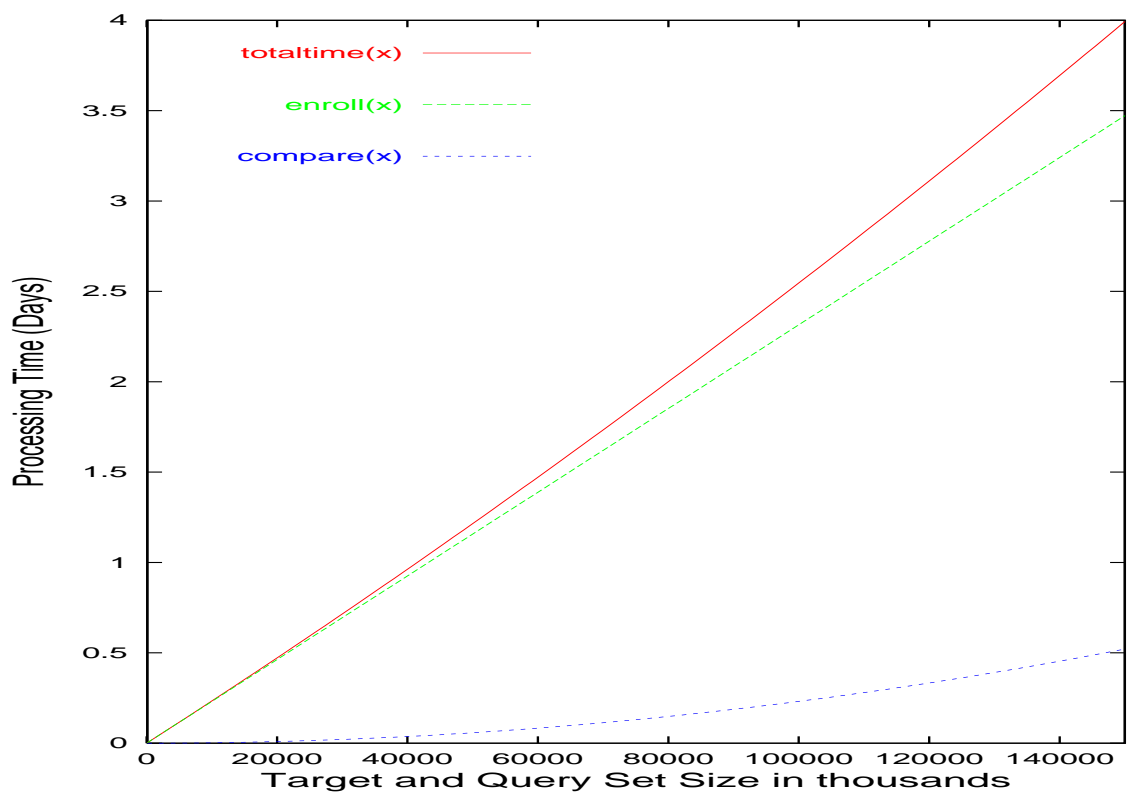

Figure 1: Processing times as a function of the target set size.

the total time. With our rate parameters the quadratic matching parts would exceed the enrollment times with $N \approx 1000000$.

The two enrollment figures in the final column were based on estimates from commercial products, and eye detection software running in-house. The computation rates were obtained using a short $\mathrm{C}++$ program, compiled with $\mathrm{g}++-\mathrm{O} 3$, running under linux 2.4 .7 on a $\mathrm{PC}$ equipped with a $1.5 \mathrm{GHz} \mathrm{Xeon}$ Pentium III and 1GB memory. The feature comparison numbers were obtained using a plain $L 2$ distance metric on feature vectors of dimension $k=256$.

Example: For the High Intensity test in which $N=M=125000, k=256$ and the rates are those shown above, the resulting total time is

$$
\begin{aligned}
T & =2 \frac{125000}{1}+125000^{2}\left(\frac{256}{1.610^{8}}+\frac{1}{2.510^{6}}+\right) \\
& =2.510^{5}+3.1310^{4} \mathrm{~s} \approx 3.26 \text { days }
\end{aligned}
$$

This figure is well within the 11 days allowed for the tests.

Note in particular that the output time is considerable and the code is not simply CPU bound. Note also that the leading factor of 2 can be ignored if the target and query sets are identical.

\section{Storage Model}

The storage needed for FRVT 2002 is broken down into three parts. Storage in this context refers to either disk space or memory depending on the architecture of the system.

1. Input: The raw imagery supplied to vendors will not exceed 5 GB.

2. Working Space: If a system typically generates templates that persist then the storage requirement is likely linear in the size of the target set, $|\mathcal{T}|$ 
Example: If the enrolled template from $\mathcal{T}$ is a $k=256$ dimensional vector then for the High Computational Intensity test with $N \approx 121000$ the target features would require $236 \mathrm{MB}$ of main memory, assuming an eight byte double precision representation.

3. Output: The protocol requires that a vendor produce similarity files that contain the $N M$ realvalued distance measures. This information must be written in a basic binary format. It is not clear that compression of this output will yield any significant reduction in storage. Thus any use of zip (or tar $-z$ ) is useful only as a bundling program.

Example: Given $N=M \approx 121000$ the the complete result will occupy about 58.6 gigabytes.

\section{Caveats}

1. System Architecture: The above models will not apply if the architecture of a system does not decompose the problem into separate enrollment and feature comparison parts. For example, a particularly naive system making $N M$ calls of an SDK function f(image1, image2) would embed $N M$ enrollments and be very expensive; using example numbers, $T(125000)=500$ years!

2. Operating System Dependencies: The empirical rate estimates quoted above are subject to measurement error. Particularly the disk IO is difficult to measure because the the operating system buffers and caches certain operations, and because of the underlying hardware (the RAID-1 striped partition used in the example above is substantially faster than a standalone SCSI disk).

3. Restart: If for any reason a recognition system ceases processing this could effect seriously effect times. Because our computations above only simulate an FRVT 2002 test without actually doing it, we cannot assert that uninterrupted processing is possible. Accordingly a mechanism for restarting an imcomplete test might be needed.

4. Video: Our simulated times above assume the existence of single-file signatures, and so does not include any overhead associated with the processing of the multi-file still-set and video signatures as defined in the FRVT 2002 Implementer's Guide. This time could be considerable. See the FRVT 2002 online FAQ for approximate numbers of images in multi-file signatures.

\section{Conclusion}

The above models may be inappropriate for various reasons including and in addition to those given in 4 Accordingly vendors should assess the computational requirements of their own systems and bring sufficient CPUs, machines, and memory so as to complete the test in the alloted time. Certain limits on hardware are described in the FRVT 2002 Test Plan.

\section{References}

[1] D. Blackburn, M. Bone, and P. J. Phillips. Facial recognition vendor test 2000. Technical report, 2001.

[2] P. J. Phillips, H. Moon, S. A. Rizvi, and P. J. Rauss. The feret evaluation methodology for face recognition algorithms. IEEE Trans. Pattern Analysis and Machine Intelligence, 22:1090-1104, 2000. 


\section{FRVT 2002 : Statement of Competence}

We have received a large number of applications for participation in FRVT 2002, most of whom will take all tests. The FRVT 2002 Evaluation Team requests you review the data in the document above, Computational Requirements of the FRVT 2002, to gain an understanding of the processing and storage resource requirements associated with each FRVT 2002 test. Based upon this information, you may decide to modify your participation request. The deadline for withdrawl from FRVT 2002 without it being noted in the final report is June 14 2002. Our goal is to ensure that you are able to complete each test that you have applied for.

Participants must submit this page, duly completed with information requested, to the Evaluation Team at frvt2002@nist.gov by 17:00 EDT on June 142002.

\begin{tabular}{|l|l|}
\hline Value & Name or Value \\
\hline Operating System + Version & \\
\hline Number and Speeds of Processors & \\
\hline Machine Memory (MB) & \\
\hline USB Connectivity (Type 1, 2) & \\
\hline $\begin{array}{l}\text { Disk type and capacity (GB) } \\
\text { (IDE, SCSI, other) }\end{array}$ & \\
\hline $\begin{array}{l}\text { Implmentation Name (Marketing or } \\
\text { or Algorithm name) }\end{array}$ & \\
\hline $\begin{array}{l}\text { Software Language } \\
\text { (C, C++, ADA etc.) }\end{array}$ & \\
\hline $\begin{array}{l}\text { Feature Space Dimension } \\
\text { (if appropriate) }\end{array}$ & \\
\hline $\begin{array}{l}\text { Time to enroll an image } \\
\text { (in seconds) }\end{array}$ & \\
\hline $\begin{array}{l}\text { Time to compare two enrolled } \\
\text { feature sets (in microseconds) }\end{array}$ & \\
\hline $\begin{array}{l}\text { Time to write 500 KB binary file } \\
\text { Name of person filing form }\end{array}$ & \\
\hline Date & \\
\hline
\end{tabular}




\title{
The HumanID Evaluation Framework
}

\author{
Version 0.99.0 \\ Initial FRVT-2002 Technical Dataset Release
}

Patrick Grother Ross J. Micheals P. Jonathon Phillips

National Institute of Standards and Technology

Information Access Division, Image Group

Gaithersburg, MD 20899

May 30, 2002 


\section{Chapter 1}

\section{Introduction}

The HumanID Evaluation Framework, or HEF, is a mechanism for the quantitative testing of biometric recognition systems. It is an extension of the FERET protocol, which defined a framework for the evaluation of face recognition technologies. The HEF, however, is general enough to apply to virtually any recognition task, and can be applied to arbitrary, heterogeneous mixtures of biometric systems.

The goal of the HEF effort is to design, implement, and deploy standards for the robust and complete documentation of the biometric system evaluation process. A mandate of the scientific method is experimental repeatability - achievement of this goal in the computer science and electrical engineering field is often thwarted by, among other things, inadequate documentation of experimental procedures.

This initial release of HEF is coupled with the 2002 Face Recognition Vendor Test, or FRVT 2002. Although FRVT 2002 has played a key role in shaping the current form of the HEF, it should be noted that they are separate, but related efforts. FRVT 2002 provides a unique opportunity for the development and implementation of an "initial" version of HEF. The lessons learned here will help determine the future shape of the HEF.

The primary focus of HEF is the documentation of the input test suites and output recognition hypotheses, and not the algorithms embedded within a particular recognition system. In various domains (character recognition, fingerprint matching, automatic target recognition), much effort has been put into the gathering of standard training and testing suites that facilitate algorithm development and subsequent evaluation. The use of test data for empirical testing is widespread in the scientific literature. The HEF is an attempt to leverage contemporary technologies for the formal description of such tests. Accordingly, the HEF defines a suite of XML-based markups for the inputs to, and outputs of, recognition systems.

The HEF is designed to facilitate black-box empirical testing. A recognition engine takes two sets of biometric signatures, the enrolled and the "unknown" test samples, and produces some form of identification data. Currently, the HEF assumes that this output data is a collection of scores, with each score indicating the similarity between a pair of signatures.

\subsection{About this Document}

This document is structured as follows. Chapter 2 discusses the mark up of signature sets - or the inputs to an evaluation. Chapter 3 discusses the mark up of similarity scores, or the outputs of an evaluation. Finally, in Chapter 4 instructions on how to build and run the example implementation are given.

This document assumes familiarity with software development, biometric systems, XML, and XML Schemas. An excellent introduction to XML Schemas is the XML Schema Primer, available from http://www.w3.org/TR/xmlschema-0/.

Throughout this document, we may use the following shorthand phrases and terms: 
- The term schema refers to an XML Schema document. The term instance document refers to an XML document that conforms to the constraints defined by some fixed schema. An instance document is "valid", or will "validate successfully" if it meets the constraints set out by a particular schema. Because an instance document might be valid under multiple schemas, or may validate with different success under different schemas, validation will often be referred to as "with respect to" or "under" a particular schema. Schemas themselves may be referred to by schema name (e.g. HEF Similarity Set Schema) or file name (e.g. "similarity-set.xsd").

- The phrase "a valid $\langle x\rangle$ (element) contains $n$ valid $\langle y\rangle$ (elements)" should be considered equivalent to, "a instance document that contains an $\langle x\rangle$ element and does not have $n$ children tags of type $\langle y\rangle$ will not successfully validate." With XML Schema, having the proper number of child elements is always a necessary, but not always sufficient, condition for successful validation.

- When referring to attributes, the phrase "attribute $a$ is a $b$ " should be considered equivalent to "an instance document that contains a $a$ attribute, and is not of type $b$, will not successfully validate." Unless specified otherwise, types (such as "boolean, "token," or "double") are as defined by the XML Schema specification, located at http://www.w3.org/XML/Schema.

It is important to note that schema validation enforces only the constraints encoded in the schema. An application may have its own set of constraints that are not reflected in the schemas. For example, a schema might enforce that a "sorted" attribute take on a "descending," "ascending," or "unsorted" value, but an application might enforce that they are actually sorted.

Participants are also directed to the following related documents:

\section{Versions}

This document describes the 0.99.0 release of the HEF FRVT-2002 Technical Dataset Release. For major and minor version numbers, the following version convention is proposed

\begin{tabular}{rrl} 
Major & Minor & \\
\hline 0 & 98 & FRVT-2002 Internal Release \\
0 & 99 & Initial FRVT-2002 Release with Request for Comments \\
1 & 0 & Final FRVT-2002 Release \\
1 & 1 & Initial Public Release
\end{tabular}

For example, the first internal release will be version 0.98.0, the third revision of the Final FRVT-2002 Release will be 1.0.2, and so on.

\section{Contact Information}

Since this initial release of HEF is in conjunction with FRVT 2002, there are some unique rules that govern the contact information regarding this version of the HEF and the FRVT 2002 Technical Datasets. The primary and preferred source of communications between participants and FRVT 2002 government personnel is the FRVT 2002 Frequently Asked Question (FAQ) page, which is located off of the FRVT 2002 website, $h t t p: / / w w w$ frot.org. A government employee has been named as the FRVT Liason. The FRVT Liason will be the primary point of contact and will manage communications with participants. Questions that are outside the purview of the FAQ can be submitted to the FRVT Liason via email at frot2002@nist.gov. All e-mails sent to the FRVT Liason and germane to the FRVT 2002 will be posted on the FAQ page. This policy ensures all participants are guaranteed equal access to information concerning FRVT 2002. Contact with the FRVT 2002 Liason other than via the FAQ areas, or contact with someone other than the non-FRVT Liason will only be allowed under extenuating circumstances. 


\section{Chapter 2}

\section{Signature Sets}

A primary goal of the HEF is to have a well-defined means of marking up sets of biometric data. This section describes the markup of "signature sets," which list the biometric data from a group of individuals. For greater flexibility, HEF provides not a single schema, but a family of schemas that may be used to validating different kinds of signature sets. Using a set of schemas allows applications to validate signature sets using domain-specific criteria, while still maintaining a high-level, or "global" consistency.

\subsection{Terminology}

In the HEF model, the following terminology describes biometric information at different grouping levels. Each human subject of interest is an individual. A collection of biometric data for a single individual makes up a signature. A collection of signatures constitutes a signature set.

For a given individual, a particular biometric recording event corresponds to a sigmember. An event in this context is typically a time-localized period during which the subject is imaged. Since a subject may have many biometric recordings, a single signature can contain one or more sigmembers. For example, in the FERET database, there are images of some subjects taken several months apart. In this case, each image is a different sigmember. In the general case a signature will be comprised of sigmembers that contain heterogenous biometric data; for example, a fingerprint and a mugshot.

A sigmember, or recording event, could also contain one or more data components. For example, a stereoscopic video might consist of two (simultaneously captured) video sequences. A dataset corresponds to a logical component of a biometric recording. It follows that a sigmember may contain one or more datasets. The precise definition of a dataset is expected to change according to the mode of biometric. For most biometrics, however, a single dataset is often sufficient.

Under the HEF, it is assumed that for each dataset, there exists a set of one or more files containing the raw biometric data of interest. Therefore, each dataset may contain one or more files. Each file corresponds to a data file that contains biometric data. Note that the HEF does not attempt to restrict the permissible file formats (JPEG, PNG, MPEG, AVI, VRML, MP3 etc) in any way.

\subsection{Detailed Example}

To illustrate the above terminology, consider the following examples. Suppose we have biometric data on three different subjects, and we wish to create a structured document that describes this data. 


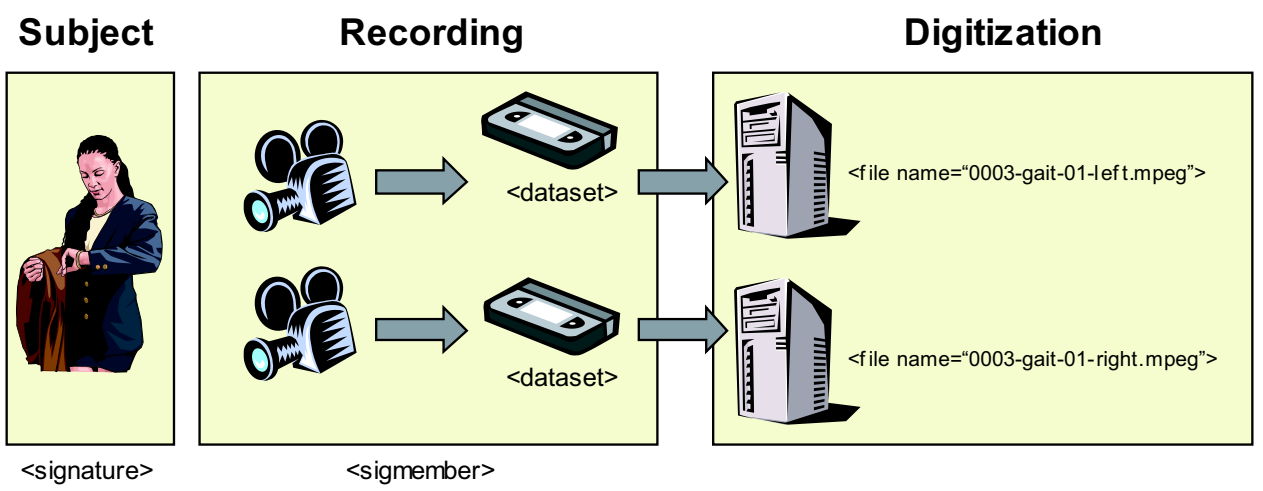

Figure 2.1: An illustration of the differences between subject 0003's signature, sigmember, dataset, and file elements.

- Subject 0001. Suppose the data to be used for subject 0001 is a single facial image. The signature for subject 0001 has a single sigmember, with a single dataset, with a single file that contains an image of subject 0001's face.

- Subject 0002. Suppose the data to be used for subject 0002 is a short video clip. The video, however, is not stored in a single file, but as a collection of five individual frames or images. The signature for subject 0002 has a single sigmember, with a single dataset, with five files that each contain a different frame. For this subject, there is only one sigmember since the video clip is from a single recording event. There is also only one dataset, since the individual frames, are part of the a larger logical component - the "video."

- Subject 0003. Suppose the data to be used for subject 0003 includes an iris scan, three facial images each taken on different days, and a stereoscopic gait video. The signature for subject 0003 therefore contains five sigmembers (one for the iris scan, three for each facial image, and one for the gait video). For the first, iris scan, sigmember, there is a single dataset with a single file that contains the iris data. Three sigmembers, for the facial imagery, each have a single dataset, each with a single file that contains facial images. The fifth sigmember (the gait video) has two datasets - one for each video. The datasets would each have a single file if the data was encoded in a single video (such as an MPEG), or would have a collection of files, where each file corresponded to a particular frame. See Figure 2.1 for an illustration.

In Section 2.2.1, we describe an example document marking up the biometric information for these three synthetic signatures. After the overview, we give a detailed treatment of each element and attribute.

\subsubsection{Document Overview}

Figures 2.2-2.3 shows an example of how one would mark up the above information using the HEF signature set schemas. The non-whitespace lines have been numbered so that we can easily reference the different parts of the document - a real signature set document would not have them.

The first line of the document is a standard XML header, used to indicate that the document is indeed a (version 1.0) XML document. If there is any whitespace before this header, then the instance document would not validate sucessfully.

Lines 2 through 5 and line 60 compose the opening and closing root elements of the document. The <signature-set> element also contains several attributes used for XML namespace "bookkeeping". We will briefly describe them, but for most signature sets, copying these lines verbatim will most likely be sufficient - it is not necessary to completely understand them. On line 2, the $x \mathrm{mlns}$ attribute defines the "target" namespace of the document. This associates the elements of the document with the string, or 
"namespace" http://www.nist.gov/humanid/hef/xml/0.99.0. On line 3, the xmlns:xsi attribute associates the

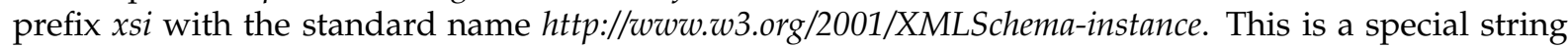
"understood" by XML parsers which allow the prefix $x$ si to be used to access XML Schema elements and attributes. Lines 4 and 5 indicate the location of the signature set schema. It is a pair that consists of a namespace (which matches the $x m l n s$ value) and the schema filename itself. For most XML parsers (like Xerces), if the schema filename is indicated with a relative path (such as line 5 in our example), then the base path is the directory in which the instance document is located, not the working directory of the application. For example, if our example instance document was located in /usr/local/HEF/example, then the XML parser would expect to find the schema in /usr/local/HEF/example/0.99.0/sigset-schemas/sigsetunrestricted.xsd.

Lines 7 through 13 describe the biometric information for the first subject - subject 0001 . This signature contains a single sigmember (lines 8 and 12), a single dataset (lines 9 and 11), and a single file (line 10). As indicated by lines 8 and 9 , the data is a JPEG facial still.

Lines 14 through 25 describe the biometric information for the second individual. Subject 0002's signature consists of a single video recording, their signature contains a single sigmember (line 15, 16, and 24), and a single dataset (lines 17 and 23). Each frame of the video clip, however, is stored in its own file, and therefore, the dataset (line 17) contains multiple file elements (lines 18-22).

Lines 26 through 59 describe the biometric information for the third subject - subject 0003 . Recall that for subject 0003 there are five separate recordings, and therefore, five separate sigmembers. Lines 27-31 are a sigmember for the iris recording information. It contains a single dataset (lines 28 and 30) and a single file (line 29). Lines 32 through 47 mark up the next three sigmembers. Since they are face stills, they are similar in structure to subject 0001's sigmember (lines 8-12). We reiterate that because they are stills taken at different times - i.e., they are different recordings - each face still is a different sigmember (as opposed to different datasets or different files). Finally, lines $48-57$ describe the stereoscopic gait video. There is only one sigmember (lines 48-57) since both videos were taken during a single biometric recording event. Each video, however, is a logical component of this recording, and therefore each corresponds to a dataset element (lines 49-52 and lines 53-56). The files themselves (one for each video) are marked up in lines 50-51 and lines 54-55).

\subsection{Document Structure}

Because of the wide variety of biometric systems, and the varying nature of the constraints that a recognition system may want to apply on a set of signatures, the HEF includes a family of related schemas that can be used "as is" for face recognition systems, or easily extended to accommodate new ones. HEF uses derived types (see Section 2.3.3) as its main vehicle for accomplishing this kind of flexibility.

Although the HEF schemas will ultimately be available online, they have been designed primarily for standalone operation (no network or web client/server access). The sigset-general.xsd schema is the main schema which defines the base and abstract types used in different signature sets. Because it contains abstract types it is not meant to be used directly by an instance document. In this section, unless otherwise specified we are referring to the schema defined by sigset-unrestricted.xsd, which is a relatively unrestricted, non-abstract redefinition of sigset-general.xsd. This schema is also be referred to in this document, and in the schema files themselves as the HEF Unrestricted Sigset Schema.

The structure of an HEF signature set document follows directly from the above grouping terminology. Each term corresponds to a tag, and the containment relationship is represented by the nesting of elements. For example, since a signature set contains multiple signatures, the <signature-set $>$ may have one or more <signature > elements as child elements - a <signature > element may have one or more $<$ sigmember $>$ elements as children - and so on.

In the remainder of this section we detail the role of each element in a signature set document. To aid understanding, we will incrementally recreate the example signature set, one element hierarchy at a time. 


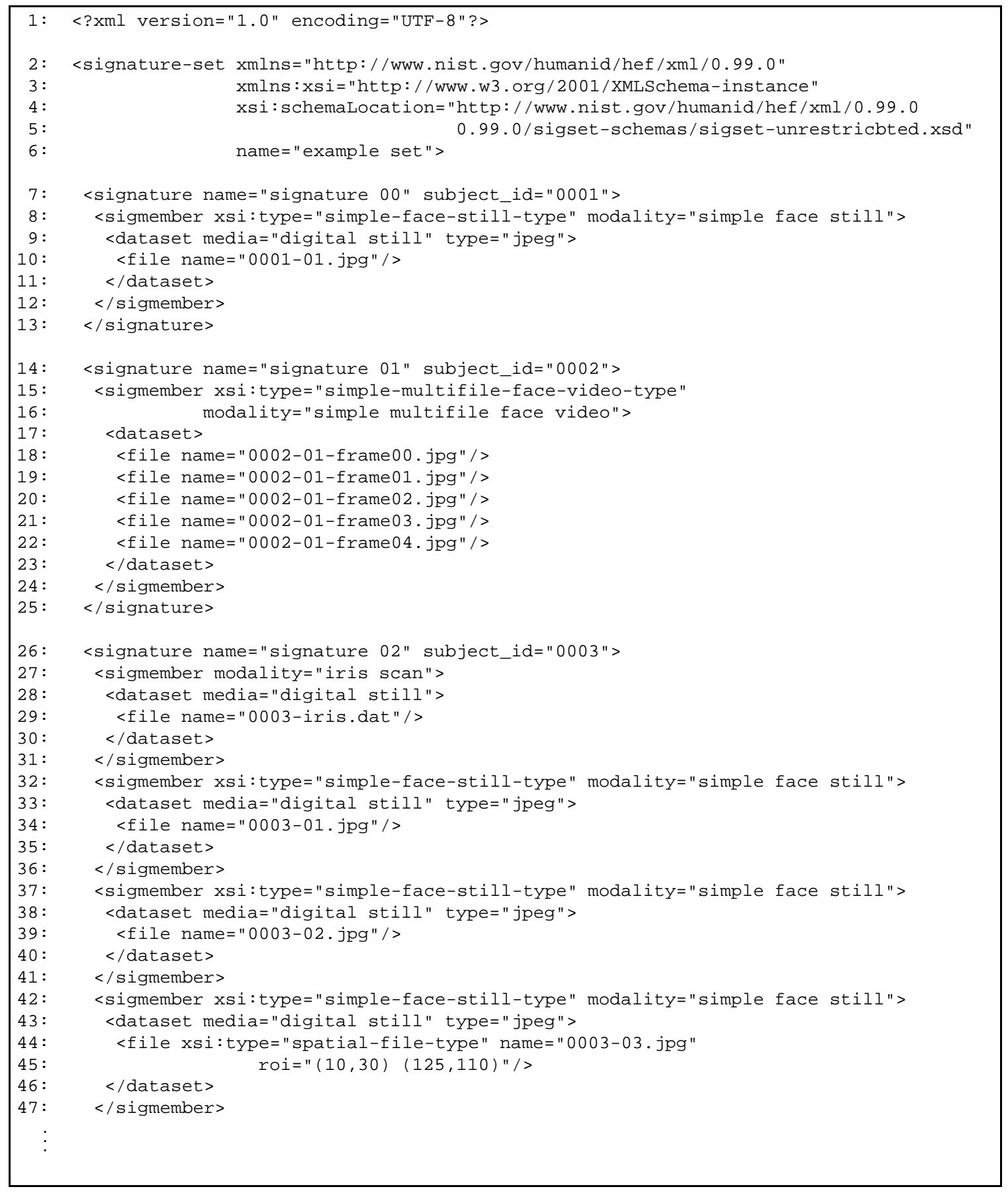

Figure 2.2: The completed XML markup of the example data (continued in Figure 2.3). 


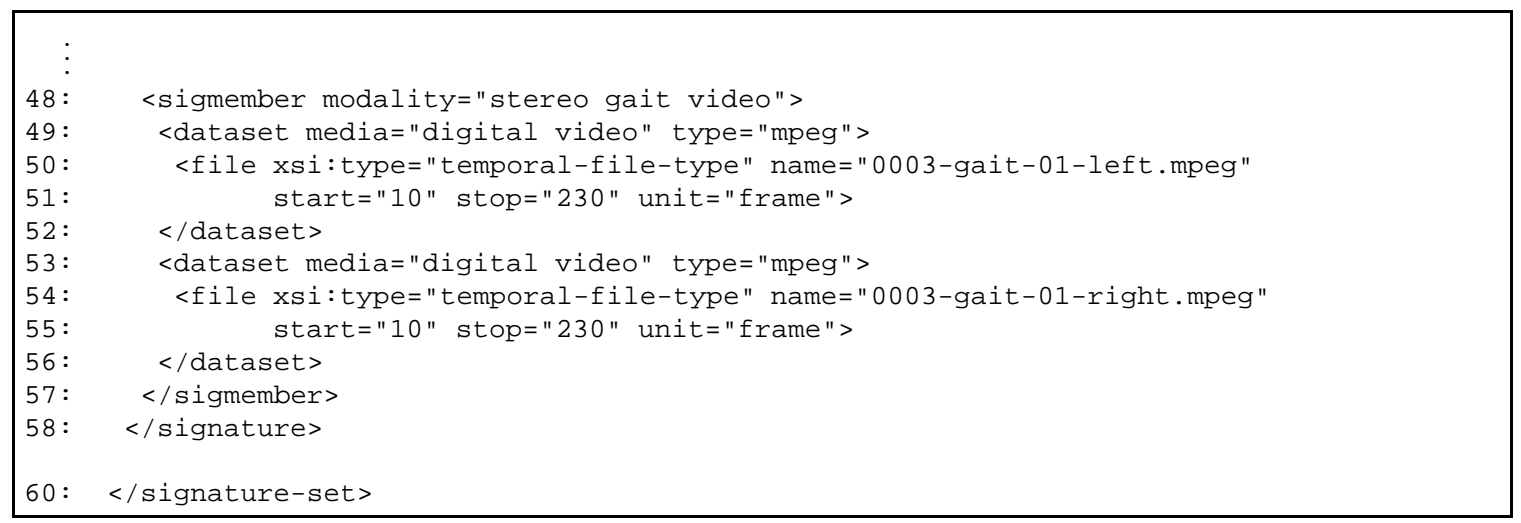

Figure 2.3: The completed XML markup of the example data (continued from Figure 2.2).

\subsubsection{Signature Set Elements}

The root tag of every valid signature set document must be <signature-set $>$. The optional name attribute is a token that may be used to name the signature set, so that an application can refer to a particular signature set by using its name, as opposed to its filename, or other characteristic. A valid <signatureset $>$ must have one or more valid child < signature $>$ elements. If a < signature-set $>$ does not have at least one < signature $>$ child element, then the document will not validate successfully.

\subsubsection{Signature Elements}

Each <signature > corresponds to a collection of biometric information for a single individual. Each signature must have a unique name which is a token that can be used to refer to a particular signature. An optional attribute subject_id is a token associated with a particular subject, as opposed to a signature. It is important to understand the difference between name and subject id. Given the nature of an evaluation, the name may or may not contain information about the subjects true identity. In an external evaluation, there may be a need to hide a subjects identity from a recognition system, and it would be expected that the subject_id attribute not be provided. However, there is still a need to provide handles for specific signatures - this is what the name attribute is for.

To ensure that both signatures and individuals can be referred to without ambiguity, within a single signature set file, no pair of <signature > elements may share the same name value. A valid <signature > contains one or more valid <sigmember $>$ elements. The enforcement of uniqueness constraints across different files is an option of an application.

Figure 2.4 is an example of a valid use of <signature-set $>$ and <signature $>$ tags based on the example signatures discussed in Section (2.1). In these examples, an ellipsis (...) represents information to be added later - they are not actually contained in the instance document itself. Since our example uses three different subjects, the root signature-set element has three signature children elements. Notice how each subject has its own set of signature tags, and each signature has its own unique name and subject id.

\subsubsection{XML Schema Types}

This subsection provides some background material on XML Schemas that will aid understanding of the nature of certain HEF XML constructions. If you have experience using and writing XML Schemas, you may want to skip to the next section.

A feature of XML Schemas is that each element and attribute is associated with a particular type. This allows the validation process to enforce type constraints on instance documents, much as a compiler enforces type constraints on source code. For example, in an XML Schema specifies that all elements 


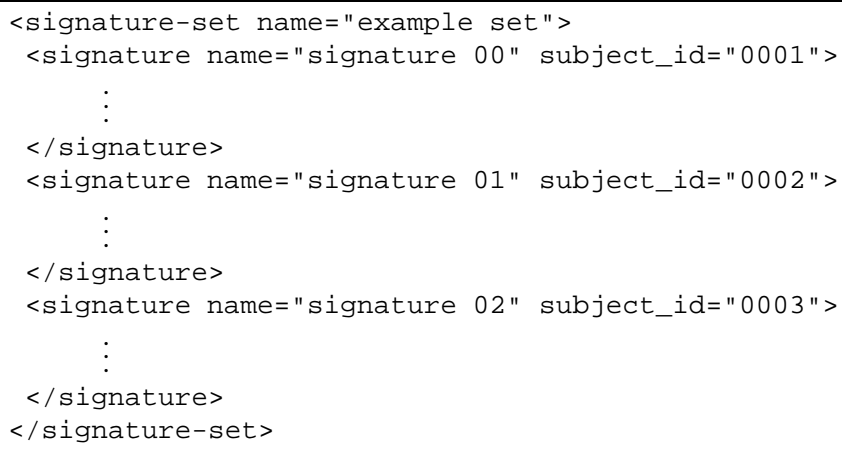

Figure 2.4: A valid nesting of <signature-set $>$ and $<$ signatures $>$.

must be of type decimal, and an instance document contains the fragment " $<$ temperature $>$ Herr Dr. Merkwuerdigichliebe </temperature $>$ " will clearly not validate successfully, since "Herr Dr. Merkwuerdigichliebe" is not a decimal value, such as "37.0".

Types in XML Schemas are not limited to such simple schemes, however. Users can generate their own complex types, create type hierarchies, and redefine new types as extensions or restricted versions of other types - much like the object oriented concept of inheritance. In XML Schemas, the "parent" or "superclass" is referred to as the base type, and the "derived", "child", or "subclass" is called the derived type.

Consider the following example (adapted from the XML Schema Primer). We start with the compound address-type type:

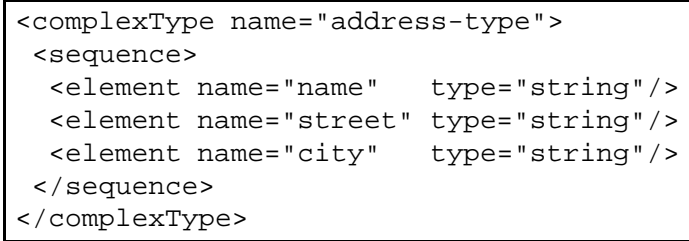

We can then use address-type as a base class for a new derived type us-address-type, which extends addresstype by including a "state" and "zip" field.

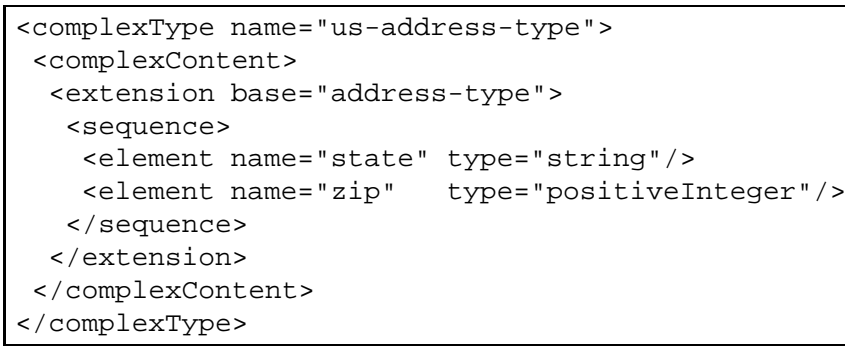

The type us-address-type is an "address derived type" of base type address-type. It has "name," "street," and "city," elements, just as address-type, but "state" and "zip" elements as well.

With XML Schemas, the association of element names and types does not occur automatically. For example, to have an element $<$ bill-to $>$ of type address-type, within a schema, we must explicitly tie them together. Because of this lack of implicit coupling with XML Schemas, in some cases, it is possible to associate multiple types with the same element, where the nature of the enforced type changes according to some explicit instruction in an instance document, or because of the context of its occurrence. We will not show how to accomplish this here. Instead, we will show how to indicate which type an element 
should be validated against, in the case there an element could be associated with potentially multiple, and therefore ambiguous, types.

To explicitly indicate that an element should be validated with respect to a specific type, one simply needs to set the xsi:type attribute to the name of desired type. The xsi:type is a special attribute shared by all XML elements, and it not parsed in the same way user-defined attributes are - it is used by the parser to indicate that a different type should be used for validating a particular element.

For example, suppose we had an XML document where the <bill-to $>$ element is of type address-type. The following fragment would be valid XML

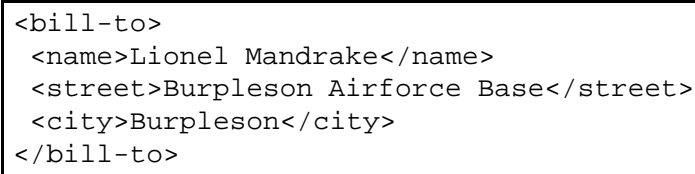

However. the fragment

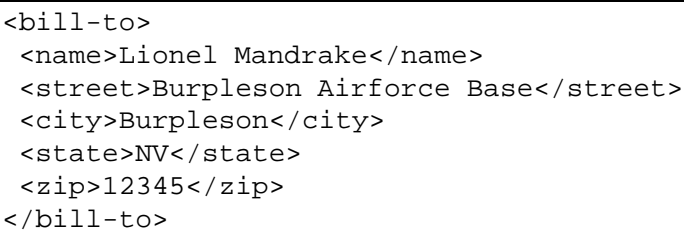

would not validate successfully, since $<$ state $>$ and $\langle z i p>$ are only appropriate elements for $u s$-addresstype, and not address-type. However, by using the xsi:type attribute, we can indicate to the parser that the us-address-type should be used, instead of the address-type. This is valid, since us-address-type is of base type address-type, which satisfies the original schema requirement that $<$ bill-to $>$ be of type address-type. The fragment

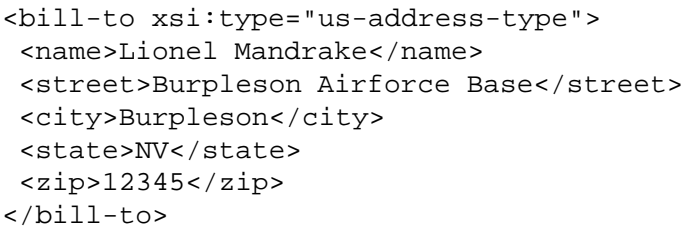

would now validate successfully. Again, note that in the xsi:type is defined in neither address-type nor us-address-type - it is a "global" attribute common to all XML schema elements.

\subsubsection{Sigmember Elements}

Each $<$ sigmember $>$ corresponds to a particular biometric recording event - the process of collecting new biometric information about a subject. There are two kinds of <sigmember $>$ types. First, a <sigmember $>$ element may be of the base type sigmember-type - a generic type that can be used to describe a recording of arbitrary mode. The other types are defined as restricted derivations of sigmember-type. Currently, there are three such types, simple-face-still-type, simple-face-video-type and simple-multifile-face-video-type.

- The simple-face-still-type is designed to be the preferred type for describing facial images stored in a single data file. Data that might be described with this type include digital stills, or scanned photographs.

- The simple-face-video-type is designed to be the preferred type for describing facial video that is stored in a single data file. An MPEG video clip might be described with this type. 
- The simple-multifile-face-video-type is designed to be the preferred type for describing facial video stored in multiple data files, typically one image or datafile per frame of video.

All of these types are accessed through the use of the <sigmember $>$ tag, but require the use of the special attribute xsi:type to indicate, to the XML parser, that they are not of the base type <sigmember-type $>$, but of a specified derived type. Typically, the derived sigmember types versions of the base type sigmembertype, but with additional constraints. If the derived types are insufficient for describing your own biometric data, either the base type sigmember-type can be used, or a new derived type could be written.

The required modality attribute describes the modality of the recording. Do not confuse this with the xsi:type attribute, which indicates to the XML parser, the proper derived type to use when validating the document. Typically, the restricted derivations of the sigmember-type (e.g., simple-face-still-type) will require that the modality attribute take on a specific value. Note that in an XML file, there is no implicit typecasting when setting the modality attribute to the value required in the derived sigmember. To validate according to the derived type, both xsi:type and the modality attribute are required. It is highly recommended that whenever possible, you use reserved/predefined type values only when using derived sigmember types.

All <sigmember > elements may also use the optional metadata attribute. This attribute is a token that an application can use to reference recording meta-information, such as sensor information, persons involved in the data collection, contact information, and so on. Although it is recommended that the value for the metadata attribute be used for filenames, there is no requirement to do so.

Returning to our common example, Figure 2.5 shows the preferred way of describing our example biometric data with <sigmember $>$ tags. For subject 0001, we choose to use the simple-face-still-type. Notice how both that xsi:type and modality attributes are included. The xsi:type indicates to the parser (in the same way that a pragma indicates to a compiler) that this particular sigmember is of derived type simple-face-still-type, and the modality indicates to our application that the sigmember is a simple face still. The simple-face-still-type is defined in such a way that the document will not validate successfully if the modality attribute does not have the value "simple face still." Due to limitations in the current version of XML schemas, setting the modality to "simple face still" cannot lead to coercion of the element from the base type sigmember-type to the derived type simple-face-still-type. Therefore, as mentioned previously, to avoid confusion, setting the value of modality to "simple face still" when using the default type sigmember-type is not recommended.

\subsubsection{Dataset Elements}

Every valid < sigmember $>$ must contain at least one $<$ dataset $>$, where each $<$ dataset $>$ corresponds to some logical component of a biometric recording. Naturally, all <dataset $>\mathrm{s}$ with a common parent should correspond to the same biometric recording. The precise definition of dataset is expected to change according to the mode of the biometric. For most biometrics, such as a facial image still, it is expected that a $<$ sigmember $>$ has a single $<$ dataset $>$. It is anticipated that more complex recordings would contain more than one <dataset $>$ (for example, one dataset per camera in the case of stereoscopic video). Until more derived sigmembers are added to HEF, it is expected that HEF users will define their own conventions for determining the nature and quantities of datasets for their own biometric recordings.

There are two optional attributes that may be used with the < dataset $>$ element. The media attribute is a token which describes the media of the original recording — "digital still", "35mm film", or "Hi-8 video" for example. The datatype attribute is a token which should be used to describe the format of the recorded data - "JPEG", "MPEG", "TIFF", and so on. Like most of the optional attributes, there is no strict convention for the values of media and datatype.

Figure 2.6 shows the proper nesting of $<$ dataset $>$ tags for our common example. Notice that except for the stereoscopic gait video, all < sigmember $>$ s have a single $<$ dataset $>$. 


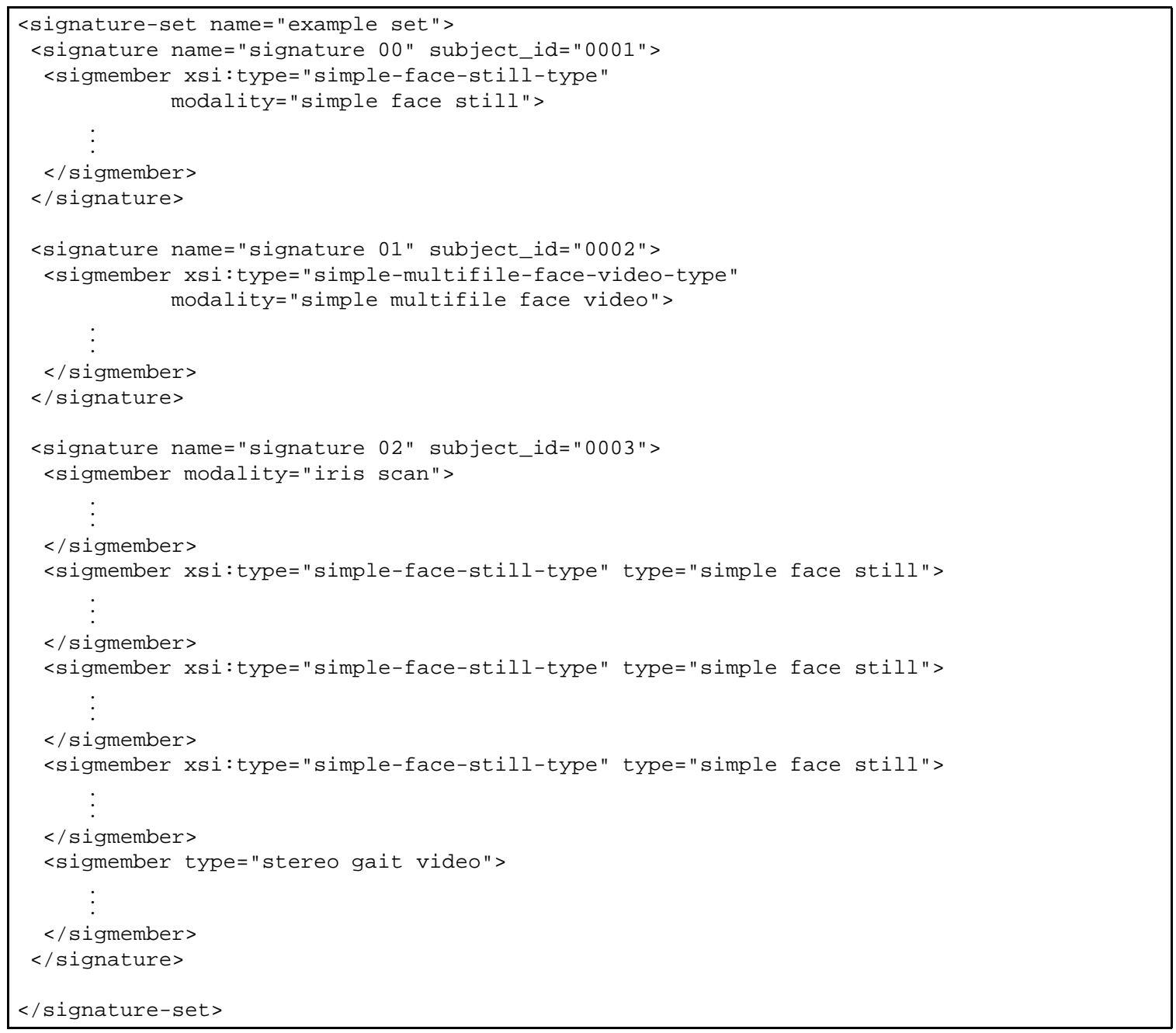

Figure 2.5: A valid nesting of <signature-set $>$, <signature $>$ and $<$ sigmember $>$ tags. 


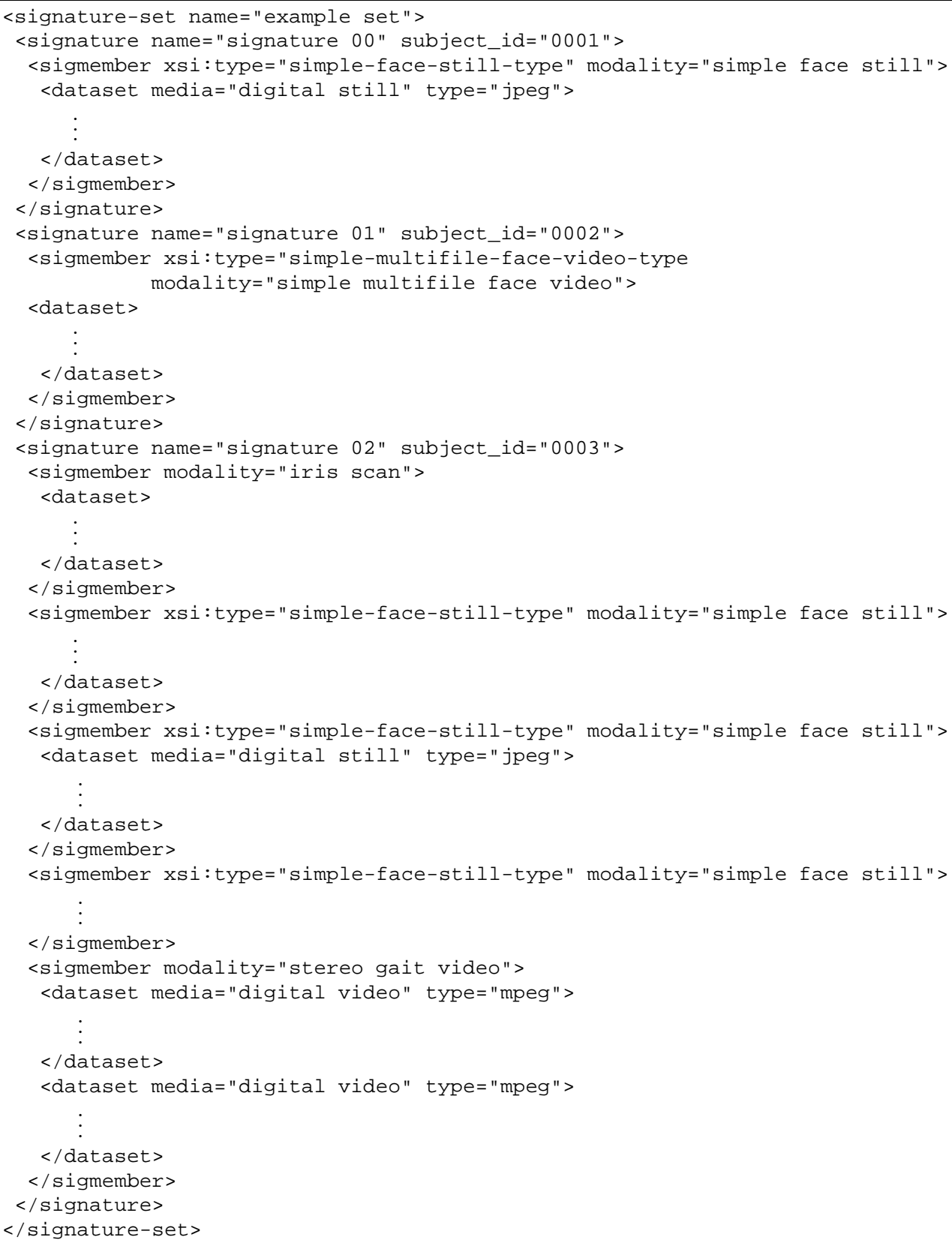

Figure 2.6: A valid nesting of <signature-set $>$, <signature $>,<$ sigmember $>$, and $<$ dataset $>$ tags. 


\subsubsection{File Elements}

Finally, we reach the bottommost tag, the $<$ file $>$ element. Each $<$ file $>$ element is terminal - if it contains any child elements, then the document will not validate successfully. Each file element is used to refer to a particular datafile, which, depending on the context of the parent tags, may contain complete or partial data of a biometric recording.

Like $<$ sigmember $>$, the $<$ file $>$ has a base and derived types. The base type, file-type is designed for general use, and has a single required attribute name which is a token that indicates the biometric data's file name. The two derived types provide mechanisms for indicating to an application more information about data of interest. For example, there may be several faces in a digital still, or several people captured in a gait video, but only one spatiotemporal region of interest.

The spatial-file-type extends the base type by offering an optional roi attribute, which is a token that can be used to specify a region of interest within the data file. The temporal-file-type is designed to be the preferred type for describing recordings with temporal information. This type requires the start and stop attributes, which are integer indexes that indicate to an application the logical beginning and ending of the biometric data. The optional unit attribute is a token to indicate the unit of measure to associate with the start and stop indexes. For example, in a video sequence, the unit value may be "frame". A type of temporal-file-type may also use the roi attribute.

Figure (2.2) shows a complete description of our example data, using all of the required tags, and the proper XML headers. There is only a single $\langle$ file $>$ tag for subject 0001, since there is only one facial image available for this subject. Subject 0002 has five $<$ file $\rangle$ tags, all part of a single $<$ dataset $\rangle$, each corresponding to a single frame of the (short) video clip. The use of $<$ file $>$ tags for subject 0003 is more complex. The iris scan, and the first two facial images use a plain $<$ file $>$. In the third face still, instead of using the default base type file-type, the derived type spatial-file-type is used, as well as a region of interest, or roi attribute. Without indicating the use of a derived type through the xsi:type tag, using an roi attribute would result in failure to validate correctly. Similarly, the derived type temporal-file-type is used to mark up the two gait videos.

\subsection{Supported Schemas}

As mentioned in Section (2), the HEF is not limited to a single schema, but a family of schemas. So far, we have limited our attention to the schema defined in sigset-unrestricted.xsd, since it is the most general schema in the family. However, there are several other schemas available for situations in which stricter constraints are desired. These schemas are briefly described here. For complete information, see the extensively commented schema files themselves.

- HEF Unrestricted Sigset Schema. The schema defined in sigset-unrestricted.xsd, and discussed in the previous section, defines the most general form of signature set. With this schema, signature sets may contain any (non-zero) number of signatures of any sigmember type. Unless otherwise specified within the document, each sigmember type may have any (non-zero) number and manner of datasets, each of which may have any (non-zero) number and manner of files.

- HEF Single Sigmember Schema. The schema defined in sigset-single-sigmember.xsd will validate documents in which every signature has one (and only one) sigmember. All documents that validate under this schema, should also validate under the HEF Unrestricted Sigset Schema.

- HEF Simple Face Still Set. The schema defined in simple-face-still-set.xsd will validate documents in which every signature has a single sigmember, and each sigmember is of derived type simpleface-still-type. This schema is designed to be used for evaluating most facial recognition systems that use still imagery. All documents that validate under this schema, should also validate under the HEF Unrestricted Sigset Schema.

- HEF Multiple Simple Face Stills Set. The schema defined in multiple-simple-face-stills-set.xsd will validate document in which every signature has one or more sigmembers where each sigmember 
is of derived type simple-face-still-type. This schema is designed to be used for evaluating most facial recognition systems capable of using multiple stills of a single subject. Note that the multiplicity is at the sigmember level, indicating that the multiple images of a subject correspond to different recording events. All documents that validate under this schema, should also validate under the HEF Unrestricted Sigset Schema.

- HEF Simple Multifile Face Video Set. The schema defined in simple-multifile-face-video-set.xsd will validate documents in which every sigmember is of derived type simple-multifile-face-video-type. This schema is intended to be used for evaluation of facial recognition systems that can operate on a video sequence where each frame has been stored in a separate file. All documents that validate under this schema, should also validate under the HEF Unrestricted Sigset Schema.

\subsection{Built-In Derived Sigmember Types}

This section provides information about the built-in derived sigmember types simple-face-still-type, simpleface-video-type, and simple-multifile-face-video-type.

\subsubsection{HEF Simple Face Still Type}

The HEF Simple Face Still Type, is defined in the file simple-face-still-type.xsd and, in instance documents, should be referred with the token "simple-face-still-type". This derived type is designed to be the preferred type for representing facial stills. A <sigmember $>$ of this type must have the attribute xsi:type set to the value "simple-face-still-type" and attribute modality set to "simple face still". Valid <sigmember $>$ elements of type simple-face-still-type" must only contain one <dataset $>$ element, which must contain a single $<$ file $>$ of type spatial-file-type. Because this is specified in the schema, there is no need to explicitly set the $<$ file $>$ 's $x$ si:type attribute in the instance document.

\subsubsection{HEF Simple Face Video Type}

The HEF Simple Face Video Type, is defined in the file simple-face-video-type.xsd and, in instance documents, should be referred to with the token "simple-face-video-type". This derived type is designed to be the preferred type for representing facial video stored in a single file. A <sigmember $>$ of this type must have the attribute xsi:type set to the value "simple-face-video-type" and attribute modality set to "simple face video". Valid <sigmember > elements of type "simple-face-video-type" must contain one < dataset $>$ element, which must contain a single $<$ file $>$ of type temporal-file-type. Because this is specified in the schema, there is no need to explicitly set the $<$ file $>$ 's $x$ si:type attribute in the instance document.

\subsubsection{HEF Simple Multifile Face Video Type}

The HEF Simple Multifile Face Video Type, is defined in the file simple-multifile-face-video-type.xsd and, in instance documents, should be referred to with the token "simple-multifile-face-video-type". This derived type is designed to be the preferred type for representing facial video where each frame is stored in a separate data file. A <sigmember $>$ of this type must have the attribute xsi:type set to the value "simple-face-video-type" and attribute modality set to "simple face video". Valid <sigmember > elements of type "simple-face-video-type" must contain one < dataset $>$ element, which must contain a one or more $\langle$ file $>$, each of type spatial-file-type. Because this is specified in the schema, there is no need to explicitly set the $<$ file $>$ 's $x$ si:type attribute in the instance document. 


\subsection{Custom Derived Sigmember Types}

In the future this section will provide instructions on how to create your own derived sigmember types, and package them so that they can be provided in the next formal release of HEF. It will also discuss the schema files themselves, including the overall design of the schema family, the files structure of the signature set schema family, and the purpose of each such file. 


\section{Chapter 3}

\section{Similarity Scores}

While the previous chapter was concerned primarily with marking up standardized input for evaluations, this brief chapter defines the XML format for describing a regular output of a face recognition system.

In the context of the HEF, a system consists of a black box recognition engine that takes two XML signature sets, the enrolled and the "unknown" test samples, and produces a set of pairwise identification scores. More formally, the protocol requires that an algorithm compare all elements of a target set, $\mathcal{T}$, with those of a query set, $\mathcal{Q}$, and report a similarity matrix, $S$, whose elements, $s_{i j}$, state some measure of the sameness of the identity of the individual in the $i$-th element of $\mathcal{T}$ and the $j$-th element of $\mathcal{Q}$. Because subsets of $\mathcal{T}$ and $\mathcal{Q}$ have different subject- or acquisition-specific properties, certain elements of $S$, corresponding to gallery (i.e. enrolled), $\mathcal{G} \subseteq \mathcal{T}$, and probe (i.e. unknown) sets, $\mathcal{P} \subseteq \mathcal{Q}$, may be used retroactively to make performance estimates on targeted recognition tasks (e.g. in face recognition, say, image acquisition issues like resolution or subject specific changes in, say, eye-wear or facial hair).

\subsection{Document Structure}

In this section, we will describe the structure of a similarity score file valid under the "HEF Similarity Set Schema". Fortunately, the structure of a file containing similarity scores is much simpler than for signature sets. Instead of a family of schemas, all valid similarity sets use the single similarity-set.xsd.

Because of the potential for running large experiments, and therefore, obtaining millions of similarity scores, similarity data from a given test may be stored in multiple files. In a standalone similarity set, all similarity data is stored in one large, monolithic file. In a multifile similarity set, data is stored within a collection of files, where each file contains the similarity scores for one a particular query signature. It is also possible, but not recommended, to have a hybrid of both types.

\subsubsection{Detailed Example}

An example will best illustrate the structure of a similarity set document. Suppose our target set consists of our three example signatures - "signature 00 ", "signature 01 ", and "signature 02 " — and our query sets contains three unknown signatures of the same subjects - "signature 00a", "signature 01a", and "signature 02a". Our similarity set file will have nine similarity scores (three targets, three queries). Figure 3.1 shows an example of how these scores would be marked up under the HEF XML formats.

Like the document from section (2.2.1) the first line of the document is the standard XML header, which indicates that the document conforms to the XML (version 1.0) standard. Leading whitespace or blank lines would render the instance document invalid. 
Lines 2 through 6 and line 27 compose the opening and closing root elements of the similarity set document. The < similarity-set> contains the same XML namespace "bookkeeping" documents as discussed in section (2.2.1), and, as indicated by line 5, uses the schema defined in the file $0.99 .0 /$ similiarityschemas/similarity-set.xsd. Line 6 indicates that this similarity set file does not depend on the presence of any other similarity file - more on this later.

In a similarity set document, the similarity scores are grouped by query signature. In other words, a particular query signature is "fixed", and the scores from all pairwise comparisons involving that query signature are indicated in the enclosing children tags. For example, since there are three signatures in the query signature set, there are three < similarity > elements in the document - the scores where the query signature is "signature 00 " are marked up on lines 7 through 14, the "signature 01" query signature is marked up on lines 14 through 20 , and the remaining "signature 02 " query signature on lines 21 through 27.

Each <similarity> element has a query attribute that indicates which query signature is fixed. Line 7 "fixes" the query signature to "signature 00 ", line 14 to "signature 01 " and line 21 to "signature 02 ". Each <similarity> element also has a <values > element which gives information about the nature of the similarity scores. For a set of "distance" scores, such as lines 9-11 and lines 23-25, lower numbers indicate stronger evidence that two signatures are from the same subject. For a set of "similarity" scores, like those in lines 16-18, lower values indicate that a two signatures are from two different subjects. The similarity or distance values themselves are marked up using the $\langle s\rangle$ element (lines 9-11, 16-18, 2325h). For example, line 9 indicates that the "distance" between target signature "signature $00 \mathrm{a}$ " and the query signature "signature 00 " is 0.02342 , line 18 indicates that "similarity" between target signature "signature $02 \mathrm{a}$ " and query signature "signature 01 " is 2.87322 , and so on.

In Figure 3.1 all of the similarity scores were stored in a single file. When an evaluation consists of hundreds or thousands of signatures, storing all the results in a single file, may become unfeasable. Therefore, there is a mechanism that allows the similarity set to be broken into a collection of files, where each file contains the values for a single query signature. The structure of such a multifile similarity set is straightfoward. Essentially, it involves replacing all the $<$ values $>$ elements with $<$ file $>$ elements. Each $<$ file $>$ element points to a separate XML file where the root element is a $<$ similarity $>$ tag, instead of a $<$ similarity-set $>$ tag.

Figures 3.2-3.5 show a multifile version of our example similarity set document. The similarity set document of Figure 3.2 is the same as Figure 3.1 except that the <values $>$ elements (lines 8-12, 15-19, 22-26 in Figure 3.1) are replaced with $<$ file $>$ elements (lines 8, 11, and 14). These $<$ file $>$ elements point to separate XML files, signature_00.xml (Figure 3.3), signature_01.xml (Figure 3.4), and signature_02.xml (Figure 3.5). Note that Figures 3.3-3.5 are using the similarity.xsd schema, and not the similarity-set.xsd schema - each of these files have a single < similarity $>$ element at their root.

\subsubsection{Similarity Set Elements}

The root of a similarity set document must be a single < similarity-set $>$ element. A valid <similarityset $>$ element must have one or more valid <similarity $>$ as child elements. The standalone attribute is a boolean used as a "hint" to indicate if, for a given test, the file contains all available similarity data. In the current similarity set schema, the value of this attribute does not effect the behavior of the validator (there is nothing encoded in the schema that may enforce a standalone constraint).

\subsubsection{Similarity Elements}

A similarity set document consists of a collection of <similarity $>$ elements. Each $<$ similarity $>$ element corresponds to a collection of pairwise comparisons, where a particular query signature is fixed, and compared against all elements in the target set. For convenience, <similarity-set $>$ elements may contain an optional name attribute which is a token that may be used to name the similarity set. A <similarityset> element may also contain the standalone attribute, which when set to true indicates that the file contains all available similarity data. 
All < similarity > elements must contain a query attribute, which is a token that indicates the name of the query signature that is being pairwise compared over all the target (a.k.a. gallery, or enrolled) signatures. Note that the signature name should be used here, not the subject_id since, by definition, within a signature set, all signatures are assigned a unique name, but may not even contain a value for the subject_id.

A <similarity $>$ element may also contain the optional meta attribute, which is a token that may be used to point to a file that contains meta-information about this particular set of comparisons (such as algorithm parameters, training information, etc.).

\subsubsection{Values and File Elements}

Each $<$ similarity $>$ element must have either a single valid $<$ values $>$ element, or, a single valid $<$ file $>$ element. The $<$ values $>$ element is used to indicate to the parser, that the raw similarity scores are to follow. Using a <file > element, however, indicates to the parser that this is not a standalone document, and that for the query signature indicated by the parent element, the similarity scores are stored in a separate file. It is expected, that this separate file will be a valid HEF Similarity (not HEF Similarity Set) document. A HEF Similarity document is a subset of the HEF Similarity Set schema. It is identical to a HEF Similarity Set document, except the root element must be a <signature $>$ element, and not a $<$ signature-set $>$ element. For multifile similarity sets, the naming convention for $<$ file $>$ elements and file names is application dependent.

For both standalone and multifile similarity sets, the raw similarity scores corresponding to a particular query signature are found in the child elements of a <values $>$ element. Each <values $>$ tag must have polarity and sort attributes. The polarity attribute must take on either the value "distance" or "similarity" depending on the nature of the comparison score returned by a particular recognition engine. If a large positive value indicates that two signatures are different, then <polarity $>$ should be set to "distance". If a large positive value indicates that two signatures are similar, then <polarity $>$ should be set to "similarity". The tag sort is used to indicate the order of the values contained in the child elements. Permitted values for sort are "ascending", "descending" and "unsorted". In the current version of HEF, the sort attribute is more of a "hint" rather than an enforcement. If the order of the values in the child elements does not match that indicated by the value of the sort attribute, by the current implementation, the document may still validate successfully.

\subsection{5 "s" Elements}

A $<$ values $>$ element contains one ore more $<s>$ element, where each $<s>$ element corresponds to a pairwise comparison of two signatures. Every $\langle s\rangle$ element must have a $n$, for name, attribute and a $v$, for value, attribute. Each pairwise comparison has a query signature and a target signature. The query signature is indicated by the value of the query attribute in the enclosing similarity element. The target signature is indicated by the value of the $\langle s\rangle$ element's $n$ attribute. Like the query attribute of the <similarity> element, the $n$ attribute is a token that should refer to the name of a signature. The $v$ attribute is used for the raw similarity or difference score itself. 


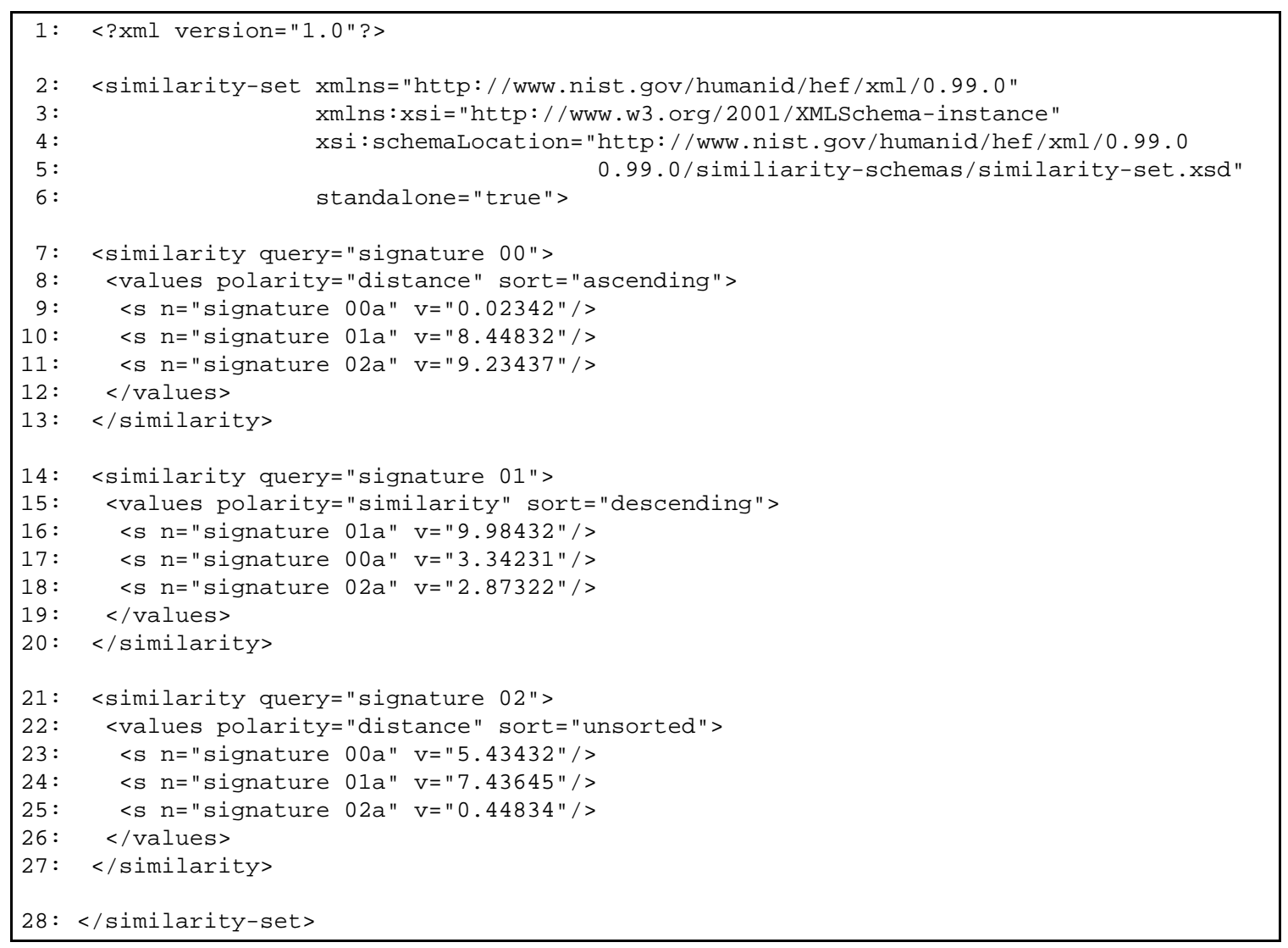

Figure 3.1: A standalone similarity set document for our example data.

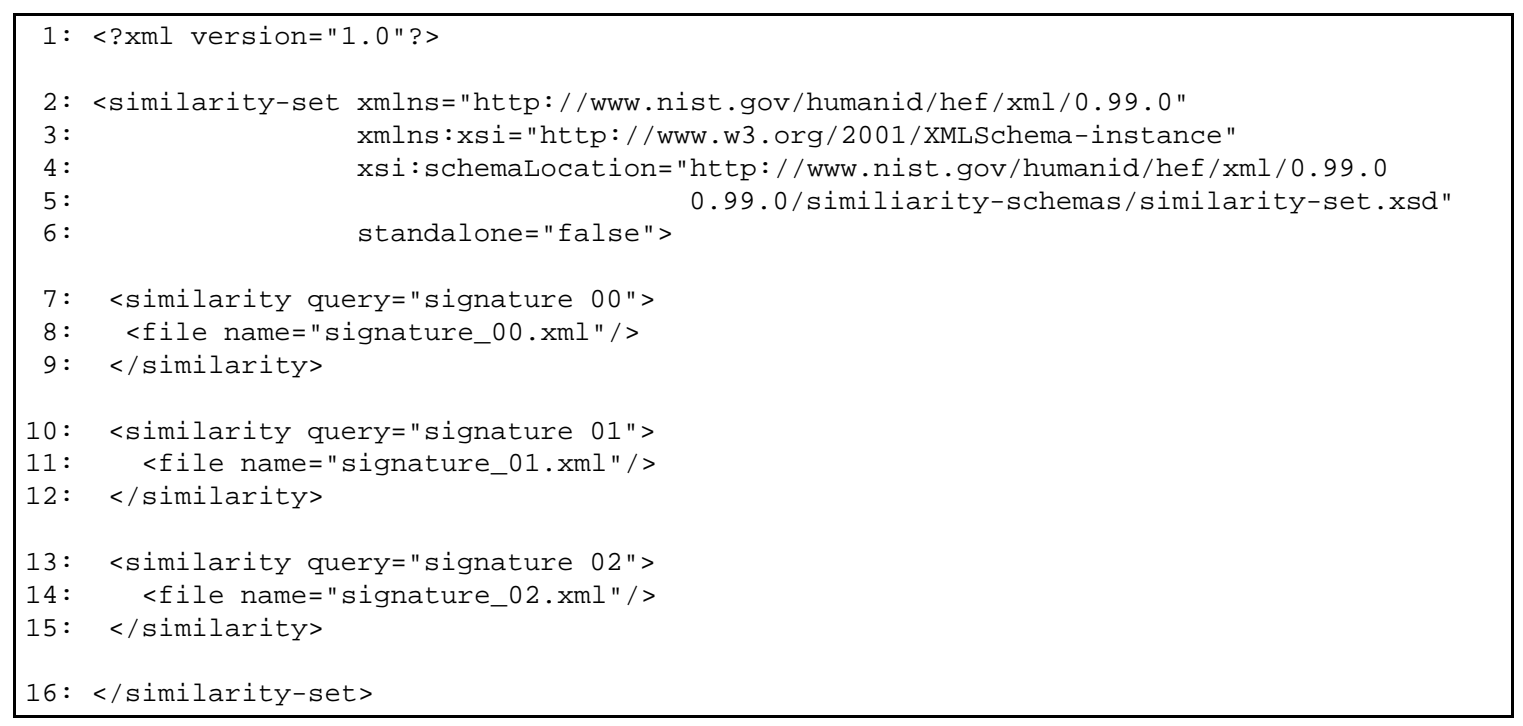

Figure 3.2: A multifile version of the similarity set document for our example data. When an evaluation has a large number of signatures, it is often useful to break the similarity data into separate files. 


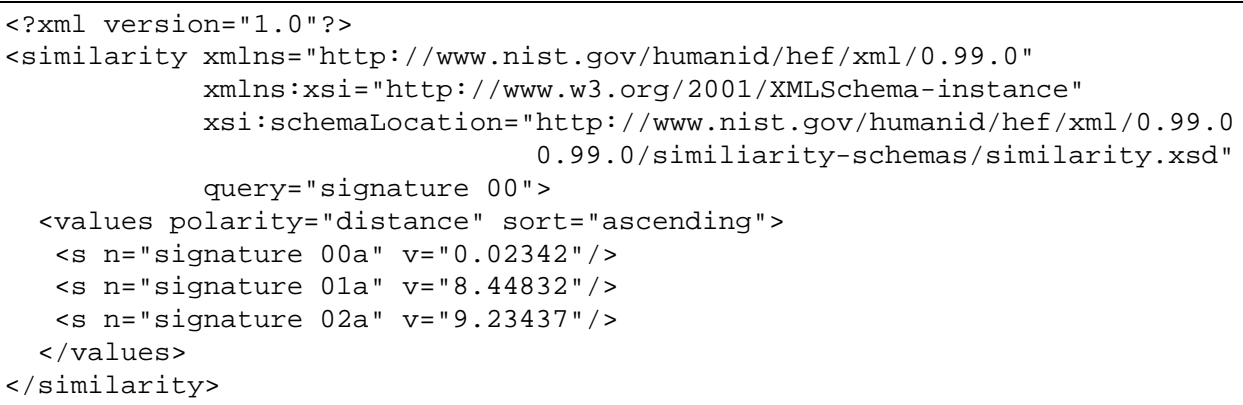

Figure 3.3: The contents of file signature_00.xml - a component of the non-standalone version of the similarity set document and the similarity scores for the query signature named "signature 00 ".

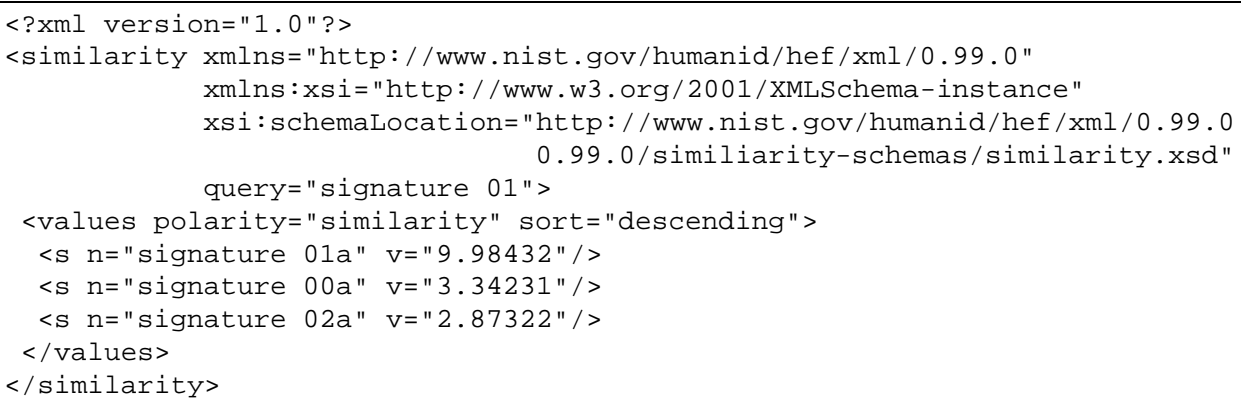

Figure 3.4: The contents of file signature_01.xml - a component of the non-standalone version of the similarity set document and the similarity scores for the query signature named "signature 01 ".

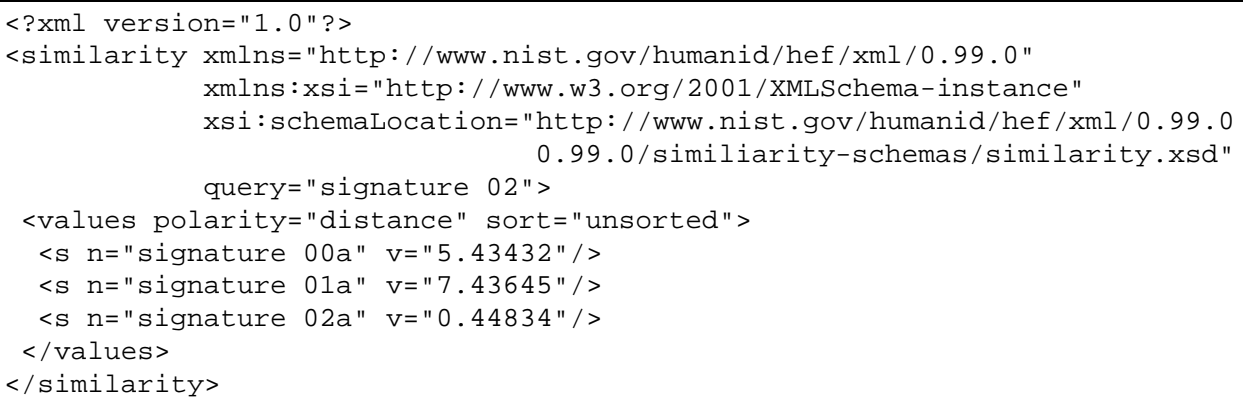

Figure 3.5: The contents of file signature_02.xml - a component of the non-standalone version of the similarity set document and the similarity scores for the query signature named "signature 02 ". 
Appendix H

FRVT 2002 Test Plan 


\section{Face Recognition Vendor Test 2002}

\section{Test Plan}

June 25, 2002

\section{Introduction}

This document describes the procedures that will be followed during the administration of Face Recognition Vendor Test 2002 (FRVT 2002). As described in the FRVT 2002 Implementer's Guide, participants will be given two sets of images to compare, from which they will produce a set of similarity files giving the matching scores for query images compared to target images. Participants will also supply a document describing their tested system(s) and associated costs. The results will be presented in the reports including a FRVT 2002 Final Report.

\section{Test Description}

\subsection{Overview}

The FRVT 2002 consists of two parts: the medium and high computational intensity tests ${ }^{1}$.

Medium Computational Intensity: This test will require the processing of approximately $7500^{2}$ signatures. It is divided into two separate parts. The first part measures performance on video style imagery. The second part resembles the FRVT 2000 evaluations using still images taken under different circumstances.

High Computational Intensity: This test will evaluate performance of a very large data set of approximately 121,000 images.

Each test will be performed separately and will have a maximum amount of time for completion. For each test, participants will provide a face recognition system complete with all necessary hardware. Time cannot be saved from one test for use in another test. Tests may be run concurrently. The FRVT 2002 will report results for complete and correctly formatted test submissions. Additionally, results for incomplete submissions may be reported where appropriate. Failure to complete a test will be noted in FRVT 2002 report(s). Results will be provided on a test-by-test basis. Prior to arriving at the test

\footnotetext{
${ }^{1}$ For a precise description of the medium and high computational intensity tests see the appropriate documentation available at http://www.frvt.org.

${ }^{2}$ The precise number, at the time of writing, remains to be determined.
} 
site, participants will have selected the tests they will take (check the website for the deadline for making such arrangements).

\subsection{Personnel}

Personnel involved with FRVT 2002 will be test agents and participants. Test agents are the government representatives and contractor assistants proctoring the evaluation. Participants are representatives of the organization whose system is being evaluated. Each organization will be allowed to send up to two people.

Each participant will assign one point of contact (POC) to interface with test agents when receiving images, submitting results, and signing the event checklist shown at the end of this document. A different POC may be assigned after first notifying the test agents. Participants are required to have a POC on site during setup and closeout procedures. They are not required to have a person on site between setup and closeout procedures. However, the FRVT 2002 evaluation team will not inform participants if their machine crashes or power goes out. If the POC is not on site when the time limit is reached to stop their program from running, then the participant will be disqualified for exceeding the time limit. There will be no penalty for finishing early and participants are free to leave the test area at any time after similarity files have been submitted and closeout procedures have been completed.

\subsection{Facilities}

Testing will take place in Building 194 at NAVSEA Dahlgren. Directions and area information has been sent to participants and is available on the "Test Plan" page of the FRVT 2002 Participant Area web site. Several participants may undergo testing at one time, possibly in the same room.

A voice-only telephone may be available for use by participants. First priority will be given to participants setting up equipment on their first day. Second priority will go to other participants with system problems. Due to the metal covering on building 194, cell phones may not work indoors.

NAVSEA Dahlgren is located approximately one hour from the nearest shopping district, so it is advisable to anticipate any spare parts that may be needed and bring them to the evaluation. FRVT 2002 Organizers recommend bringing your equipment with you. If necessary, parts may be shipped to the following address.

Duane Blackburn

DoD Counterdrug Technology Development Program Office

NSWCDD Code B07

1470 Room 1101

17320 Dahlgren Road

Dahlgren, VA 22448 


\subsection{Computer Requirements}

The following table shows the maximum number of desktop computers allowed for each test.

\begin{tabular}{|l|l|}
\hline Test & Desktop Computers Allowed \\
\hline Medium Computational Intensity & 1 \\
\hline High Computational Intensity & 3 \\
\hline
\end{tabular}

For the High Computational Intensity Test, the three (maximum) computers may be networked using a participant-supplied local area network (LAN) to perform operations in parallel. Wireless networking will not be allowed.

A desktop computer is defined to be a computer with case dimensions and power requirements similar to commercially available computers for an individual's use. Multiple processors are allowed inside that case and will be counted as a single desktop computer. Other configurations approximating a single desktop may be allowed on a case-by-case basis, but must be coordinated with the FRVT 2002 Liaison prior to participant arriving at the test site (check the website for the deadline for making such arrangements).

Participants must supply power strips to accommodate all hardware associated with submitted systems as well as uninterruptible power supplies, as needed. Systems should be setup and tested in advance so that no design, coding, or debugging is necessary at the evaluation site. Only minor configuration changes such as mapping of USB hard drive letters will be allowed.

Participants must install virus-scanning software on all submitted systems and scan with the latest virus definitions on the first day of their evaluation in the presence of a test agent.

\subsection{System Description Documents}

Participants will be required to submit a five-page (maximum) document, in electronic form, on the first day of their testing that describes the submitted system(s). This document will be a section in the final report that will be released to the public. This is the participant's opportunity to describe their system(s) and costs to those that may read the FRVT 2002 report. This document must be submitted on the first day of testing.

Target and query sets will not be distributed to the participants unless this document has been received. Failure to meet this requirement will effectively disqualify a participant. This document must, at a minimum, adequately address the following:

- Provide an overview of the submitted system(s)

- Provide a component list for the submitted system(s)

- Provide a detailed cost breakdown of the submitted system(s)

- Detail any modifications specifically for the purpose of FRVT 2002 
Additionally, participants must use the following guidelines for submitting system description documents.

1. Use Microsoft Applications: All participants should ensure their reports are submitted in a Microsoft Office application format. For instance, word processing documents should be submitted in Word, charts and spreadsheets should be submitted in Excel, etc. Using Microsoft applications will ensure uniformity and facilitate compilation of the final report.

2. Send All Original Documentation: Send original documents for anything that is to be inserted into your report. For instance, send original Excel spreadsheets, PowerPoint slides, and image files (such as PS, EPS, JPG, TIF, etc.). Once the final report is compiled, it will be converted to PDF format for distribution to the community. Sending original documentation ensures uniformity and accuracy when the report is compiled and converted to PDF format.

3. Use Standard Fonts: Use either "Arial" or "Times" fonts (NOT Times Roman) in your reports (except for equations).

4. Embed All Fonts: Not all fonts are available on all machines. If you must use a font that is not standard with Microsoft (for instance, with equations, etc.), you should embed the font in the document so it will not change when the document is converted to PDF format.

5. PDF or Hard Copy: Include either a PDF file or hard copy of your document so your desired layout may be preserved.

\subsection{Time Limits}

Each test selected by a participant must be completed within a designated time limit. The following table shows the time limit for each test.

\begin{tabular}{|l|l|}
\hline Test & Time Limit \\
\hline Medium Computational Intensity & 264 hours \\
\hline High Computational Intensity & 264 hours \\
\hline
\end{tabular}

The time allotted for each test includes the time necessary to copy the target and query sets from the supplied USB hard drive(s) to the participant hard drive(s) and to copy the resulting output back to the USB hard drive(s). Participants will setup their system(s) when arriving on their start date then process a small set of sample images and return the results on the supplied USB hard drive(s). Test agents will then verify that the results are in the proper format then distribute the main body of images. The evaluation period will start at the time the main body of images is released and end 264 hours later. The evaluation period must begin by 1700 EDT on the participant's start date.

The FRVT 2002 will report results for complete and correctly formatted test submissions. Additionally, results for incomplete submissions may be reported if appropriate. Systems may run continuously during the allotted time, but access to the testing area will be limited to the hours of 0900 through 1700 EDT each test day, including weekends. During that time, participants may monitor their system(s) once each hour, including weekends, with the exception of the participant's start date, during which full time access 
will be allowed from 0900 through 1700 EDT. In the event of an overnight system crash, participants will not be allowed to restart their system(s) until 0900 EDT the following day. Participants are encouraged to implement their system(s) in a manner that allows restarting from the point where a crash occurred rather than restarting from the beginning.

In the event of a power outage, time will be added to the evaluation period of all affected participants. If the outage occurs between 0900 and 1700 EDT, the amount of time added will be the amount of time that the power was out. If a power outage occurs outside these hours, the amount of time added will cover the period from the start of the outage until 0900 EDT the next day.

\subsection{Images}

FRVT 2002 participants will be given a large database of images on the day they arrive for testing. The images will be provided via IDE hard drives in USB 2.0 enclosures. The drives will contain a single $80 \mathrm{~GB}$ partition containing a NTFS file system. Any participant requiring a different configuration must contact the FRVT 2002 Liaison prior to arrival at the test site (check the website for the deadline for making such arrangements). A separate hard drive will be used for each test. If required, a separate laptop computer may be used to facilitate transfer to/from the USB hard drive and face recognition system(s).

Participants will be required to use two separate partitions or hard drives, one for the OS and face recognition algorithms, and the other for working space. Participants will be allowed to copy the images onto their working space partition or drive only. Any intermediate files derived from the images must also be stored on the working space partition or drive.

\subsection{Similarity Files}

Participants must produce matching results in the form of similarity files that will be submitted to the test agents at the end of the evaluation. Participants will be allowed to store similarity files on their working space partition or drive only. At test completion, the similarity files must be transferred to the same USB hard drive(s) used for distributing the image files. If required, a separate laptop computer may be used to facilitate transfer to/from the USB hard drive(s) and face recognition system(s).

Participants must return the media to the government at test completion and perform a low-level format on their working partitions and drives. A test agent will assure that none of the images, or data derived from the images, are still resident on the test computer. The inspection will involve, at a minimum, the government deleting files generated during testing and wiping free space on all disk drives.

\subsection{Video Recording}

Testing activities will be recorded using video cameras for documentation of the evaluations. Footage from this documentation will not be made available to the public without review and comment from any participant that is named in the video. 


\subsection{Schedule}

Participants will begin and end their evaluation period on staggered dates in order to prevent traffic jams while equipment is being moved into the test area. Participants will report to the test area at 0900 EDT on their scheduled start date. The USB hard drive(s) containing the images to be processed will be distributed the same day, after the participant has completed system setup and sample output has been verified. Participants may return the complete results on the hard drives to the test agents at any time within 264 hours after the images have been released. Participants will be allowed to monitor their systems once each hour from 0900 through 1700 EDT while the test is in progress, including weekends, with the exception of the participant's start date, during which full time access will be allowed from 0900 through 1700 EDT.

\section{Test Procedures}

The evaluation will proceed according to the procedures detailed in the following subsections. Certain events will be recorded using the event checklist shown at the end of this document. A test agent and participant POC will initial the checklist as events occur. Each participant will assign one spokesperson to perform this function.

\subsection{Setup}

1. Test area opens at 0900 EDT.

2. Participant provides system description document(s) to test agent.

3. Participant and test agent initial checklist indicating that a system description document was submitted, in its proper form, for each system.

4. Participant sets up computer system(s) in test area.

5. Test agent records available hard drive space on checklist.

6. Participant runs participant-supplied virus scanning software on each system.

7. Participant and test agent initial checklist acknowledging available hard drive space and the fact that a virus scan has been successfully completed.

8. Test agent releases USB hard drive(s) containing sample images to participant when system setup is complete.

9. Participant copies the set of sample images from USB hard drive to working space of face recognition system, performs matching to produce similarity files, then submits results to test agent using same USB hard drive.

10. Test agent verifies that similarity files are in proper format.

11. Participant and test agent initial checklist indicating that sample similarity files are in the proper format.

12. Test agent releases USB hard drive containing main body of images.

13. Participant and test agent initial checklist indicating that the main body of images has been released to participant. At this point, the time is recorded and the evaluation period begins. The evaluation period must begin on or before 1700 EDT on the participant's start date.

\subsection{Evaluation Period}

1. Participant copies images for medium and/or high computational intensity tests from USB hard drive(s) to their system(s). 
2. Participant begins matching query images against target images.

3. Participant may monitor system(s) once each hour between the hours of 0900 and 1700 EDT each testing day to handle system crashes, with the exception of the participant's start date, during which full time access will be allowed from 0900 through 1700 EDT.

4. Participant copies resulting similarity files to USB hard drive(s).

\subsection{Completion}

1. Participant returns USB hard drive(s) containing images and similarity files to test agent.

2. Test agent verifies a random sampling of similarity files to confirm that they are in the proper format.

3. If the similarity files are not in the proper format, participant may make corrections and resubmit as many times as needed until the evaluation period ends.

4. When similarity files have been successfully verified, participant and test agent initial checklist indicating that USB hard drive(s) have been returned and that a random sampling of similarity files has been verified.

5. Test agent copies all similarity files from the USB hard drive to a RAID server then makes additional copies using other media.

6. Test agent inspects all hard disks of submitted system(s), deletes images, derived files, and similarity files then wipes drive free space.

7. Participant and test agent initial checklist indicating that hard disks have been inspected and free space has been wiped.

8. Participant and test agent sign checklist. 


\section{Organization Name:}

\section{Event}

System description documents submitted

System 1

System 2

System hard drive free space recorded and virus scan successfully completed

Computer 1 (bytes):

Computer 2 (bytes):

Computer 3 (bytes):

Computer 4 (bytes):

Sample similarity files verified to be in proper format

Medium Computational Intensity

High Computational Intensity

USB hard drive(s) released to participant (timer starts)

Medium Computational Intensity

High Computational Intensity

Random sampling of similarity files verified to be in proper format.

Medium Computational Intensity

High Computational Intensity

USB hard drive(s) returned to test agent

Medium Computational Intensity

High Computational Intensity

System hard drives inspected, free space recorded and wiped Computer 1 (bytes):

Computer 2 (bytes):

Computer 3 (bytes):

Computer 4 (bytes):

Test Agent Signature / Date

Participant Signature / Date

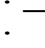

\section{Test Agent Participant Date / Time \\ Initials Initials}
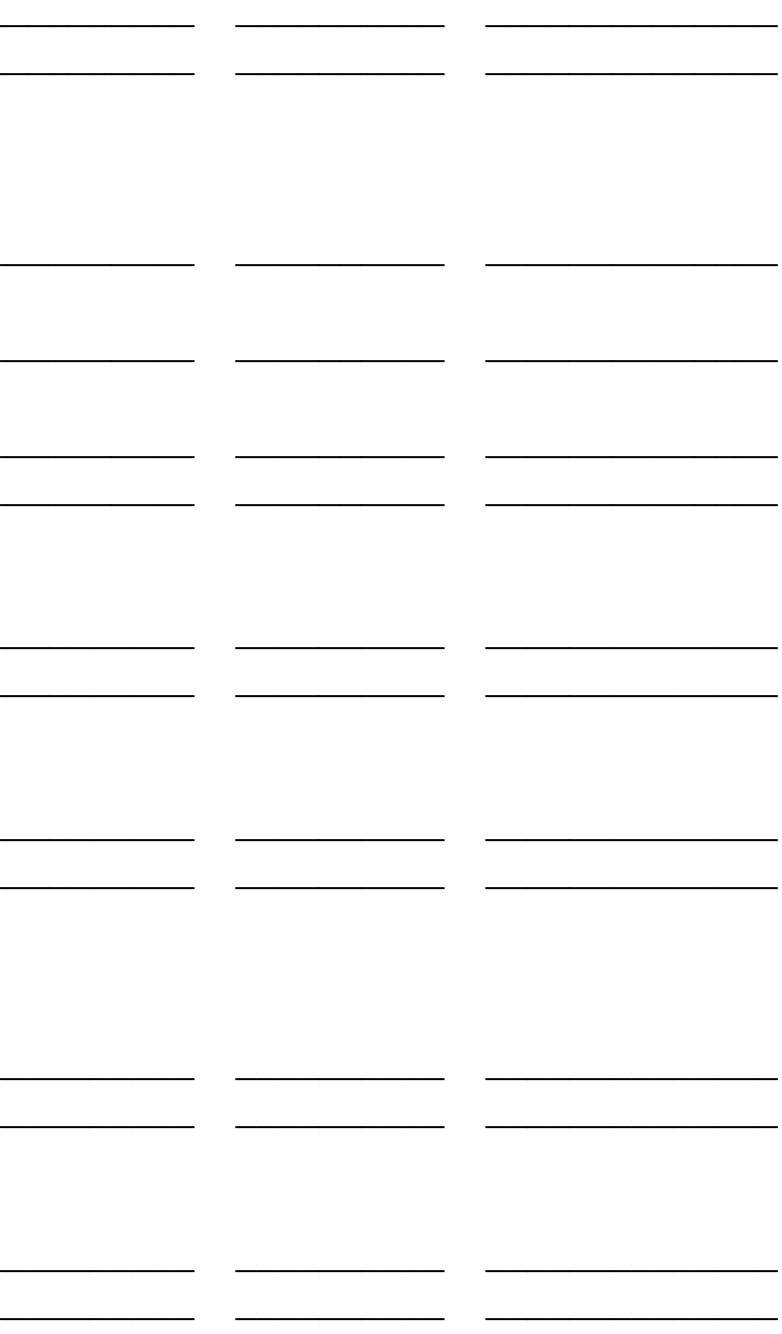


\section{Appendix I : FRVT 2002 Imagery}

\section{I.1 Introduction}

This appendix describes the imagery used in the Face Recognition Vendor Test 2002 (FRVT 2002). It discusses the image sets used in both the High and Medium Computational tests, and includes examples of the images, both stills and video sequences, that were used in the various studies described in the main body of the report. This appendix gives brief descriptions of the acquisition, processing, properties and use of the images in each subset. Some common aspects of all images of the FRVT 2002 corpus are that they contain the face of exactly one individual, and that the face (and neck and part of the chest) is the only foreground object, and that the image was acquired in the visible spectrum in color. All the images provided to FRVT 2002 participants are in standard JPEG format, regardless of whether they ware acquired that way.

\section{I.2 High Computational Intensity Test Database}

Imagery used for the High Computational Intensity Test is a subset of a much larger collection furnished to the FRVT2002 Evaluation Team by the Visa Division of the Department of State. That collection consisted of approximately 6.8 million images from about 6.1 million visa applicants who received various nonimmigrant visas in Mexico over the period 1996 to 2002. The data supplied to NIST is itself a random subset of all images gathered in Mexico during that period. The imagery was obtained in the field and in large quantities such that its utility in quantifying face recognition performance is for the most part unreproachable. As discussed below the images are of good quality, gathered in a consistent manner. This homogeneity leaves natural anthropmetric population variation as the leading cause of error in the systems tested. This is in contrast to much of the academic literature in which vision systems are designed to handle particular difficulties in images such as non-frontal views, or non-uniform or low contrast illumination.

The vast majority of the images were collected at United States consular offices using standard issue digital imaging apparatus whose specification remained fixed over the collection period. Consular officials who collected the images instructed the applicant to face the camera. The result is a set of well-posed (i.e. frontal to within 10 degrees) images of cooperative subjects usually with a mouth-closed neutral facial expression. The subject usually occupies a quarter of the image area. The top of the subject's shoulders is almost always visible. The background is universally uniform, with a white background in most cases. White balance artifacts occur less than 5\% of the time. Figure I-1 shows images of the authors at NIST, included here in place of actual State images because privacy issues preclude their inclusion. 

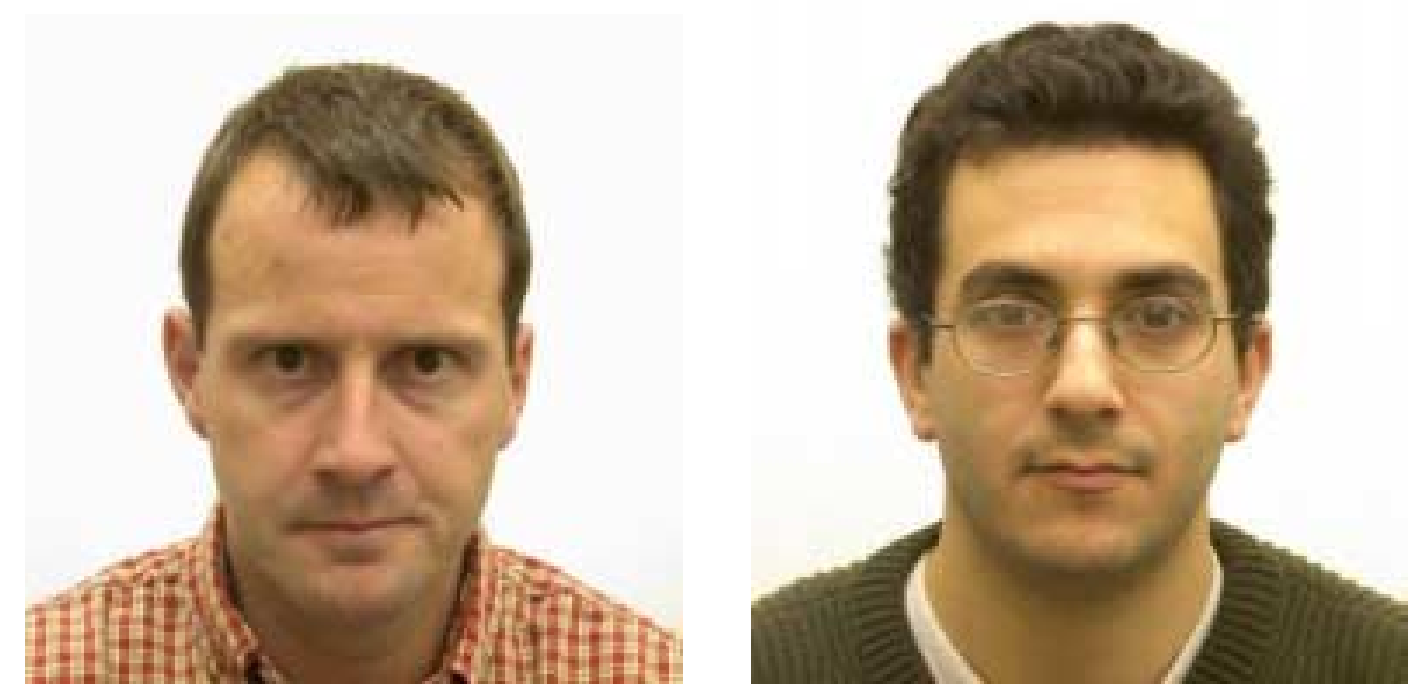

Figure I-1. Images of NIST personnel included here as reasonable representations of those used in the FRVT 2002 High Computational Intensity test.

Metadata The images, as supplied to the FRVT 2002 Evaluation Team, were accompanied by image and subject specific information: subject id, age, country of birth and nationality, image acquisition date and consular office code. Because sex information was not available NIST inspected one image of each of the 37,440 individuals used in HCInt, and keyed either male, female, or indeterminate. This latter category was used in 89 cases where the there individual's sex could not readily be determined.

\section{I.2.1 The FRVT2002 High Computational Intensity Subset}

We applied a sequence of image and population specific selection criteria to the supplied State Corpus to arrive at the HCInt subset. The original State set contains 6.8 million images of 6.1 million subjects. The HCInt subset contains 121,589 images from 37,440 persons which we obtained as follows. We retained only color images, discarding greyscale images for which the precision was one byte per pixel, or for which $\mathrm{R}=\mathrm{G}=\mathrm{B}$ for all pixels; second we retained only those images of height and width 300 and 252 pixels; and third we kept only those images that did not produce even very minor JPEG file format irregularities as reported by our Independent JPEG Group March 1998 implementation. These three steps reduced the number of candidate images by about $8.2 \%$ of the original total.

We then invoked two population specific criteria. The first is to consider only those images of person who were 18 years of age or older at the time they first applied for a visa. This obviates legal complications involved with the use of minors, and also removes effects arising from the rapid change of appearance associated with childhood and adolescent growth. Secondly, and most significantly, we retained only those persons with three or more images. This multiple images per person criterion is essential to a recognition evaluation. We chose to use three or more images as this led to a final database of size that is tractible in the FRVT 2002 test.

The result is a collection of color images, all of size height 300 and width 252 pixels, stored as standard JPEG images with a mean file size $9.5 \mathrm{kB}$. The mean interoccular distance, as reported by a known effective eye location algorithm, is 75.2 pixels. The images given to participants were byte-for-byte the same as those provided by the Department of State; they were not recompressed, resized or in any way processed by NIST.

Selection Biases The criteria described above impart selection biases on the population. For example, the demographic structure of the whole State corpus is plotted with that of the partial set in the figure I-2. It 
shows that persons born through the 1960s are under-represented by our study, but those born after 1967 are over-represented. However our study reports performance on the population who applied for visas on three or more occasions. In that sense the appropriate population density we should compare against is that from which persons apply on TWO or more occasions.

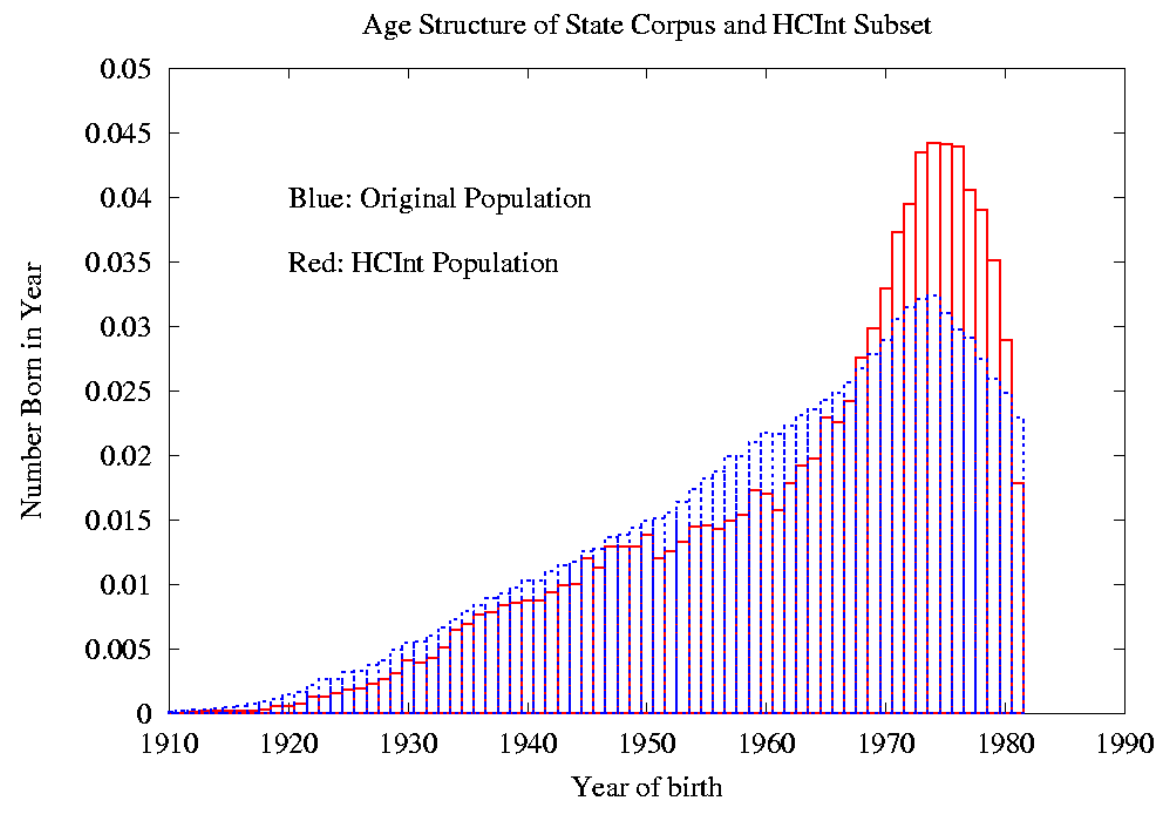

Figure I-2. Age structures of the entire State corpus and the HCInt subset

If recognition performance is affected by age of the subject then an under- or over-representation in our sample would change the results somewhat. It should be noted, however, that this effect depends on the application; if the recognition task is a new one-to-many search on an entire corpus, then the number of images per person is irrelevant.

Other distributions were also affected by our selection criteria. For instance $62 \%$ of the population used in HCInt were issued visas at just three of the consular offices, whereas in the State corpus as a whole that figure is just $37 \%$ for the same three offices. This concentration occurred because repeat applicants preferentially attend a select few consular offices. Given the uniformity of the imaging apparatus across offices, any changes in the recognition performance estimates is likely to be small. This latter assertion can be tested using the framework we have.

Metadata information is vital to recognition evaluations as it allows recognition performance to be broken out by category, for example by age, or by time between image acquisition dates.

Subject ID Given the vital nature of subject identity in recognition performance testing, this section discusses the derivation and integrity of the HCInt ID metadata field. The ID field itself is a 32 character hexadecimal string (i.e. 128 bits) from the MD5 hash function applied to the concatenation of four metadata strings, namely the subjects name, date and place of birth, and nationality.

There are two types of error that generally affect identity information in biometric databases. First, is that several different persons have the same ID, and, second, that a single person has more than one ID. In the case of HCInt the process by which two or more persons have the same ID is that the four metadata fields 
are identical. This is clearly an unlikely coincidence in a general population and can be expected to introduce errors much less than the recognition variation observed over disjoint populations in the FRVT 2002 results. Another mechanism for this class of error is that the hash function produced the same output string, a hash-collision, for different inputs. This is an even more unlikely scenario given the quality of the MD5 cryptographic algorithm and the number of trials.

The second class of error is, at first glance, more problematic. If a typographical error occurred during the data-entry phase of the subject enrollment then two IDs would be obtained from the same person. However for HCInt this event would, via MD5, yield a "new" person with only one image, and these individuals will be excluded by the criterion that we only use persons with three or more images. Likewise if an individual within in the State corpus has applied under two different names, then this will not be revealed in FRVT 2002 unless it has occured three or more times. We have validated this finding by inspection of the most similar ostensibly non-matching images, as reported by the leading FRVT 2002 systems, and found no errors of this kind.

Nationality As we stated above the State imagery has unreproachable size and real-world provenance. The notable exception to this is that the images are from one nation, and although the population is large enough to span anthropological face variation in Mexicans we cannot strictly extend the conclusions to, say, a Scandinavian population. Although the entire corpus contains images of persons of 185 nationalities and Mexicans comprise $98.76 \%$ of them, the HCInt subset contains images of persons of 66 nationalities of which $96.59 \%$ or 36165 are Mexican. The decrease in the proportion of Mexican nationality indicates that the non-Mexican population in Mexico applies more frequently for visas. The next most represented nationality within the HCInt subset is Chinese at $1.68 \%$ or 628 persons. This fraction was considered insufficient to evaluate the effect of nationality (and ethnicity) on recognition performance.

Footnote to the Results Many of the FRVT 2002 results are based on a gallery of 37437 individuals. This is three fewer than the number of persons present in the HCInt subset described above. This difference arose because one FRVT 2002 participant produced incomplete similarity files for a single image of those three persons. The FRVT 2002 committee elected to remove those three subjects from all scoring computations.

\section{I.3 Medium Computational Intensity Test Database}

In contrast to the HCInt image set, the Medium Computational Intensity image set is composed of a heterogeneous set of still images and video sequences of subjects in a variety of, poses, activities and illumination conditions. The images originate from two distinct sources. The first is the joint National Institute of Standards and Technology (NIST), Naval Surface Warfare Center (NSWC, Dahlgren), University of South Florida (USF) still face image corpus gathered between 1999 and 2002. The second, from University of Texas, Dallas, consists of video and stills, taken in 2001. Both sets of images are partitions of the hBase database being assembled as part of DARPA's Human Identification at a Distance Program ${ }^{1}$. Each piece of imagery there is accompanied by ground truth information describing its acquisition and properties. The images of the MCInt were supplied to participants in two disjoint partitions. The first contained 7722 still images from 1477 individuals; the second contained 1477 video sequences and stills, from 63 subjects.

The two partitions share in common that the subjects appearing in the images are all unpaid, informed, and consenting volunteers. For privacy reasons the data was gathered anonymously; a volunteer's name was never recorded and is instead replaced by a randomized integer identifier that is used to label all the imagery ever taken of that individual. IDs are maintained over time by having subjects self-identify from a previous image.

\footnotetext{
${ }^{1}$ See P. Jonathon Phillips, "Human Identification Technical Challenges", in Image Processing for Biometrics in the International Conference on Image Processing (ICIP), 2002, Rochester, New York.
} 


\section{I.3.1 The NIST-NSWC-USF Collection.}

This collection is comprised of images taken indoors and ourdoors. Although the images were taken over more than three years at three sites they were acquired in almost identical circumstances such that their geometric and photometric characteristics are approximately the same. This was achieved by using consumer grade autofocus digital CCD cameras (Sony Mavica) in to photograph individuals against a background of photographic supply $18 \%$ gray paper, illuminated alternately under a two photoflood arrangement, and an ambient overhead fluorescent setting.

Although the imagery itself has consistent properties the subject populations of the three sites differ. The USF segment consists of subject population of diverse ethnicity that is distinct from the older predominantly caucausian mix at NIST and NSWC. The collection is therefore not free from unavoidable selection biases.

Four images of each subject are used in the MCInt study. The first two, under the two photofloods have the subject with a neutral then an alternate facial expression. The latter two are a corresponding pair obtained under ambient lighting. In most cases the alternative expression is classified as a smile, but in a significant fraction other expressions are present.

The images of figure I-3 form the gallery and probe sets used for testing changes in expression, overhead lighting and outdoor lighting, and elapsed time. The leftmost image is drawn from the gallery of the earliest examples of neutral expression two-light frontal stills from all the persons in the NIST-NSWC-USF collection. The second image is a corresponding image from a later collection. The third shows a subject with an alternative facial expression taken on the same day as his gallery image. Finally the last image shows fluorescent room lighting, without changes in the camera's white balance setting or facial expression. The images are either $240 \times 320$ or $256 \times 341$ in size with an average compressed JPEG file size of approximately $18 \mathrm{kB}$. The median interoccular distance is 51.2 pixels.

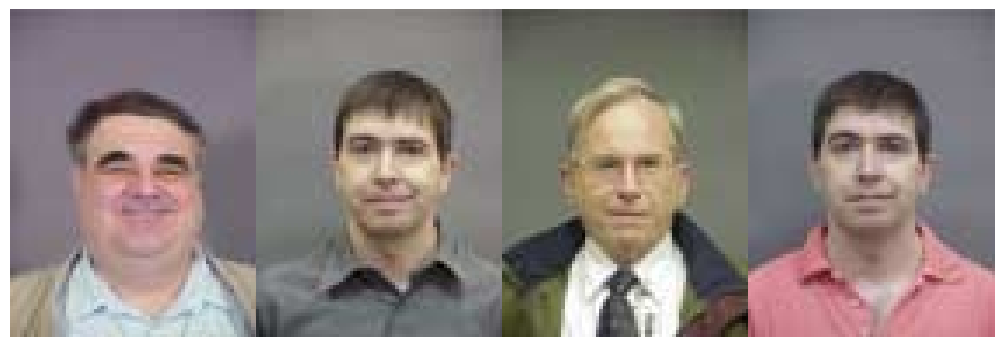

Figure I-3. Four sample indoor images from the NIST-NSWC-USF Collection used in the MCInt test.

Outdoor Still Images The outdoor stills are characterized by changing background and directional sunlight illumination. A small fraction of the images were taken with cloud cover. Each subject stood facing the camera at a distance of 2 meters from a zoomed Sony Mavica digital camera in auto-focus mode. In all cases subjects were asked to provide a neutral facial expression. Figure I-4 shows two images taken on the same-day as those in Figure I-3, then two more recent samples for studying indoor outdoor and time delay effects. All images have size 240x320 pixels and compressed JPG file size of about $26 \mathrm{kB}$. The median interoccular distance is 45 pixels. 

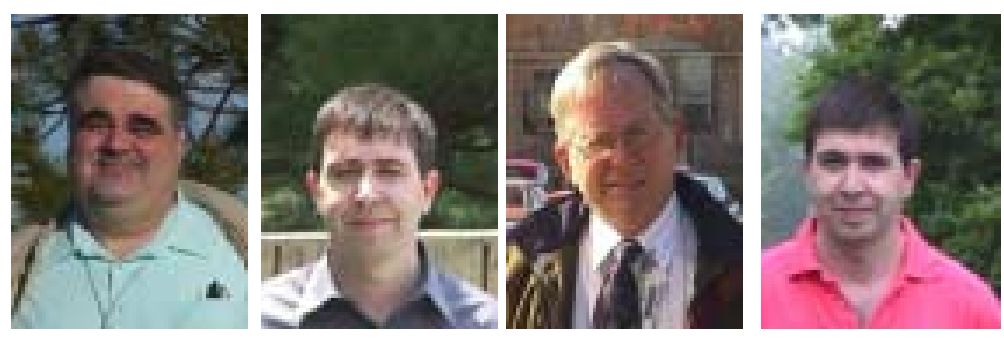

Figure I-4. Four sample outdoor images from the NIST-NSWC-USF Collection used in the MCInt test.

Frontal Reconstructions The MCInt image set included partitions included to test the efficacy of using 3D morphable models as a preprocessing step in the recognition of non-frontal images. The concept is that a non-frontal image can be fit to a 3D model of human heads, and then a frontal view can be generated. Specifically by starting from an example set of 3D face images, a morphable face model is derived by transforming the shape and texture of the examples into a vector space representation. New faces and expressions can be modeled by forming linear combinations of the prototypes. Shape and texture constraints derived from the statistics of example faces can be used to guide manual modeling or automated matching algorithms. This method ${ }^{2}$ has been advanced by Thomas Vetter and his group at the University of Freiburg, and funded recently by DARPA's Human Identification at a Distance program.

To investigate the utility of the Vetter method, NIST departed from its standard two-light source illumination protocol and instead used a single photoflood source situated behind and above the camera on the optical axis. This was done to satisfy a single light source assumption of the Freiburg model. NIST consulted with Volker Blanz in Freiburg, supplied him with nearly 800 of the new NIST images and incorporated the original and frontal reconstructions into the MCInt set. Examples of the original images are shown in Figure I-5. Reconstructions are shown in Figure I-6. The images used for the test were reduced from their original size to have width 256 and height 341 pixels. The average file size is $14.7 \mathrm{kB}$.
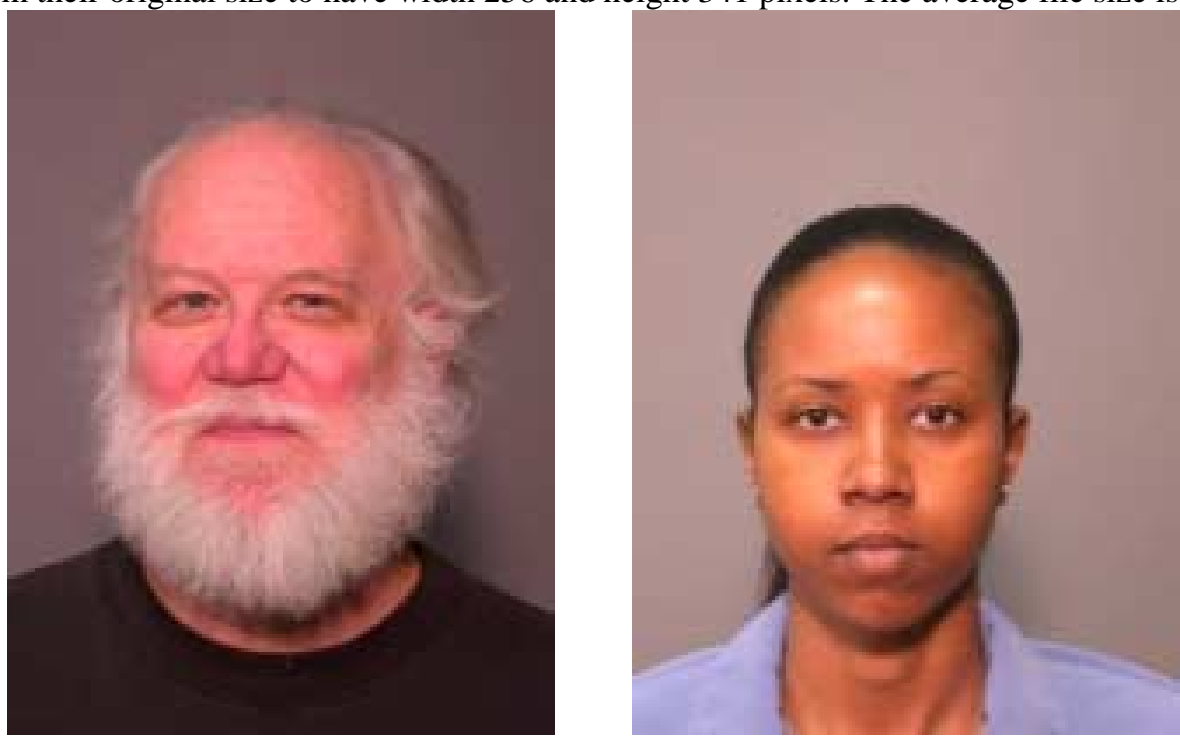

Figure I-5. Frontal images taken from persons at NIST using a single light source (and ambient overhead lighting). The use of a single light source is assumed by the 3D morphable model used to remove pose variation from non-frontal images.

\footnotetext{
2 See Volker Blanz and Thomas Vetter, A Morphable Model For The Synthesis Of 3D Faces", Siggraph 1999, Computer Graphics Proceedings, Pages 187-194, ed. Alyn Rockwood, Addison Wesley Longman, Los Angeles.
} 


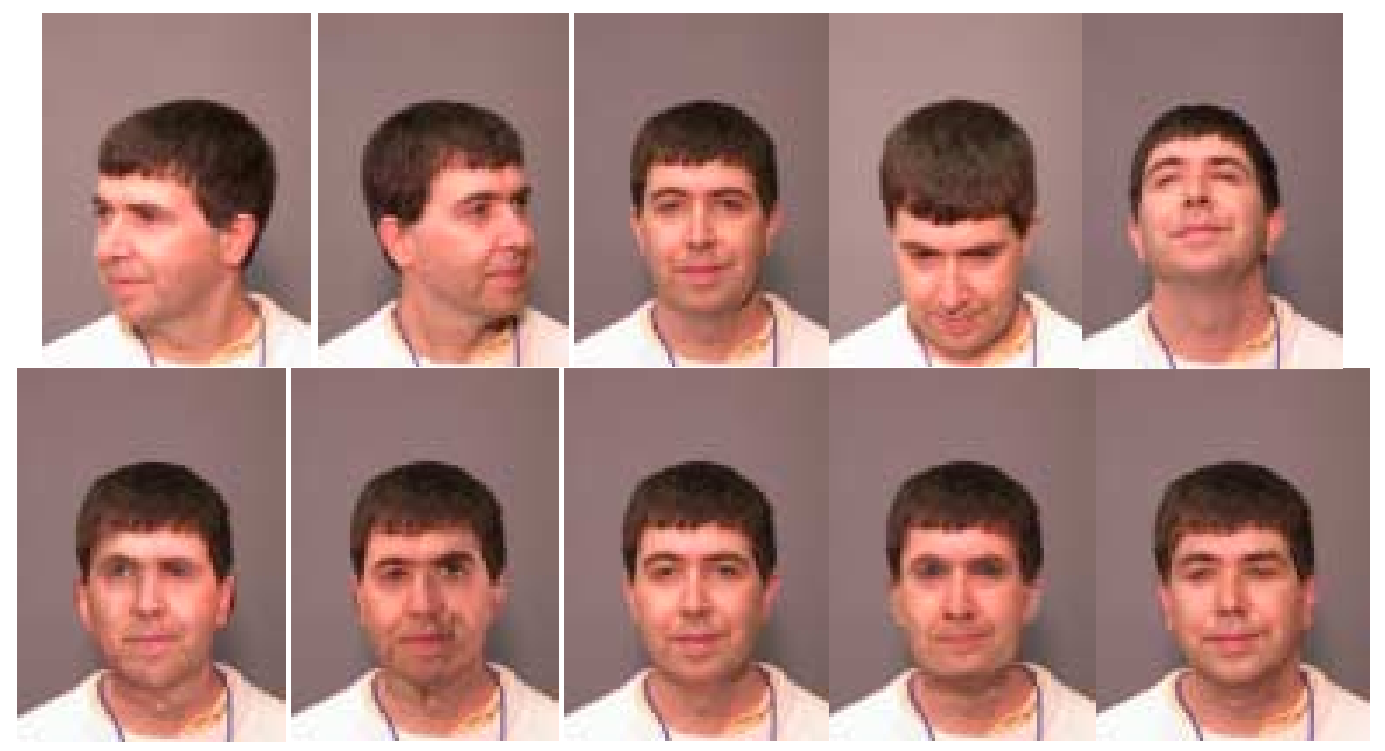

Figure I-6. The top row shows original images as matched against those in the gallery of Figure I-5. The subject, illuminated by one light source, is looking left and right at about 45 degrees, and up and down at about 30 degrees. The second row shows the corresponding frontal reconstructions from the 3D morphable model.

\section{I.3.2 Imagery from the University of Texas at Dallas}

A database of face and person imagery was created at The University of Texas at Dallas for use in human memory experiments that test the importance of natural face and body animation, as well as posture. Closerange video clips and static images were taken of more than a hundred individuals on at least two different occasions to make duplicate sets separated by one week to six months. We used a 63-person subset who appeared on two occasions. The image file size is 720 by 480 pixels, with file size $22 \mathrm{kB}$.

Facial Speech Videos Two five-second video clips were taken of each individual speaking, first in a neutral way, then in an animated way. Figure I-7 shows two examples; all images have width 520 pixels and height 480 pixels, and the filesize is $16 \mathrm{kB}$.
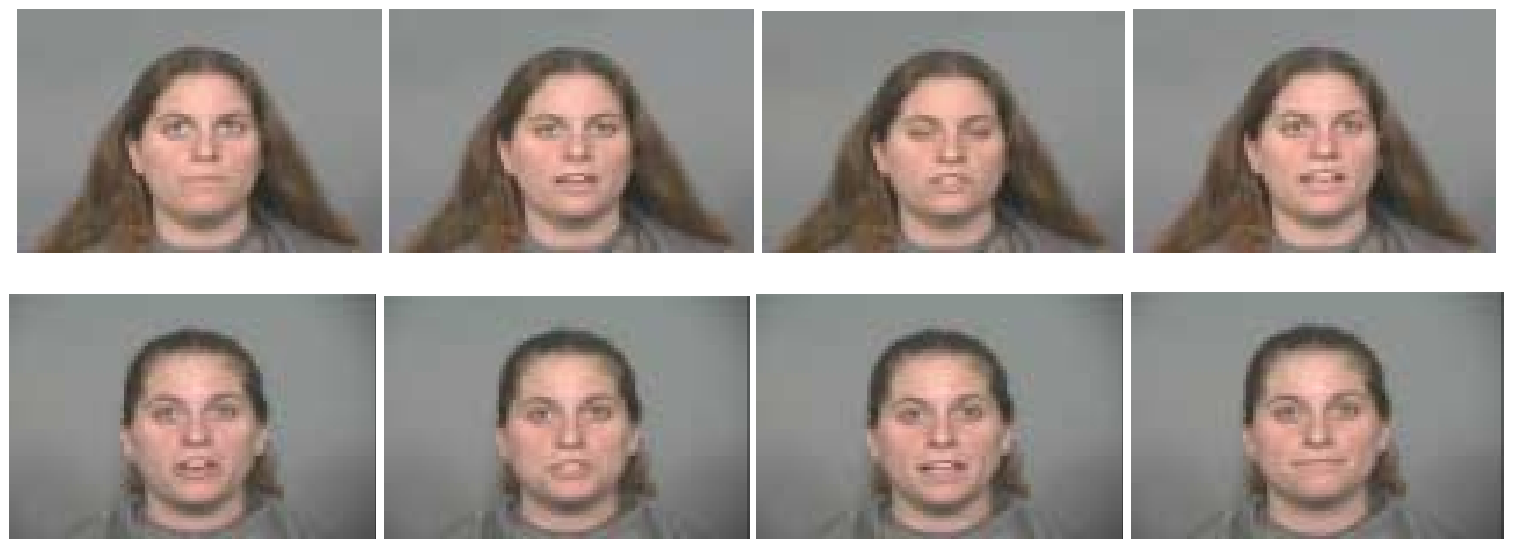

Figure I-7. The rows show selected frames from examples of the UT Dallas "facial speech" videos lasting 150 frames. The two rows show the subject gathered on different occasions. 


\section{This page intentionally blank.}




\section{Appendix J}

Normalization in FRVT 2002 


\section{FRVT 2002 Normalization Specifications}

\section{Introduction}

As stated on the Face Recognition Vendor Test (FRVT) 2002 website (www.frvt.org), the FRVT 2002 uses the Sep96 FERET evaluation protocol (Phillips et al. [1]). The key innovation in the Sep96 FERET evaluation was the introduction of virtual galleries and probe sets. Virtual galleries and probe sets allow for the measurement of performance for different categories of image from one set of similarity files.

Using normalized similarity scores is an idea that has emerged in the biometrics field. Normalization is most commonly used in fingerprint matching. Classically, similarity scores are computed by comparing two images and reporting a measure of similarity between the two faces in those images. Normalization adjusts the scores based on all the images in a gallery. An overview of normalization is presented in section 2 , which precedes a detailed description of how FRVT 2002 participants may proceed with such an option if they elect to do so.

\section{Normalization}

To make the idea of normalization more concrete, consider the following notation. For details on the notation and terms, see Phillips et al. (2000). Let $G$ be a gallery that is a subset of a target set $T$, and $p$ a probe from a probe set $P$ that is a subset of a query set $Q$. Let $S$ be the matrix of nonnormalized similarity scores between target set $T$ and query set $Q$. Let $S^{G}{ }_{P}$ be the set of similarity scores between probe and all signatures in $G$, and $|G|$ be the number of signatures in gallery $G$. The set $S_{P}^{G}$ consists of non-normalized scores. One possible normalization function, $f_{1}$, takes $S_{P}^{G}$ as input, and gives as output, a set of normalized scores $N_{P}^{G}$ - i.e. $\mathrm{N}_{\mathrm{P}}^{\mathrm{G}}=\mathrm{f}_{1}\left(\mathrm{~S}^{\mathrm{G}}\right)$ where $N^{G}{ }_{P}$ is a new set of $|G|$ normalized similarity scores between probe $p$ and all images in $G$. The set of normalized score $N_{P}^{G}$ is computed separately for each $p$ in $P$, and the performance scores are computed from $|\mathrm{P}|$ sets of normalized scores.

A second possible normalization function takes into account the similarity scores between all pairs of gallery images. Let $S^{G G}$ be a $|G|$ by $|G|$ similarity matrix between all pairs of gallery images in $G$. A possible normalization function, $f_{2}$, takes input $S^{G G}$ and $S_{P}^{G}$ and outputs $N_{P}^{G}$ i.e. $N_{P}^{G}=f_{2}$ $\left(S^{G G}, S_{P}^{G}\right)$.

\section{Implementation}

Specific guidelines for the use of normalization in FRVT 2002 are given below. Normalization is a post-test administration procedure that will be executed by NIST after similarity files have been submitted. Participants who elect to utilize the FRVT 2002 normalization option will need to provide NIST with an object file that contains the result of the compilation of $C$ code of specific functions. For intellectual property reasons vendors must not, at any time, submit source code, nor any details of any algorithm to the FRVT 2002 committee.

1. Tasks: Participants may submit up to six functions: zero, one, or two for each of the identification, verification, and watch list tasks. To ensure proper interoperability, the calling semantics cannot be varied from those described below. 
2. Object Files: The functions will be supplied as a single compiled object (i.e. .o) file that can be run on the FRVT 2002 analysts' computers performing the scoring. All code should be written in ISO C. Other languages can only be used if the call semantics are identical to those for C. Particularly $\mathrm{C}++$ must not be used as is because of nonstandardization of function name mangling.

3. Linux: The object files will be linked into programs that run on Intel-based machines running the Linux operating system ${ }^{1}$. To minimize interoperability problems, we recommend using a recent Linux distribution such as RedHat 7.x, Mandrake 8.x, SuSE 8.x, or Gentoo 1.x.

4. Report: The FRVT 2002 will report both normalized and non-normalized performance scores.

5. Responsibility: Participants are responsible for providing a normalization function that works with the FRVT 2002 scoring system.

6. Resources: The normalization functions need to work within a reasonable time, and within reasonable memory. The normalization functions will need to be called each time a probe is processed. This means that the function is embedded in the heart of the scoring code. Use of normalization cannot cause a significant delay in the scoring process. Note, to process a gallery of 10,000 images and probe set of 10,000 images will require calling the normalization routine 10,000 , and each call will contain 10,000 similarity scores.

7. Side-Effects: The normalization functions must have no unspecified inside effects such as sending data to standard output or standard error, writing files to disk, etc.

8. Usage: The FRVT 2002 organizers will not tailor galleries to fit specific properties of a normalization function. For example, if a normalization function assumes that a gallery is homogeneous in terms of image quality or properties of the subjects appearing therein, the FRVT 2002 organizers do not guarantee that all galleries will be homogeneous.

\subsection{Function Specifications}

Participants may submit an object file containing up to six different normalization functions --zero, one, or two functions for each of the identification, verification, and watch list tasks. Each task may use a function with the either of two prototypes, $F 1$ and $F 2$, as detailed below. The function name itself is discussed in section 3.2.

void F1(const unsigned int g_in, const float ${ }^{*} s P G \_i n$, float $\left.^{*} s P G \_o u t\right) ;$

where

g_in is the number of elements in the gallery;

$s P_{-}$in is a pointer to the similarity scores for a given probe or gallery (i.e., a onedimensional array);

$s P$ s_out $_{\text {is }} \quad$ where the normalized similarity scores are to be written.

Or, with the $F 2$ call signature:

void F2(const unsigned int g_in, const float* $s G G \_i n$, const float* $s P G \_i n$, float* $\left.s P G \_o u t\right)$;

\footnotetext{
${ }^{1}$ Linux is a freely-downloadable, Unix-like operating system, available inexpensively online and in bookstores. See http://www.tldp.org/for more information on how to obtain, install, and use Linux.
} 
where $s G G$ in is a pointer to a matrix of similarity values between all pairs of gallery elements. Because this matrix may be not be symmetric it contains all elements represented as an array of floats. The (i,j)-th element is accessed using sGG in[j * $g$ in $+i]$ and corresponds to the $i$-th element from the j-th similarity file; this implies column major ordering of the data, with each column of the matrix coming from a single similarity file.

Participants may assume that the proper storage for sPG_out, g_in * sizeof(float), has already been allocated. Storage for these variables will be allocated outside of the functions.

Within time and memory limitations, performance scores will be generated for normalization functions provided. Because of potential time and memory limitations associated with F2 class functions, it recommended that vendors provide an F1 function for each F2 function.

\subsection{Naming Conventions}

The name of the functions that a participant may submit in their object file is determined by:

1. the name of the participant

2. the application (i.e., identification, verification, watch list)

3. the signature's structure (i.e., F1() or F2())

Each participant and application has its own abbreviation. Participants will be e-mailed their own particular abbreviation by the FRVT 2002 liaison. The application abbreviations are:

$\begin{array}{ll}\text { Identification } & \text { ident } \\ \text { Verification } & \text { verif } \\ \text { Watch List } & \text { watch }\end{array}$

Each call signature will take the following general form.

void [participant code] [application] [function type](const int $\mathrm{g}, \ldots$...)

where [participant code] is the participant's three-letter code, [application] is the target application, or context within which the normalization code should be used, and [function type] is the structure of the function (i.e., F1() or F2()).

The name of the submitted object file must be [participant code]-norm.o. The participant may submit code to be used during scoring on identification, verification and watch list testing. For example, consider that a generic participant, whose three-letter code is NST, provides a F1() structured function for identification and a F2() function for the watch list task. Their full declarations would be

and

$$
\text { void NST_ident_F1(const unsigned int, const float }{ }^{*} \text {, float *); }
$$

void NST_watch_F2(const unsigned int, const float ${ }^{*}$, const float ${ }^{*}$, float ${ }^{*}$ );

The NST code would be written into a single source file named NST-norm.c. The following command would be used to compile the normalization code:

$$
\text { helix\% gcc }-03 \text {-I./ } \quad \text {-c NST-norm.c } \quad \text {-o NST-norm.o }
$$

The flag -c suppresses linking, -I./ indicates that the code can include header files from the current directory, $-\mathrm{O} 3$ enables optimization, and -o directs the output to the specified file. Note the use of gcc not $\mathbf{g + +}$ here. Also note that gcc running under the Cygwin package on non-Linux platforms does not interoperate with the linux runtime environment. The code must not be 
compiled with the $-g$ flag. This prevents meaningful symbols being visible to the FRVT 2002 analysts. The code should be as self-contained as possible. Any non-standard library code should be included, and to avoid link problems, private functions and variables should be declared static in a participant's code.

Sample code is shown in the box below; note that it does not necessarily perform a useful normalization - it is included for illustration of data access and interoperability only.

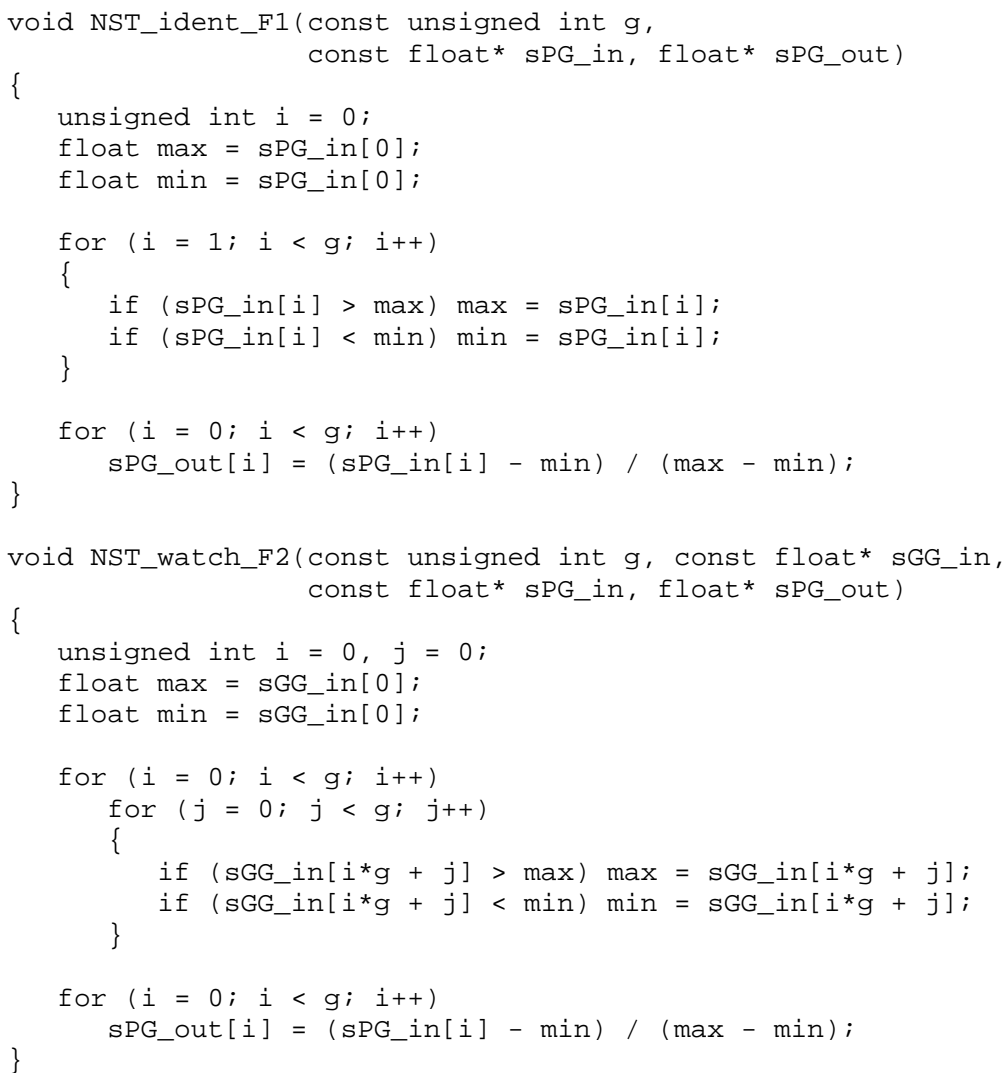

The resulting object file should be sent to frvt2002@nist.gov according to a timetable that the FRVT 2002 administrator will send with the participants' three-letter participant abbreviation. The object file submission will initiate a correspondence with an FRVT 2002 analyst to verify the behavior of the normalization routines.

\section{References}

[1] P. J. Phillips, H. Moon, S. A. Rizvi, and P. J. Rauss. The FERET Evaluation Methodology for Face Recognition Algorithms. IEEE Trans. Pattern Analysis and Machine Intelligence, 22: pp. 1090-1104, 2000. 


\section{This page intentionally blank.}




\section{Appendix K}

Development Image Set

The following images were provided to FRVT 2002 Participants as examples of images they would use during the FRVT 2002. A larger database of FERET images was also provided for participant's use during the pre-evaluation check of their APIs to ensure compatibility with the FRVT 2002 scoring code. Images have been non-uniformly resized for printing purposes. 

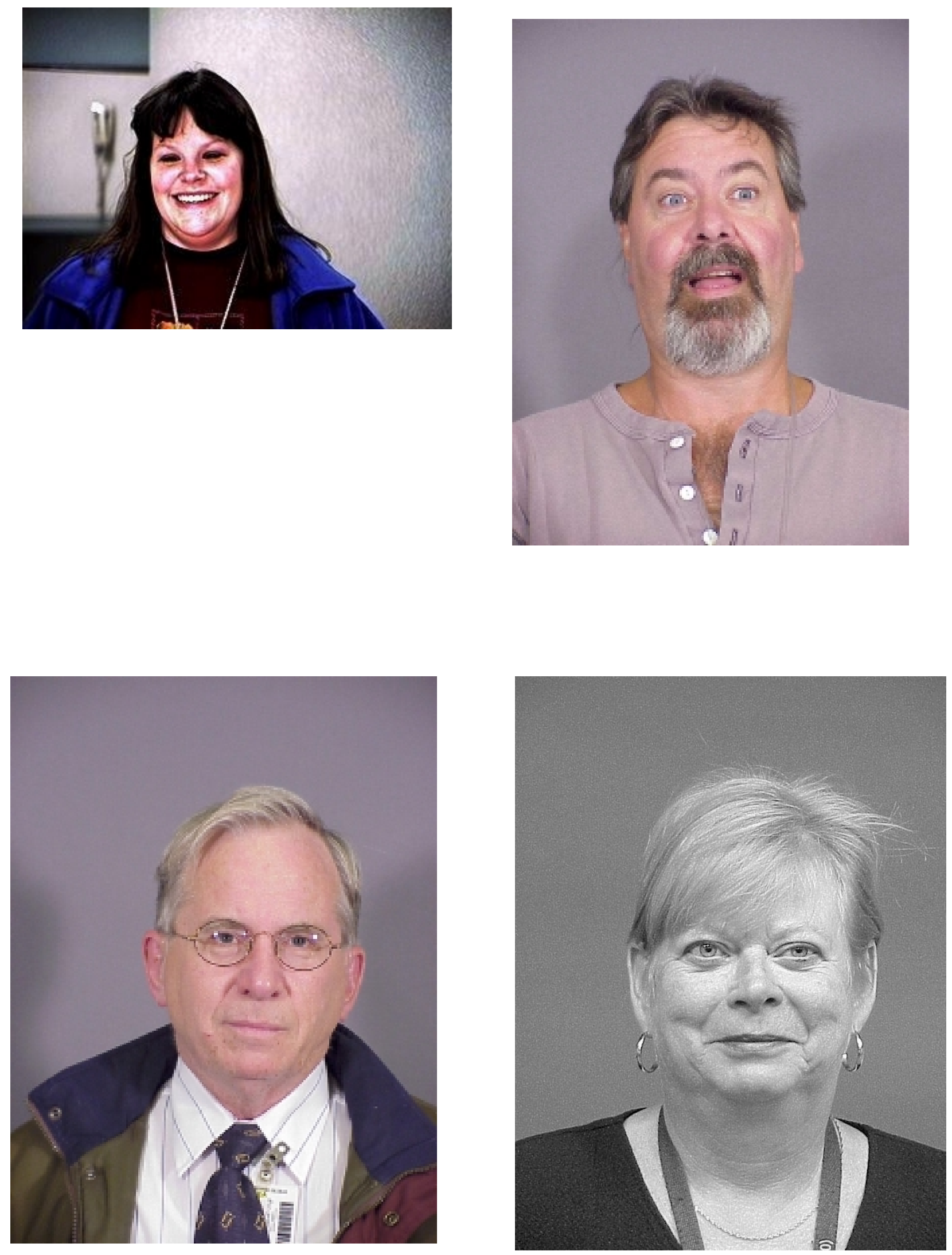

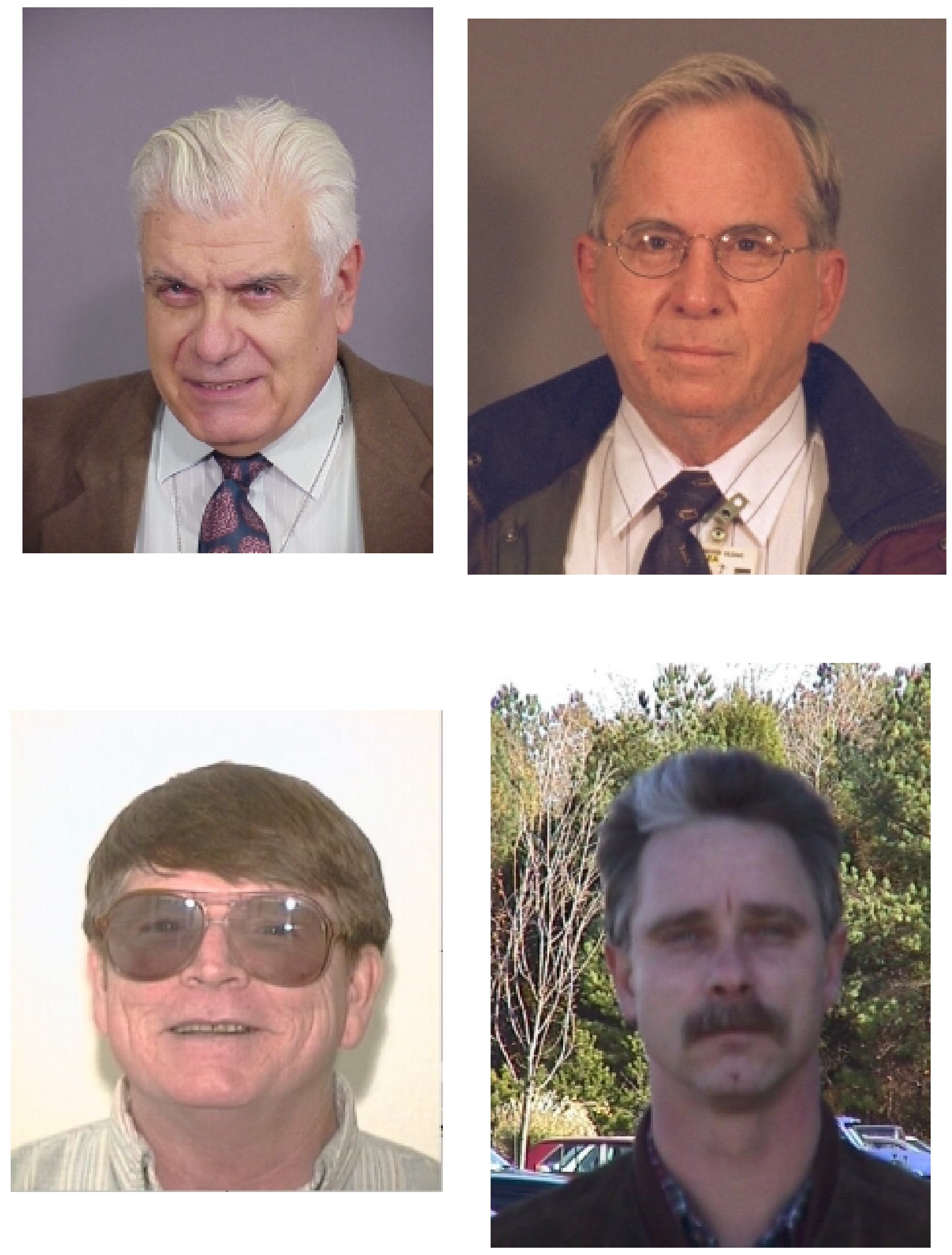

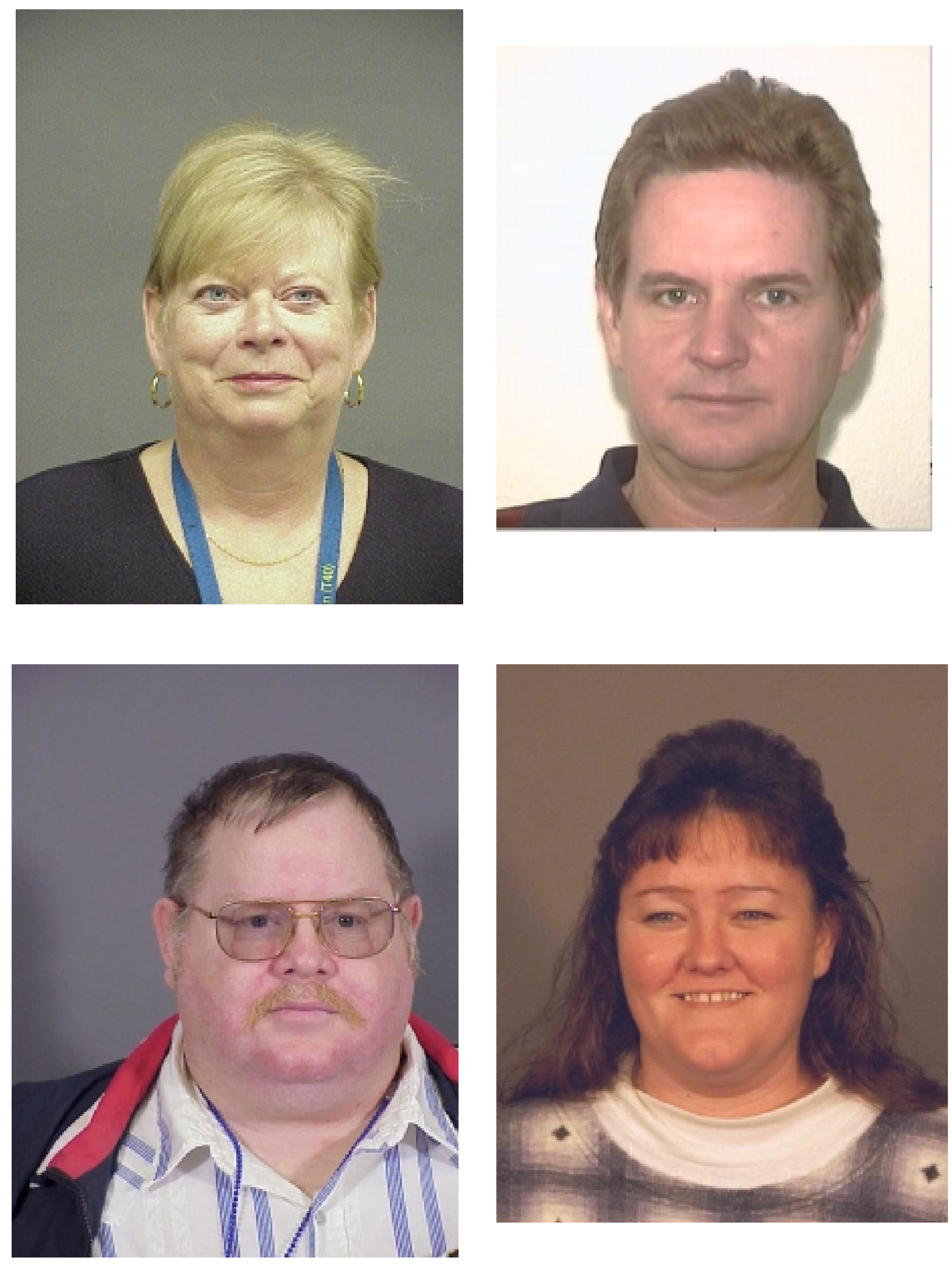

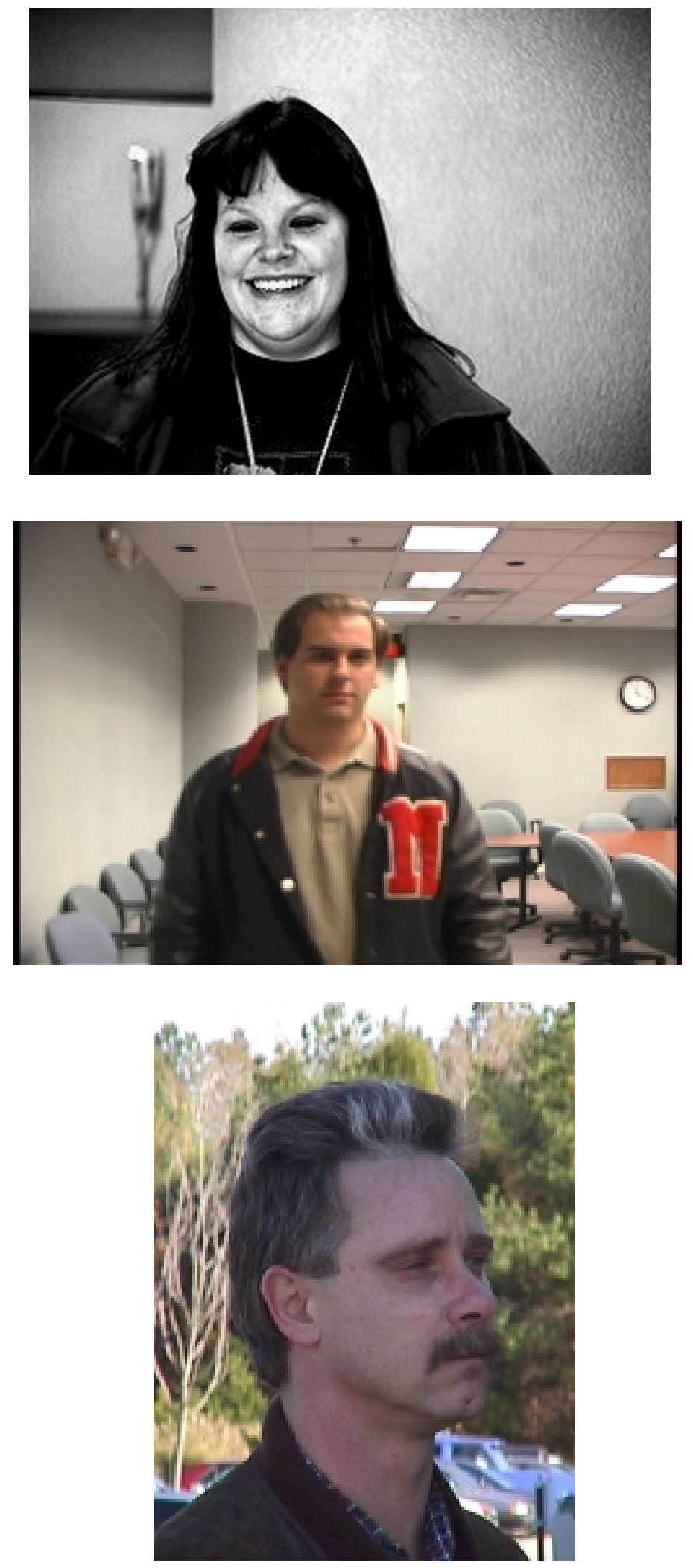


\section{This page intentionally blank.}




\section{Appendix L}

In order to clearly present an assessment of the state-of-the-art in face recognition, it was necessary in the Evaluation Report to report summary statistics of performance. Appendix L presents full cumulative match charateristics (CMC) and receiver operating characteristics (ROC) for all MCInt experiments (Section 8 and Appendix A.4 in the Evaluation Report). Also, this appendix presents HCInt verification error ellipses ROCs (Figure 8) for all participants. 


\section{L.1 HCInt Verification Error Ellipses Experiment}

Figure 8 in the FRVT 2002: Evaluation Report showed standard error ellipses for verification performance for Cognitec, Eyematic and Identix. The standard error was computed from twelve HCInt small galleries of size 3,000. The centerline is the ROC performance for the aggregate for all twelve galleries. The ellipses are two times the standard deviation at selected performance points, and the points clustered around the ellipses are the performance of one of the twelve galleries at the selected performance point. Figure L.1 through Figure L. 8 show error ellipses for each of the HCInt participants separately, and Figure L.9 show error ellipses for all participants on one graph.

\begin{tabular}{cc}
\hline Participant & Figure \\
\hline Cognitec & Figure L.1 \\
C-Vis & Figure L.2 \\
Dream-Mirh & Figure L.3 \\
Eyematic & Figure L.4 \\
Identix & Figure L.5 \\
Imagis & Figure L.6 \\
Viisage & Figure L.7 \\
Visionsphere & Figure L.8 \\
All Participants & Figure L.9 \\
\hline
\end{tabular}

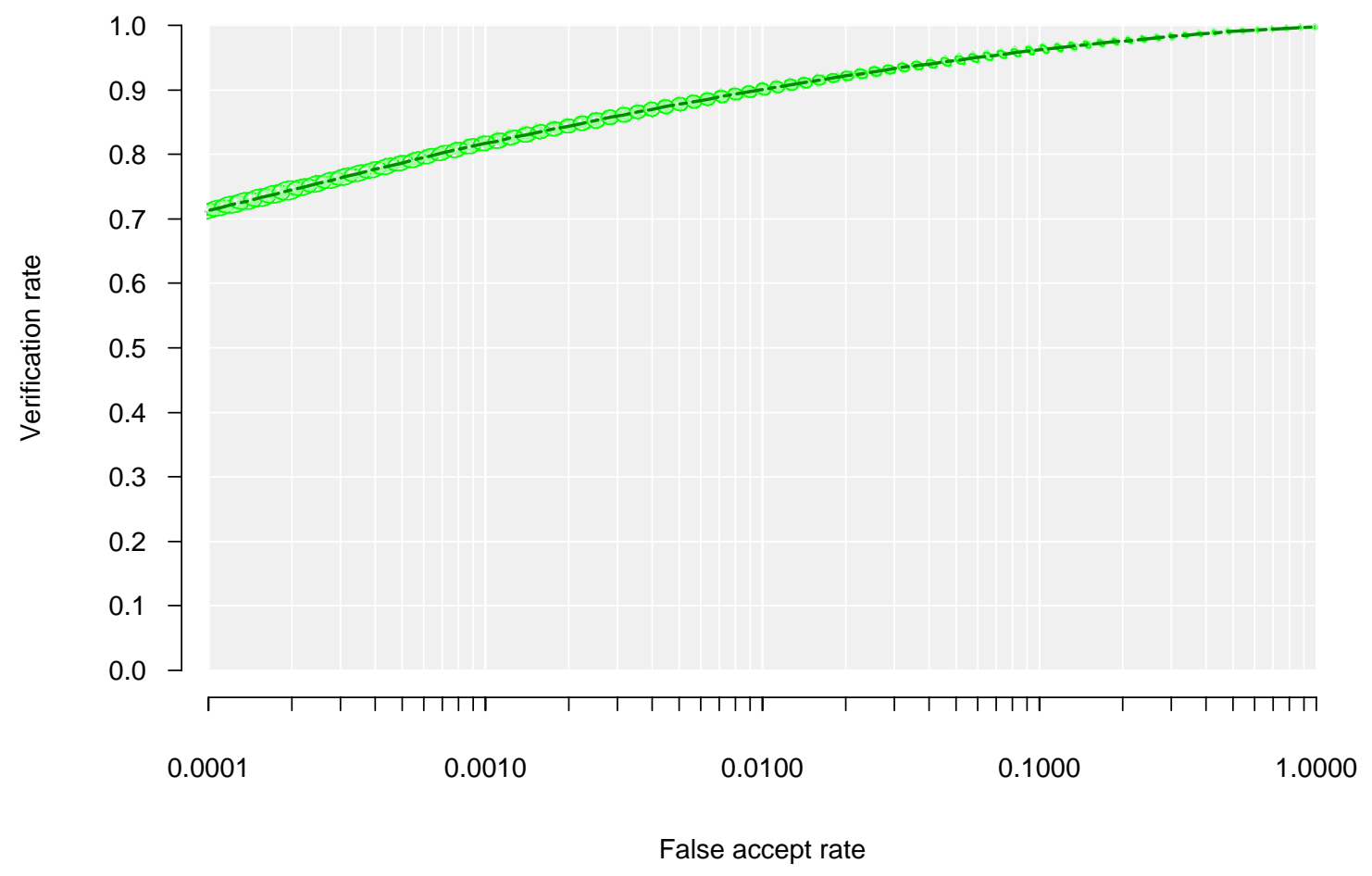

Figure L.1 - Cognitec Verification Error Ellipses. This figure corresponds to Figure 8 in the FRVT 2002: Evaluation Report. 


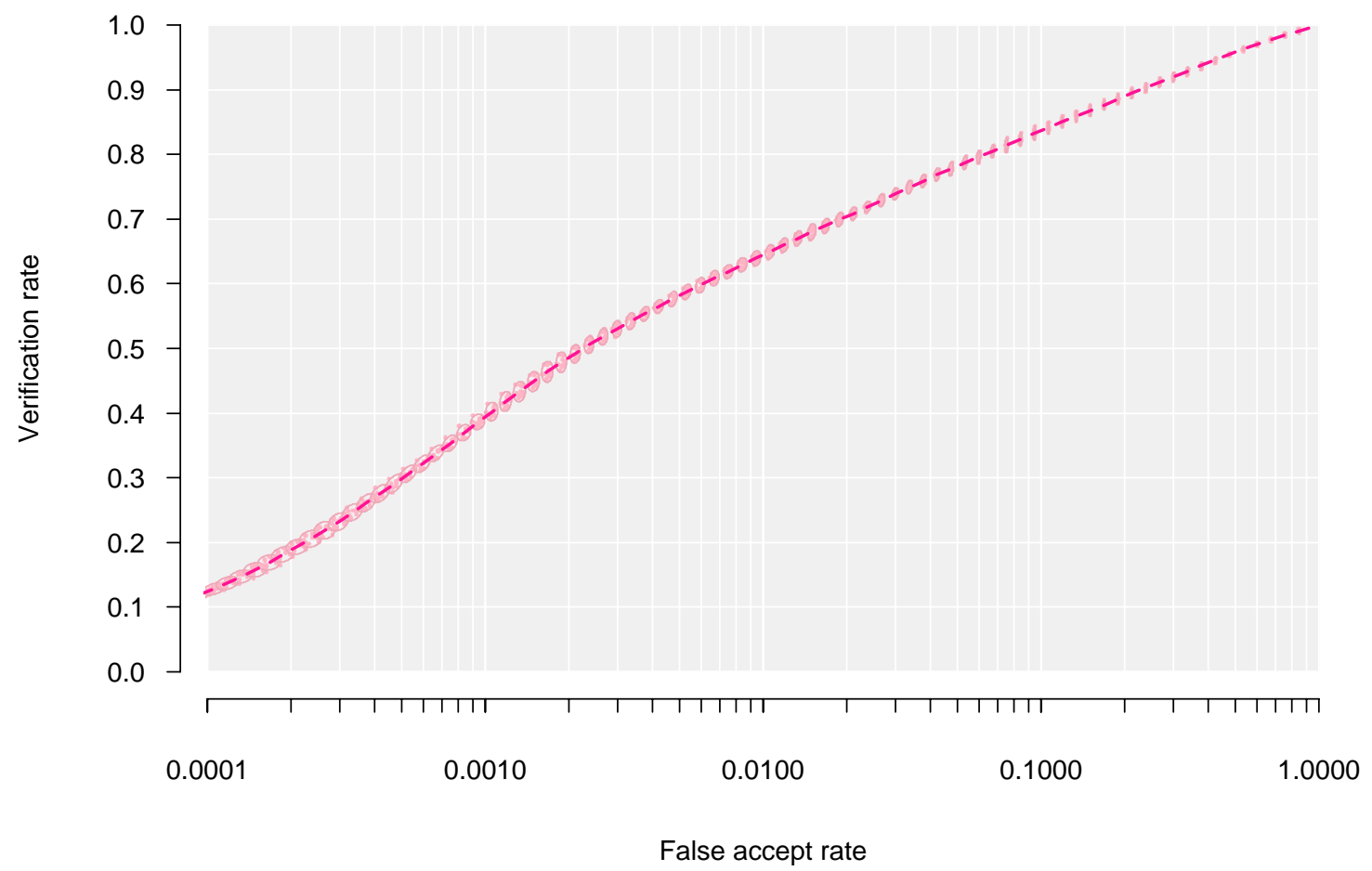

Figure L.2 - C-VIS Verification Error Ellipses. This figure corresponds to Figure 8 in the FRVT 2002: Evaluation Report.

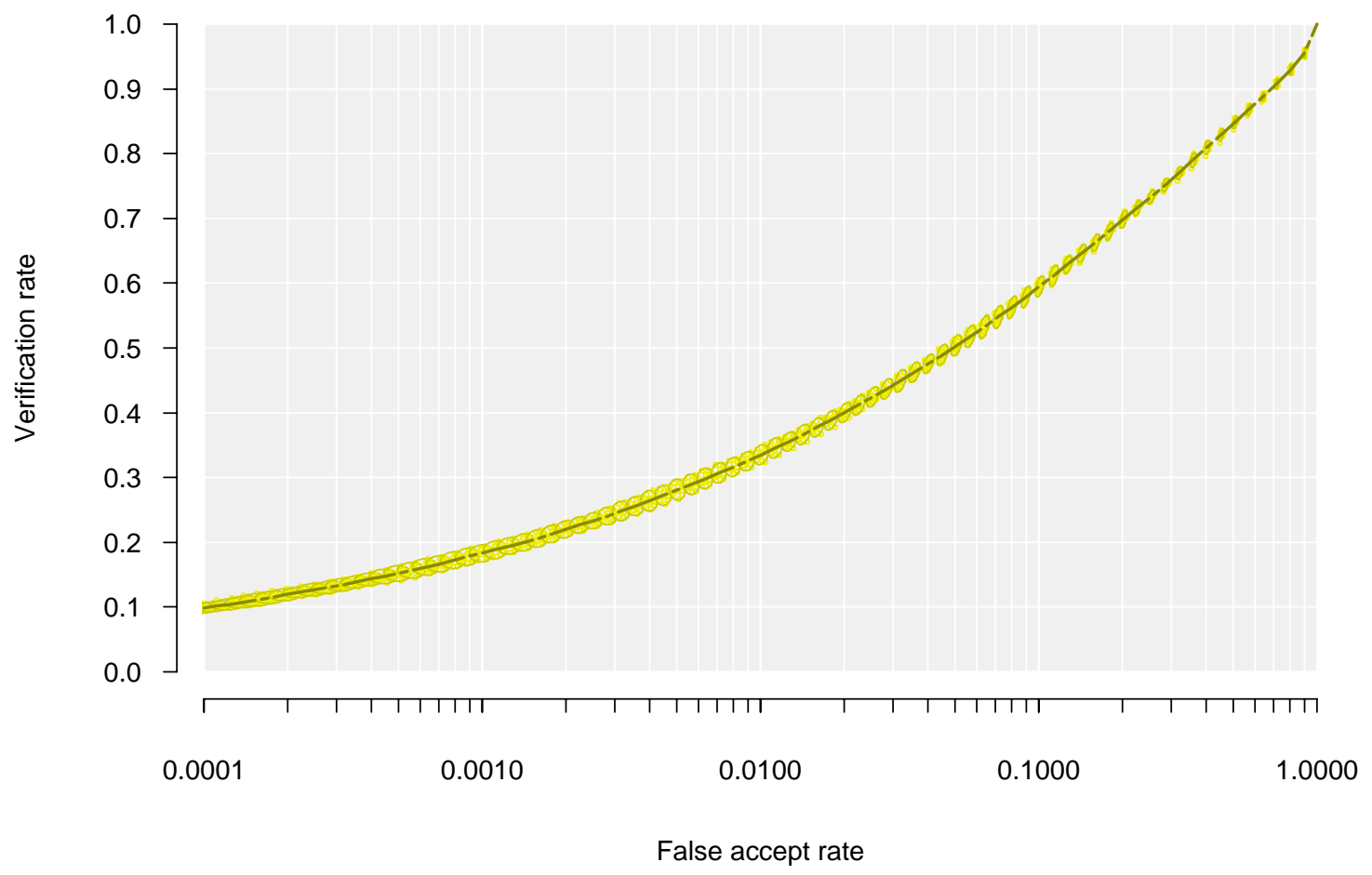

Figure L.3 - Dream Mirh Verification Error Ellipses. This figure corresponds to Figure 8 in the FRVT 2002: Evaluation Report. 


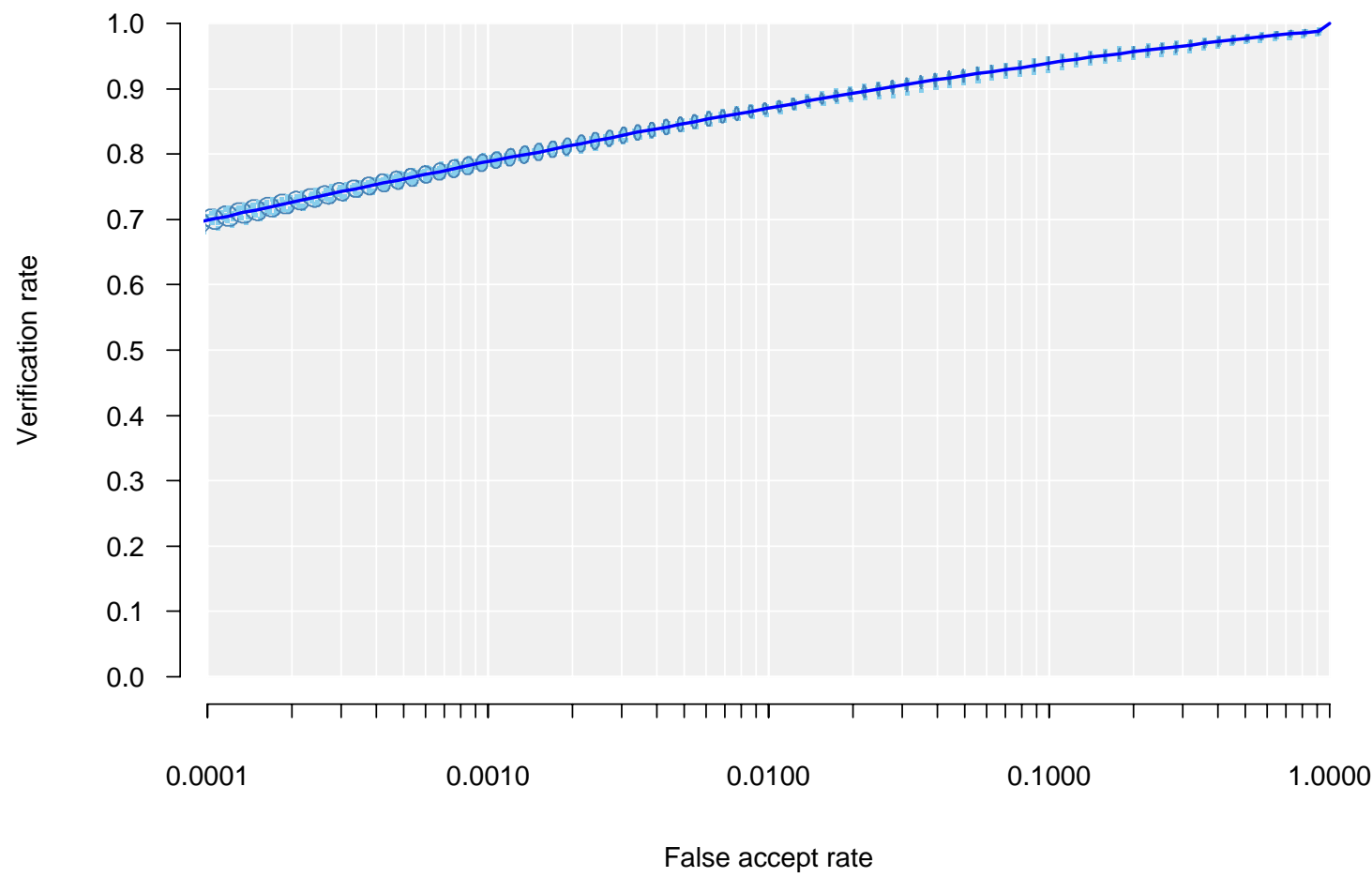

Figure L.4 - Eyematic Verification Error Ellipses. This figure corresponds to Figure 8 in the FRVT 2002: Evaluation Report.

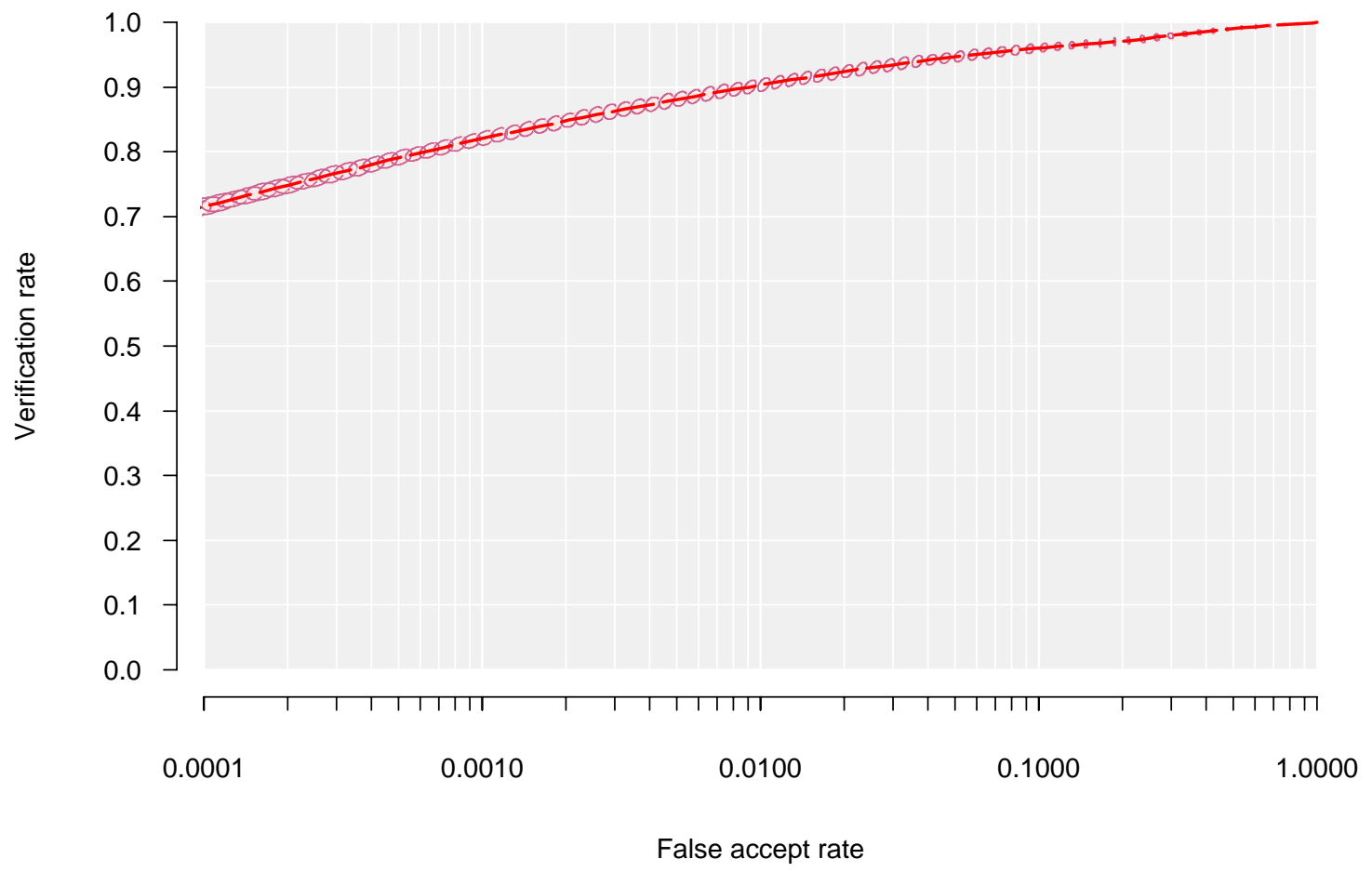

Figure L.5 - Identix Verification Error Ellipses. This figure corresponds to Figure 8 in the FRVT 2002: Evaluation Report. 


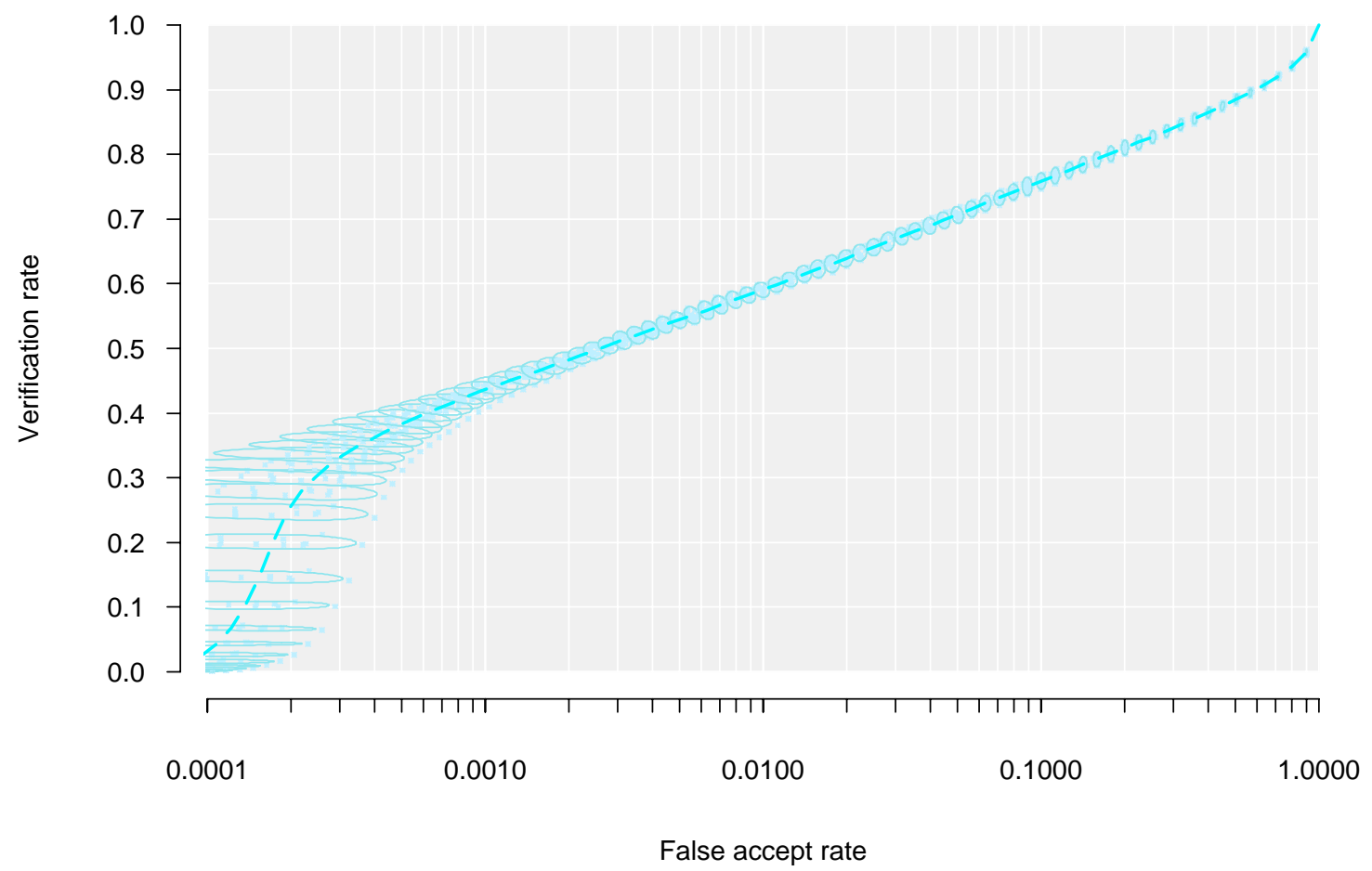

Figure L.6 - Imagis Verification Error Ellipses. This figure corresponds to Figure 8 in the FRVT 2002: Evaluation Report.

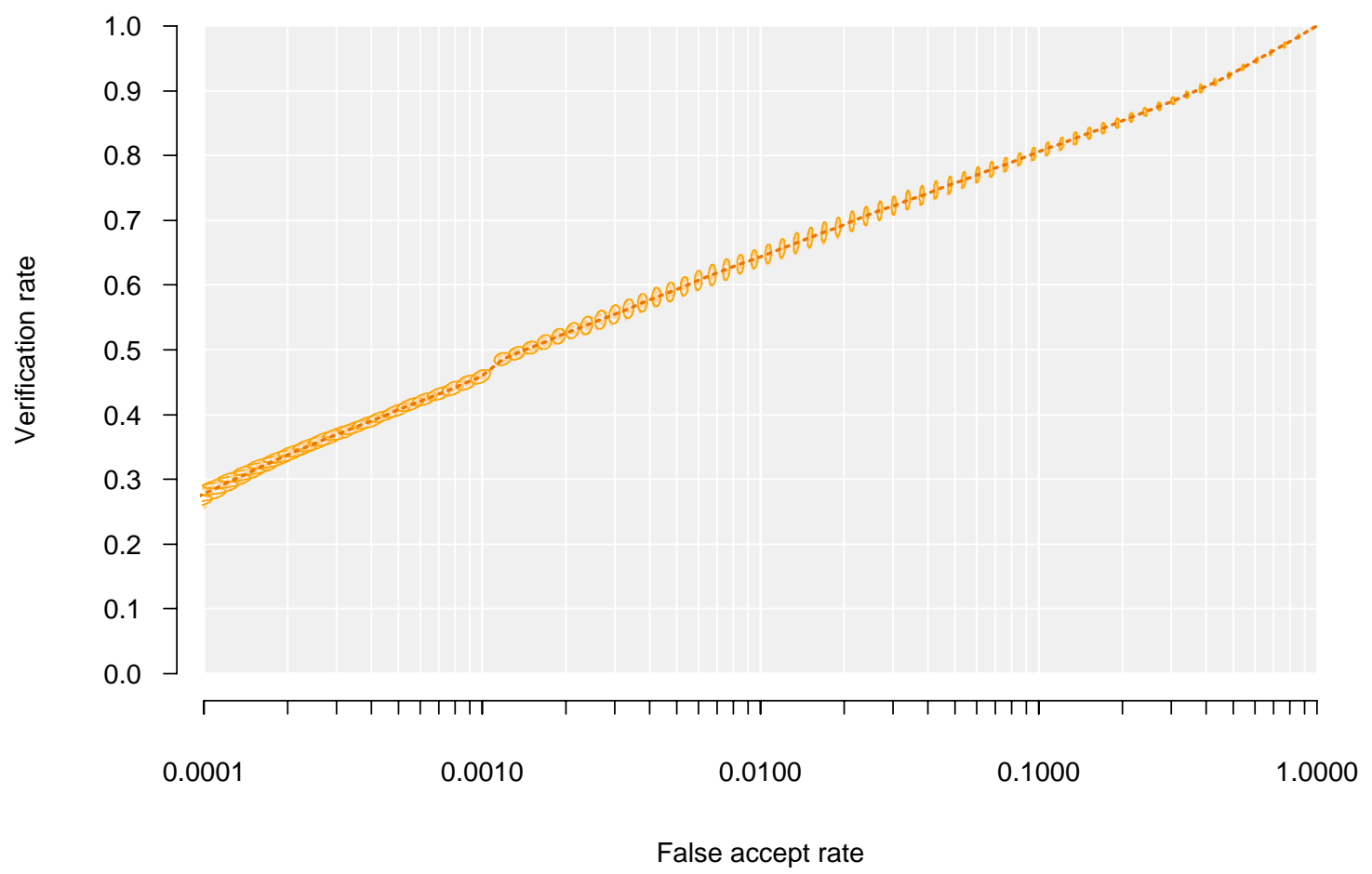

Figure L.7 - Viisage Verification Error Ellipses. This figure corresponds to Figure 8 in the FRVT 2002: Evaluation Report. 


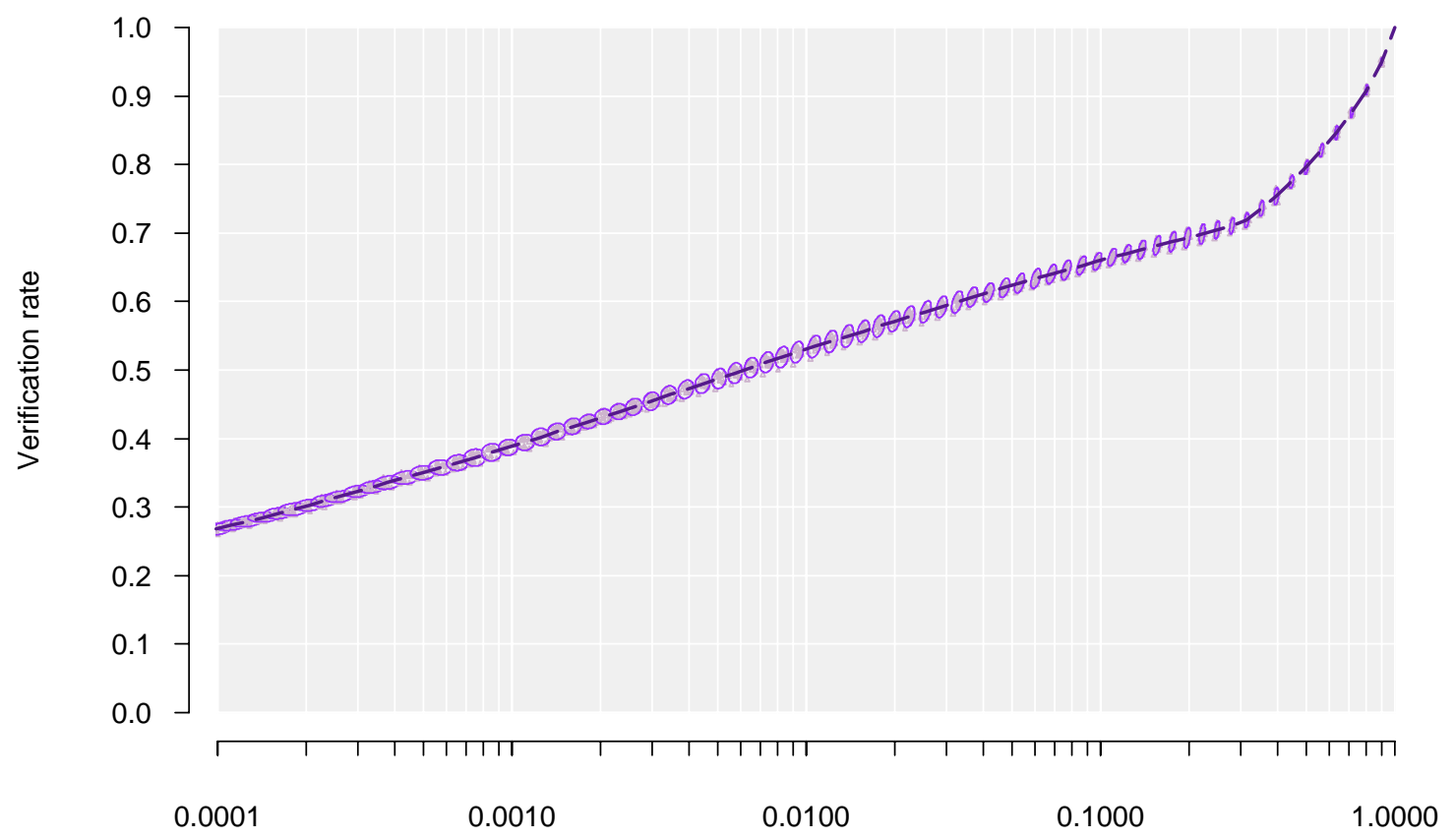

False accept rate

Figure L.8 - VisionSphere Verification Error Ellipses. This figure corresponds to Figure 8 in the FRVT 2002: Evaluation Report.

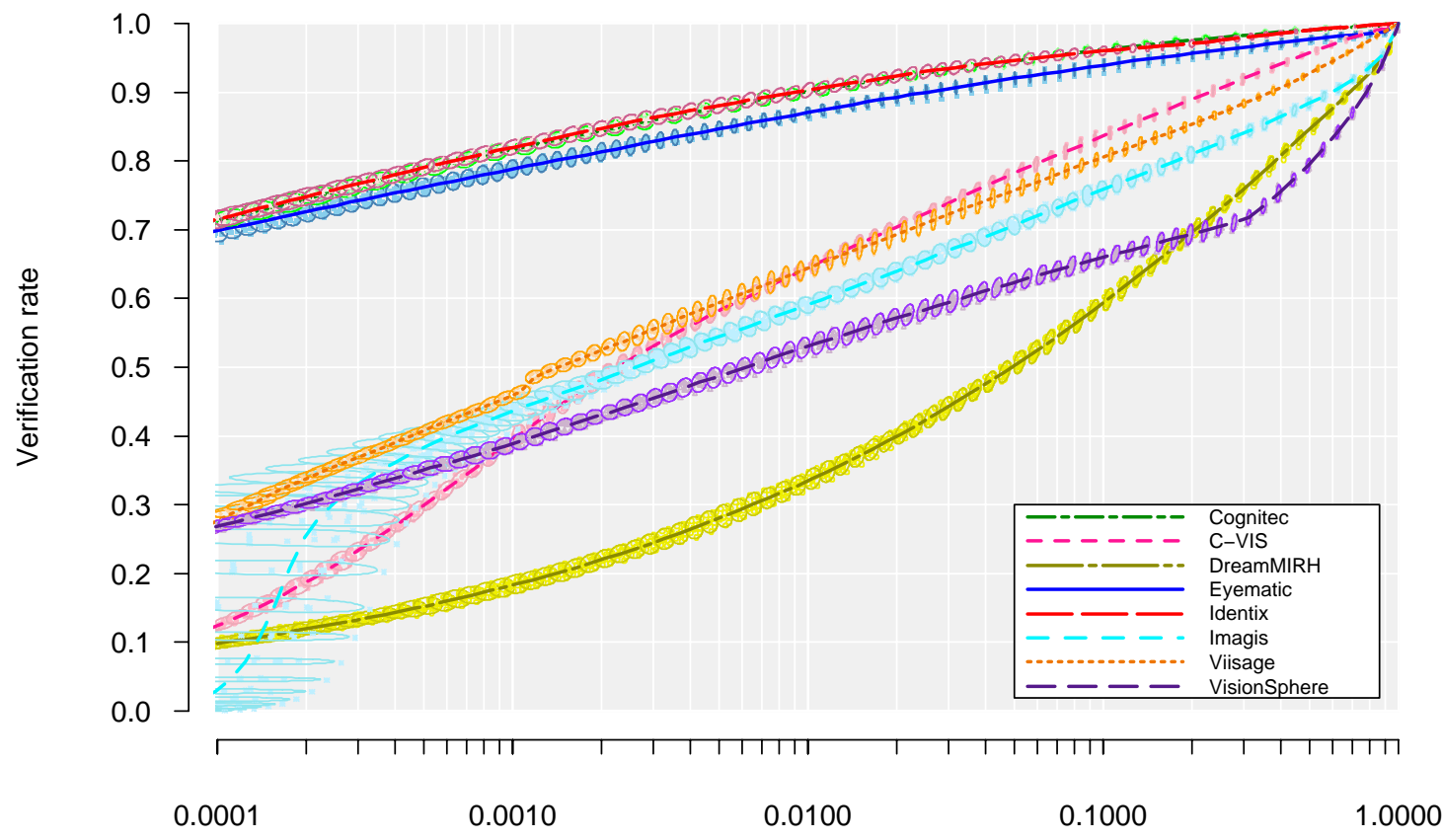

False accept rate

Figure L.9 - Verification Error Ellipses for all Participants. This figure corresponds to Figure 8 in the FRVT 2002: Evaluation Report. 


\section{L.2 Normalization Experiment}

Figure 9 in the FRVT 2002: Evaluation Report highlighted changes in performance between normalized and non-normalized verification performance. Figure L.10 through Figure L. 13 provide full normalized and non-normalized ROC curves from this experiment.

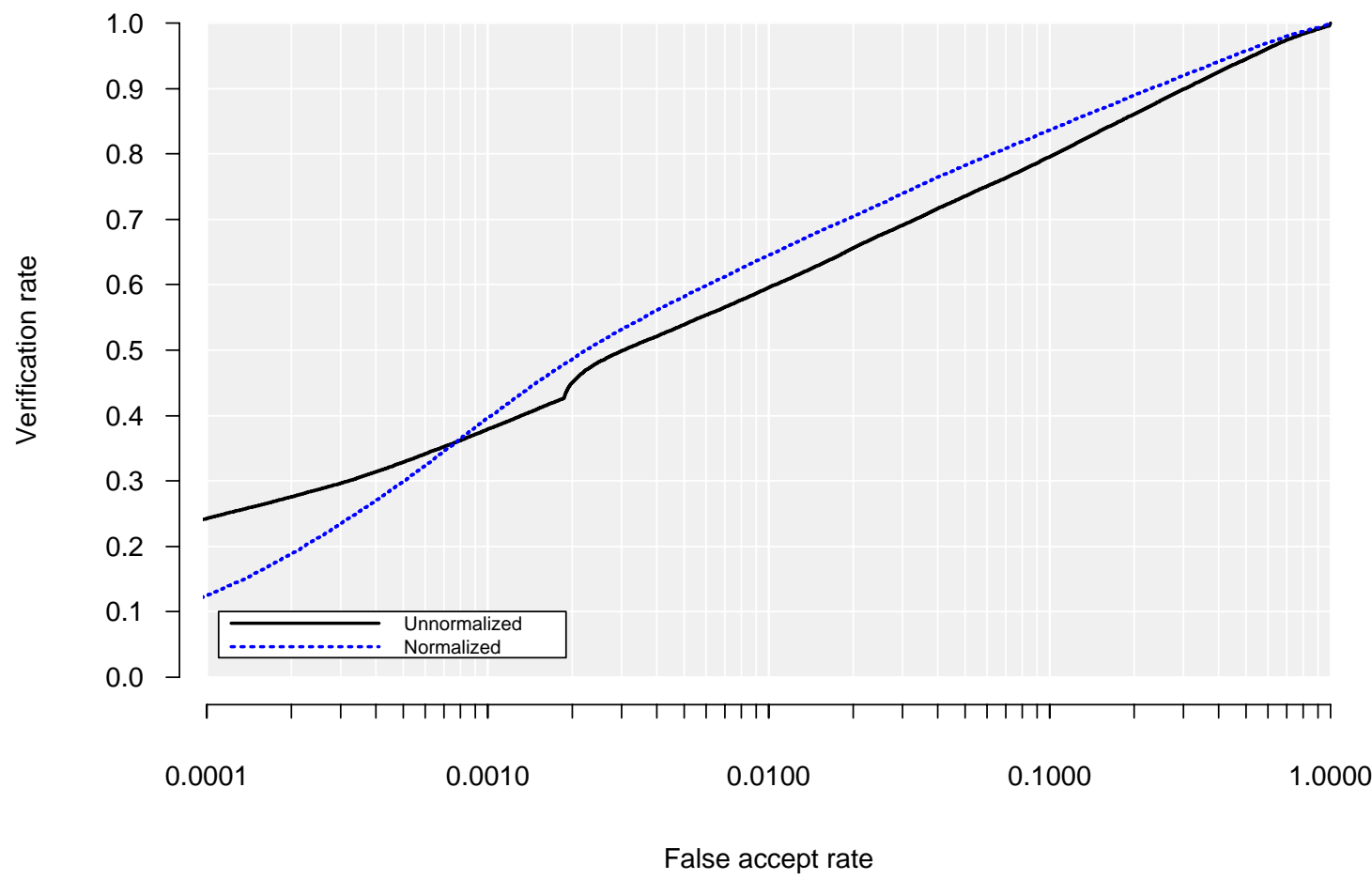

Figure L.10 - Normalized and non-normalized ROC for C-Vis. This figure corresponds to Figure 9 in the FRVT 2002: Evaluation Report. 


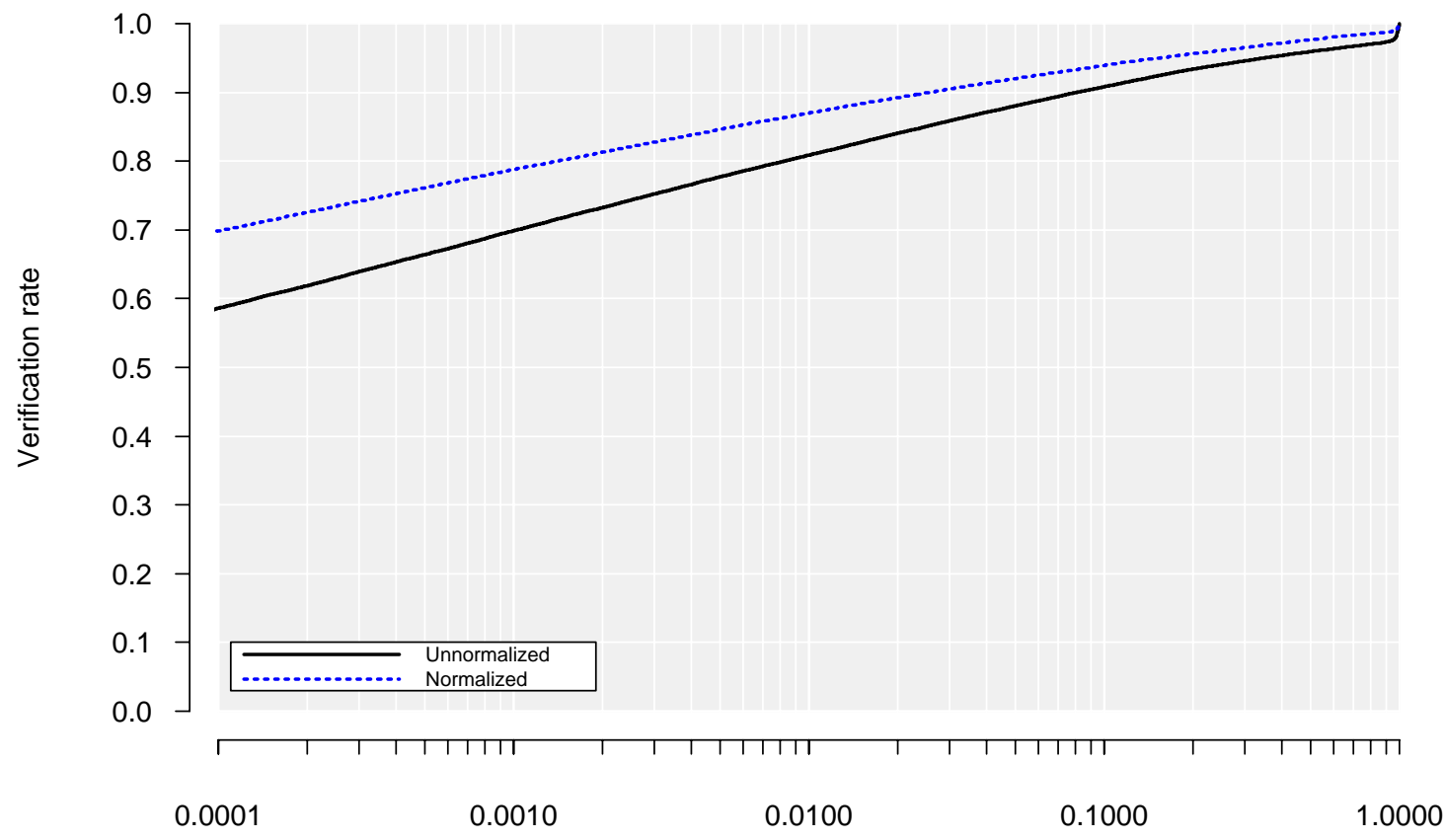

False accept rate

Figure L.11- Normalized and non-normalized ROC for Eyematic. This figure corresponds to Figure 9 in the FRVT 2002: Evaluation Report.

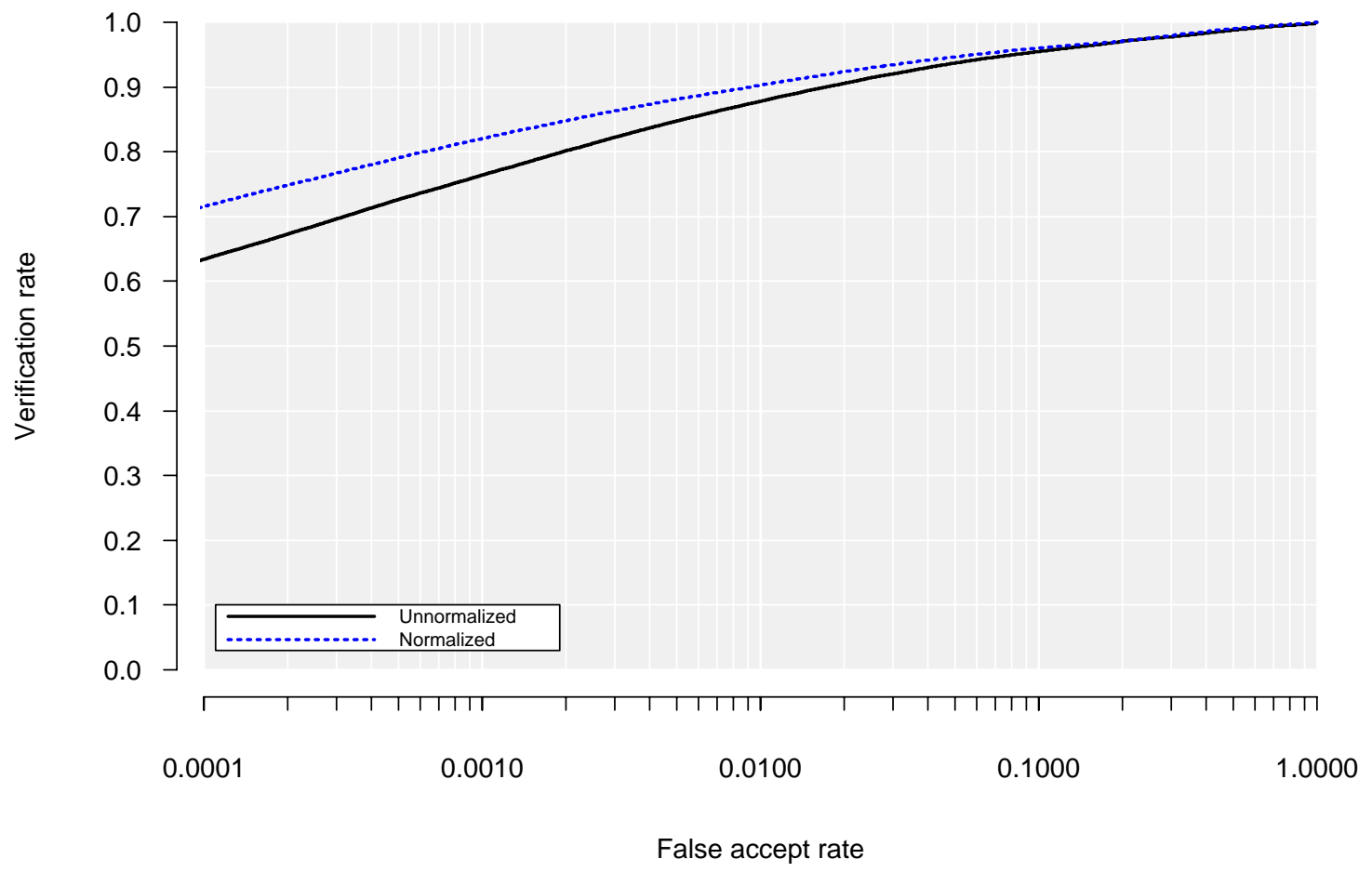

Figure L. 12 - Normalized and non-normalized ROC for Identix. This figure corresponds to Figure 9 in the FRVT 2002: Evaluation Report. 


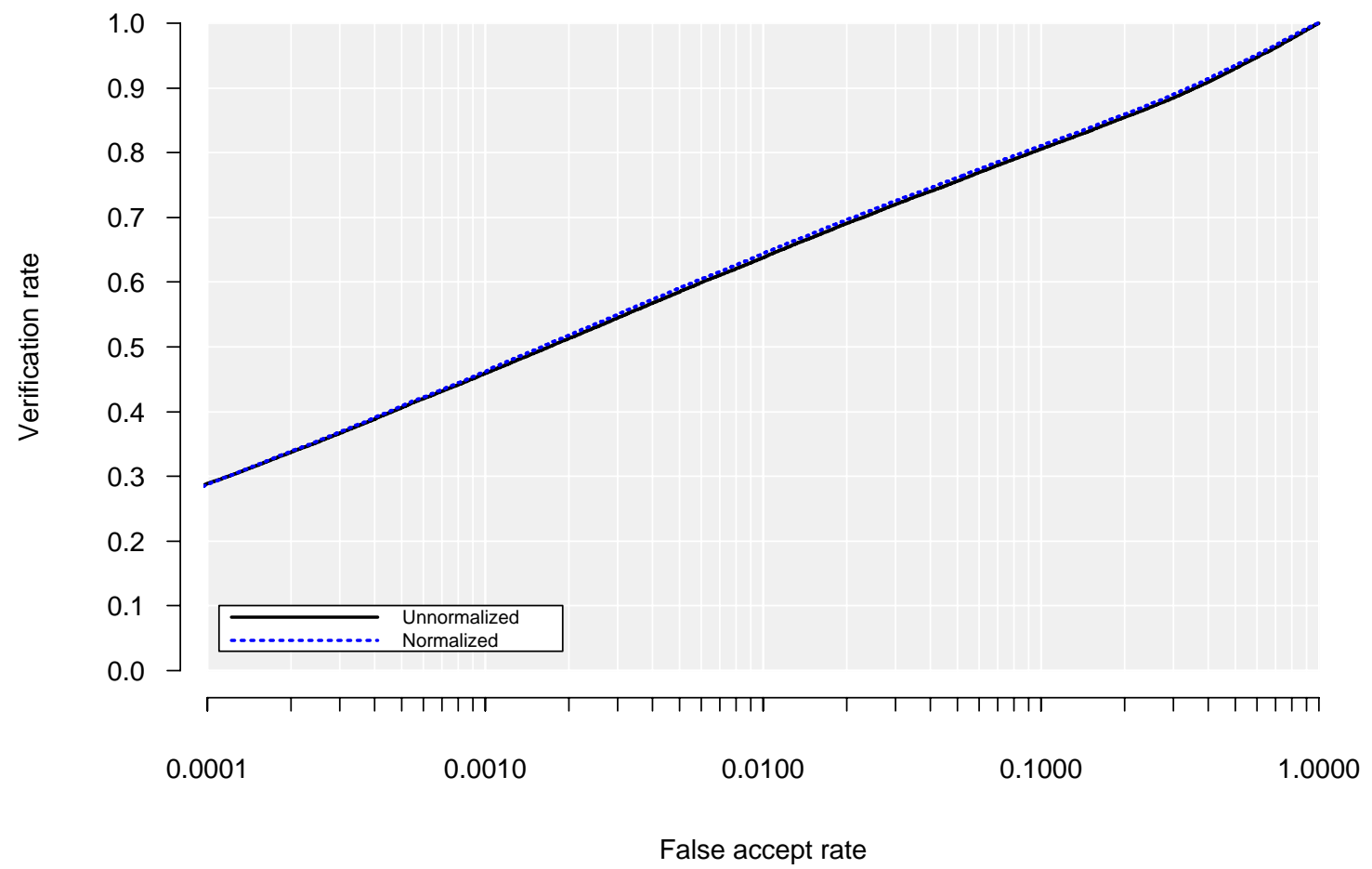

Figure L. 13 - Normalized and non-normalized ROC for Viisage. This figure corresponds to Figure 9 in the FRVT 2002: Evaluation Report. 


\section{L.3 MCInt Frontal Face Experiment}

Figure 22 in the FRVT 2002: Evaluation Report was a comparison of system performance for the verification task using various frontal face probes. Figure 28 showed identification results for the same experiments. Figure L.14 through Figure L.23 provide full ROC and CMC charts for each lighting condition, as outlined in the following table.

\begin{tabular}{ccc}
\hline Lighting Condition & $\begin{array}{c}\text { Performance } \\
\text { Curve }\end{array}$ & $\begin{array}{c}\text { Figure } \\
\text { Number }\end{array}$ \\
\hline Indoor (same day) & ROC & Figure L.14 \\
& CMC & Figure L.15 \\
Indoor (same day, overhead) & ROC & Figure L.16 \\
& CMC & Figure L.17 \\
Indoor (different day) & ROC & Figure L.18 \\
& CMC & Figure L.19 \\
Outdoor (same day) & ROC & Figure L.20 \\
& CMC & Figure L.21 \\
Outdoor (different day) & ROC & Figure L.22 \\
& CMC & Figure L.23 \\
\hline
\end{tabular}




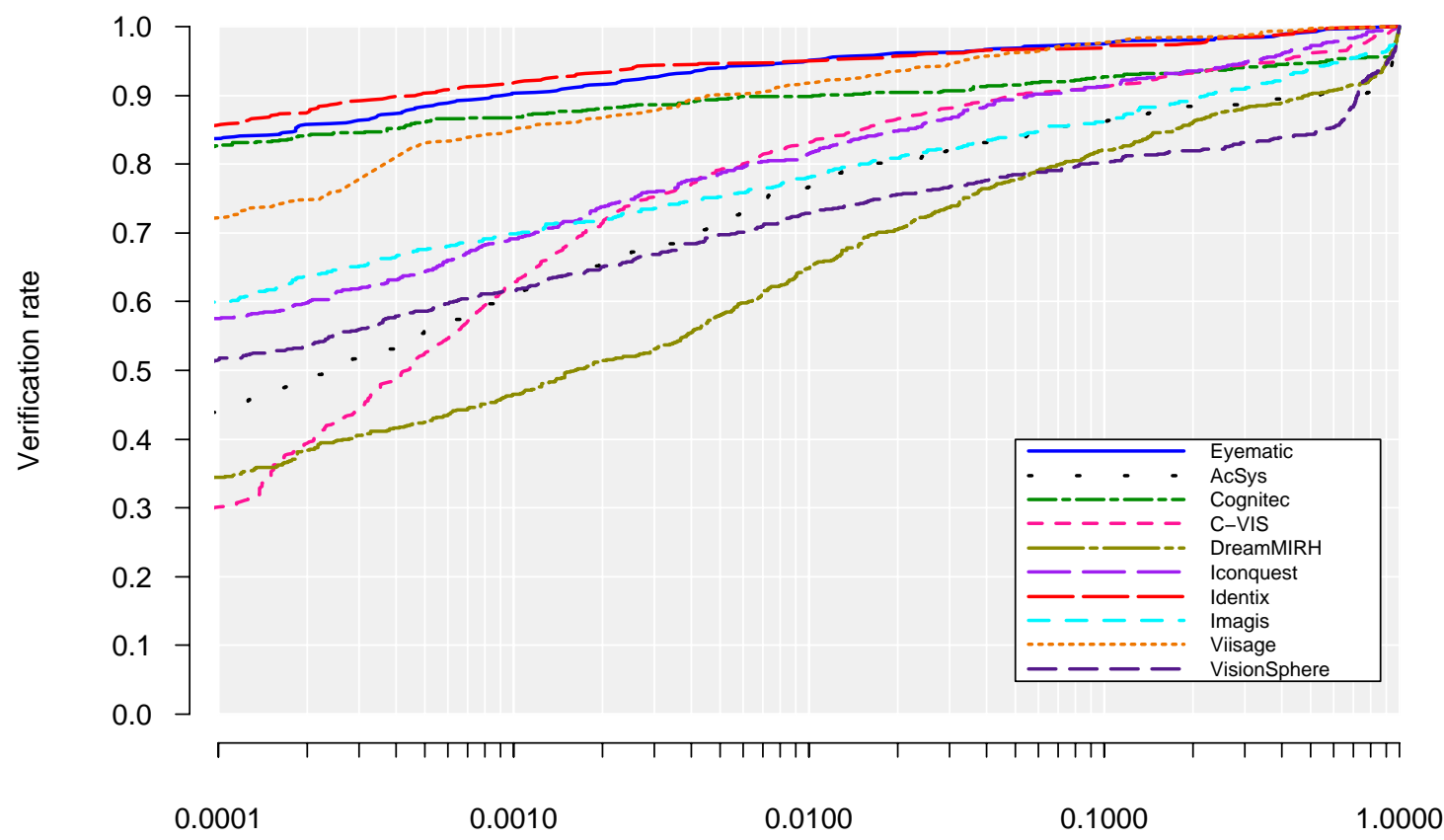

False accept rate

Figure L.14 - Verification Performance for Indoor (same day) Probes. This figure corresponds to Figure 22 in the FRVT 2002: Evaluation Report.

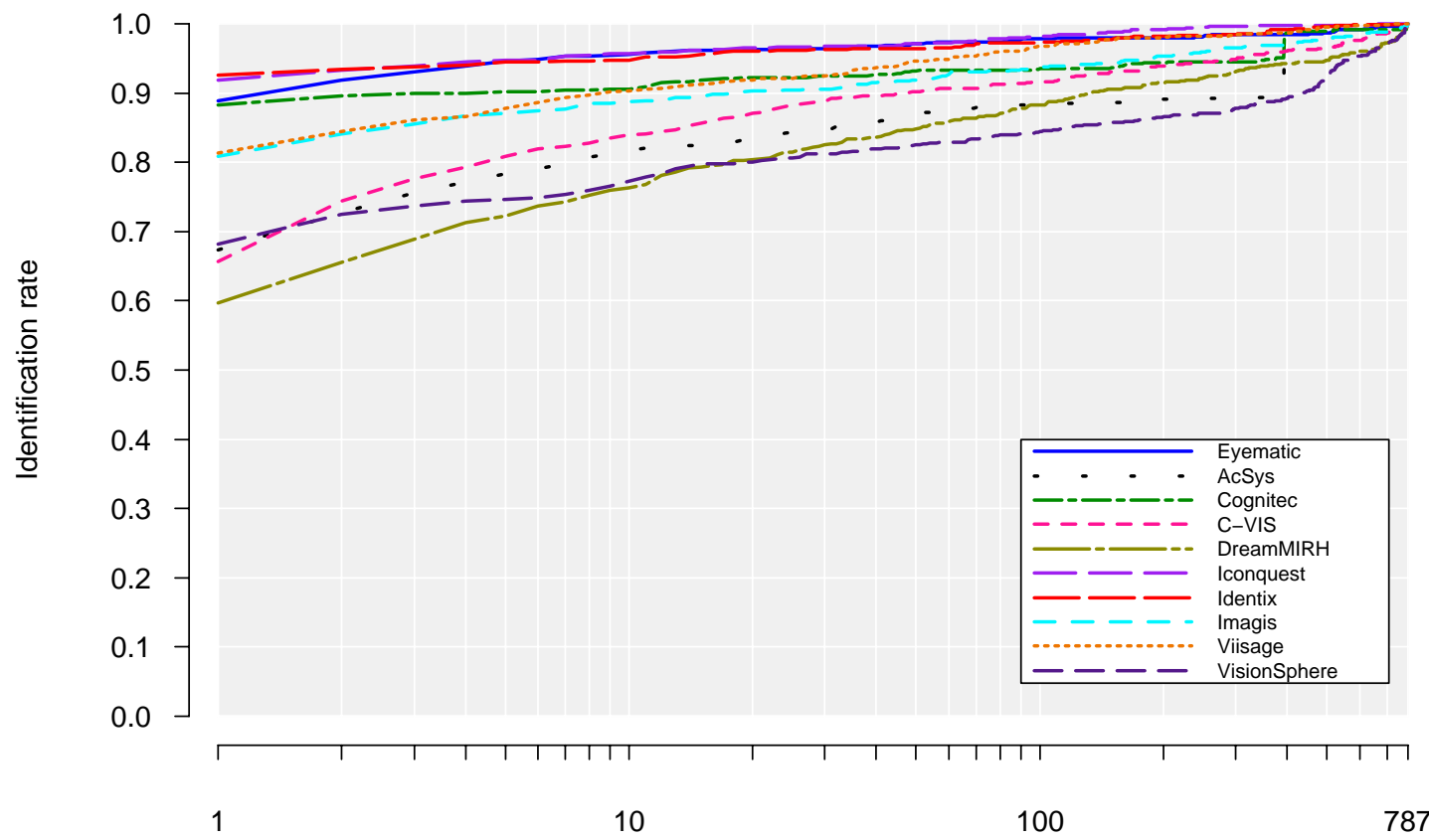

Rank

Figure L.15 - Identification Performance for Indoor (same day) Probes. This figure corresponds to Figure 28 in the FRVT 2002: Evaluation Report. 


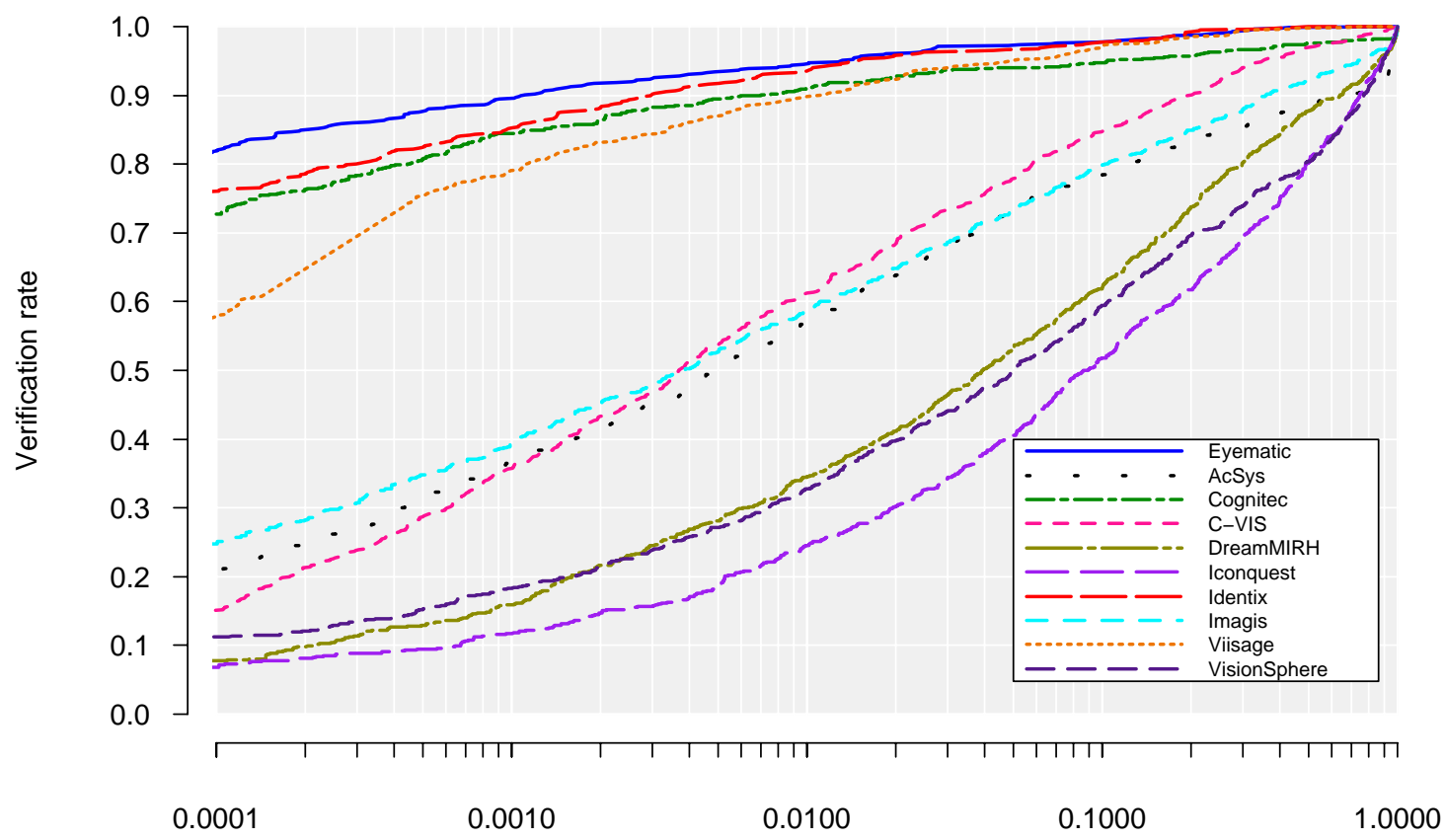

False accept rate

Figure L.16 - Verification Performance for Indoor (same day, overhead) Probes. This figure corresponds to Figure 22 in the FRVT 2002: Evaluation Report.

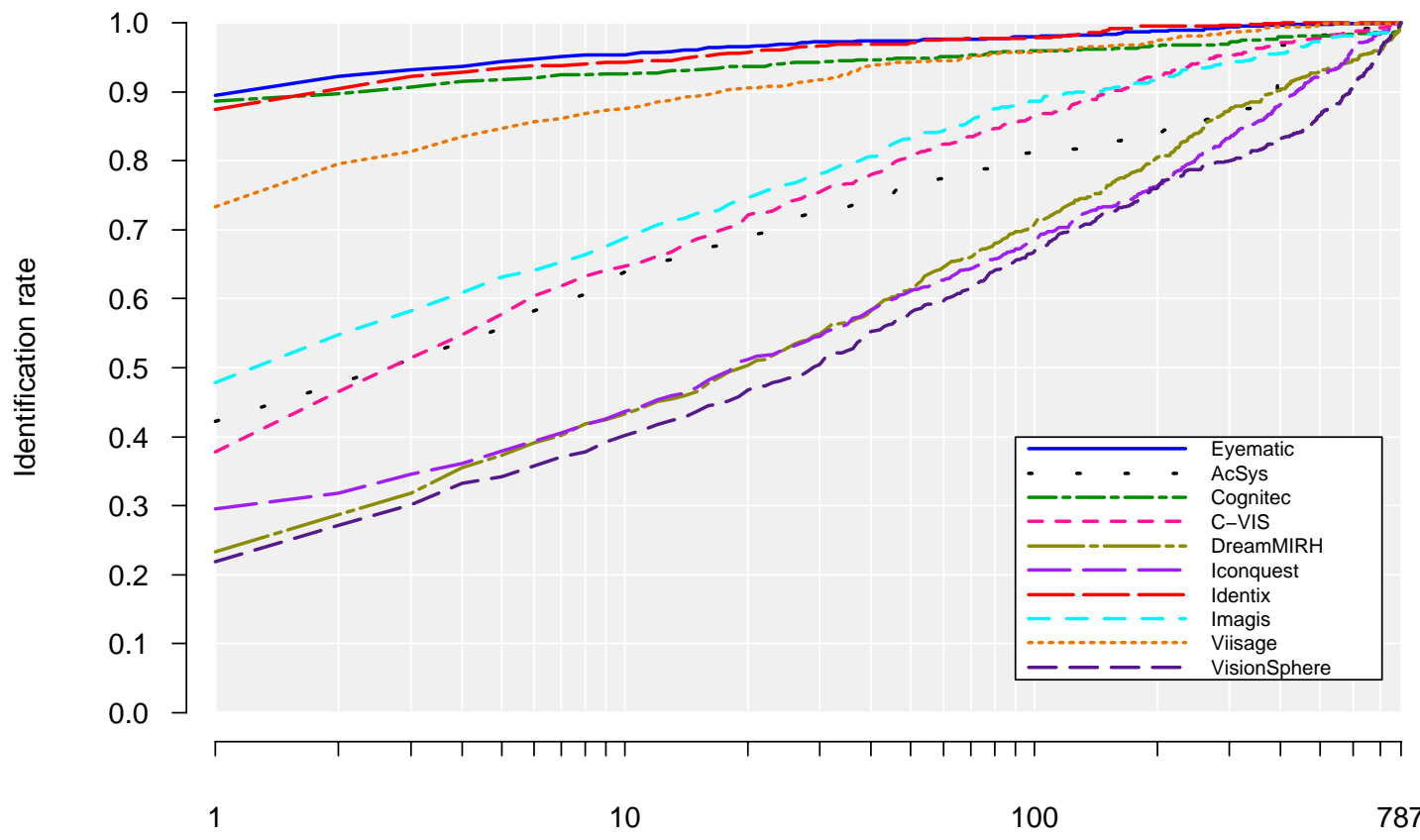

Rank

Figure L.17 - Identification Performance for Indoor (same day, overhead) Probes. This figure corresponds to Figure 28 in the FRVT 2002: Evaluation Report. 


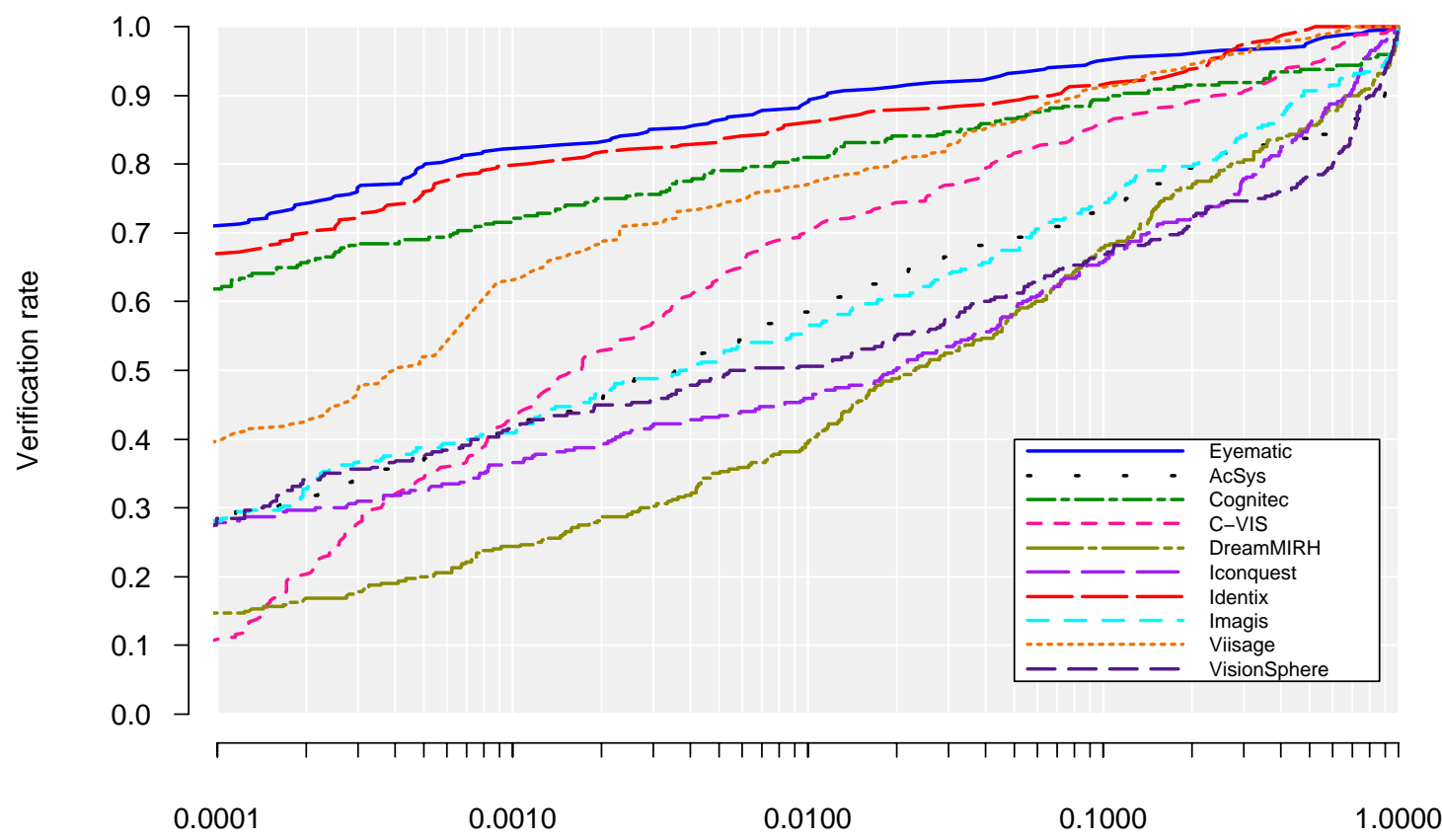

False accept rate

Figure L.18 - Verification Performance for Indoor (different day) Probes. This figure corresponds to Figure 22 in the FRVT 2002: Evaluation Report.

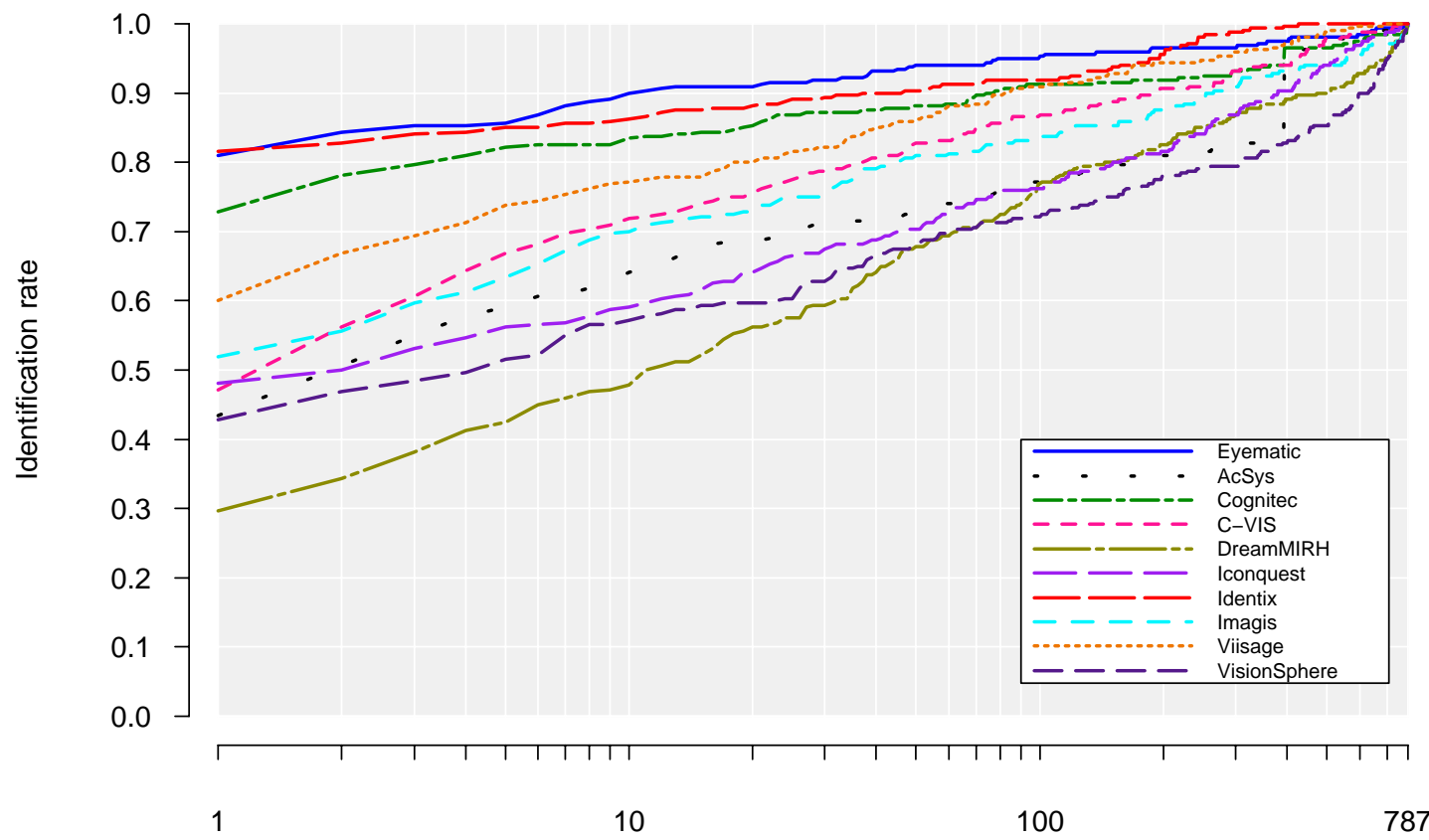

Rank

Figure L.19 - Identification Performance for Indoor (different day) Probes. This figure corresponds to Figure 28 in the FRVT 2002: Evaluation Report. 


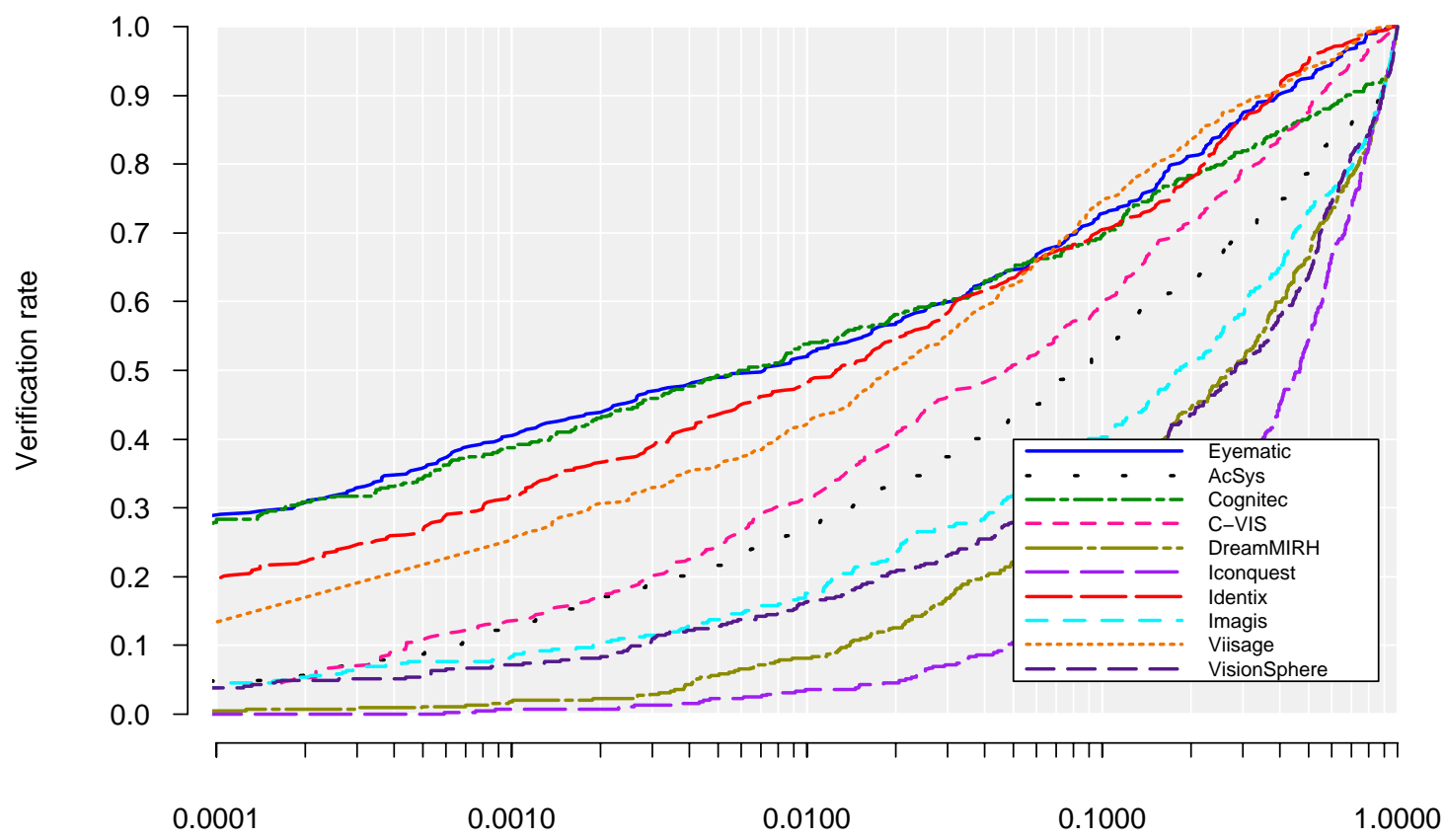

False accept rate

Figure L.20 - Verification Performance for Outdoor (same day) Probes. This figure corresponds to Figure 22 in the FRVT 2002: Evaluation Report.

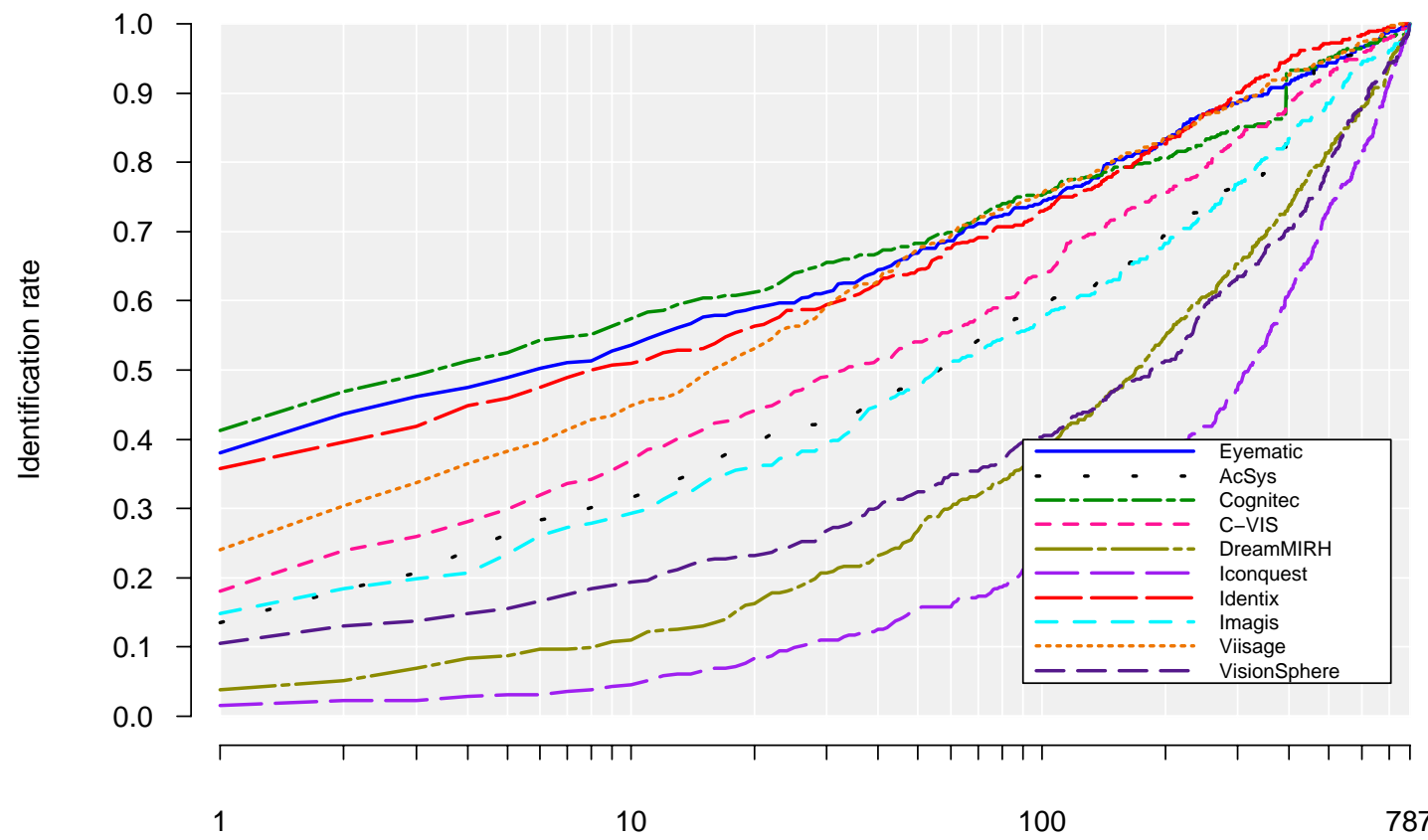

Rank

Figure L.21 - Identification Performance for Outdoor (same day probes). This figure corresponds to Figure 28 in the FRVT 2002: Evaluation Report. 


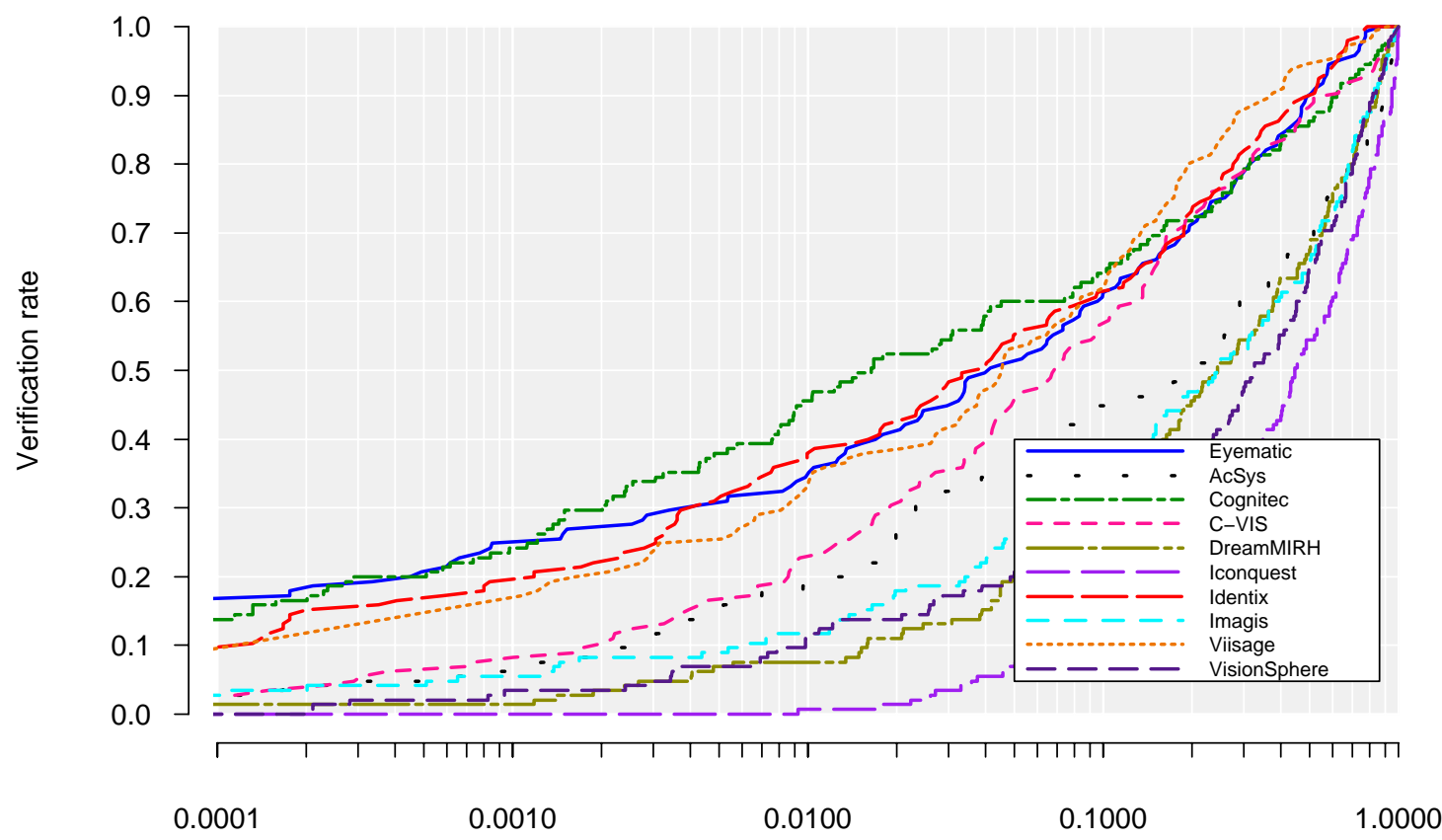

False accept rate

Figure L.22 - Verification Performance on Outdoor (different day) Probes. This figure corresponds to Figure 22 in the FRVT 2002: Evaluation Report.

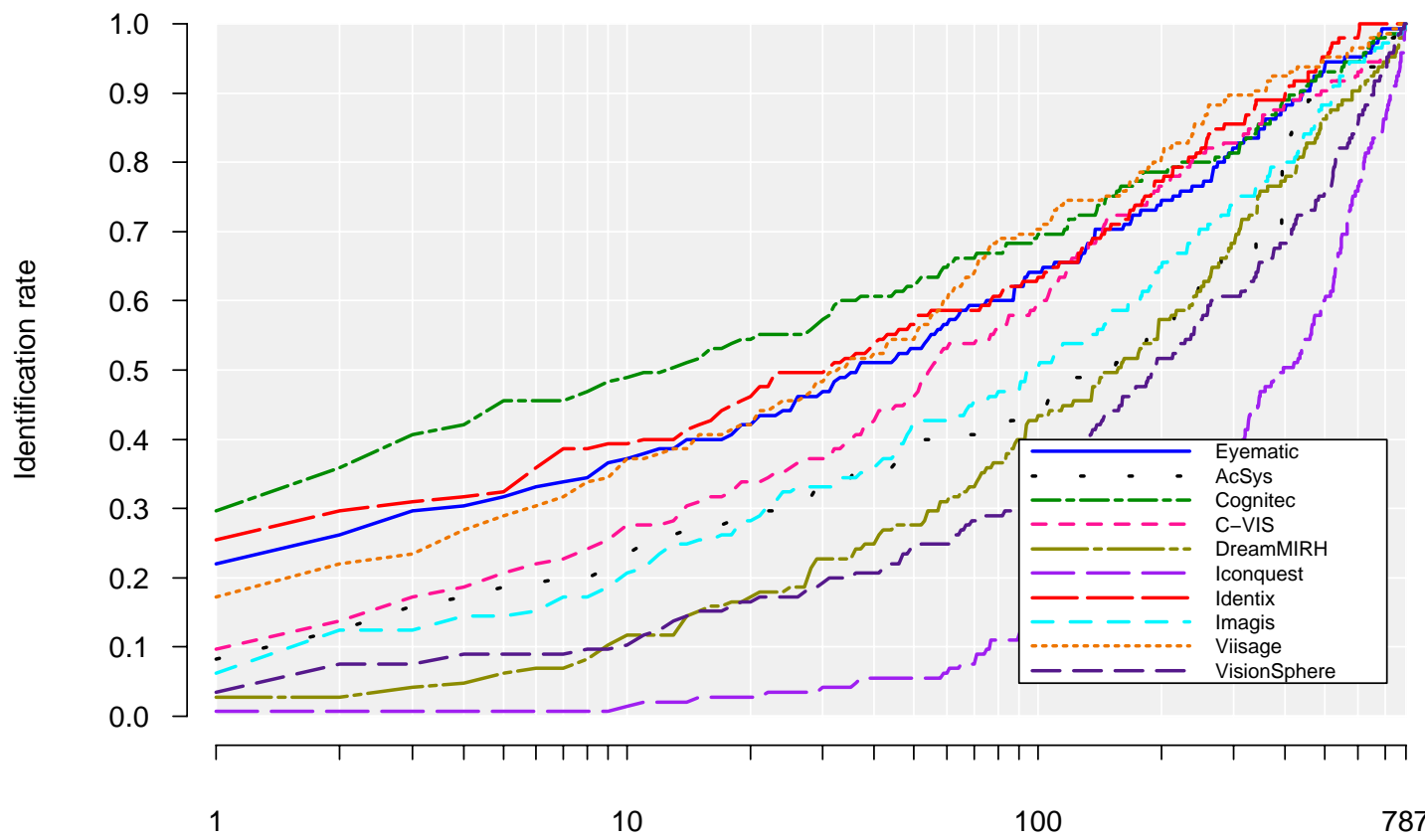

Rank

Figure L.23 - Identification Performance on Outdoor (different day) Probes. This figure corresponds to Figure 28 in the FRVT 2002: Evaluation Report. 


\section{L.4 MCInt Morphable Model Experiment}

Figure 23 in the FRVT 2002: Evaluation Report was a comparison of verification system performance using probes of non-frontal poses as well as 3D Morphable reconstructions. Figure 29 showed identification performance for the same experiments. Figure L. 24 through Figure L.41 provide full ROC and $\mathrm{CMC}$ charts for each pose and reconstruction, as outlined in the following table.

\begin{tabular}{ccc}
\hline Pose & $\begin{array}{c}\text { Performance } \\
\text { Curve }\end{array}$ & $\begin{array}{c}\text { Figure } \\
\text { Number }\end{array}$ \\
\hline \multirow{2}{*}{ Front (Morph) } & ROC & Figure L.24 \\
& CMC & Figure L.25 \\
ROL & ROC & Figure L.26 \\
& CMC & Figure L.27 \\
45L (Morph) & ROC & Figure L.28 \\
& CMC & Figure L.29 \\
$45 \mathrm{R}$ & ROC & Figure L.30 \\
& CMC & Figure L.31 \\
45R (Morph) & ROC & Figure L.32 \\
& CMC & Figure L.33 \\
$30 \mathrm{U}$ & ROC & Figure L.34 \\
& CMC & Figure L.35 \\
30U (Morph) & ROC & Figure L.36 \\
& CMC & Figure L.37 \\
30D & ROC & Figure L.38 \\
& CMC & Figure L.39 \\
30D (Morph) & ROC & Figure L.40 \\
& CMC & Figure L.41 \\
\hline
\end{tabular}




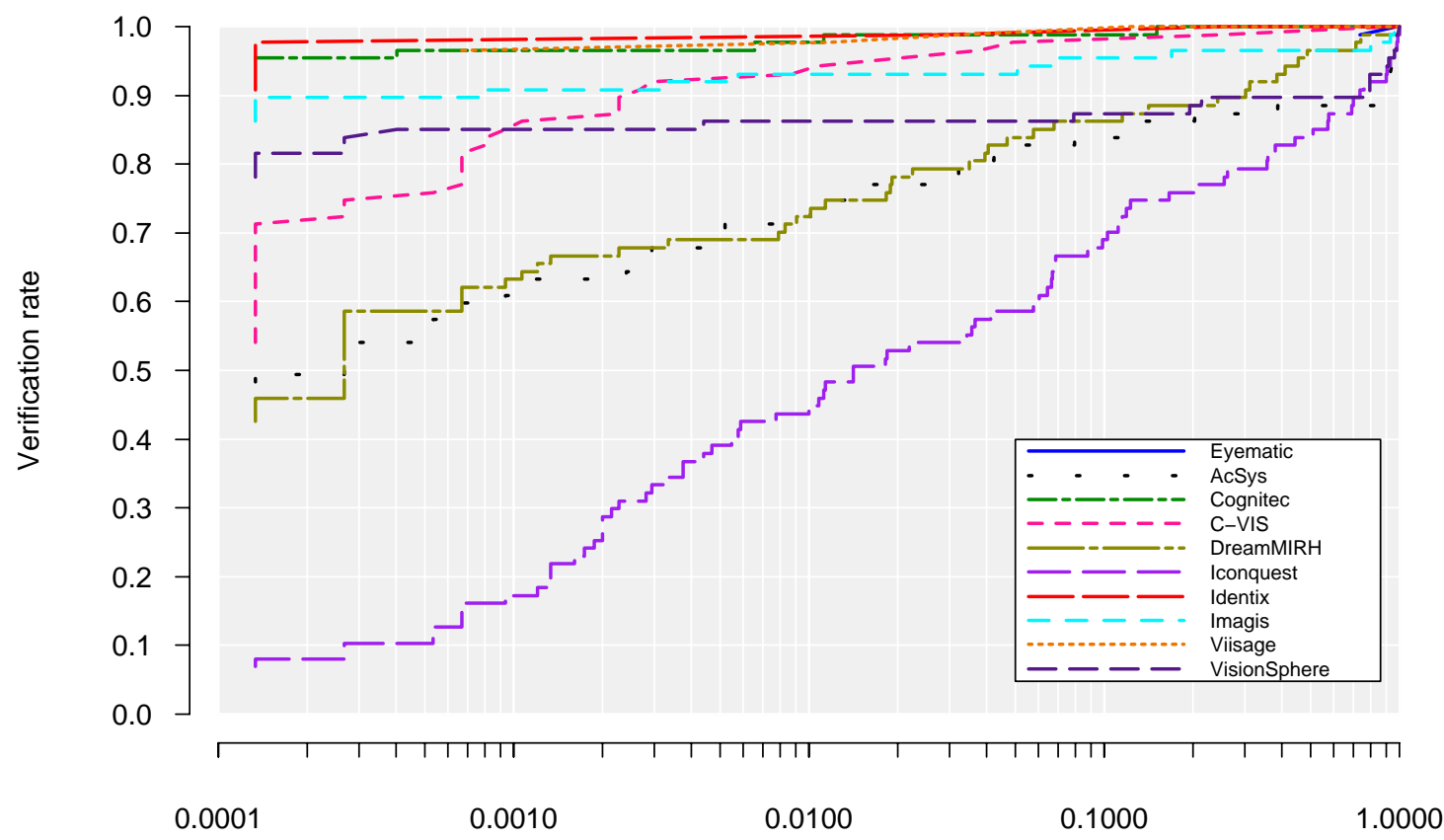

False accept rate

Figure L.24 - Verification Performance on Front (Morph) Probes. This figure corresponds to Figure 23 in the FRVT 2002: Evaluation Report.

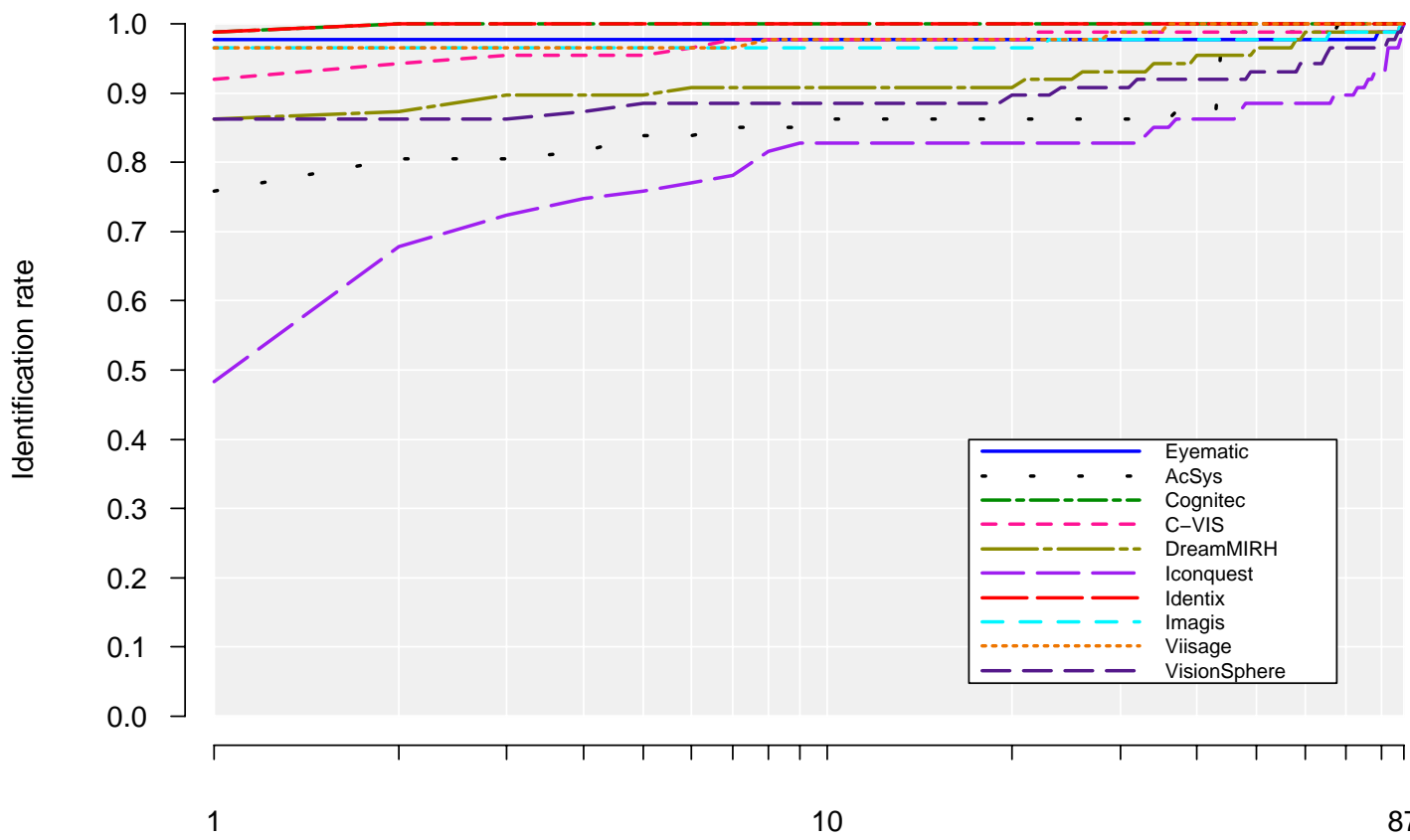

Rank

Figure L.25 - Identification Performance on Front (Morph) Probes. This figure corresponds to Figure 29 in the FRVT 2002: Evaluation Report. 


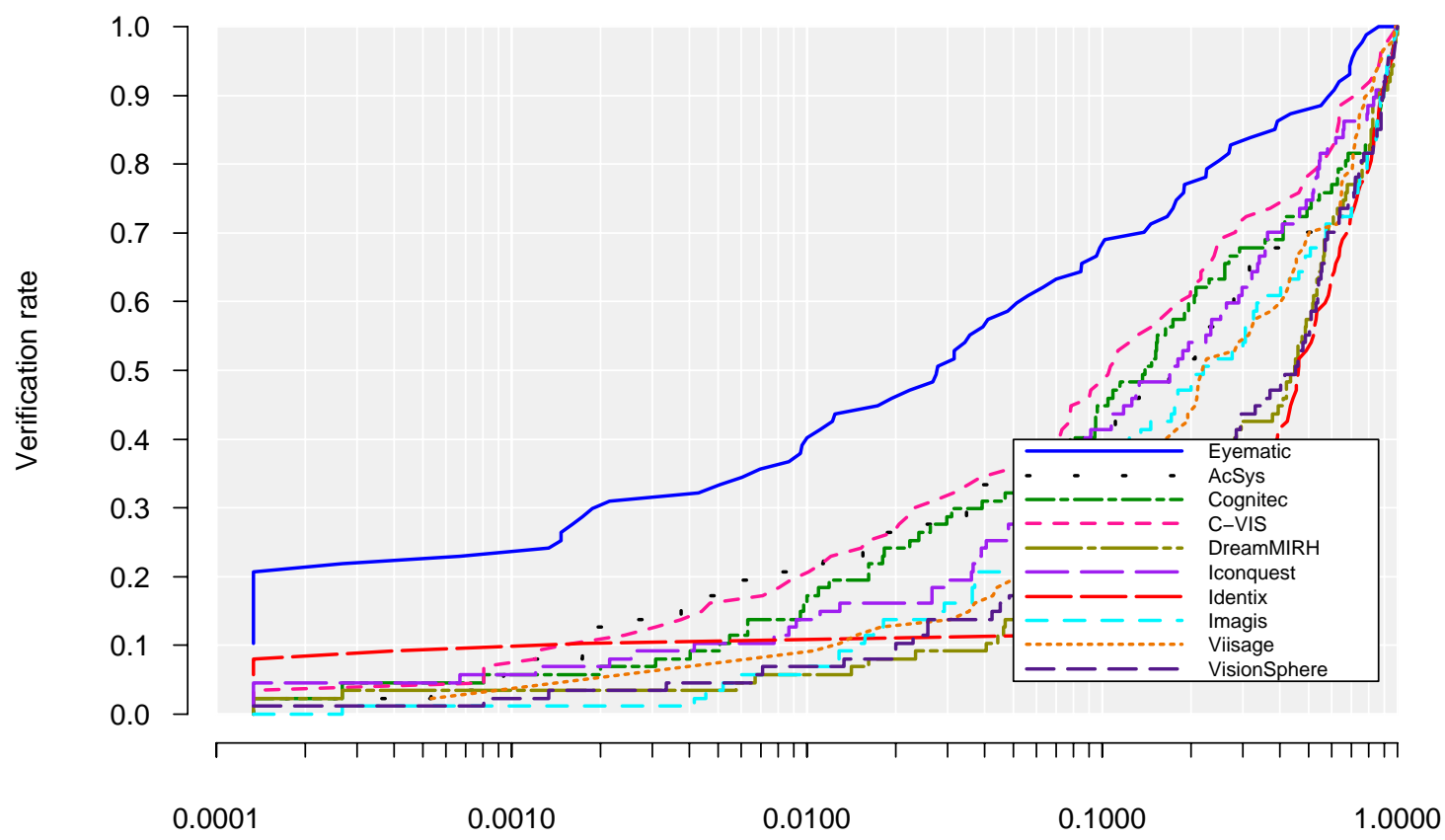

False accept rate

Figure L.26 - Verification Performance on 45 Degree (Left) Pose. This figure corresponds to Figure 23 in the FRVT 2002: Evaluation Report.

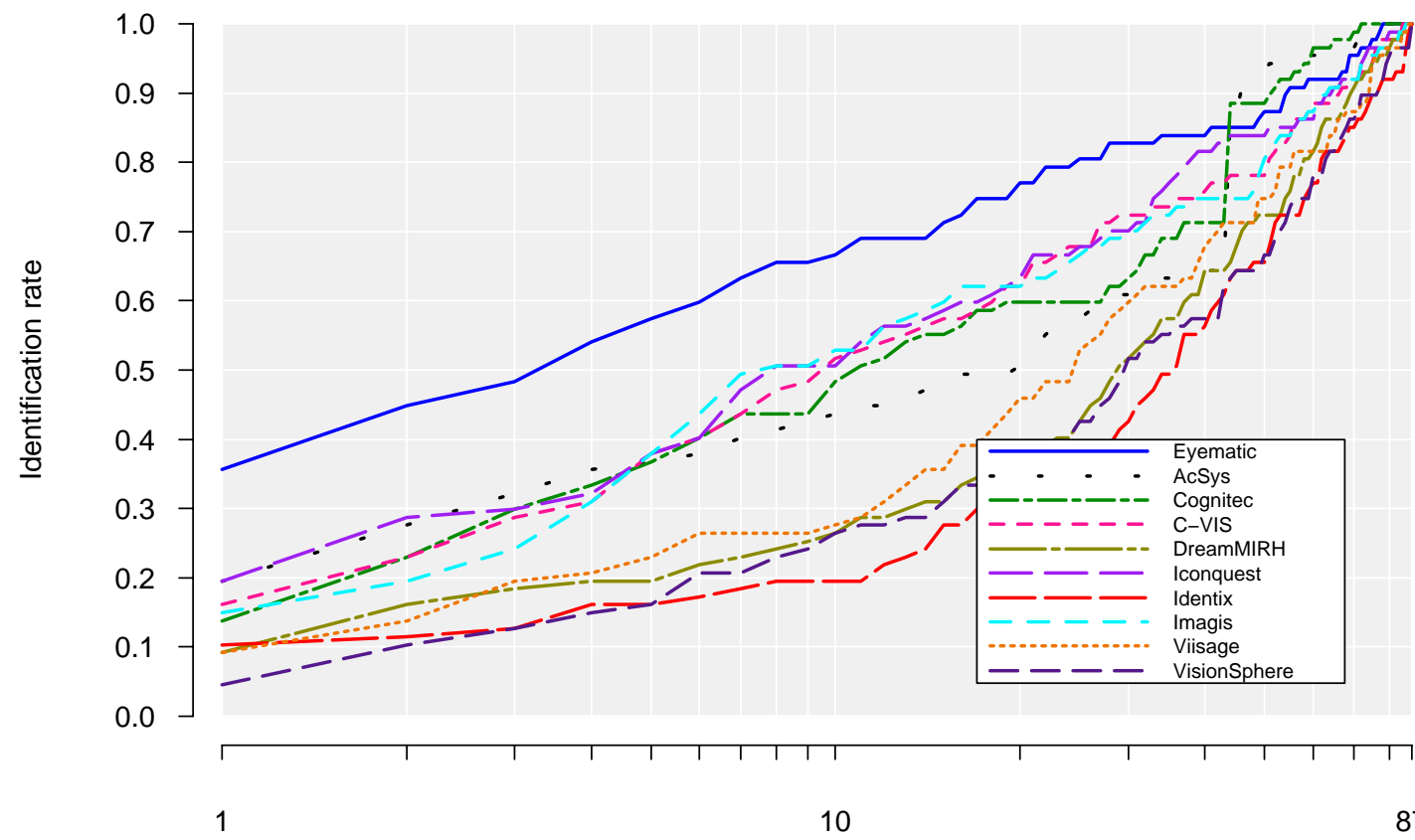

Rank

Figure L.27 - Identification Performance on 45 Degree (Left) Pose. This figure corresponds to Figure 29 in the FRVT 2002: Evaluation Report. 


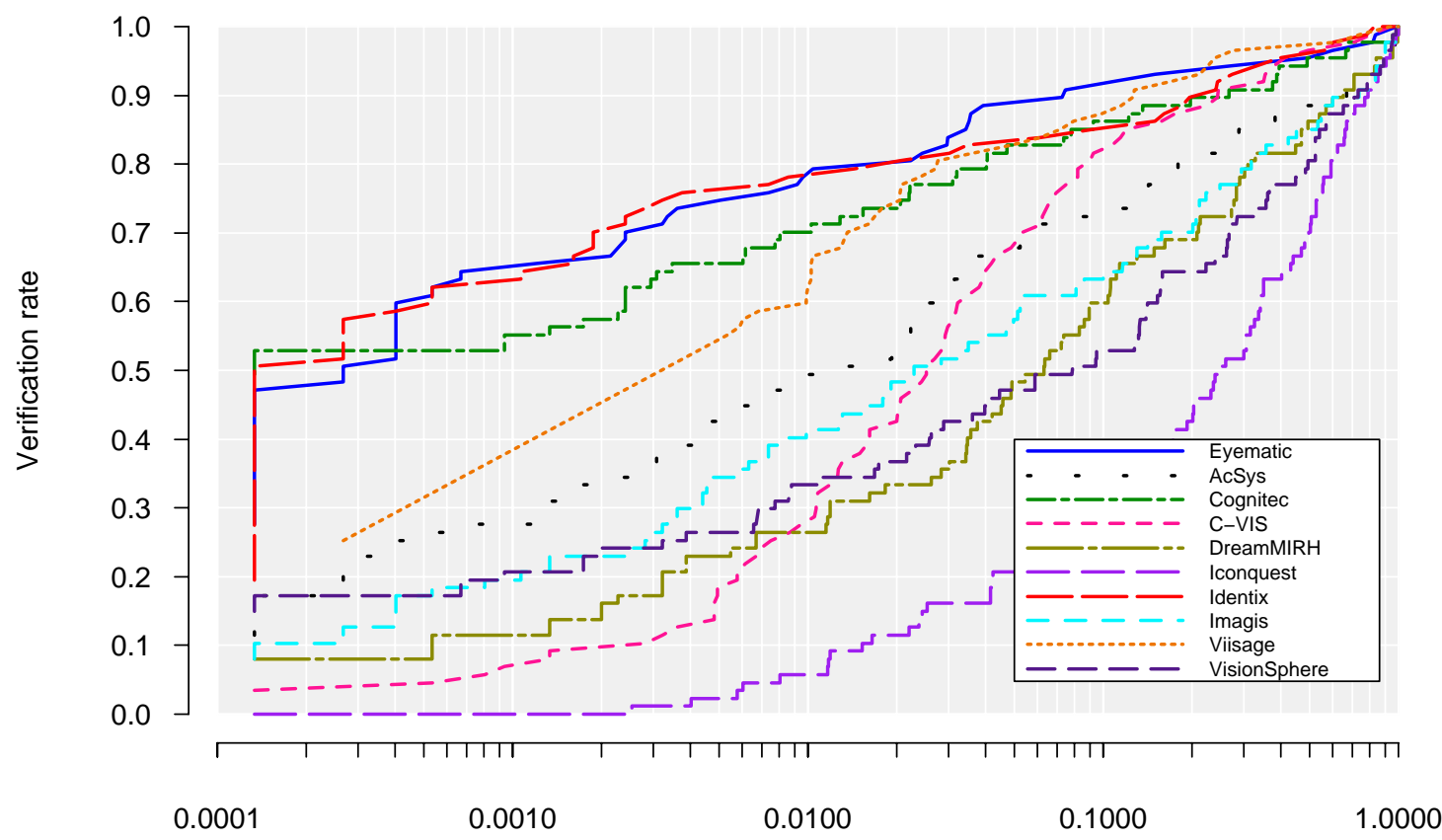

False accept rate

Figure L.28 - Verification Performance on 45 Degree (Left, Morphed) Probes. This figure corresponds to Figure 23 in the FRVT 2002: Evaluation Report.

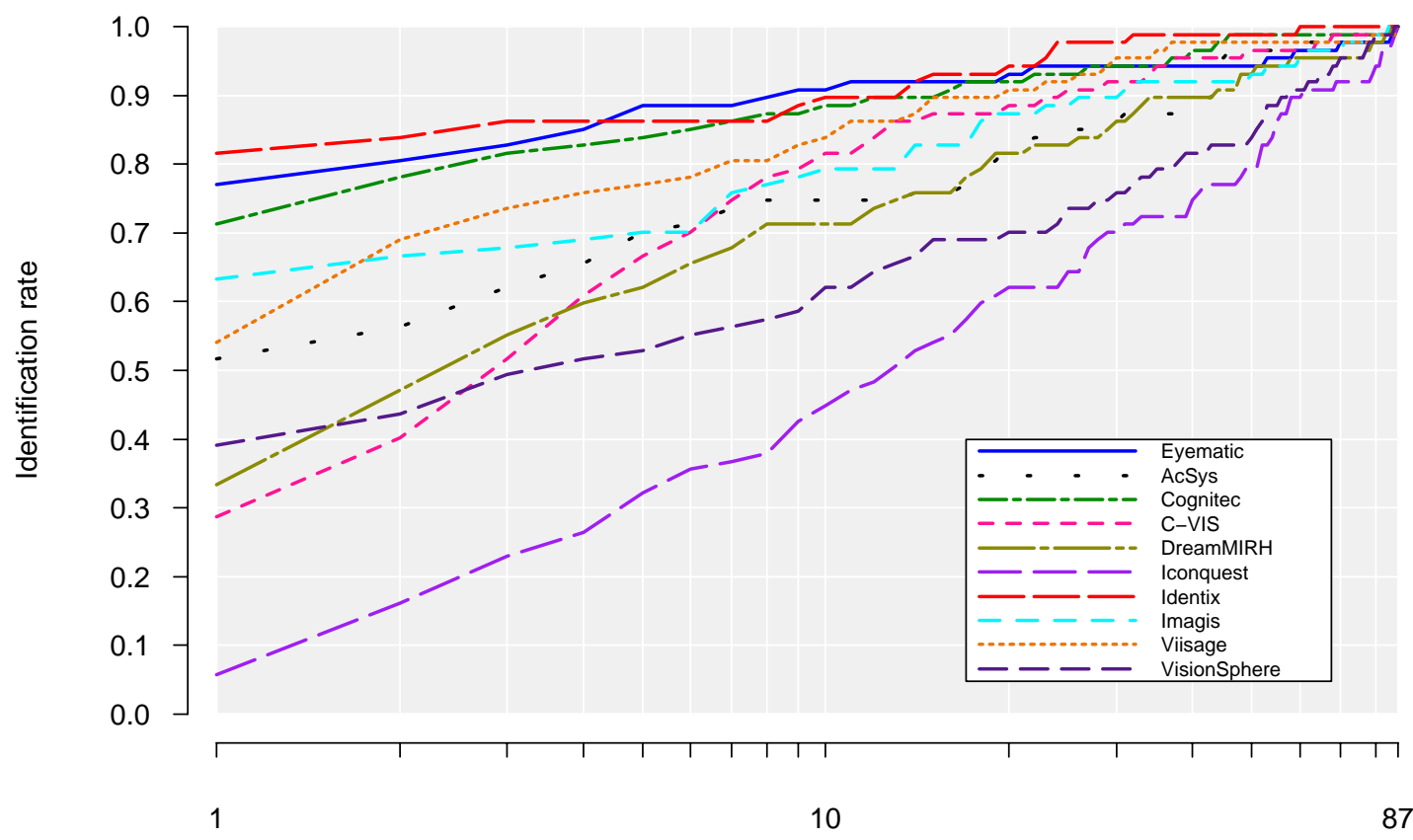

Rank

Figure L.29 - Identification Performance on 45 Degree (Left, Morphed) Probes. This figure corresponds to Figure 29 in the FRVT 2002: Evaluation Report. 


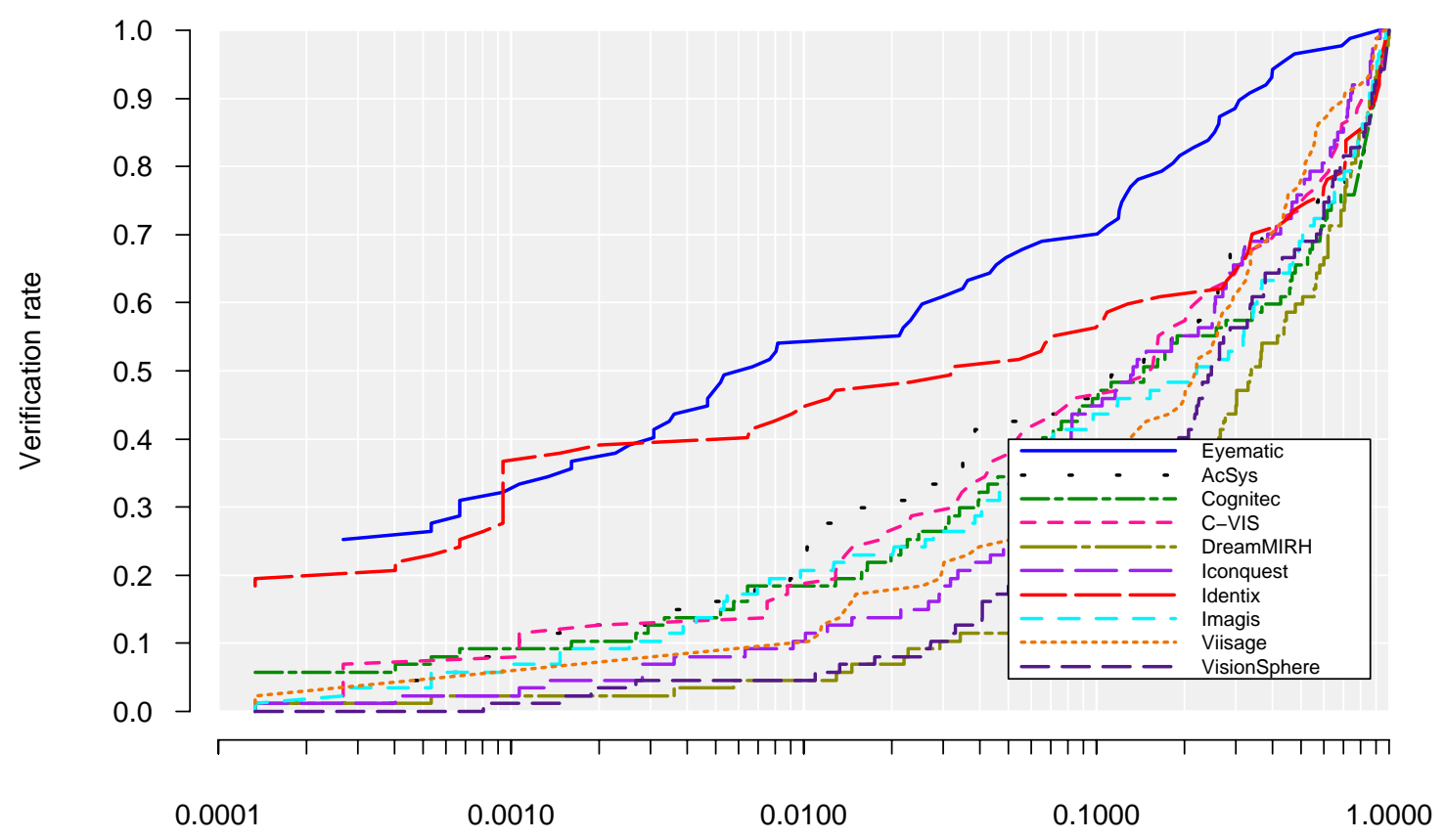

False accept rate

Figure L.30 - Verification Performance on 45 Degree (Right) Probes. This figure corresponds to Figure 23 in the FRVT 2002: Evaluation Report.

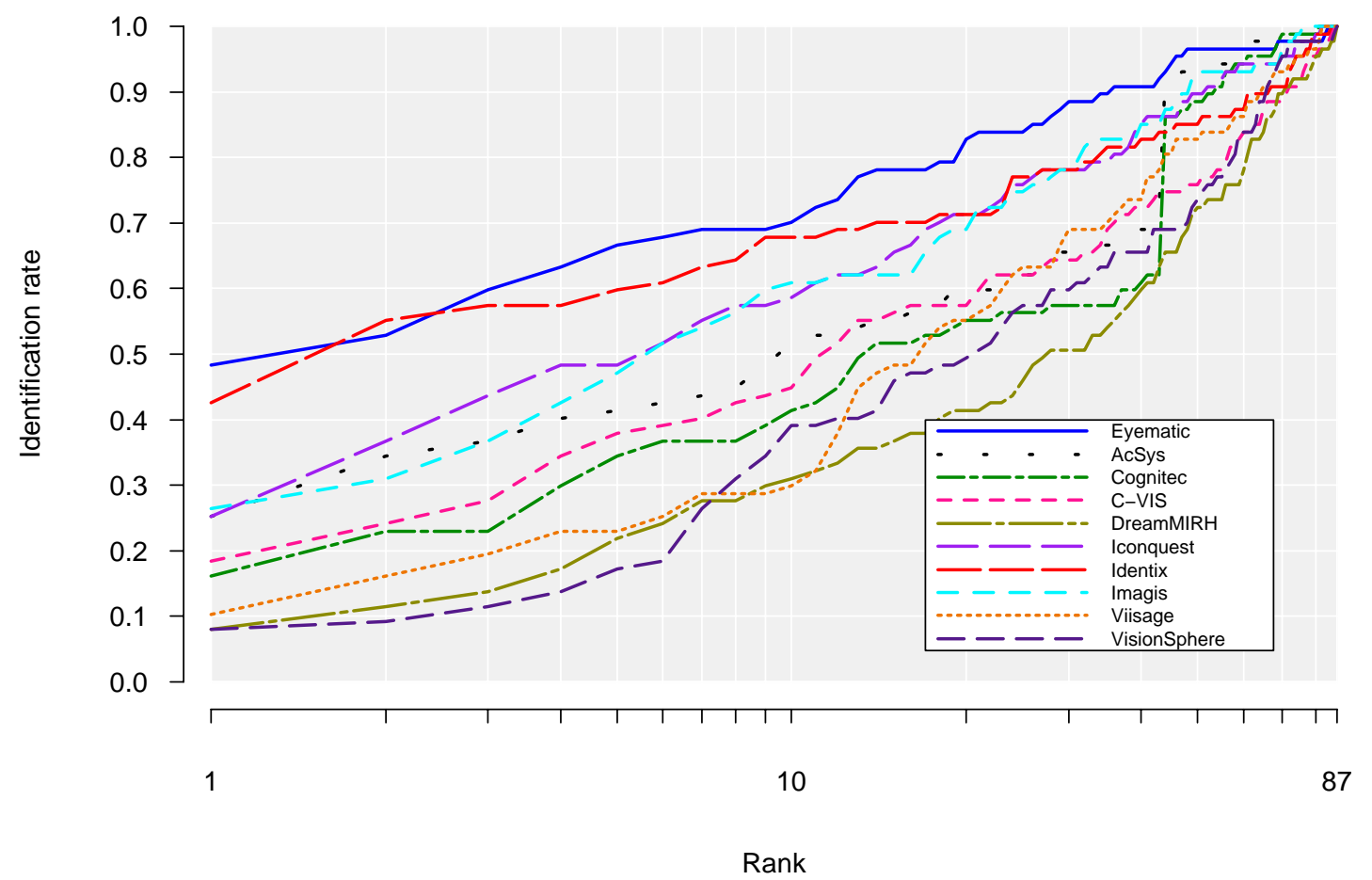

Figure L.31 - Identification Performance on 45 Degree (Right) Probes. This figure corresponds to Figure 29 in the FRVT 2002: Evaluation Report. 


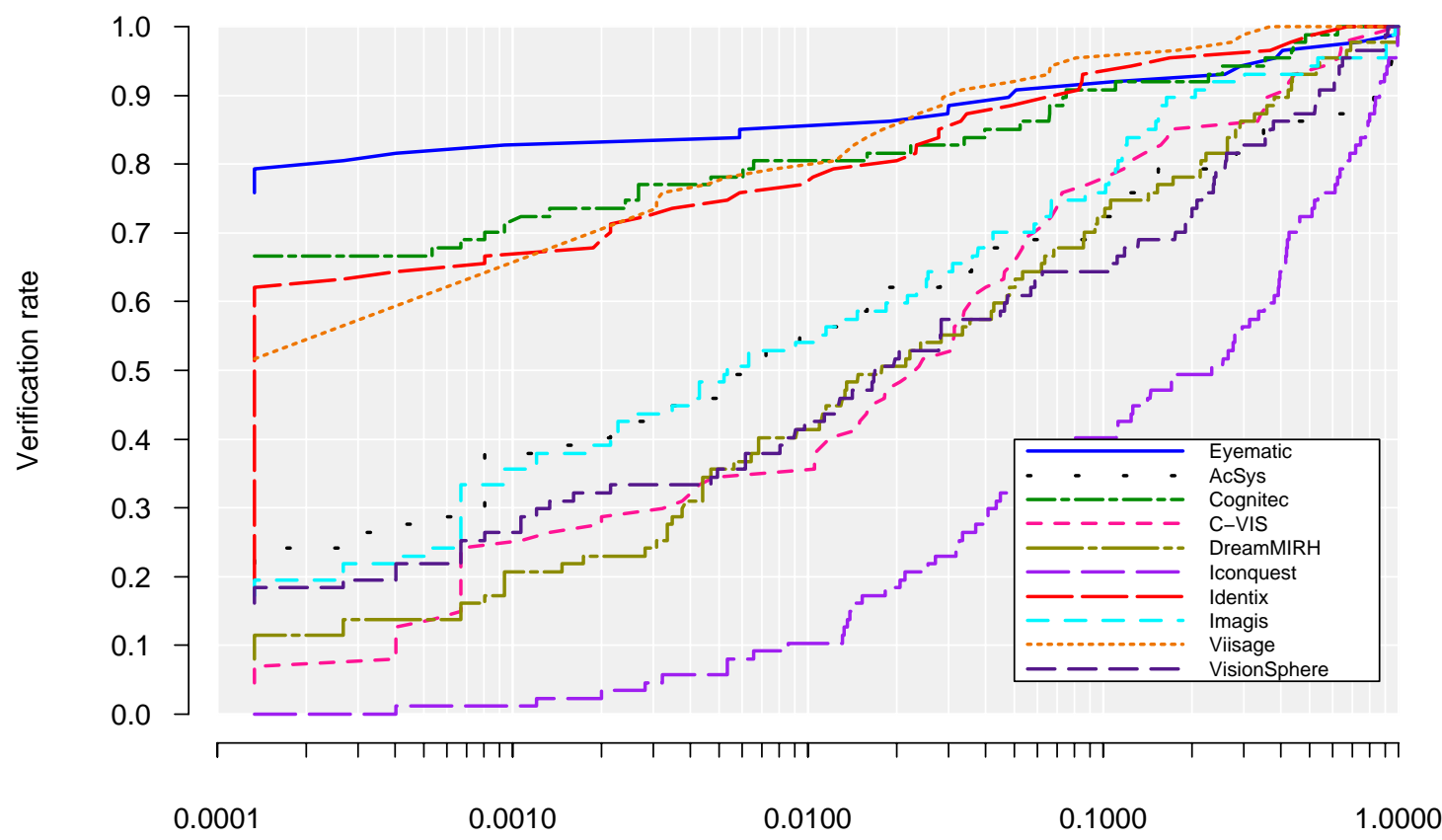

False accept rate

Figure L.32 - Verification Performance of 45 Degree (Right, Morphed) Probes. This figure corresponds to Figure 23 in the FRVT 2002: Evaluation Report.

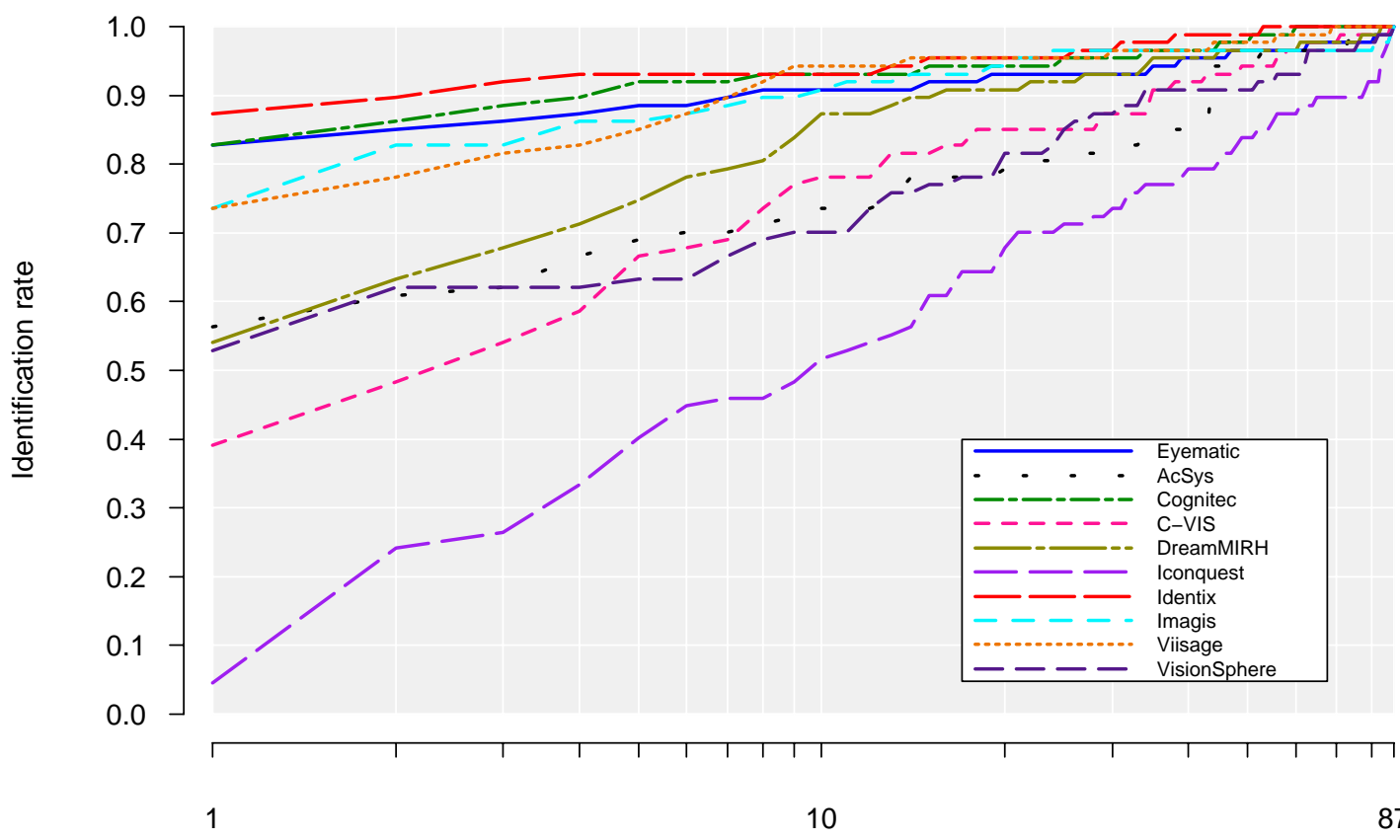

Rank

Figure L.33 - Identification Performance of 45 Degree (Right, Morphed) Probes. This figure corresponds to Figure 29 in the FRVT 2002: Evaluation Report. 


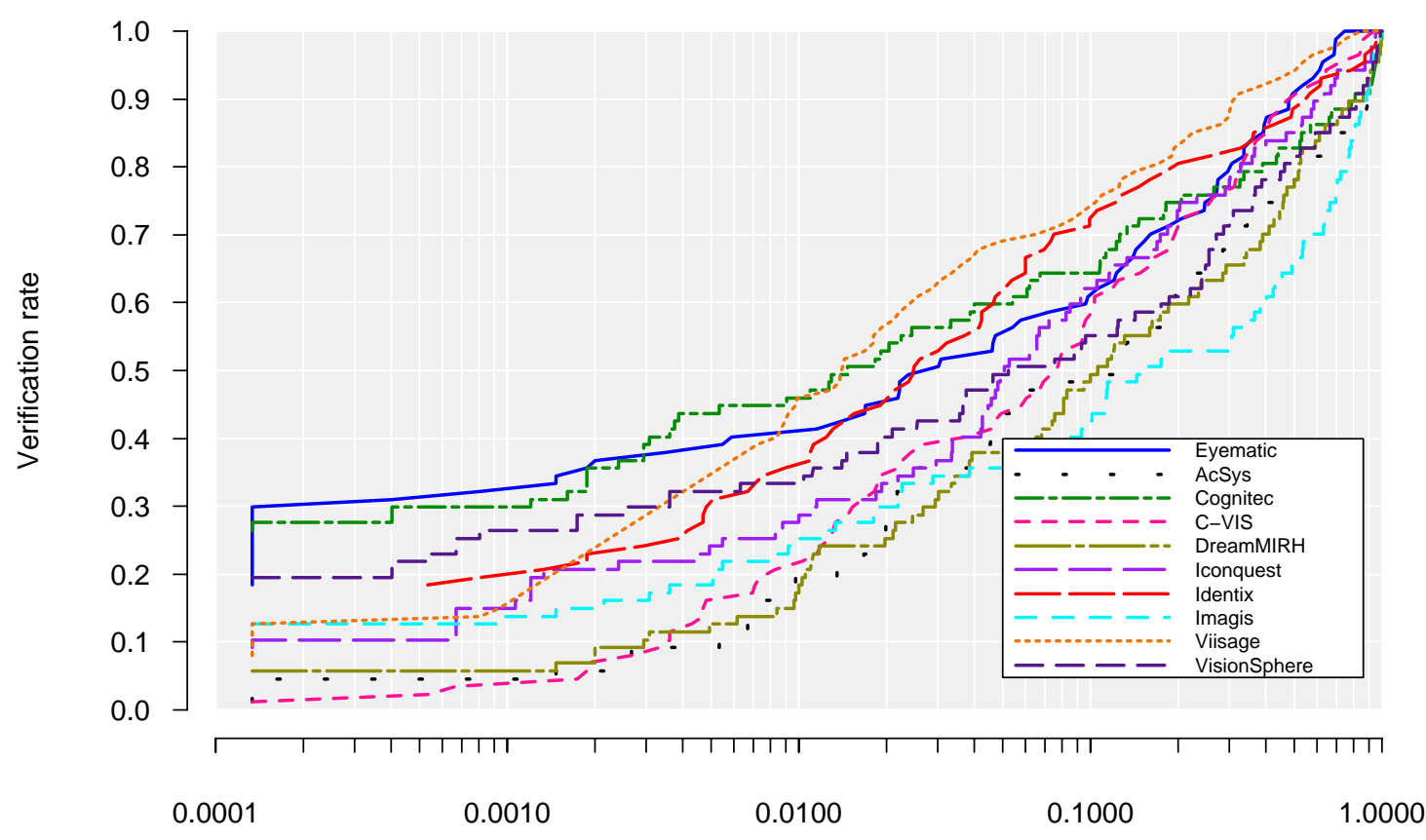

False accept rate

Figure L.34- Verification Performance of 30 Degree (Up) Probes. This figure corresponds to Figure 23 in the FRVT 2002: Evaluation Report.

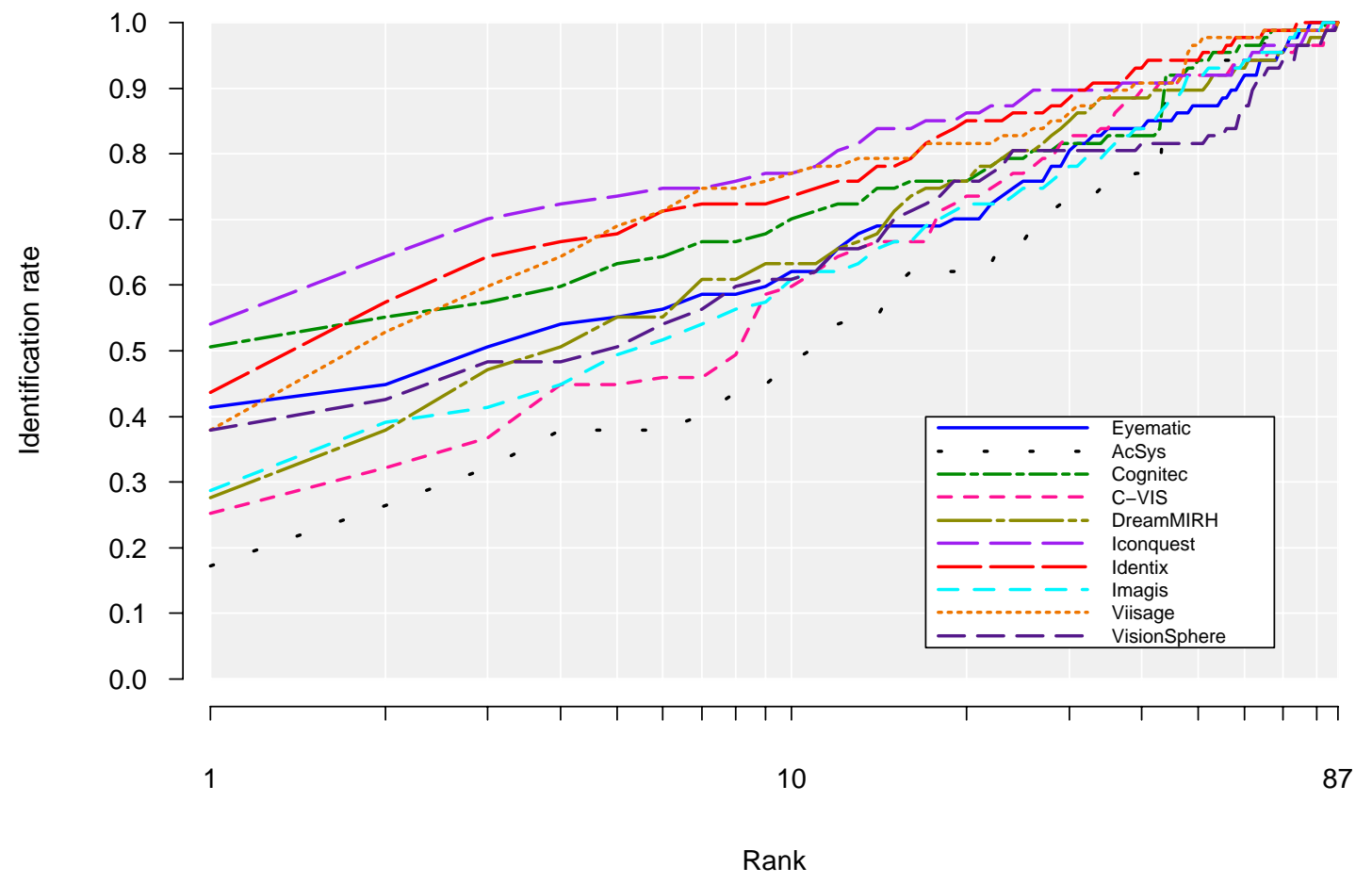

Figure L.35 - Identification Performance of 30 Degree (Up) Probes. This figure corresponds to Figure 29 in the FRVT 2002: Evaluation Report. 


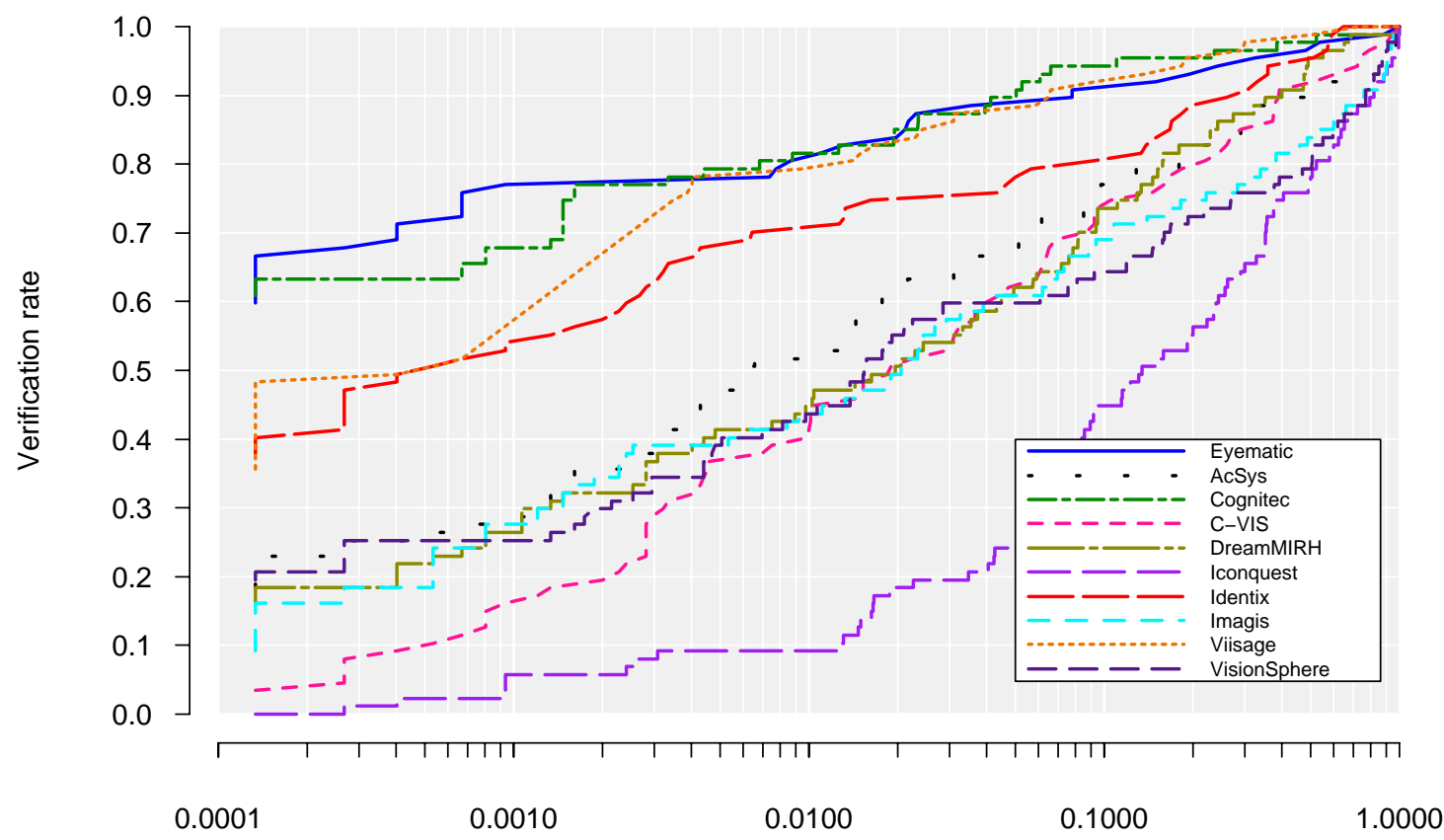

False accept rate

Figure L.36 - Verification Performance of 30 Degree (Up, Morphed) Probes. This figure corresponds to Figure 23 in the FRVT 2002: Evaluation Report.

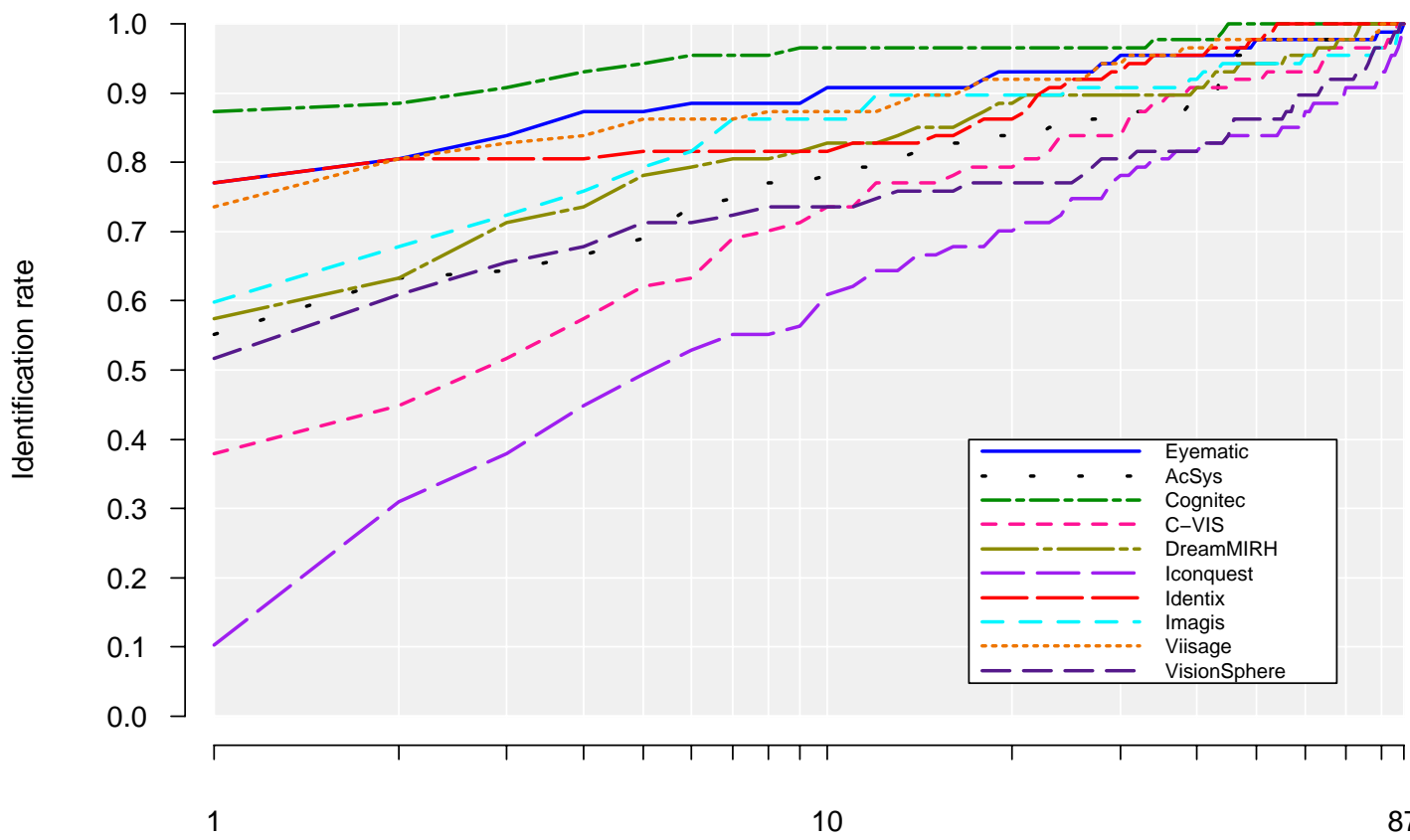

Rank

Figure L.37 - Identification Performance of 30 Degree (Up, Morphed) Probes. This figure corresponds to Figure 29 in the FRVT 2002: Evaluation Report. 


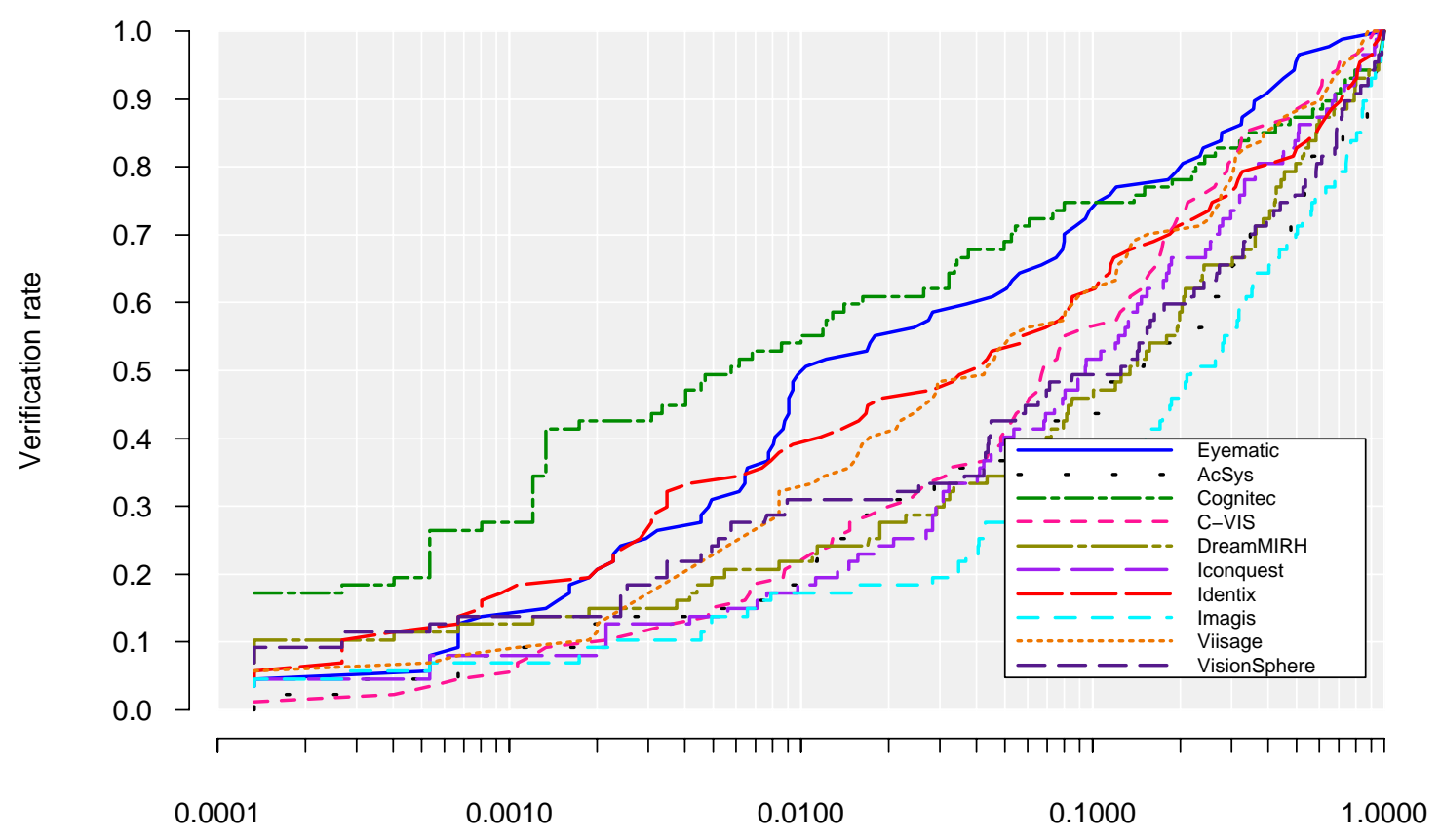

False accept rate

Figure L.38 - Verification Performance of 30 Degree (Down) Probes. This figure corresponds to Figure 23 in the FRVT 2002: Evaluation Report.

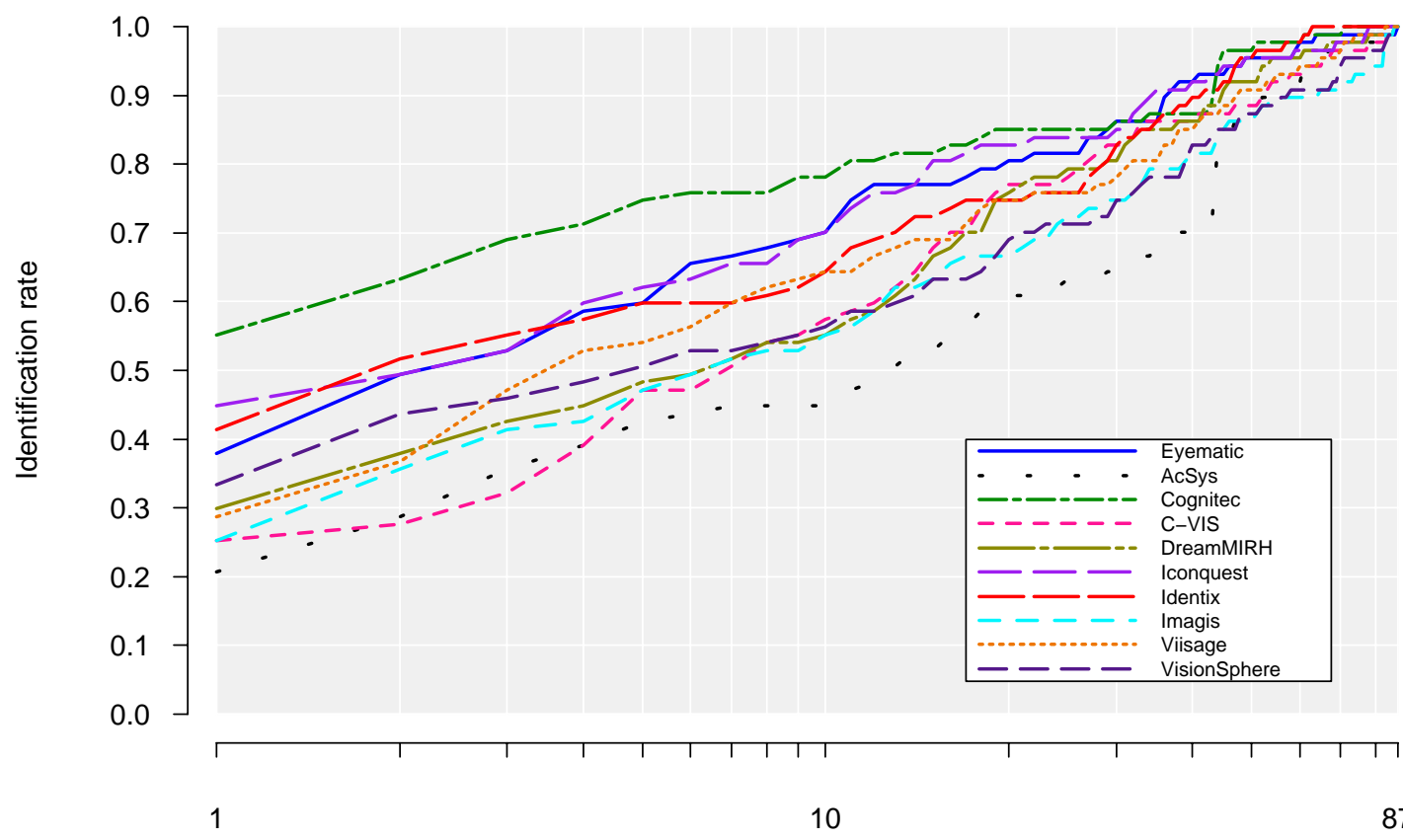

Rank

Figure L.39 - Identification Performance of 30 Degree (Down) Probes. This figure corresponds to Figure 29 in the FRVT 2002: Evaluation Report. 


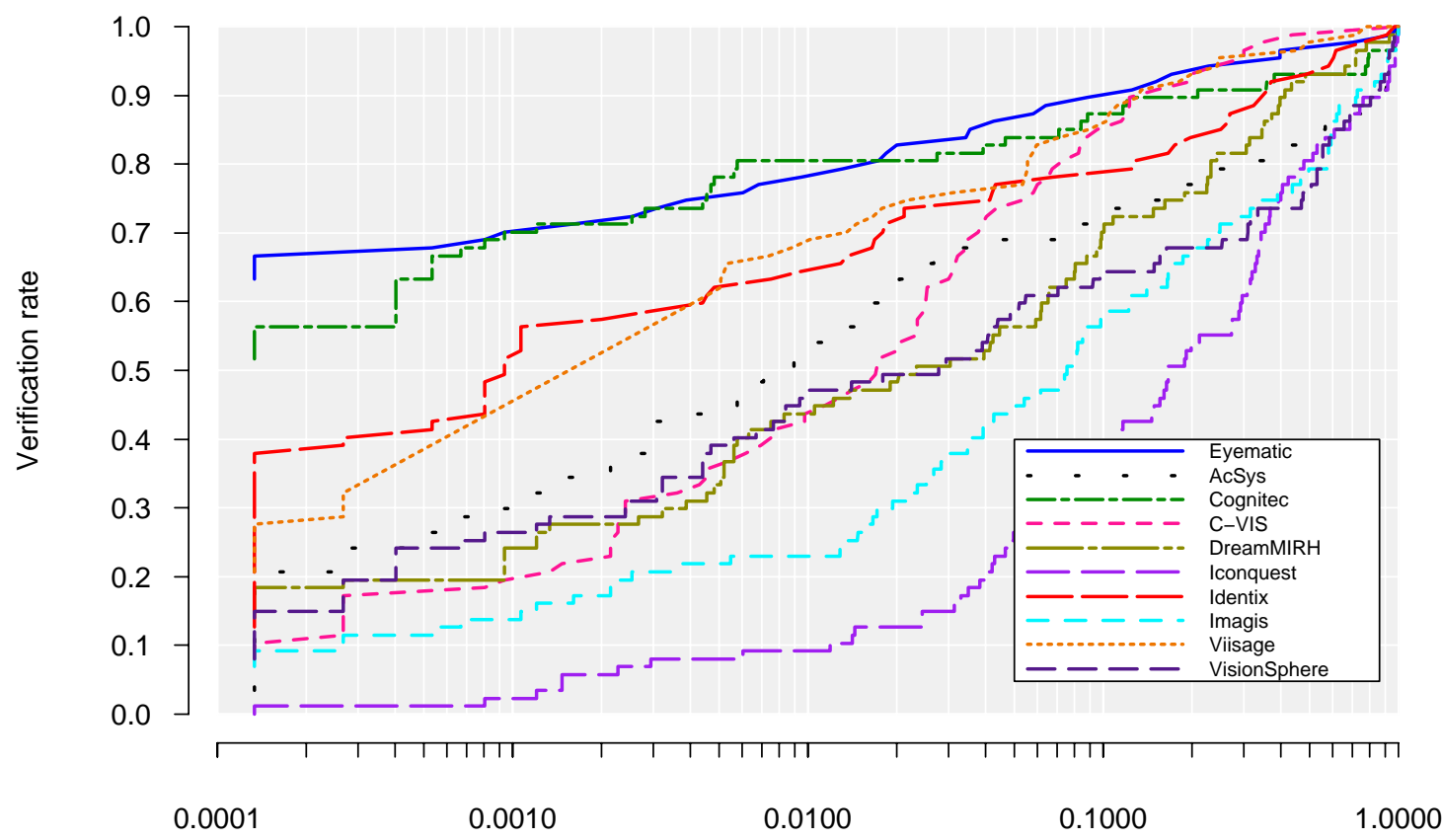

False accept rate

Figure L.40 - Verification Performance for 30 Degree (Down, Morphed) Probes. This figure corresponds to Figure 23 in the FRVT 2002: Evaluation Report.

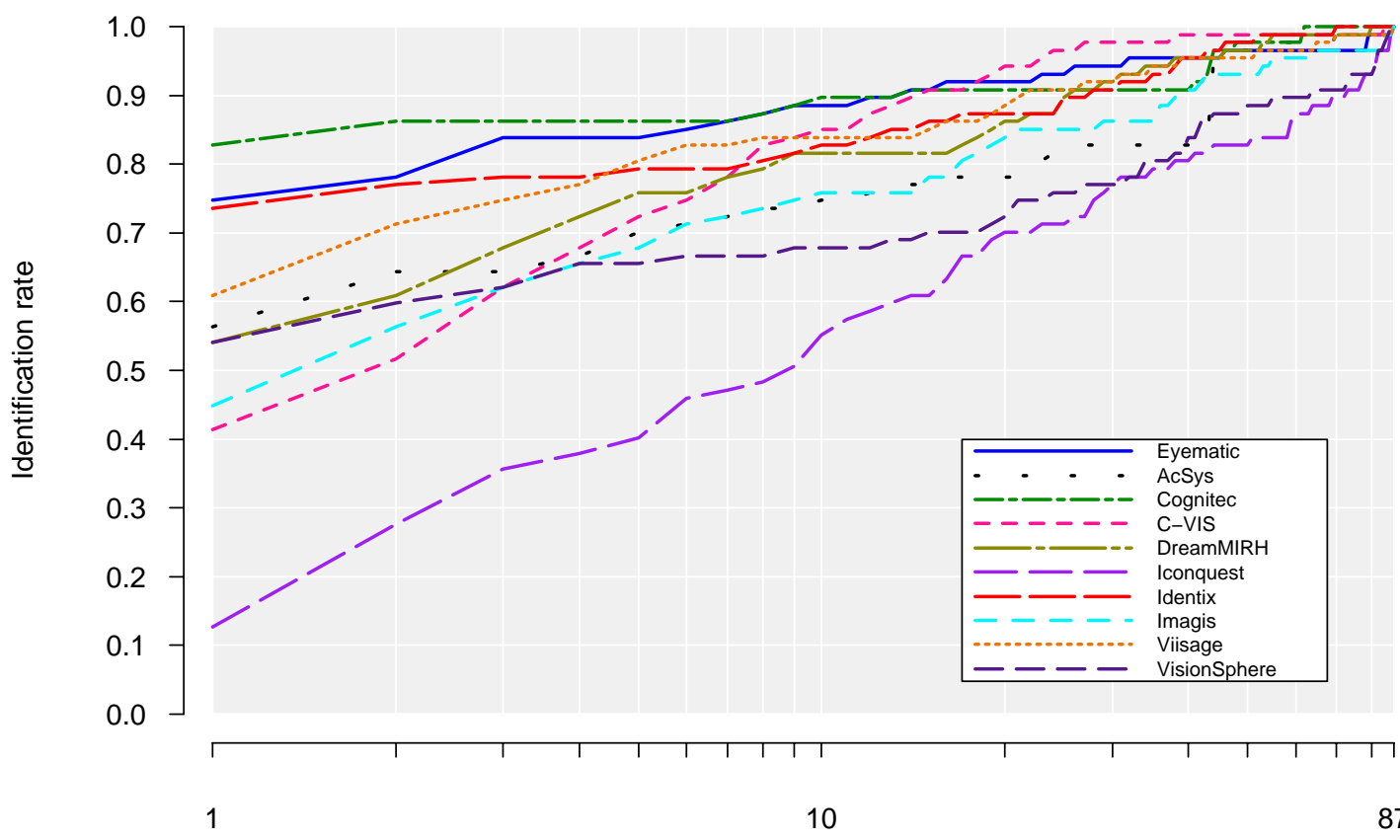

Rank

Figure L.41 - Identification Performance for 30 Degree (Down, Morphed) Probes. This figure corresponds to Figure 29 in the FRVT 2002: Evaluation Report. 


\section{L.5 MCInt Still-Video Experiment}

Figure 24 in the FRVT 2002: Evaluation Report was a comparison of system performance using still and video probes. Figure 30 showed identification performance for the same experiments. Figure L.42 through Figure L.45 provide full ROC and CMC charts for each. 


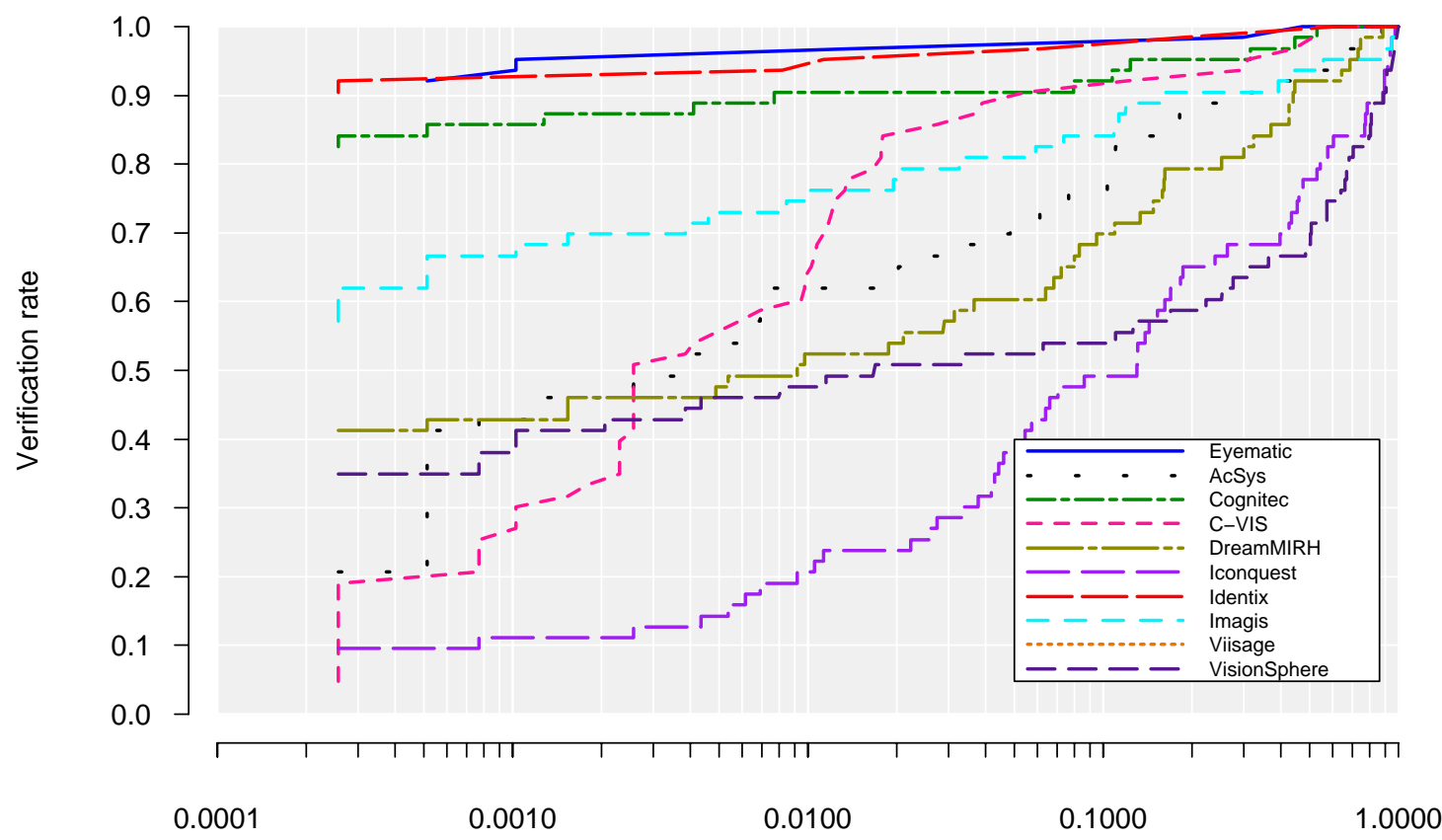

False accept rate

Figure L.42 - Verification Performance for Frontal (Still, Different Day) Probes. This figure corresponds to Figure 24 in the FRVT 2002: Evaluation Report.

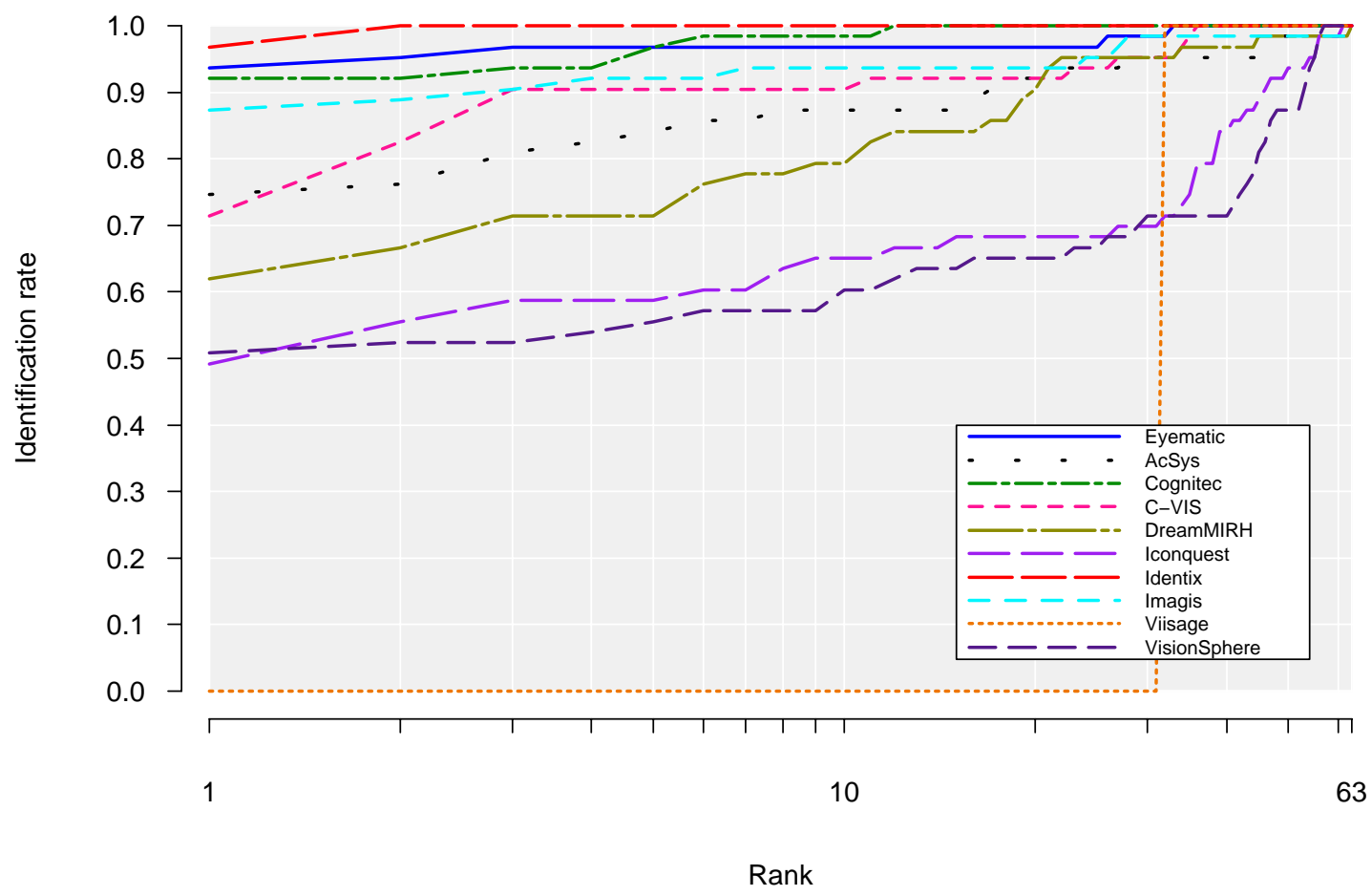

Figure L.43 - Identification Performance for Frontal (Still, Different Day) Probes. This figure corresponds to Figure 30 in the FRVT 2002: Evaluation Report. 


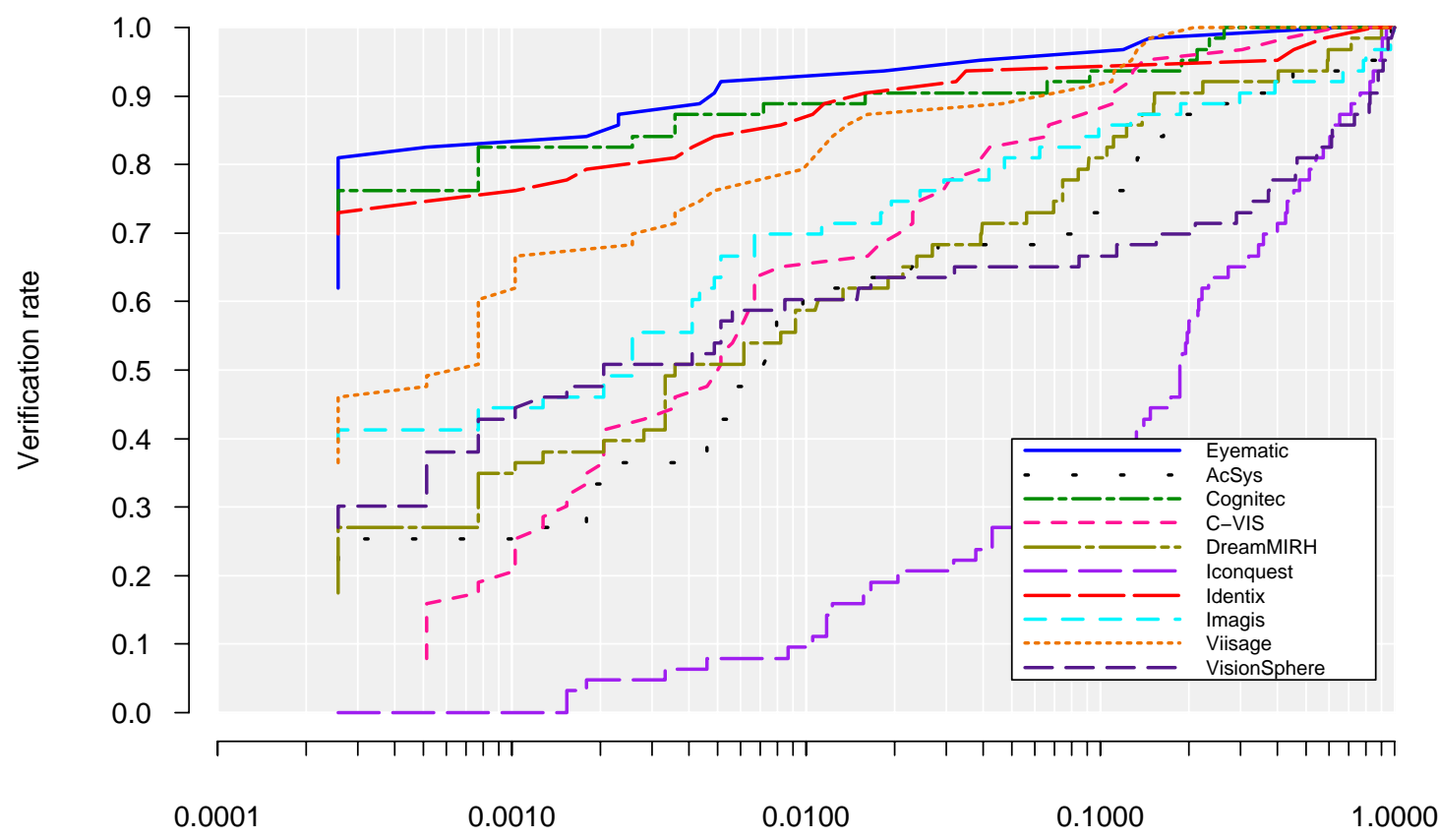

False accept rate

Figure L.44 - Verification Performance for Frontal (Speech "Video", Different Day) Probes. This figure corresponds to Figure 24 in the FRVT 2002: Evaluation Report.

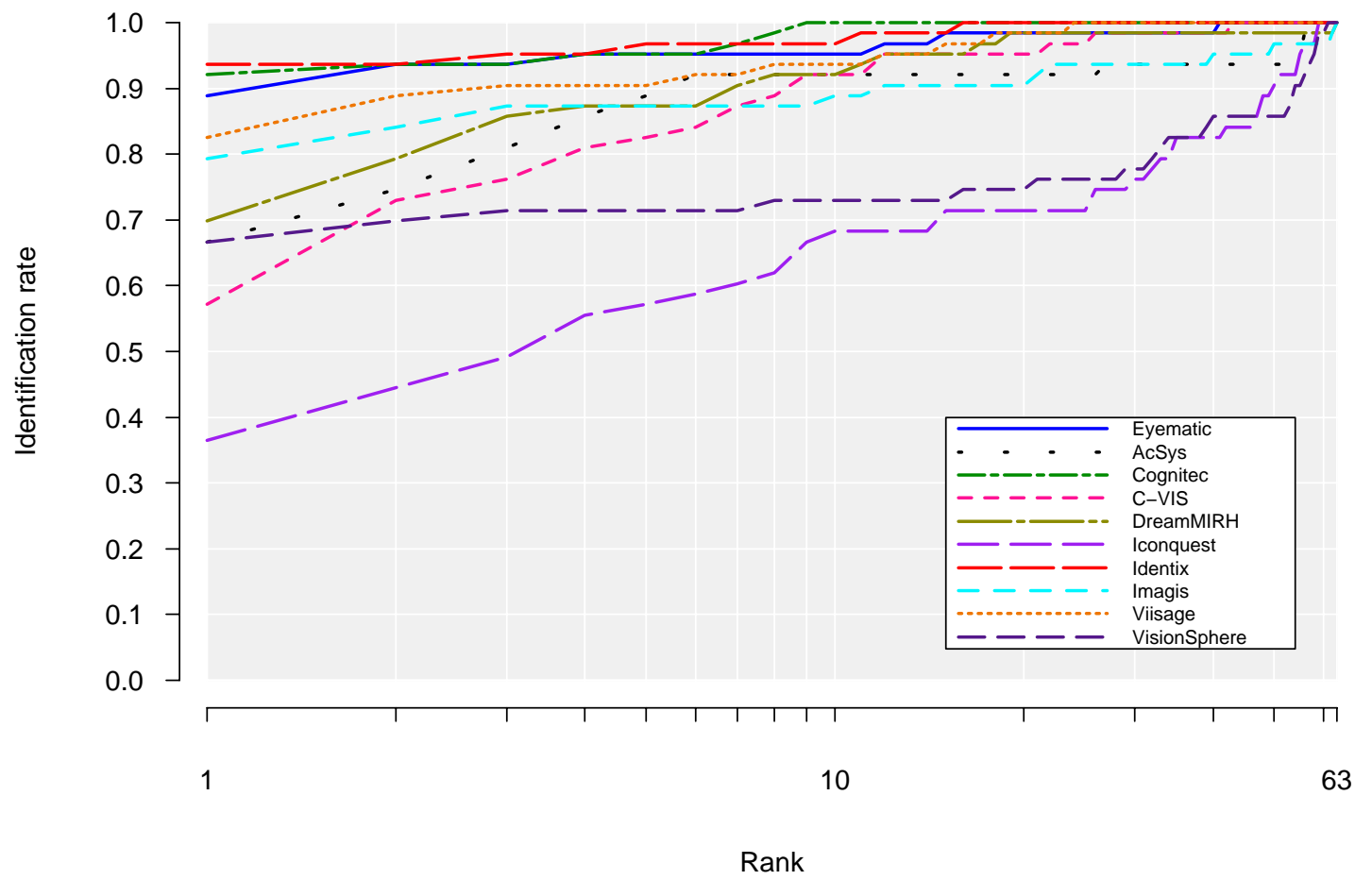

Figure L.45 - Identification Performance for Frontal (Speech "Video", Different Day) Probes. This figure corresponds to Figure 30 in the FRVT 2002: Evaluation Report. 


\section{L.6 HCInt Images in the MCInt Subtest.}

FRVT 2002 Organizers placed a small number of images from the corpus that comprised the HCInt sub-test into the MCInt sub-test. The experiment in section L. 6 has:

512 images in the gallery

512 images in the probe

1024 images total.

These are images of 512 randomly selected individuals from the HCInt corpus. The image in the gallery is the earliest image of that person, while the probe entry is the most recent image of that person.

This experiment was developed to see if any participants that only took the MCInt experiment could potentially do well on HCInt imagery and to see if there are any major deviations between scores for those participants that took both the MCInt and HCInt tests.

Verification and Identification performance for these images in the MCInt sub-test are provided in Figure L.46 and Figure L.47. 


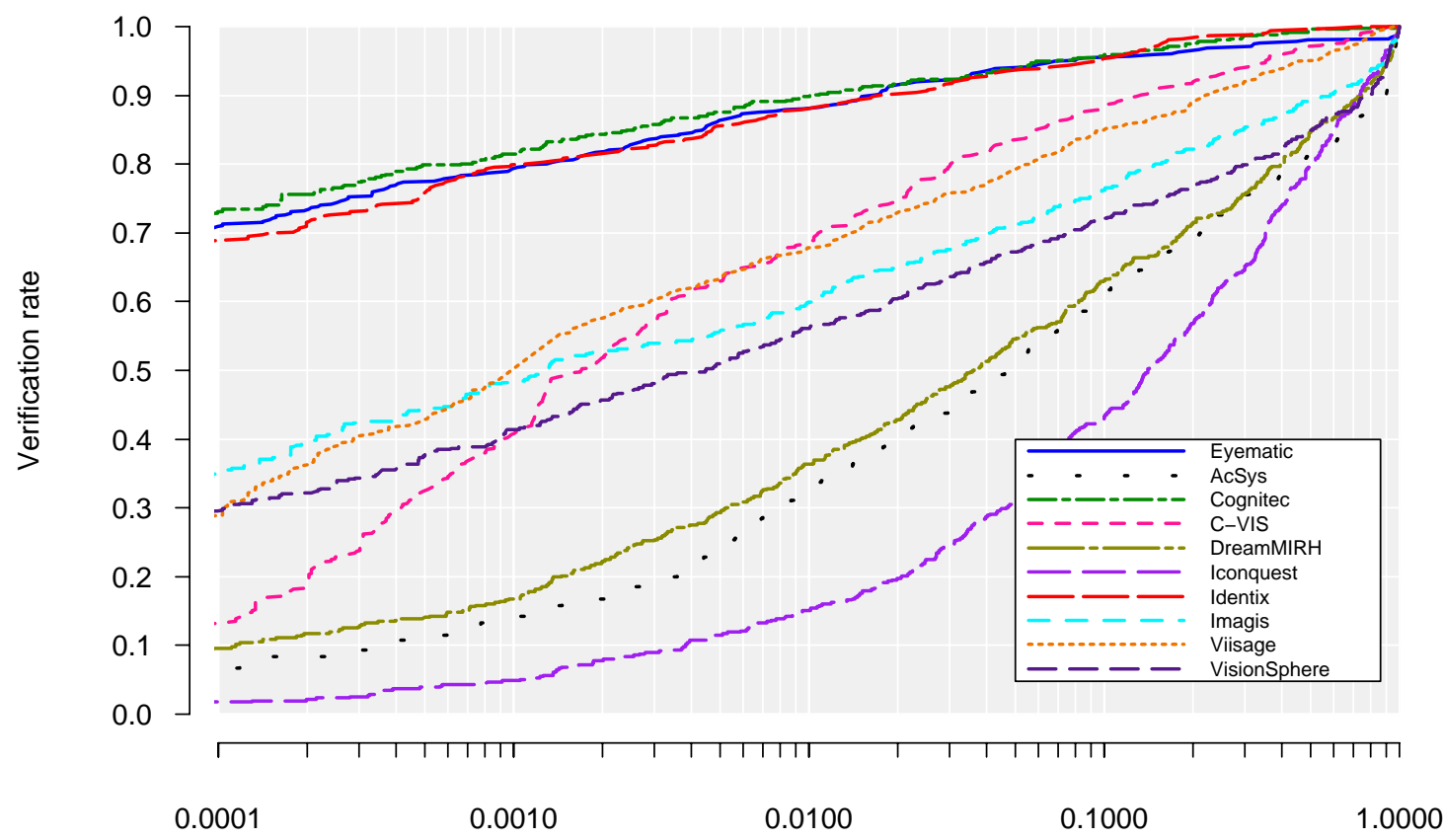

False accept rate

Figure L.46 - Verification Performance of Images from the HCInt Corpus in the MCInt sub-test.

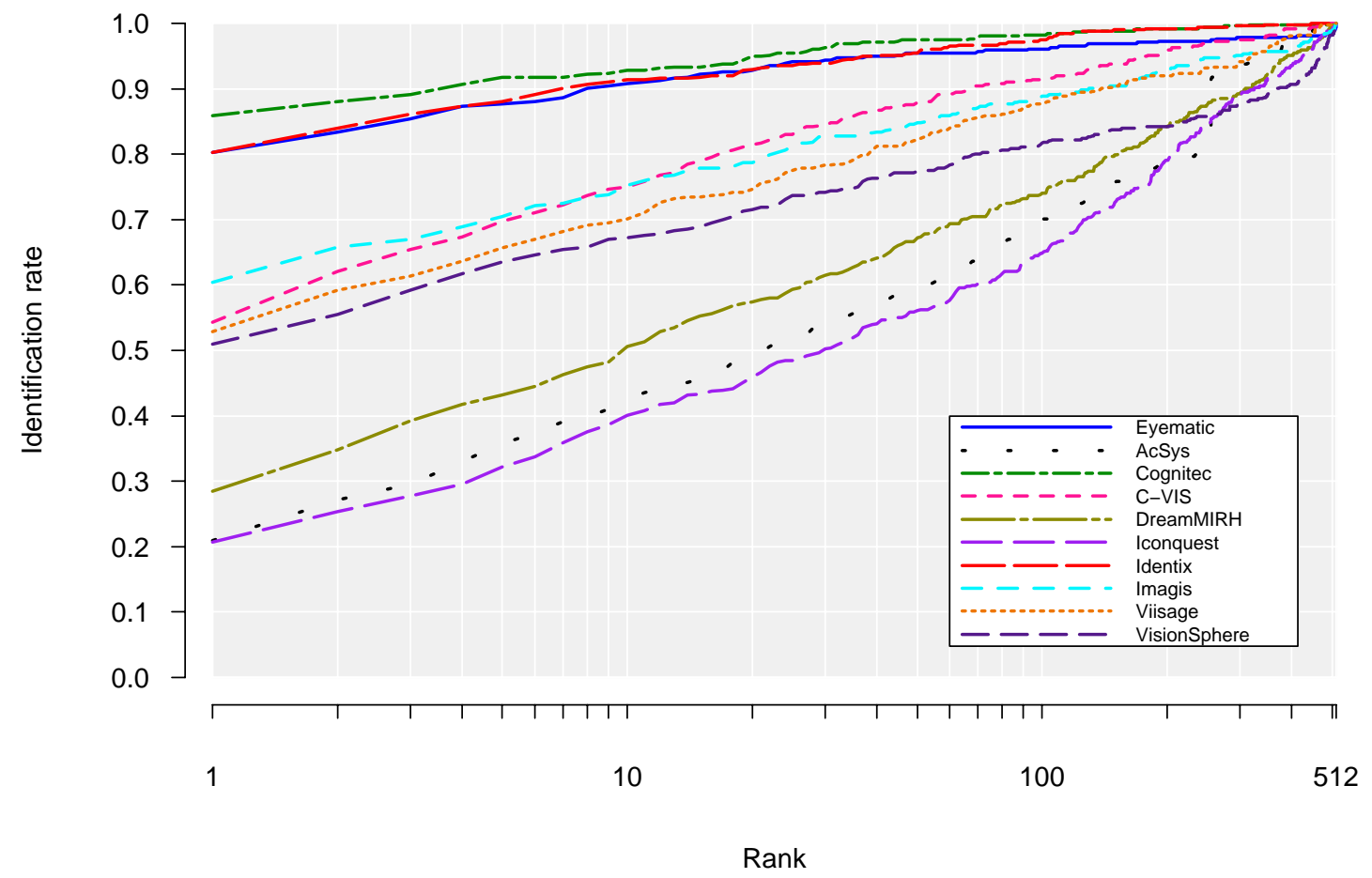

Figure L.47 - Identification Performance of Images from the HCInt Corpus in the MCInt sub-test 


\section{Appendix M}

\section{Participant Product Descriptions}

FRVT 2002 Participants were required to submit a paper describing their face recognition approach, modifications made to current systems for FRVT 2002 and an estimated cost of the system(s) used in FRVT 2002. These documents were turned in to FRVT 2002 organizers prior to beginning the FRVT 2002 on-site evaluations.

Participant documents are the opinions of the Participants and are provided in this text for reference. Inclusion of these documents does NOT imply that FRVT 2002

Authors/Sponsors/Supporters agree with any statements or derivative results within those documents. The identification of any commercial product or trade name does not imply endorsement or recommendation by the National Institute of Standards and Technology or any other FRVT 2002 Author/Sponsor/Supporter. 


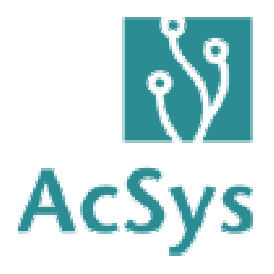

AcSys Biometrics Corporation

399 Pearl Street

Burlington, ON L7R 2 M8 - Canada

Phone: (905) 634-4111

Fax: (905) 634-1101

E-mail: info@nxsgrp.com

Web Site: www.acsysbiometricscorp.com

\section{AcSys Watchlist Application}

\section{Overview}

AcSys Biometrics Corp. is pleased to participate in the 2002 Facial Recognition Vendor Test. AcSys Watchlist, the prototype we are introducing for FRVT 2002, applies the speed, accuracy, and intelligence of AcSys face recognition technology to a 2-D watch list application. AcSys Watchlist is our prototype for a new product line that represents an entirely new direction for AcSys face recognition technology.

\section{AcSys Watchlist versus AcSys FRS}

Our mature product line, AcSys FRS, is not compatible with the test procedures proposed by FRVT 2002. The focus of the AcSys FRS product line is on the reliable authentication of identity. It accomplishes this authentication through the comparison of a number of images (representing a three-dimensional view of the individual) against a biometric template that is itself a product of a substantial number of images. This technique allows AcSys FRS to verify faces presented at a variety of angles.

This three-dimensional approach to face recognition makes AcSys FRS an ideal technology in access control situations and for other applications where individuals wish to authenticate a claimed identity, or are otherwise attempting to be recognized in a cooperative manner within a live environment. The AcSys FRS product line is designed to provide unparalleled speed and accuracy in such situations using an approach to authentication that is radically different from that of other face recognition technologies.

AcSys FRS is designed to continue to learn an individual's face over time and provide quick, accurate, and reliable authentication of the individual time after time. In contrast, AcSys Watchlist looks for degrees of resemblance between a single facial image (or small set of images) and a database of biometric templates.

\section{AcSys Watchlist Functionality}

AcSys Watchlist is a tool for performing two-way analysis of similarities between facial images (on a query list) and biometric templates (on a target list). Whereas our established AcSys FRS line of products works on a 3-D principle, examining facial images within live video to determine or authenticate identity, AcSys Watchlist:

- Compares a 2-D still image and/or live video to biometric templates

- Compares a biometric templates against 2-D images

- Returns a list of top matches, along with similarity values (scores), and displays associated images

- Creates biometric templates from sets of image files

- Updates biometric templates with new images without increasing the size of the template

The system's ability to build biometric templates from a large number of images and update the templates with additional images without increasing template size makes it a scalable solution 
Acsys Biometrics

for all watch list applications. The system's ability to recognize faces will improve with the addition of more images to templates, but the size of the template database will not grow.

AcSys Watchlist is designed to assist officials in the performance of background checks by allowing them to compare images captured for visa and license applications against databases of existing visa and license applications and watch list databases.

AcSys Watchlist includes the same set of utilities that have made the AcSys FRS line of products easy to use and administer:

- FaceDatabase - Allows you to administer target information

- FaceTrainer - Creates and updates templates from facial images and live video placed in a training queue

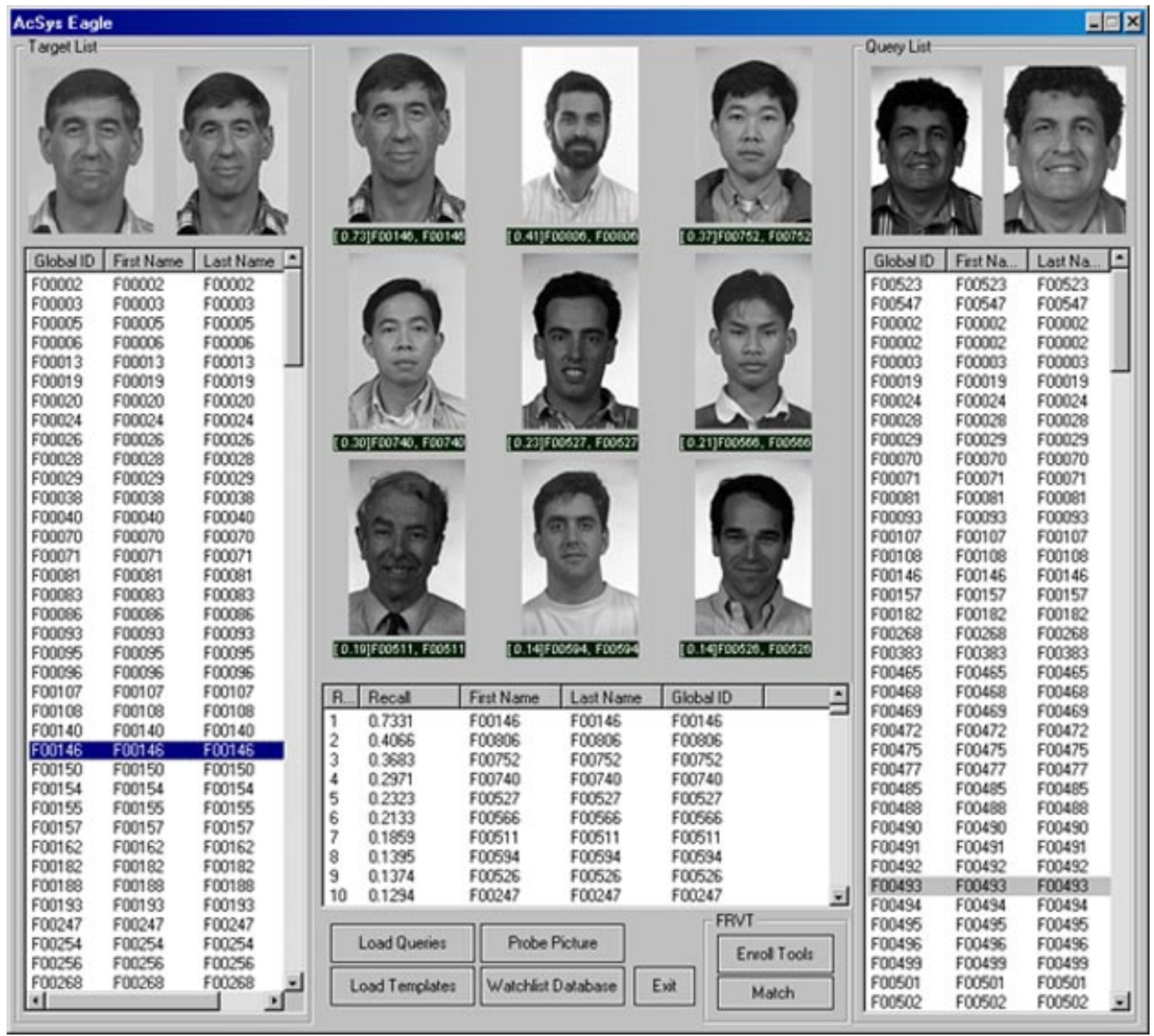

Figure 1: The AcSys Watchlist Main Interface 


\section{Component List}

System includes a video capture board, even though it is not required for this test, as image capture is out of scope.

\section{Hardware}

- Intel Pentium III $1 \mathrm{GHz}$ CPU x 2 installed

- ASUS CUV4X-D Motherboard

- 512 MB SDRAM @ 133 MHz

- Winnov PCI Videum Capture Board

- SoundBlaster $128 \mathrm{PCl}$

- ATI Rage XL Pro 2x AGP

- 3com 3C905C-TX NIC

- Maxtor 5T040H4 Hard Drive

- CD ROM

- 3.5 Floppy Drive

\section{Software}

\section{Operating System:}

AcSys Face

Recognition Software:
- Windows XP Pro

- AcSys Watchlist

- AcSys FaceTrainer

- AcSys FaceDatabase

\section{Cost Breakdown}

When the system is released to general availability, software costs will start at approximately US $\$ 25,000$, with peripheral modules (e.g., database, administrative tools, capture and control units, training stations, enrolment stations) to be priced separately.

\section{Modifications for FRVT 2002}

1. The Target List and Query List were renamed for clarity.

2. Two options were added to the application specifically for FRVT 2002:

- Enroll Tools: This option allows you create biometric templates from images and add the templates to the Target List.

- Match: This option runs the FRVT 2002 test process. Specifically, it performs a one-tomany comparison of each template in the Target List against each signature in the Query List and generates a similarity file for each signature. 


\title{
The Systems Used by Cognitec in the FRVT 2002
}

\author{
Cognitec Systems GmbH \\ An der Flutrinne 12 \\ D-01139 Dresden
}

Germany

\section{Overview of the systems}

Cognitec participated in the Face Recognition Vendor Test 2002 with two different systems, one for the High Computational Intensity (HClnt) test, the other for the Medium Computational Intensity (MCInt) test. The systems differed only in the computer hardware: a PC with a fast processor and $1 \mathrm{~GB}$ of main memory for the $\mathrm{HClnt}$ test, a notebook computer for the MCInt test. The software was the same in both systems and mainly consisted of two command line tools running under Windows 2000 and written specially for the Vendor Test using Cognitec's FaceVACS ${ }^{\circledR}$ Software Development Kit (FaceVACS $®$ SDK).

\subsection{FaceVACS\& SDK}

The FaceVACS ${ }^{\circledR}$ SDK provides functions for using all parts of Cognitec's face recognition engine such as face finding, eyes finding, creation of a biometric template from one or more images (enrollment), 1:1 comparison (verification) and 1:n comparison (identification) of an image against templates. The SDK thus gives integrators the opportunity to use high quality face recognition technology in a broad range of applications.

The main features of FaceVACS ${ }^{\circ}$ SDK are:

- State of the art face recognition API for easy integration of biometric technology into a broad range of applications

- Support for biometric operations: enrollment, verification and identification from image streams (live video) and image collection (image databases)

- $\mathrm{C}++$ interface to support modern paradigms of software development:

- Object oriented design

- Extensibility and modularity

- Abstract interfaces support easy adaption to various video streaming hardware and databases

- Detailed documentation including API reference, user guide containing installation and redistribution manuals

- Fully documented examples illustrating the main use cases and variation points 
FaceVACS ${ }^{\circledR}$ SDK is available for Linux (glibc 2.x) and various Windows platforms $(95 / 98 / \mathrm{Me} / \mathrm{NT} 4.0 / 2000 / \mathrm{XP})^{1}$.

\subsection{Face Recognition Technology}

The FaceVACS $\AA$ face recognition engine processes each image (8 bit greyscale) as follows (see also the two block diagrams below):

- Face localization: The image is analyzed to determine the position and size of one or more faces. (In all of the following steps, we assume that only a single face is found.)

- Eye localization: The positions of the eye centers in the face are determined.

- Image Quality Check: The quality of the face image is checked to see whether it is sufficient for the steps that follow.

- Normalization: The face is extracted from the image and is scaled and rotated such that an image of fixed size results, with the eye centers at fixed positions in that image.

- Preprocessing: The normalized image is preprocessed with standard techniques such as histogram equalization, intensity normalization, and others.

- Feature extraction: In the preprocessed image, features are extracted that are relevant for distinguishing one person from another.

- Construction of the reference set: During enrollment the facial features of (usually) several images of a person are extracted and combined into a reference set, also called the "biometric template".

- Comparison: For verification, the set of extracted features is compared with the reference set of the person who the person in the image just processed is claimed to be; for identification, the feature set is compared to all stored reference sets, and the person with the largest comparison value is selected; in both cases the recognition is considered successful if the (largest) comparison value - which is interpreted as a similarity value - exceeds a certain threshold value.

\footnotetext{
${ }^{1}$ Windows 95 is only supported as a target platform for applications developed with the SDK, not as a platform for the development itself.
} 


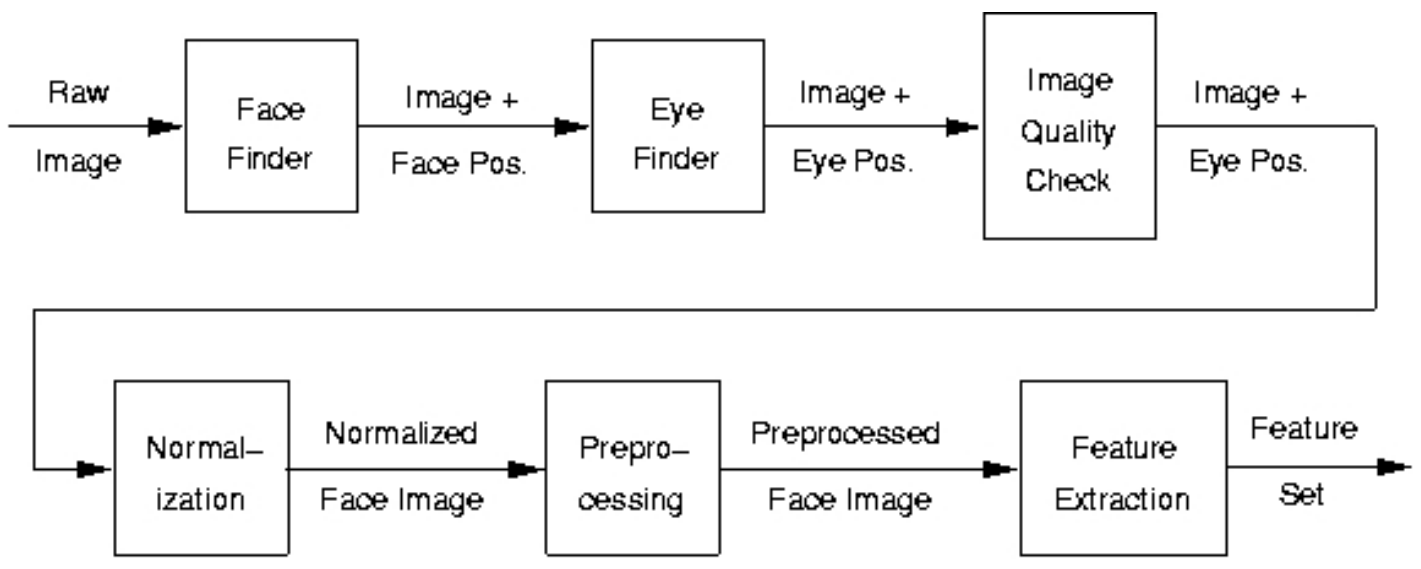

Figure 1: FaceVACS architecture: Feature set creation

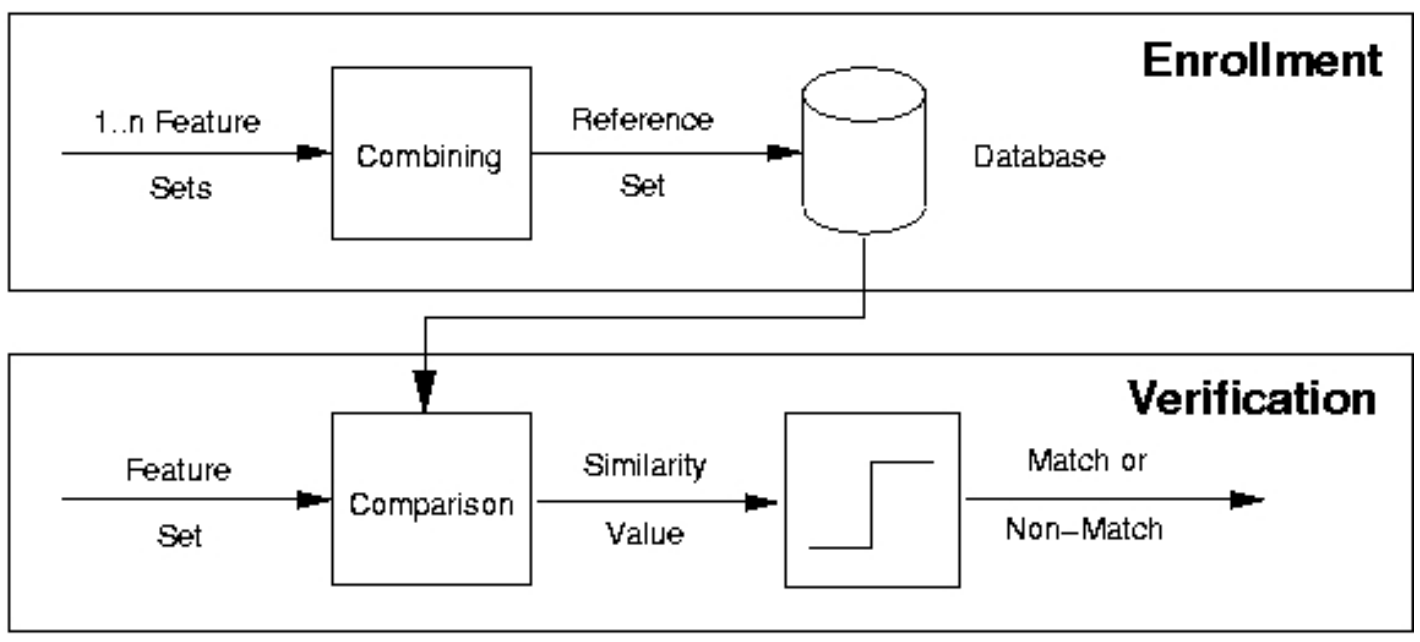

Figure 2: FaceVACS architecture: enrollment and verification

In addition, FaceVACS has a "live check" to ensure that the face in front of the camera is a real one and not just a photograph. To this end, the changes in appearance occurring during movement of the face (rotations around the vertical axis in particular) are exploited. Due to the special 3D structure of a real face, those changes are quite different for a real face than for a photo. So when the user wants to pass the live check, he or she should briefly rotate his or her head back and forth.

For more details see the technology description on Cognitec's website (http://www.cognitec-systems.com). 


\section{Component list and cost breakdown}

The submitted systems had in common the software, consisting mainly of two simple command line tools (about 500 lines of $\mathrm{C}++$ code) that can be quickly written on the basis of the FaceVACS ${ }^{\circledR}$ SDK. The FaceVACS ${ }^{\circledR}$ SDK is currently priced at $\$ 3,800^{2}$.

\subsection{System for HClnt test}

The following table shows the components and the cost breakdown of the PC used for the HCInt test (all prices are average mail order prices in Germany without tax as of July 18th 2002):

\begin{tabular}{|l|l|r|}
\hline Item & Vendor & Price \\
\hline $\begin{array}{l}\text { Case } \\
\text { Power Supply 300W }\end{array}$ & $\begin{array}{l}\text { SilentMax } \\
\text { Fortron }\end{array}$ & $\begin{array}{r}\text { (case + power supply) } \\
\text { Mainboard } \\
\text { MSI K7N 420Pro }\end{array}$ \\
\hline MSI & $\$ 120$ \\
\hline CPU Cooler & AMD & $\$ 95$ \\
\hline $\begin{array}{l}\text { RAM 2x512MB } \\
\text { PC266 CL2 DDR }\end{array}$ & AMD & $\$ 25$ \\
\hline $\begin{array}{l}\text { HDD 80 GB } \\
\text { IBM IC35L080VA }\end{array}$ & Infineon & $\$ 310$ \\
\hline DVD/CDROM & IBM & $\$ 86$ \\
\hline USB 2.0 Controller & & $\$ 65$ \\
\hline
\end{tabular}

The total system price (SDK + PC) thus amounts to $\$ \mathbf{4 , 7 1 1}$.

\subsection{System for MCInt test}

The notebook computer used for the MCInt test is a Fujitsu-Siemens Lifebook C 6555 equipped with the following hardware components:

- CPU Pentium III $650 \mathrm{MHz}$

- HDD $10 \mathrm{~GB}$

- SDRAM $128 \mathrm{MB} 100 \mathrm{MHz}$

- FDD

\footnotetext{
${ }^{2}$ In addition, if an SDK customer plans to sell applications developed with the SDK, runtime royalties have to paid to Cognitec for each sold copy of the application software; those royalties are fixed individually in a contract between the customer and Cognitec.
} 
- CDROM drive

- 4 MB Video RAM

In February 2001, the price for this notebook was DM 4,985 (German Marks) without VAT. Nowadays (July 2002), an equivalent or even somewhat more powerful notebook should be available for less than $\$ 1,800$, making the total system price (SDK + Notebook) be less than $\mathbf{\$ 5 , 6 0 0}$.

\section{Modifications for the purpose of FRVT 2002}

The only modification, that was done for efficiency reasons, was the extension of the FaceVACS ${ }^{8}$ SDK interface to allow the comparison of two biometric templates (in the current release, FaceVACS ${ }^{\circ}$ SDK 1.2, only comparison between a template and a set of images is possible). This allowed to exploit the fact that in the HClnt test, query and target set were identical; so, the whole processing chain consisting of, among other steps, face finding, eyes finding, and feature extraction had to be executed only once for each image. With the interface of the current release, each image would have had to be processed twice, once as target image and once as query image. The comparison results, however, would have been exactly the same. 


\section{FaceSnap RECORDER - General Overview}

The FaceSnap RECORDER digitizes standard video sources and performs real-time pattern recognition on the captured images. The FaceSnap RECORDER detects human faces in the video stream and stores a user-adjustable image region around each face. $A$ powerful image database facilitates easy navigation and search on large numbers of stored images. For analyzing the face image database, interactive and automatic search functions are provided. As an option, the FaceSnap RECORDER can perform real-time facial identification. The FaceSnap RECORDER is a novel solution for person-oriented video surveillance, access monitoring and other applications requiring face image recording and face recognition.

The FaceSnap RECORDER targets the market for intelligent video surveillance. It captures and stores face images in near video real-time. That is, any standard video source (cameras, VCRs ...) can be analyzed for one or more persons of whom the face is visible in the image. The face images are stored in a real-time data base. The user can select images as reference faces and then search all the recorded data for images that may match the reference image(s).

The most important applications for the FaceSnap RECORDER are in law enforcement (e.g. police observations), in casinos, airports, border control, and banks.

The FaceSnap RECORDER is a complete ready-to-use system comprising a powerful PC platform and a factory installed software.

It is currently shipped in three versions:

Standard, "law enforcement" (LEF), and LEF Portable.

The FaceSnap RECORDER also is designed for operating in a network.

That is, several FaceSnap RECORDER may be accessed from a central server and other systems can easily be interfaced to a FaceSnap RECORDER using standard internet protocols.

A powerful real-time facial identification system can be setup by connecting a FaceCheck SERVER to a FaceSnap RECORDER over a network link.

There are several modes of operation:

- Standard recording mode

The FaceSnap RECORDER screen shows the live camera image and a number of most recently recorded persons. The user can switch between a number of different video inputs. The captured face images are displayed in a normalized format and can be stored in real-time on the local disk.

- Replay mode

In replay mode, the user can easily navigate through a large amount of face image data. Images are time stamped and can be directly accessed through their time keys or the graphical event histogram that provides an overview of the temporal distribution of recorded images. 
- Search mode

In search mode, the user can define image groups as a general purpose tool for data management. In addition, image groups are used to define training sets for face identification. Automatic functions for image pattern recognition are provided to search the face image database and to support content-based data management.

- Training mode

The FaceSnap RECORDER can be trained to recognize faces based on one or more sample images collected by the user. In training mode, an image group can be imported to establish or extend a training set for a particular person. There are two hyper classes of persons: a) the class of all persons known to the FaceSnap RECORDER and b) the class of all persons the FaceSnap RECORDER is supposed to look for.

- $\quad$ Selective recording mode (FaceCheck SERVER)

Face images captured by a FaceSnap RECORDER operating in recording mode can be sent to a second FaceSnap RECORDER over a high-speed 100BaseTX network connection in real-time. The second system, which has to be put in the selective recording mode, receives the stream of face images and tries to identify all persons belonging to the hyper class of "active/interesting" people (watch list). Only those images for which the similarity value exceeds a user-defined threshold are displayed and optionally stored in the database.

\section{Technology}

The most important technology built into the FaceSnap RECORDER is the automatic selection of human faces in an image. The underlying technical problem is to detect and localize face-like patterns in a digitized image of an arbitrary scene. Moreover, the computer has to complete this search within fractions of a second since the person may not look straight toward the camera much longer than that. An additional requirement FaceSnap was designed to meet is the independence of object cues like motion or color. FaceSnap looks for face patterns in an image, not for regions showing a certain color range or being in motion relative to the image background. FaceSnap solves these problems by using a fuzzy abstract face model in combination with a neural net classifier. The outcome of the face finding stage are face image windows which are ranked according to a faceness value indicating how much the selected image portion resembles a human face

The face detection (face spotting) of the FaceSnap RECORDER uses the FaceSnap technology as described above. The model-based pattern recognition has a processing rate of up to 25 images per second, depending on CPU type and size of the source image. Frontal views of human faces are preferred with a rotational tolerance of ca. $+/-15$ degrees. Tolerance to deviations of the frontal face pose can be adjusted.

The face identification of the FaceSnap RECORDER uses the FaceCheck technology. The pattern recognition for face identification is based on user-provided training sets. The facial feature extraction is designed for frontal face views. Compensation of image rotation works up to $+/-15$ degrees. Based on the location of prominent facial features, the face images are first geometrically normalized and then normalized with respect to the gray level

FRVT2002 • System Description Document 
distribution. From the normalized images, feature vectors are produced and then used for classification. The classification algorithm is based on a combination of the elastic net matching technique and specialized neural net classifiers.

The face recognition technology used for the product FaceSnap RECORDER has been developed by C-VIS and partly by the University of Bochum. All intellectual property rights are owned by C-VIS.
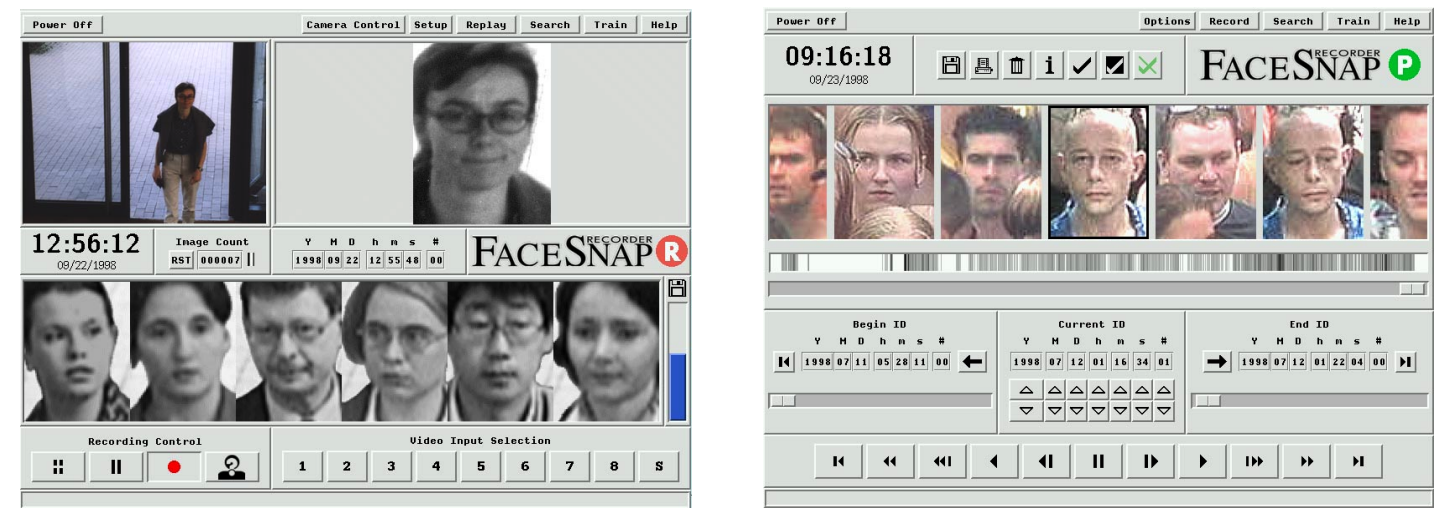

Screenshots of standard recording mode (left) and replay mode (right).
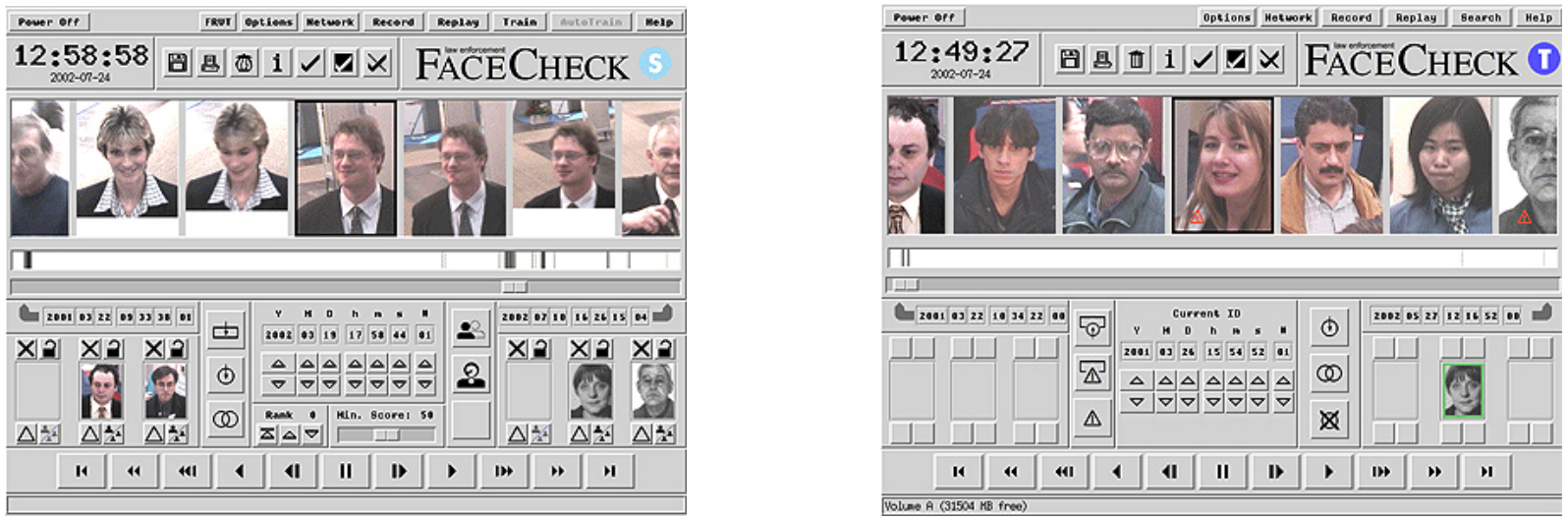

Screenshots of search mode (left) and training mode (right)

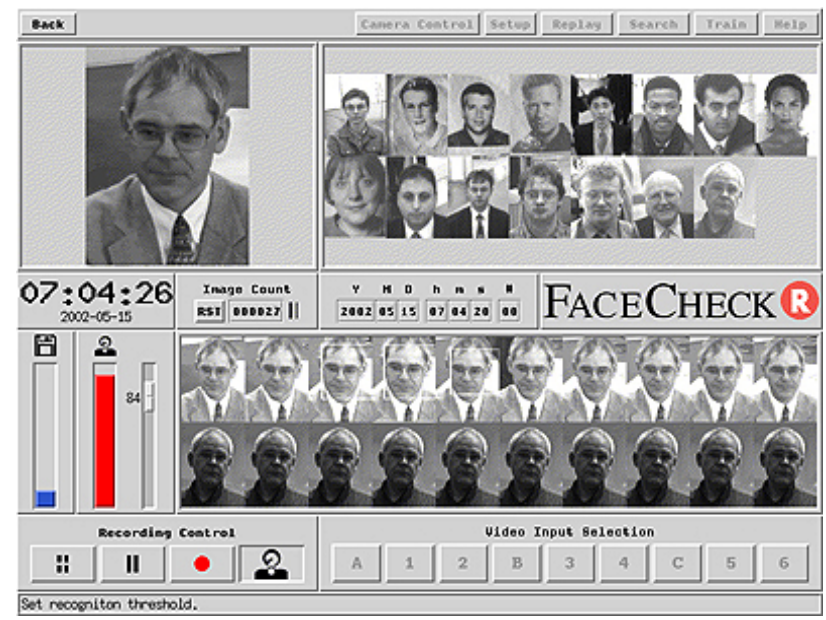

Screenshot of the selective recording mode (FaceCheck SERVER) 


\section{Systems submitted to the FRVT2002}

The facial recognition vendor test 2002 does not include any test with real-time video input. Therefore the FaceCheck SERVER option of the FaceSnap RECORDER was not part of the systems that were submitted by C-VIS.

The systems submitted by C-VIS for the FRVT2002 are all of the product model "FaceSnap RECORDER law enforcement". C-VIS used a total of three systems for the test.

The component lists of the submitted systems are given below. The table also states the price of the products.

\begin{tabular}{|c|c|c|c|c|c|}
\hline Product Model & $\begin{array}{l}\text { System } \\
\text { Price } \\
\text { [Euro] }\end{array}$ & Enclosure & Processors / RAM & $\begin{array}{l}\text { Other } \\
\text { Components }\end{array}$ & $\begin{array}{c}\text { Test } \\
\text { used for }\end{array}$ \\
\hline $\begin{array}{l}\text { FaceSnap } \\
\text { RECORDER LEF } \\
\text { portable }\end{array}$ & 10450 & $\begin{array}{l}\text { ATX- } \\
\text { Portable } \\
\text { with } \\
12,1 " \text { TFT }\end{array}$ & $\begin{array}{l}\text { Dual Pentium III, } \\
\text { 1.4 GHz, } 512 \text { MB }\end{array}$ & $\begin{array}{l}\text { CDROM, } \\
\text { LS120, } \\
60 \text { GB HDD, } \\
\text { PCl video } \\
\text { frame grabber, } \\
\text { 10/100 Ethernet } \\
\text { Adapter }\end{array}$ & $\mathrm{HCl}$ \\
\hline $\begin{array}{l}\text { FaceSnap } \\
\text { RECORDER LEF }\end{array}$ & 8800 & $\begin{array}{l}\text { ATX- } \\
\text { Desktop }\end{array}$ & $\begin{array}{l}\text { Dual Pentium III, } \\
\text { 1.1 GHz, } 512 \mathrm{MB}\end{array}$ & $\begin{array}{l}\text { 80 GB HDD, } \\
\text { 10/100 Ethernet } \\
\text { Adapter }\end{array}$ & $\mathrm{HCl}$ \\
\hline $\begin{array}{l}\text { FaceSnap } \\
\text { RECORDER LEF }\end{array}$ & 8800 & $\begin{array}{l}\text { ATX- } \\
\text { Mini-Tower }\end{array}$ & $\begin{array}{l}\text { Single } \\
\text { AMD Athlon 1900+, } \\
256 \text { MB }\end{array}$ & $\begin{array}{l}\text { CDROM, FDD, } \\
80 \text { GB HDD, } \\
\text { PCl video } \\
\text { frame grabber, } \\
10 / 100 \text { Ethernet } \\
\text { Adapter }\end{array}$ & $\mathrm{MCl}$ \\
\hline
\end{tabular}

The standard products uses for the FRVT2002 had to be modified to meet the special requirements of the Test Plan and the "FRVT 2002 Implementer's Guide".

The significant modifications are:

- User interface option for choosing "FRVT2002 operating modes".

- Special "Import Mode" that uses XML documents for controlling the image access.

- Special mode for generating scoring results in the FRVT2002 file format.

- Normalization of the scoring results with an external function.

- Special matching mode with reduced accuracy for the High Computational Intensity Test. 


\section{Summary Description Documents Dream Mirh Co., Ltd.}

\section{Overview of System}

The Dream Mirh FRVT2002 system consists of hardware and software. The hardware is a generic standalone style workstation. One workstation is used for the high computational intensity test and another for the medium computational intensity test. The software is used for all tests using the MIRH Eye Face Recognition Engine.

\section{Hardware}

The structure of FRVT 2002 appears on $<$ Table 1>. <Table 1> compares the MIRH Eye Engine with the commercial package, MIRH Eye Surveillance system.

\begin{tabular}{|l|l|l|}
\hline & \multicolumn{1}{|c|}{ FRVT 2002 } & \multicolumn{1}{|c|}{ Commercial (Surveillance) } \\
\hline Computer & $\begin{array}{l}\text { HP x-4000 } \\
\text { Pentium IV 2.2GHz (Single) }\end{array}$ & Pentium IV 2.0GHz (Single) \\
\hline Main Memory (RAM) & 4GB & 512MB \\
\hline OS & Windows 2000 Server & Windows 2000 Server \\
\hline Input Condition & JPEG format image & Any CCD Camera or Photo \\
\hline
\end{tabular}

Table 1: Hardware Configuration

FRVT 2002 uses the widely used HP Workstation x-4000 model system. The breakdown in price is as follows:

HP x-4000 Workstation

Pentium IV 2.2GHz Dual CPU

4GB RDRAM Memory

Wildcat $611064 \mathrm{M}$

Price: $\$ 4,000$

17 " TFT LCD Monitor

Price: $\$ 700$ 


\section{Windows 2000 Server}

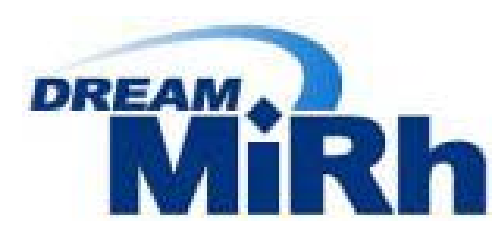

Price: $\$ 1,000$

For the windows series or Red Hat Linux operation systems and hardware, basic configurations can be expanded upon the user's request.

\section{Software}

The software used in FRVT 2002 was developed for users wishing to use facerecognition technology. Its functions are based on MIRH Eye SDK (Software Development Kit) 2.0. MIRH Eye SDK takes image input from a camera or any other image, finds the facial area, compares that with the data stored in the database, discriminates and verifies the data. This SDK can be used with the Windows series and Visual $\mathrm{C}++$ as it has optimal codes for better compatibility program development, thus ensuring a faster and more powerful operation. The structure and hardware for the MIRH Eye SDK is as follows:

MIRH Eye SDK has the following 3 components.

Detection: Finds the face in the entire image

Verification: 1:1 method. An ID is entered with the facial image

Identification: $1: \mathrm{N}$ method. Only the facial image is entered so that the system needs to search the database for the match.

MIRH EYE SDK use the following hardware

PCs with Windows 9x, 2000, NT, XP operating systems

Intel Pentium III 500MHz or higher

64 RAM or higher 
The software engine used for FRVT 2002 is the basic MIRH Eye SDK Engine that has been modified for the test environment. The structure and process for the FRVT 2002 is show in the flow chart. 〈Picture 1>

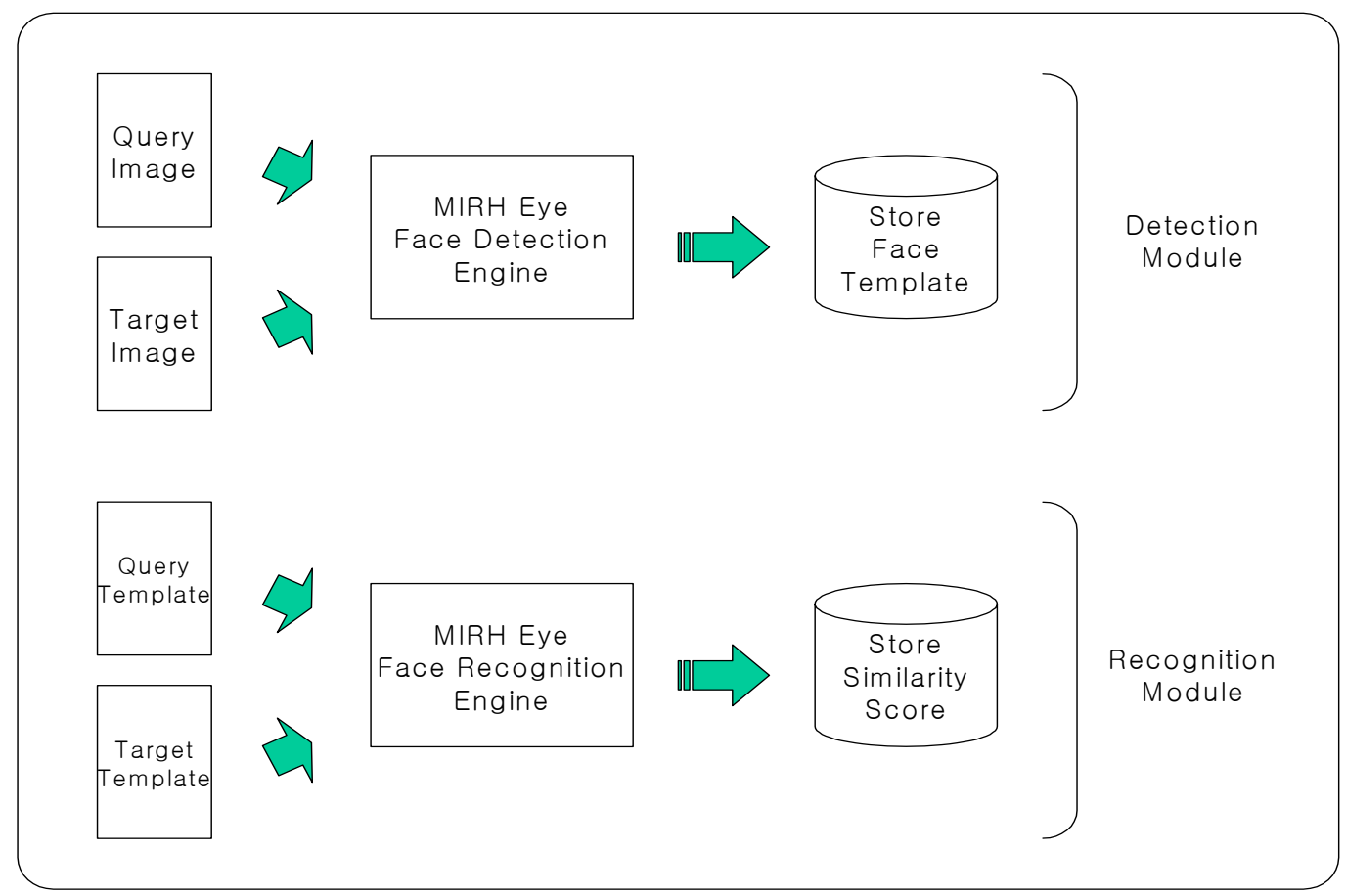

\section{$<$ Picture 1>: MIRH Eye Engine flow chart}

The MIRH Eye Engine that was designed for the FRVT 2002 has two main processes Detection and Identification. In the detection process, the images on the query/target list are uploaded, the facial area is found, and a template is made for the images to be stored in xml form. After all the images are loaded in the database, the Identification process automatically begins. The detector converts all images into template. Recognizer takes one template from Query, and stores the degree of resemblance based on the comparison with all templates in the target in the similarity file. After all Query images are running the test ends.

\section{For further information, please contact:}

Donghoon Jeon

Dream Mirh Co., Ltd.

\section{R \& D Center}

\section{Senior Engineer}

Email : smallove@dreammirh.com http://www.dreammirh.com/ 


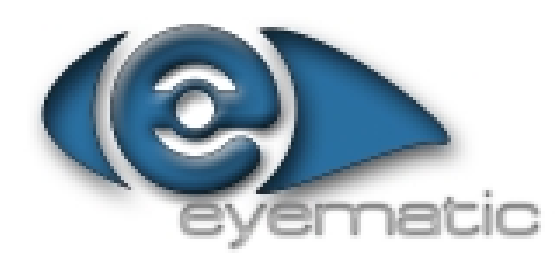

\section{Face Recognition System Description Eyematic Interfaces Inc.}

Face Recognition Vendor Test, July 2002

Eyematic's visual sensing technology is made available through a Software Development Toolkit (SDK). The toolkit bases on Eyematic's visual sensing software, called EPL (Eyematic's Primary Library). Specific capabilities are Face detection in images, Head or Local Feature tracking in sequences, Facial Feature Analysis and Human Identification through Face Recognition. The SDK's core component is a C++ based API consisting of image processing modules.

The API was developed to allow easy Integration of Eyematic's technology in applications such as Access Control Systems, Credit Card Authorization Terminals, Point of Sales Systems, Baggage/ Passenger Matching and surveillance Systems.

OMRON Corporation (Japan) is Eyematic's largest partner integrating face recognition applications based on the SDK.

\section{EPL-API SDK Overview}

\subsection{Components}

The API architecture was designed with emphasis on modularity, adaptability and convenience for the application developer. It provides a generic system of data processing components (modules) that can be linked together to a pipeline. Each module is optimized for a specific image-processing task. Sub sequential modules process information provided by preceding modules. The data set being passed through consists of an image and image related information. Still images as well as video stream images flow through all processing stages and are gradually enriched by face related information (face position, head pose, bounding rectangle, local feature positions, etc) as the data passes each module (Fig. 1,3). Processing modules can be individually combined to serve specific requirements such as fast processing on video streams or extensive search on still images. The pipeline concept allows optimizing CPU usage on multi processor platforms as well as using multiple computers by running each module in a separate thread or process.

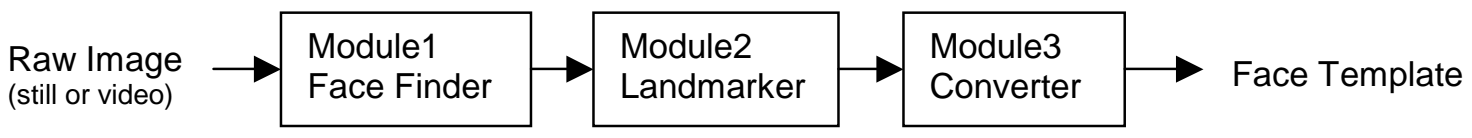

Figure 1: Typical set of SDK Modules used for face recognition. 


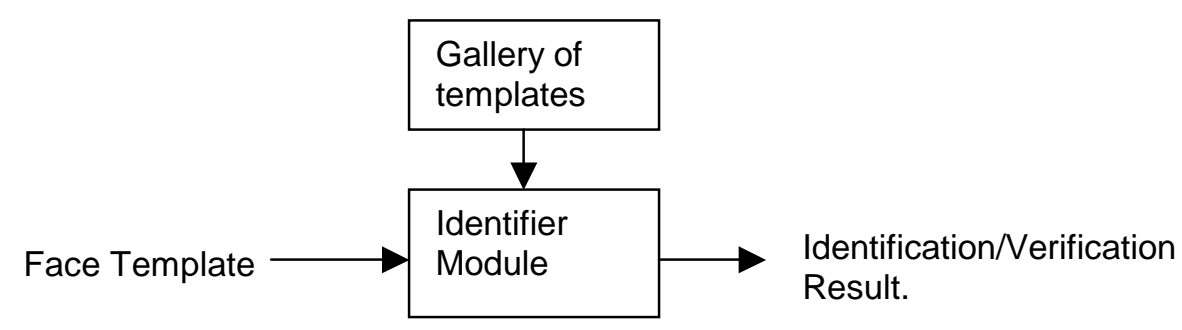

Figure 2: Typical set of SDK Modules used for face recognition.

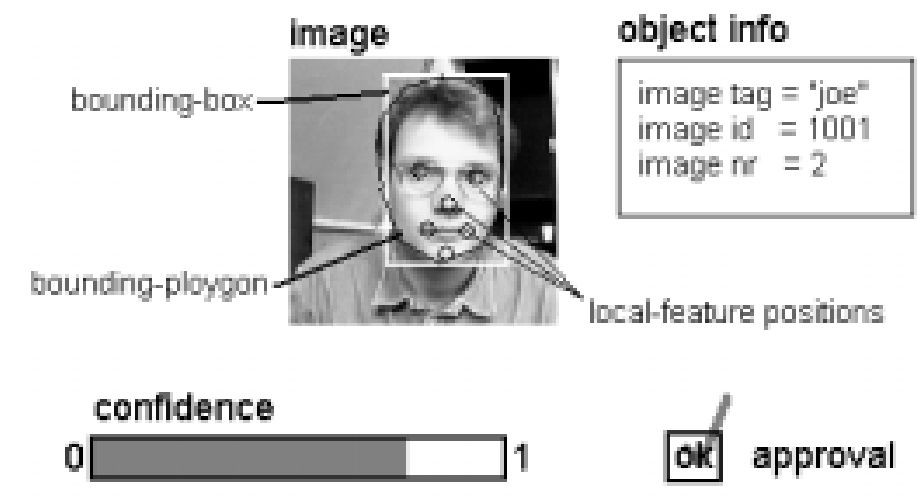

Figure 3: Data set being passed through modules.

In order to build a face recognition system, the system integrator would combine modules of 4 categories:

\begin{tabular}{|l|l|}
\hline Module Category & Description \\
\hline Face Finder & Locates the face on the image \\
\hline Landmarker & Finds local feature positions \\
\hline Converter & Extracts a face template from the image \\
\hline Identifier & $\begin{array}{l}\text { Recognizes/Verifies a person by comparing a face template to a gallery } \\
\text { of templates. }\end{array}$ \\
\hline
\end{tabular}

A combination of Face Finder, Landmarker and Converter specifies a face enrollment system (Fig. 1). The face template obtained would either be stored in a gallery or identified/verified by matching it to entries of a gallery (Fig. 2). Face Finder and Landmarker modules produce a confidence value that provides a measure of quality for face detection. This is particularly important when processing video stream data. Here this measure is used as filter to select images that do display a face in sufficient quality. 


\subsection{Underlying Technology}

The face recognition system uses Gabor Wavelet Image Transformation and Neural Networks. Face Detection Modules employ a general face model to localize a face in the image. A 3D representation of the head copes with pose variations. The general face model is learned from a large database of sample images. The face models supplied with our standard modules cover a wide variety of environmental conditions (illumination, poses, expressions, etc). Sometimes an adaptation to specific conditions is desired. For this purpose a special training tool can be provided.

\subsection{Technical Specifications}

For each category we have developed a variety of modules, which represent different accuracy and speed levels. The processing speed per image typically varies between $200 \mathrm{~ms}$ and $1000 \mathrm{~ms}$ depending on configuration used ${ }^{-1}$. Modules of the same category can be exchanged without the need to adapt source code. The size of a face template can range from 1 Kbytes to 6 Kbytes depending on the type of Converter used. The typical Identification/Verification speed is about $90 \mu \mathrm{s}$ (microseconds) for a single template-to-template match ${ }^{2}$ based on a template size of 4 Kbytes. Usage of lower template sizes will yield significantly higher processing speeds at reduced accuracy.

\subsection{Target Platforms}

The EPL software has been developed with high emphasis on portability. The underling code was written in strict accordance with standards that ensure portability to multiple platforms. Time critical routines are optimized in using platform specific resources to ensure maximum performance. The API itself complies with the ANSI C, C++ Standard. For high performance and desktop applications conventional desktop PCs can be used. For low cost and low size embedded systems, we have developed an embedded EPL-API based on Texas Instruments Digital Signal Processors.

Full functionality is available on MS Windows based Platforms using Intel Pentium (or similar) Processors.

Operating System: Windows 95, 98, ME, 2000, XP

Recommended Development Environment: MS Visual C++ 6.0

Slightly reduced functionality is available for Linux/Unix based Platforms.

Recommended Compiler: g++ or compatible

A special embedded version of the SDK is available for the DSP Families TMS320C6200, C6400, and $\mathrm{C} 6700$.

Recommended Development Environment: TI Code Composer Studio

\subsection{System Recommendation}

The system requirements depend on desired processing speed and gallery size. We recommend using a MS Windows Intel Platform:

CPU: $\quad$ Pentium 3 at $800 \mathrm{MHz}$ or better

RAM: $\quad 256$ Mbytes or higher

\footnotetext{
${ }^{1}$ Speed value refers to Face Finder, Landmarker and Converter pipeline on a Single CPU Platform: Intel P4, 2GHz (or similar).

${ }^{2}$ Measured on single CPU Platform: Intel P4, 2GHz (or similar).
} 


\section{FRVT2002 Test Application}

The FRVT2002 Application uses the EPL Module concept described above for still image processing. We selected a template size of about $4 \mathrm{Kbytes} /$ image for both subtests (HCINT and MCINT). We bypassed the Identifier module and compared face templates directly to avoid some unneeded processing, which the Identifier Module would normally do automatically (sorting of similarities, normalization, etc). For video data we used the filtering method mentioned in the paragraph above.

For both subtests we chose face detection module configurations that take between $0.5 \mathrm{~s}$ and 0.8 s processing time for each image on a single computer. (S. Paragraph 3 for Hardware Specs.)

Part of training data of face detection module used for FRVT2002 was taken from the FERET database of facial images collected under the FERET program.

Ref.: P. J. Phillips, H. Wechsler, J. Huang, and P. Rauss, "The FERET database and evaluation procedure for face recognition algorithms," Image and Vision Computing J, Vol. 16, No. 5, pp 295-306, 1998.

\section{FRVT2002 Component list and cost breakdown}

\subsection{Computers}

Three desktop computers were prepared to run the test. Two computers were dedicated to HCINT. One computer was prepared for MCINT and HCINT. The computers used for HCINT were connected via $100 \mathrm{MB} / \mathrm{s}$ Ethernet Network during the time HCINT was running. We used Windows 2000 as operating system on all machines.

\begin{tabular}{|c|c|c|c|}
\hline Computer Specification (Excl. Monitor) & Quantity & $\begin{array}{l}\text { Unit Price } \\
\text { (Excl. Tax) }\end{array}$ & Total Price \\
\hline $\begin{array}{l}\text { DELL Dimension } 8200 \text { (Single Processor) } \\
\text { Intel Pentium } 4 \text { Processor at } 2.53 \mathrm{GHz} \\
1024 \text { MB PC800 RDRAM } \\
80 \text { GB } 7200 \text { RPM Ultra ATA Hard Drive }\end{array}$ & 2 & $\$ 2,456.00$ & $\$ 4,912.00$ \\
\hline $\begin{array}{l}\text { Compaq EVO } \\
\text { Intel Pentium } 4 \text { Processor at } 2 \mathrm{GHz} \\
\text { Southland Compaq } 1024 \text { MB RAM } \\
40 \text { GB Hard Drive }\end{array}$ & 1 & $\$ 1,482.98$ & $\$ 1,482.98$ \\
\hline
\end{tabular}

\subsection{Accessories}

The following accessory items were prepared:

\begin{tabular}{|l|l|l|l|}
\hline Accessory Specification / Usage & Quantity & $\begin{array}{l}\text { Unit Price } \\
\text { (Excl. Tax) }\end{array}$ & Total Price \\
\hline $\begin{array}{l}\text { Netgear Ethernet Hub 100MB/s } \\
\text { To connect computers }\end{array}$ & 1 & $\$ 169.95$ & $\$ 169.95$ \\
\hline $\begin{array}{l}\text { Belkin KVM Switch/Cable Omnvm } \\
\text { To switch single monitor, keyboard, mouse between } \\
\text { computers. }\end{array}$ & 1 & $\$ 226.32$ & $\$ 226.32$ \\
\hline $\begin{array}{l}\text { LACIE 80GB USB 2.0 Hard Drive } \\
\text { Extra Data Storage Device (if needed) }\end{array}$ & 1 & $\$ 203.68$ & $\$ 203.68$ \\
\hline
\end{tabular}




\subsection{Face Recognition Software}

Our Business Model for the API SDK is to license it to System Integrators. The price consists of an upfront license fee and royalties depending on (but not limited to) field of view, geographical region and exclusivity.

We offer evaluation licenses to customers who might be interested in testing our system. The fee for an evaluation license of the API SDK Software and Modules to replicate the FRVT2002 test setup would be approximately $\$ 10,000$. The actual amount can fluctuate depending on market conditions, special requests, etc.

We gladly refer inquiries about applications using Eyematic's visual sensing technology to OMRON Corporation (Japan). URL: http://www.society.omron.com/faceid/index.html

\section{Contact:}

Eyematic Interfaces Inc.

9800 S. La Cienega Blvd., $5^{\text {th }}$ Fl.

Inglewood, CA 90301

USA

www.eyematic.com 


\section{Iconquest}

3525 Piedmont Road

Building 7, Suite 600

Atlanta, GA 30305

\section{System Description for FRVT 2002 Face Recognition Vendor Test}

July 24, 2002

Iconquest's submission to FRVT 2002 consists of proprietary software, IQ Imagematcher ${ }^{\mathrm{TM}}$, hosted on a Dell Precision 340 desktop computer.

\section{Description of IQ ImageMatcher ${ }^{\mathrm{TM}}$ Software}

IQ ImageMatcher ${ }^{\mathrm{TM}}$ is software for personal computers that combines a fractal image comparison algorithm with both a graphical user interface and a script driven interface.

The image comparison algorithm returns a number $\mathrm{M}(\mathrm{Q}, \mathrm{T})$ for each pair of images $\mathrm{Q}$ and $\mathrm{T}$.

The methodology is to partition Q into relatively small pixel blocks and to determine whether each such block can be better represented as a contractive transformation of a somewhat larger block chosen from $\mathrm{Q}$ or chosen from $\mathrm{T}$. The transformations are chosen from among compositions of spatial rotation and spatio-spectral scaling and translation. In the simplest case, $\mathrm{M}(\mathrm{Q}, \mathrm{T})$ can be chosen as the ratio of number of partition blocks better represented from $\mathrm{T}$ to total number of partition blocks. Typically though, we scale up $\mathrm{T}$ somewhat and normalize to insure that this process results in $\mathrm{M}(\mathrm{Q}, \mathrm{T})=1$ when $\mathrm{T}=$ Q.

This approach is an example of unconstrained image comparison where no assumption of structure is imposed on the underlying imagery. In contrast are constrained image comparison applications such as optical character recognition, fingerprint identification, biometric face recognition, robotic vision for quality control and automatic target recognition.

One expects unconstrained systems to be applied at least in cases where the imagery is of too poor quality for structure differentiation necessary in a constrained system, where there is not sufficient imagery available for development of constrained systems or when there is not sufficient time for development of a constrained system in response to some immediate application need.

The IQ ImageMatcher ${ }^{\mathrm{TM}}$ approach has several advantages in unconstrained image matching. First, $\mathrm{M}(\mathrm{Q}, \mathrm{T})$ exhibits continuity relative to spatial and spectral

Phone : 404-264-8060_Email: asloan@mediabin.com Fax: 404-264-8313 
transformations built from rotation, scaling and translation, i.e., if $\mathrm{S}$ is such a transformation that doesn't rotate, scale or translate much, then $\mathrm{M}(\mathrm{Q}, \mathrm{S}(\mathrm{T}))$ will tend to be close to $\mathrm{M}(\mathrm{Q}, \mathrm{T})$. In addition, if a portion of $\mathrm{Q}$ is similar to a portion of $\mathrm{T}$, then $\mathrm{M}(\mathrm{Q}, \mathrm{T})$ can be relatively large in comparison to $\mathrm{M}\left(\mathrm{Q}^{\prime}, \mathrm{T}\right)$, when $\mathrm{Q}^{\prime}$ is unlike $\mathrm{T}$.

$\mathrm{M}$ can be extended to sets of imagery in a number of useful ways. For example, if $\mathrm{T}=$ $\left\{\mathrm{T}_{1}, \mathrm{~T}_{2}, \ldots \mathrm{T}_{\mathrm{N}}\right\}$ where each $\mathrm{T}_{\mathrm{i}}$ is an similar view of one object, define

(1) $\operatorname{ANY}(Q, T)=\operatorname{maximum}\left\{\mathrm{M}\left(\mathrm{Q}, \mathrm{T}_{\mathrm{i}}\right): \mathrm{I}=1,2, \ldots, \mathrm{N}\right\}$

(2) $\operatorname{ALL}(\mathrm{Q}, \mathrm{T})=\operatorname{minimum}\left\{\mathrm{M}\left(\mathrm{Q}, \mathrm{T}_{\mathrm{i}}\right): \mathrm{I}=1,2, \ldots, \mathrm{N}\right\}$

Increasing the number of images in T representing very different views of an object increases the likelihood that $\operatorname{ANY}(\mathrm{Q}, \mathrm{T})$ will be high when $\mathrm{Q}$ is any view of the object.

Increasing the number of images in T representing very similar views of an object decreases the likelihood that $\operatorname{ALL}(\mathrm{Q}, \mathrm{T})$ will be high when $\mathrm{Q}$ is not a similar view of the same object.

Using WHOLE $(\mathrm{Q}, \mathrm{T})=\operatorname{minimum}\{\mathrm{M}(\mathrm{Q}, \mathrm{T}), \mathrm{M}(\mathrm{T}, \mathrm{Q})\}$ instead of $\mathrm{M}(\mathrm{Q}, \mathrm{T})$ makes it harder to get a high score when $\mathrm{Q}$ contains only a portion of the object completely represented in an image $\mathrm{T}$.

The IQ ImageMatcher ${ }^{\mathrm{TM}}$ software implements these and other related functions.

One application of IQ ImageMatcher ${ }^{\mathrm{TM}}$ is to pre-screening. In a typical pre-screening application, there is a Watch List of important objects and classes of objects, whose presence or absence in Query imagery is important, in varying degrees, to determine. Prescreening is used when the amount of unknown Query imagery exceeds the capacity of available image analysts or security personnel to review. For example, if analysts can only review $10 \%$ of available imagery in a given time frame, then how should those $10 \%$ be selected? Pre-screening selects the imagery most likely to be most important. The continuity mentioned above can be used to direct to collection of a database of Target imagery and the construction of generalizations of $\mathrm{M}$ similar to the examples given above to define a procedure which selects imagery more likely to be representative of important objects and classes of objects.

In applications where a database of Target imagery is available prior to use of software, the database can be analyzed and a more optimal measure can be computed by using maximum and minimum values of $M$ across various subsets of images in the database representing similar instances of a person and different instances of a person.

As a simulation of matching with less than ideal imagery, a FERET thumbnail database of 7249 images was segmented for faces and each segmented face was scaled to 96 pixels in area, while maintaining the aspect ratio of the segmented image. The first image of

Phone : 404-264-8060_Email: asloan@mediabin.com $\quad$ Fax: 404-264-8313 
each of the 695 individuals was used to create 695 Query Sets, each consisting of 1 image. The remaining 6554 images constituted a fixed Target set. ImageMatcher ${ }^{\mathrm{TM}}$ was run 695 times, once for each Query set and the same Target set. We set Success(N) to be the fraction of the 695 Query images for which the set \{those Target images having the $\mathrm{N}$ highest scores \} contained at least one Target image representing the Query image individual and found

$\frac{\text { Image Size }}{" 8 \times 12 "}, \quad \frac{\text { Success }(1)}{.65} \quad \frac{\text { Success(20) }}{.87} \quad \frac{\text { Success }(50)}{.92} \quad \frac{\text { Success }(100)}{.95}$

\section{Modifications for the Face Recognition Test}

The ability to handle image signatures in an XML format was added to comply with Test requirements.

An image segmentation pre-processing module was added to search for the largest object and enclose in a bounding rectangle since the FRVT 2002 test protocol guarantees that the face is the largest object in each image.

IQ Imagematcher ${ }^{\mathrm{TM}}$ has a parameter which determines the output size of an image scaling pre-preprocessing module. This output size can dramatically affect the computation time. We used the each of 256 images in the FERET_query data set to create 256 Query sets and all the 256 images in the FERET_target data set to create a Target set. The FERET_query and FERET_target data sets were supplied in the June HEF supplement. We ran IQ Imagematcher ${ }^{\mathrm{TM}}$ to test the effect of the rescaling the original imagery by segmenting each image for a face and rescaling the segmented images to an area equivalent to the following Image Sizes while attempting to maintain the aspect ratio and found

\begin{tabular}{|c|c|c|c|}
\hline Image Size & $\underline{\text { Success }(1)}$ & $\underline{\operatorname{Success}(20)}$ & $\underline{\operatorname{Success}(50)}$ \\
\hline $6 \times 9$ & .25 & .56 & .71 \\
\hline $8 \times 12$ & .36 & .70 & .78 \\
\hline $12 \times 18$ & .41 & .69 & .78 \\
\hline $16 \times 24$ & .50 & .75 & .82 \\
\hline $24 \times 36$ & .59 & .77 & .85 \\
\hline $32 \times 48$ & .63 & .77 & .86 \\
\hline
\end{tabular}

Here Success $(\mathrm{N})$ is the fraction of the 256 Query images for which the system selected the correct Target image among the set of 256 Target images having the $\mathrm{N}$ highest scores.

Typically, performance would improve with the use of higher resolution images and the improvement changes are smoother as the resolution increases. The 1536 pixel image area, corresponding to $32 \times 48$, was selected to insure that the Test be completed in the specified time limit. The scaling module was invoked to resize each segmented Target

Phone : 404-264-8060_Email: asloan@mediabin.com $\quad$ Fax: 404-264-8313 
and Query image to have 1536 pixels, while maintaining the aspect ratio of the segmented imagery.

The higher resolution original image data provided in the Test was only used to convert to greyscale and to segment. The image matching was done only with the " 32 x 48 " imagery.

In order to reduce computation, rotations were not included in the collection of contractive transformations considered by the software.

Color information was only used to create grayscale imagery and in the segmentation step.

In cases where the signature was a still set or a video only 2 image representatives were used. This number was chosen to control the computation load, so that the Test could be completed within the constraints provided. The video was turned into a still set by selecting every tenth image. The image representatives for each still set were selected to maximize the value of $M(,$,$) when applied to different images in the still set.$

With $T=\left\{T_{1}, T_{2}, \ldots T_{N}\right\}$ and $Q=\left\{Q_{1}, Q_{2}, \ldots, Q_{P}\right\}$ being the selected images, the reported similarity scores are computed as:

Maximum $\left\{\mathrm{M}\left(\mathrm{Q}_{\mathrm{i}}, \mathrm{T}_{\mathrm{j}}\right)\right.$ for $\mathrm{i}=1,2, . ., \mathrm{P}$ and $\left.\mathrm{j}=1,2, \ldots, \mathrm{N}\right\}$

and for the test $\mathrm{N}$ and $\mathrm{M}$ were either 1 or 2 .

\section{System Description}

Hardware

Dell Precision 340 Desktop

2.4 GHz/533 MHz Front Side Bus, P4 Processor, 512 Full Speed Cache

1 GB PC800 @ 400MHz ECC, RAMBUS memory

nVidia Quadro 2 EX, 32 MB Graphics Card

Two 36 GB SCSI U160/M Hard Drives

SCSI Ultra 160/M Controller

3.5" 1.44 Floppy Drive

1394 Controller Card

16x DVD and 24xCDRW

Windows 2000 Pro

Cost $\$ 4,313$

\section{Software}

IQ ImageMatcher - development version, not separately priced.

Phone : 404-264-8060_Email: asloan@mediabin.com $\quad$ Fax: 404-264-8313 


\section{ident}

Identix Corporation

One Exchange Place, Suite 800

Jersey City, New Jersey 07302

Phone: 201-332-9213

Fax: 201-332-9313

http://www.identix.com

E-mail: info@identix.com

\subsection{Overview of Submitted Systems}

\section{Facelt ${ }^{\circledR}$ Technology used in Facial Recognition Vendor Test 2002}

All Identix Corporation face recognition products and solutions are derived from the algorithms in the Facelt ${ }^{\circledR}$ Identification Software Developers Kit (SDK).

\section{THE FACEIT ${ }^{\circledR}$ ENGINE HAS A NUMBER OF CAPABILITIES}

Face Detection Detects faces in static images or video streams, even in complex scenes or backgrounds.

Segmentation Crops, rotates, and re-scales faces out of an image.

Image Quality Evaluates the quality of a captured facial image for recognition.

Faceprint Creation Generates templates as small as 84 bytes.

Face Recognition One-to-one (verification) or one-to-many (identification).

These capabilities are packaged as

1. C++ classes and COM objects, included in the Software Developers Kits (SDKs), and Facelt ${ }^{\circledR}$ add-on software modules.

2. ABIS (Automated Biometric Identification System), a scalable one-to-many search engine and application framework.

3. Argus, a scalable real-time video watchlist system with capabilities to integrate into video surveillance infrastructures.

4. IBM/Informix Datablade.

5. Third-party applications such as the IBM screen saver, the Sony/Keyware screensaver, etc. 


\section{identio}

\section{FACIAL RECOGNITION TECHNOLOGY BENEFITS}

- Accurate

- Passive

- Convenient

- Human Readable Audit Trail

- Non-Invasive

- Uses Existing Photographs and Databases

\section{APPLICATIONS}

\section{Authentication}

Computer and Network Security, Token Replacement

Access Control, Banking, Border Control

\section{Criminal Justice Systems}

Mugshot and Booking Systems

\section{Human ID at a Distance}

Surveillance, Smart CCTV, Human Traffic

Control, Image Indexing and

Post-Event Analysis

\section{Identity Fraud}

Voter Registration, National IDs, Passports, Drivers' Licenses, Employee IDs

Facelt ${ }^{\circledR}$ Face Recognition Technology Airport Application An Overview: Facelt ${ }^{\circledR}$ Access Controlled Points

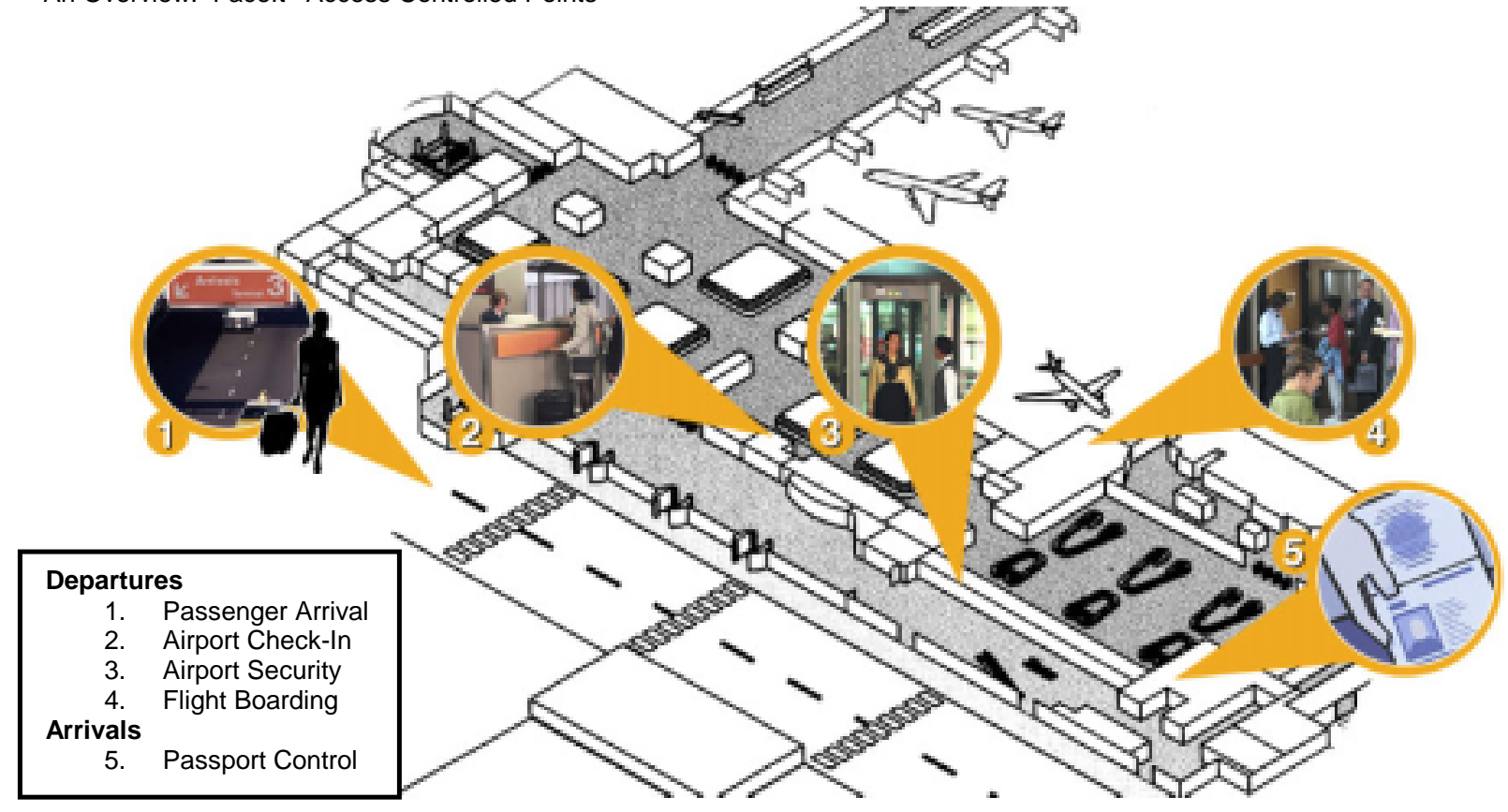




\section{identix}

\section{WORKING WITH FACEIT ${ }^{\circledR}$}

\section{Technology and Pricing}

Facelt ${ }^{\circledR}$ technology is initially provided in the form of software developers kits and modules. Once the solution is completed, a license agreement is then structured in accordance with the application or market segment. Evaluation opportunities are available to pre-qualified entities.

\section{What is Facelt ${ }^{\circledR}$ ?}

An award-winning enabling technology for detecting and recognizing human faces.

Facelt ${ }^{\circledR}$ is used by Identix's business partners to build a variety of finished products and solutions such as mugshot and booking systems, self-service financial kiosks, identity fraud database systems, access control solutions, network security applications and many others.

\section{How It Works}

\section{The Core of the Facelt ${ }^{\circledR}$ Engine: The Local Feature Analysis (LFA) Algorithm}

Fundamental to any facial recognition system is the way in which faces are coded. Initial studies into this field resulted in a global approach, known as eigenfaces. This theory suggests that faces can be synthesized from a set of master global facial templates, called eigenfaces. Combining co-efficients from the various eigenfaces yields a unique identity for every given face, similar to the composition of colors from the primary colors, red, blue and yellow. However, due to the fact that the eigenfaces are global in nature, they do not compensate for real-world variability with respect to lighting, pose, expression, facial hair, etc. Moreover, different sets of global templates apply for different ethnic groups and races, making the results significantly reliant on knowing which set of templates to apply and highly dependent on color and shading.

On the other hand, the approach used by Identix, called Local Feature Analysis (LFA) is based on local features that make up part of the face that differentiate between individuals. These features combine the texture information of a face image with information of the underlying anatomical bone structure. Identity is determined by combining information of these features in a statistically meaningful way. 


\section{dentix}

Because its makeup is based on local features, the LFA faceprint is very resistant to changes in lighting, skin tone, eyeglasses, aging, facial expression, hairstyle, pose, etc.

There are roughly 80 degrees of freedom on the face that differentiate between humans. These are encoded in both the Identix "vector" faceprint, which is 84 bytes in size, and the Identix "Intensive" faceprint, with is $3.5 \mathrm{~K}$ in size.

\section{Application and Installations}

CCTV Town Centers -- Taking a high-tech approach to fighting crime, London's borough of Newham has tied over 300 cameras into a central control room where the Facelt ${ }^{\circledR}$ system identifies known criminals.

Information Security -- IBM provides Facelt ${ }^{\circledR}$ screensaver with all shipments of its snap-on Ultraport camera, designed for the A, T and X series Thinkpad laptops.

Eliminating Voter Fraud -- Facelt ${ }^{\circledR}$ is an integral component of the Mexican Federal Election Institute's on-going program to eliminate duplicate voter registrations.

\section{FACEIT $^{\circledR}$ AT WORK: SELECT PARTNERS}

See how other partners are implementing Facelt ${ }^{\circledR}$ technology at: http://www.identix.com/partners

Our professional services group is also available to assist in developing enhanced Facelt ${ }^{\circledR}$ software modules to meet specific project requirements.

\subsection{Component Lists and Cost Breakdown for Systems}

\section{Medium Computational Intensity Test}

The system configuration used for the Medium Computational Intensity Test (MCINT) consisted of:

\begin{tabular}{|l|r|r|}
\hline Component & Quantity & Cost (U.S. Dollars) \\
\hline $\begin{array}{l}\text { Dell Precision 530 } \\
\text { Workstation Dual 2.4GHz }\end{array}$ & 1 & 4258 \\
\hline Dell Model M782 Monitor & 1 & 229 \\
\hline Identix Facelt & & 10,000 \\
\hline
\end{tabular}




\section{identcu}

Identification SDK

Total Cost

14,487

\section{High Computational Intensity Test}

The system configuration used for the High Computational Intensity Test (HCINT) consisted of:

\begin{tabular}{|l|r|r|}
\hline Component & Quantity & Cost (U.S. Dollars) \\
\hline $\begin{array}{l}\text { IBM xSeries 360 Server } \\
\text { Quad 1.6GHz }\end{array}$ & 3 & 111,870 \\
\hline $\begin{array}{l}\text { APC Smart-UPS } \\
\text { 2200VA }\end{array}$ & 1 & 916 \\
\hline USB 80GB Hard drive & 1 & 200 \\
\hline Ethernet 10/100 Switch & 2 & 84 \\
\hline Cables for USB Drive & 2 & 50 \\
\hline USB PCI Card & 1 & 25 \\
\hline Dell P793 Monitor & 1 & 259 \\
\hline KVM Switch 4-way & 1 & 75 \\
\hline Identix Facelt & & 10,000 \\
Identification SDK & 1 & 123,479 \\
\hline Total Cost & & \\
\hline
\end{tabular}

Note that we do not recommend using Quad servers because of the cost. The same system performance can be achieved using 12 inexpensive rank-mounted single CPU machines at $\$ 1,200$ each. This reduces the cost to roughly $\$ 27,000$.

\subsection{Modifications Specifically for the FRVT 2002 Test}

A test application was written to perform both the medium and high intensity tests. This test application invoked the Face $\mathrm{t}^{\circledR}$ Identification SDK. The test application included the following functionality specifically for the FRVT 2002 test:

- Reading and parsing the FRVT 2002 target and query XML files.

- Crash recovery to recover from power outages or other problems during the test.

- Support for running the face matching on three computers in parallel.

- Writing binary similarity files conforming to the FRVT 2002 Test specifications.

- Displaying status information on the Microsoft Windows console and writing status information periodically to log files. 


\section{ID-2000 System Description - Imagis Technologies Inc.}

\section{System Overview}

ID-2000 uses a deformable surface model to locate a face, normalize it, render it using various light source positions and find values for the pitch, yaw and roll of the head. The modeling allows the face detection to work accurately with an infinite number of face shapes (not just the "universal facial shapes" that are pre-determined by Eigenface-based approaches). Spectral analysis algorithms are then used to transform the detected face object into an encode array. The encode array can then be matched against other encode arrays in a database.

\begin{tabular}{|c|c|}
\hline Platforms Supported & Windows 98 / NT / 2000 / ME/ XP \\
\hline Architectures Supported & Client Server, Application Server, Stand-alone \\
\hline Databases Supported & ODBC/ADO compliant databases such as Oracle, SQL Server, MSAccess. \\
\hline Database Size & $\begin{array}{l}\text { Unlimited. Note: some file based databases such as Microsoft Access } \\
\text { have an inherent upper limit. Access has a size limit of } 1 \mathrm{~GB}\end{array}$ \\
\hline Input & $\begin{array}{l}\text { Accepts any source of visual signals including photographs, live or } \\
\text { recorded video. Also works with artist rendered images. }\end{array}$ \\
\hline Faceprint Size & 278 bytes. (269 MB of RAM per million records). \\
\hline Speed & $\begin{array}{l}\text { Calculations based on a single } 1.7 \mathrm{GHz} \text { Intel Pentium } 4 \text { processor with } \\
512 \mathrm{MB} \text { of RAM (1 Million records requires } 269 \mathrm{MB} \text { of available RAM). } \\
\text { Face finding and encoding using a } 200 \times 250 \text { image: } 500-800 \\
\text { milliseconds. One-to-many matching: } 60 \text { million per minute. The ID-2000 } \\
\text { Server is multi-threaded so that it can respond to an unlimited number of } \\
\text { simultaneous requests and so that it can fully utilize multiple processor } \\
\text { machines. }\end{array}$ \\
\hline Live Video Capture & $\begin{array}{l}\text { Video capture is implemented through Microsoft Video for Windows } \\
\text { (VFW). Any VFW compatible image capture system is all that is required } \\
\text { for live video capture. The ImgWatch control provides a complete live } \\
\text { video face recognition solution. Separate Authentication and Identification } \\
\text { modes (as well as motion detection and scene verification modes). } \\
\text { Images (or sequences of images) can be captured using the VFW capture } \\
\text { window provided with the client ActiveX control. }\end{array}$ \\
\hline Pose & $\begin{array}{l}\text { ID-2000 finds faces where both eyes are visible, typically up to } 25 \\
\text { degrees from frontal. Forthcoming releases of ID-2000 will address } \\
\text { greater angles with yaw, tilt and roll. }\end{array}$ \\
\hline Eyeglasses & $\begin{array}{l}\text { Can work with eyeglasses provided the eyes are visible and not occluded } \\
\text { by dark tints or glare. Thick frames may adversely affect search results. }\end{array}$ \\
\hline Lighting & $\begin{array}{l}\text { Does not require special lighting or background. Optimal performance is } \\
\text { achieved in diffuse ambient lighting. In addition, performance is best if the } \\
\text { subject is not back-lit, although this can be compensated for with gain } \\
\text { control on the video camera. ID-2000. }\end{array}$ \\
\hline
\end{tabular}




\begin{tabular}{|l|l|}
\hline Background & $\begin{array}{l}\text { Finds faces in any background, plain or cluttered. Recognition is } \\
\text { independent of background. Face recognition from live video is } \\
\text { independent of background motion and / or camera motion }\end{array}$ \\
\hline $\begin{array}{l}\text { Image Color, Depth and } \\
\text { Resolution }\end{array}$ & $\begin{array}{l}\text { Functions with equal performance on color or gray scale images. } \\
\text { Requires minimum 8-bit image depth. }\end{array}$ \\
\hline Tracking & $\begin{array}{l}\text { Tracks a face over time while encoding and querying the database for } \\
\text { matches }\end{array}$ \\
\hline Segmentation & $\begin{array}{l}\text { Crops faces from background } \\
\text { Watch Mode enables a face in a video stream to be captured, encoded, }\end{array}$ \\
\hline Surveillance & \\
\hline
\end{tabular}

ID-2000 is Imagis' proprietary face recognition software technology. ID-2000 is a full ActiveX control and Server Service Executable developed with Microsoft Visual C++ 6.0 and ATL 3.0. The control is insertable and is functional with the majority of clients, including (but not limited to) VBA, VB, C++, Delphi, Internet Explorer and Netscape web browsers. The Server is an NT Service (or Tray Application on Win9X) that runs as a background process on the same machine as the Server Database is located.

The Client / Server architecture is shown in the diagram below:

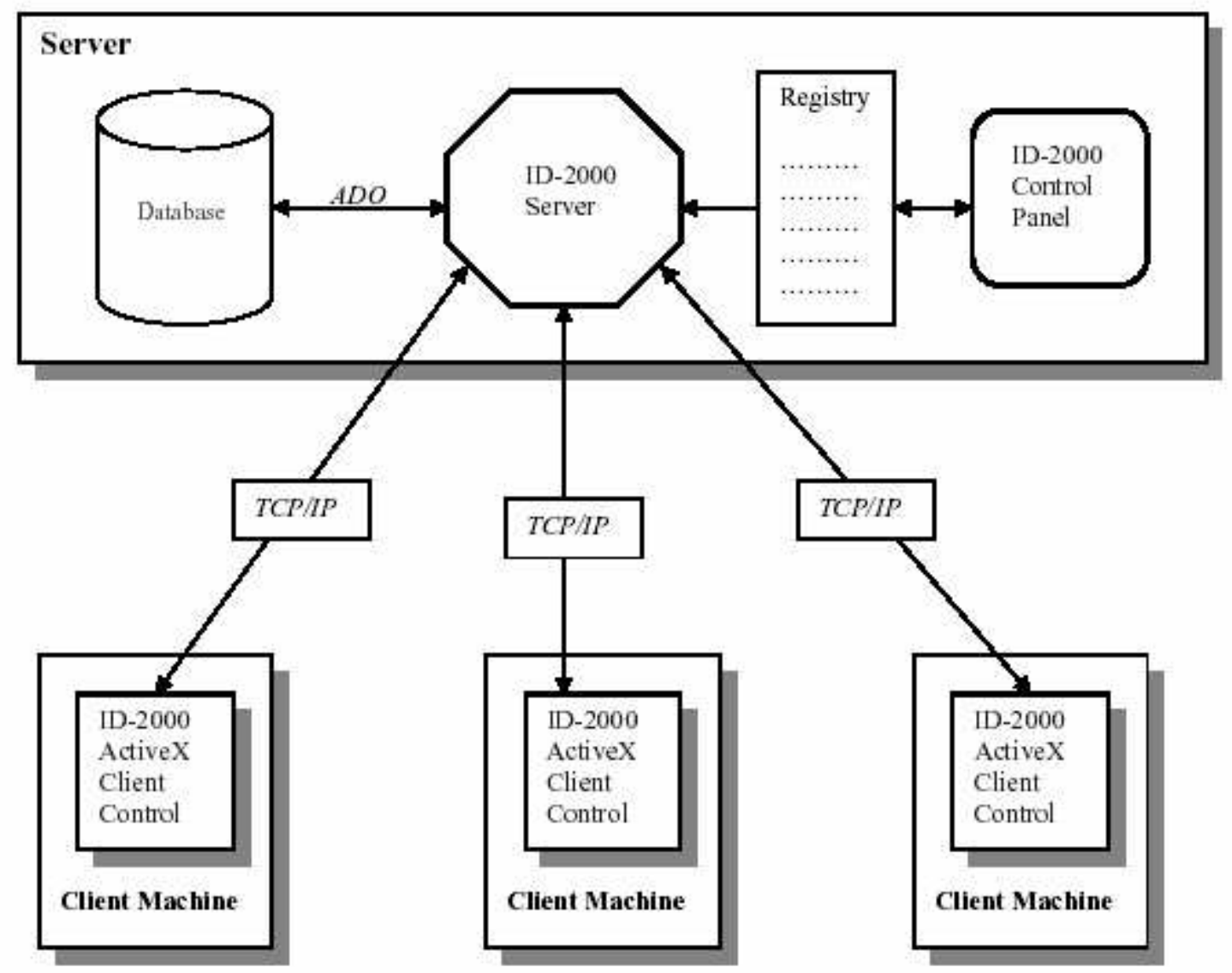


The ID-2000 SDK is modeled after the client-server architecture. An ID-2000 client connects to an ID-2000 server and asks the server to do something such as find a person or encode a face. The server fulfills the request and sends the results back to the client. Under the client-server architecture, the server, in response to client requests performs the majority of processing. The client-server architecture provides the following benefits:

- An ID-2000 administrator is free to place ID-2000 servers wherever he or she wishes, with little regard to the location of client. The server can be located in the same room as a client or in another room on an entirely different continent. The location of the server is normally a well-kept secret.

- Because the server performs the majority of work in a client-server application, to increase client's responsiveness, it is often sufficient to increase the speed of the computer hosting the ID-2000 server.

- Client-server applications are ideal for use in low-bandwidth environments such as wireless mobile applications. It requires only a few hundred bytes of information exchanged to ask an ID-2000 server to find an individual. Client applications never talk to the ID-2000 server directly using TCP/IP sockets or other low-level communication primitives. Instead, they call methods and examine properties on the ID-2000 client control.

The ID-2000 server can use data in MS-Access, SQL Server 7+, Oracle 8+ and most ODBC compatible databases. The only requirements are:

- Records in the database be identified by a numeric primary key

- That the images managed by the database be in JPEG format

- That the server have write access to a single BLOB field to store the image encoding.

Pictures can be stored externally to the database in separate files, or stored in the database as binary large objects (BLOBs). Encodings must be stored in BLOB fields and be one of the following data types:

- Database Encode Array Data Type

- Microsoft Access OLE Object

- SQL Server 7+ Image

- Oracle 8+ BLOB

The ID-2000 server is implemented as a Windows NT service that uses encrypted TCP/IP as its transport. ID-2000 clients connect to the ID-2000 server on an administrator-defined protocol port number. The protocol port number defines the ID-2000 database available to the ID-2000 client. It is possible to set up different ID-2000 Servers on different protocol ports. For example, arrest and booking system may wish to keep its adult database separate from its juvenile database. To accomplish this, an ID-2000 administrator would set up two databases, each on a separate protocol port. This way, there is no chance that a juvenile would be returned inadvertently on a search of an adult database.

An ID-2000 server may be deployed where access to the database must be controlled. For this reason, a number of security features are available. The ID-2000 server administrator can restrict access to the ID-2000 server by username and password, by Internet address or both. For example, it is possible limit access to the ID-2000 server to John, Mary, Bob, James, and Darryl from computers whose Internet addresses are in the range of (142.104.6.5 .. 142.104.6.15). 
Unauthorized attempts to access the server are logged in the ID-2000 event log that the ID-2000 server administrator can examine periodically.

\section{Component List}

\section{a) Medium Computational Intensity Test}

Hardware

- DELL Optiplex (Single Processor)

- Intel Pentium 4 Processor at $1.7 \mathrm{GHz}$

- 512 MB SDRAM

- 20GB 7200 RPM Hard Drive

- Cost - $\$ 1600$ US

\section{Software}

- ID-2000 Server Service $\$ 25,000$ US

- ID-2000 ActiveX Client Control (for static images) $\$ 500-\$ 3500$ US per client depending on number of concurrent connections required. Note: Only one concurrent connection was used in the Medium Computational Intensity Test

- ImgWatch ActiveX Client Control (for live video) $\$ 500-\$ 3500$ US per user depending on number of concurrent connections required. Note: Video data for the test was provided using previously captured frame clips, therefore this control was not used.

b) High Computational Intensity Test

Hardware

- DELL Optiplex (Single Processor)

- Intel Pentium 4 Processor at $2.2 \mathrm{GHz}$

- 1024 MB SDRAM

- 80GB 7200 RPM Hard Drive

- Cost - $\$ 2200$ US

Software

- ID-2000 Server Service $\$ 25,000$ US

- ID-2000 ActiveX Client Control (for static images) $\$ 500-\$ 3500$ US per client depending on number of concurrent connections required. Note: Only one concurrent connection was used in the High Computational Intensity Test

- ImgWatch ActiveX Client Control (for live video) $\$ 500-\$ 3500$ US per user depending on number of concurrent connections required. Note: Video data for the test was provided using previously captured frame clips, therefore this control was not used.

\section{Modifications for FRVT 2002}

The system used in FRVT 2002 contains some improvements to the face fitting routines. 


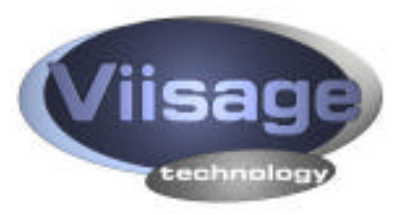

\subsection{Viisage Technology Core Facial Recognition Technology}

The facial recognition technology developed at the Massachusetts Institute of Technology (MIT) and exclusively used by Viisage Technology employs "Eigen faces", which are characteristics of a person's face, and maps the facial image into a multi-dimensional face space. Using special techniques developed by Viisage the Eigen faces are used to provide high speed facial matching to one or many candidate faces in a database.

\section{Figure 1.1: Eigen Faces}

The multidimensional Eigen space itself is determined through a separate process at Viisage Technology that is typically done only once. This process begins with a large diverse population of thousands of facial images. For each of these images, the head and eyes are located; the image is standardized, and then masked. The resulting ensemble of localized, standardized and masked images

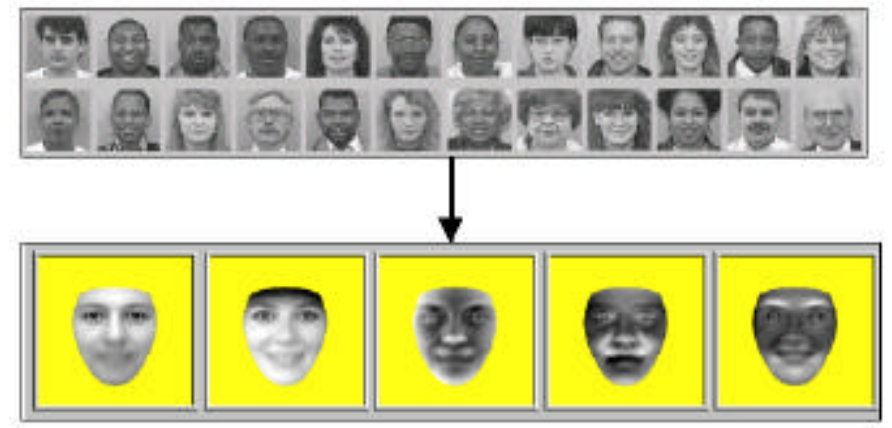
are then processed with a mathematical technique called Principal Components Analysis. The result of Principal Components Analysis a set of face-like images called Eigen faces. Each Eigen face is mathematically independent/orthogonal to all others, and is an independent degree of freedom for describing faces. In other words, these face-like images are the most efficient set of building blocks needed to build any face. Figure 1.1 shows the first five Eigen faces that result when Principal Components Analysis is performed on a large sample of face images.

After standardization and masking, the image is projected into the multidimensional Eigen space of facial recognition as shown in Figure 1.2. The result of this projection of the facial image onto the Eigen face templates is a set of Eigen coefficients, which together form an Eigen vector. The multidimensional Eigen space is constructed of 128 mathematically orthogonal coordinates and each coordinate is representative of a single characteristic Eigen face.

The first coefficient of the image being enrolled is calculated through the projection of that image onto the primary Eigen space coordinate, which is also referred to as the average Eigen face. Once determined, the first coordinate projection is subtracted from the original image in order to produce a residual image. This residual image is then projected onto the second designated

Figure 1.2: Face Space

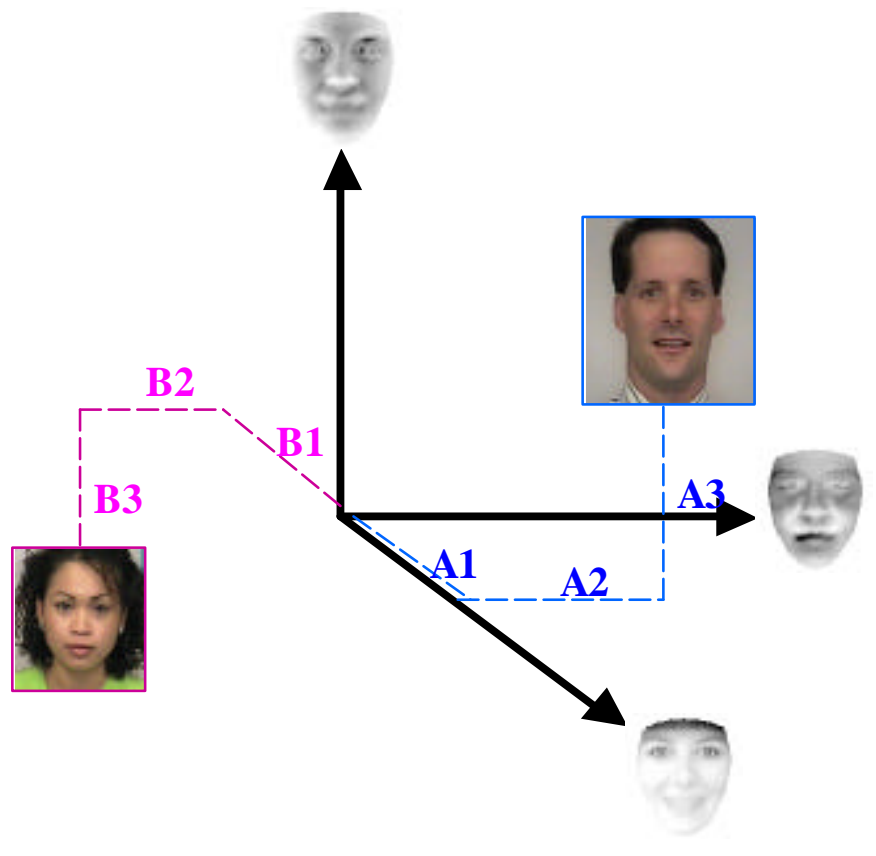




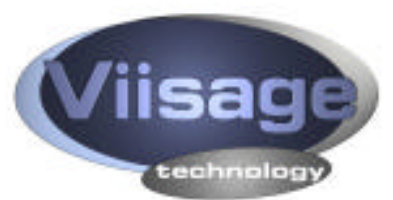

Eigen space coordinate and thus the second coefficient is obtained. Then the second projection is subtracted from the previous residual image in order to produce a new residual image. Successively, each new residual image is projected onto the next coordinate. Each projection subtracted produces a further deconstructed residual image.

This process of projecting the resulting residual image onto each coordinate produces a total of 128 characteristic Eigen coefficients. Together this set of characteristic coefficients represents a complete vector projection in the facial recognition Eigen space. The combination of these 128 coefficients with respect to their corresponding Eigen face images produces a reconstructed masked image that can be viewed and verified as being visually very similar to the original masked image.

The captured or scanned digital image is converted to Eigen coefficients (simply described as facial features) and submitted to the Facial Recognition Search Engine, which returns results in real-time to the requesting client. These results comprise the closest matches found for the individual. They are ranked in order with the closest match shown first.
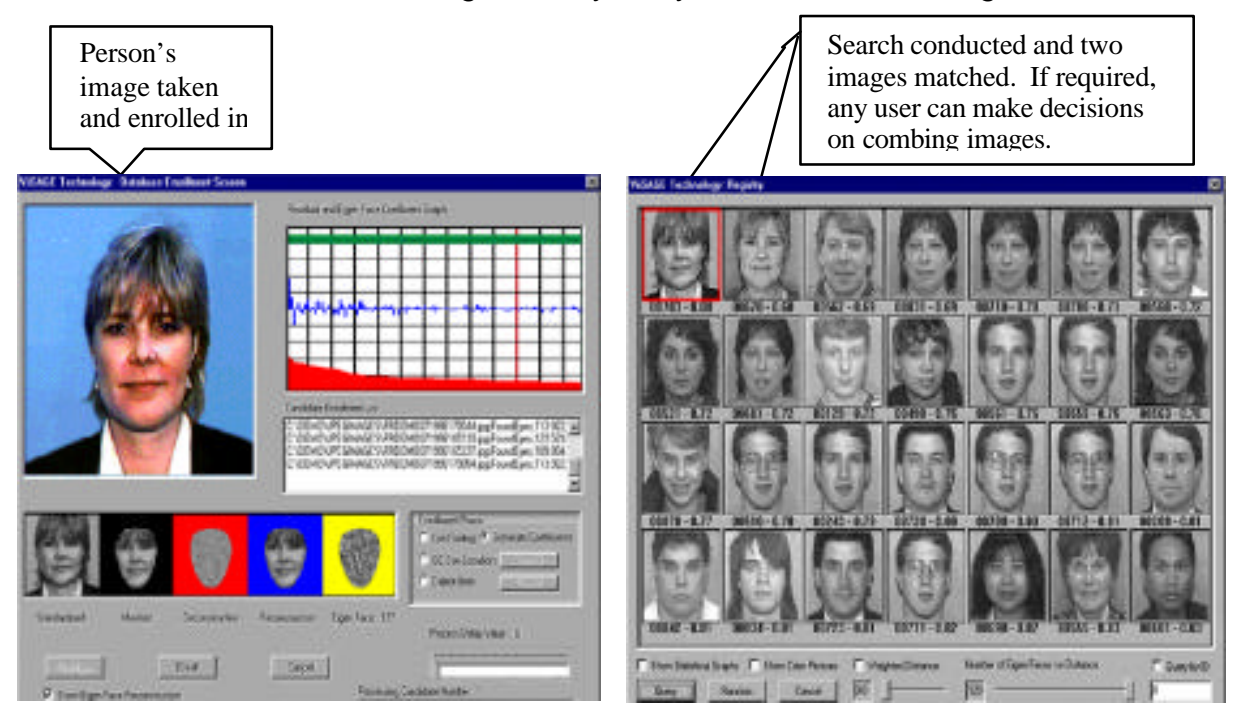

\subsection{The Viisage Technology Facial Recognition Software Development Kit}

Viisage Technology has developed its facial recognition technology into a SDK that provides the fundamental facial recognition algorithms for development of applications that perform facial recognition. This FRSDK is used internally by Viisage Technology to produce surveillance, access control, and facial database search applications for its customers. The FRSDK itself is a product that empowers its users to provide state-of-the-art facial recognition in their own applications.

\subsection{The Viisage Technology FRVT2002 Software}

The facial recognition applications used by Viisage Technology in the FRVT2002 test are based on our FaceEXPLORER ${ }^{\mathrm{TM}}$. These applications consist of an automatic enrollment application and an automatic matching application. The enrollment application is customized to process the FRVT2002 XML scripts to enroll the images into an Oracle based facial recognition database. The matching application is customized to perform the comparisons specified in XML, using the data in the Oracle database, and write the results in files as specified in the XML scripts.

\subsection{Component List}

\subsection{Medium Computational Intensity \\ Dell WorkStation 340}


2.53GHz Pentium 4

1 GB ECC memory

80 GB ATA-100 IDE Hard drive

Microsoft Windows 2000 Professional operating system

CD-ROM, and CD-RW drives

Price: $\$ 2507$

\subsection{High Computational Intensity}

3 Dell WorkStation 530

Xeon $2.4 \mathrm{GHz}$ processor

1 GB ECC Memory

80 GB ATA-100 IDE Hard drive

Microsoft Windows 2000 Professional operating system

CD-ROM drive

Price: $\$ 4615$ dual CPU.

13 Com 8 port switch

Price: $\$ 125$

\subsection{Software}

FaceEXPLORER ${ }^{\mathrm{TM}}$ : typically Viisage Technology provides a custom quote. Pricing is complex and requires knowledge of the legacy operating systems in which one plans to convert prior database images as well as the manner in which the facial recognition technology may be used.

Our FaceEXPLORER ${ }^{\mathrm{TM}}$ system normally is a networked solution and not sold on a stand-alone basis. The product is shipped and licenses provided either per computer or per image it is application dependent. A baseline system would require, at a minimum, one software key, which would be priced $\$ 10,000$.

FaceEXPLORER ${ }^{\mathrm{TM}}$ typically runs on Windows 2000 , NT or XP platform. Viisage Technology would prefer to suggest the recommended components that would be integrated into a system such as cameras, lighting, backdrops,

framegrabbers, databases, signature capture devices, printers and consumables. 


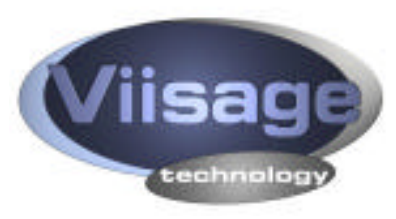

\section{Viisage can search a database containing millions of images and find a match in seconds.}

\section{FaceEXPLORER $^{\mathrm{TM}} \cdot$ Identity Fraud Prevention}

Viisage's FaceEXPLORER ${ }^{\mathrm{TM}}$ applications provide a real answer to the exploding problem of identity theft and fraud. Identity theft is one of the fastest growing crimes in America, claiming 1.7 million victims in 2001. Viisage has deployed the world's largest face recognition application, supporting a database containing ten million images, growing by 15,000 images a day, where State law enforcement officials maintain the integrity of their drivers' license database by decreasing the ability for individuals to fraudulently obtain multiple credentials under different names. Applications also include law enforcement booking and investigative systems, voter registration, social services benefits, weapons permits and immigration programs all of which reduce identity fraud. The technology searches millions of images in seconds - no competitor can match Viisage's performance in size, scope and functionality. 


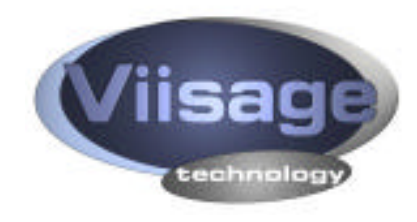

"Viisage's system has met and exceeded our expectations, resulting in the need for us to expand our current licenses. The level of support we have received from Viisage confirms that we have partnered with the right company."

\section{A. Muwonge \\ Uganda Secretary of the \\ Electoral Commission}
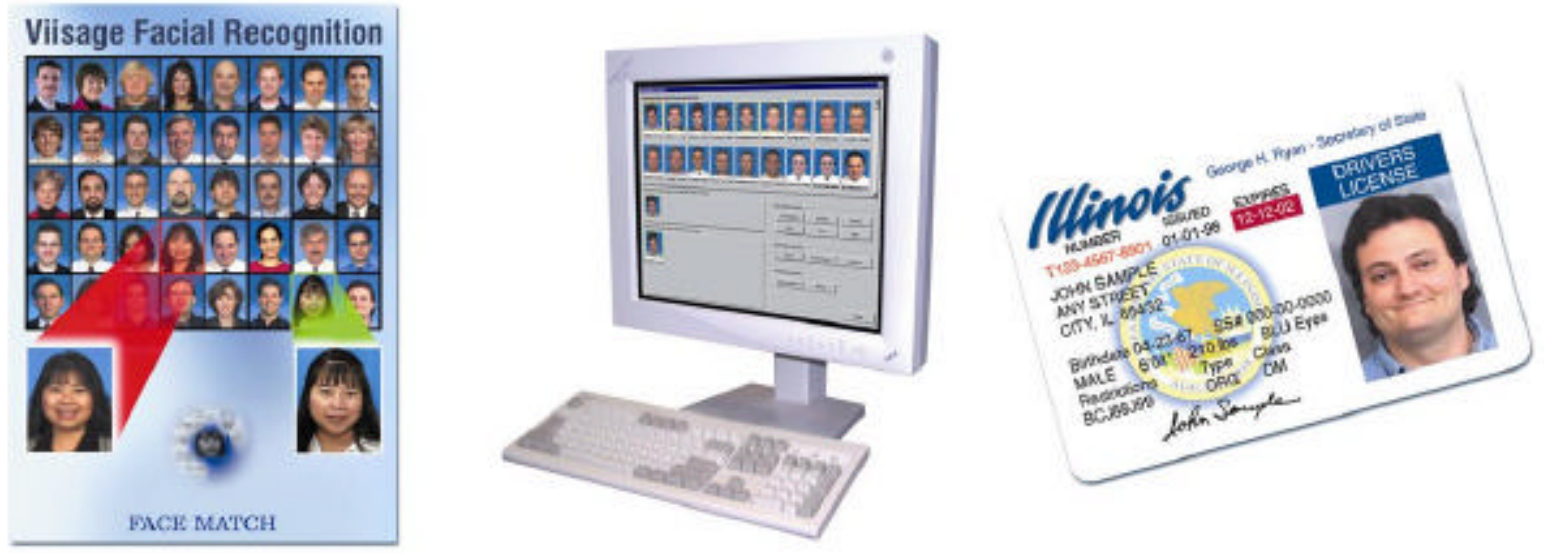


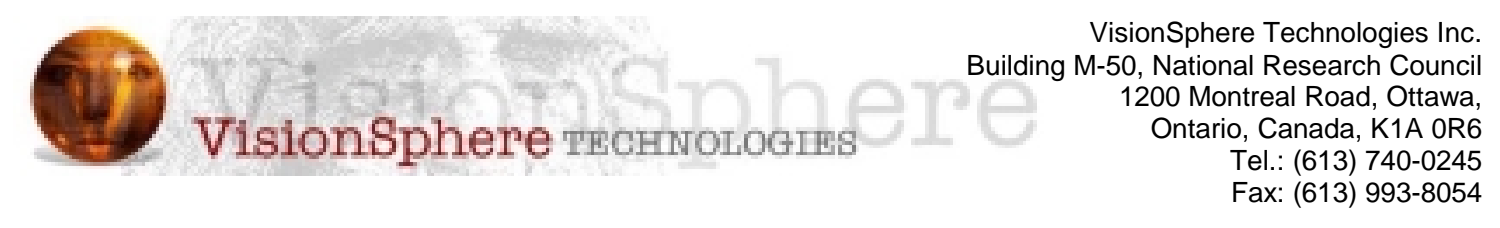

\section{Overview of VisionSphere's UnMask Technology}

\section{How does UnMask work?}

VisionSphere's products use algorithms and techniques based and developed on more than 30 years of research experience by Dr. Martin Levine of McGill University, the Chief Scientist of VisionSphere Technologies Inc.

Every face verification or recognition system must rely on the same three steps:

- Face detection and location of key features

- Extraction of facial descriptors

- Comparison of extracted information to a database

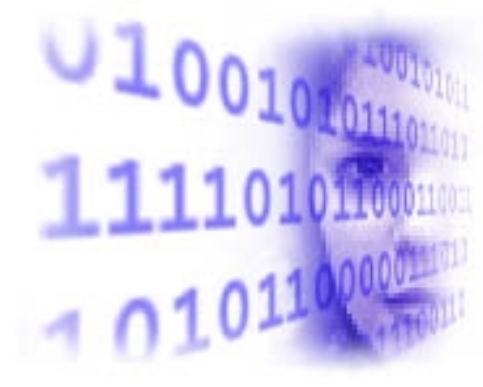

The first component of the system, which locates the face and its key features, determines the robustness of the system. The more advanced the face detection algorithms, the more invariant the system is to changes in head rotation, colour, brightness, hairstyle and facial expression. As a result, the face location and feature-finder components effectively determine how useful the face recognition system will be in the final application.

With the goal of constructing the most reliable and robust face recognition system possible, VisionSphere's UnMask takes the simplest and most effective approach to face processing. Initially, it locates the face and the eyes in the image automatically, using proprietary search algorithms. Next, the software normalizes and crops the image to provide excellent invariance to variations in lighting, head rotation, facial expression, and hairstyle. The image has now been made comparable to other face images and is ready to be analysed.

The second component of the system, which extracts the required information needed for comparing face images, determines the ultimate accuracy of the face recognition system. This stage computes a unique facial code for each individual.

For the second stage, VisionSphere's software uses a unique Holistic Feature Code (HFC). This provides a strong discriminating capability for comparing faces with very high confidence rates at fast processing speeds.

The third component of the system compares the coded information with data stored in a database. The percentage of false positives and false negatives determines the performance of this component of the system. Databases of face images that are used for law enforcement typically contain large numbers of images. For this reason, the speed and accuracy of the comparison system is critical to the usefulness of any face recognition system.

For the third stage, VisionSphere's software compares the two faces using a proprietary distance function. This function stresses the significant differences between faces and is another important factor in the system's high confidence rate and excellent speed 
Our product line is currently composed of three separate but related products:

UnMask Plus is a software Al (artificial intelligence) system for identification and removal of duplicate or multiple images from large photoID databases.

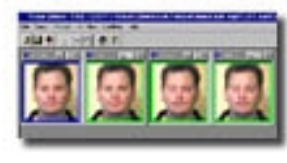

It's $\mathbf{M e}$-- an advanced automatic computer logon authentication system. The system hardware and software components cannot only be used to verify the identity of a network or workstation user at logon time, they can also be used as part of e-Business verification, video conferencing, etc.

FaceCam -- VisionSphere's layered, biometric, user-verification terminal, designed and built specially for secure access applications, such as physical access control, time and attendance and registration systems

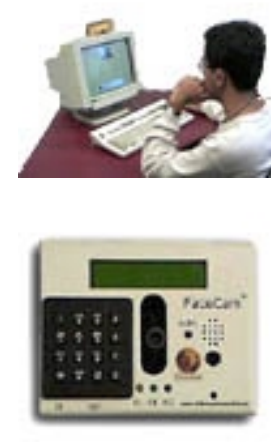

\section{Face Recognition Vendor Test 2002}

For the FRVT2002, two custom console-based test application written with our face recognition SDK were used for the Medium and the High Computational Intensity Tests. In general, the test application first locates the eye locations of all the images in the gallery set and the test set. Then, one face template is generated from each image in both sets. Next, it performs the matches between the face templates from the gallery set and the test set. Finally, the matching results were written in the form of similarity files according to the FRVT2002 specifications.

\section{Component list}

- Applications built using Visionsphere Technologies face recognition Software Developers Kit.

- One (1) computer for the Medium Computation Intensity Test (a single Pentium $41.7 \mathrm{GHz}$ processor with 512Mb PC133 SDRam with a 60GB Western Digital Hard Disk)

- Three (3) computers for the High Computation Intensity Test (Dual Pentium Xeon 2.0GHz processors with 1GB DDRRam). Both used a 80GB Ultra ATA-100 (7200 RPM) hard drive and ran Windows 2000 Professional with Service Pack 2. Three high-end computers were used for the High Computational Intensity Test because of the nature of this test (finding the precise location of faces in 121589 images and performing nearly $15,000,000,000$ comparisons in 264 hours).

\section{Cost of the systems used in FRVT 2002}

FRVT Medium Computational Intensity Test

One computer with a single Intel Pentium $41.7 \mathrm{GHz}$

Can $\$ 1430$

processor and 512Mb Ram

Unmask Identification SDK

Call Sales

FRVT High Computational Intensity Test

Three computers with dual Xeon 2.0GHz processors and 1024Mb Ram Unmask Identification SDK

Can $\$ 3,700$ each

Call Sales 


\section{This page intentionally blank.}




\section{Appendix N}

\section{Participant Comments on FRVT 2002: Evaluation Report}

FRVT 2002 Participants were provided a pre-release copy of the FRVT 2002: Evaluation Report and FRVT 2002: Technical Appendices on 1 March 2003. They were invited to submit a 6-page (maximum) document that serves as their "position paper" on the evaluation and the results. The deadline for submission of these papers was noon EST on 10 March 2003.

Participant documents are the opinions of the Participants and are provided in this text for reference. Inclusion of these documents does NOT imply that FRVT 2002 Authors/Sponsors/Supporters agree with any statements or derivative results within those documents. The identification of any commercial product or trade name does not imply endorsement or recommendation by the National Institute of Standards and Technology or any other FRVT 2002 Author/Sponsor/Supporter. 


\section{AcSys Biometrics Corp $\quad \begin{aligned} & \text { AcSys Biometrics Corp } \\ & 399 \text { Pearl Street }\end{aligned}$

\section{FRVT 2002 -AcSys Vendor Comments}

AcSys Biometrics Corp 


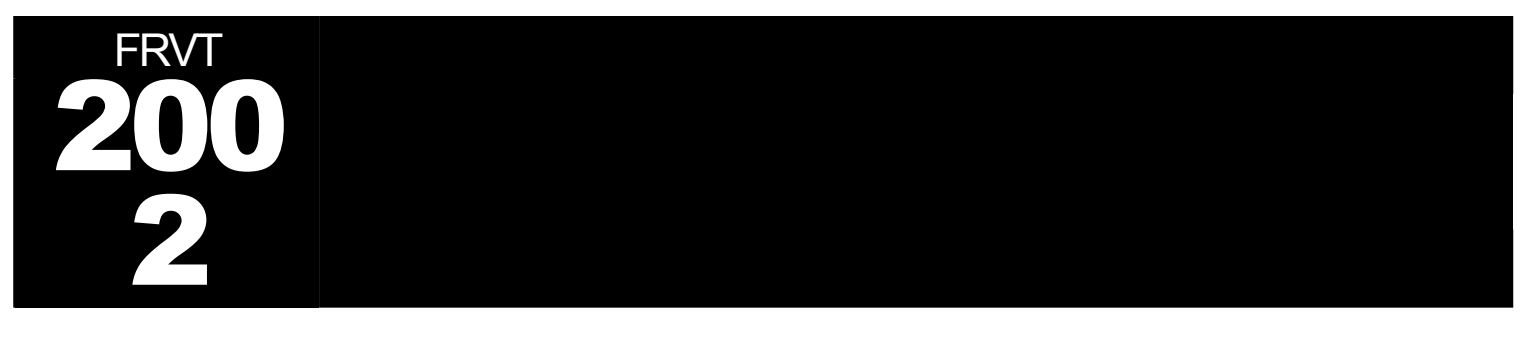

\section{AcSys Biometrics Corp}

\section{Executive Summary}

AcSys Biometrics Corp would like to thank the members of the FRVT 2002 testing group for allowing AcSys to participate in this review and evaluation of facial recognition technologies.

AcSys Biometrics participated in this evaluation utilizing a pure proof of concept application based on of our Facial Recognition Software (FRS) engine. This engine was developed for physical and logical access control using live video during facial identification and enrollment. Our participation in this test allowed us to gather information about testing procedures for static image (2D) comparison, gain access to large 2D databases for the concept application and gather performance information that would be used in developing a commercial application for the 2D image market segment.

The performance results received from this test matched our expectations considering that the AcSys application, while still in its infancy, has been designed from the perspective of operation from continuous video feed, rather than static 2D images. With the information gathered from this evaluation and the recent FRS engine enhancements, the new AcSys $2 \mathrm{D}$ product that is being produced will we expect to substantially exceed the performance results of this test.

On the following pages we will address these issues;

1) Review of testing procedures

2) Product Status at time of evaluation

3) Obstacles for the AcSys software within the evaluation

4) Reasons for entering only the Medium Computational test

5) AcSys Face Recognition engine improvements since test

6) Information on How AcSys FRS works

7) Summary 


\section{1) Review of Testing Procedures}

The testing procedure was setup to enroll multiple images of the same person using different identity tags. The same dataset is used as both target and query set. This is an understandable way of doing the test because several statistics can be calculated from the same enrollment. This does give the AcSys 1.6 engine a disadvantage because the system as configured expects enrolled images of a person to have the same identity tag.

The AcSys FRS system is based upon machine learning, which operates by countertraining images of the individual against images of different but similar looking individuals. The result of having different ID tags for the same individual inhibits the operation of our system as it results in images of the same individual being used in both the training and counter training sets during generation of the biometric template.

For the MCInt - Still Images dataset, there are 9 images of each person containing different identity tags.

\section{2) Product status at time of Evaluation}

When AcSys was asked to join the FRVT 2002 testing the current Face Recognition engine was AcSys FRS version 1.6. This engine was developed and used for physical or logical access control using live video feed. Enrollment was done by capturing 30-100 pictures in a fully automated enrollment process while moving towards the camera over several feet, in order to capture significant variations in head pose and ambient lighting.

The application of matching static picture (2D) facial images (versus continuous video feed) had not been attempted by us prior to this test. At the time of the test, the AcSys system analyzed gray scale facial images extracted at a $32 \times 32$ pixel resolution. The extracted facial image is processed through a mapping function and does not measure specific features. The product employed in this test was designed for low pixel resolution video feed with the user typically at a distance of from 5 to 20 feet from the camera $(\sim 20$ pixels between the eyes).

\section{3) Obstacles for the AcSys software within FRVT}

FRVT 2002 Evaluation Protocol - Rule 2

"To be able to compute performance for multiple galleries and probe sets requires that multiple signatures of a person are placed in both the target and query sets. This leads to the second rule: Each signature in the target and query sets is considered to contain a unique face. In practice, this rule is enforced by giving every signature in the target and query sets a unique random identifier."

"Each signature in the target and query sets is considered to contain a unique face." The faces are not actually unique, but they are considered unique. 
This way of testing creates a problem for the AcSys 1.6 engine in the way that facial biometric templates are generated.

The AcSys 1.6 engine is based upon machine learning, and as such counter-trains images of an individual against images of other similar individuals within the database, iteratively refining this separation of faces. In the FRVT 2002 test there existed several pictures of the same individual, containing different identities. This causes the AcSys 1.6 engine to counter-train the same individual against images of that same individual, thus inhibiting system performance.

\section{4) Reasons for entering only Medium Computational}

Our business at that time was focused solely on physical and logical access based upon continuous video feed. No development effort had been expended on analysis and use of high-resolution static images. Current developments are underway oriented towards this application area. AcSys decided on entering the MCInt test to focus on gathering information about testing procedures that would allow us to improve the engine accuracy for single picture enrollment. Our next step will be to deploy a FRS solution the High Computational tests utilizing some of the information gathering in the round.

\section{5) AcSys Face Recognition engine improvements since test}

Changes to the AcSys FRS 2D and multi-dimensional image software where already underway at the time this test took place and more will be added based on some of the information gathered in FRVT 2002.

We are integrating the following improvements into the AcSys FRS engine.

- $\quad$ Automated 3D morphing of static 2D images

- Increased resolution of pixel array for facial image analysis

- Application of N-tuple logic to analyze and classify distinct facial regions. The approach employed at the time of this test analyzed a single region of the face.

- Optimization of FRS algorithm for high-resolution static picture (2D) facial image recognition.

- Optimized processing of assembly templates to achieve pattern matching rates of 500,000 images/sec.

\section{6) Overview of How the AcSys Face software works.}

The AcSys Facial Recognition system is based upon a proprietary machine learning approach that uses non-linear complex domain analysis. Logical units referred to as "cell assemblies" are applied to separate tasks such as facial tracking, determination of head orientation and facial identification.

The face-tracking cell is trained using a database of up to several thousand facial images 
and the neural network component applies a synaptic pruning and regrowth operation to optimize the selection of inputs and adjust complex "weights". Inputs to the cell assemblies are simply the raw image or frequency domain coefficients of the raw image, with prior normalization applied. The learning process used in this test required a minimum of 100 synaptic pruning and regrowth cycles, or approximately 10 seconds of processing.

The process is generic in that, same cell assembly structures are used to perform the various recognition tasks i.e. facial tracking, classification, etc.

\section{7) Summary}

Acsys Biometrics core facial engine is based on an advanced learning solution that can be modified or can train and learn independently to compensate for new variables submitted to the system. Having said that, the Acsys FRS system performed well considering no new training solutions or modifications were made to the beta test system. If Acsys would have been allowed to submit the images with the appropriate ID tags, and had the systems run the appropriate training routine on those images, the AcSys FRS solution would have produced substantially higher results.

The FRVT 2002 was a very comprehensive evaluation of facial recognition technologies and AcSys was pleased to take part, and AcSys will also offer its new products for further evaluation for the FRVT group due to the unique nature of our solution. This will allow the people who test face applications to gain a better understanding of the true strengths of the AcSys FRS engine, and allow AcSys to continue to enhance its security solutions.

AcSys, like most technology companies, will continually upgrade and modify our solutions to meet the ever-changing needs of the environments they are being deployed in. This test has allowed AcSys to understand another type of testing routine that is being developed to evaluate facial recognition software and we will continue to train our product to achieve the highest results in all of the possible testing environments currently being developed around the world. 


\title{
Comments on the FRVT 2002 Report
}

\author{
Cognitec Systems GmbH \\ An der Flutrinne 12 \\ D-01139 Dresden \\ Germany
}

\section{Summary}

Cognitec was invited to participate in the FRVT 2002 in April 2002. We took this opportunity and provided tools based on the then latest state of our FaceVACS technology. We are very pleased with Cognitec's performance compared to the other participants. These excellent results confirm that our technology is second to none.

The FRVT 2002 report presents many important results relevant to the practical use of face recognition systems. One issue, however, could have been treated in a better way for a meaningful comparison of technologies, and this would have demonstrated Cognitec's superb comparative performance even more clearly. While the reported identification performance allows the direct comparability between the participants, this is not the case for verification and watch list experiments due to the fact that the non-normalized performances are not compared directly in most of the figures. The performance scores for the participants who provided normalization functions are 'optimized' for the gallery, while the scores for the participants that did not were achieved making no assumptions about the data. We strongly believe that an evaluation based primarily on the non-normalized scores is more appropriate for real-world problems.

Cognitec did not submit normalization functions to FRVT 2002 even knowing that normalization does improve our performance under certain conditions. Based on the organizers' announcement that "FRVT 2002 will report both normalized and non-normalized performance scores" (see Appendix J, section 3), we assumed that the non-normalized scores would also be presented for all participants. Under this assumption we saw no need whatsoever to submit normalization functions for two reasons. Firstly, we think that normalization should not be regarded as being part of core face recognition technology. In our opinion, it is just a way of optimizing the results for a specific experiment but does not tackle the classical pattern recognition problem that the learned parameters of some function do not 'generalize' properly. This becomes more evident on noticing that it is possible to define effective normalization functions independent of the classification task. Secondly, the applicability of normalization functions in real-world scenarios is limited.

We indeed used normalization in the past. With normalized scores, Cognitec's performance in the verification and watch list experiments would have been significantly higher. In the following section we show the results we have obtained on the public FERET database as an example.

Aside from our critical remarks, we strongly believe that FRVT 2002 is a major milestone in the field of biometric evaluation technology. The FRVT 2002 report shows the improvements achieved in the last years as well as the weaknesses and the directions where future research is needed.

In the next section, we comment on Cognitec's performance results. After a deeper discussion of the normalization issue, we go into the results of the HClnt and MCInt tests in more detail. We also provide data on the hardware used and on the time spent for those tests. Section 3 lists some improvements of Cognitec's FaceVACS technology that we achieved since the time of the FRVT 2002, both in recognition performance and in processing speed. We close with some concluding remarks in section 4. 


\section{Comments on the Performance Results}

The testing protocol implemented by FRVT 2002 addressed the evaluation of state-of-the-art facial recognition technology for different use cases and applications. The high computational intensity test was the most important test of FRVT 2002 as it measured the performance on 121,589 images of more than 37,000 persons.

Some of the tests aimed at the impact of three techniques for improving the performance: three-dimensional morphable models, normalization of similarity scores, and the use of video sequences for face recognition.

\subsection{Normalization vs. Non-normalization}

At first, the organizers considered normalization a post-processing technique for improving performance. Unfortunately, the presentation of some performance results mixes the normalized scores of some participants with the non-normalized scores of others.

Cognitec was among the participants that did not submit normalization functions even knowing that they improve the performance under very specific conditions. This is confirmed by several experiments we conducted. The impact of our normalization function for our algorithm is shown in figure 1.

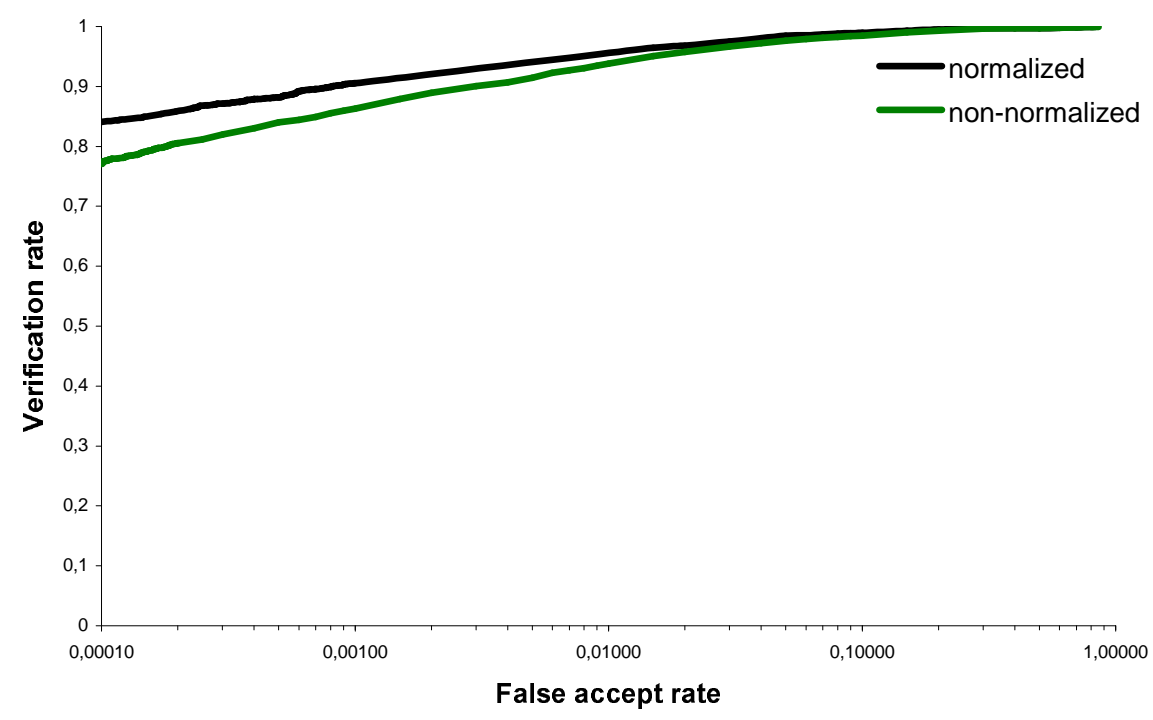

Figure 1. Normalized versus non-normalized results for Cognitec's algorithm on images from the FERET database (gallery: fa subset; probes: subsets fb (different facial expression), rb and rc (different head pose), and bk (different illumination); for additional information on these subsets, see $\mathrm{http}: / / \mathrm{www}$.itl.nist.gov/iad/humanid/feret/feret_master.html).

Based on the organizers' announcement that 'FRVT 2002 will report both normalized and non-normalized performance scores" (see Appendix J, section 3), we assumed that also diagrams would be included showing only non-normalized performance for the different technologies. This certainly is a fairer and more direct way of comparing the performance of the participants. Said assumption was the main reason why, finally, we decided not to provide score normalization.

Another reason is the limited applicability of normalization. In general, we think it is desirable that the result of a comparison between a probe and a gallery image does not depend on what other images are in the gallery. In the last paragraph on page 9 of the FRVT 2002 report, the authors also address this issue and ask: "When normalization is applied, is 
verification still ' 1 to 1 ' matching?" Certainly it is not! Normalization requires that a probe be compared with a gallery and therefore access to the gallery has to be provided for the comparison of just two templates. Normalization not only demands more computing power for verification (as many comparisons have to be performed as images are in the gallery), but also the assessment of the performance of a system becomes a very tough practical problem when the gallery at hand changes over time.

There are applications in which the gallery is not available and thus are not amenable to normalization. An example is an access control system comprising smartcards containing the biometric template and terminals that take a live image of the cardholder and perform a verification using that live image and the template on the card. In such a system, the gallery is distributed over the smartcards and cannot be used at verification time for normalization. Access to the gallery can also be restricted due to privacy policies.

In our opinion, score normalization lies outside the face recognition process. It can be advantageous in some situations while in others simply it is not or might even cause problems. For example, in identification, normalization is irrelevant ${ }^{1}$.

It should be possible to use a generic method for generating normalization functions based on the estimation of the similarity score distributions for a range of galleries and probe sets. This is exemplified by the normalization function we have implemented, which works as follows:

A normal distribution function is fitted to the set of scores. From the parameters of that distribution function, the parameters of the uniquely determined linear transform are computed that maps the fitted normal distribution to the standard normal distribution (i.e. zero mean, unit variance). That linear transform is then applied to all scores in the set.

Obviously, that normalization function can be used for any non-normalized scores whose distribution is near to a normal distribution, independent of the specific classification task. Thus for an objective comparison of core face recognition technologies, only the nonnormalized results should be used. Figure 2 shows the non-normalized verification performance ROC on the HCInt large gallery and probe set for Cognitec and the second and third best performers.

While the non-normalized scores for verification in the HCInt test are reported for all participants (for the participants who submitted normalization functions these results are shown in Appendix L, figures L.10 through L.13), there are some results for which no corresponding results based on non-normalized scores are provided. Therefore we consider the reported data incomplete; the missing data would have been very valuable for a meaningful comparison of the participants.

\footnotetext{
${ }^{1}$ This holds if the order of the similarity scores between faces is not changed. However, we do not see how changing the order could make sense, and it seems indeed that the submitted normalization functions do not change it.
} 


\section{$<$ Cognitec}

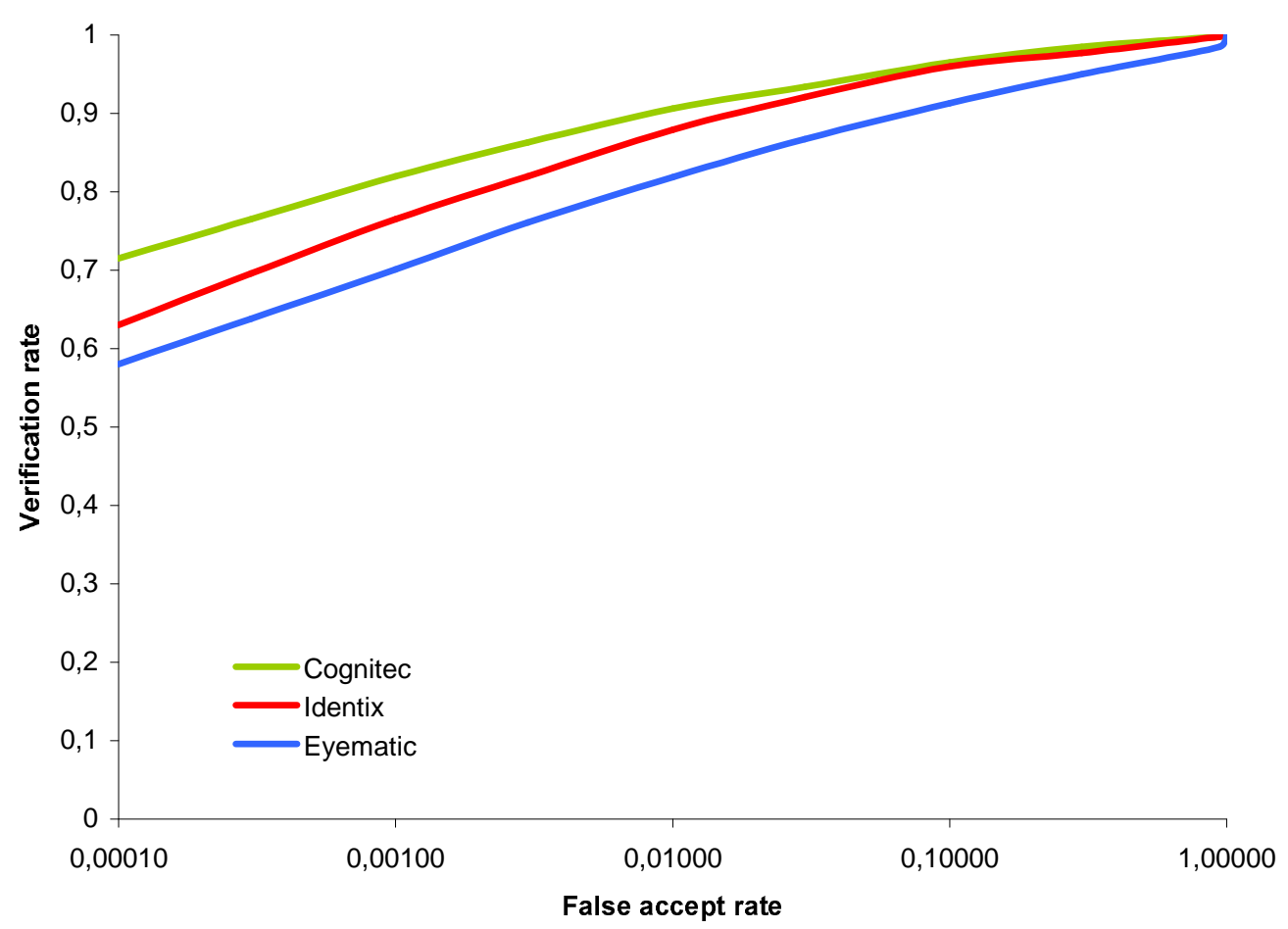

Figure 2. Non-normalized verification performance ROC on the $\mathrm{HClnt}$ large gallery and probe set for Cognitec and the second and third best performers.

\subsection{High Computational Intensity Test}

The high computational intensity test (HCInt) was the most important test in FRVT 2002. As the report says, it aimed at the evaluation of the performance of state-of-the-art systems on extremely challenging real-world problems. The excellent performance of our technology in these experiments confirms the top position of our products to satisfy best the demands of the market.

We found interesting that our algorithms discern males better than females. In our opinion, this is not a property of our algorithms. We have observed that women's facial appearance is more homogeneous than men's.

\subsection{Medium Computational Intensity Test}

The medium computational intensity (MCInt) test assessed the impact of further techniques for improving the performance of current technologies: three-dimensional morphable models, and the use of video sequences for face recognition.

Unfortunately, the comparison of non-normalized results for those experiments is not possible as the non-normalized data is missing in the report for those participants that used normalization.

\section{Three-dimensional morphable models}

Cognitec's algorithms were not trained specifically to deal with faces rotated by more than 15 degrees. The devised experiment shows that a large improvement can be achieved by morphing non-frontal faces to their frontal view. Although these techniques still require considerable computing power, they could become feasible in the near future. 
Care has to be taken when interpreting these results because the measured performance is not only determined by the facial recognition system but also by the quality of the morphing system.

\section{Video images}

Cognitec's FaceVACS technology does not exploit any properties related to video imagery. Therefore, it is not surprising that these experiments did not show an improvement of Cognitec's performance.

\subsection{Processing Speed and Hardware}

The FRVT 2002 did not directly address the evaluation of computing requirement and processing speed. In real-world applications though, this is a very important aspect. In table 1 we give the hardware used for the tests and the figures for the time spent on each of the tasks. This shows that our technology is also very competitive regarding computing requirements and processing speed. Further details of the hardware can be found in Cognitec's system description in Appendix M.

Table 1. Times spent on the HCInt and MClnt tests ${ }^{2}$

\begin{tabular}{ccccccc}
\hline Test & $\begin{array}{c}\text { \# of } \\
\text { signatures }\end{array}$ & $\begin{array}{c}\text { \# of } \\
\text { comparisons }\end{array}$ & $\begin{array}{c}\text { Time to } \\
\text { enroll }\end{array}$ & $\begin{array}{c}\text { Time to } \\
\text { compare }\end{array}$ & $\begin{array}{c}\text { Comparisons } \\
\text { per second }\end{array}$ & Hardware \\
\hline HCInt & 121,589 & $\sim 15$ billions & 12 hours & 104 hours & $\sim 40,000$ & Athlon XP @ 1.7 GHz \\
\hline MClnt & 7,722 & $\sim 60$ millions & 6.5 hours & 1 hour & $\sim 16,600$ & Mobile Pentium III @ 650 Mhz \\
\hline
\end{tabular}

\section{Improvements of the FaceVACS technology since the test}

The FRVT 2002 took place eight months ago. For facial recognition, one of the most challenging biometrics around, this is a long time, as the level of development efforts remains very high. Cognitec keeps its focus on the improvement of FaceVACS and its underlying algorithms and techniques.

\subsection{Increased Performance}

We increased the detection rate and the accuracy of our face and eye finder by employing better feature detectors and by employing better methods for learning the parameters of the classifiers. In addition, we improved the recognition algorithm, leading to lower overall error rates and less performance differences between faces of different ethnicities.

\subsection{Current Matching Speed}

We could improve the speed of the comparison function from former 40,000 comparisons per second achieved during FRVT 2002 up to 500,000 comparisons per second on a single Pentium $4 @ 2.4 \mathrm{GHz}$. This was achieved through code optimizations and through using optimized functions that take advantage of the latest SSE2 instruction sets on modern processors for the most time consuming operations.

\footnotetext{
${ }^{2}$ The given times include the time spent on file $\mathrm{I} / \mathrm{O}$. In particular, the enrollment time includes the time for reading the images and writing the templates, and the comparison time includes the time for reading the templates and writing the similarity scores. The enrollment time given for the MCInt test actually refers to enrolling the signature set twice, once as target set and once as query set.
} 


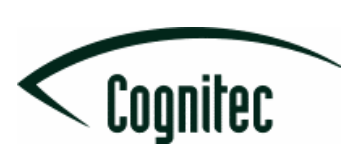

\section{Conclusions}

Cognitec is very pleased that the high quality of our face recognition technology FaceVACS was confirmed by the FRVT 2002. We have been developing this technology since 1995, and will continue to improve its recognition performance and to extend its range of applications. To an increasing degree, this will encompass application areas for which other biometrics are currently preferred.

Both the biometrics industry and potential customers in government and industry will be very grateful for the results of FRVT 2002. So far it has been difficult for them to assess the different face recognition technologies on a common and neutral basis; this is what FRVT 2002 has provided with very demanding and real-world data. We think that this type of evaluation should be repeated on a regular basis.

Cognitec thanks the organizers and sponsors of the FRVT 2002 for the opportunity to participate and for the excellent and independent work they did.

Remarks on this commentary are appreciated and should preferably be sent as e-mail to info@cognitec.com. 


\section{C.VIS C-VIS Computer Vision und Automation $\mathrm{GmbH} \cdot$ Page 1 of 1}

\section{Comments from C-VIS on the Facial Recognition Vendor Test 2002}

The FRVT 2002 is an important technology evaluation. Its results provide valuable insights into the state of the art in facial recognition. However, the test will only partially give a potential user of automated facial recognition an answer on whether this technology fulfills his expectations.

The results of the High Computational Intensity test (HCI) are valid only for images of an ID photo type. Among the $\mathrm{HCI}$ images that we saw, no one showed a face in front of a structured non-uniform background and no images came from typical video sources, for example. As a consequence, a HCI test on a realistic mix of image types may have very different results both in terms of the general capabilities of the technology and for the individual vendors.

The main product of C-VIS, the FaceSnap RECORDER, has been designed for real-time surveillance applications. The kind of image data we are confronted with in typical applications is often quite different to most of the images used in the FRVT 2002. In the previous FRVT in the year 2000 there was an attempt to include in the test a realistic application scenario. In our opinion, the short video sequences from files that were included in the FRVT2002 are not an equal substitute to a real video surveillance test. As a suggestion for the future, a recognition experiment from a digital video footage of at least a few hours length should become part of the FRVT.

There are a number of important real world applications for which customers seek a complete and viable solution. In a real application scenario, the success or failure of a particular system may not depend primarily on the performance of a single "core technology". Instead, the quality of interaction of all system components and the suitability of the system design for a particular purpose becomes crucial. A commercial system should be tested against its claims with respect to particular applications. For future tests, it would be desirable to exactly identify the most important applications of facial recognition and to tailor test procedures to them.

In the FRVT2002, C-VIS has confirmed its position as one of the leading players in this market. The priorities of our technology developments are driven by actual customer needs. A test like the FRVT2002 can of course not reflect the whole spectrum of challenges encountered in practical applications. However, we hope that the FRVT team continues its excellent work, making the FRVT a regular benchmarking service for the commercial face recognition community.

Bochum, March 10, 2003

Prof. Dr. Thomas Zielke

President

C-VIS GmbH 


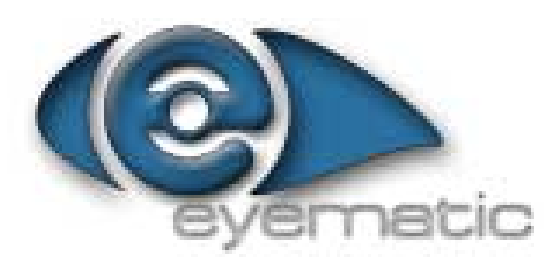

Comment on FRVT2002 Evaluation Report

Eyematic Interfaces Inc.

March 2003

After reviewing the FRVT 2002 evaluation report and considering our on-site experience, Eyematic is very pleased with the overall results. The test was conducted in a well-organized manner, and special care was given to ensure equal opportunities to all vendors, which gives high credibility to the test results. We appreciate the straightforward way in which the test data was described and presented. Assistance from the FRVT Team was pleasant and helpful to complete all test runs without any problems for which we needed only half of the allotted time.

In this statement we would like to discuss our motivation for our system's configuration and consequences for the test result of HCINT. We will then consider aspects of MCINT, add a few remarks about technical advances we have made since July 2002 and finally give a few suggestions for future face recognition tests.

\section{Importance of Adaptive Systems}

Considering the diversity of applications made possible through face recognition technology, a reader of the evaluation results must understand that the variety of possible conditions under which images can be acquired are practically endless and a public test can not yet be seen as comprehensive for all aspects of use. Specific face recognition applications always impose specific requirements to the underlying technology. Image data that was acquired under controlled conditions like plain background and well-distributed illumination clearly represents the ideal scenario, which is optimal to investigate effects related to the variability of faces. But the value of a system performing well under these conditions still remains questionable with respect to applications like "Access Control" or "Surveillance" where we are confronted with unstable environmental conditions like illumination-variation, pose, etc. These conditions have a great influence on the system's performance; despite efforts to control them through hardware (e.g. artificial light). Secondly, even in so-called "Cooperative Scenes" we must make allowances for the quality level of cooperation in the absence of a human operator. This is particularly important if the application is built for convenience: A 'fast lane' for frequent flyers; access control replacing the use of ID cards or typing an ID number; quick validation of credit card or passport; etc. To properly face this challenge, the fundamental technology must become adaptable.

We understand ourselves as a supplier of the general core technology. Our customers integrate it into their applications. This requires us to allow our customers freedom in choosing the environmental conditions and still provide them with software solutions that can cope with such conditions. For years Eyematic has undertaken significant $R \& D$ efforts to solve these problems. We have developed methods that allow a customer to adapt our system to any condition, even those unknown to us, without imposing additional restrictions in the generality of usage or raising the complexity of our API. One of our most successful solutions is an automatic training tool that can optimize the entire face recognition system to specific scene conditions through sample images with some ground truth position information. Recent developments allow such training even without ground truth data. 


\section{Details about our system configuration for FRVT2002}

The FRVT2002 did not allow any sort of adaptation or training during the test. Since no specific information about the image conditions was disclosed, we chose to configure our system for high invariance to pose, size, background texture and some illumination as it occurs for access control systems. Our customers frequently face these conditions. To limit the influence of background texture we suppressed hair and head-shape cues for recognition. However, such cues can be beneficial when the faces are presented before untextured background (as it was apparently the case in the HCINT) ${ }^{1}$. After reviewing the evaluation report, we came to the conclusion that our configuration was good for most data used in MCINT but it was sub-optimal for HCINT. Additionally, since our largest customer is located in Asia, our face databases have a large portion of Asian faces. This might have given our system a slight bias.

\section{MCINT Test Results}

This test mimics better than the HCINT test the conditions present in typical applications such as access control or passport authentication.

\section{Indoor-Outdoor Test}

Our scores in all indoor conditions reflect our optimization for indoor access control systems. We are pleased to have reached highest rank here. For outdoor conditions it is possible to raise the performance through training, however, we admit that reaching an acceptable level is not at all easy under the influence of direct sunlight.

\section{Head Pose Test}

We are pleased to have reached highest or second highest scores in all pose (morph) experiments (Evaluation Report Fig. 23). However, 45 degrees head rotation is rather extreme for systems designed for frontal faces and for most practical purposes we found that tolerance to pose variations of $+/-20$ degrees is sufficient. We would like to point out that our SDK can be adapted to any dominant pose as long as enough recognizable facial features remain visible (We do not require visibility of both eyes). This is necessary for applications where, due to camera mounting limitations, faces can only be viewed under an angle and consequently seldom appear frontal.

\section{Still-Video Test}

Our SDK includes specific features that were designed for optimized performance on video or still-set data. Unfortunately we introduced a bug in the FRVT2002 test setup that was discovered a few weeks after the test was finished. It affected the performance of the Still-Video Test. Despite this error we are pleased to learn that we still reached the best score (Evaluation Report Fig. 24).

\section{Technical Advances since July 2002.}

About $3 / 4$ year has elapsed since we prepared the product description for FRVT2002 as presented in Appendix $\mathrm{M}$ of the Evaluation Report. In the meantime we have reduced the size of a face template from about 4 Kbytes (this was used in the test) to less than 1.5 Kbytes without any loss of accuracy. With small loss of accuracy we now offer template sizes below 1 Kbytes. This has

\footnotetext{
${ }^{1}$ This might explain why among the three best scoring vendors our system showed the least sensitivity towards gender (Evaluation Report Figures 17 through 19).
} 
drastically increased the template matching speed and its memory economy for large galleries. We also increased the overall processing speed by over $50 \%$ without any loss of accuracy.

\title{
Suggestions for future face recognition tests
}

\section{More emphasis on real-world scenarios}

While the design of HCINT was excellent to answer questions regarding temporal, gender and age effects or the use of large databases, it did not address typical challenges of real-world applications, which are: Illumination variation, pose variation, occlusion, noise, lens distortion and other. MCINT covers some of these issues but not sufficiently due to its size limitation. We also suggest (re-) introducing fine-grained tests for pose variation $(10,20,30, \ldots$ degrees rotation) as well as variable head size.

\section{Evaluation of methods for Adaptable Systems}

Unless our computers become several orders of magnitude faster and the software much smarter, there will always be a need for adaptability to specific scenes. A system that achieves peak accuracy on any possible image containing a face under any possible condition without adaptation does not seem realistic for the near future. Consequently vendors that have a flexible system produce scores that depend on its configuration rather than its principal capability. We suggest introducing a session in which a percentage of image data is extracted from the test data set and given to the vendor for a limited time before the actual generation of similarities begins. The vendor should be allowed to use the image data in order to train his system in order to properly configure it for the subsequent test. This simply mimics the situation that is prevalent in most real world applications.

\section{Different presentation of results}

For tests where systems reach a very high score (e.g. Evaluation Report Fig. 23 (Front)), results like $99 \%$ and $98 \%$ verification rate are hardly distinguishable in the chart although they would represent significantly different performances. We suggest plotting against "Failure Rate" with using a logarithmic scale.

We would like to give thanks to all FRVT sponsors and organizers for making this test possible.

\author{
Contact: \\ Eyematic Interfaces Inc. \\ 9800 S. La Cienega Blvd., $5^{\text {th }}$ Fl. \\ Inglewood, CA 90301 \\ USA \\ www.eyematic.com
}




\section{IconQuest}

www.iconquesttech.com

\section{Comments on the Facial Recognition Vendor Test 2002}

\section{OVERVIEW}

IconQuest has developed an image recognition system, IQ MatchMaker ${ }^{\mathrm{TM}}$, which is focused on solving recognition problems with poor quality images. For example, such images may be very small, blurred, partially obscured, or photographed through dirty windows. Poor quality images arise routinely in the real world.

IQ MatchMaker ${ }^{\mathrm{TM}}$ is not based on an underlying structure characteristic of faces, but applies to general imagery. For example, by simply changing the Probe and Gallery images to photos of planes and reconnaissance photos of airfields the same software used in FRVT 2002 can locate a variety of aircraft in the reconnaissance photo.

In order to apply the general image recognition engine in IQ MatchMaker ${ }^{\mathrm{TM}}$ to face images, a segmentation algorithm was incorporated as an option into the software.

In FRVT 2002, IconQuest made only minimal use of the full size imagery provided by the Sponsor. Specifically, the full size imagery was only used for conversion from color to gray scale, histogram equalization and segmentation. Once these tasks were accomplished, the images were scaled down using pixel averaging to smaller images, each containing no more than 1536 pixels. In most cases, the scaled-down imagery contained substantially less than 1536 pixels. For comparison, note that 1536 pixels could come from an image of resolution $32 \times 48$, while the MCInt images provided by the Sponsor varied in resolution between $240 \times 320$ and 720 x 480. IconQuest made no further use of the full size imagery. Only the scaled down images were used in the image comparison and analysis phases of recognition. Therefore, all results relating to IconQuest in the FRVT 2002 Evaluation Report are for segmented images having an area containing no more than 1536 pixels.

\section{PROGRESS SINCE THE FRVT 2002}

\section{(A) Identification}

IconQuest tested its IQ MatchMaker ${ }^{\mathrm{TM}}$ technology to see the dependence of image size and Identification performance. 695 Probes consisting of the first image of an individual in the FERET database of thumbnails and a Gallery of another 6554 images in the FERET thumbnail database were used. Pixel averaging was used to scale down the FERET thumbnail images. The results for Rank 1 are:

Image Size (pixel area) Identification Rate

$8 \times 8$

.72

$11 \times 13 \quad .72$

$21 \times 22 \quad .90$

$37 \times 41 \quad .97$


These results represent a significant improvement over the performance of the system as it existed in July of 2002, reflecting substantial modifications to the software since FRVT 2002. Additional results on small, blurred imagery are contained in the March 2003 Advanced Imaging article Through a Glass, Darkly : Image Recognition with Poor Quality Imagery.

(B) Verification

IconQuest had done no Verification performance testing prior to the FRVT 2002. Since that time, an algorithm for re-normalizing similarity scores has been added which substantially improves verification performance. A number of specific tests were conducted using segmented FERET thumbnails which were then scaled down using pixel averaging. An example that illustrates the improvement in Verification performance follows. A Gallery consisting of the first image of each of the first 100 individuals and Probes consisting of the second image of each of the first 608 individuals was created by scaling the segmented FERET thumbnails down to 21 x 22 pixels. An operating threshold was set to reach a false accept rate of .01. Without re-normalization, the system produced a false alarm rate of $46 \%$. With re-normalization, the false alarm rate was reduced to $26 \%$.

\section{COMMENTS/ANALYSIS OF FRVT 2002 RESULTS}

(1) Identification

IQ MatchMaker ${ }^{\mathrm{TM}}$ performed well in Identification Performance for Indoor (same day) Probes; see Figure L.15 where IconQuest results varied between best, second best and third best as rank varied.

(2) Verification

(a) IQ MatchMaker ${ }^{\mathrm{TM}}$ performed relatively better in the Identification tests than in the Verification tests. This is not surprising since, as noted in (B) above, IconQuest conducted no verification testing prior to the FRVT 2002. Testing was critical in optimizing the software to perform better in identification tasks.

(b) As noted in the FRVT 2002 Evaluation Report, (page 19), normalization of similarity scores increases verification rates. Of the five participants who scored highest in verification tests (see Figure 22 on page 33 of the Evaluation report), four submitted normalization functions which were used in computing verification results. As noted in (B) above, IconQuest developed a normalization function only after the FRVT 2002 time limit to submit such functions expired. Based on results typical of those noted in (B) above, substantial improvements related to verification performance are expected to result from the use of IconQuest's current normalization function.

(c) Despite the lack of optimization based on verification testing prior to FRVT 2002 and the lack of a normalization function, IQ MatchMaker ${ }^{\mathrm{TM}}$ tended to $^{-}$ perform mid-range in verification tests, when it performed well in identification tests; see, for example, L.14 \& L.15 and L.34 \& L.35

(3) Pose

(a) IQ MatchMaker ${ }^{\mathrm{TM}}$ performed relatively well in the identification tests of pose variation. For example in Rank 1 Identification performance, IconQuest was: 
(i) best in 30 Degree Up; (ii) second best in 30 Degree Down; (iii) tied for second best in 45 Degree (Left); and (iv) tied for fourth best in 45 Degree (Right).

(b) As noted in the Overview, all of IconQuest's results are based on similarity scores obtained from images which were scaled down to contain no more than 1536 pixels. The pose results are remarkable since they represent comparisons of images resulting from the composition of pose distortions and significant scaling transformations.

(4) Lighting

(a) Changes in lighting (e.g., Indoor (same day) Probes Vs. Indoor (same day, overhead) Probes challenged the system. Since we are dealing with a general image recognition engine, as opposed to a face recognition system, an ideal solution to this problem would allow recognition despite changes in illumination in general imagery. This represents an area of on-going interest.

(b) As noted in the overview, all of IconQuest's results are based on similarity scores obtained from images which were scaled down to contain no more than 1536 pixels. Therefore the image variation resulting from the lighting changes is really the result of compositions of the lighting distortions and significant scaling transformations.

(5) Background

Samples of Outdoor imagery contained in the Evaluation Report display a much greater background complexity than those samples of the Indoor imagery. IconQuest selected an image segmentation algorithm on the basis of its speed. This non-proprietary algorithm was selected after testing on a large number FERET images. The FERET images tend to have backgrounds closer to these Indoor imagery samples than to the Outdoor imagery samples. The segmentation algorithm, which we selected, is fast, but doesn't work as well for images with complex backgrounds. We expect that this was not an appropriate choice of segmentation algorithm for a number of FRVT 2002 images with complex backgrounds and provides at least a partial explanation of the variation in performance between Indoor and Outdoor imagery.

(6) Temporal Variations

Analysis of the variation between same-day and different-day results isn't really possible without access to the relevant image data. In the case of Outdoor images, we expect that changes in natural illumination from day to day might offer a partial explanation.

(7) $\underline{\text { Video }}$

In the Abstract of the Face Recognition Vendor Test 2002 Evaluation Report, the Sponsor observed that "face recognition from video sequences offers only a limited increase in performance over still images." We feel that the results supporting this conclusion, at least from the vantage of IQ MatchMaker ${ }^{\mathrm{TM}}$, 
may well reflect the lack of a video database similar in variation to the FERET database for use in optimizing the software, and that rapid and substantial performance improvement would result from acquisition of such a video database.

(8) Morphable Models

(a) The Morphable Models ("MM") approach is an example of a more general approach in which images undergo pre-processing transformations. Despite the lack of utility of employing the specific morphable model used in FRVT 2002 together with IQ MatchMaker ${ }^{\text {TM }}$, we believe other transformational approaches can have great value.

(b) The MM tests of MCInt seem to be of a different nature than the rest of the FRVT 2002 tests. FRVT 2002 was a time-limited test in that each participant had to complete production of similarity scores in no more than 264 hours. Except for the MM tests, the Probe and Gallery images represent real world images and participants are free to spend as much time as they like preprocessing images, but their pre-processing time is counted toward the test time. However, the MM tests reflect mandating a specific pre-processing step (performed by the Sponsor), but without counting the time it takes to carry out that step towards the overall test time. Without counting this time, it is not possible to support the conclusion of the Evaluation Report that "Results show that three-dimensional morphable models. ... increase performance" especially in the context of a time-limited test. In addition, results show that the use of MM decreases performance in at least one important case: frontal imagery. In more detail:

(i) Utility of MM and Computation Time: The computation time, MMTime, expended by the Sponsor in the MM pre-processing should be given. Since Participants were not given external pose information concerning Gallery images, they would have had to apply MM preprocessing to all images. To the extent MMTime is negligible, then the objection raised here is minor. If MMTime is significant, then the objection raised here is material. For example, if a Participant added MMTime to the 264 hour time limit and its performance increased more than the increase observed by using MM in the 264 hour test period, then the proper conclusion would be that "Morphable Models decrease performance", at least in the context of a time-limited applications represented by FRVT 2002.

(j) Utility of MM and Pose Statistics: FRVT 2002 test results (see Fig. L25 and Fig. L-24) show that Morphable Models decrease identification and verification performance on frontal imagery. The extent of decrease varies considerably among Participants. Whether or not MM pre-processing is practically useful then clearly depends on the statistics of pose variations in an image population.

(k) Utility of MM and Test Structure: Except for MM subtests, FRVT 2002 tests the performance of face recognition alternatives in a situation where a user has no advance knowledge of Gallery images. 
In many real world situations, the user has a database of known imagery and is attempting to perform Image Recognition ("IR") functions such as identification, verification or watch functions on unknown Probe images. In such situations, Gallery images are known in advance and can be subject to substantial pre-processing and any time limit would apply only to performing the IR functions on Probes. Then it would be reasonable not to count MM pre-preprocessing time as part of the time limit. In such a test, Participants would be given Gallery images in advance of the test and should be able to choose how to pre-process the Gallery imagery. Then one could evaluate the state-of-the art in pre-processing and decide whether MM increases performance more than other pre-preprocessing techniques. 


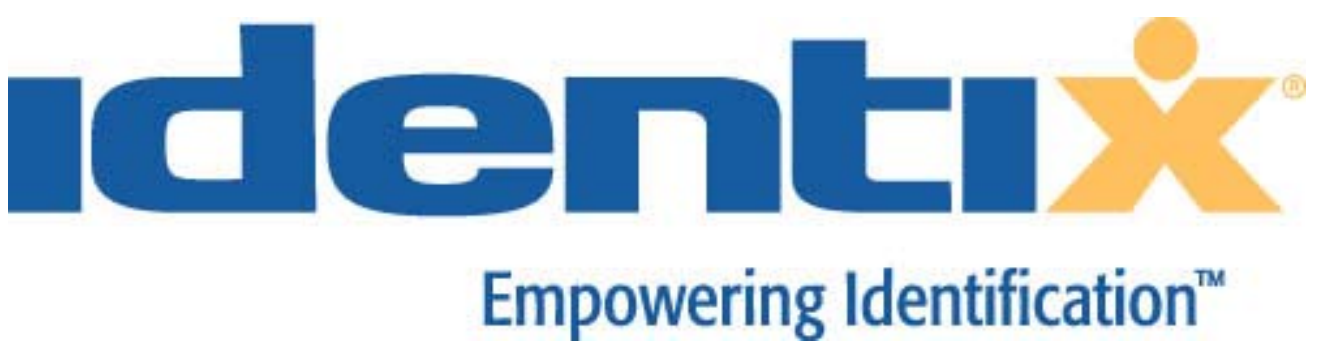

\section{Comments on FRVT 2002 \\ Identix FRVT2002 Team}

March 10, 2003

$\begin{array}{lr}\text { INTRODUCTION } & 2\end{array}$

PERFORMANCE STRATIFICATION 2

FACIAL RECOGNITION VALIDATED FOR NATIONAL ID SYSTEMS 2

FURTHER RESULT HIGHLIGHTS

VARIATIONS BETWEEN TEST RESULTS AND TRUE PERFORMANCE 3

HARDWARE AND COST VARIATIONS BETWEEN TEST SYSTEM AND REAL SYSTEMS

WHY CHOOSE IDENTIX? 


\section{Introduction}

The results of the FRVT 2002 tests demonstrate Identix Facelt technology to be a leading performer in all categories. Through the various tests, facial recognition has been validated for large one-to-many applications (i.e., national ID) and verification applications. The tests have also shown the significant value that facial recognition can deliver in watch list applications. Most significantly for the field of biometrics, the tests show that facial recognition biometrics do work, and they can substantially augment fingerprint applications when fused together.

While there are a few variations between the test results and actual performance which is discussed briefly below, the evaluation overall paints a very strong picture for Facelt technology and Identix as a whole, which stands alone among the top performers in being a large, stable, US based publicly traded company. Identix is a multi-biometric company with over 20 years of real world experience, established partnerships with a large number of systems integrators, card manufacturers and other biometric solution providers.. We have worldwide facial recognition and fingerprint biometric deployments for national ID, drivers' license, voter registration, background checking, physical and logical access control, social security payments and airport security.

\section{Performance Stratification}

The results of the FRVT 2002 tests show that there are at present three top tier technologies: Identix, Cognitec and Eyematic. This top tier membership was observed consistently for Identification, Verification, and Watch list tests. The best of breed technologies outperform all others regardless of task.

The close clustering of results for these three companies indicates a convergence of performance and thus, suggests the maturation of the field of automated facial recognition. Such clustering has not been observed in previous tests.

The convergence of results does not mean that further improvements in performance should not be expected. As pointed out in the FRVT2002 report, performance gains have been significant over every previous test period. Furthermore, internal testing of current (March 2003) Identix algorithms demonstrates performance gains over the (June 2002) algorithm used in FRVT2002.

\section{Facial Recognition Validated for National ID Systems}

For the first time, the US Government has published the performance of face recognition for large databases (>100,000 images). This report should be considered a highly reliable validation for those interested in implementation of face for applications such as identity card programs and border control. While the expected performance of the system will vary with the quality of the database, these tests shows that for real-world face images and real-world size databases, face recognition works.

The conclusion that the performance of one-to-many identification lookup decreases only linearly with the logarithm of database size (p. 44) is very good news. This important result has been extended internally at Identix to database sizes of up to 10 million. These results indicate that face recognition is a viable option for state and even national ID systems. 


\section{IDENTIX INCORPORATED}

Note that performance will be further enhanced when demographics are used to segment the database into bins, thus reducing search size before performing the biometric search.

\section{Further Result Highlights}

Face Recognition Works. In general, the results of the various sub-tests show that face recognition technology:

- Works well for one-to-many identification with real-world databases.

- Works very well for indoor verification.

- $\quad$ Provides significant value for watch list (surveillance) applications.

Improved Accuracy. Identix is particularly pleased about the improvement in accuracy that our technology has demonstrated since the last FRVT in 2000.

Minimal Sensitivity to Aging. Contrary to popular belief, Figure 16 shows that face recognition performance is only slightly sensitive to temporal variations (changes over time). Thus, we would argue that template aging is not a significant problem for adults.

Unaffected by Indoor Lighting. The indoor lighting variation problem has been solved. Facelt is unaffected by changes in lighting for images taken indoors (see leftmost two data points in Fig. 22).

Suitable for Fusion with Fingerprint. As discussed by the authors (see Fig. 25) facial recognition provides greater accuracy than single fingerprint (circa 1998) for verification applications requiring relatively low security (FAR of 0.01 or lower). This cross-over result, together with the finding that face has reached a level of maturity sufficient for large-scale one-to-many applications, suggests that fusion of biometrics is an idea whose time has come.

Identix offers both facial and fingerprint solutions, and has the expertise to develop and deploy dual and fused biometric systems where these provide the best solution.

\section{Variations Between Test Results and True Performance}

While overall we are very impressed with the scientific validity of FRVT2002, we briefly wish to list slight variations between true Identix performance and that measured by the test.

Current Technology Delivers Improved Performance. Identix prides itself on active research and development, so that already our performance is better than what is shown in this report.

Use of Video Can Benefit Facial Performance. Identix challenges the assertion that video is not an advantage for facial recognition. The source of confusion on this point is that the video used in the still versus video test was unrealistic, containing either pure pose change or pure opened/closed mouth (talking) change. In order to properly test the impact of video, sequences would contain natural variations in resolution, lighting, pose and expression associated with humans moving through space.

Video also offers many opportunities to perform real-time registration of facial features. Registration errors are a primary source of recognition failure.

For surveillance applications, Identix offers a finished product, Facelt ARGUS. We expect that Facelt ARGUS performance exceeds what is shown in Figure 12, because the 
technology used in Facelt ARGUS has been optimized to take advantage of the data that real-world video applications provide.

Tuning to Different Populations is Not Required. We disagree with the notion that algorithms should be tuned to data sets, as discussed on page 40 . Our core Facelt matching algorithm does not contain any information about race, age or gender, thus the technology used in the large identification test has no parameters to tune. Optimization is performed dynamically in the context of normalization (discussed in detail in the Appendix A of the main report).

Normalization Does Improve Performance for One-to-Many Applications. The benefit of normalization for identification was not fully tested in the present evaluation because the type of normalization that was allowed in this test (see Appendix A of main report) was restricted to routines that do not change the rank order of the one-to-many search result. The FRVT 2002 test givers did not allow a more appropriate normalization for identification.

\section{Hardware and Cost Variations Between Test System and Real Systems}

Use of Identix facial technology does not require the high cost hardware that was used in this test.

Our one-to-many product, Facelt ABIS (Automated Biometric Information System), utilizes a set of separate, low cost units to perform face finding, template creation, load balancing and matching. For the present tests, more than the maximum allowed number of these minicomputers would have been required. Note that Facelt ABIS is a modern three-tier http/XML system that scales with database size, number of searches required per unit time, distance between hardware components and number of users. Again, Facelt ABIS relies on the lowcost single CPU components mentioned above, which we refer to as Biometric Appliances.

For large-scale identification systems, the total cost of ownership includes many business factors other than hardware cost. The costs underlying an AFIS (fingerprint) system are an example of this fact.

\section{Why Choose Identix?}

Other than the obvious requirement for superior technology, those contemplating a choice between vendors should also consider the following important factors:

Business factors - Identix is US-based; it is a large, stable, publicly traded organization, arguably the only facial company in that class. Identix is a multi-biometric company with over 20 years of real world experience, established partnerships with a large number of systems integrators, card manufacturers and other biometric solution providers.. We have worldwide facial recognition and fingerprint biometric deployments for national ID, drivers' license, voter registration, background checking, physical and logical access control, social security payments and airport security.

Technical factors - Identix is technically active: we have a strong R\&D group, an active presence in standards organizations, and we are the inventors of algorithms and thus dedicated to their continued improvement, as opposed to having licensed technology which is static and now out of date.

Identix offers core technology and products for both facial and fingerprint matching technologies, of key importance for those considering a solution that will rely on fusion of the two. 
Product factors - Identix provides modern implementations that take advantage of the latest technologies: mature finished off-the-shelf products and software developer kits. Also, Identix has a Professional Services organization, which can be of tremendous value in performing tasks leading up to real world implementation, including development of customized software applications or finished systems, provision of benchmarking services and acceptance testing, and data analysis services.

Identix offers facial solutions for verification, one-to-many and surveillance in the form of both finished products and SDKs.

For facial one-to-many, we offer:

- $\quad$ Facelt ABIS, a cutting-edge scalable facial image search system akin to an AFIS that delivers speed, accuracy and throughput based on customer requirements.

- $\quad$ IBIS, a wireless, mobile solution for finger and face ID in the field; ideal for law enforcement and border patrol.

- $\quad$ Facelt Identification SDK - COM-based Win32 SDK for building one-to-many search applications.

For facial surveillance (watch list applications), we offer:

- $\quad$ Facelt ARGUS, an off-the-shelf facial surveillance and identification system that provides watch list and alarm capabilities for enhancing new and existing CCTV systems. Facelt ARGUS scales to any number of cameras and can be set up remote from the location under surveillance.

- $\quad$ Facelt MultiFace SDK - add-on to Identification SDK for building watch list applications: enables capture of faces from live video to input to search engine.

For facial one-to-one, we offer:

- $\quad$ Facelt Verification SDK - Win32 SDK for building access control applications; uses COM and ActiveX

\section{Final Comments}

Identix is pleased with the results of FRVT2002. In particular, we are happy with the progress that we have made since the last evaluation and with the validation of facial for large-scale one-to-many applications.

Identix applauds the momentous effort by the evaluating body. We feel that the tests were well organized and that the results are expertly presented. We value our continued strong relationship with the agencies and personnel involved in carrying out this evaluation. 
Imagis welcomed the opportunity to participate in the Face Recognition Vendor Test 2002 and appreciates the efforts made by the administrators to provide a uniform academic laboratory test environment for face recognition accuracy assessment.

Last summer's test -- Imagis' first experience with the FRVT -- provided a snapshot of the state of the industry at that time. The test was particularly useful to Imagis in that it suggested to us a number of ways in which we could achieve significant improvements to our then-current FR algorithms. In addition, it provided us with valuable insights into the problem of balancing the need to achieve acceptable levels of accuracy while at the same time preserving our technology's intrinsic ability to search large databases quickly - a factor that is often cited by our customers as a critical requirement in real world implementations of FR applications.

The release of ID-2000 Version 9.0 in the fall of 2002 reflected these improvements. This version not only achieved a substantial increase in accuracy by correcting certain limitations identified during the internal benchmark procedures that preceded the FRVT test itself, it also proved that significant accuracy advancements are achievable on an ongoing basis as a result of the inherently extensible nature of our core imaging algorithms. These improvements have been, and will continue to be, implemented in upcoming software releases. For example, major users around the world have already acclaimed the level of real-world performance achieved by the post-FRVT release of the Imagis' ID-2000 Version 9.0. Imagis has also recently demonstrated a 64-bit version of ID-2000, further enhancing the technology's ability to search large databases (i.e. more than two million images) without impeding the speed requirements demanded by our customers. Imagis is the only face recognition vendor that supports a 64bit environment.

For FRVT 2002, Imagis used customer-ready software with an encode array size of 1,400 bytes, operating on a single processor. This still achieved overall performance levels that compared favorably with other vendors, some of whom required multi-processor systems and encode arrays exceeding 4,000 bytes. A forthcoming release of the software will allow the user to choose larger encode sizes suitable for environments in which encode time and match speed are less important than accuracy. A true symmetric multiple processor (SMP) version of the Imagis' ID-2000 application will be available by mid-year 2003.

We invite all interested parties to evaluate our Version 9.0 release. While laboratory testing provides a good basis for discussion, nothing can substitute for a real-world test deployment using one's own data and imagery. In such an environment, users can measure the impact that speed and database size have on the operational processes of their own organization. To discuss a pilot deployment, please visit www.imagistechnologies.com or call 1.604.684.2449. 


\section{Viisage Technology Position Paper on the FRVT 2002 Report}

The FRVT 2002 represents a significant advance in the testing of facial recognition technology. As such, the FRVT 2002 team is to be commended for how they conducted the test, the indepth report they generated, and the useful conclusions and trends they identified.

We participated in the FRVT 2002 using a commercial version of our FaceEXPLORER application. It was only customized to handle the inputs and outputs as dictated by the FRVT test specifications. No technology was customized for this test.

After reviewing our performance results, we were greatly surprised by the relative performance of Viisage. We do not believe that these test results represent the performance of our applications. The results our many customers obtain do not match with what the curves would otherwise suggest. Without a more detailed analysis of our particular results, we find it hard to reconcile these differences. However, in light of some of the figures in the report, there is troubling evidence that something was wrong with our test setup, either due to human error or a problem with the software.

We will continue to investigate what went wrong with our test software and hope to be able to offer a more informative appraisal when completed.

The primary evidence is Figures 24 and 30 in the "FRVT 2002: Evaluation Report" and the supporting Figures L.42 and L.43 from Section L.5, MCInt Still-Video Experiment, in the accompanying "FRVT 2002: Technical Appendices." These figures compare the performance between still imagery and video for "different day" signatures with a gallery of 63 signatures, one per person, under both verification and identification scenarios. In Figure L.42, there is no verification curve for Viisage. In Figure L.43, the identification curve is a strange step-shaped function.

According to the FRVT evaluation team, these anomalies were both caused by our software generating the same score for all matches in this particular test. Clearly, this indicates a problem with our system. These results also surprised the FRVT evaluation team as the "FRVT 2002: Evaluation Report" says they manually checked the Viisage similarity files for

confirmation. We would like to thank the FRVT evaluation team for including this manual check.

Unfortunately, at this time, we are unable to determine the source of this problem and whether or not it could have affected the results from the other tests. Given that the images are presented to the system in a presumably randomized order, we must assume that it could have affected the results of the other tests in some unknown way.

We therefore must conclude that all the results for Viisage are in question and do not represent the performance of our software. 


\section{FRVT 2002}

The FRVT 2002 was managed very professionally from beginning to end by a dedicated team of people who showed competence, flexibility, and fairness in all their interactions with the participants. It was certainly a very important milestone in the field of face recognition for the raw size of the test database used, the number of participants, and the careful selection of test sets to allow for the evaluation of the sensitivity of the algorithms to various parameters such as aging and gender.

Indirectly, FRVT also tested the speed of the systems: for the high computational test, everyone had to process about 15 billion face comparisons within 264 hours, not including the time to detect the faces and code them. This represents more then 15,000 comparisons per second.

In order to complete the FRVT in the allotted time, we had to use a custom solution where we used our best comparison algorithm only a certain percentage of the time, using another, less efficient but faster, algorithm to pre-sort the pictures. The results obtained do not completely represent the full power of this algorithm when used in verification applications where more time can be used for the match.

Since FRVT, many improvements have been implemented to our face recognition algorithms to be able to deal with a larger volume of data. The comparison speed has been increased to be about 15 times faster than what was used in the competition. The raw recognition performance has also been improved significantly in the process. A recent test ran on an in-house face database of 5,800 pictures shows an increase of the recognition performance ( $F A R=0.1 \%$ ), $81 \%$ over the software used in FRVT. We are confident this new technology would now perform very well under strict time constraints as imposed by the competition.

\section{Static Images \& Live Image Verification}

Our system was originally designed to perform a one-to-one verification in which verification speed is not crucial. A verification application that takes up to one second for the face- verification part of the verification process is usually acceptable to a user. Once scrubbed, a database of facial images that does not contain multiples of duplicate images is used in numerous applications such as passport or border control to authenticate the user standing before a facial authentication device. However, there are several factors that adversely affect the performance of a verification system that are not applicable to a static image test such as the FRVT. One of those factors is the effect of ambient illumination found on a live human face. In a static image test such as that of the FRVT, illumination stayed constant in the pairs of images tested. However, this is not the case in real life situations. To cope with this problem, VisionSphere has developed and integrated into UnMask, controls that adapt the Company's camera's sensors, to varied ambient illumination found on a face. The camera sensors are embedded into the Company's several facial authentication devices.

This technology permits our cameras, embedded in our facial authentication devices, FaceCam, and our USB desktop camera, to automatically adjust their sensor settings to obtain a good image of the face, independently of the background. This process significantly improves the recognition performance in certain lighting conditions.

In a pilot project conducted on behalf of Transport Canada, in the summer of 2002, at the Ottawa International Airport, our software integrated with, and used with a FaceCam, was deployed to control door access to a restricted area for 170 airport employees over a 7-week period. Access was granted successfully by our integrated solution $96.4 \%$ of the times and not a single instance of false acceptance was reported in approximately 2,700 authentications over a seven-week period. A liveliness test (eye blink detection) was also used to confirm that the employee granted access was a human and not a photograph of that employee. 


\section{Conclusion}

In spite of the excellent organization of the tests, FRVT did not evaluate the ability of the systems to deal with live verification under varied lighting conditions to verify an average cooperative user under constraints other then raw number of matches per second. The FRVT test compares static images to static images. The images are compared to locate duplicate or multiple images, which for certain applications, results in the removal or location of duplicate or multiple images of the same person. After the removal of duplicate or multiple images, the facial-image database can be considered clean.

In a real world situation, the clean database would be used to authenticate/verify a live person in varied ambient illumination, against his/her facial image template stored in the clean facial-image template database. The FRVT did not, however, take that into consideration, i.e., it did not authenticate/verify users in real-world conditions (varied ambient illumination).

When face-recognition software is rated by tests such as FRVT, consideration should be given to authenticating/verifying a live person against his/her facial image templates stored in the clean database. A static image "ONLY" test such as the FRVT does not truly reflect how face recognition technology will perform in real world situations.

March 10, 2003 


\section{Appendix 0}

Additional Information on the 3D Morphable Model Used in FRVT 2002 


\author{
Generating Frontal Views \\ from Single, Non-Frontal Images \\ Volker Blanz ${ }^{1}$, Thomas Vetter $^{2}$ \\ ${ }^{1}$ Max-Planck-Institut für Informatik, \\ Stuhlsatzenhausweg 85, 66123 Saarbrücken, Germany \\ ${ }^{2}$ Universität Basel, Departement Informatik, \\ Bernoullistr. 16, 4056 Basel, Switzerland
}

\begin{abstract}
This paper describes a method for generating frontal views of faces, given single images at arbitrary poses and illuminations. The system is based on a Morphable Model of 3D faces, which represents face-specific information extracted from a dataset of $3 \mathrm{D}$ scans. It estimates the $3 \mathrm{D}$ shape of novel faces from the input images, and completes surface regions that are not visible in the image, due to self occlusion. The reconstructed 3D surface covers the face, the ears, and the upper half of the neck.

To generate the frontal views of the reconstructed faces at a standard position, pose, and size, the system uses 3D computer graphics. In the frontal image, the algorithm uses standard lighting conditions, after having compensated the effects of illumination in the original image. The novel drawing parameters are extracted from an example image. This image also provides standard hair, shoulders, and background, which are added to all transformed frontal views.
\end{abstract}

\title{
1. Introduction
}

Many face recognition algorithms perform best if the gallery and probe images are frontal views. In some applications, however, image data are taken at oblique viewing directions, such as images from cameras on ATMs. Often, authorities have not more than one random snapshot of a suspect.

To achieve robust recognition across a wider range of viewpoints, we propose a system that computes frontal views from arbitrary images. The result of this non-trivial preprocessing step can then be used by state-of-the-art face recognition systems.

Clearly, the transformed frontal views can only be estimates of the true appearance of the person: The shading information in an image, in general, is not sufficient to recover 3D depth in a unique way. Moreover, shading effects in images of faces are difficult to distinguish from variations in skin complexion, for example in the region around the eyes. Finally, parts of the face may be invisible in the input image, due to self-occlusion.

Our strategy to estimate the faces' frontal appearance, therefore, relies heavily on class-specific knowledge about human faces. This information is derived in an automated way from a dataset of textured 3D scans of faces. 3D shape of faces is 
reconstructed by finding the combination of example faces that is as similar as possible to the input image. We can then redraw the reconstructed shape in a frontal view, using $3 \mathrm{D}$ computer graphics.

In the following sections, we briefly summarize the Morphable Model and the algorithm for $3 \mathrm{D}$ shape reconstruction. In Section 4 , we describe the procedure that was applied to the test images, and show a set of examples.

\section{Morphable Model of 3D Faces}

The Morphable Model of 3D faces[Vetter and Poggio, 1997, Blanz and Vetter, 1999] is a vector space of $3 \mathrm{D}$ shapes and textures (surface colors), spanned by a set of examples, and capturing the variations found within this set. It is derived from a dataset of 200 textured Cyberware (TM) laser scans. The set is balanced with respect to male and female faces. The persons are aged between 18 and 45 years, and except for one Asian female, all are Caucasian. Previous work [Blanz et al., 2002] indicates that the model may well be applied to reconstruct $3 \mathrm{D}$ shape from images of a wider ethnic variety.

The shape and texture vectors are defined such that any linear combination of examples

$$
\mathbf{S}=\sum_{i=1}^{m} a_{i} \mathbf{S}_{i}, \quad \mathbf{T}=\sum_{i=1}^{m} b_{i} \mathbf{T}_{i}
$$

is a realistic face, given that $\mathbf{S}, \mathbf{T}$ are within a few standard deviations from their averages. Each vector $\mathbf{S}_{i}$ is the $3 \mathrm{D}$ shape, stored in terms of $x, y, z$-coordinates of all vertices $k \in\{1, \ldots, n\}$ of a high-resolution $3 \mathrm{D}$ mesh:

$$
\mathbf{S}_{i}=\left(x_{1}, y_{1}, z_{1}, x_{2}, \ldots, x_{n}, y_{n}, z_{n}\right)^{T} .
$$

In the same way, we form texture vectors from the red, green, and blue values of all vertices' surface colors:

$$
\mathbf{T}_{i}=\left(R_{1}, G_{1}, B_{1}, R_{2}, \ldots, R_{n}, G_{n}, B_{n}\right)^{T} .
$$

In the conversion of the laser scans into shape and texture vectors $\mathbf{S}_{i}, \mathbf{T}_{i}$, it is essential to establish dense point-to-point correspondence of all scans with a reference scan, to make sure that vector dimensions in $\mathbf{S}$, $\mathbf{T}$ describe the same point, such as the tip of the nose, in all faces. Dense correspondence is computed automatically with an algorithm derived from optical flow (for details, see [Blanz and Vetter, 1999]). Finally, we perform a Principal Component Analysis (see [Duda et al., 2001]) to estimate the probability distribution of faces around their average, and we replace the basis vectors $\mathbf{S}_{i}, \mathbf{T}_{i}$ in Equation (1) by an orthogonal set of eigenvectors.

\section{Fitting the Morphable Model to Images}

In order to reconstruct a $3 \mathrm{D}$ face from an image, we fit the Morphable Model to the input image in an analysis-by-synthesis loop: At each iteration, the current model parameters, together with 3D computer graphics techniques, define a model image. Let $I_{r, \text { model }}(x, y), I_{g, \text { model }}(x, y), I_{b, \text { model }}(x, y)$ be the red, green, and blue channels of this image. The goal of the fitting algorithm is to make it as similar as possible to the input image: In a gradient descent, the algorithm minimizes the image difference

$$
E_{I}=\sum_{x} \sum_{y} \sum_{c \in\{r, g, b\}}\left(I_{c, \text { input }}(x, y)-I_{c, \text { model }}(x, y)\right)^{2}
$$


with respect to the following model parameters: shape coefficients, texture coefficients, 3D position, 3D angle, focal length, red, green and blue components of ambient and parallel light, direction of that parallel light, and color offsets, gains, and

contrast. For details, see [Blanz and Vetter, 1999].

In the current version, the system is initialized by providing $2 \mathrm{D}$ image positions of some feature points, such as the tip of the nose, or the corners of the eyes. The algorithm then converges automatically, and produces the following results: shape and texture coefficients, 3D shape and texture $\mathbf{S}, \mathbf{T}$, pose parameters, such as head position, orientation, and illumination conditions.

The fact that parameters for pose and illumination are recovered from the image, and that they are represented separately and explicitly just as in computer graphics, implies that we can modify any of them independently, and draw the face from any new angle and under any new illumination.

Since the linear combination of textures $\mathbf{T}_{i}$ cannot reproduce all local characteristics of the novel face, such as moles or scars, which in fact may be highly relevant for recognition, we extract the person's true texture from the image wherever it is visible. This is done by an illumination-corrected texture extraction algorithm [Blanz and Vetter, 1999]. The boundary between the extracted texture and the predicted regions (where the true texture is occluded in the input image) was still visible in some of the images used in this test. We have meanwhile improved our algorithm to achieve a smooth transition, based on a reliability criterion for texture extraction.

None of the 3D faces that form the Morphable Model has facial hair. Still, texture extraction captures the beard in a texture map, and reproduces it on the frontal view, even if the beard's thickness is neglected in this transformation. In the same way, eyeglasses are mapped on the facial surface by the algorithm. Recently, we have extended the model to handle open-mouth facial expressions, but this was not yet applied to the FRVT02 data.

\section{Standard Hair-Style and Shoulders}

Since our model covers neither hair, nor shoulders, nor the scene behind the head, we cannot produce an estimate of these image regions in the transformed frontal view. As a result, we could leave the image blank around the face, ears, and top of the neck. However, these images would be quite different from the type of images that most face recognition systems are designed for, so the programs might at least require readjustment. In particular, this would be true if transformed and real frontal view images occured in the same test.

Therefore, we decided to render the reconstructed faces into a standard frontal view of one person, so all transformed images have the same hair-style, shoulders, and background (Figure 1).

As an alternative, we could render the faces into their individual frontal view images, which are part of the database. However, this would not reflect the realistic face recognition scenario, where nothing more than the oblique view is available. In fact, if we rendered the faces into the frontal views stored in the gallery, a simple comparison of some pixels around the faces would give away the identities in a trivial way.

As a standard frontal view, we selected an image with short, dark hair, that looks realistic on most faces. With additional gender recognition, which may be based on the $3 \mathrm{D}$ face reconstruction, it would be possible to select different standard images for different genders (or ethnic groups). 

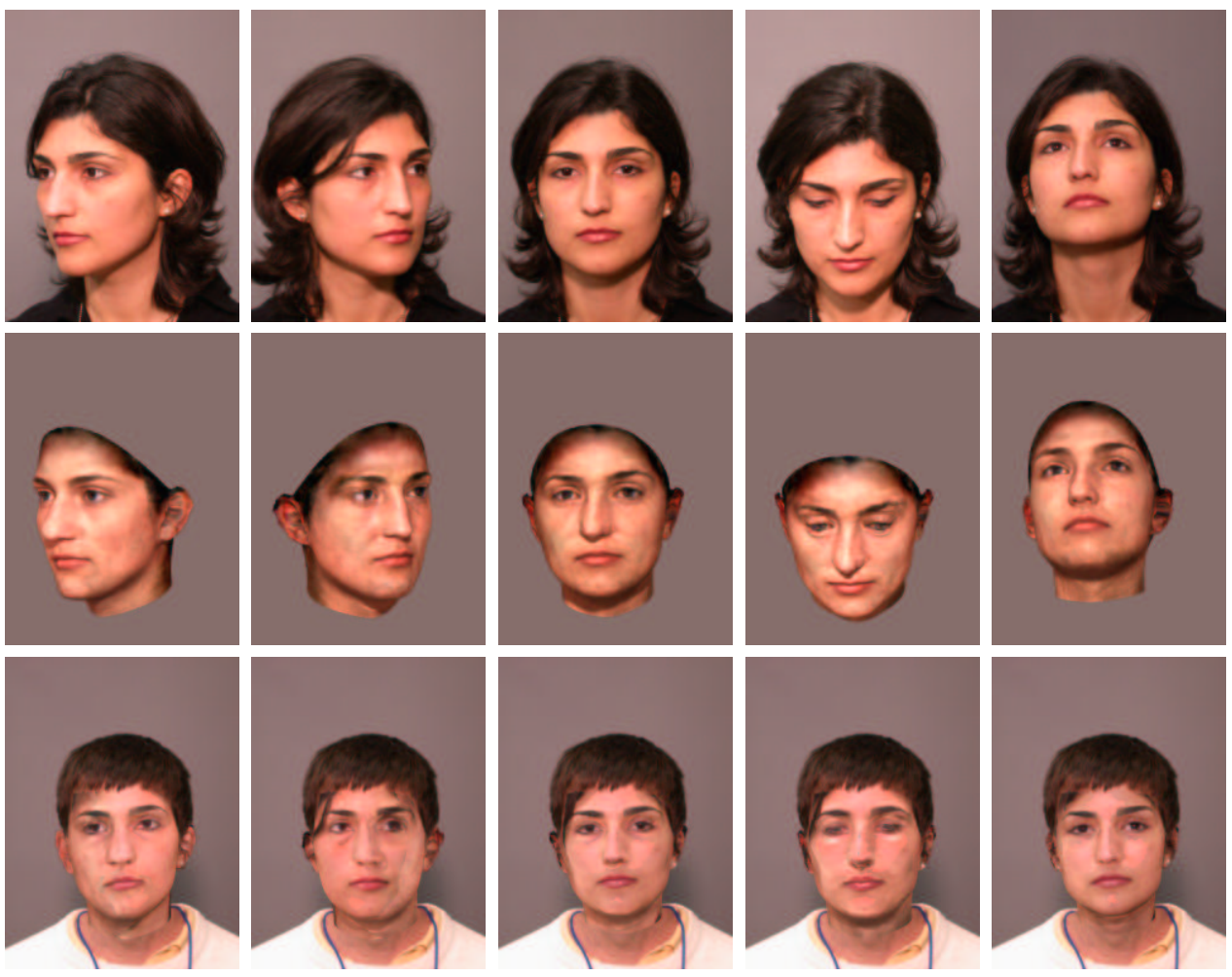

Figure 1: From the original images (top row), we recover 3D shape (second row), by finding an optimal linear combination of example faces. Mapping the texture of visible face regions on the surface and rendering it into a standard background, which is a face image that we selected, produces transformed front views (bottom row). Note that the frontal-to-frontal mapping, which served as a baseline test, involves hairstyle replacement (bottom row, center).

In order to determine head size, position, orientation and illumination of the standard image, we fitted the Morphable Model to the image, as described in the previous section. To be able to draw hair in front of the forehead, we manually defined a transparency map that is opaque on the hair, and transparent everywhere else, for the standard image.

Given a novel input image, we perform the following steps:

1. Manually define feature points, such as the tip of the nose, the corners of the eyes, or any points along the occluding contours on the cheeks. For FRVT02, we defined an average number of 11 feature points to ensure optimum quality. However, 6 points are often sufficient. In future systems, these points can be found by an automated feature detection algorithm.

2. Run the optimization ( 4 minutes on a Pentium 4 at $2 \mathrm{GHz}$ ).

3. Render the 3D face in front of the standard image, using the parameters for position, orientation, size, and illumination of the standard image.

4. Draw the hair in front of the forehead, using the transparency map. 


\section{Conclusions}

We have presented a method to handle images taken at arbitrary viewpoints with state-of-the-art face recognition systems. The combination of our reconstruction algorithm with other recognition systems may reduce their sensitivity to pose angle considerably. In the current state, our method requires manual labelling of a small number of feature points in the image. We anticipate that this labelling is no major problem in many applications, for example if a relatively small number of suspects' images have to be added to a gallery that is used for search. Combined with a feature detection algorithm, our system will be fully automated.

Acknowledgements: Philipp Georg has labelled the feature points in the input images. The 3D face scans were scanned by Niko Troje at Max-Planck-Institut für Biologische Kybernetik, Tübingen, Germany, in the department of Heinrich Bülthoff.

\section{References}

[Blanz et al., 2002] Blanz, V., Romdhani, S., and Vetter, T. (2002). Face identification across different poses and illuminations with a $3 \mathrm{~d}$ morphable model. In Proc. of the 5th Int. Conf. on Automatic Face and Gesture Recognition, pages 202-207.

[Blanz and Vetter, 1999] Blanz, V. and Vetter, T. (1999). A morphable model for the synthesis of 3d faces. In Computer Graphics Proc. SIGGRAPH'99, pages 187-194, Los Angeles.

[Duda et al., 2001] Duda, R., Hart, P., and Stork, D. (2001). Pattern Classification. John Wiley \& Sons, New York, 2nd edition.

[Vetter and Poggio, 1997] Vetter, T. and Poggio, T. (1997). Linear object classes and image synthesis from a single example image. IEEE Trans. on Pattern Analysis and Machine Intelligence, 19(7):733-742. 